\title{
Cyclisierungsreaktionen mehrfach ungesättigter Systeme - Theorie und Experiment -
}

\author{
DISSERTATION \\ Zur Erlangung des Doktorgrades \\ der Mathematisch-Naturwissenschaftlichen Fakultäten \\ der Georg-August-Universität zu Göttingen
}

vorgelegt von

Matthias Prall

aus Kassel

Göttingen 2002 
D7

Referent: Prof. Dr. Peter R. Schreiner

Korreferent: Priv.-Doz. Dr. Christoph Schneider

Tag der mündlichen Prüfung: 20.06.2002 
Die vorliegende Arbeit wurde unter der Leitung von Herrn Prof. Dr. Peter R. Schreiner in der Zeit von Juni 1998 bis Juni 2002 am Institut für organische Chemie der Georg-AugustUniversität zu Göttingen angefertigt.

Ich möchte mich bei meinem Lehrer Herrn Prof. Dr. Peter R. Schreiner herzlich für die interessante Themenstellung, die anregenden Diskussionen und die fortwährende Unterstützung im Verlauf dieser Arbeit bedanken. 
Meiner Familie 
$1 \quad$ Einleitung

2 Teil I: Theoretische Berechnungen thermischer

Umlagerungen

2.1 Grundlagen der Computer-Chemie ………………………………... 7

2.2 Wahl der Methode …………………………………………………….... 14

2.3 Cope-ähnliche Umlagerungen ………………………………………….. 20

2.4 Cyclisierungen ……………………………………………………………….. 33

2.4.1 Die Cyclisierung von Hex-3-en-1,5-diin: Was gibt es neben der "Bergman-Cyclisierung" ?

2.4.2 Die Cyclisierung von Hepta-1,2,4-trien-6-in: "Myers-Saito"-,

"Schmittel"- und weitere Cyclisierungen

2.4.3 Die Cyclisierung von Hexa-1,3-dien-5-in

("Hopf-Cyclisierung")

2.4.4 Die Cyclisierung von Hexa-1,3,5-trien

("[ 66$]$-disrotatorische Cyclisierung")

2.4.5 Die Cyclisierung von Octa-1,2,4,6,7-pentaen

2.4.6 Die Cyclisierung von Hepta-1,2,4,6-tetraen

2.5 Schlußbetrachtung

3 Teil II: Experimente zur kationisch induzierten

Cyclisierung 100

3.1 Grundlagen 100

3.2 Synthese der Cyclisierungs-Ausgangsverbindungen 107

3.3 Kationisch induzierte Additions- und

Cyclisierungsreaktionen 116

3.4 Schlußbetrachtung 
5 Experimentalteil

6 Literaturverzeichnis

$7 \quad$ Anhang

7.1 Abbildungen der ${ }^{1}$ H-NMR Spektren

7.2 Abbildungen der ${ }^{13} \mathrm{C}$-NMR Spektren

7.3 Molekülverzeichnis

7.4 Abkürzungen und Akronyme 202

7.5 Publikationsliste 204

Danksagung 205

Lebenslauf 206 


\section{Einleitung}

Reaktionen, die unter Umlagerung des Kohlenstoffgerüsts und gleichzeitiger Verschiebung von Mehrfachbindungen ungesättigte Moleküle ineinander überführen können, sind sowohl in der präparativen Chemie als auch in der Pharmazie von herausragender Wichtigkeit. Bekannte Beispiele thermischer Umlagerungen aus der organischen Chemie sind die Cope-Umlagerung von 1,5-Hexadien-Derivaten 1,1,2 die Claisen-Umlagerungen von Allylvinylethern 2 zu Pent-4-enal-Derivaten 3,-5 sowie die Diels-Alder-Reaktion zwischen Dien 4 und Dienophil 5 zu Cyclohexenen 6 $^{6-8}$ und die thermische, disrotatorische Cyclisierung von 1,3,5-Hexatrien 7 zu Cyclohexadien-Derivaten 8 (Abb. 1). ${ }^{9-11}$

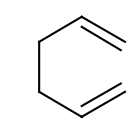

1<smiles>C=CCOCC=C</smiles>

2

$\checkmark$

4<smiles>C=CC=CC=C</smiles>

7
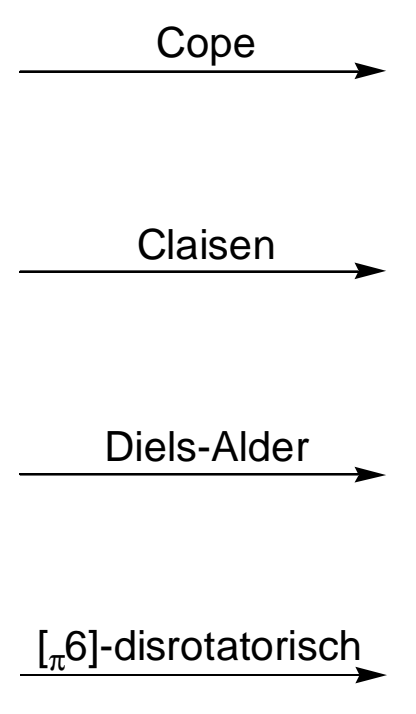

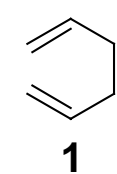

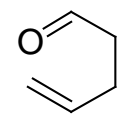

3<smiles>C1=CCCCC1</smiles>

6<smiles>C1=CCCC=C1</smiles>

8

Abbildung 1. Verschiedene Umlagerungen von ungesättigten Verbindungen

In den letzten Jahren fanden auch thermische Cyclisierungen, die Produkte mit ungepaarten Elektronen liefern, Eingang in die organische Synthese. Insbesondere sind hier die Bergman-Cyclisierung von Hex-3-en-1,5-diin-Derivaten 9 ("Endiine") zu 1,4Didehydrobenzolen 10 ("Bergman-Produkte") ${ }^{12-15}$ und die Myers-Saito-Cyclisierung von Hepta-1,2,4-trien-6-in-Derivaten 11 ("Eninallene") zu $\alpha, 3$-Didehydrotoluolen 12 ("MyersSaito-Produkte") zu nennen (Abb. 2). ${ }^{16-19}$ Obwohl die Reaktionen in Abbildung 1 und Abbildung 2 auf den ersten Blick nichts miteinander $\mathrm{zu}$ tun haben, liegt ihnen doch ein gleiches Prinzip zu Grunde. Bei genauerer Betrachtung handelt es sich bei der Bergman- und Myers-Saito-Cyclisierung formal um nichts anderes als Abwandlungen der Cope-Umlagerung mit ungesättigterem Charakter. 


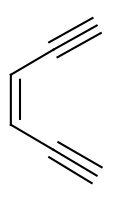

9<smiles>C#C/C=C\C=C=C</smiles>

11

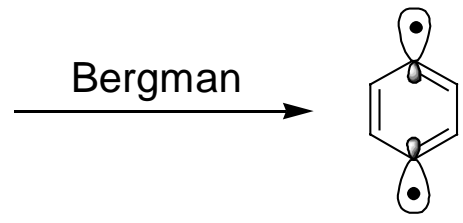

10

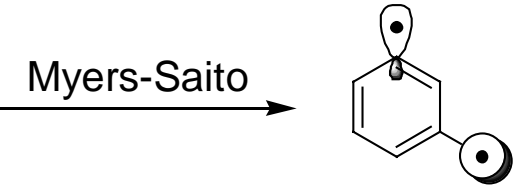

12

Abbildung 2. Bergman- und Myers-Saito-Cyclisierung

Die Bergman- und Myers-Saito-Cyclisierungen sind vor allem in der Naturstoffsynthese von Bedeutung, seit bekannt ist, daß die DNA-schädigenden Eigenschaften einiger Antitumor-Antibiotika, wie z.B. Calicheamicin $\gamma_{1}^{\mathrm{I}}$ 13, Dynemicin 14 und Neocarzinostatin 15, auf die Cyclisierung und die damit einhergehende Biradikalbildung solcher Endiin- und Eninkumulen-Untereinheiten zurückzuführen sind (Abb. 3). ${ }^{20-25}$

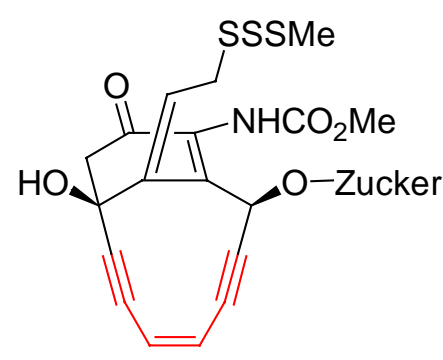

13

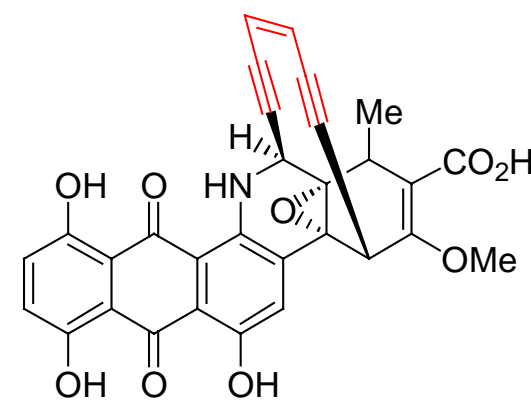

14<smiles>COc1cc2ccccc2cc1C1COC(=O)O1</smiles>

15

Abbildung 3. Calicheamicin $\gamma_{1}^{\mathrm{I}}$ 13, Dynemicin 14 und Neocarzinostatin 15

Die normalerweise endotherm verlaufende Bergman-Cyclisierung ${ }^{26}$ wird in diesen Molekülen durch Erhöhung der Ringspannung ausgelöst. Durch einen intramolekularen Angriff wird die Struktur von 13 soweit verändert, daß die Reaktion exotherm verläuft, um Ringspannung abzubauen (Abb. 4). Das entstehende hochreaktive Biradikal 16 abstrahiert dann zwei HAtome von der DNA, die daraufhin abgebaut wird. ${ }^{20-24,27,28}$ Im Neocarzinostatin 15 wird die Ringspannung durch einen intermolekularen Angriff erreicht, der zur Ausbildung des hochgespannten Eninkumulen-Gerüstes 17 führt. ${ }^{29}$ 


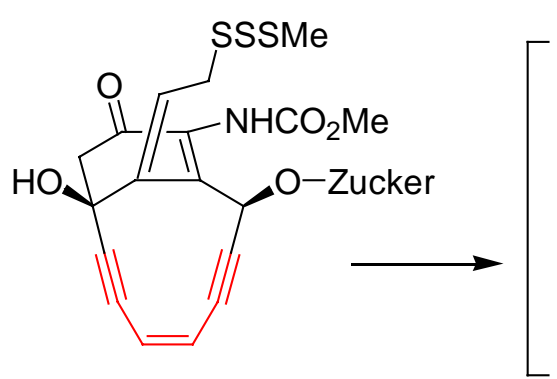

13
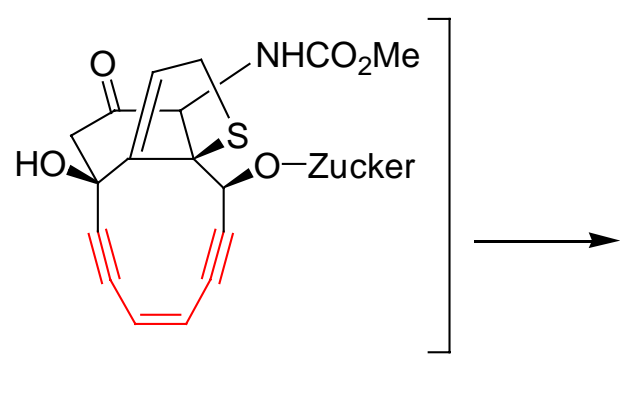

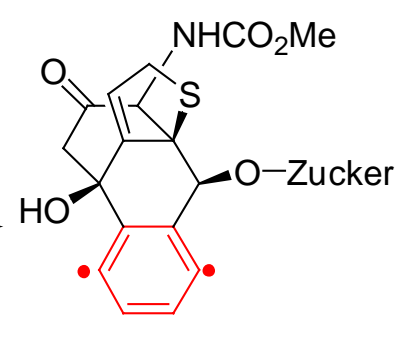

16

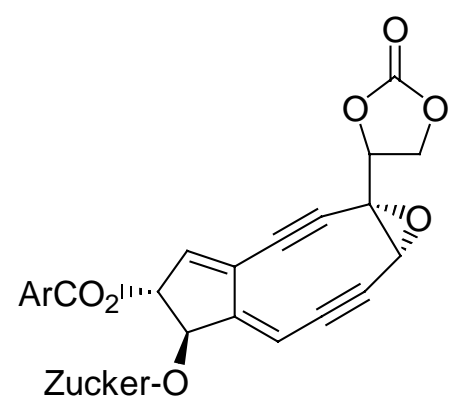

15

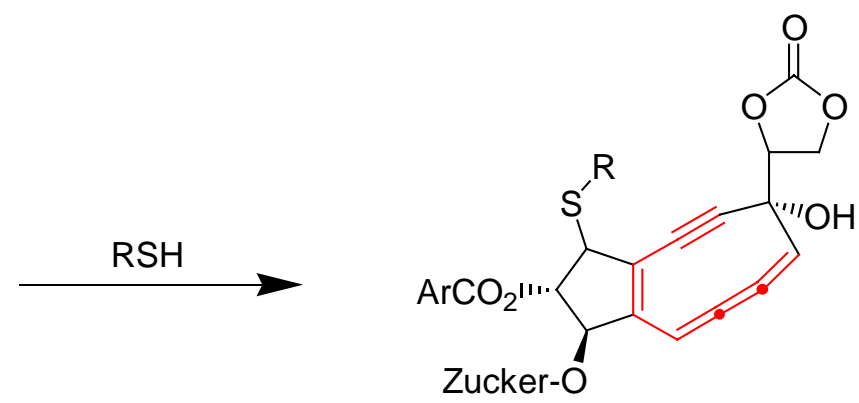

17

Abbildung 4. Erhöhung der Ringspannung und damit der Reaktivität von Calicheamicin $\gamma_{1}^{\mathrm{I}} \mathbf{1 3}$ und Neocarzinostatin 15

Ziel der präparativen und pharmazeutischen Chemie ist es, solche Endiin-Antibiotika zu synthetisieren, die unter normalen physiologischen Bedingungen inaktiv sind und die sich in Tumorzellen anreichern. Dort sollen sie aktiviert werden und ihr zerstörerisches Werk vollbringen, indem die Tumorzell-DNA irreparabel geschädigt, und dadurch die Tumorzelle abgetötet wird. Dieser Aktivierungs-Schalt-Mechanismus ("Trigger-Mechanismus") kann wie bei den Naturstoffen durch Erhöhen der Spannung im Ring, 30-36 durch Metallkomplexierung $37-41$ oder aber durch gezielte Substituentenmuster funktioneller Gruppen erreicht werden. ${ }^{42}$

Zum besseren Verständnis der Reaktivität mehrfach ungesättigter Systeme und besonders auch der Naturstoffe, ist es vor der Synthese wichtig, die Grundlagen der Umlagerungen an einfachen Stammsystemen herauszuarbeiten. Dazu ist die Computerchemie ein exzellentes Mittel und kann viele unnötige Experimente vermeiden. Mit Hilfe der Computerchemie können die Stammsysteme eingehend untersucht und von allen Seiten beleuchtet werden. Anschließend können Veränderungen an diesem System vorgenommen werden, die nach Auswertung der gewonnenen Daten sinnvoll erscheinen. Die Effekte dieser Veränderungen können dann auf ihre Nützlichkeit hin untersucht werden, bevor die vielversprechendsten Strukturen synthetisiert werden. 
Neben den zahlreichen thermisch initiierten Cyclisierungen, gibt es einige wenige Beispiele in der Literatur, bei denen sie auch kationisch, anionisch und radikalisch eingeleitet werden konnte. ${ }^{43-47}$ Die dabei erhaltenen Produkte entsprechen in der Struktur im Allgemeinen nicht denen der thermischen Cyclisierungen. Ein gutes Beispiel ist die Cyclisierung von 1,2-Bis(phenylethinyl)benzol 18 (Abb. 5). Während die thermische Cyclisierung über das schon bekannte substituierte Bergman-Produkt 19 ein substituiertes Naphthalinderivat 20 ergibt, ${ }^{48}$ werden bei der induzierten Cyclisierung zwei diastereomere Benzfulvenderivate $(\boldsymbol{Z})$-21 und $(\boldsymbol{E})$-21 erhalten. ${ }^{45}$

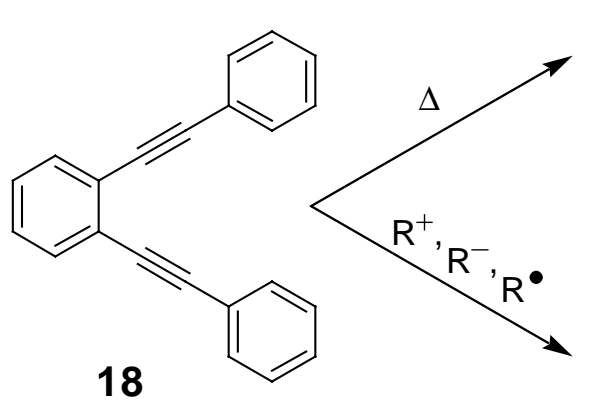

$(Z)-21$

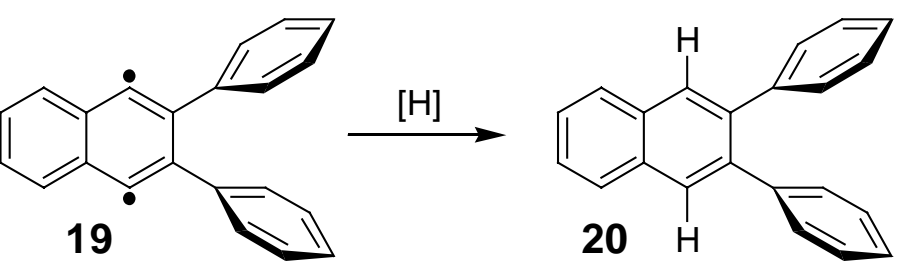

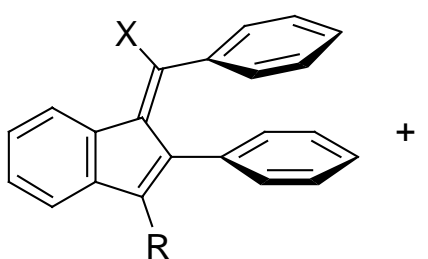

$R$

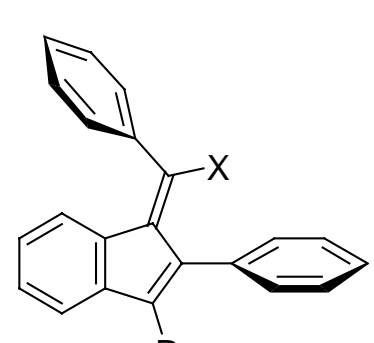

$(E)-21 R$

Abbildung 5. Thermische und kationisch induzierte Cyclisierungen von 1,2-Bis(phenylethinyl)benzol 18

Die vorliegende Arbeit verfolgt zwei Ziele. In einem ersten, theoretischen Teil wird mit Hilfe der Computerchemie die Familie der Cope-ähnlichen Umlagerungen und Cyclisierungen eingehend durchleuchtet (Abb. 6). Obwohl einige Einzelstudien, insbesondere über die Cope-Umlagerung, bereits existieren, und obwohl Hopf ${ }^{49}$ und Houk ${ }^{50}$ bereits ein System hinter den Cyclisierungen erkannt hatten, wurde eine systematische Studie über den Familienzweig 1 bisher noch nicht durchgeführt. Für die nicht bekannten Mitglieder dieses Familienzweiges werden in dieser Arbeit präzise Vorhersagen über die Energetiken und Produkte der Umlagerungen gemacht. Für Familienzweig 2, besonders auf dem Gebiet der Bergman-Reaktion, ${ }^{32,33,51-57}$ gibt es ebenfalls schon einige Studien. Diese Ergebnisse wurden noch einmal nachempfunden, um die Anwendbarkeit und Qualität der gewählten Methoden zu demonstrieren. Das Hauptaugenmerk liegt jedoch auf den Cyclisierungen, die bisher noch nicht systematisch untersucht wurden. Neben den experimentell bekannten Cyclisierungspfaden, soll dabei auch nach anderen, vielleicht unwahrscheinlicheren, aber nicht unmöglichen Cyclisierungen gesucht werden. Gleichzeitig wird der Effekt der Benzannelierung auf 
die Reaktivität der Mitglieder des Familienzweiges 2 untersucht. Weitere Schwerpunkte liegen auf der Auswirkung von Ringspannung auf die normalerweise endotherm ablaufenden Reaktionen, sowie auf den Substituenteneffekten auf die Bergman-Cyclisierung, beides zum Zweck der Reaktivitätserhöhung und -kontrolle.

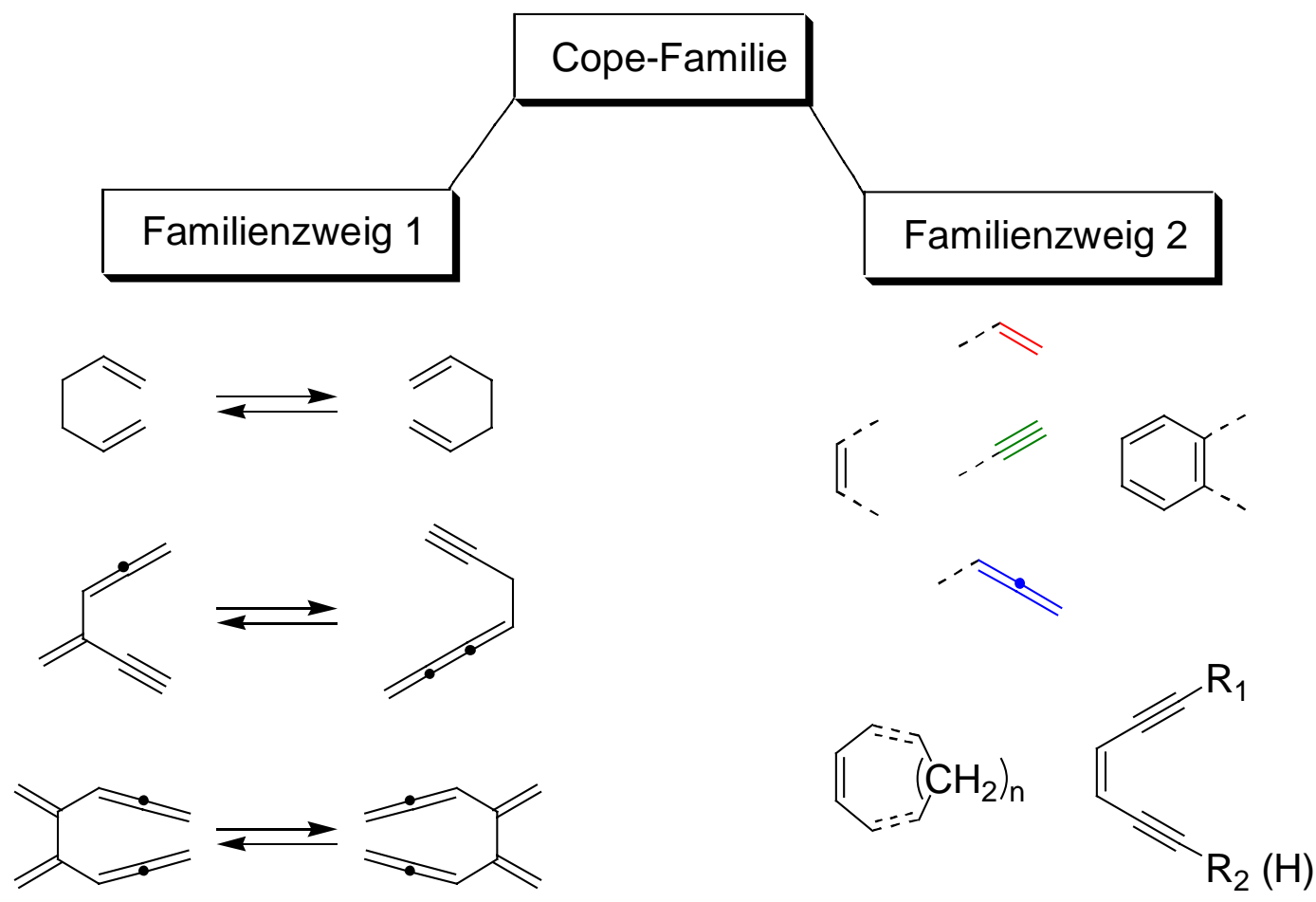

Abbildung 6. Die beiden Familienzweige der Cope-Familie mit einigen Beispielen

In einem zweiten, praktischen Teil wird die Reaktivität sorgsam ausgewählter, mehrfach ungesättigter Systeme gegenüber Elektrophilen untersucht und anhand dieser Ergebnisse versucht, eine Methode zu erarbeiten, die Reaktionen bisher nicht untersuchter Moleküle vorherzusagen. Bereits bekannte Ergebnisse werden zum Teil reproduziert, um deren Charakterisierung zu vervollständigen. Eine entsprechende Veränderung, Erweiterung oder Vergrößerung der Systeme, wie z.B. die Substanzen 22, 23, 24 und 25, sollte eine interessante Vielfalt von "Zipper"-Reaktionen hervorbringen (Abb. 7). Insbesondere die Regioselektivität bei mehreren möglichen Cyclisierungsarten, die unterschiedlichen Reaktivitäten der Doppel- und Dreifachbindungen, sowie die Abhängigkeit der Produktgeometrie von den Substituenten, sind die Schwerpunkte dieser Studie. 
<smiles>C(#Cc1cccc(C#Cc2ccccc2)c1C#Cc1ccccc1)c1ccccc1</smiles>

22<smiles>C(#Cc1ccccc1C#Cc1ccccc1C#Cc1ccccc1)c1ccccc1</smiles>

23<smiles>[X]c1ccccc1C#Cc1ccccc1-c1ccccc1</smiles><smiles>OC(F)(F)C#Cc1ccccc1C=C(c1ccccc1)c1ccccc1</smiles>

25

Abbildung 7. Verschiedene ungesättigte Systeme für "Zipper"-Reaktionen 


\section{Teil I: Theoretische Berechnungen thermischer Umlagerungen}

\subsection{Grundlagen}

Durch quantenmechanische Berechnungen ist es im Prinzip möglich, alle chemischen Phänomene quantitativ vorherzusagen. Die einzigen Daten, die man für solche Berechnungen benötigt, sind eine kleine Anzahl physikalischer Konstanten (die Lichtgeschwindigkeit im Vakuum, das Planck'sche Wirkungsquantum und die Massen und Ladungen der Elektronen und Kerne). Die Lösung der quantenmechanischen Gleichungen für ein chemisches System könnte man als $a b$ initio-Chemie bezeichnen (,ab initio” Lat. = "von vorne herein”). Die $a b$ initio-Chemie (oder Quantenchemie) ist unabhängig von Experimenten, abgesehen von der experimentellen Bestimmung der erwähnten, notwendigen physikalischen Konstanten.

In der Praxis stellt es sich jedoch heraus, daß die Lösung der quantenmechanischen Gleichungen ein so riesiges numerisches Problem darstellt, daß die berechneten Ergebnisse nicht immer die exakte Genauigkeit eines Experimentes erreichen. Die genäherten Lösungen der Schrödinger-Gleichung sind jedoch so genau, daß mit ihrer Hilfe Geometrien und Eigenschaften von Molekülen berechnet werden können, die dann zur Bestimmung und Vorhersage der Reaktivitäten und Selektivitäten von Reaktionen in einem gewissen Fehlerrahmen verwendet werden können. Die Qualität der Ergebnisse hängt dabei von der Größe des Moleküls, der verwendeten ab-initio Methode, dem Basissatz und der Computerpower ab. Im folgenden sollen kurz die in dieser Arbeit verwendeten Methoden vorgestellt werden.

Hartree-Fock (HF). ${ }^{58,59}$ Die Hartree-Fock-Methode basiert auf dem Konzept des selbstkonsistenten Feldes (self consistent field, SCF). Ausgangspunkt ist die SchrödingerGleichung für die Wellenfunktion eines Systems:

$$
\hat{H} \Psi=E \Psi
$$

Unter der Annahme ruhender Kerne (Born-Oppenheimer-Näherung), kann der HamiltonOperator in den Beitrag aus der Kernabstoßung

$$
V_{N N}=\sum_{A} \sum_{B>A} \frac{q_{A} q_{B}}{r_{A B}}
$$

und den elektronischen Beitrag unterteilt werden. Für ein $n$-Elektronensystem ergibt sich dieser zu 


$$
\hat{H}_{\text {elek }}=-\frac{1}{2} \sum_{i}^{n} \nabla_{i}^{2}+\sum_{i}^{n} V\left(r_{i}\right)+\sum_{i}^{n} \sum_{j>i}^{n} \frac{1}{\left|r_{i}-r_{j}\right|}
$$

wobei der erste Term die kinetische Energie der Elektronen, der zweite die potentielle Energie der Elektronen im Feld der Kerne und der dritte die potentielle Energie der Elektronen im Feld der anderen beschreibt. Da es sich dabei um ein Vielelektronensystem handelt (klassisches Mehrkörperproblem), ist dieses nicht analytisch lösbar und ein iterativer Prozeß wird notwendig, in dem nicht jedes Elektron mit jedem wechselwirkt, sondern jedes Elektron mit dem effektiven Gesamtfeld aller anderen Elektronen. Dabei werden folgende Schritte durchlaufen:

1. Begonnen wird mit einem Satz einfacher ,geschätzter" Orbitale für alle Elektronen.

2. Ein Elektron wird ausgewählt und das Potential, in dem es sich bewegt, wird bestimmt, indem die Wechselwirkung mit dem effektiven Gesamtfeld berechnet wird.

3. Die Schrödinger-Gleichung wird für dieses Potential gelöst, was ein neues Orbital für das Elektron ergibt.

4. Die Schritte 2. und 3. werden für alle anderen Elektronen wiederholt.

5. Mit dem neuen Satz von Orbitalen werden die Schritte 2. bis 4. solange wiederholt, bis die Gesamtenergie des Systems konstant bleibt (selbstkonsistent).

Da die genäherte Energie im Laufe des Prozesses immer kleiner wird und sich asymptotisch einem Grenzwert annähert, der größer oder gleich der korrekten Energie ist, gehorcht diese Methode dem Variationsprinzip.

$$
E_{0} \leq \frac{\left\langle\Psi^{\prime}|\hat{H}| \Psi^{\prime}\right\rangle}{\left\langle\Psi^{\prime} \mid \Psi^{\prime}\right\rangle}
$$

Dichtefunktionaltheorie (DFT). ${ }^{60}$ Die grundlegende Idee der DFT ist, daß die Energie eines elektronischen Systems in Termen der Elektronenwahrscheinlichkeitsdichte $\rho$ geschrieben werden kann. ${ }^{61}$ Die elektronische Energie ist dann ein Funktional der Elektronendichte.

$$
E^{D F T}[\rho]=T_{S}[\rho]+E_{n e}[\rho]+J[\rho]+E_{X C}[\rho]
$$

wobei der erste Term die kinetische Energie der Elektronen, der zweite die Anziehung zwischen Elektronen und Kernen und der dritte die Coulomb-Wechselwirkungen zwischen den Elektronen beschreibt. Der vierte Teil ist der sogenannte Austausch-Korrelations-Term, 
in dem die Austauschwechselwirkungen der Elektronen und die Interaktion der Orbitale zusammengefaßt sind. Den eindeutigen Zusammenhang zwischen Vielteilchen-Wellenfunktion und Elektronendichte zeigt das Hohenberg-Kohn-Theorem. ${ }^{61}$ Kohn und Sham konnten dann das Vielteilchenproblem in ein effektives Einteilchenproblem überführen, indem sie Orbitale ähnlich denen der HF-Theorie einführten. ${ }^{62}$ Natürlich muß auch die KohnSham-Gleichung wie die SCF-Gleichnung iterativ gelöst werden. Ein DFT-Funktional besteht immer aus zwei Teilen: dem Austausch- (z.B. B ${ }^{63}, \mathrm{~S}^{61,62,64}$, G9661,62,64-66, B367) und dem Korrelationsfunktional (z.B. LYP68,69, PW9170, VWN ${ }^{71}$ ), die alle frei miteinander kombinierbar sind (z.B. BLYP, SVWN). Zu unterscheiden sind dabei noch die "reinen" und die Hybrid-DFT Methoden. Während z.B. BLYP eine reine Methode darstellt, ist in B3LYP das Austauschfunktional B3 ein Hybrid, das aus dem reinen B und Termen aus der HFRechnung besteht. Die geeignete Wahl der Kombination hängt dabei vom zu beschreibenden System ab und sollte vor jeder Anwendung auf ihre Tauglichkeit geprüft werden (nähere Einzelheiten siehe nächstes Kapitel).

Møller-Plesset-Störungsrechnung (MPn). ${ }^{72}$ Die Verwendung der Störungstheorie zur Abschätzung der Korrelationsenergie geht auf einen Vorschlag von Møller und Plesset zurück, den im Rahmen der Hartree-Fock-Näherung verwendeten Hamiltonoperator als ungestörten Operator anzusehen. Die Differenz zwischen diesem und dem exakten Hamiltonoperator ist dann der Störoperator.

$$
\widehat{H}=\hat{H}^{(0)}+\hat{V}
$$

Dabei wird unterstellt, daß der Beitrag des Störoperators klein ist im Verhältnis zu dem des ungestörten Operators. Die exakte Grundzustandsenergie, sowie die Wellenfunktion werden in Potenzreihen der Form

$$
\begin{gathered}
E_{0}=E^{(0)}+\lambda E^{(1)}+\lambda^{2} E^{(2)}+\ldots+\lambda^{k} E^{(k)} \\
\text { und } \Psi_{0}=\Psi^{(0)}+\lambda \Psi^{(1)}+\lambda^{2} \Psi^{(2)}+\ldots+\lambda^{k} \Psi^{(k)}
\end{gathered}
$$

mit den Störtermen $k$-ter Ordnung entwickelt. Der Energieterm nullter Ordnung ist gleich der Summe der Orbitalenergien, die Summe aus nullter und erster Ordnung ist die HF-Energie. Die Störenergie zweiter Ordnung kann dann relativ einfach berechnet werden. Ein gravierendes Problem ist, daß der bei Routineberechnungen übliche Abbruch der Energieentwicklung mit der zweiten Ordnung (MP2) dazu führt, daß die so berechneten Energien nicht mehr dem Variationsprinzip unterliegen. 
Multikonfigurations-Methoden (CASSCF). ${ }^{73-75}$ Moleküle, die elektronisch verschiedene Resonanzstrukturen oder nicht eindeutige Spinzustände besitzen, können nur durch Multikonfigurationsmethoden richtig beschrieben werden. Ozon kann beispielsweise durch zwei ionischen und eine biradikalische Strukturen beschrieben werden (Abb. 8). Während die ionischen Strukturen beide durch dieselbe Konfiguration beschrieben werden können, ist für die biradikalische eine zweite notwendig.

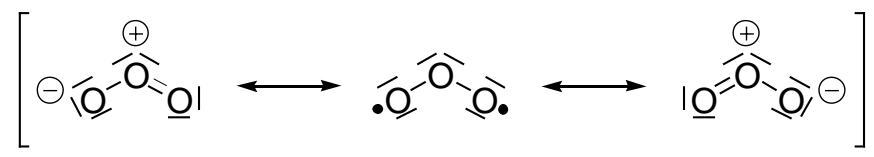

Abbildung 8. Resonanzstrukturen von Ozon

In diesen Fällen wird jeder Resonanzstruktur eine eigene Wellenfunktion zugeschrieben und mit einem Koeffizienten versehen, der die Wichtung dieser Struktur anzeigt. Die Koeffizienten und die Orbitale werden dann im Rahmen der Rechnung optimiert, wobei die Summe aller Koeffizienten zu jederzeit eins sein muß. Für die am häufigsten verwendete Methode des vollständig aktiven Raumes (complete active space self consistent field, CASSCF), wird nur ein Satz wichtiger Konfigurationen ausgewählt, die den aktiven Raum aufspannen, in dem die Elektronen dann in alle möglichen Zustände verteilt werden (Abb. 9). Da die Auswahl der kritischen Konfigurationen nicht einfach ist, führt die unsachgemäße Verwendung häufig zu falschen oder nicht sinnvollen Ergebnissen.

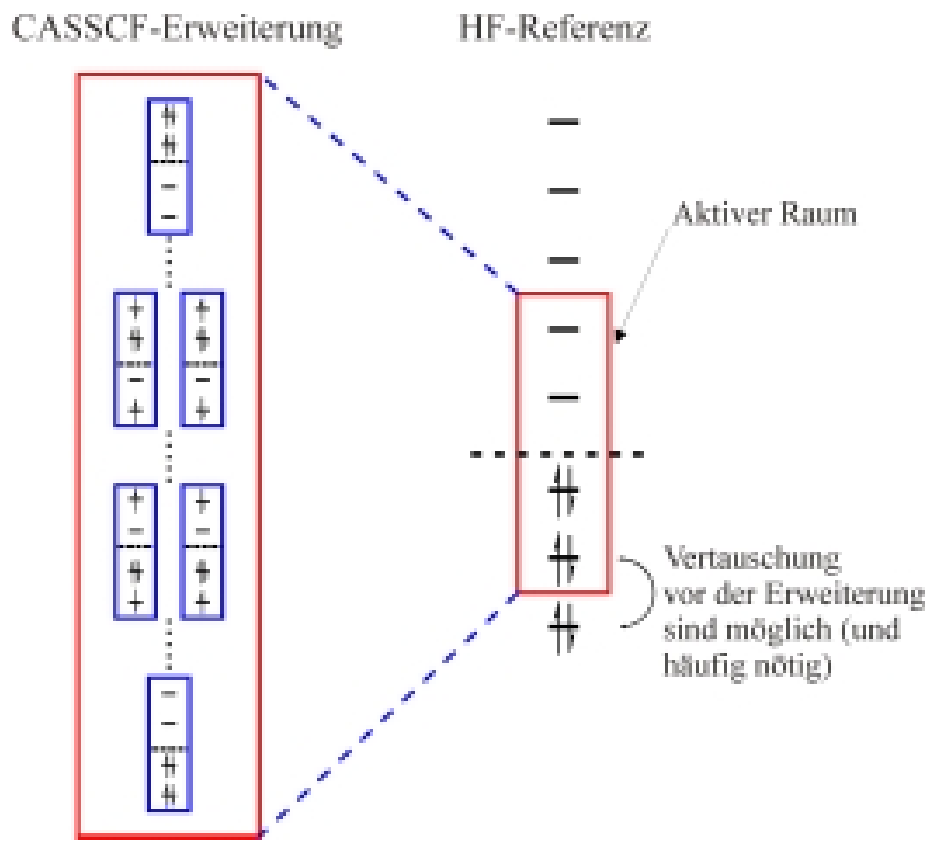


Coupled Cluster Methoden (CC). ${ }^{76,77}$ In der coupled cluster-Theorie wird die Wellenfunktion als Reihe entwickelt. Dabei wird die Referenzwellenfunktion (HF) durch den sogenannten cluster-Operator expandiert, der sich aus der Summe aller möglichen clusterAnregungsoperatoren zusammensetzt, die wiederum durch die cluster-Amplituden gewichtet sind.

$$
\Psi^{C C}=e^{T} \Phi^{H F}=\left(\sum_{k=0}^{K} \frac{1}{k !} \widehat{T}^{k}\right) \Phi^{H F} \text { und } \widehat{T}=\widehat{T}_{1}+\widehat{T}_{2}+\widehat{T}_{3}+\ldots+\widehat{T}_{L}
$$

Die am Häufigsten verwendete Methode ist der CCSD(T)-Ansatz, der die singles und doubles Amplituden (Anregungen) beinhaltet ${ }^{78}$ und die triples störungstheoretisch miteinbezieht. ${ }^{79}$ Diese Methode gibt äußerst exakte Ergebnisse für geschlossenschalige Moleküle, hat jedoch die Nachteile, daß sie sehr zeitaufwendig ist und nicht dem Variationsprinzip folgt. Eine Verbesserung für offenschalige Spezies bringt der BD(T)-Ansatz mit Brueckner-Orbitalen, ${ }^{80-}$ 83 die erst in Anwesenheit der Korrelationsstörung optimiert (rotiert) werden, bis die singlesAmplitude null ist, bevor die Wellenfunktion expandiert wird.

Basissätze. ${ }^{84}$ Basissätze sind die mathematische, approximative Darstellung von Orbitalen. Zunächst wurden die sogenannten Slater-Orbitale (Slater-type-orbitals, STOs) verwendet, die den Orbitalen der exakten Lösung für das Wasserstoffatom sehr nahe kommen.

$$
\chi_{\zeta, n, l, m}^{S T O}(r, \Theta, \varphi)=N Y_{l, m}(\Theta, \varphi) r^{n-1} e^{-\zeta r}
$$

Da jedoch mathematisch Gauß-Funktionen leichter zu integrieren sind als die STOs, ging man dazu über, jedes STO durch ein Produkt mehrerer primitiver Gauß-Funktionen (PGTOs) zu beschreiben (Abb. 10).

$$
\chi_{\zeta, n, l, m}^{G T O}(r, \Theta, \varphi)=N Y_{l, m}(\Theta, \varphi) r^{(2 n-2-l)} e^{-\zeta r^{2}}
$$

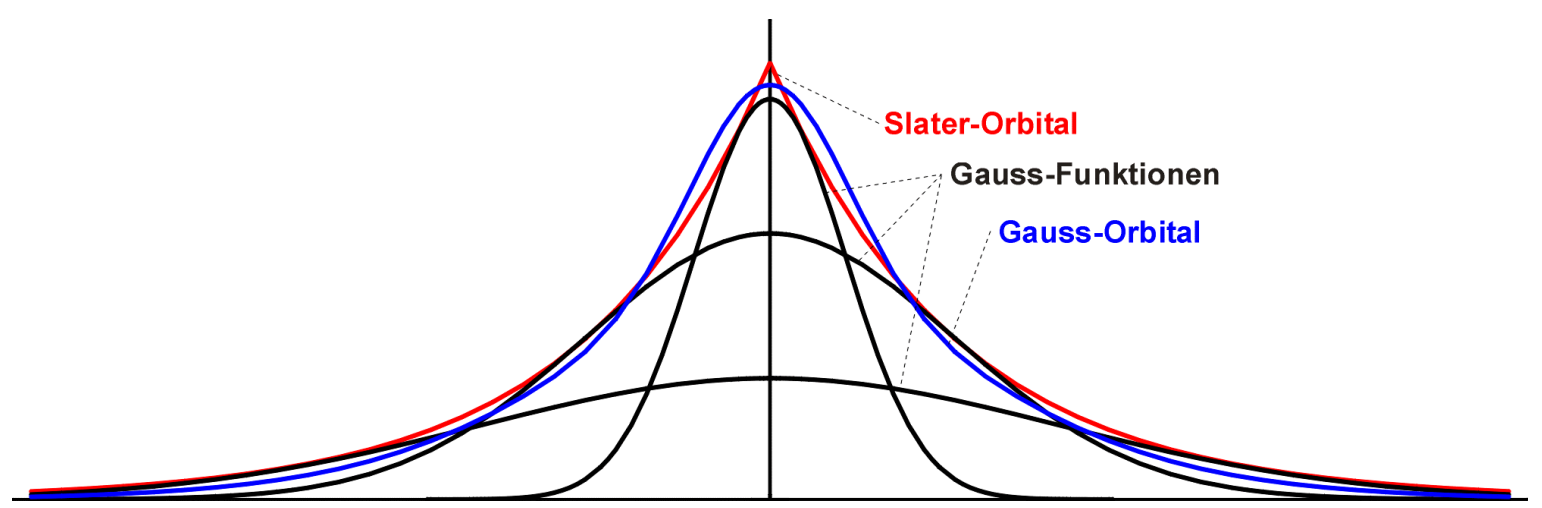

Abbildung 10. Abbildung eines STOs und eines aus drei primitiven GTOs zusammengesetztes GTO 
Der minimale Basissatz (STO-3G) enthält die Anzahl von Basisfunktionen, die zur Beschreibung der Atome gerade ausreicht (z.B. H: 1s; C:1s, 2s, 2px, 2py, 2pz). Minimale Basissätze repräsentieren atomähnliche Orbitale fester Größe.

Split-valence Basissätze verwenden zwei oder mehrere Basisfunktionen für jedes Valenz-Orbital (z.B. 3-21G $\rightarrow$ H: 1s, 1s'; C: 1s, 2s, 2s', 2px, 2px', 2py, 2py', 2pz, 2pz'), wodurch die zur Beschreibung anisotroper Elektronenverteilungen nötige, zusätzliche Flexibilität in den Orbitalen zur Verfügung gestellt wird (Abb. 11). Die Anzahl solcher Gruppen von Basisfunktionen wird auch als double-, triple-, usw. $\xi$ (Zeta, DZ, TZ, usw.) bezeichnet.

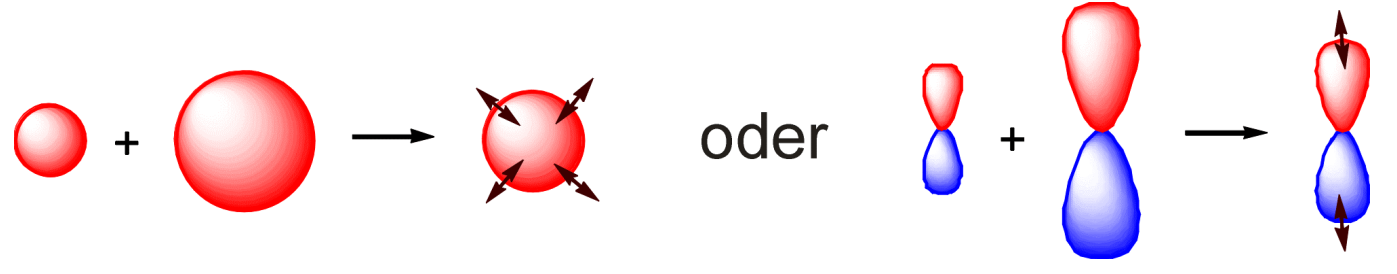

Abbildung 11. Flexibilitätsgewinn in split-valence Basissätzen

Die Einführung polarisierter Basissätze erlaubt die Beeinflussung der Gestalt der Orbitale. Das wird erreicht, indem Orbitale mit einer höheren Nebenquantenzahl hinzugefügt werden (z.B. p-Funktionen für Wasserstoff und d-Funktionen für die Elemente der zweiten Periode, Abb. 12). Dies wird als * oder (d) bzw. ** oder (d,p) für d-Funktionen an Schweratomen, bzw. zusätzlich p-Funktionen für Wasserstoff, in der Basissatzbezeichnung vermerkt (z.B. 6-31G*).

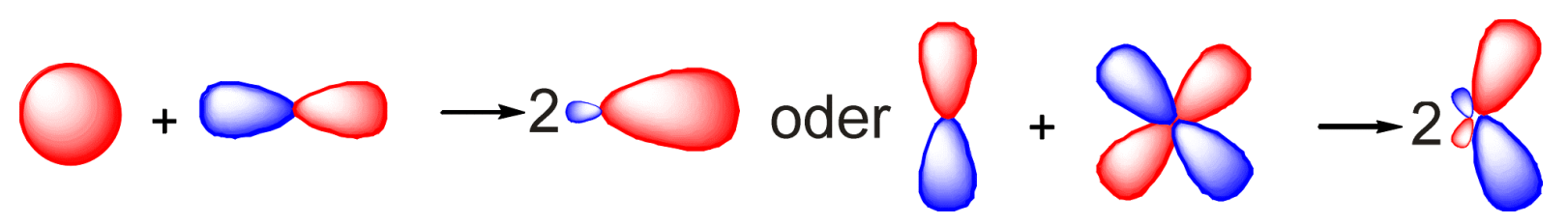

Abbildung 12. Polarisierung der Orbitale durch Einmischung von Orbitalen höherer Nebenquantentzahlen

Bei Rechnungen zur Beschreibung von Anionen, Wasserstoffbrücken oder angeregten Zuständen, bei denen die Elektronen auch weit von den Atomkernen entfernt sein können, ist manchmal die Verwendung zusätzlicher, diffuser Funktionen notwendig. Dabei werden Orbitale mit großer räumlicher Ausdehnung zugefügt. Dies wird als + bzw. ++ für diffuse sund p-Funktionen an Schweratomen bzw. zusätzlich diffuse s-Funktionen für Wasserstoff in der Basissatzbezeichnung vermerkt (z.B. 6-31++G**). 
Die Zahlen für die Pople-Basissätze sollen am Beispiel 3-21G erläutert werden. Die drei bedeutet dabei, daß die Rumpforbitale (wie bei STO-3G) durch drei primitive Gaussfunktionen beschrieben werden. Die kompakteren Valenzorbitale werden durch zwei, die diffuseren durch eine Gaussfunktion beschrieben, was die folgenden zwei Zahlen erklärt.

Zur Bestimmung hochwertiger Energien werden sogenannte Einzelpunktenergien berechnet, d.h. von sehr guten Geometrien aus HF- oder DFT-Berechnungen wird mit hochkorrelierten Methoden nur einmal die Wellenfunktion ermittelt und ohne Geometrieoptimierung die Energie der Wellenfunktion bestimmt. Für solche Berechnungen sollten korrelationskonsistente (correlation-consistent, cc) Basissätze, wie z.B. Dunnings cc-pVXZ (correlation-consistent polarized valence X-zeta), verwendet werden, die eigens für solche Fälle optimiert wurden. ${ }^{85}$

Verfahren. Die Rechnungen im Rahmen dieser Arbeit wurden mit den Programmpaketen GAUSSIAN9486 und GAUSSIAN98 ${ }^{87}$ durchgeführt. Die Strukturen wurden durch Frequenzrechnungen als Grundzustand (NImag $=0$ ) oder Übergangszustand erster Ordnung (NImag $=1$ ) identifiziert. Aus denselben Rechnungen stammen die thermischen Korrekturen und die Nullpunkt-Schwingungsenergie (zero point vibrational energy, ZPVE), die zu Berechnungen von $\Delta \mathrm{H}_{0}$ Enthalpien unkorrigiert eingesetzt wurden. Die Wahl der Methode und des Basissatzes für die Berechnungen wird im Folgenden beschrieben. 


\subsection{Wahl der Methode}

Die Beschreibung organischer Reaktionen, die biradikalische Zwischenprodukte oder Übergangszustände beinhalten, sind und bleiben eine der großen Herausforderungen der Quantenchemie.88-93 Die Schwierigkeiten entstehen durch die Tatsache, daß bei biradikalischen Spezies mit einer kleinen Singulett-Triplett-Separation $\left(\Delta \mathrm{E}_{\mathrm{ST}}\right)$ die angenäherte Wellenfunktion in manchen Fällen eine niedrigere elektronische Energie erreichen kann, wenn sie die Raum- oder Spinsymmetrie der exakten Wellenfunktion bricht. ${ }^{94-96}$ Aus diesem Grund sind Ein-Referenz Techniken wie die uneingeschränkte (unrestricted, U) Hartree-Fock (HF) Methode 58,59,97 oder störungstheoretische Ansätze (Møller-Plesset, MPn), ${ }^{72,98}$ in denen eine diskrete elektronische Konfiguration von einer einfachen bzw. einer gestörten Referenzwellenfunktion beschrieben wird, für solche Fälle nicht geeignet. Methoden, die die ordnungsgemäße Mischung der nahe beieinander liegenden Valenzstrukturen erlauben, sind im Allgemeinen extrem zeitaufwendig. Standardmethoden für Problemfälle bei Raum- oder Spinsymmetriebruch sind Multireferenz-Techniken, wie die Methode des vollständig aktiven Raumes (CASSCF, complete active space self consistent field) $73,75,99,100$ oder die Multireferenz-Konfigurations-Wechselwirkungsmethode (MR-CI, multi reference configuration interaction). ${ }^{101-103}$ Eine weitere Möglichkeit bietet der Brueckner doubles (BD) ${ }^{80-83,104}$ Ansatz, einer coupled cluster Methode, ${ }^{78,79,105,106}$ bei der die Molekülorbitale in Gegenwart der Korrelations-Störung rotiert werden, bis die resultierende Clusteramplitude der ersten Anregung verschwindet. Diese Prozedur vermeidet die Probleme, die manchmal im Falle sehr kleiner Singulett-Triplett-Separation bei der herkömmlichen coupled cluster Methode ohne modifizierte Molekülorbitale auftreten können. ${ }^{55}$ Diese Methoden können sehr erfolgreich eingesetzt werden, da sogar ein großer Teil der nichtdynamischen Elektronenkorrelation durch die angenäherte Wellenfunktion beschrieben wird, was einen Symmetriebruch verhindert.

Eine Alternative zu diesen äußerst genauen, jedoch auch äußerst zeitaufwendigen Methoden ist die Dichtefunktionaltheorie (DFT), ${ }^{107}$ da sie, im Vergleich mit der HF Theorie, weniger anfällig für Raum- und Spinsymmetriebruch ist. ${ }^{108-112}$ In neuerer Literatur wird die Nützlichkeit dieses Ansatzes für die Beschreibung biradikalischer Spezies, wie sie in homolytischen Spaltungen von Bindungen oder Cyclisierungsreaktionen vorkommen, eindrucksvoll demonstriert. ${ }^{57,113-121}$ Es wurde hervorgehoben, daß diese Theorie mit Schwierigkeiten bei Spinsymmetriebruch effizient umgehen kann und Ergebnisse mit ausreichend hoher Genauigkeit ohne den Zeitaufwand der Multireferenzmethoden liefert. 
Darüber hinaus erlaubt der uneingeschränkte Ansatz mit gestörtem Spin (unrestricted brokenspin, BS-U), in dem die Raum- und Spinsymmetrie der Wellenfunktion durch Mischung der Grenzorbitale gestört wird, die Einbeziehung derselben statischen Elektronenkorrelation. ${ }^{52,122,123}$ Diese Methode erlaubt die Beschreibung von reinen oder fast reinen, offenschaligen Singulett-Zuständen ohne die Probleme, die mit teilweise besetzten Orbitalen zusammenhängen, und sie verhindert das Kollabieren der uneingeschränkten zur beschränkten Wellenfunktion. Ältere Publikationen, die Biradikale wie z.B. das paraDidehydrobenzol 10 als reine, geschlossenschalige Singuletts behandeln, sollten deshalb nicht mehr als Referenz verwendet werden. ${ }^{32,33,52,119}$ Dieses Ergebnis wird durch die Tatsache unterstützt, daß die Struktur von 10 berechnet auf dem BS-UBLYP Niveau ${ }^{63,68,69,124}$ näher an der CASSCF (8,8)-Geometrie liegt als die entsprechende RBLYP-Geometrie (Abb. 13). Die RBLYP-Geometrie scheint dabei eher ein Bis-Allen (1,2,4,5-Cyclohexatetraen, 26) zu beschreiben. Deutlicher wird der Unterschied noch beim $\alpha, 2$-Didehydrotoluol, bei dem die BS-UBLYP Rechnung wie erwartet das planare Biradikal 12 liefert, die RBLYP-Rechnung jedoch ein aus der Ebene verdrehtes Allen 27.

Die Frage, ob die DFT auf ein Multireferenz-Problem angewendet werden kann, ist nicht allgemeingültig zu beantworten. Die zugrunde liegende Frage ist, ob es eine diskrete Elektronenkonfiguration gibt, die die vollständige Elektronendichte für ein offensichtlich multireferentes Problem produziert. Dies ist eine Fall-zu-Fall Entscheidung, die nicht a priori getroffen werden kann und die für jeden Fall neu überprüft werden muß. Für die biradikalischen Systeme in dieser Arbeit soll im Folgenden gezeigt werden, daß die Verwendung der DFT-Methode zulässig ist und, verglichen mit dem Experiment, gute Ergebnisse liefert.

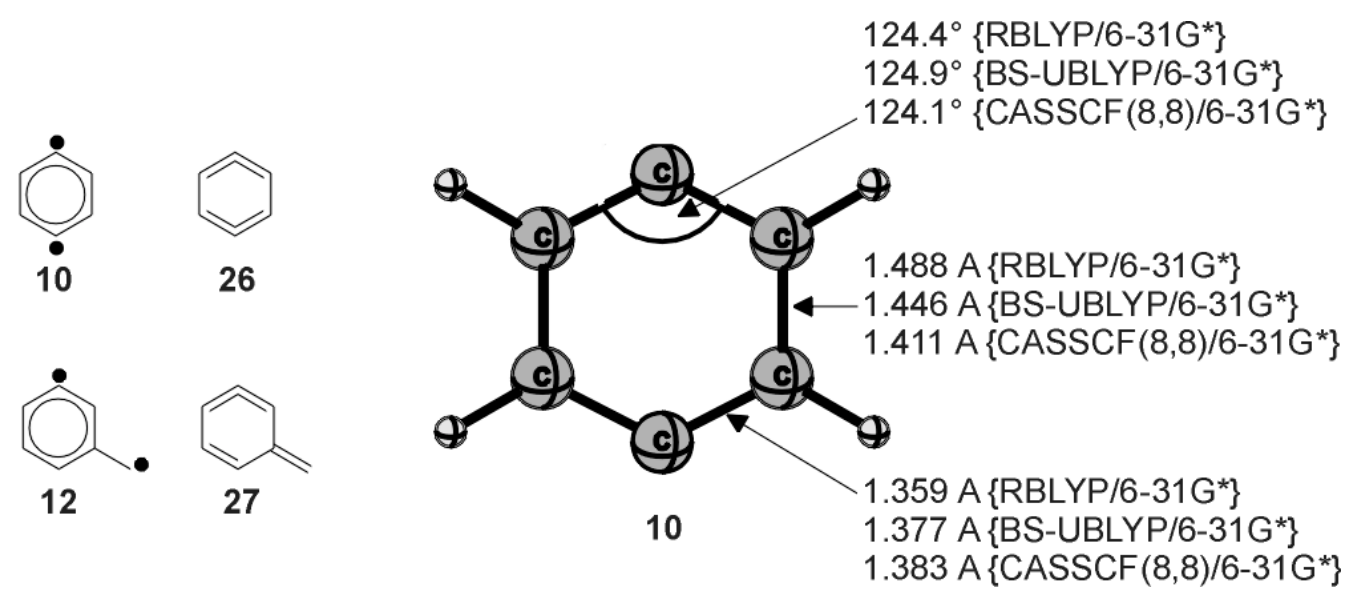

Abbildung 13. Strukturelle Parameter für para-Didehydrobenzol 10 mit verschiedenen Methoden 
Da viele Produkte und Übergangszustände der diskutierten Reaktionen einen mehr oder weniger ausgeprägten Biradikalcharakter haben, was an den kleinen Singulett-TriplettAbständen zu sehen ist, ${ }^{95,96}$ sind auf HF basierende Einzelreferenzmethoden für die Beschreibung dieser Umlagerungen nicht geeignet, während Multireferenzmethoden zu zeitaufwendig für große Geometrieoptimierungen sind. Wie schon diskutiert, eröffnet die DFT einen annehmbaren Kompromiß für diese quantenmechanisch anspruchsvollen Systeme. ${ }^{32,33,52,55,95,96,119}$ Um zu stabilen, unbeschränkten Lösungen zu gelangen und um die räumliche und die $\alpha / \beta$-Symmetrie zu zerstören, wird deshalb ein BS-U Ansatz für alle offenschaligen Singuletts verwendet.

Es gibt noch immer viele Diskussionen über das geeignetste DFT-Funktional, was auch die Frage nach einer Unterscheidung zwischen reinen und Hybridfunktionalen miteinschließt. Obwohl einige wenige systematische Vergleiche $\mathrm{zu}$ anderen Beispielen existieren, ${ }^{57}$ wurde speziell für das vorliegende Problem eine große Anzahl von Ergebnissen aus gebräuchlichen DFT-Varianten mit den experimentellen Daten für die Barrieren und Enthalpien der Bergman- und Myers-Saito-Reaktion verglichen. Um einen ordnungsgemäßen Vergleich mit dem Experiment zu gewährleisten, wurden beide Reaktionsenthalpien bei 298.15 K, die Barriere der Bergman-Reaktion bei 470.15 K und die der Myers-Saito-Reaktion bei $343.15 \mathrm{~K}$ bestimmt, was den experimentellen Temperaturen entspricht. Die kleinsten Fehlerquadrate der berechneten, im Vergleich zu den experimentellen Daten der HF-, MP2-, CCSD(T)-, BD(T)- und DFT-Berechnungen, einschließlich der Austauschfunktionale B (Becke 1988), ${ }^{63}$ S (Slater), ${ }^{61,62,64}$ MPW (Barons modifiziertes PW), ${ }^{125}$ G96 (Gills 1996), $, 65,66$ B3 (Beckes drei Parameter Hybrid) ${ }^{67}$ und der Korrelationsfunktionale LYP (Lee, Yang und Parr), ${ }^{68,69}$ PW91 (Perdew und Wang 1991), ${ }^{70}$ P86 (Perdew 1986), ${ }^{126}$ PL (Perdews nicht gradienten-korrigiert), ${ }^{127}$ VWN (Vosko, Wilk und Nosair), ${ }^{71}$ VWN5 ${ }^{71}$ sind in Abb. 14 zusammengefaßt.

Während die besten Ergebnisse von Kombinationen mit dem Korrelationsfunktional von Lee, Yang und Parr (LYP) erhalten werden, hat die Wahl des Austauschfunktionals einen geringeren Effekt auf die Qualität der Ergebnisse. Nur Slaters Austauschfunktional (S) leidet an fehlender Gradientenkorrektur und ergibt deshalb schlechte Ergebnisse. Da schon früher gezeigt wurde, daß die Lösungen von reinen DFT Funktionalen für Schwierigkeiten bei Symmetriebruch generell stabiler sind als die von Hybridfunktionalen (z.B. B3LYP), ${ }^{122}$ sind MPWLYP, G96LYP und BLYP die besten Kandidaten für den Kompromiß zwischen chemischer Genauigkeit und rechnerischem Aufwand. Frühere Abhandlungen über die Bergman-Cyclisierungen und verwandte Ringschlußreaktionen verwendeten die BPW9155,119 
und BLYP $32,33,124$ Funktionale. Da die Qualität des relativ neuen G96LYP Funktionals noch nicht an einem ausreichend großen Satz an Referenzmolekülen getestet wurde, ist BLYP die Methode der Wahl zur Untersuchung der Umlagerungen in dieser Arbeit.

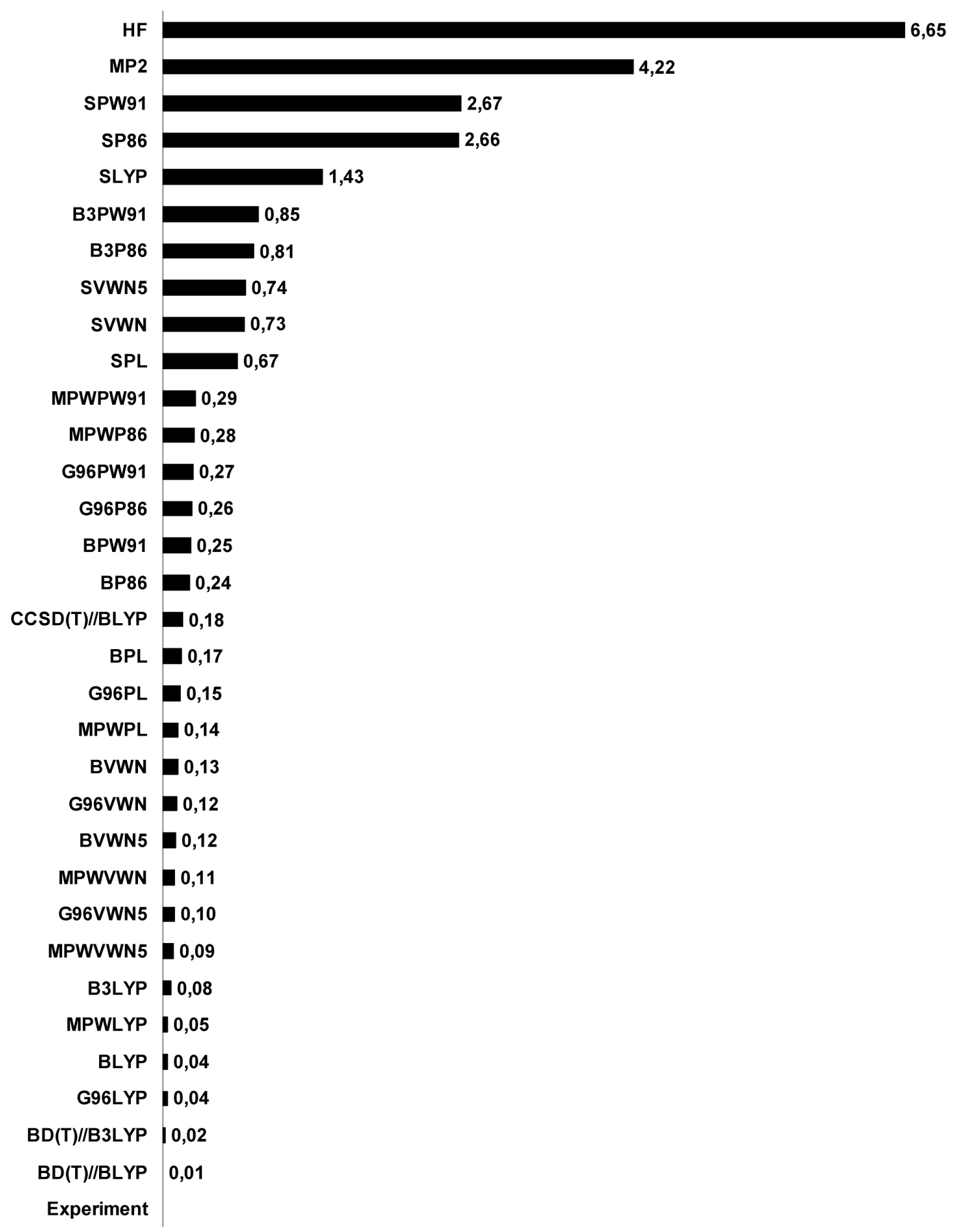

Abbildung 14. Vergleich der berechneten und experimentellen Ergebnisse der Bergman- und Myers-SaitoReaktion für eine große Zahl von Methoden (BS-U für die Produkte, mit einem 6-31G* Basissatz für DFT, HF und MP2 und cc-pVDZ für die coupled cluster Berechnungen). Jeder Eintrag ist aus vier berechneten und vier experimentellen Daten zusammengesetzt. Dargestellt ist das normierte, kleinste Fehlerquadrat für jede Methode. 
Die Basissatzabhängigkeit wurde ebenfalls durch systematische Vergrößerung des Basissatzes untersucht (Abb. 15). Wie schon mehrfach bei DFT-Rechnungen festgestellt, ist das Verhalten bei größeren Basissätzen, insbesondere für reine Funktionale (BLYP und G96LYP), nicht vorhersagbar. In diesem Fall wird das Ergebnis bei der Vergrößerung der Basis von 6-31G*128 zu 6-311G** schlechter, dasselbe gilt für den Schritt von cc-pVDZ zu cc-pVTZ. ${ }^{85}$ Das Hybrid-Funktional B3LYP hingegen verhält sich eher traditionell, d.h. eine größere Basis verbessert das Ergebnis. Für die im Rahmen dieser Arbeit berechneten, teilweise bis zu 18 Schweratome enthaltenen Strukturen stellt die Kombination aus dem BLYP Funktional und dem 6-31G* Basissatz einen sehr guten Kompromiß für die Optimierung der Strukturen und Berechnung der Energien dar.

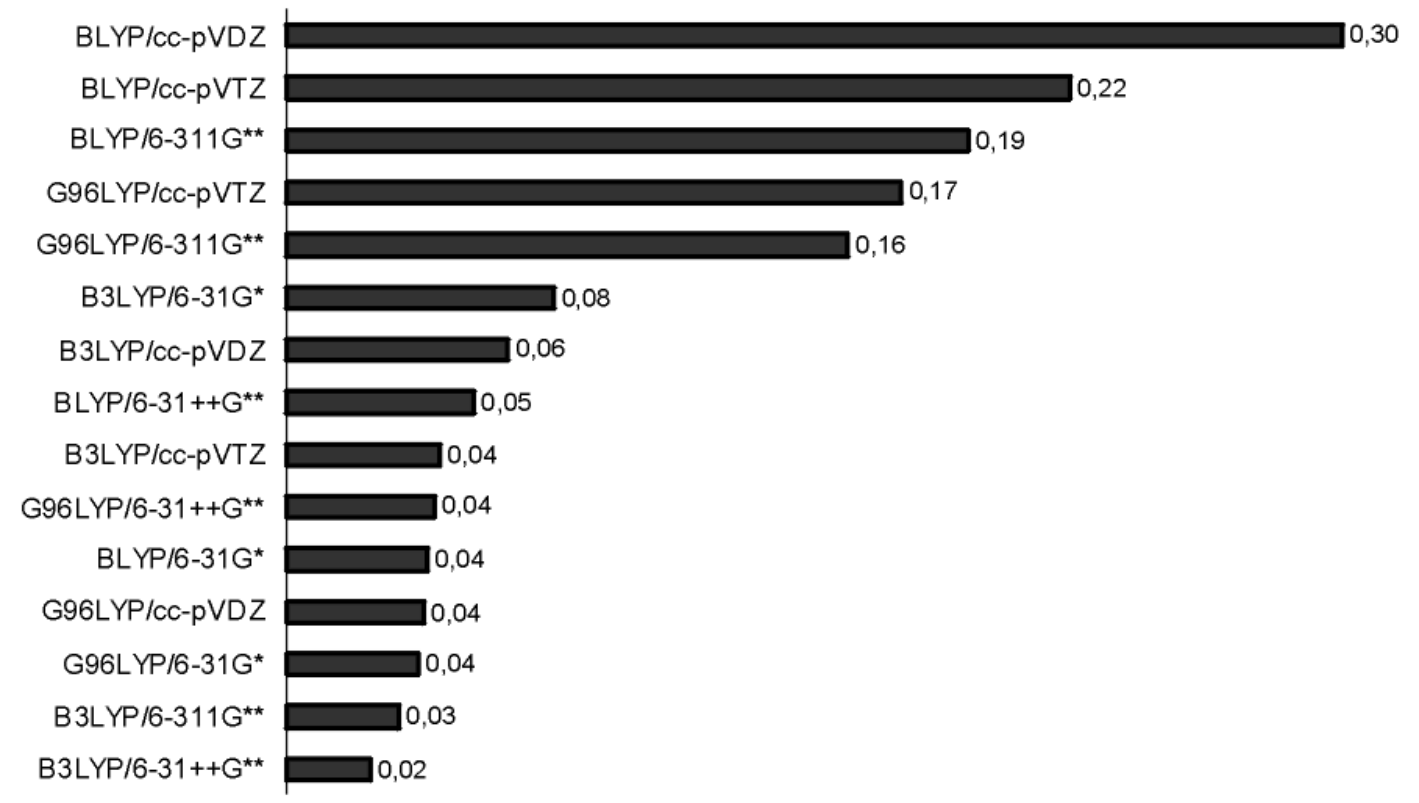

Abbildung 15. Vergleich der berechneten und experimentellen Ergebnisse der Bergman- und Myers-SaitoReaktion für die besten drei DFT-Methoden unter Verwendung verschiedener Basissätze. Dargestellt ist das normierte, kleinste Fehlerquadrat für jeden Eintrag.

Wie in Abbildung 15 gezeigt, wird die Qualität der Ergebnisse durch EinzelpunktBerechnungen auf dem BD(T)-Niveau unter Verwendung der BLYP/6-31G* Geometrien weiter verbessert. Für die offenschaligen Systeme wird die Energie basierend auf der BS-UHF Wellenfunktion ermittelt, die durch die Verwendung der optimierten Brueckner-Orbitale, in denen der Anteil der ersten Anregung Null ist, die experimentellen Werte sehr gut reproduziert. Im Gegensatz dazu ergeben $\operatorname{CCSD}(\mathrm{T})$ Berechnungen mit unmodifizierten Orbitalen schlechte Ergebnisse, was wahrscheinlich am hohen Anteil der ersten Anregung 
liegt, die im offenschaligen Singulett an sich verbotenen ist, dort aber in die Berechnung eingeht.

Wie die Daten in Tabelle 1 zeigen, reproduziert das rechnerisch wenig aufwendige BLYP/6-31G* die experimentellen Daten schon recht gut, während die $\mathrm{BD}(\mathrm{T})$ Resultate, für die Dunnings korrelationskonsistenter Doppel- $\zeta$ Basissatz (cc-pVDZ) verwendet wird, nahezu innerhalb der Fehlerbreite des Experiments liegen. Mit dieser überzeugenden Übereinstimmung zwischen Theorie und Experiment, sollen im Verlauf dieser Arbeit präzise Vorhersagen über bisher noch nicht durchgeführte Reaktionen gemacht werden. Dabei werden bei den Stamm- und benzannelierten Systemen die BLYP- von BD(T)-Rechnungen unterstützt, während alle anderen, größeren Systeme nur mit BLYP behandelt werden sollen.

Tabelle 1. Vergleich einiger berechneter und experimenteller $\Delta \mathrm{H}^{\ddagger}$ und $\Delta \mathrm{H}_{298}$ Werte (in kcal $\left.\mathrm{mol}^{-1}\right)$.

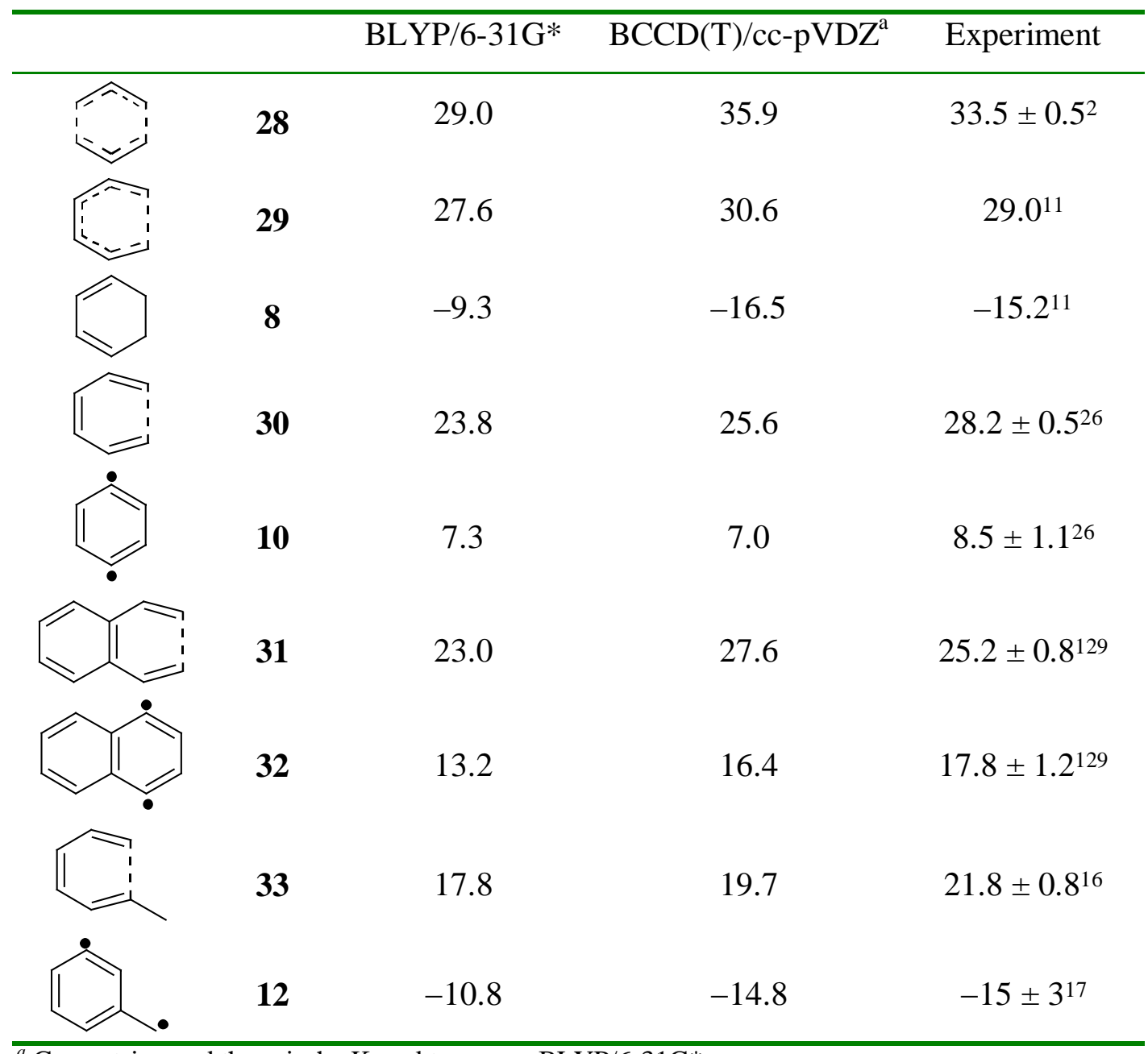

${ }^{a}$ Geometrien und thermische Korrekturen aus BLYP/6-31G* 


\subsection{Cope-ähnliche Umlagerungen}

Die Computerchemie kann zur Validierung, Interpretation und Voraussage von experimentellen Ergebnissen verwendet werden, wobei der letzte Aspekt der nützlichste, jedoch am wenigsten benutzte ist. Echte Voraussagen sind schwer zu machen und benötigen vorher einer genauen Validierung der eingesetzten Methode durch den Vergleich mit genau bekannten experimentellen Werten. Hilfreich sind in diesem Zusammenhang bereits bekannte Reaktionen und Strukturen, die den Untersuchten ähnlich genug sind, um eine sichere Interpretation und Extrapolation der Ergebnisse zu gewährleisten ("Reaktions- und Strukturfamilien").

Die Gruppe der $\left[3_{\mathrm{S}}, 3_{\mathrm{S}}\right]$-sigmatropen Umlagerungen, von denen die Cope-Umlagerung von 1,5-Hexadien 1 wahrscheinlich die geläufigste ist, ${ }^{2}$ bildet eine solche Familie. Sie enthält viele bekannte ${ }^{130-136}$ aber auch bisher unbekannte Transformationen, die teilweise auf den ersten Blick nicht als zur Cope-Familie zugehörig zu erkennen sind, jedoch in einem umfassenderen Rahmen zweifellos dazugehören. Ein solches Beispiel ist die BergmanCyclisierung von (Z)-Hexa-3-en-1,5-diin 9,12-15 das sich zwar im Reaktionsprofil deutlich von der Cyclisierung von 1 unterscheidet, aber dennoch im Grunde eine Cope-ähnlich Umlagerung ist (Abb. 16). Beim Einordnen der verschiedenen Reaktionen in die CopeFamilie müssen nun die Unterschiede in den Energieprofilen erklärt werden, z.B. warum die Cope-Umlagerung ein einstufiger Prozeß ohne Zwischenprodukt ist, während das Zwischenprodukt der Bergman-Cyclisierung eindeutig als offenschaliges Biradikal identifiziert wurde. Um eindeutige Vorhersagen über die Reaktivitäten und Cyclisierungsmechanismen bisher unbekannter Mitglieder der Cope-Familie machen zu können, muß geklärt werden, wann eine biradikalische Zwischenstufe (oder Produkt) zu erwarten ist und wann nicht.

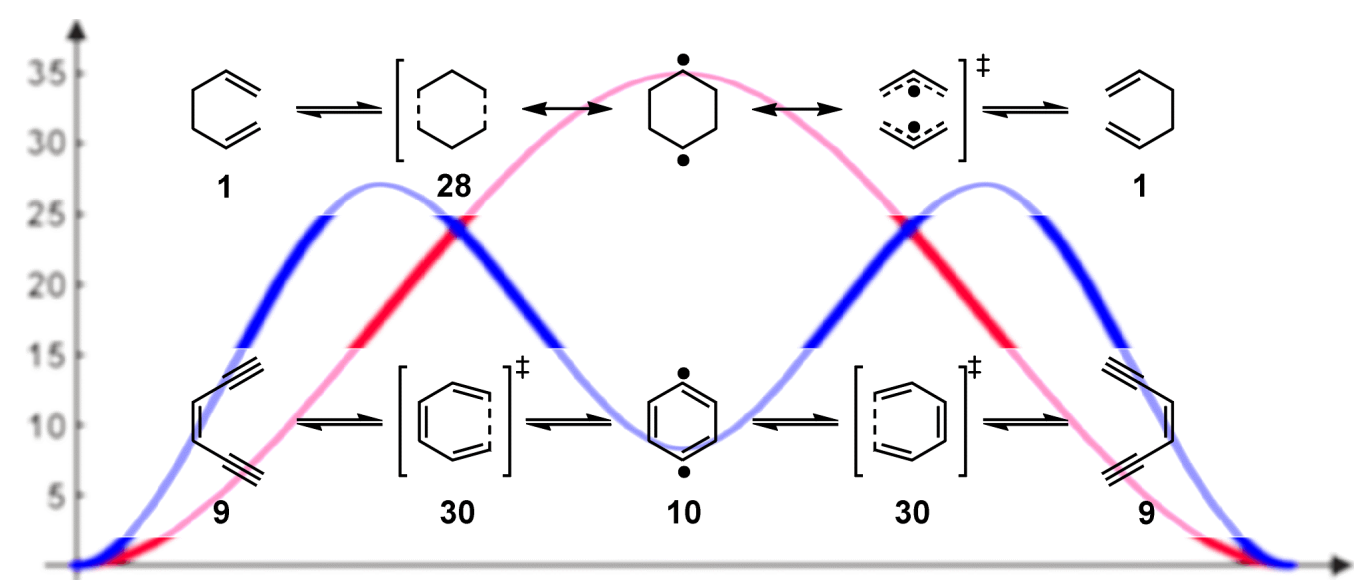

Abbildung 16. Cope- und Bergman-Umlagerung einschließlich der Energieprofile 
Generell muß zwischen zwei Fällen unterschieden werden: einem delokalisierten "aromatischen" Übergangszustand, in dem Bindungsbruch und Bindungsbildung simultan ablaufen, und einem biradikalischen stabilisierten Zwischenprodukt, in dem Bindungsbruch und Bindungsbildung in zwei aufeinander folgenden Schritten stattfinden. Für den genannten Cope-Bergman-Fall wurde auf vielen fortgeschrittenen Methoden ein aromatischer Übergangszustand für die Cope-Reaktion gefunden. ${ }^{137-146}$ Auf verschiedenen DFT-Niveaus konnte auch eine biradikalische Zwischenstufe lokalisiert werden, die aber in der Energie nur $\sim 0.2 \mathrm{kcal} \mathrm{mol}^{-1}$ unter der des Übergangszustand liegt. ${ }^{139}$ Für die Bergman-Reaktion konnte hingegen eindeutig eine biradikalische, aromatische Zwischenstufe gefunden werden, die nach der einen oder anderen Seite zum Endiin öffnen kann. ${ }^{54,147,148}$ Die Situation ist also ähnlich wie bei der Cope-Umlagerung mit dem Unterschied, daß es wirklich eine biradikalische Zwischenstufe gibt.

Ein ähnlicher Vergleich kann zwischen der Cyclisierung von 1,2,6-Hexatrien 34 und (Z)-Hepta-1,2,4-trien-6-in 11 (Myers-Saito-Cyclisierung) gezogen werden (Abb. 17). Biradikalische Zwischenprodukte wurden experimentell für beide Reaktionen nachgewiesen, jedoch in verschiedenem Ausmaß. Während das $\alpha, 2$-Didehydrotoluol (12, Myers-SaitoProdukt) quantitativ über $\mathbf{3 3}$ aus $\mathbf{1 1}$ gebildet wird, ${ }^{16-19}$ findet nur ein Teil der Umlagerung von 34 über die biradikalischen Zwischenstufe 35 statt, ein anderer Teil jedoch über einen konzertierten Prozeß.131 Die Strukturen 12 und 27 sind elektronische Isomere (Elektronomere), d.h. ein offenschaliges und eine geschlossenschaliges Singulett. Das allenische 27 zeigt jedoch noch einmal die Ähnlichkeit beider Reaktionen im Vergleich zu 36.

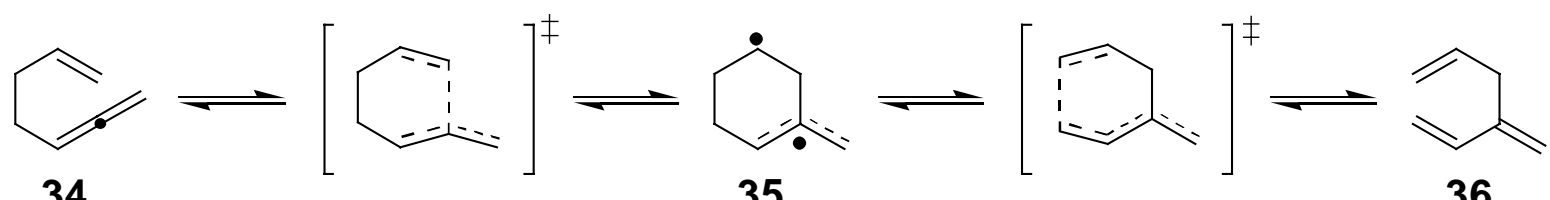

$35 \quad 36$

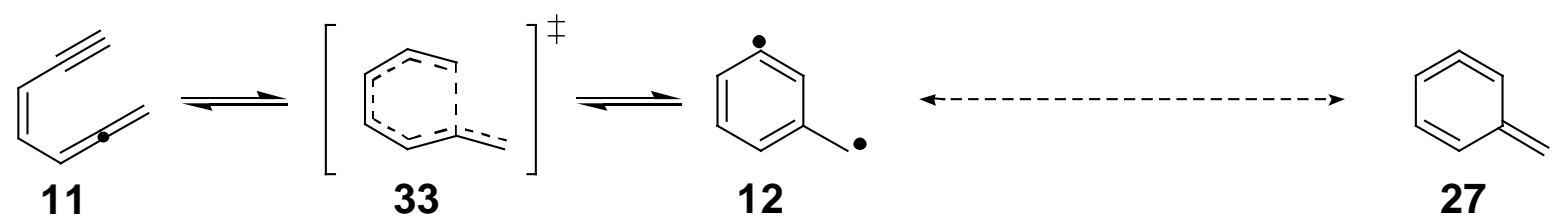

Abbildung 17. Umlagerung von 1,2,6-Hexatrien 34 und Myers-Saito-Cyclisierung von 11 
Die Cope-Familie läßt sich grob in zwei Familienzweige unterteilen. Der erste Zweig besteht aus den Einzelbausteinen I bis VIII, die sich über eine Einfachbindung (gestrichelt) in jeder beliebigen Kombination zu den Cope-Edukten und -Produkten zusammensetzen lassen (Abb. 18). Durch den Bruch dieser Einfachbindung und das Ausbilden einer neuen Bindung über einen sechsgliedrigen Übergangszustand oder ein sechsgliedriges, biradikalisches Zwischenprodukt, führt jeder Molekülbaustein einzeln die unten aufgeführte Umlagerung aus. Durch die Kombination der Bausteine ergeben sich die Cope-Moleküle 1, 34 und 36 bis 69, die sich durch die Umlagerung ineinander überführen lassen, d.h. Edukt und Produkt sein können (Abb. 19). Die zweite Familie IX bis XI hat weniger Bausteine, da diese über eine Doppelbindung verknüpft werden und deshalb die Auswahl der ungesättigten Gruppen eingeschränkt ist. Durch die Anwesenheit dieser Doppelbindung lagern diese Moleküle auch nicht ineinander um, sondern ergeben sechsgliedrige Cyclisierungsprodukte, die nicht wieder öffnen (einzige Ausnahme ist, wie schon beschrieben, das Endiin 9). Die folgenden Aussagen beschäftigen sich zunächst mit dem Familienzweig eins, bevor dann in weiteren Kapiteln auf die Mitglieder des zweiten Zweiges eingegangen wird.

\section{Cope-Familienzweig 1:}

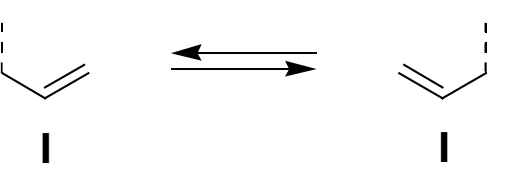

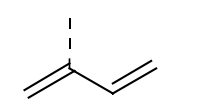

II

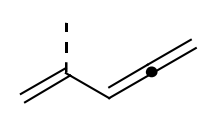

IV
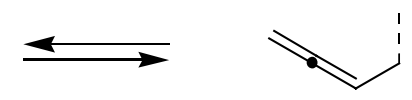

III

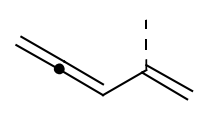

IV

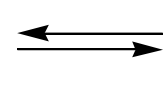

V<smiles>C#CC(=C)C</smiles>

VII

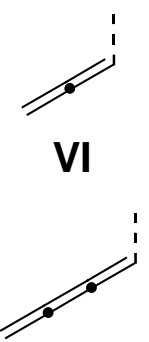

VIII

Cope-Familienzweig 2:
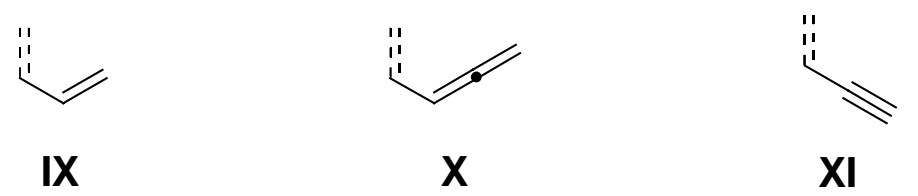

Abbildung 18. Die Einzelbausteine der zwei Zweige der Cope-Familie 


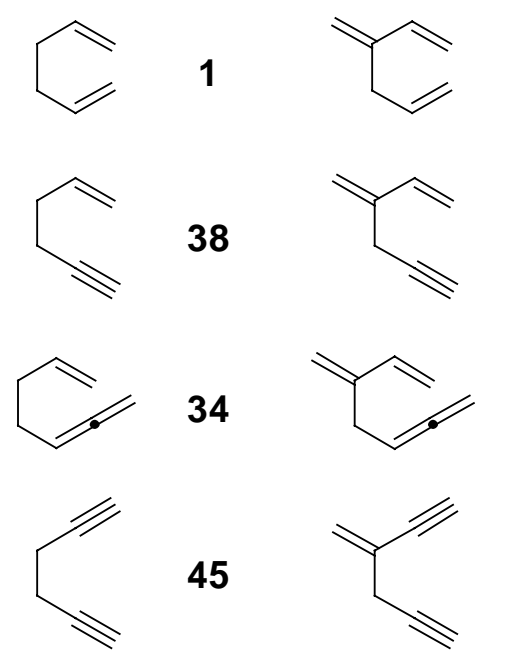

36

39<smiles>C#CC(=C)CC=C</smiles>

40<smiles>C#CC(=C)C(=C)C=C</smiles>

41

42<smiles>C=C1CC=CC2=CC1C=C2</smiles><smiles>C=CC(=C)C(=C)C=C</smiles><smiles>C#CC(=C)C(=C)C#C</smiles>

\section{7}<smiles>C#CCC(=C)C=C</smiles><smiles>C#CC(=C)C(=C)C=C</smiles>

51<smiles>C1=CCCCC1</smiles><smiles>C=C1C=CC=CC(=C)CC1</smiles>
53<smiles>C=C1C=CC(=C)C(=C)C=C1</smiles><smiles>C=CCC=C</smiles>

55<smiles>C=CC(=C)C=C</smiles>

56<smiles>C=CCC=CC</smiles>

57<smiles>C=CC(=C)C=C</smiles>

58<smiles>C#CCC=C</smiles>

59<smiles>C#CC(=C)C=C</smiles><smiles>C#CCC=C</smiles>

61<smiles>C#CC(=C)C=C=C</smiles>

62<smiles>C=CCC=C</smiles>

63<smiles>C=CC(=C)C=C</smiles><smiles>C1=CCC=CC1</smiles>

65<smiles>C=CC(=C)C=CC</smiles>
66<smiles>C=CC=C</smiles>

67<smiles>C=C=CC=C=C</smiles><smiles>C=C=C=CC=C=C</smiles>

69

Abbildung 19. Die Mitglieder des ersten Zweiges der Cope-Famile.

Die Überführung der Moleküle ineinander, ihre Energien und ob sie Zwischenprodukte bilden oder über einstufige Umlagerungen ablaufen, läßt sich am besten durch die Matrix in Abbildung 21 veranschaulichen. In ihr sind alle möglichen Mitglieder der CopeFamilie vertreten und ihrem Umlagerungspartner eindeutig zugeordnet. Da ein Molekül sowohl Edukt als auch Produkt sein kann, sind die Moleküle mehrfach vertreten. Die Verwendung der Matrix wird in Abbildung 20 beschrieben. Aus der Zeile und Spalte der Edukt-Einzelbausteine wird das Molekül von Interesse zusammengesetzt. Am Schnittpunkt 
dieser Zeile und Spalte ist dann eine entsprechende Zwischenstruktur abgebildet. Dort sind drei Fälle zu unterscheiden:

1. Wenn die abgebildete Struktur ein Übergangszustand ist und in der rechten oberen Ecke ein ÜZ-Symbol steht, bedeutet dies eine einstufige Umlagerung.

2. Wenn die abgebildete Struktur ein Biradikal ist und in der rechten oberen Ecke ein ÜZSymbol steht, bedeutet dies, daß die Reaktion über einen einstufigen Mechanismus und eine biradikalische Zwischenstufe stattfinden kann und beide Wege auch in unterschiedlichen Ausmaßen beschritten werden.

3. Wenn die abgebildete Struktur ein Biradikal ist und in der rechten oberen Ecke eine Zahl steht, bedeutet dies eine Reaktion ausschließlich über eine biradikalische Zwischenstufe.

Die Zuordnung der Energiewerte ist in Abbildung 20 dargestellt und kann Tabelle 2 entnommen werden. Folgt man der Zeile und Spalte weiter, so kommt man zu den ProduktBausteinen aus denen dann das Zielmolekül zusammengesetzt werden kann. 

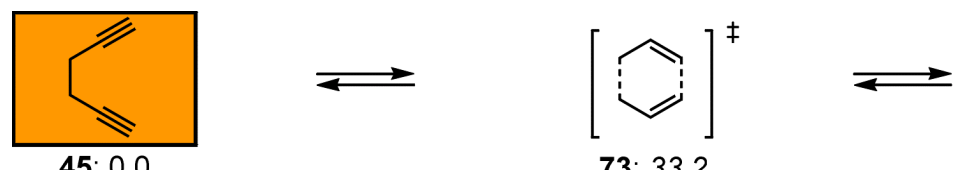

73: 33.2
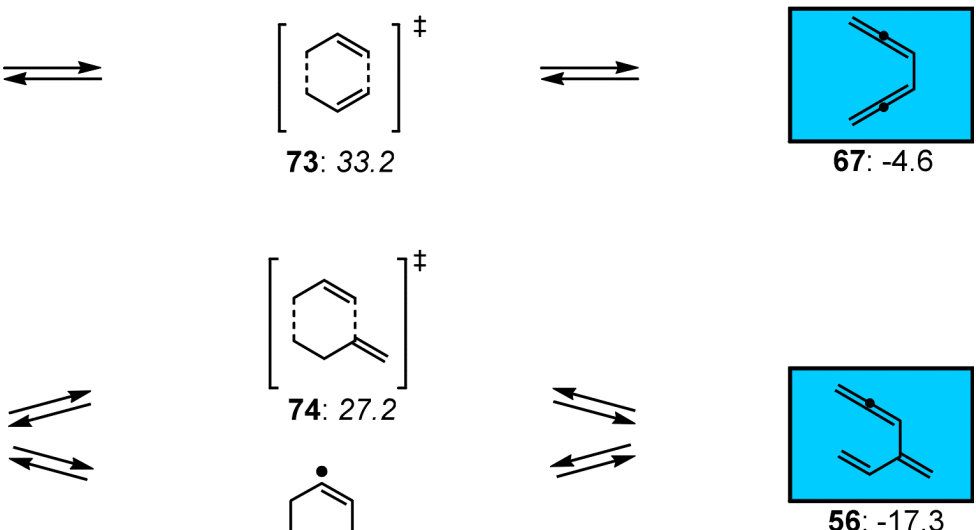

74: 12.1
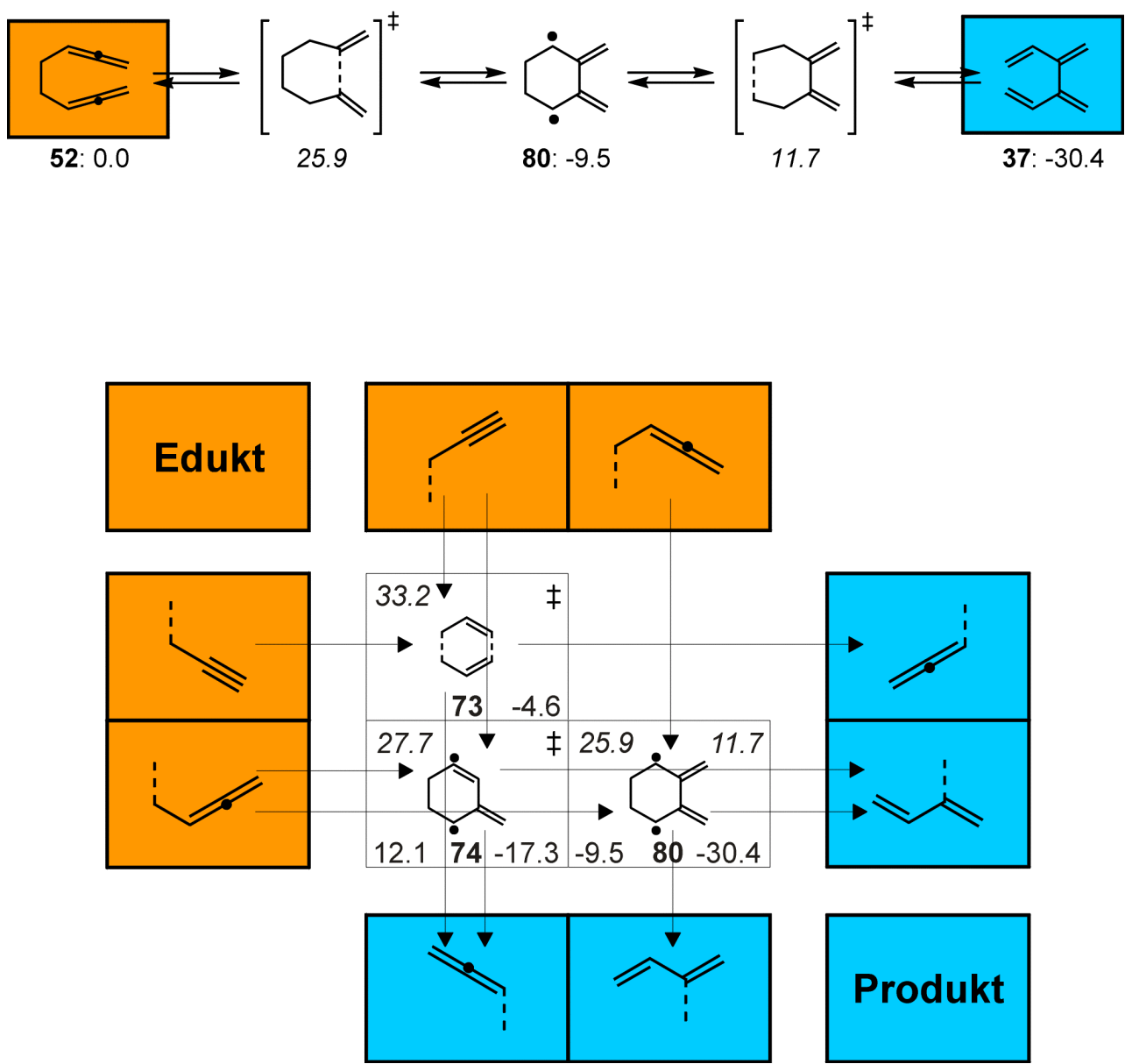

Abbildung 20. Erklärung zur Verwendung der Cope-Matrix 


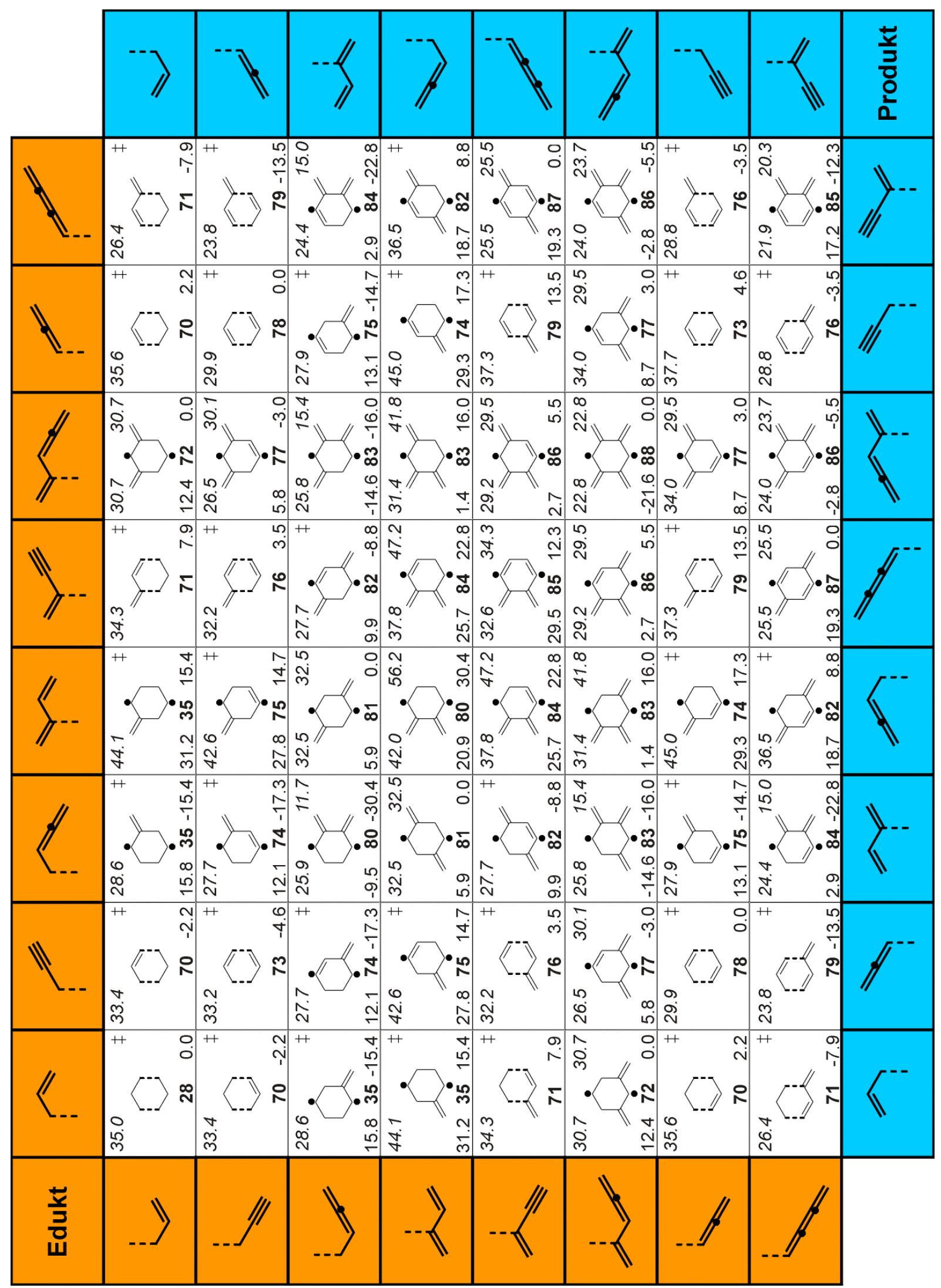

Abbildung 21. Die Matrix der Cope-ähnlichen Umlagerungen ("Cope-Familie") 
Tabelle 2. Energien $\left(\Delta \mathrm{H}_{0}\right.$ bzw. $\left.\Delta \mathrm{H}_{0}{ }^{\ddagger}\right)$ der Umlagerungen der Cope-Familie (in kcal mol ${ }^{-1}$ )

\begin{tabular}{|c|c|c|c|c|c|c|c|}
\hline \multicolumn{2}{|c|}{ Edukt } & \multirow{2}{*}{$\frac{\mathrm{TS}_{\text {Zweistufig }}}{-}$} & \multicolumn{2}{|c|}{ Bir / TS $\mathrm{T}_{\text {Einstufig }}$} & \multirow{2}{*}{$\frac{\mathrm{TS}_{\text {Zweistufig }}}{-}$} & \multicolumn{2}{|c|}{ Produkt } \\
\hline 1: & 0.0 & & 28: & 35.0 & & 1: & 0.0 \\
\hline 34: & 0.0 & - & 35: & $15.8 / 28.6$ & - & 36: & -15.4 \\
\hline 36: & 0.0 & - & 35: & $31.2 / 44.1$ & - & 34: & 15.4 \\
\hline 37: & 0.0 & 42.0 & 80: & 20.9 & 56.2 & 52: & 30.4 \\
\hline 38: & 0.0 & - & 70: & 33.4 & - & 55: & -2.2 \\
\hline 39: & 0.0 & - & 75: & $27.8 / 42.6$ & - & 63: & 14.7 \\
\hline 40: & 0.0 & - & 71: & 34.3 & - & 57: & 7.9 \\
\hline 41: & 0.0 & 37.8 & 84: & 25.7 & 47.2 & 65: & 22.8 \\
\hline 42: & 0.0 & 32.5 & 81: & 5.9 & 32.5 & 42: & 0.0 \\
\hline 43: & 0.0 & 30.7 & 72: & 12.4 & 30.7 & 43: & 0.0 \\
\hline 44: & 0.0 & 31.4 & 83: & 1.4 & 41.8 & 53: & 16.0 \\
\hline 45: & 0.0 & - & 73: & 33.2 & - & 67: & -4.6 \\
\hline 46: & 0.0 & - & 76: & 32.4 & - & $68:$ & 3.5 \\
\hline 47: & 0.0 & 32.6 & 85: & 29.5 & 34.3 & $68:$ & 12.3 \\
\hline 48: & 0.0 & - & 74: & $12.1 / 27.7$ & - & 56 : & -17.3 \\
\hline 49: & 0.0 & - & 82: & $9.9 / 27.7$ & - & $58:$ & -8.8 \\
\hline 50: & 0.0 & 26.5 & 77: & 5.8 & 30.1 & 64: & -3.0 \\
\hline 51: & 0.0 & 29.2 & 86: & 2.7 & 29.5 & 66: & 5.5 \\
\hline $52:$ & 0.0 & 25.9 & 80: & -9.5 & 11.7 & 37: & -30.4 \\
\hline 53: & 0.0 & 25.8 & 83: & -14.6 & 15.4 & 44: & -16.0 \\
\hline 54: & 0.0 & 22.8 & 88: & -21.6 & 22.8 & 54: & 0.0 \\
\hline $55:$ & 0.0 & - & 70: & 35.7 & - & 38: & 2.24 \\
\hline $56:$ & 0.0 & - & 74: & $29.3 / 45.0$ & - & 48: & 17.3 \\
\hline 57: & 0.0 & - & 71: & 26.4 & - & 40: & -7.9 \\
\hline $58:$ & 0.0 & - & 82: & $18.7 / 36.5$ & - & 49: & 8.8 \\
\hline 59: & 0.0 & - & 78: & 29.9 & - & 59: & 0.0 \\
\hline 60 : & 0.0 & - & 79: & 37.3 & - & 61: & 13.5 \\
\hline 61: & 0.0 & - & 79: & 23.8 & - & 60: & -13.5 \\
\hline $62:$ & 0.0 & 25.5 & 87: & 19.3 & 25.5 & 62: & 0.0 \\
\hline 63: & 0.0 & - & 75: & $13.1 / 27.9$ & - & 39: & -14.7 \\
\hline 64: & 0.0 & 34.0 & 77: & 8.7 & 29.5 & 50: & 3.0 \\
\hline 65: & 0.0 & 24.4 & 84: & 2.9 & 15.0 & 41: & -22.8 \\
\hline 66: & 0.0 & 24.0 & 86: & -2.8 & 23.7 & 51: & -5.5 \\
\hline 67: & 0.0 & - & 73: & 37.7 & - & 45: & 4.6 \\
\hline $68:$ & 0.0 & - & 76: & 28.8 & - & 46: & -3.5 \\
\hline 69: & 0.0 & 21.9 & 85: & 17.2 & 20.3 & 47: & -12.3 \\
\hline
\end{tabular}


Die Ergebnisse der Berechnungen sind aus Tabelle $2 \mathrm{zu}$ entnehmen und können nicht alle im Detail diskutiert werden. Deshalb soll im Folgenden nur auf einige Beispiele eingegangen werden und anhand derer eine Erklärung für hohe bzw. niedrige Reaktivität gegeben werden. Eine Untergruppe der Cyclisierungen kann ausschließlich $\pi, \pi$-Biradikale bilden und beinhaltet deshalb auch keine Dreifachbindungen (Abb. 22). Die Reaktivität hängt dabei maßgeblich von den Ausgangsverbindungen ab. Gibt es in ihnen schon energiereiche Bausteine, wie in diesen Beispielen den Allenteil, ist die Barriere von dieser Seite aus natürlich geringer als die der Rückreaktion. Wie man deutlich sehen kann, haben alle Verbindungen auf der linken Seite, mit Ausnahme der entarteten Umlagerungen von 1, 42, 43 und 54, jeweils mehr Allenbindungen als die der rechten Seite. Bei den Verbindungen auf der linken Seite geht die Reaktivität ganz deutlich mit der Stabilisierung des Zwischenproduktes einher. Bei 1 gibt es keine biradikalische Zwischenstufe, sondern nur den ÜZ 28, der keinerlei Stabilisierung besitzt und deshalb auch eine hohe Barriere hat. Bei den Molekülen 42, 43 und 52 findet die Cyclisierung ausschließlich über Zwischenprodukte statt. Die Biradikale 71, 80 und 79 können sich über zwei exocyclische Doppelbindungen stabilisieren und sind deshalb echte Zwischenprodukte. Da alle drei Biradikale etwa gleich gut stabilisiert sein sollten, können die Unterschiede in den Barrieren nur auf die Eduktstabilitäten zurückzuführen sein. Die Cyclisierung von $\mathbf{3 4}$ kann sowohl über einen konzertierten Prozeß als auch über eine biradikalische Zwischenstufe 35 ablaufen. Durch die Stabilisierung mittels Delokalisierung über eine exocyclische Doppelbindung sollte die Barriere eigentlich höher sein als bei 42, 43 und 52. Sie liegt jedoch dazwischen und ist wahrscheinlich auf eine Eduktdestabilisierung zurückzuführen. Eine weitere Erniedrigung der Barriere gibt es bei 53, da das Biradikal 83 bereits von drei Doppelbindungen stabilisiert wird, und die Reihe gipfelt schließlich in der Cyclisierung von 54, dessen Zwischenprodukt 88 eigentlich das Endprodukt ist, denn durch die hohe Stabilisierung wird es $21.6 \mathrm{kcal} \mathrm{mol}^{-1}$ exotherm gebildet und wird sich nicht mehr öffnen. Die rechte Seite hängt stärker von der Eduktstabilität ab. Die Butadien-Einheit scheint dort einen stabilisierenden Einfluß auf die Edukte zu haben, so daß die Barrieren alle (mit Ausnahme der entarteten Umlagerungen) höher sind als bei ihrem Gegenpart. 


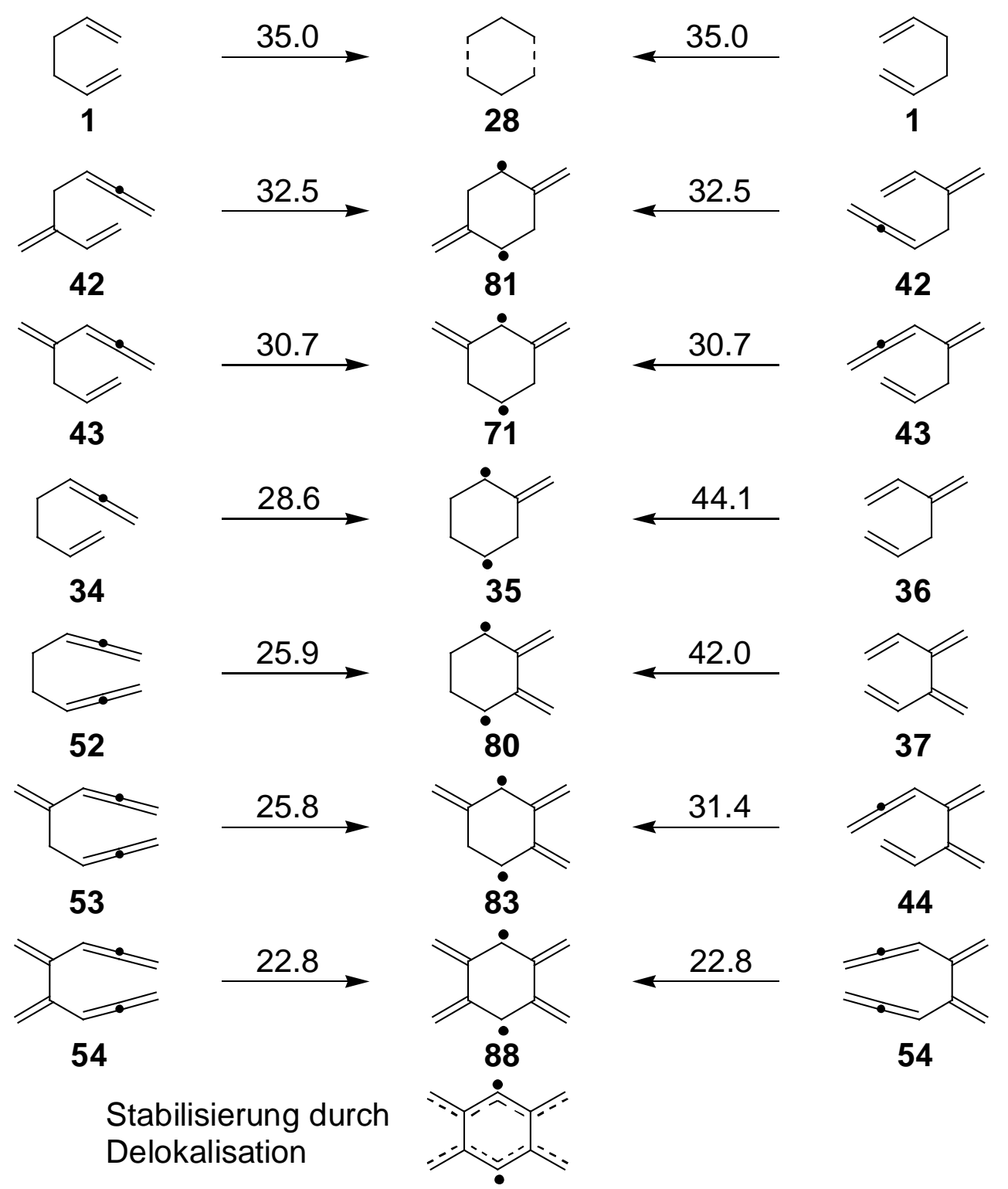

Abbildung 22. Umlagerungen der reinen $\pi, \pi$-Biradikale ohne Dreifachbindungen

Bei den Molekülen, die bei der Cyclisierung reine $\sigma, \sigma$-Biradikale bilden könnten, die also Dreifachbindungen und Allen- bzw. Kumuleneinheiten direkt an der zu brechenden bzw. zu knüpfenden Bindung haben, zeigt sich ein ähnliches Bild (Abb. 23). Die Umlagerungen von 45 und 59 laufen über einen konzertierten Prozeß ab und sind im Übergangszustand nicht stabilisiert. Die folgenden Moleküle besitzen alle eine energiereiche Kumuleneinheit, die die Edukte in der Energie destabilisiert und die Cyclisierung erleichtert. Während $\mathbf{6 1}$ und $\mathbf{6 8}$ ebenfalls über einen konzertierten Übergangszustand ablaufen, gibt es bei der Cyclisierung von 62 und 69 eine biradikalische Zwischenstufe. Die Stabilisierung der Biradikale ist in diesem Fall nicht ganz so einfach wie bei den $\pi, \pi$-Biradikalen, kann aber über teilweise Aromatisierung durch die exocyclischen Doppelbindungen und durch die Bildung von 
Elektronomeren erklärt werden. Die niedrigste Barriere hat 69 mit den zwei energiereichen Kumuleneinheiten und der teilweise stabilisierten, biradikalischen Zwischenstufe 85. Auf der rechten Seite sind die Dreifachbindungen offensichtlich ernergieärmer, da die Cyclisierungen von der rechten Seite her alle (wiederum abgesehen von den entarteten Umlagerungen) höhere Barrieren besitzen als ihre entsprechenden Gegenstücke.

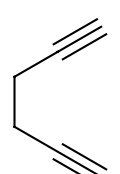

45

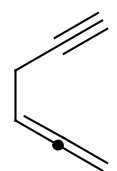

59

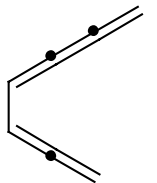

68

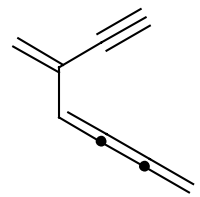

62<smiles>C#CCCCCCCCCCCCCC</smiles>

61<smiles>c1ccccc1</smiles>

69

\section{8}

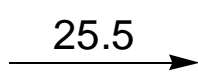

23.8

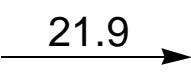

Stabilisierung durch exocyclische Doppelbindung und Elektronomere<smiles>[C]1C=CC=C1</smiles>

73

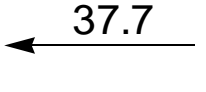<smiles>C1=CCCC=C1</smiles>

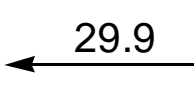

78<smiles>C=C1C=CC=CC1</smiles>

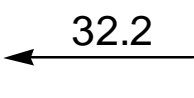

76<smiles>C=c1ccc(=C)cc1</smiles>

87

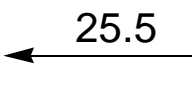<smiles>C=C1C=CC=CC1</smiles>

79

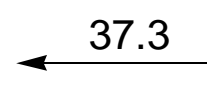<smiles>C=c1ccccc1=C</smiles>

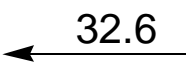

85

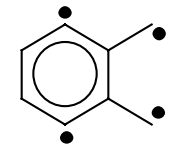

67

59

46

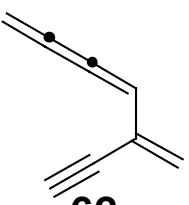

62

60

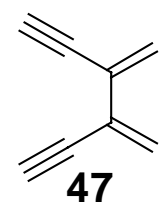

Abbildung 23. Umlagerungen der reinen $\sigma, \sigma$-Biradikale mit Dreifachbindungen und Allen- bzw. Kumuleneinheiten an der zu brechenden bzw. zu knüpfenden Bindung 
Gemischte Zustände haben in der Regel Energien zwischen oder nahe den reinen Zuständen (Abb. 24). Die zwei Beispiele zeigen, wie Mischungen aus reinen $\pi, \pi$ - und $\sigma, \sigma-$ Zuständen ermittelt werden können. Biradikalische Zwischenprodukte sind im Allgemeinen zu erwarten, wenn dieses durch zwei oder mehr exocyclische Doppelbindungen stabilisiert wird. Die $\sigma, \sigma$-Zustände mit einer exocyclischen Doppelbindung ergeben Übergangszustände, die $\pi, \pi$ - und die gemischten Zustände können über beide Reaktionspfade ablaufen (Übergangszustand und biradikalisches Intermediat). Umlagerungen bei Molekülen ohne Stabilisierung durch exocyclische Doppelbindungen laufen nur über Übergangszustände ab.

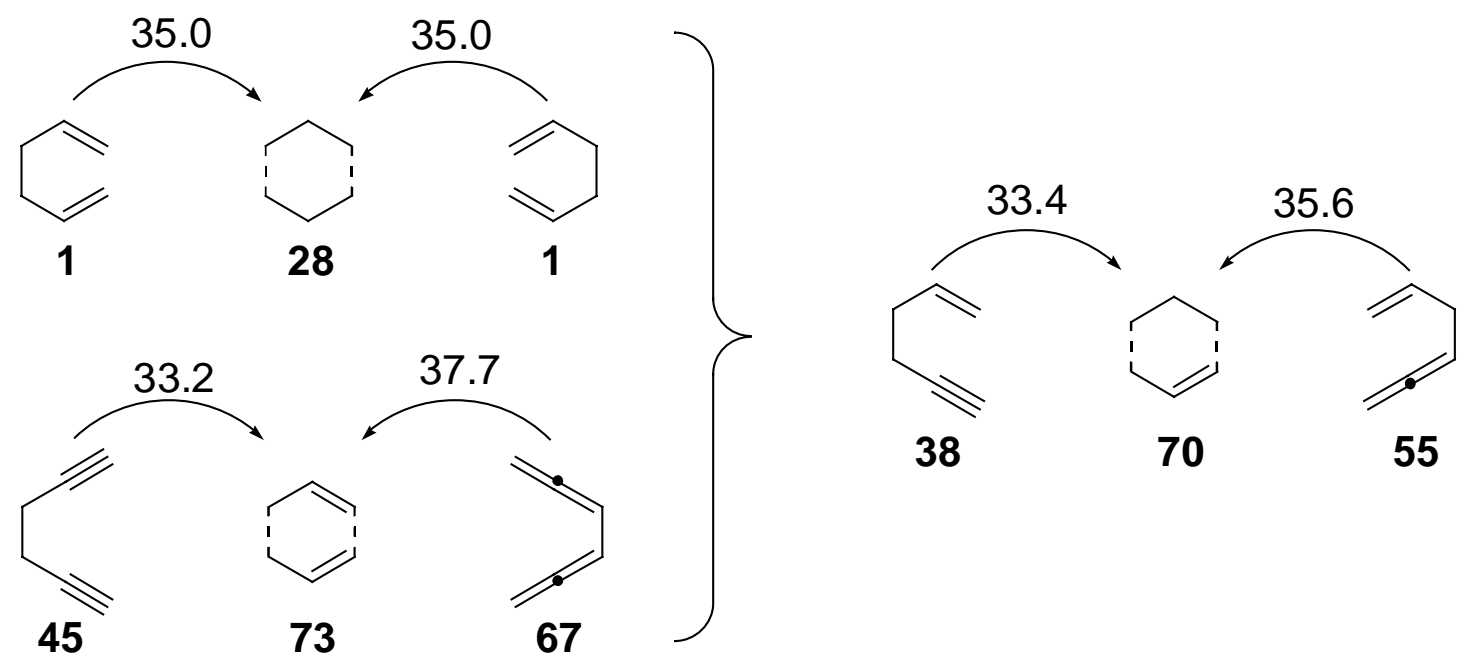<smiles>C=CC(=C)C(=C)C=C</smiles>

54<smiles>C=C1CC(=C)C(=CC)C(=C)C1=C</smiles>

88<smiles>C=c1ccc(=CC=CC)c(=C)c1=C</smiles>

69 85<smiles>[R6]C1C=CC(=C)C(=C)C(=C)C=C1</smiles>

54
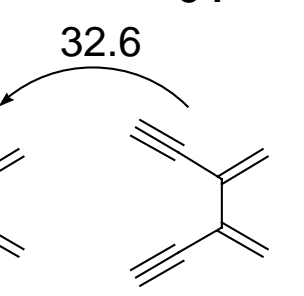

47

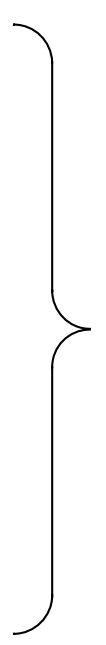

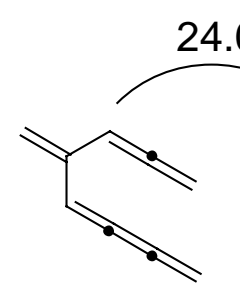

66<smiles>C=c1ccc(=C)c(=C)c1=C</smiles>

86<smiles>C#CC(=C)C(=C)C=C=C</smiles>

51

Abbildung 24. Die Energie gemischter Zustände ergibt sich ungefähr aus der Energie der reinen Zustände. 
Der zweite Cope-Familienzweig umfaßt nur sechs Mitglieder, da die Kombinationsmöglichkeiten durch die zusätzliche Doppelbindung eingeschränkt sind (Abb. 25). Diese ist auch dafür verantwortlich, daß die Mitglieder dieses Zweiges nicht umlagern, sondern cyclisieren, d.h. ein cyclisches Produkt bilden und kein cyclisches Intermediat (Ausnahme: die Bergman-Reaktion von 9 ohne Radikalfänger, siehe oben). Es gelten aber dieselben Regeln wie zuvor: die Edukte 7, 92 und 94 ohne Dreifachbindungen ergeben formal $\pi, \pi$ Biradikale, die jedoch wegen der Konjugation über die zusätzliche Doppelbindung rekombinieren und die geschlossenschalige Produkte 8, 93 und 95 bilden. Die gemischten Moleküle 11 und 89 ergeben die $\sigma, \pi$-Biradikale 12 und 91, man kann ihnen aber auch die geschlossenschaligen Strukturen 27 und $\mathbf{8 8}$ zuweisen (Elektronomere). Das symmetrische 9 mit zwei Doppelbindungen schließlich ergibt das $\sigma, \sigma$-Biradikal 10 oder sein Elektronomer 26. Wie die Cyclisierungen und Energetiken dieser Reaktionen im einzelnen aussehen und ablaufen, soll in den folgenden sechs Kapiteln ausführlich besprochen werden.

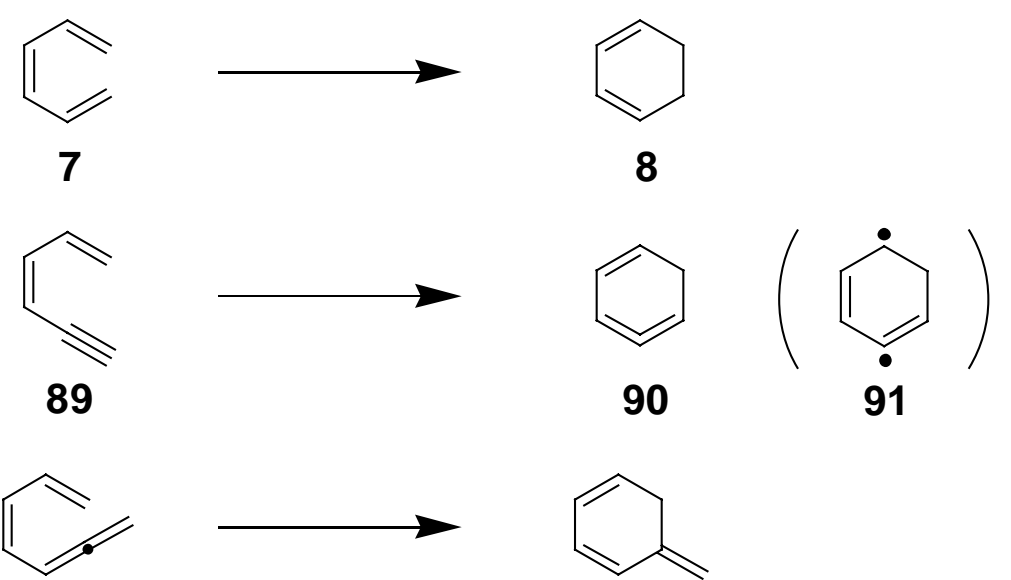

92<smiles>C#C/C=C\C#C</smiles><smiles>C#C/C=C\C=C/C=C</smiles>

11<smiles>C=C=C=CC=C=C</smiles>

94
93

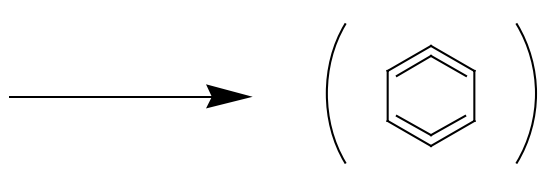

26

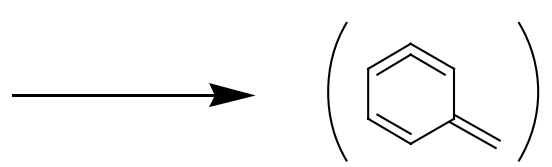

27<smiles>Cc1ccc(C)cc1</smiles>

10<smiles></smiles>

12
Abbildung 25. Mitglieder des zweiten Cope-Familienzweigs. 


\subsection{Cyclisierungen}

2.4.1 Die Cyclisierung von Hex-3-en-1,5-diin: Was gibt es neben der "Bergman"-Reaktion ?

Eine Gruppe bakterieller Produkte, zu denen auch Calicheamicin $\gamma_{1}^{\mathrm{I}} 13$ zählt, wurde in den 60er Jahren isoliert und zeigte starke antibiotische und tumorschädigenden Eigenschaften. ${ }^{20-25}$ Allen gemeinsam ist ein zehngliedriger Kohlenwasserstoffring, der eine Doppel- und zwei Dreifachbindungen enthält - die Endiin-Einheit.

Die pharmakologische Aktivität von 13 ist auf die Erhöhung der Ringspannung durch intramolekulare Michael-Addition zurückzuführen (Abb. 26). Unter diesen Bedingungen cyclisiert der Endiin-Teil des Moleküls spontan bei Körpertemperatur zu einem aromatischen Benzol-Biradikal 16, welches Wasserstoffatome von der Doppelhelix der DNA abstrahiert und damit zum Tod der Zelle führt.20-24,27,28

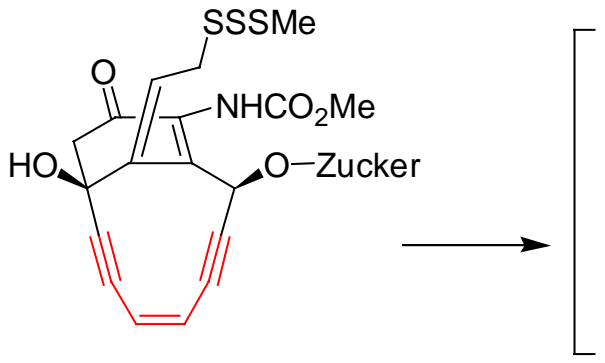

13
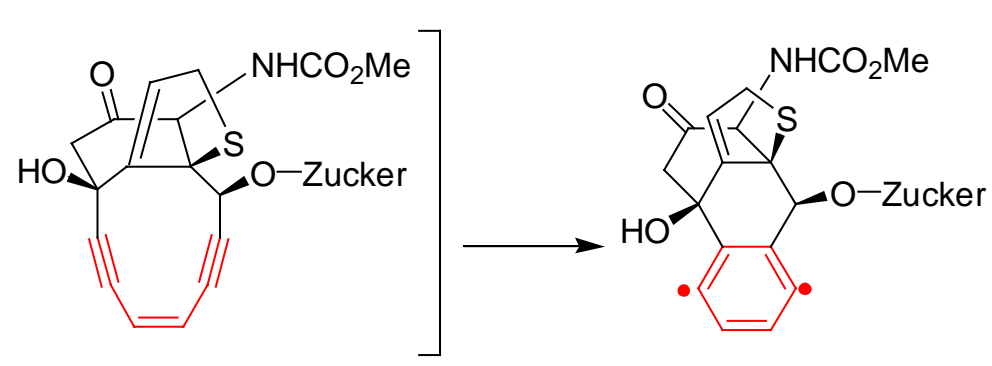

16

Abbildung 26. Die Cyclisierung von Calicheamicin $\gamma_{1}^{\mathrm{I}} \mathbf{1 3}$

Im Jahre 1972 konnte Bergman zeigen, daß die $C^{1}-C^{6}$ Cyclisierung von (Z)-Hex-3-en1,5-diin ("Endiin", 9) über die Zwischenstufe des 1,4-Didehydrobenzol-Biradikals ("Bergman-Produkt", 10) Benzol 96 ergibt (Abb. 27). ${ }^{12-15}$ Der Ringschluß zum aromatischen nichtkonjugierten $\sigma, \sigma$-Biradikal 10 ist dabei um $8.5 \pm 1.1 \mathrm{kcal} \mathrm{mol}^{-1}$ endotherm und findet erst bei hohen Temperaturen statt $\left(\mathrm{t}_{1 / 2} \approx 1 \mathrm{~h}\right.$ bei $\left.155^{\circ} \mathrm{C}\right) .{ }^{26}$ Da die folgende Bildung von zwei neuen $\mathrm{CH}$-Bindungen durch Abstraktion von einem geeigneten $\mathrm{H}$-Donor hoch exotherm verläuft, ist die Reaktion insgesamt irreversibel.

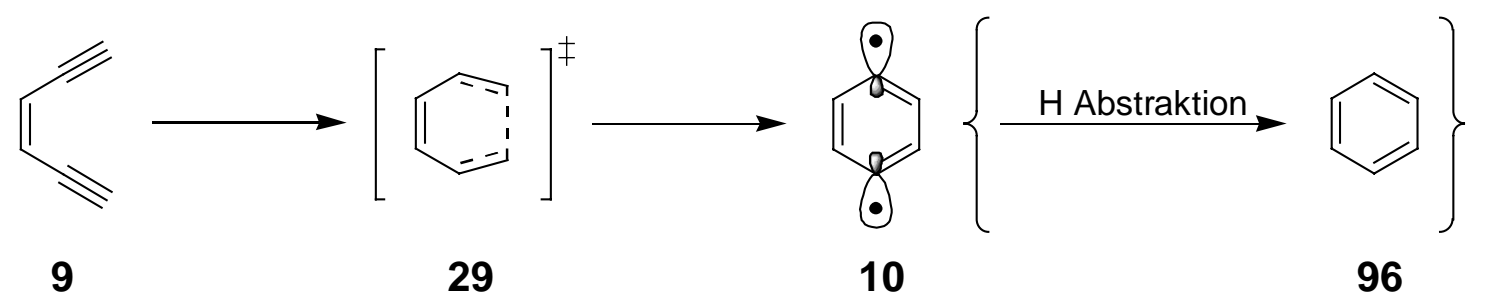

Abbildung 27. Die $C^{1}-C^{6}$ Cyclisierung von (Z)-Hex-3-en-1,5-diin 9 
Der Grund, warum die Cyclisierung der natürlichen Antibiotika bei niedrigeren Temperaturen, z. B. bei Körpertemperatur, abläuft, ist die Erhöhung der Ringspannung vor der eigentlichen Reaktion. ${ }^{24}$ Obwohl natürliche Antibiotika durch ihre Eigenschaften potentielle Antitumor-Medikamente sein sollten, sind sie leider wenig selektiv und lassen nur eine geringe Reaktionskontrolle zu. Daher sollten Endiine gefunden werden, die einen „Schalter“-Mechanismus beinhalten, der die Reaktivität nur innerhalb der Tumorzelle ,an“und „ab“-stellen kann, z. B. durch Veränderung der Ringspannung32,33 oder geeignete Substituenten, die protoniert/deprotoniert werden können. ${ }^{53}$ Auf diesem Wege könnten Moleküle mit physiologischen Eigenschaften generiert werden, die ihre zerstörerischen Eigenschaften nur dort einsetzen, wo sie benötigt werden.

In diesem Kapitel sollen die Cyclisierungseigenschaften des Stammsystems 9 eingehend untersucht und neue Cyclisierungen, die neben der bekannten Bergman-Reaktion stattfinden können, eingeführt werden. Insbesondere soll dabei auf eine interessante $C^{1}-C^{5}$ Cyclisierung eingegangen werden, die zum Fulven-Biradikal 97 führt (Abb. 28). Weiterhin wird der Effekt der Benzannelierung (98) untersucht sowie der Einfluß von Ringspannung in den Endiinringen 99a-h mit Ringgrößen von $7(n=1)$ bis $14(n=8)$ und verschiedenster Substituenten in den monosubstituierten 100a-t und disubstituierten Endiinen 100aa-tt auf die Barriere und Reaktionsenthalpie.

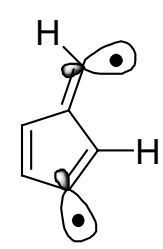

97

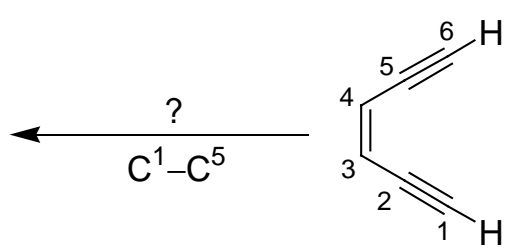

9<smiles>c1ccccc#1</smiles>

99a-h

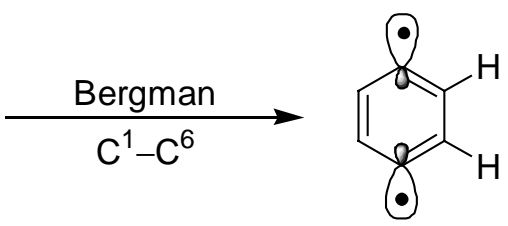

10<smiles>C#Cc1ccccc1C#C</smiles>

98

$$
n=1,2,3,4,5,6
$$

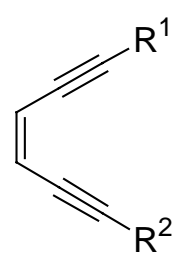

100a-t: $R^{1}=$ Subst.; $R^{2}=H$ 100aa-tt: $R^{1}=R^{2}=$ Subst.

Abbildung 28. Die Cyclisierungen des Endiin Stamm-, sowie weitere untersuchte Systeme

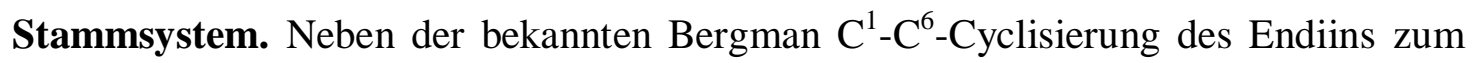
1,4-Didehydrobenzolbiradikal $\mathbf{1 0}$ (6-endo-dig) lassen sich theoretisch noch zwei weitere 
Cyclisierungsreaktionen formulieren. Der $C^{1}-C^{5}$-Ringschluss (5-exo-dig) würde dabei das 3,6-Didehydrofulven-Biradikal 97 erzeugen, während der $C^{2}-C^{5}$-Ringschluss (4-exo-dig) das

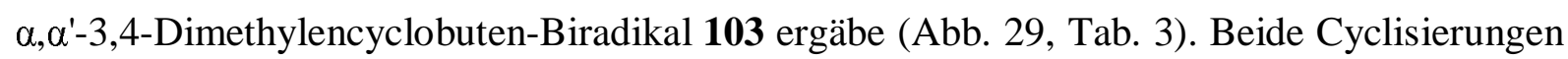
des Stammsystems zum fünf- und viergliedrigen Ring sind experimentell bisher noch nicht beobachtet worden.

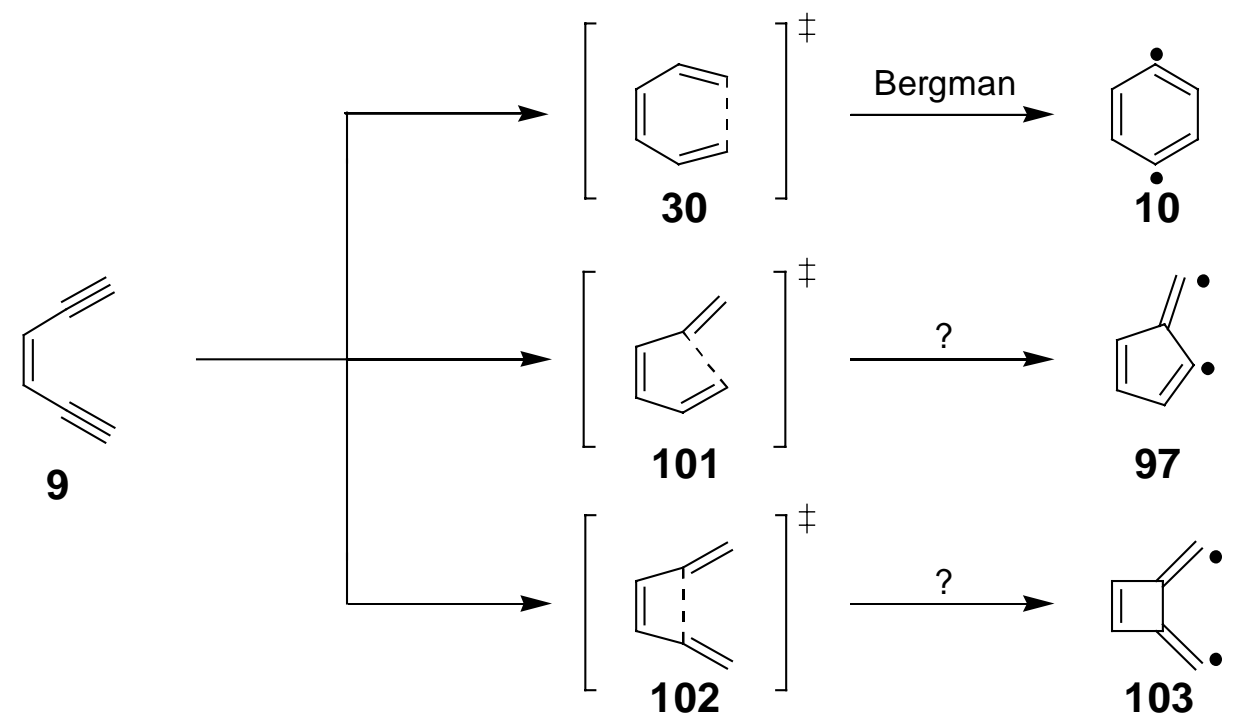

Abbildung 29. Die verschiedenen, theoretisch denkbaren Cyclisierungsarten des Endiin 9

Die Bergman-Reaktion ist experimentell ${ }^{12-15,26,40,54,148-150}$ sowie computerchemisch $^{32,33,51,52,55,56,74,151-154}$ sehr gut untersucht. Die experimentellen Werte für die Barriere $\left(28.2 \pm 0.5 \mathrm{kcal} \mathrm{mol}^{-1}\right)^{26}$ und die Reaktionsenthalpie $\left(8.5 \pm 1.1 \mathrm{kcal} \mathrm{mol}^{-1}\right)^{26}$ lassen sich mit BLYP/6-31G* (25.2 $\mathrm{kcal} \mathrm{mol}^{-1} ; 8.5 \mathrm{kcal} \mathrm{mol}^{-1}$ ) relativ gut beschreiben, besser jedoch mit BD(T)/cc-pVDZ Einzelpunkt-Energien auf die BLYP Geometrien $\left(27.1 \mathrm{kcal} \mathrm{mol}^{-1} ; 8.3 \mathrm{kcal}\right.$ $\mathrm{mol}^{-1}$ ). Deswegen soll im Folgenden nur auf die BD(T)-Energien eingegangen werden.

Die $C^{1}-C^{5}$ Cyclisierung des Stammsystems zum Fulven-Biradikal 97 ist wesentlich endothermer und hat eine höhere Barriere als die Bergman-Reaktion. Der Grund dafür liegt in der fehlenden Stabilisierung, da weder Aromatisierungs-Energie (wie bei der BergmanReaktion) noch andere Radikalstabilisierungs-Energien (z.B. $\pi$-Konjugation) gewonnen werden. Es entstehen zwei lokalisierte, isolierte Radikalzentren. Dementsprechend ist die Barriere der $C^{1}-C^{5}$ Cyclisierung (42.1 kcal mol-1) $15 \mathrm{kcal} \mathrm{mol}^{-1}$ höher und die Reaktionsenthalpie (39.6 kcal mol${ }^{-1}$ ) liegt $31 \mathrm{kcal} \mathrm{mol}^{-1}$ über der der Bergman-Reaktion. Deshalb wird, in Übereinstimmung mit allen experimentellen Beobachtungen, bei der thermischen Cyclisierung von 9 keine Bildung von Fulvenen beobachtet. Nichtsdestotrotz ist es wichtig, 
diesen Reaktionspfad zu studieren, da durch geeignete Veränderungen am Edukt die Bildung des Bergman-Produktes zugunsten des Fulven-Produktes zurückgedrängt werden könnte.

Einen Hinweis auf die Existenz dieser $C^{1}-C^{5}$ Cyclisierung gibt die Bildung von Indeno[2,1-a]inden-Derivaten 106 aus den sym-Dibenzo-1,5-cyclooctadien-3,7-diin-Derivaten 104, einem cyclischen Endiin-Verwandten (Abb. 30). ${ }^{155}$ Als Zwischenstufe wurde die Biradikal-Spezies 105 vorgeschlagen, die formal ein Dimeres des Fulven-Biradikals 97 ist.<smiles>[R]Oc1c(C)c(C)c(O)c2c1C#Cc1c(O)c(C)c(C)c([R])c1C#C2</smiles>

104

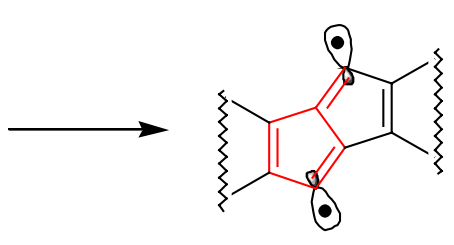

105<smiles>[R]Oc1c(C)c(C)c(O)c2c3c(ccc12)-c1c([R])c(C)c(C)c([R])c1-3</smiles>

106

Abbildung 30. Bildung von Indeno[2,1-a]inden-Derivaten 106 aus den sym-Dibenzo-1,5-cyclooctadien-3,7diin-Derivaten 104 via biradikalische Zwischenstufe 105

Der $C^{2}-C^{5}$ Ringschluß zum Dimetylencyclobuten Biradikal 103 ist aufgrund seiner ebenfalls fehlenden Stabilisierungsenergie und der zusätzlichen Ringspannung eines ungesättigten viergliedrigen Rings noch endothermer. Die Reaktionsenthalpie liegt mit 69.0 kcal mol ${ }^{-1}$ schon fast $42 \mathrm{kcal} \mathrm{mol}^{-1}$ über der des Bergman-Produktes 10 und $27 \mathrm{kcal} \mathrm{mol}^{-1}$ über der des Fulven-Biradikals 97. Der Übergangszustand dieser Cyclisierung konnte trotz größter Anstrengungen nicht lokalisiert werden, liegt aber höchstens $5 \cdot 10^{-3} \mathrm{kcal} \mathrm{mol}^{-1}$ oberhalb der Energie von 103. Dieser Reaktionspfad kann deshalb als experimentell detektierbar ausgeschlossen werden.

Tabelle 3. Barrieren und Reaktionsenthalpien $\left(\Delta_{0} \mathrm{H}\right)$ der Cyclisierungspfade von Endiin 9 (in kcal mol ${ }^{-1}$ )

\begin{tabular}{ccc}
\hline Struktur & BLYP/6-31G* & $\mathrm{BD}(\mathrm{T}) / \mathrm{cc}-\mathrm{pVDZ}$ \\
\hline $\mathbf{9}$ & 0.0 & 0.0 \\
$\mathbf{3 0}$ & 25.2 & 27.1 \\
$\mathbf{1 0}$ & 8.5 & 8.3 \\
$\mathbf{1 0 1}$ & 41.0 & 42.1 \\
$\mathbf{9 7}$ & 41.3 & 39.6 \\
$\mathbf{1 0 2}$ & - & - \\
$\mathbf{1 0 3}$ & 68.8 & 69.0 \\
${ }^{\mathrm{a}}$ Geometrien und thermische Korrekturen aus BLYP/6-31G*.
\end{tabular}


Das Fulven-Biradikal kann in zwei Isomeren vorkommen, die sich in der Stellung des Wasserstoffatoms an der exocyclischen Doppelbindung unterscheiden (Abb. 31). Die (Z)Konfiguration (Z)-97 ist dabei $2.7 \mathrm{kcal} \mathrm{mol}^{-1}$ stabiler als das (E)-Isomer (E)-97, da im ersteren Fall eine stabilisierende Interaktion zwischen dem Radikal-sp ${ }^{2}$-Orbital an $\mathrm{C}^{6}$ und dem antibindenden $C^{4}-C^{5}$ Orbital stattfinden kann. Die entsprechende Interaktion ist in $(\boldsymbol{E})-97$ kleiner, was sich auch in der kürzeren Bindungslänge der Akzeptorbindung bemerkbar macht

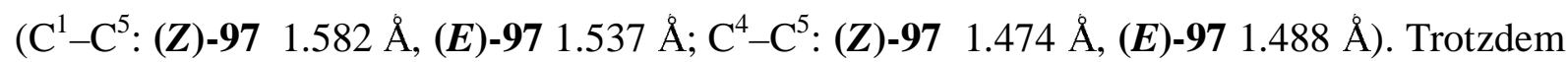
kann (E)-97 eine wichtige Rolle in der Cyclisierung von Endiin spielen, wenn große Substituenten ihre Repulsion minimieren müssen.
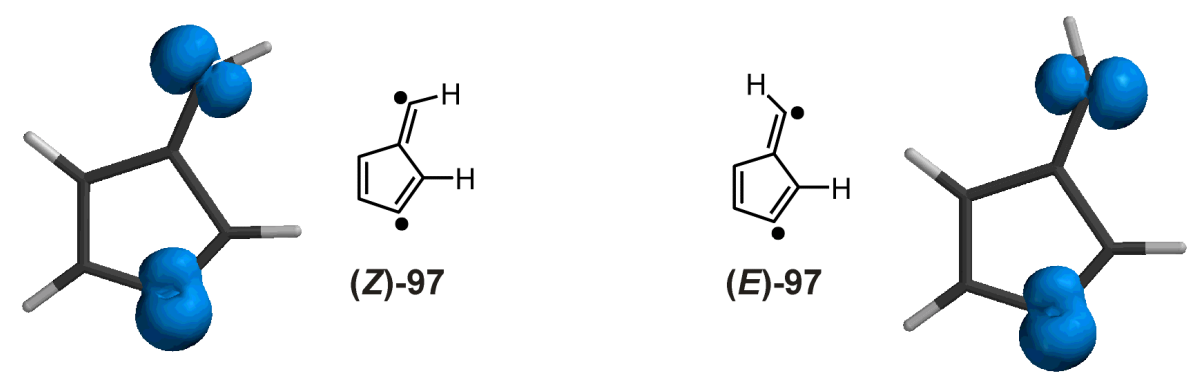

Abbildung 31. Spindichten des (Z)-97 und (E)-97 Fulven Biradikals

Im Hinblick auf die experimentelle Bestätigung der Cyclisierung zum FulvenBiradikal erscheint es als geeignet, die acetylenischen Wasserstoffatome durch raumbeanspruchende Gruppen zu substituieren. Die Repulsion dieser ortho-angeordneten Gruppen in einem entstehenden Bergman-Produkt sollte dann diesen Reaktionspfad gegenüber dem einer Fulven-Cyclisierung energetisch ungünstiger machen (Abb. 32).
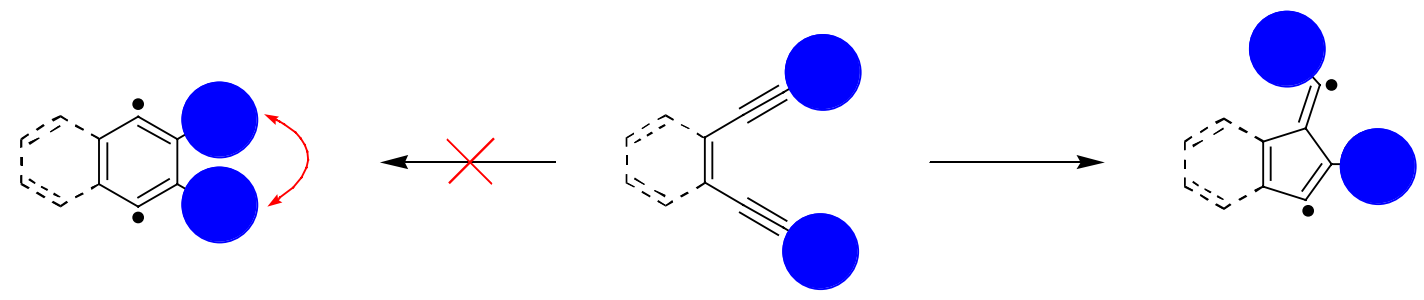

Abbildung 32. Schematische Darstellung einer möglichen Synthesemethode von Fulven-Derivaten aus disubstituierten Endiinen

Im Gegensatz zur Bergman Reaktion hat der Übergangszustand der $\mathrm{C}^{1}-\mathrm{C}^{5}$ Cyclisierung einen hohen Biradikalcharakter. Optimierungen entlang des Reaktionspfades zeigen, daß ein ÜZ nur existiert, wenn eine broken spin offenschalige Wellenfunktion (BS- 
UBLYP) verwendet wird. Für den geschlossenschaligen Fall (RBLYP) ergibt sich eine stetig ansteigende Energie für kleinere $C^{1}-C^{5}$ Abstände ohne Maximum (Abb. 33). Am ÜZ ergibt sich damit eine Energielücke zwischen dem offen- und geschlossenschaligen Ansatz (grauer Kasten oben, Abb. 33). Der Übergangszustand der $\mathrm{C}^{1}-\mathrm{C}^{6}$ Cyclisierung (Bergman) zeigt hingegen, wie schon in anderen Studien angemerkt, keinen Biradikalcharakter. Dies wird durch das Fehlen der Energielücke am Hochpunkt der Energiehyperflächen der offen- und geschlossenschaligen Ansätze eindeutig belegt (grauer Kasten unten, Abb. 33).

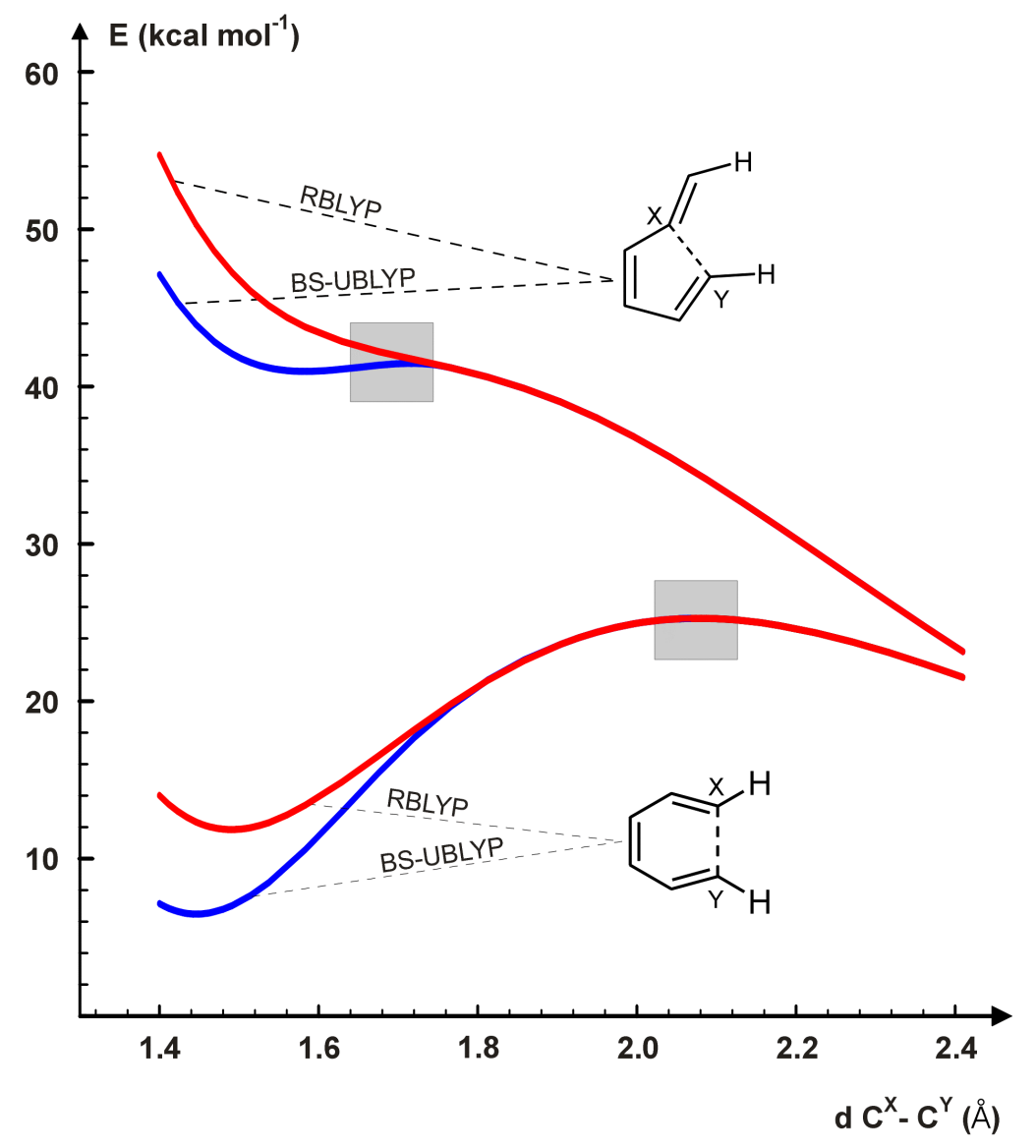

Abbildung 33. Offen- und geschlossenschalige $C^{1}-C^{5}$ und $C^{1}-C^{6}$ (Bergman) Cyclisierung von 9 berechnet mit BLYP/6-31G*

Die Standardbildungsenthalpie $\Delta_{\mathrm{f}} \mathrm{H}^{\ominus}$ von 97 kann durch eine isodesmische Gleichung bestimmt werden (Tab. 4). Diese Methode wurde bereits zur Bestimmung der Standardbildungsenthalpie des Bergman Produktes verwendet, wobei auf dem BD(T)/ccpVDZ Niveau ein Wert von $137.5 \pm 2.0 \mathrm{kcal} \mathrm{mol}^{-1}$ errechnet wurde. Der Vergleich mit dem experimentellen Wert von $138.0 \pm 1.0 \mathrm{kcal} \mathrm{mol}^{-1}$ zeigt, daß die Qualität dieser Methode sehr gut ist. Unter denselben Bedingungen kann $\Delta_{\mathrm{f}} \mathrm{H}^{\ominus}$ von 97 mit $172.0 \pm 1.0 \mathrm{kcal} \mathrm{mol}^{-1}$ vorhergesagt werden. 
Tabelle 4. Isodesmische Gleichung zur Bestimmung der Standardbildungsenthalpie $\Delta_{\mathrm{f}} \mathrm{H}^{\ominus}$ von $97\left(\Delta_{\mathrm{f}} \mathrm{H}^{\ominus}\right.$ Benzol $=19.81 \mathrm{kcal} \mathrm{mol}^{-1}, 156$ Fulven $=53.6 \mathrm{kcal} \mathrm{mol}^{-1},{ }^{156}$ Didehydrobenzol $\left.=138.0 \pm 1.0 \mathrm{kcal} \mathrm{mol}^{-1}\right)^{124}$

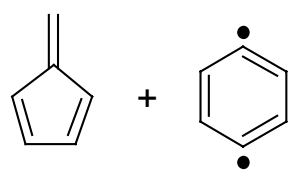

10

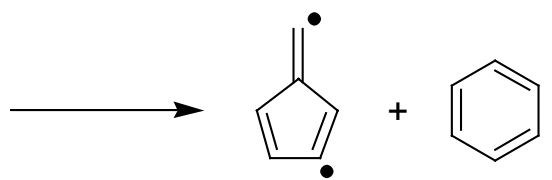

97

\begin{tabular}{lcc}
\hline & $\Delta_{R} \mathrm{H}$ & $\Delta_{\mathrm{f}} \mathrm{H}^{\ominus}$ \\
\hline BLYP/6-31G* & 0.02 & $171.8 \pm 1.0$ \\
BCCD(T)/cc-pVDZ & 0.23 & $172.0 \pm 1.0$ \\
\hline${ }^{\mathrm{a}}$ Geometrien und thermische Korrekturen aus BLYP/6-31G*
\end{tabular}

Benzannelierung. Das benzannelierte Endiin (1,2-Diethinylbenzol, 98) folgt den gleichen Reaktionspfaden wie 9, aber die Barrieren und Enthalpien sind verändert (Tab. 5). Wie auch experimentell beobachtet, ist die Bergman-Cyclisierung, die zu 1,3-Didehydronaphthalin 32 führt, energetisch ungünstiger als die des Stammsystems. ${ }^{129,150,156}$ Der Ursprung dafür liegt in der geringeren aromatischen Stabilisierungsenergie verglichen mit zwei Benzolringen. Der $\mathrm{C}^{1}-\mathrm{C}^{6}$ Ringschluß des Stammsystems erzeugt ein aromatisches aus einem nichtaromatischen System und erhält dafür die volle aromatische Stabilisierung eines Benzolringes. Das benzannelierte System enthält schon ein aromatisches System, die Cyclisierung zu 32 erhält deshalb nicht die volle aromatische Stabilisierung. Dies kann anhand der Exothermie $\left(-9.4 \mathrm{kcal} \mathrm{mol}^{-1}\right)$ der folgenden isodesmischen Gleichung nachvollzogen werden:

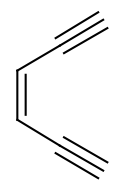

9

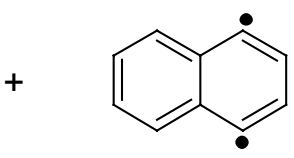

32<smiles>C#Cc1ccccc1C#C</smiles>

98

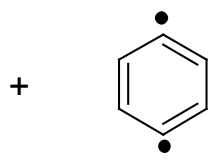

10

Als Konsequenz daraus liegt der ÜZ $31\left(29.2 \mathrm{kcal} \mathrm{mol}^{-1}\right) 2.1 \mathrm{kcal} \mathrm{mol}^{-1}$ oberhalb dem der Stammreaktion und $32\left(17.6 \mathrm{kcal} \mathrm{mol}^{-1}\right)$ wird $9.4 \mathrm{kcal} \mathrm{mol}^{-1}$ endothermer gebildet als 10 (Abb. 34). Wie in der Stammreaktion hat auch 31 keinerlei Biradikalcharakter.

Eine leichte Erhöhung der Ringspannung durch Benzannelierung macht die Cyclisierung zu 108 (70.9 $\left.\mathrm{kcal} \mathrm{mol}^{-1}\right) 2.0 \mathrm{kcal} \mathrm{mol}^{-1}$ endothermer als in der Stammreaktion. In diesem Falle konnte jedoch der Übergangszustand 107 lokalisiert werden, liegt aber mit $71.8 \mathrm{kcal} \mathrm{mol}^{-1}$ nur wenig oberhalb von $\mathbf{1 0 8}$ (Abb. 34). 
Das benzannelierte Derivat des Fulven-Biradikals (1-Methylen- $1 H$-inden-Biradikal, 110) ist nur unwesentlich gegenüber der Stammreaktion stabilisiert $\left(0.9 \mathrm{kcal} \mathrm{mol}^{-1}\right)$, dasselbe gilt für den entsprechenden Übergangszustand $109\left(41.6 \mathrm{kcal} \mathrm{mol}^{-1}\right)$ der nur $0.5 \mathrm{kcal} \mathrm{mol}^{-1}$ niedriger liegt (Abb. 34). Das ist nicht unerwartet, da durch die Cyclisierung kein aromatisches System gebildet wird, d.h. die aromatische Stabilisierung ist vor und nach der Cyclisierung gleich (keine für die Stammreaktion und die eines Benzolringes im benzannelierten Falle). Dies kann wiederum durch die folgende, nahezu thermoneutrale isodesmische Gleichung $\left(1.3 \mathrm{kcal} \mathrm{mol}^{-1}\right)$ gezeigt werden:

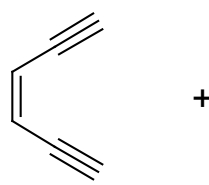

9<smiles>C=C1C=Cc2ccccc21</smiles>

110

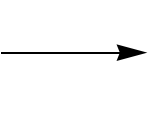

Der Übergangszustand 109 hat wie im Stammsystem einen ausgeprägten Biradikalcharakter.

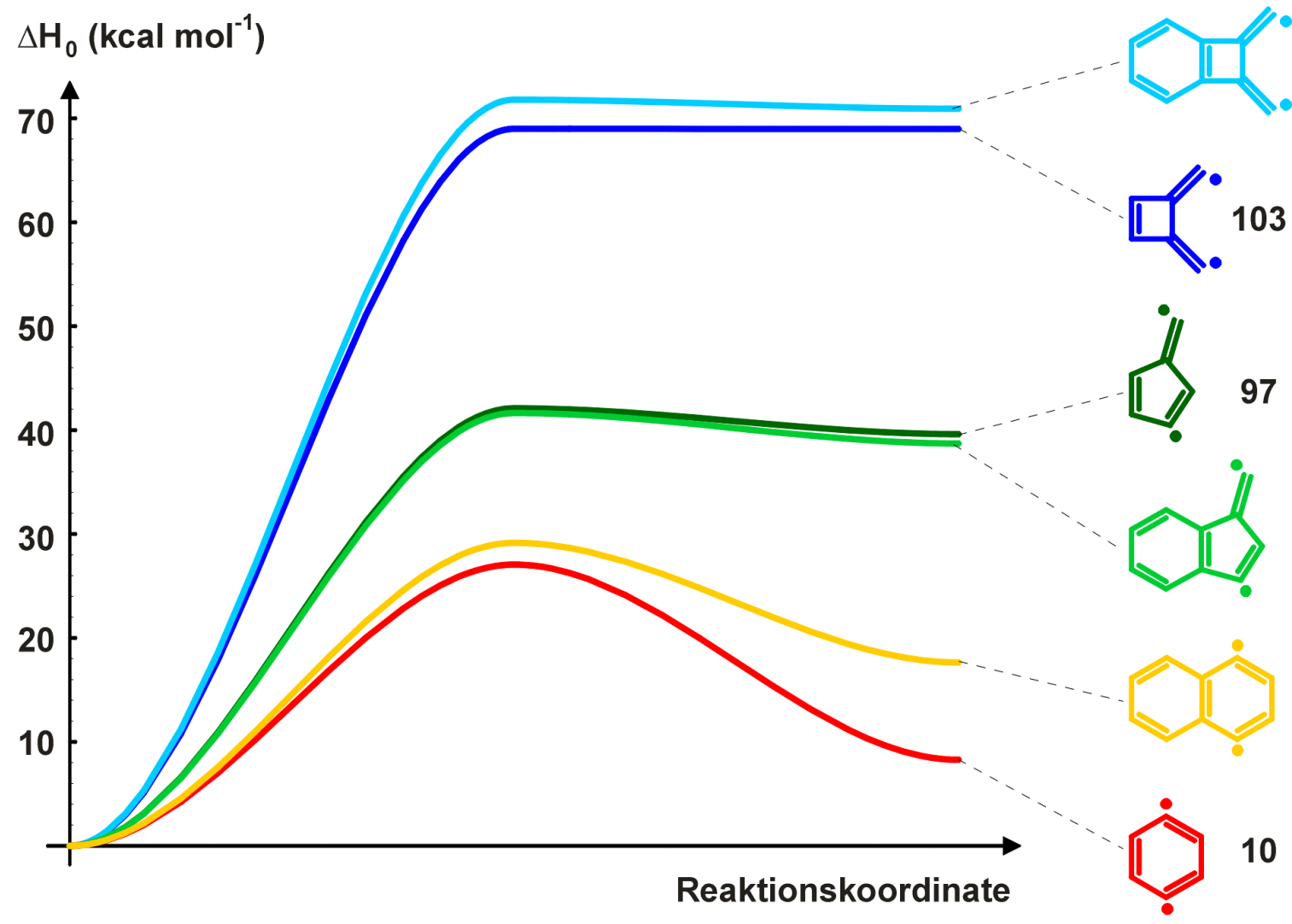

Abbildung 34. Vergleich der Energiehyperflächen für die Cyclisierungsreaktionen von Stamm- 9 und benzanneliertem System 98 auf BCCD(T)/cc-pVDZ//BLYP/6-31G* Niveau 
Tabelle 5. Barrieren und Reaktionsenthalpien $\left(\Delta_{0} \mathrm{H}\right)$ der Cyclisierungspfade von 1,2-Diethinylbenzol 98 (in kcal mol${ }^{-1}$ )

\begin{tabular}{ccc}
\hline Struktur & BLYP/6-31G* & BD(T)/cc-pVDZ \\
\hline $\mathbf{9 8}$ & 0.0 & 0.0 \\
$\mathbf{3 1}$ & 24.6 & 29.2 \\
$\mathbf{3 2}$ & 14.4 & 17.6 \\
$\mathbf{1 0 9}$ & 37.2 & 41.6 \\
$\mathbf{1 1 0}$ & 37.4 & 38.7 \\
$\mathbf{1 0 7}$ & 67.3 & 71.8 \\
$\mathbf{1 0 8}$ & 67.9 & 70.9 \\
\hline
\end{tabular}

${ }^{\text {a }}$ Geometrien und thermische Korrekturen aus BLYP/6-31G*.

Ringspannung. ${ }^{32,33}$ Wie schon bei den Naturstoffen zu beobachten, ist die Einbettung der Endiin-Einheit in ein Ringgerüst und der damit einhergehende Aufbau von Ringspannung eine Möglichkeit, das Cyclisierungsverhalten bei der Bergman-Reaktion zu verändern. ${ }^{20-}$ 24,27,28,30,31,34-36 Ist die Ringspannung, aufgebaut durch die zwei linearen Acetylen-Einheiten, größer als die Reaktionsbarriere, sollte es sogar bei Raumtemperatur zu spontaner Cyclisierung kommen. ${ }^{36}$ Fehlt bei der Reaktion jedoch ein geeigneter Radikalfänger, so kann das Radikal 10 wieder zum Endiin 9 zurückreagieren, und zwar, wie aus Experimenten mit deuteriertem Endiin 111 bekannt, in die eine oder die andere Richtung. ${ }^{14}$ Beim Endiin selbst gibt es keinen Unterschied in der Struktur der beiden vom Didehydrobenzol zurückerhaltenen Edukte, wohl aber bei den cyclischen Endiinen, bei denen das Biradikal 112a-h zum endocyclischen 99a-h und zum exocyclischen Endiin 113a-h zurückumlagern kann (Abb. 35).

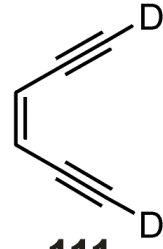

111

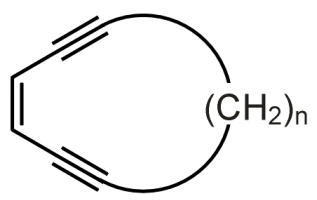

99a-h
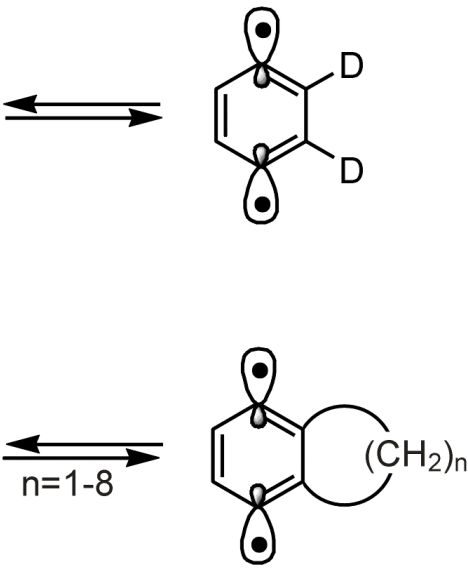

112a-h
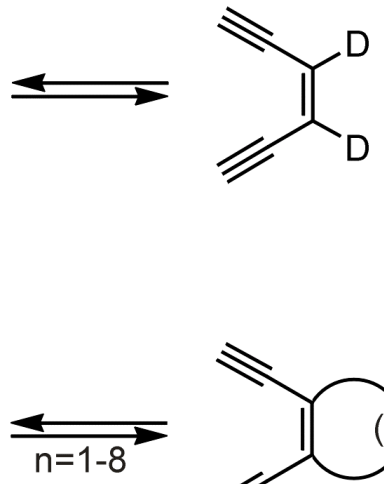<smiles>C#CC1=C(C#C)CCCC1</smiles>

113a-h

Abbildung 35. Cyclisierung und Umlagerung von Dideuteroendiin 111 und der Ringendiine 99a-h und 113a-h 
Im folgenden Abschnitt soll zunächst der Effekt der Ringspannung auf das Cyclisierungsverhalten endocyclischer Endiine 99a-h untersucht werden. Anschließend werden die exocyclischen Endiine 113a-h sowie das Öffnungsverhalten der Biradikale 112a$\mathbf{h}$ hin zu beiden Endiinspezies näher betrachtet.

Eine generelle Tendenz in der Cyclisierung der endocyclischen Endiine 99a-h ist die abnehmende Reaktivität mit zunehmender Ringgröße (Abb. 36, Tab. 6). Die Ringe können dabei in drei Kategorien eingeteilt werden:

- Ringe, die Biradikale mit kleinen Alkyl-Ringen bilden (99a,b; $\mathrm{n}=1,2)$

- Ringe, die Biradikale mit mittleren Alkyl-Ringen bilden (99c,d,e; $\mathrm{n}=3,4,5)$

- Ringe, die Biradikale mit großen Alkyl-Ringen bilden (99f,g,h; $\mathrm{n}=6,7,8)$

Die beiden sieben- und achtgliedrigen Endiine 99a,b müßten bei der Cyclisierung ein hochgespanntes Benzocyclopropen- 112a bzw. Benzocyclobuten-Biradikal 112b ausbilden. Im Falle von 99a ist dies nicht möglich, es konnte kein entsprechendes Biradikal lokalisiert werden. Im Falle von 99b liegt das Biradikal 112b mit einer Energie von $12.4 \mathrm{kcal} \mathrm{mol}^{-1}$ etwas über dem der Stammreaktion, die Barriere 114b liegt jedoch bei nur $13.4 \mathrm{kcal} \mathrm{mol}^{-1}$, was weit unterhalb der der Stammreaktion liegt. Allerdings ist die Barriere der Rückreaktion zu 99b so klein, daß keine Cyclisierung stattfindet. Ein Hinweis darauf, daß 99b stabil bei Raumtemperatur ist, zeigt das ebenfalls stabile, bereits bekannte Benzocyclooct-1-en-3,7-diin, das benzannelierte Derivat von 99b, das sich beim Erhitzen zersetzt, statt zu cyclisieren.

Das neungliedrige Endiin 99c bildet im Biradikal 112c einen nahezu ungespannten Cyclopentenring aus und sollte deshalb die Ringspannung des Edukts zur Verringerung der Barriere voll ausnutzen können. Tatsächlich hat 99c mit einer Barriere von $12.9 \mathrm{kcal} \mathrm{mol}^{-1}$ und einer Reaktionsenergie von $4.5 \mathrm{kcal} \mathrm{mol}^{-1}$ die niedrigsten Werte aller untersuchten Endiine und 99c sollte deshalb bei Raumtemperatur spontan cyclisieren. Der zehngliedrige Ring 99d bildet ebenfalls einen nahezu ungespannten Cyclohexenring aus, hat jedoch das ungespanntere Edukt aufgrund des größeren Ringes. Deshalb hat 99d eine höhere Barriere (114d: $21.8 \mathrm{kcal} \mathrm{mol}^{-1}$ ) und Energie (112d: $9.5 \mathrm{kcal} \mathrm{mol}^{-1}$ ), die jedoch beide noch unterhalb der des Stammsystems liegen. Wie experimentell bekannt und rechnerisch bestätigt, cyclisiert 99d bei Raumtemperatur langsam, bei $50^{\circ} \mathrm{C}$ jedoch glatt und ergibt mit einem geeigneten Radikalfänger Tetrahydronaphthalin. Der elfgliedrige Ring 99e liegt in seiner Barriere schon knapp (114e: $27.6 \mathrm{kcal} \mathrm{mol}^{-1}$ ) und in der Energie (112e: $14.7 \mathrm{kcal} \mathrm{mol}^{-1}$ ) deutlich oberhalb der des Stammsystems und cyclisiert deshalb erst bei sehr hohen Temperaturen. 
Die Energien der weiteren Endiine 99f-h liegen erheblich höher, was darauf zurückzuführen ist, daß bei der Cyclisierung ungünstige, große acht- bis zehngliedrige Cycloalkenringe gebildet werden. Für 99f liegen Barriere (114f: $39.2 \mathrm{kcal} \mathrm{mol}^{-1}$ ) und Reaktionsenergie (112f: $27.1 \mathrm{kcal} \mathrm{mol}^{-1}$ ) schon weit oberhalb der Stammreaktion, was eine thermische Cyclisierung praktisch ausschließt. Dasselbe gilt für 99g (114g: $38.3 \mathrm{kcal} \mathrm{mol}^{-1}$; 112g: $29.4 \mathrm{kcal} \mathrm{mol}^{-1}$ ) und 99h (114h: $42.6 \mathrm{kcal} \mathrm{mol}^{-1}$; 112h: $\left.31.9 \mathrm{kcal} \mathrm{mol}^{-1}\right)$, die mit geringen Abständen folgen.

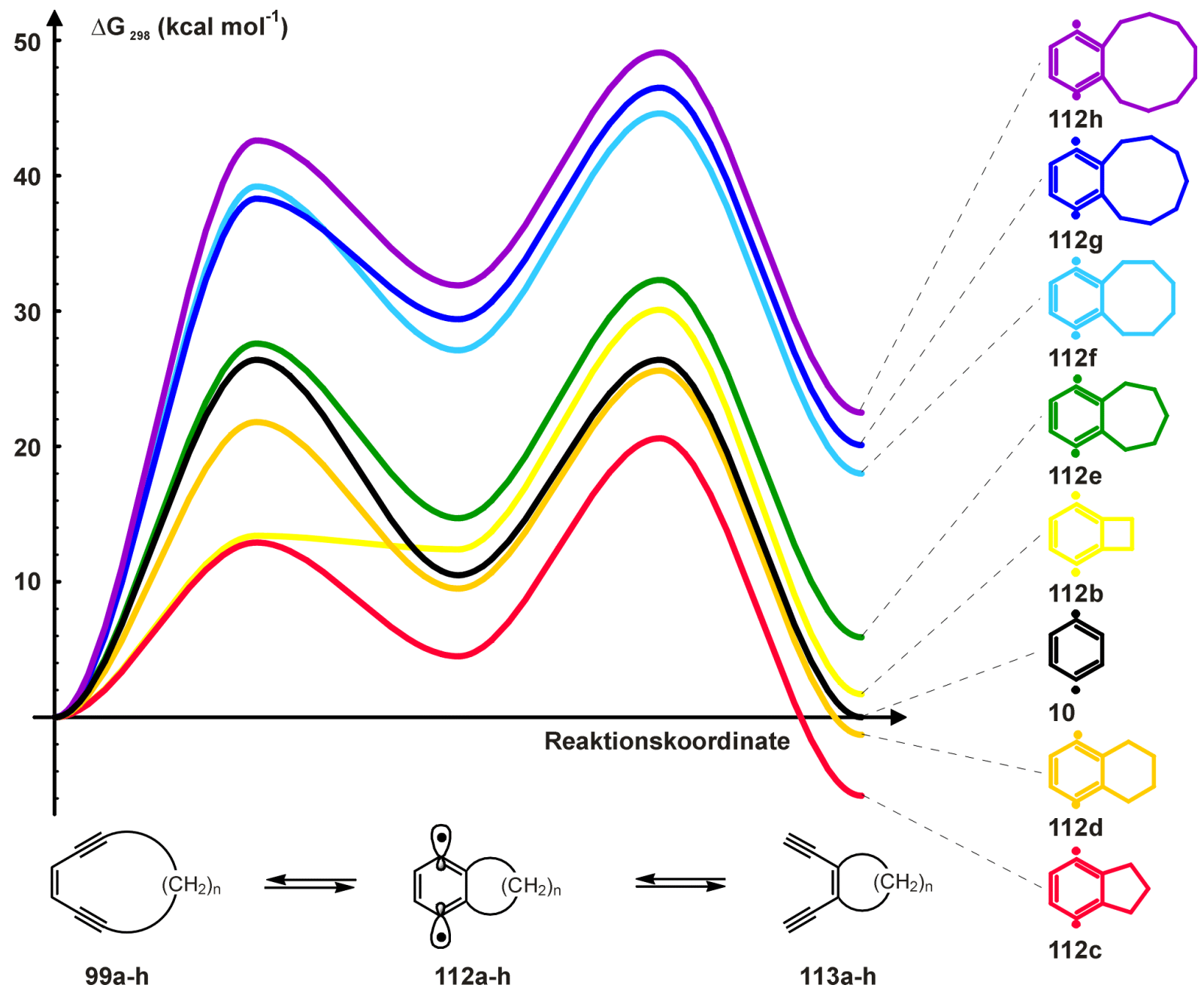

Abbildung 36. Energiediagramm für die Cyclisierung und Ringöffnung von endocyclischen Endiinen 99b-h

Die exocyclischen Endiine weisen eine ganz andere Tendenz auf. Bei allen Cyclisierungen von 113b-h liegen die Barrieren 115a-h in einem Bereich von $2 \mathrm{kcal} \mathrm{mol}^{-1}$ beieinander, ohne 113b, das man als Sonderfall aufgrund des aufgeweiteten Winkels der Acetyleneinheiten betrachten kann, sogar in einem Bereich von nur $0.5 \mathrm{kcal} \mathrm{mol}^{-1}$ um die Barriere des Stammsystems (Abb. 37, Tab. 6. Zur besseren Darstellung zeigt Abb. 37 dieselben Energiewerte wie Abb. 36, jedoch mit anderem Nullpunkt). Die Reaktionsenergien 
zu 112b-h verteilen sich ebenfalls in einem Bereich von $2 \mathrm{kcal} \mathrm{mol}^{-1} \mathrm{um}$ die Energie des Stammsystems, wobei die kleineren Ringe 112b-d näher an dem Wert des Stammsystems liegen, während die Werte der größeren Ringe 112e-h etwas darunter liegen $\left(\sim 1 \mathrm{kcal} \mathrm{mol}^{-1}\right)$. Man kann also davon ausgehen, daß die Annelierung von Cycloalkenringen an die Doppelbindung des Endiins keinerlei Effekt auf die Energetik der Bergman-Reaktion verglichen mit dem Stammsystem hat.

Ausgehend von den Bergman-Biradikalen 112b-h ist die Barriere der Ringöffnung hin $\mathrm{zu}$ den exocyclischen Endiinen 113b-h immer höher als $\mathrm{zu}$ den entsprechenden endocyclischen Endiinen 99b-h. In Abwesenheit eines geeigneten Radikalfängers sollte es also möglich sein, die endocyclischen Endiinen über die Zwischenstufe der Biradikale 112b-h aus den exocyclischen herzustellen, während der umgekehrte Weg nicht möglich ist. Im zweiten Fall erfolgt nur die Rückreaktion zu den endocyclischen 99b-h.

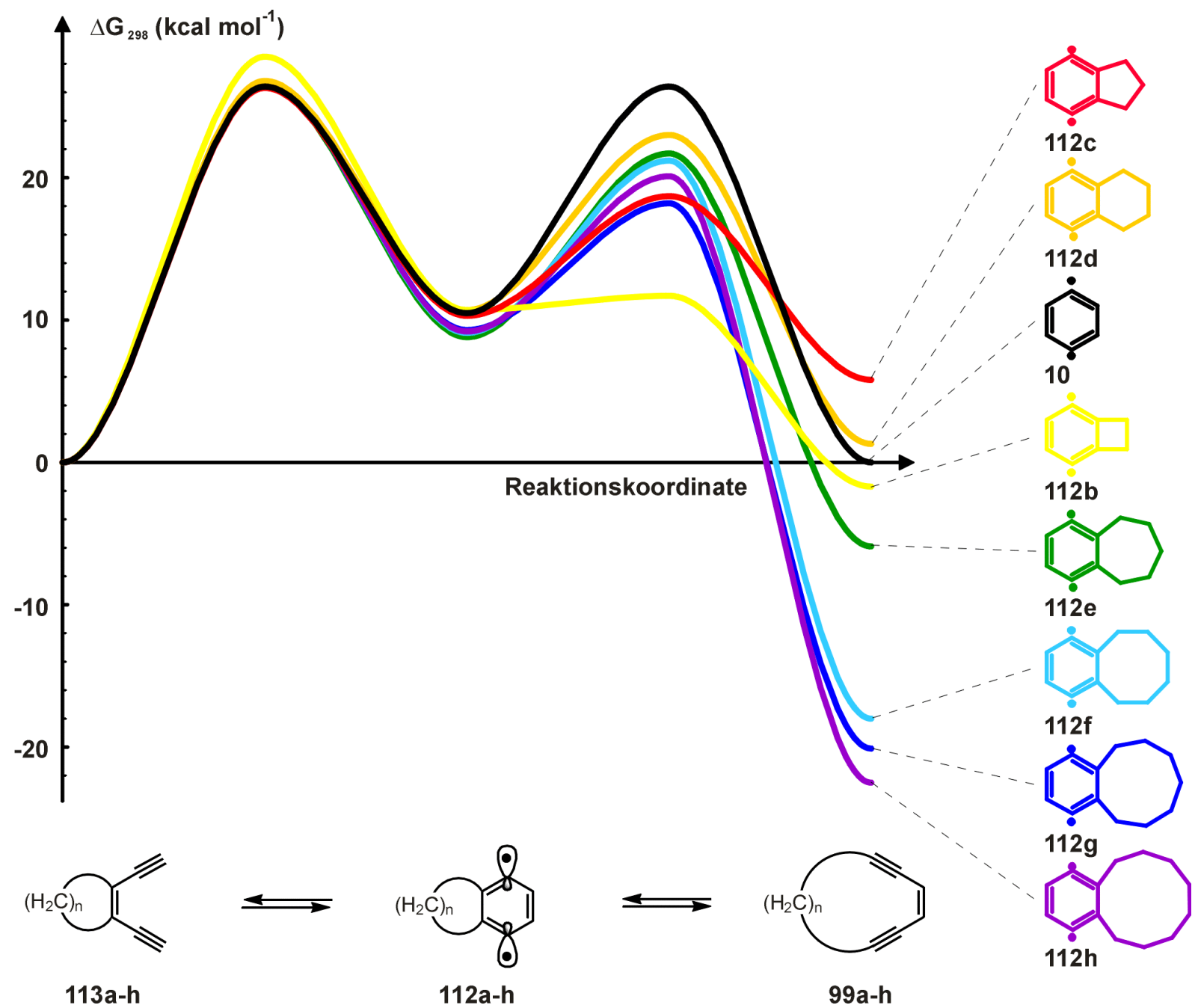

Abbildung 37. Energiediagramm für die Cyclisierung und Ringöffnung von exocyclischen Endiinen 113b-h 
Tabelle 6. Barrieren und Reaktionsenthalpien $\left(\Delta \mathrm{G}_{298}\right)$ der Endiine 99a-h und 113a-h, berechnet auf dem BLYP/6-31G* Nivau (in kcal mol ${ }^{-1}$ )

\begin{tabular}{|c|c|c|c|c|c|c|}
\hline Ringgröße & & 99 & 114 & 112 & 115 & 113 \\
\hline \multirow[t]{2}{*}{ 7-Ring } & $\mathbf{a}$ & 0.0 & - & - & - & -4.3 \\
\hline & & 4.3 & - & - & - & 0.0 \\
\hline \multirow[t]{2}{*}{ 8-Ring } & b & 0.0 & 13.4 & 12.4 & 30.1 & 1.7 \\
\hline & & -1.7 & 11.7 & 10.7 & 28.5 & 0.0 \\
\hline \multirow[t]{2}{*}{ 9-Ring } & c & 0.0 & 12.9 & 4.5 & 20.6 & -5.8 \\
\hline & & 5.8 & 18.7 & 10.3 & 26.3 & 0.0 \\
\hline \multirow[t]{2}{*}{ 10-Ring } & d & 0.0 & $21.8^{\mathrm{a}}$ & 9.5 & 25.6 & -1.3 \\
\hline & & 1.3 & 23.0 & 10.7 & 26.8 & 0.0 \\
\hline \multirow[t]{2}{*}{ 11-Ring } & $\mathbf{e}$ & 0.0 & 27.6 & 14.7 & 32.2 & 5.9 \\
\hline & & -5.9 & 21.7 & 8.8 & 26.5 & 0.0 \\
\hline \multirow[t]{2}{*}{ 12-Ring } & f & 0.0 & 39.2 & 27.1 & 44.6 & 18.0 \\
\hline & & -18.0 & 21.2 & 9.1 & 26.6 & 0.0 \\
\hline \multirow[t]{2}{*}{ 13-Ring } & g & 0.0 & 38.3 & 29.4 & 46.5 & 20.1 \\
\hline & & -20.1 & 18.2 & 9.3 & 26.5 & 0.0 \\
\hline \multirow[t]{2}{*}{ 14-Ring } & h & 0.0 & 42.6 & 31.9 & 49.1 & 22.9 \\
\hline & & -22.5 & 20.1 & 9.5 & 26.6 & 0.0 \\
\hline \multirow{2}{*}{\multicolumn{2}{|c|}{ Stammsystem 9}} & 0.0 & 26.4 & 10.4 & 26.4 & 0.0 \\
\hline & & 0.0 & 26.4 & 10.4 & 26.4 & 0.0 \\
\hline
\end{tabular}

Substitution. Neben der Ringspannung ${ }^{30-36}$ und der Komplexierung durch Metalle, ${ }^{37-}$ ${ }^{41}$ sollte durch die Substitution eines oder mehrerer Wasserstoff-Atome im Endiin eine weitere Möglichkeit gegeben sein, die Reaktivität der Cyclisierung zu kontrollieren, vielleicht sogar zu steuern und sie "an"- oder "ab"-schalten zu können. Um den Einfluß von Substituenten auf die Barriere und die Enthalpie der Bergman-Reaktion zu untersuchen und vorherzusagen, wurden die acetylenischen Wasserstoffatome im Endiin durch einige funktionelle Gruppen einfach und zweifach ersetzt. Die Substituenten wurden dabei so gewählt, daß ein breites Spektrum an $\sigma^{-}$und an $\pi$-akzeptierenden und -donierenden Spezies sowie Mischformen untersucht wurden, um eine breite theoretische Basis zu gewährleisten (Abb. 38). 


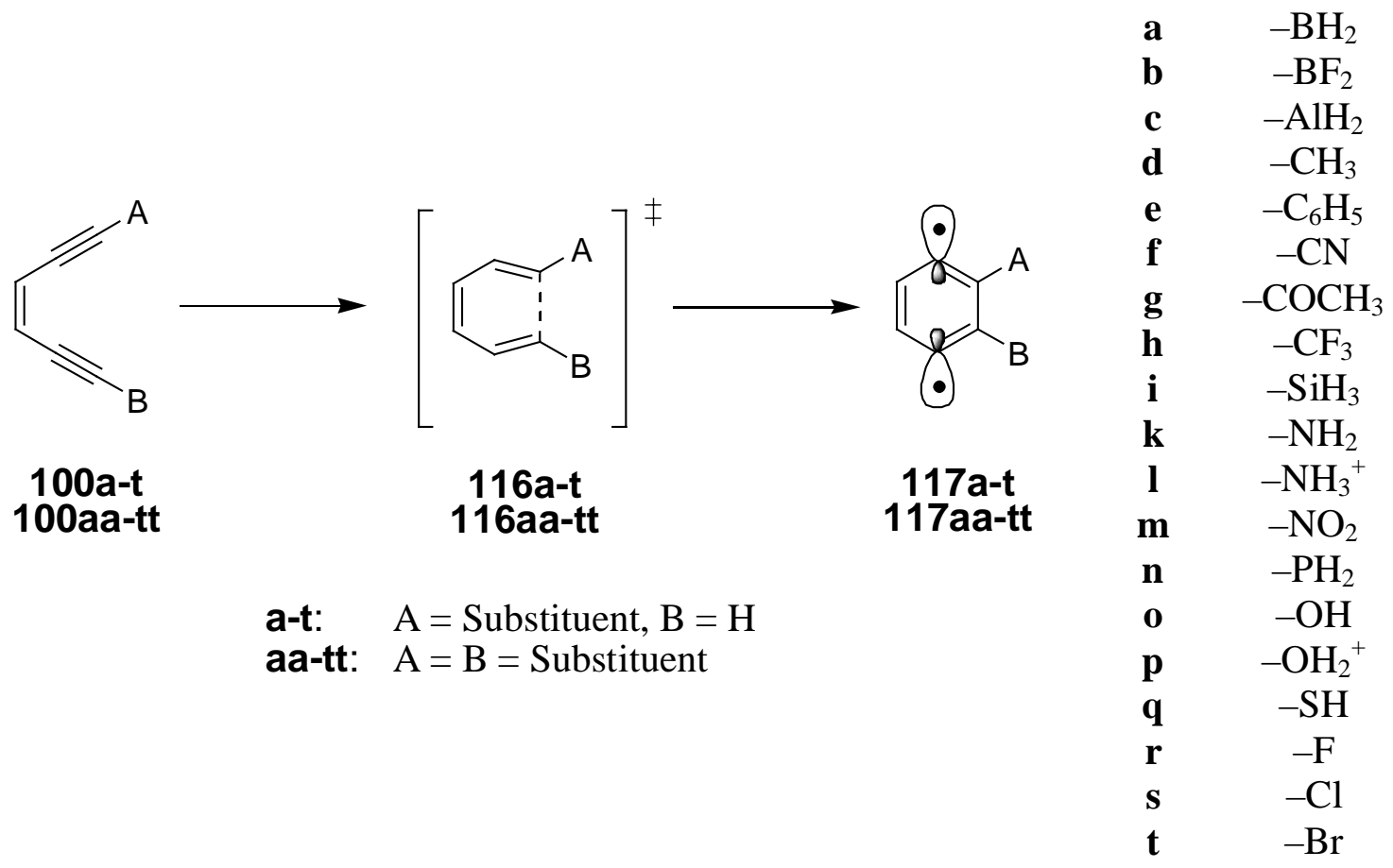

Abbildung 38. Ausgewählte substituierte Endiine 100a-t und 100aa-tt

Im Allgemeinen sollte eine Reaktivitätssteigerung der Endiine durch die Stabilisierung der entsprechenden biradikalischen Produkte oder durch Destabilisierung der Edukte erreicht werden. Die folgende isodesmische Gleichung kann als Indikator für den Effekt des jeweiligen Substituenten A angesehen werden:

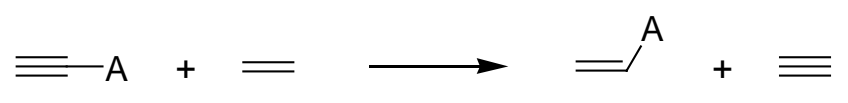

Eine positive Reaktionsenergie $\left(\mathrm{E}_{\mathrm{stab}}\right)$ charakterisiert dabei die Stabilisierung der Dreifachbindung verglichen mit der Doppelbindung durch den Substituenten A. Daraus kann auf eine ähnliche Stabilisierung der Endiin-Einheit geschlossen werden, was die BergmanReaktion energetisch ungünstiger, verglichen mit dem Stammsystem, macht (Tab. 8). Eine negative $E_{\text {stab }}$ hingegen weist auf die Destabilisierung des Endiins hin und sollte die BergmanReaktion energetisch begünstigen. Die Korrelation zwischen $\mathrm{E}_{\text {stab }}$ und den Barrieren bzw. den Reaktionsenthalpien der Cyclisierungen zeigt eine lineare Beziehung für die monosubstituierten Endiine (Korrelationsfaktor für die Barrieren: 0.88; für die Enthalpien: 0.97; Abb. 39) 


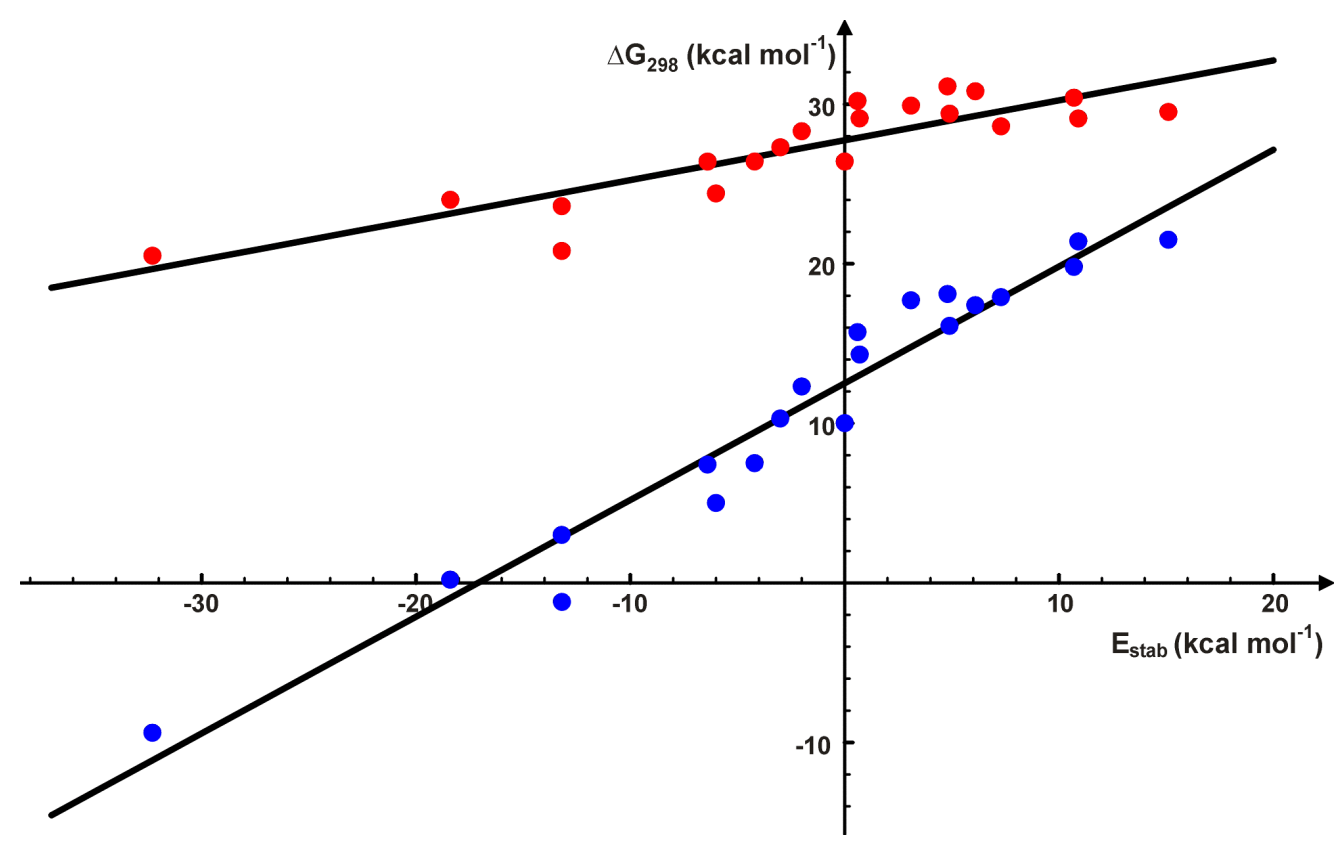

Abbildung 39. Korrelationsdiagramm für die Barrieren (rote Punkte) und die Reaktionsenthalpien (blaue Punkte) der monosubstituierten Endiine 100a-t mit $\mathrm{E}_{\text {stab }}$

Für die disubstituierten Endiine ist die Korrelation nicht so einfach. Da sich die zwei Substituenten in ortho-Position gegenseitig beeinflussen, ist die Korrelation zwischen $\mathrm{E}_{\text {stab }}$ und den Enthalpien schlecht (0.74) während es zwischen $\mathrm{E}_{\text {stab }}$ und den Barrieren keine Korrelation gibt (0.14). Die Energie der gegenseitigen Interaktion $\mathrm{E}_{\text {inter }}$ kann anhand der folgenden isodesmischen Gleichung bestimmt werden:<smiles>O=C(O)c1ccccc1I</smiles><smiles>c1ccccc1</smiles><smiles>C=C</smiles>

2<smiles>Ic1ccccc1</smiles>

Eine negative $E_{\text {inter }}$ zeigt dabei einen energieerhöhenden Einfluß der Substituenten (z.B. sterische oder elektrostatische Abstoßung, Drehung aus der Ebene), eine positive $E_{\text {inter }}$ einen energieerniedrigenden Einfluß (z.B. Wasserstoffbrückenbildung) bezogen auf das entsprechende monosubstituierte Endiin (Tabelle 9).

Die Singulett-Triplett Aufspaltung $\Delta \mathrm{E}_{\mathrm{ST}}$ zeigt weder mit den Barrieren noch mit den Enthalpien eine Korrelation. Eine generelle Tendenz besteh darin, daß bis auf zwei Ausnahmen alle Biradikal-Singuletts niedriger in der Energie sind als die entsprechenden Tripletts (negativer Wert für $\Delta \mathrm{E}_{\mathrm{ST}}$ ) und daß für die meisten disubstituierten Didehydrobenzole $\Delta \mathrm{E}_{\mathrm{ST}}$ kleiner ist als für die entsprechenden monosubstituierten (Tabelle 9). 
Wie erwartet korrelieren die Abstände $d$ der C-Atome der neu entstehenden Bindung in den Übergangszuständen und die Reaktionsenthalpien gut miteinander (je niedriger die Reaktionsenthalpie, desto länger $d$, Tabelle 9).

Substituenten an den vinylischen Positionen des Endiins wurden ebenfalls exemplarisch untersucht, stellten sich dort allerdings, im Bezug auf die Reaktivität, als wesentlich weniger effektiv dar als an den acetylenischen Positionen. ${ }^{157}$ Substitution an der Doppelbindung ergab (in einem Bereich von $\pm 2 \mathrm{kcal} \mathrm{mol}^{-1}$ ) die gleichen Barrieren und Enthalpien wie das Stammsystem, unabhängig vom Substituenten.

Orbitalanalysen. Eine NBO-Analyse (natural bond orbitals $=$ natürliche Bindungsorbitale) der partiellen Ladungen in Edukten, Übergangszuständen und Produkten zeigte leider nur eine schlechte Korrelation zwischen den Ladungen der Kohlenwasserstoffreste und den Barrieren bzw. den Enthalpien. Generell haben positiver geladene Kohlenwasserstoffreste niedrigere Barrieren und Reaktionsenthalpien (Tab. 9). Dieser offensichtliche Mangel an Korrelation zwischen Ladung und Barrieren ist auf Unterschiede der Orbitalgeometrien zurückzuführen. Die Orbital-Analyse identifizierte zwei unterschiedliche Gruppen von Substituenten. Eine Gruppe hat $\sigma$-artige HOMOs und $\pi$-artige HOMO-1s und die andere Gruppe $\pi$-artige HOMOs und $\sigma$-artige HOMO-1s (Abb. 40).

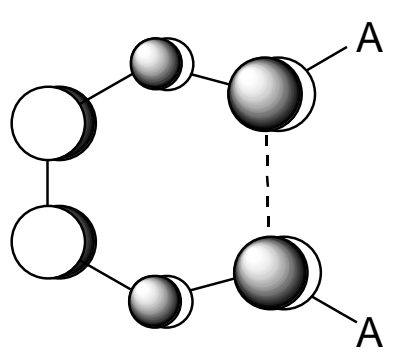

$\pi$-artiges HOMO

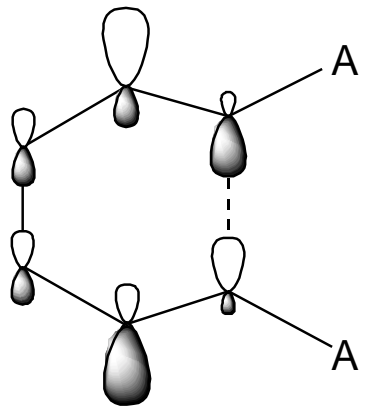

$\sigma$-artiges HOMO

Abbildung 40. $\pi$ - und $\sigma$-artige HOMOs der Übergangszustände

Da beide MO-Besetzungen eine große Rolle bei der Bildung des Übergangszustandes spielen, können Substituenten die Barriere zusätzlich verkleinern, wenn sie:

- $\pi$-Donor-Eigenschaften haben und die Elektronendichte im bindenden $\pi$-artigen Orbital erhöhen.

- $\sigma$-Akzeptor-Eigenschaften haben und die Elektronendichte im antibindenden $\sigma$ artigen Orbital erniedrigen. 
Tabelle 9 zeigt deutlich, daß $\sigma$-Akzeptor und $\pi$-Donor Substituenten (z.B. $-\mathrm{F},-\mathrm{Cl}$, $-\mathrm{Br},-\mathrm{OH})$ die Cyclisierungsbarriere und die Enthalpie generell erniedrigen, während $\pi$ Akzeptor und $\sigma$-Donor Substituenten (z.B. $-\mathrm{BH}_{2},-\mathrm{AlH}_{2}$ ) den umgekehrten Effekt haben. ${ }^{48,147}$ Für $\sigma$ - und $\pi$-Akzeptoren, wie $-\mathrm{NO}_{2}$, überwiegt der $\sigma$-Akzeptor Einfluß.

Die Substituenteneffekte werden im Folgenden in der Reihenfolge der Hauptgruppen des Periodensystems (III. Hauptgruppe, IV. Hauptgruppe, Pniktiden, Chalkogene, Halogene) und innerhalb einer Gruppe in der Reihenfolge der Perioden ausführlich beschrieben.

III. Hauptgruppe $(B, A l)$. Aufgrund seiner starken $\pi$-akzeptierenden Eigenschaften hat des Boranyl-Endiin (100a) eine herabgesetzte Reaktivität verglichen mit 9, da der ÜZ destabilisiert wird. Schwerer wiegt jedoch der $\sigma$-Donor Effekt des Boranylsubstituenten, der für eine Stabilisierung von 100a $\left(\mathrm{E}_{\text {stab }}=+10.9 \mathrm{kcal} \mathrm{mol}^{-1}\right)$ sorgt, so daß sowohl Barriere (116a: $29.1 \mathrm{kcal} \mathrm{mol}^{-1}$ ) als auch die Energie von 117a $\left(21.4 \mathrm{kcal} \mathrm{mol}^{-1}\right)$ erhöht sind. Diboranyl-Endiin 100aa ist noch weniger reaktiv, da zwei Boranylgruppen das Endiin gegenüber dem ÜZ stabilisieren (116aa: $37.2 \mathrm{kcal} \mathrm{mol}^{-1}$ und 117aa: $35.8 \mathrm{kcal} \mathrm{mol}^{-1}$ ). Difluorboranyl-Endiin 100b ist etwas reaktiver (116b: $28.6 \mathrm{kcal} \mathrm{mol}^{-1}$; 117b: $17.9 \mathrm{kcal} \mathrm{mol}^{-1}$ ) als 100a, weil die $\sigma$-Donor-Eigenschaft durch die Fluor-Atome herabgesetzt ist, jedoch unreaktiver als 9. Doppelt substituiertes 100bb ist weniger reaktiv als 100b (116bb: $34.5 \mathrm{kcal}$ $\mathrm{mol}^{-1}$; 117bb: $29.9 \mathrm{kcal} \mathrm{mol}^{-1}$ ), da sich die ortho-Substituenten gegenseitig behindern und aus der Ebene drehen müssen.

Alanyl-Endiin 100c hat eine mit 100a vergleichbare Reaktivität, da die $\pi$-Azeptorstärke ab-, die $\sigma$-Donorstärke jedoch zunimmt. Deshalb sind Barriere (116c: $29.5 \mathrm{kcal} \mathrm{mol}^{-1}$ ) und Energie (117c: $21.5 \mathrm{kcal} \mathrm{mol}^{-1}$ ) nahzu gleich. Im Dialanyl-Endiin 100cc sind Barriere und Energie (116cc: $30.6 \mathrm{kcal} \mathrm{mol}^{-1}$; 117cc: $29.8 \mathrm{kcal} \mathrm{mol}^{-1}$ ) kleiner als erwartet, weil durch gegenseitige Beeinflussung eine Alanyl-Gruppe aus der Ebene gedreht ist und sich eine $\mathrm{Al}{ }^{\cdots} \mathrm{H}^{\cdots} \mathrm{Al}$ Brücke ausbilden kann.

IV. Hauptgruppe $(\mathrm{C}, \mathrm{Si})$. Substituenten dieser Gruppe erhöhen generell Barriere und Reaktionsenergie der Bergman-Reaktion, verglichen mit dem Stammsystem. Die MethylGruppe stabilisiert die Dreifachbindung stärker als die Doppelbindung, ${ }^{33}$ was zu einer Stabilisierung des Methyl-Endiin 100d und deshalb zu einer Erhöhung der Barriere (116d: $\left.29.4 \mathrm{kcal} \mathrm{mol}^{-1}\right)$ und der Energie von 117d (16.1 $\left.\mathrm{kcal} \mathrm{mol}^{-1}\right)$ führt. Im Dimethyl-Endiin 100dd spielen zusätzlich sterische Effekte eine Rolle. Die beiden Methyl-Gruppen in orthoPosition in 117dd stoßen sich gegenseitig ab und erhöhen dadurch zusätzlich die Barriere (116dd: $36.3 \mathrm{kcal} \mathrm{mol}^{-1}$ ) und die Energie (117dd: $24.2 \mathrm{kcal} \mathrm{mol}^{-1}$ ). Phenyl-Endiin 100e ist ebenfalls von der Phenyl-Gruppe stabilisiert und deshalb weniger reaktiv als das 
Stammsystem (31.1 kcal mol ${ }^{-1}$ und $18.1 \mathrm{kcal} \mathrm{mol}^{-1}$ für 116e und 117e) trotz des negativen induktiven Effektes der Phenylgruppe. Ein zweiter Phenylsubstituent führt zu einer deutlichen Erhöhung der Barriere und Energie von 100ee (116ee: $41.6 \mathrm{kcal} \mathrm{mol}^{-1}$; 117ee: $32.1 \mathrm{kcal} \mathrm{mol}^{-}$ ${ }^{1}$ ), da das Edukt durch zwei Phenylgruppen stabilisiert wird, während ÜZ und Produkt durch sterische Hinderung destabilisiert werden.

Substituenten, die Heteroatome enthalten, welche durch Mehrfachbindung an ein Kohlenstoff-Atom gebunden sind, zeigen deutliche $\pi$-Akzeptor Fähigkeiten. Sie stabilisieren deshalb die Edukte, während die ÜZ destabilisiert werden, was einer geringerer Reaktivität, verglichen mit dem Stammsystem, entspricht (100f, 100ff, 100g, 100gg). Im TrifluormethylEndiin 100h kompensieren sich die stabilisierenden Effekte der Methylgruppe und die destabilisierenden des Halogens (siehe unten), so daß 100h nur eine wenig höhere Barriere (116h: $27.3 \mathrm{kcal} \mathrm{mol}^{-1}$ ) und Reaktionsenergie (117h: $10.3 \mathrm{kcal} \mathrm{mol}^{-1}$ ) hat als 9. Doppelt substituiertes 100hh hat eine etwas höhere Barriere (116hh: $32.9 \mathrm{kcal} \mathrm{mol}^{-1}$ ) und Energie (117hh: $15.6 \mathrm{kcal} \mathrm{mol}^{-1}$ ), jedoch liegen beide noch weit unterhalb der entsprechenden Energien für 100dd. Dies ist auf die teilweise Kompensation von sterischen durch elektronische Effekte zurückzuführen, die 117hh ausschließlich aufgrund der höheren $\sigma$ akzeptierenden Eigenschaften der $-\mathrm{CF}_{3}$-Gruppe gegenüber 117dd energetisch begünstigen.

Wie erwartet ist Silanyl-Endiin 100i, aufgrund des stärkeren $\sigma$-Donors $-\mathrm{SiH}_{3}$, weniger reaktiv als sein Methyl-substituiertes Pendant 100d. Da Silane und Borane ähnliche Eigenschaften aufweisen sind Barriere und Energie von 100i $\left(30.4 \mathrm{kcal} \mathrm{mol}^{-1}\right.$ und $19.8 \mathrm{kcal}$ mol $^{-1}$ ) vergleichbar mit denen von 100a. Disilanyl-Endiin 100ii leidet unter der sterischen Abstoßung der beiden Silanylgruppen und hat deshalb erhöhte Werte für Barriere (116ii: 39.2 kcal mol${ }^{-1}$ ) und Energie (117ii: $32.6 \mathrm{kcal} \mathrm{mol}^{-1}$ ).

Pniktiden $(N, P)$. Amino-Endiin 100k zeigt eine geringfügig niedrigere Reaktivität als das Stammsystem (116k: $28.3 \mathrm{kcal} \mathrm{mol}^{-1}$; 117k: $\left.12.3 \mathrm{kcal} \mathrm{mol}^{-1}\right)$. Für Diamino-Endiin 100kk sind Barriere (116kk: $26.6 \mathrm{kcal} \mathrm{mol}^{-1}$ ) und Reaktionsenergie (117kk: $12.8 \mathrm{kcal} \mathrm{mol}^{-1}$ ) kaum unterschiedlich zu 100k und 9. In diesen Reaktionen scheinen sich die $\pi$-Donor- und die $\sigma$ Akzeptor-Fähigkeiten der Substituenten sowie alle sterischen Einflüsse zu kompensieren. ${ }^{147}$

$\mathrm{Da}-\mathrm{NH}_{3}{ }^{+}$über keine $\pi$-Donor- sondern nur über große $\sigma$-Akzeptor-Eigenschaften verfügt, hat Ammonium-Endiin 1001 eine relativ kleine Barriere (116l: $24.0 \mathrm{kcal} \mathrm{mol}^{-1}$ ) und eine nahezu thermoneutrale Reaktionsenergie (117l: $0.2 \mathrm{kcal} \mathrm{mol}^{-1}$ ). Doppelt substituiertes Bisammonium-Endiin $\mathbf{1 0 0 1 l}$ zeigt leider nicht den erwarteten Reaktivitätsanstieg, der sich bei 1001 abzuzeichnen schien, sondern im Gegenteil eine drastische Erhöhung von Barriere (116ll: $\left.50.7 \mathrm{kcal} \mathrm{mol}^{-1}\right)$ und Reaktionsenergie von $117 \mathrm{ll}\left(25.4 \mathrm{kcal} \mathrm{mol}^{-1}\right)$, was dieses System 
zum am wenigsten reaktiven aller untersuchten macht. Der Grund ist in der Abstoßung der beiden positiv geladenenen ortho-ständigen Ammonium-Gruppen in 117ll zu suchen.

Nitro-Endiin $\mathbf{1 0 0 m}$ ist ein Beispiel für die Überlagerung von $\sigma$ - und $\pi$-akzeptierndem Effekt, wobei der $\sigma$-Effekt überwiegt und sich in einer niedrigeren Barriere (116m: $23.6 \mathrm{kcal}$ mol $^{-1}$ ) und Reaktionsenergie (117m: $3.0 \mathrm{kcal} \mathrm{mol}^{-1}$ ) relativ zu 9 bemerkbar macht. DinitroEndiin 100mm ist weniger reaktiv; Barriere (116mm: $\left.27.8 \mathrm{kcal} \mathrm{mol}^{-1}\right)$ und Energie $(\mathbf{1 1 7} \mathbf{m m}$ : $4.4 \mathrm{kcal} \mathrm{mol}^{-1}$ ) sind gegenüber dem monosubstituierten Fall wegen sterischer Hinderung erhöht (O而 Abstand: $2.85 \AA$ ).

Der Phosphan-Substituent ist ein schlechter $\sigma$-Akzeptor und deshalb hat PhosphanylEndiin 100n eine höhere Barriere (116n: $30.8 \mathrm{kcal} \mathrm{mol}^{-1}$ ) und Energie (117n: $17.4 \mathrm{kcal} \mathrm{mol}^{-1}$ ) als das Stammsystem. Diphosphanyl-Endiin 100nn hat eine noch höhere Barriere und Energie (116nn: $37.4 \mathrm{kcal} \mathrm{mol}^{-1}$ und 117nn: $27.1 \mathrm{kcal} \mathrm{mol}^{-1}$ ) was einhergeht mit doppelter Substitution und somit doppelter Stabilisierung von 100nn.

Chalkogene $(O, S)$. Da der Hydroxy-Substituent ein guter $\sigma$-Akzeptor ist, sollte Hydroxy-Endiin 1000 eine höhere Aktivität als 9 zeigen. Tatsächlich sind Barriere (116o: $24.4 \mathrm{kcal} \mathrm{mol}^{-1}$ ) und Energie (117o: $5.0 \mathrm{kcal} \mathrm{mol}^{-1}$ ) erniedrigt, was auf die Destabilisierung des Edukts und die Stabilisierung des ÜZ zurückzuführen ist. Da die Hydroxy-Gruppe ein sterisch wenig anspruchsvoller Substituent ist, findet im Dihydroxy-Endiin 1000 wenig Repulsion statt und ist somit noch reaktiver als das monosubstituierte. Mit einer so niedrigen Barriere und der fast thermoneutralen Reaktionsenergie (116oo: $19.8 \mathrm{kcal} \mathrm{mol}^{-1}$; 117oo: 0.8 kcal mol ${ }^{-1}$ ) sollte $\mathbf{1 0 0 0 o}$ bereits bei Raumtemperatur schnell und leicht cyclisieren. Die protonierte Hydroxy-Gruppe zeigte sich als ein viel besserer $\sigma$-Akzeptor als die nichtprotonierte, und daher ist auch die Reaktivität von 100p gegenüber 1000 gesteigert. Mit einer Barriere von $20.5 \mathrm{kcal} \mathrm{mol}^{-1}$ (116p) und einer Energie von $-9.4 \mathrm{kcal} \mathrm{mol}^{-1}$ (117p) ist 100p das reaktivste aller untersuchten monosubstituierten Endiine. Doppelte Protonierung hingegen erhöht die Barriere (116pp: $34.0 \mathrm{kcal} \mathrm{mol}^{-1}$ ) und Energie (117pp: $-1.8 \mathrm{kcal} \mathrm{mol}^{-1}$ ) von 100pp aufgrund der Abstoßung der positiv geladenen Oxonium-Gruppen. Der Effekt ist nicht so groß wie in 117ll, da 117pp eine Konformation einnehmen kann, in der die ungünstigen $\mathrm{H}^{\cdots} \mathrm{H}^{\mathrm{H}}$ Repulsionen minimiert sind.

Die Reaktivität des Mercapto-Endiins 100q ist niedriger als die von 9, da die Thiolgruppe aufgrund weniger ausgeprägter $\sigma$-Akzepor-Fähigkeiten das Endiin relativ zu 100o stabilisiert und den ÜZ destabilisiert (116q: $29.1 \mathrm{kcal} \mathrm{mol}^{-1}$; 117q: $14.3 \mathrm{kcal} \mathrm{mol}^{-1}$ ). Dimercapto-Endiin 100qq ist doppelt stabilisiert was eine noch niedrigere Reaktivität (116qq: $33.9 \mathrm{kcal} \mathrm{mol}^{-1}$; 117qq: $20.3 \mathrm{kcal} \mathrm{mol}^{-1}$ ) darstellt. 
Halogene $(F, C l, B r)$. Da alle Halogene sowohl gute $\sigma$-Akzeptoren als auch gute $\pi$ Donoren sind, können hier niedrigere Barrieren erwartet werden. Das Fluor-Endiin 100r hat eine Barriere von $20.8 \mathrm{kcal} \mathrm{mol}^{-1}$ (116r) und cyclisiert exergonisch (117r: $\left.-1.2 \mathrm{kcal} \mathrm{mol}^{-1}\right)$; damit ist es das reaktivste ungeladene, monosubstituierte Endiin dieser Studie. Da der FluorSubstituent sehr klein ist, gibt es wenig sterische Abstoßung im Cyclisierungsprodukt des Difluor-Endiin 100rr, d.h. sowohl Barriere (116rr: $16.9 \mathrm{kcal} \mathrm{mol}^{-1}$ ) als auch Energie (117rr: $-8.9 \mathrm{kcal} \mathrm{mol}^{-1}$ ) sind nochmals im Vergleich zum monosubstituierten Endiin reduziert. Damit hat 100rr die kleinste Barriere aller untersuchten substituierten Endiine, was nicht nur dazu führt, daß 100rr äußerst reaktiv ist, es ist sogar instabil bei Raumtemperatur und cyclisiert spontan zum Biradikal 117rr. Die Reaktionsenergie ist nur geringfügig höher als die von 100p, dem am exergonischsten cyclisierenden Endiin.

Chlor- (100s) und Brom-Endiin (100t) haben gleiche Barrieren (116s und 116t: 26.4 kcal $\mathrm{mol}^{-1}$ ), die mit der von 9 nahezu übereinstimmen. Zurückzuführen ist das auf die Kompensation von zunehmender $\pi$-donierender und abnehmender $\sigma$-akzeptierender Eigenschaft beim $-\mathrm{Br}$, verglichen mit $-\mathrm{Cl}$. Die Reaktionsenergien liegen etwas niedriger als bei 9 (117s: $7.4 \mathrm{kcal} \mathrm{mol}^{-1} ; \mathbf{1 1 7 t}$ : $7.5 \mathrm{kcal} \mathrm{mol}^{-1}$ ). Doppelte Substitution ändert die Energien von 100ss und 100tt, im Vergleich zu ihren monosubstituierten Derivaten, nicht.

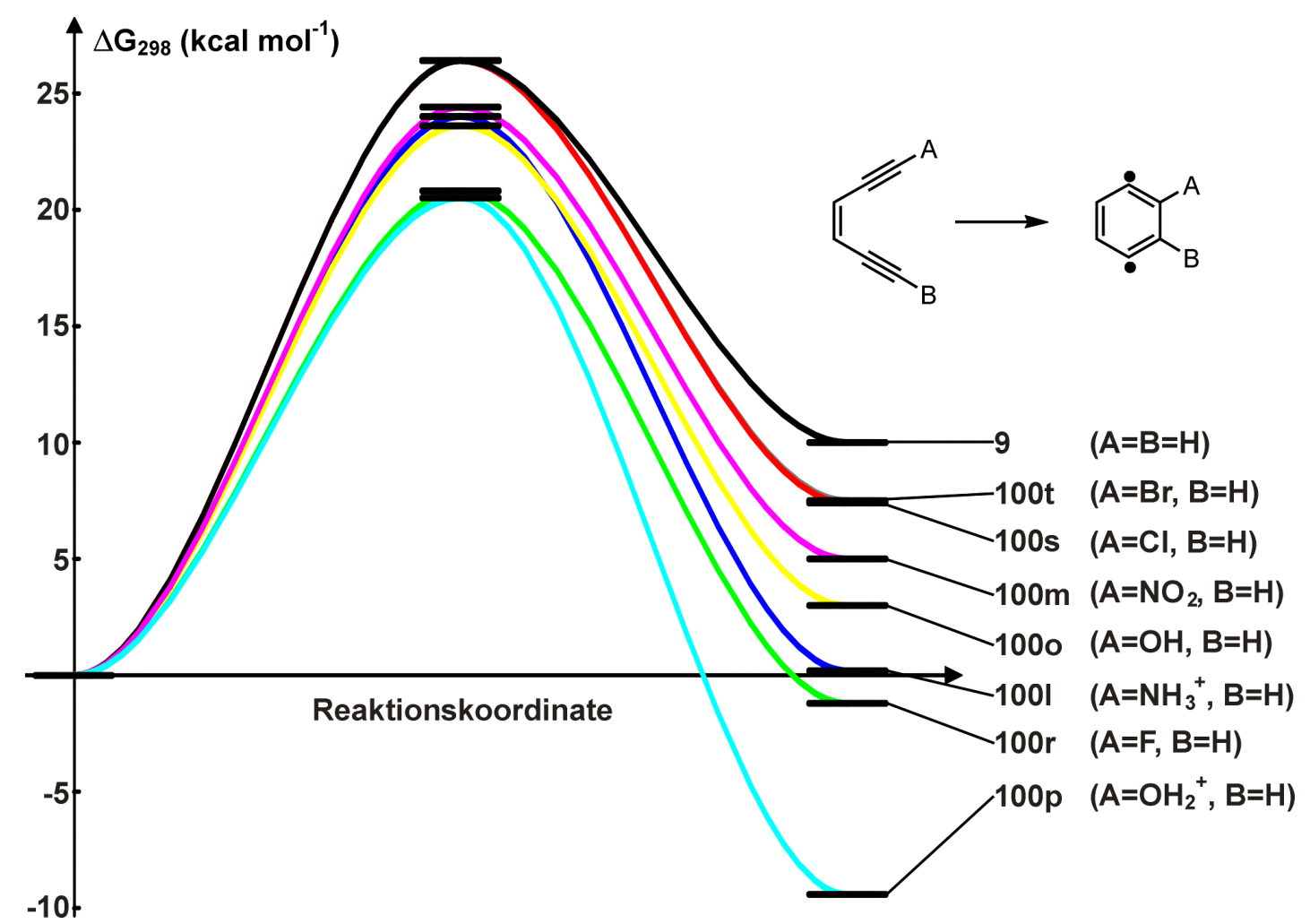

Abbildung 41. Potentialhyperflächen der Cyclisierungen monosubstituierter Endiine mit erhöhter Reaktivität verglichen mit dem Stammsystem 9 


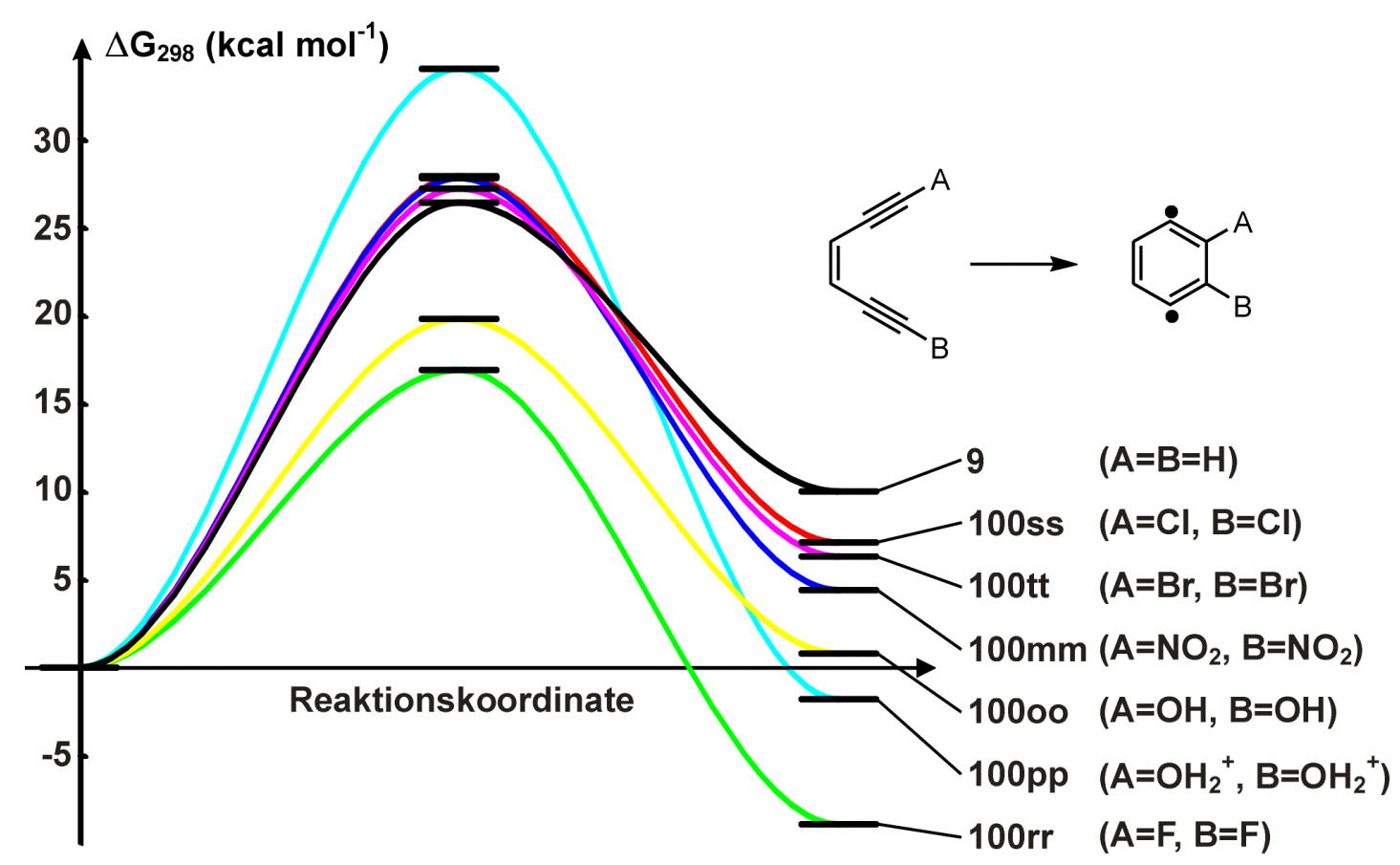

Abbildung 42. Potentialhyperflächen der Cyclisierungen disubstituierter Endiine mit erhöhter Reaktivität (Ausnahme: 100pp) verglichen mit dem Stammsystem 9

Zusätzlich zu den Reaktivitätsunterschieden durch Substituenten in der BergmanReaktion wurden die Effekte auf die $C^{1}-C^{5}$-Cyclisierung untersucht, die zu substituierten offenschaligen Singulett-Fulvenbiradikalen 118 führen sollten (Abb. 43). ${ }^{158}$ Tatsächlich konnten Didehydrofulven-Biradikale 118 für $\mathrm{R}=-\mathrm{CN}$ (ff), $-\mathrm{COCH}_{3}(\mathbf{g g}),-\mathrm{CF}_{3}(\mathbf{h h}),-\mathrm{NH}_{2}$ $(\mathbf{k k}),-\mathrm{NH}_{3}{ }^{+}(\mathbf{l l}),-\mathrm{NO}_{2}(\mathbf{m m}),-\mathrm{OH}(\mathbf{o o}),-\mathrm{OH}_{2}{ }^{+}(\mathbf{p p}),-\mathrm{F}(\mathbf{r r})$ und $-\mathrm{Cl}$ (ss) identifiziert werden. Sie liegen jedoch alle energetisch viel ungünstiger als die entsprechend substituierten Didehydrobenzol-Biradikale 117 um mit der Bergman-Cyclisierung konkurrieren zu können, da alle Fulven-Reaktionsenergien bereits höher oder wenigsten gleich hoch sind wie die entsprechenden Bergman-Barrieren (Tab. 7).<smiles>[R]C1C(=O)C=CC1=CC</smiles>

118

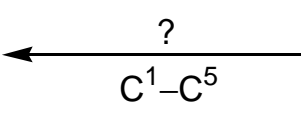

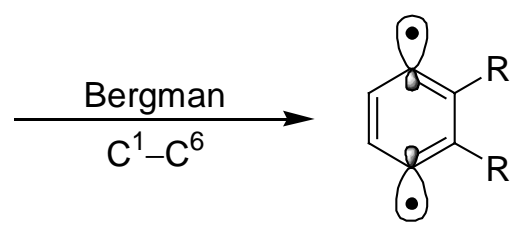

117

Abbildung 43. Cyclisierungen der substituierten Endiine 100 zu Bergman- 117 und Fulven-Biradikal 118 
Tabelle 7. Berechnete Enthalpien $\left(\Delta \mathrm{G}_{298}\right)$ für die Bergman- und Fulven-Cyclisierungen der disubstituierten Endiine 116 (in $\mathrm{kcal} \mathrm{mol}^{-1}$ ).

\begin{tabular}{lccccc}
\hline \multicolumn{1}{c}{$\mathrm{R}$} & & ÜZ 116 & Bergman 117 & Fulven 118 & $\Delta \Delta \mathrm{G}(\mathbf{1 1 8}-\mathbf{1 1 7})$ \\
\hline$-\mathrm{H}$ & $\mathbf{3}$ & 26.4 & 10.0 & 42.0 & 32.0 \\
$-\mathrm{CN}$ & $\mathbf{f f}$ & 36.8 & 27.4 & 42.3 & 14.9 \\
$-\mathrm{COCH}_{3}$ & $\mathbf{g g}$ & 29.7 & 21.3 & 32.6 & 11.3 \\
$-\mathrm{CF}_{3}$ & $\mathbf{h h}$ & 32.9 & 15.6 & 43.1 & 27.5 \\
$-\mathrm{NH}_{2}$ & $\mathbf{k k}$ & 26.6 & 12.8 & 30.7 & 17.9 \\
$-\mathrm{NH}_{3}{ }^{+}$ & $\mathbf{l l}$ & 50.7 & 25.4 & 58.8 & 33.4 \\
$-\mathrm{NO}_{2}$ & $\mathbf{m m}$ & 27.8 & 6.0 & 25.3 & 19.3 \\
$-\mathrm{OH}$ & $\mathbf{o o}$ & 19.8 & 0.8 & 27.5 & 26.7 \\
$-\mathrm{OH}_{2}{ }^{+}$ & $\mathbf{p p}$ & 34.0 & -3.2 & 32.7 & 35.9 \\
$-\mathrm{F}$ & $\mathbf{r r}$ & 16.9 & -8.9 & 24.3 & 33.2 \\
$-\mathrm{Cl}$ & $\mathbf{s s}$ & 27.9 & 7.8 & 32.9 & 25.1 \\
\hline
\end{tabular}


Tabelle 8. Berechnete Enthalpien $\left(\Delta \mathrm{G}_{298}\right)$ für die Cyclisierungen der mono- und disubstituierten Endiine 100 (in kcal mol ${ }^{-1}$ )

\begin{tabular}{|c|c|c|c|c|c|c|c|}
\hline & & & Endiin $\mathbf{1 0 0}$ & ÜZ 116 & Produkt 117 & Stab.Energie & $\mathrm{d}$ \\
\hline & A & $\mathrm{B}$ & $\Delta \mathrm{G}_{298}$ & $\Delta \mathrm{G}_{298}$ & $\Delta \mathrm{G}_{298}$ & $\Delta \mathrm{E}_{\mathrm{stab}}$ & \\
\hline 3 & $-\mathrm{H}$ & $-\mathrm{H}$ & 0.0 & $26.4^{\mathrm{a}}$ & $10.0^{b}$ & 0.0 & 12 \\
\hline $\mathbf{a}$ & $-\mathrm{BH}_{2}$ & $-\mathrm{H}$ & 0.0 & 29.1 & 21.4 & +10.9 & - \\
\hline $\mathbf{a a}$ & $-\mathrm{BH}_{2}$ & $-\mathrm{BH}_{2}$ & 0.0 & 37.2 & 35.8 & & - \\
\hline b & $-\mathrm{BF}_{2}$ & $-\mathrm{H}$ & 0.0 & 28.6 & 17.9 & +7.3 & - \\
\hline bb & $-\mathrm{BF}_{2}$ & $-\mathrm{BF}_{2}$ & 0.0 & 34.5 & 29.9 & & - \\
\hline c & $-\mathrm{AlH}_{2}$ & $-\mathrm{H}$ & 0.0 & 29.5 & 21.5 & +15.1 & - \\
\hline cc & $-\mathrm{AlH}_{2}$ & $-\mathrm{AlH}_{2}$ & 0.0 & 30.6 & 29.8 & & - \\
\hline d & $-\mathrm{CH}_{3}$ & $-\mathrm{H}$ & 0.0 & 29.4 & 16.1 & +4.9 & 159 \\
\hline dd & $-\mathrm{CH}_{3}$ & $-\mathrm{CH}_{3}$ & 0.0 & 36.3 & 24.2 & & 160 \\
\hline $\mathbf{e}$ & $-\mathrm{C}_{6} \mathrm{H}_{5}$ & $-\mathrm{H}$ & 0.0 & 31.1 & 18.1 & +4.8 & 161 \\
\hline ee & $-\mathrm{C}_{6} \mathrm{H}_{5}$ & $-\mathrm{C}_{6} \mathrm{H}_{5}$ & 0.0 & $41.6^{\mathrm{c}}$ & 32.1 & & 162 \\
\hline $\mathbf{f}$ & $-\mathrm{CN}$ & $-\mathrm{H}$ & 0.0 & 29.9 & 17.7 & +3.1 & - \\
\hline ff & $-\mathrm{CN}$ & $-\mathrm{CN}$ & 0.0 & 36.8 & 27.4 & & - \\
\hline $\mathbf{g}$ & $-\mathrm{COCH}_{3}$ & $-\mathrm{H}$ & 0.0 & 30.2 & 15.7 & +0.6 & - \\
\hline gg & $-\mathrm{COCH}_{3}$ & $-\mathrm{COCH}_{3}$ & 0.0 & 29.7 & 18.2 & & 149 \\
\hline $\mathbf{h}$ & $-\mathrm{CF}_{3}$ & $-\mathrm{H}$ & 0.0 & 27.3 & 10.3 & -3.0 & - \\
\hline hh & $-\mathrm{CF}_{3}$ & $-\mathrm{CF}_{3}$ & 0.0 & 32.9 & 15.6 & & - \\
\hline $\mathbf{i}$ & $-\mathrm{SiH}_{3}$ & $-\mathrm{H}$ & 0.0 & 30.4 & 19.8 & +10.7 & 163 \\
\hline ii & $-\mathrm{SiH}_{3}$ & $-\mathrm{SiH}_{3}$ & 0.0 & 39.2 & 32.6 & & 164 \\
\hline $\mathbf{k}$ & $-\mathrm{NH}_{2}$ & $-\mathrm{H}$ & 0.0 & 28.3 & 12.3 & -2.0 & - \\
\hline $\mathbf{k k}$ & $-\mathrm{NH}_{2}$ & $-\mathrm{NH}_{2}$ & 0.0 & 26.6 & 12.8 & & - \\
\hline 1 & $-\mathrm{NH}_{3}{ }^{+}$ & $-\mathrm{H}$ & 0.0 & 24.0 & 0.2 & -18.4 & - \\
\hline Il & $-\mathrm{NH}_{3}{ }^{+}$ & $-\mathrm{NH}_{3}{ }^{+}$ & 0.0 & 50.7 & 25.4 & & - \\
\hline $\mathbf{m}$ & $-\mathrm{NO}_{2}$ & $-\mathrm{H}$ & 0.0 & 23.6 & 3.0 & -13.2 & - \\
\hline $\mathbf{m m}$ & $-\mathrm{NO}_{2}$ & $-\mathrm{NO}_{2}$ & 0.0 & 27.8 & 4.4 & & - \\
\hline $\mathbf{n}$ & $-\mathrm{PH}_{2}$ & $-\mathrm{H}$ & 0.0 & 30.8 & 17.4 & +6.1 & - \\
\hline nn & $-\mathrm{PH}_{2}$ & $-\mathrm{PH}_{2}$ & 0.0 & 37.4 & 27.1 & & 165 \\
\hline $\mathbf{o}$ & $-\mathrm{OH}$ & $-\mathrm{H}$ & 0.0 & 24.4 & 5.0 & -6.0 & - \\
\hline oo & $-\mathrm{OH}$ & $-\mathrm{OH}$ & 0.0 & 19.8 & 0.8 & & - \\
\hline $\mathbf{p}$ & $-\mathrm{OH}_{2}{ }^{+}$ & $-\mathrm{H}$ & 0.0 & 20.5 & -9.4 & -32.3 & - \\
\hline pp & $-\mathrm{OH}_{2}{ }^{+}$ & $-\mathrm{OH}_{2}^{+}$ & 0.0 & 34.0 & -1.8 & & - \\
\hline $\mathbf{q}$ & $-\mathrm{SH}$ & $-\mathrm{H}$ & 0.0 & 29.1 & 14.3 & +0.7 & - \\
\hline $\mathbf{q q}$ & $-\mathrm{SH}$ & $-\mathrm{SH}$ & 0.0 & 33.9 & 20.3 & & - \\
\hline $\mathbf{r}$ & $-\mathrm{F}$ & $-\mathrm{H}$ & 0.0 & 20.8 & -1.2 & -13.2 & - \\
\hline $\mathbf{r r}$ & $-\mathrm{F}$ & $-\mathrm{F}$ & 0.0 & 16.9 & -8.9 & & - \\
\hline $\mathbf{S}$ & $-\mathrm{Cl}$ & $-\mathrm{H}$ & 0.0 & 26.4 & 7.4 & -6.4 & - \\
\hline SS & $-\mathrm{Cl}$ & $-\mathrm{Cl}$ & 0.0 & 27.9 & 7.1 & & - \\
\hline $\mathbf{t}$ & $-\mathrm{Br}$ & $-\mathrm{H}$ & 0.0 & 26.4 & 7.5 & -4.2 & - \\
\hline $\mathbf{t t}$ & $-\mathrm{Br}$ & $-\mathrm{Br}$ & 0.0 & 27.2 & 6.3 & & 166 \\
\hline
\end{tabular}


Tabelle 9. Ausgewählte Eigenschaften der Cyclisierung substituierter Endiine

\begin{tabular}{|c|c|c|c|c|c|c|c|}
\hline & A & B & $\Delta \mathrm{E}_{\mathrm{ST}}{ }^{\mathrm{a}}$ & $d^{\mathrm{b}}$ & $E_{\text {inter }}{ }^{c}$ & HOMO & $\begin{array}{c}\text { Ladung des } \\
\text { KW-Teils }\end{array}$ \\
\hline 3 & $-\mathrm{H}$ & $-\mathrm{H}$ & -4.1 & 2.077 & 0.00 & $\sigma$ & -0.51 \\
\hline $\mathbf{a}$ & $-\mathrm{BH}_{2}$ & $-\mathrm{H}$ & -5.4 & 1.975 & & $\sigma$ & -0.49 \\
\hline $\mathbf{a a}$ & $-\mathrm{BH}_{2}$ & $-\mathrm{BH}_{2}$ & -5.1 & 1.792 & -5.4 & $\sigma$ & -0.50 \\
\hline $\mathbf{b}$ & $-\mathrm{BF}_{2}$ & $-\mathrm{H}$ & -4.9 & 2.009 & & $\sigma$ & -0.59 \\
\hline bb & $-\mathrm{BF}_{2}$ & $-\mathrm{BF}_{2}$ & -5.3 & 1.914 & -4.5 & $\sigma$ & -0.69 \\
\hline c & $-\mathrm{AlH}_{2}$ & $-\mathrm{H}$ & -6.8 & 1.992 & & $\sigma$ & -0.81 \\
\hline cc & $-\mathrm{AlH}_{2}$ & $-\mathrm{AlH}_{2}$ & -12.1 & 1.834 & 5.4 & $\sigma$ & -1.11 \\
\hline d & $-\mathrm{CH}_{3}$ & $-\mathrm{H}$ & -3.8 & 2.023 & & $\sigma$ & -0.31 \\
\hline dd & $-\mathrm{CH}_{3}$ & $-\mathrm{CH}_{3}$ & -2.7 & 1.963 & -1.2 & $\sigma$ & -0.11 \\
\hline e & $-\mathrm{C}_{6} \mathrm{H}_{5}$ & $-\mathrm{H}$ & -3.5 & 2.001 & & $\sigma^{\mathrm{d}}$ & -0.28 \\
\hline ee & $-\mathrm{C}_{6} \mathrm{H}_{5}$ & $-\mathrm{C}_{6} \mathrm{H}_{5}$ & -2.1 & 1.905 & -5.2 & $\sigma^{\mathrm{d}}$ & -0.06 \\
\hline f & $-\mathrm{CN}$ & $-\mathrm{H}$ & -3.6 & 1.976 & & $\sigma$ & -0.25 \\
\hline ff & $-\mathrm{CN}$ & $-\mathrm{CN}$ & -2.8 & 1.889 & -4.0 & $\sigma$ & -0.03 \\
\hline g & $-\mathrm{COCH}_{3}$ & $-\mathrm{H}$ & -3.1 & 2.045 & & $\sigma$ & -0.28 \\
\hline gg & $-\mathrm{COCH}_{3}$ & $-\mathrm{COCH}_{3}$ & -4.8 & 2.014 & 0.7 & $\sigma^{\mathrm{d}}$ & -0.13 \\
\hline $\mathbf{h}$ & $-\mathrm{CF}_{3}$ & $-\mathrm{H}$ & -3.5 & 2.052 & & $\sigma$ & -0.31 \\
\hline $\mathbf{h h}$ & $-\mathrm{CF}_{3}$ & $-\mathrm{CF}_{3}$ & -1.8 & 2.009 & -5.0 & $\sigma$ & -0.12 \\
\hline $\mathbf{i}$ & $-\mathrm{SiH}_{3}$ & $-\mathrm{H}$ & -5.4 & 2.001 & & $\sigma$ & -0.69 \\
\hline ii & $-\mathrm{SiH}_{3}$ & $-\mathrm{SiH}_{3}$ & -5.9 & 1.914 & -3.0 & $\sigma$ & -0.88 \\
\hline $\mathbf{k}$ & $-\mathrm{NH}_{2}$ & $-\mathrm{H}$ & -2.8 & 2.050 & & $\pi$ & -0.26 \\
\hline $\mathbf{k k}$ & $-\mathrm{NH}_{2}$ & $-\mathrm{NH}_{2}$ & -1.6 & 2.029 & 0.1 & $\pi$ & 0.03 \\
\hline 1 & $-\mathrm{NH}_{3}{ }^{+}$ & $-\mathrm{H}$ & -2.2 & 2.139 & & $\pi$ & 0.04 \\
\hline ll & $-\mathrm{NH}_{3}{ }^{+}$ & $-\mathrm{NH}_{3}{ }^{+}$ & 0.2 & 2.041 & -95.8 & $\sigma$ & 0.56 \\
\hline $\mathbf{m}$ & $-\mathrm{NO}_{2}$ & $-\mathrm{H}$ & -2.2 & 2.091 & & $\sigma$ & -0.05 \\
\hline $\mathbf{m m}$ & $-\mathrm{NO}_{2}$ & $-\mathrm{NO}_{2}$ & -0.9 & 2.072 & -11.9 & $\sigma^{\mathrm{d}}$ & 0.32 \\
\hline $\mathbf{n}$ & $-\mathrm{PH}_{2}$ & $-\mathrm{H}$ & -4.1 & 2.007 & & $\sigma$ & -0.59 \\
\hline nn & $-\mathrm{PH}_{2}$ & $-\mathrm{PH}_{2}$ & -3.3 & 1.934 & -1.4 & $\sigma$ & -0.50 \\
\hline o & $-\mathrm{OH}$ & $-\mathrm{H}$ & -2.4 & 2.083 & & $\pi$ & -0.13 \\
\hline $\mathbf{0 0}$ & $-\mathrm{OH}$ & $-\mathrm{OH}$ & -1.1 & 2.097 & -1.3 & $\pi$ & 0.27 \\
\hline $\mathbf{p}$ & $-\mathrm{OH}_{2}{ }^{+}$ & $-\mathrm{H}$ & -1.0 & 2.220 & & $\sigma$ & 0.19 \\
\hline pp & $-\mathrm{OH}_{2}{ }^{+}$ & $-\mathrm{OH}_{2}^{+}$ & 0.7 & 2.207 & -97.7 & $\sigma$ & 0.83 \\
\hline $\mathbf{q}$ & $-\mathrm{SH}$ & $-\mathrm{H}$ & -3.0 & 1.999 & & $\sigma$ & -0.51 \\
\hline $\mathbf{q q}$ & $-\mathrm{SH}$ & $-\mathrm{SH}$ & -1.7 & 1.950 & -0.8 & $\pi$ & -0.52 \\
\hline $\mathbf{r}$ & $-\mathrm{F}$ & $-\mathrm{H}$ & -2.1 & 2.131 & & $\pi$ & -0.02 \\
\hline $\mathbf{r r}$ & $-\mathrm{F}$ & $-F$ & -0.5 & 2.170 & -3.6 & $\pi$ & 0.47 \\
\hline $\mathbf{S}$ & $-\mathrm{Cl}$ & $-\mathrm{H}$ & -2.2 & 2.064 & & $\pi$ & -0.39 \\
\hline SS & $-\mathrm{Cl}$ & $-\mathrm{Cl}$ & -0.6 & 2.032 & -2.2 & $\pi$ & -0.27 \\
\hline $\mathbf{t}$ & $-\mathrm{Br}$ & $-\mathrm{H}$ & -2.2 & 2.060 & & $\pi$ & -0.46 \\
\hline $\mathbf{t t}$ & $-\mathrm{Br}$ & $-\mathrm{Br}$ & -0.6 & 2.029 & -1.0 & $\pi$ & -0.42 \\
\hline
\end{tabular}

in $\mathrm{kcal} \mathrm{mol}^{-1}$; ein positiver Wert bedeutet einen Singulett-Grundzustande

${ }^{\mathrm{b}}$ Abstand der C-Atome im ÜZ, die die neue Bindung bilden, in $\AA$

${ }^{c}$ in $\mathrm{kcal} \mathrm{mol}^{-1}$

${ }^{\mathrm{d}}$ Wegen fehlender Planarität nicht eindeutig identifiziert. 
2.4.2 Die Cyclisierung von Hepta-1,2,4-trien-6-in: "Myers-Saito"-, "Schmittel"- und weitere Cyclisierungen

Die antibiotische Wirkung von Calicheamicin 13 beruht, wie bereits gezeigt wurde, auf der Änderung der Ringspannung und der damit verbundenen Erhöhung der Reaktivität der Endiin-Einheit. ${ }^{20-25}$ Das Endiin 9 cyclisiert, wie von Bergman gezeigt, ${ }^{12}$ zu Didehydrobenzol, einem Biradikal, das hochreaktiv ist und bei den Naturstoffen zum Tod der angegriffenen Zelle durch Zerstörung der DNA führt (Abb. 44).

Ein Antibiotikum mit der gleichen Wirkung, Neocarzinostatin, wurde 1961 aus Streptomyces carzinostaticus extrahiert ${ }^{167}$ und besteht aus einem aktiven Chromophor 15, gebunden an ein 113-Aminosäuren-langes Apoprotein. ${ }^{21,25}$ Das Chromophor besitzt dabei aber nicht die typische Endiin-Einheit, was bedeutet, daß in diesem Fall eine andere Radikalreaktion stattfinden muß (Abb. 44). Das Chromophor 15 wird dabei zunächst durch ein Thiol aktiviert, und aus dem relativ ungespannten neungliedrigen Diin-Ring bildet sich ein hochgespannter Enin-Kumulen-Ring 17, der sofort zum Biradikal 119 cyclisiert ${ }^{29}$ und wie 13 dabei die DNA zerstört, indem es H-Atome von Adenin- oder Thymin-Einheiten entfernt. ${ }^{168,169}$

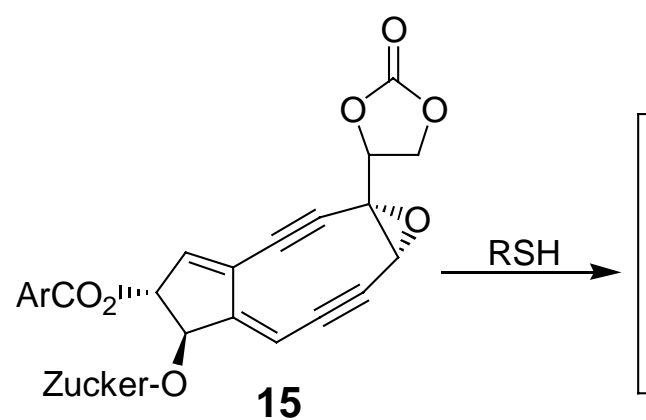<smiles>C=CC1=C(C#CC(C)(C)O)C(S)CCC1</smiles>

17

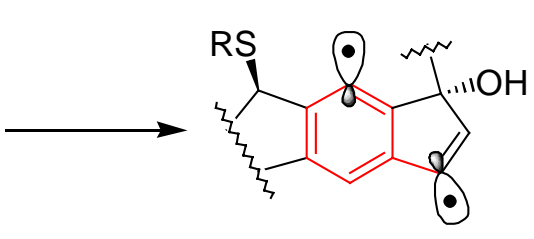

119

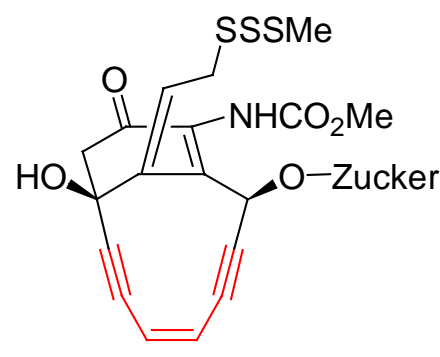

13

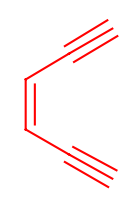

9

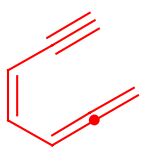

11

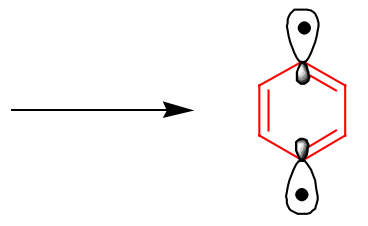

10

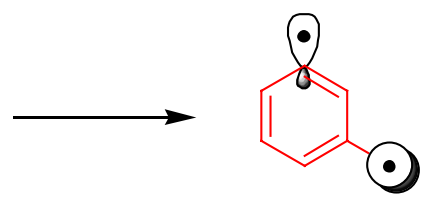

12

Abbildung 44. Cyclisierung des Neocarzinostatin-Chromophors 15, das Endiin-Antibiotikum Calicheamicin 13 und die Reaktion der Endiin- 9 und der Eninallen-Einheit 11 
Im Jahr 1989 konnten Myers und (unabhängig davon) Saito zeigen, daß das Eninallen 11 eine ähnliche Reaktion wie der Enin-Kumulen-Körper 120 von 17 eingeht (Abb. 45). ${ }^{16-19}$ Die $\mathrm{C}^{2}-\mathrm{C}^{7}$ Cycloaromatisierung ("Myers-Saito Cyclisierung") von $\mathbf{1 1}$ ergibt $\alpha, 3-$ Didehydrotoluol 12, ein $\sigma, \pi$-Biradikal, das durch benzylische $\pi$-Konjugation stabilisiert wird. Im Gegensatz zu dem nicht konjugierten $\sigma, \sigma$-Bergman-Biradikal 10, das eine Endothermie von $8.5 \pm 1.1 \mathrm{kcal} \mathrm{mol}^{-1}$ besitzt, ${ }^{26}$ wird deshalb das Myers-Saito-Produkt 12 exotherm $(-15 \pm$ 3 kcal mol ${ }^{-1}$ ) gebildet. ${ }^{17}$ Für beide Reaktionen ist dabei der Gewinn von Stabilisierungsenergie durch Ausbildung eines aromatischen Ringsystems aus einem offenkettigen Molekül die treibende Kraft. Während 10 nur von $\sim 21 \mathrm{kcal} \mathrm{mol}^{-1}$ Aromatisierungsenergie stabilisiert wird, gewinnt 12 noch zusätzliche $\sim 13 \mathrm{kcal} \mathrm{mol}^{-1}$ aus benzylischer $\pi$-Konjugation. ${ }^{170,171}$

Obwohl das konjugierte $\pi$-System von 11 weitere Cyclisierungen zulassen sollte, konnten lange Zeit nur Myers-Saito-Produkte isoliert werden, bis Schmittel 1995 zeigte, daß substituierte 11 durch einen neuen $C^{2}-C^{6}$ Ringschluß ("Schmittel-Cyclisierung") Methylfulven-Derivate 121 ausbilden können (Abb. 45). ${ }^{172-177}$ Obwohl es sich bei $\mathbf{1 2 1}$ ebenfalls um ein $\sigma, \pi$-Biradikal handelt, ist die treibende Kraft der Schmittel-Reaktion offensichtlich nicht die Cycloaromatisierung. Da ihr die Aromatisierungsenergie fehlt, verläuft die Reaktion im Stammsystem $\sim 10 \mathrm{kcal} \mathrm{mol}^{-1}$ endotherm und wurde daher noch nicht beobachtet. ${ }^{124,178,179}$ Durch große Reste an Stelle des acetylenischen Wasserstoffs (Phenyl, tert-Butyl oder Trimethylsilyl) ${ }^{172,180,181}$ konnte jedoch die Reaktion zum Myers-Saito-Produkt durch sterische Wechselwirkung soweit zurückgedrängt werden, daß sich Schmittel-Produkte bildeten (Abb. $45)$.

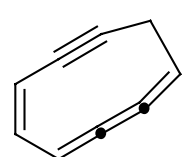

120

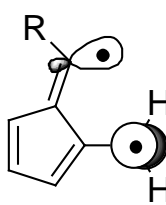

121

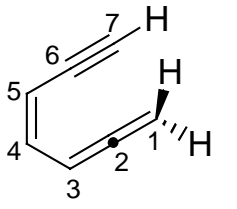

11

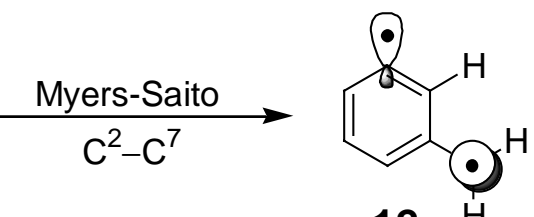

12 
Da im Eninallen 11 mehr ungesättigte C-Atome vorhanden sind als beim Endiin 9, sollte es rein hypothetisch auch mehr Cyclisierungsmöglichkeiten geben. Im Gegensatz zu

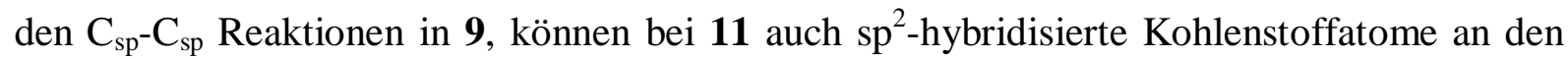
Cyclisierungen teilnehmen. Im folgenden Kapitel werden zunächst die beiden bekannten Myers-Saito- und Schmittel-Reaktionen eingehend untersucht, danach wird auf die weiteren möglichen Cyclisierungen eingegangen. Im weiteren Verlauf wird der Effekt der Benzannelierung auf 122 untersucht, bevor als letztes der Effekt, den die Ringspannung in den cyclischen Eninallenen 123a-f auf die Barrieren, Reaktionsenthalpien und die Regioselektivität des Ringschlusses hat, betrachtet wird (Abb. 46).
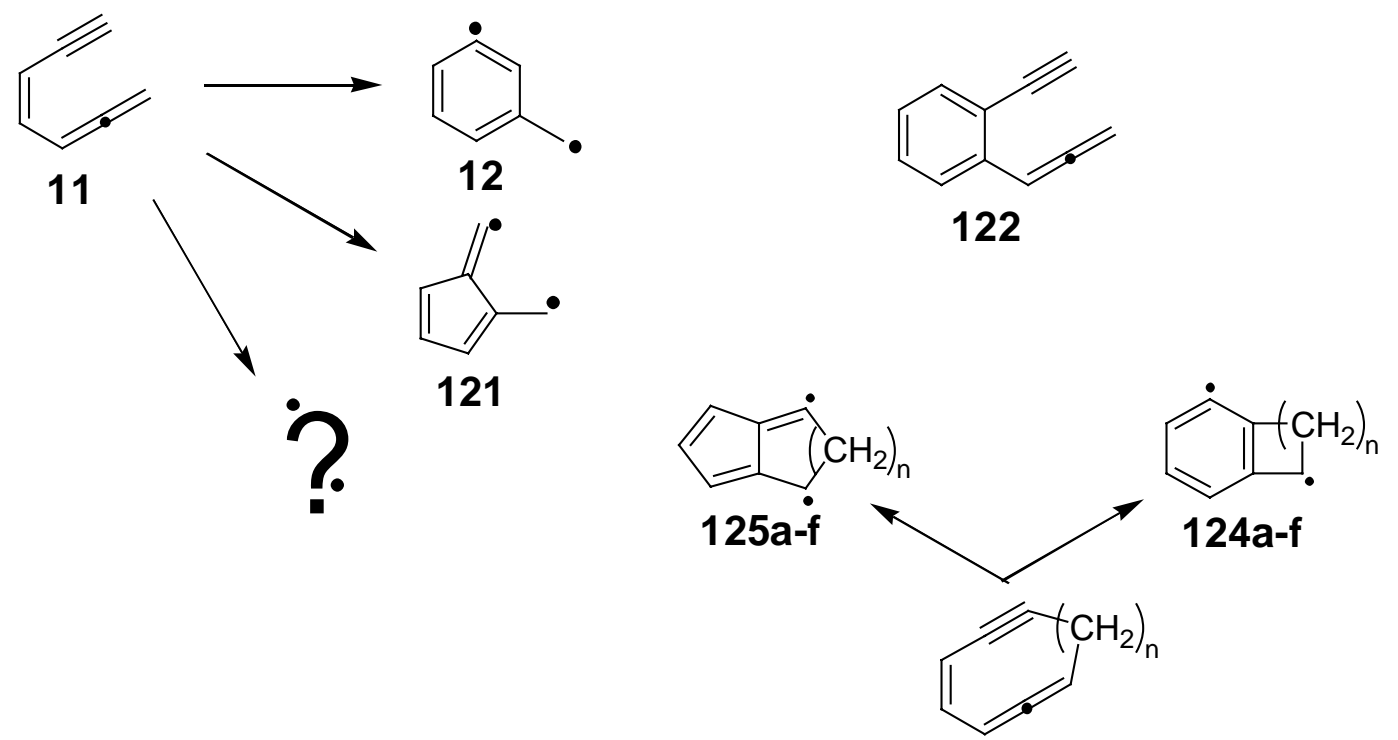

123a-f

Abbildung 46. Cyclisierung des Eninallen Stammsystems 10 sowie weitere Systeme

Stammsystem. Neben den bereits beschriebenen Cyclisierungen von 11 zu Schmittel121 und Myers-Saito-Produkt 12 lassen sich noch vier weitere Ringschlußreaktionen formulieren (Abb. 47). Die Produkte lassen sich grob in zwei Gruppen einteilen: Die zwei bereits bekannten 12 und 121 bilden die Gruppe der $\sigma, \pi$-Biradikale, die durch die Reaktion von zwei sp-hybridisierten C-Atomen entstehen. Die zweite Gruppe sind die vier $\sigma, \sigma-$ Biradikale 126, 127, 128 und 129, die aus der Reaktion von einem sp- mit einem $\mathrm{sp}^{2}$ hybridisierten C-Atom hervorgehen. Da den Radikalen der zweiten Gruppe, wie auch dem Bergman-Produkt, die $\pi$-Konjugation und zusätzlich auch die Aromatisierungsenergie fehlt, ist mit ihnen höchstens bei höheren Energien zu rechnen. Zunächst sollen deshalb die bereits bekannten Reaktionen im Detail besprochen werden, bevor auf weitere Cyclisierungen Bezug genommen wird. 


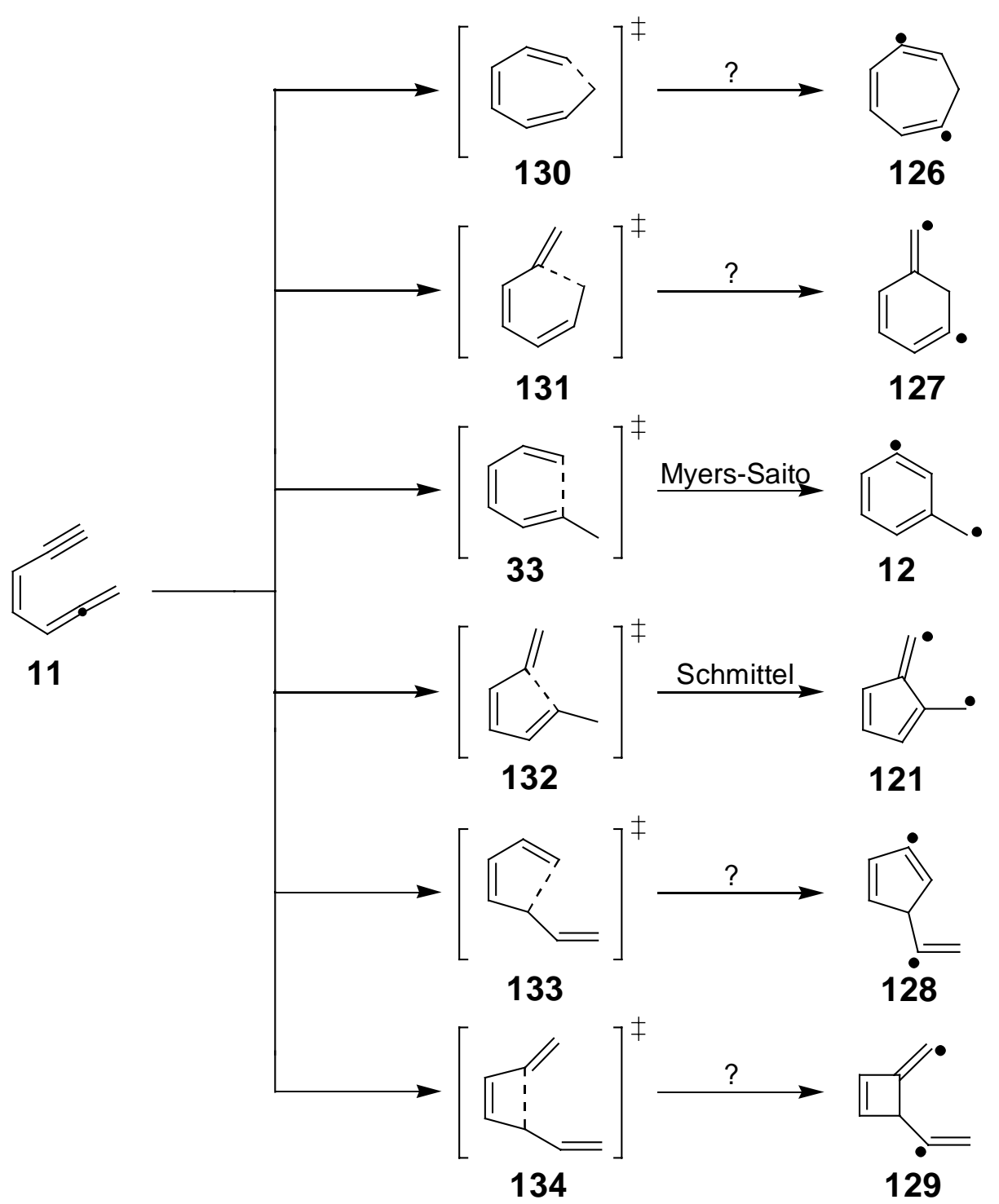

Abbildung 47. Die theoretisch denkbaren Cyclisierungsarten des Eninallen 11

Wie der Übergangszustand der Bergman-Reaktion haben auch die ÜZ der MyersSaito- und Schmittel-Reaktion keinen Biradikalcharakter, ${ }^{178,179}$ was sich zum einen daraus ergibt, daß die Energien der beschränkten und der unbeschränkten Berechnungen gleich sind, und zum anderen, daß der Erwartungswert des Spinoperators bei der unbeschränkten Wellenfunktion Null ist. Die experimentelle Barriere der Myers-Saito-Reaktion $(21.8 \pm 0.5$ $\left.\mathrm{kcal} \mathrm{mol}^{-1}\right)^{16}$ wird durch BLYP mit $18.8 \mathrm{kcal} \mathrm{mol}^{-1}$ einigermaßen gut beschrieben, während $\mathrm{BD}(\mathrm{T})$ mit $20.7 \mathrm{kcal} \mathrm{mol}^{-1}$ sehr gut liegt (Tab. 10). Bei der Schmittel-Reaktion gibt es zwei Übergangszustände $(\boldsymbol{E})$-132 und $(\boldsymbol{Z})$-132 zu zwei Produkten $(\boldsymbol{E})$-121 und $(\boldsymbol{Z})$-121, die sich, wie bei der $\mathrm{C}^{1}-\mathrm{C}^{5}$-Cyclisierung des Endiins 9, in der Stellung des Wasserstoffatoms an der exocyclischen Doppelbindung unterscheiden (Abb. 48). Die Barriere zu (E)-121 liegt dabei 11.4 bzw. $12.6 \mathrm{kcal} \mathrm{mol}^{-1}$ über 33 [BLYP: $30.2 \mathrm{kcal} \mathrm{mol}^{-1}$; BD(T): $33.3 \mathrm{kcal} \mathrm{mol}^{-1}$ ], die zu 
(Z)-121 nur 7.4 bzw. $8.1 \mathrm{kcal} \mathrm{mol}^{-1}$ über 33 [BLYP: $26.2 \mathrm{kcal} \mathrm{mol}^{-1}$; BD(T): $28.8 \mathrm{kcal} \mathrm{mol}^{-1}$ ]. Wie bereits angedeutet, ist diese höhere Barriere dafür verantwortlich, daß im Stammsystem diese Cyclisierung noch nicht beobachtet wurde.

Bei den Produkten gibt es für beide Reaktionspfade offen- und geschlossenschalige Lösungen, die sich sowohl in der Energie als auch in der Geometrie unterscheiden (Abb. 48). Das biradikalische Myers-Saito-Produkt 12 wird exotherm gebildet und stimmt mit $-9.6 \mathrm{kcal}$ $\mathrm{mol}^{-1}$ für BLYP recht gut und mit $-13.6 \mathrm{kcal} \mathrm{mol}^{-1}$ für $\mathrm{BD}(\mathrm{T})$ hervorragend mit dem experimentellen Wert von $15 \pm 3 \mathrm{kcal} \mathrm{mol}^{-1} 17$ überein (Tabelle 10). Das geschlossenschalige allenische Produkt 27 kann ebenfalls über den ÜZ 33 erreicht werden, liegt aber 5.2 bzw. 3.3 $\mathrm{kcal} \mathrm{mol}^{-1}$ für BLYP bzw. BD(T) über 12 und ist deshalb energetisch ungünstiger. Es ist eindeutig von dem planaren, $C_{S}$-symmetrischen 12 zu unterscheiden, da die Allen-Teilstruktur für eine Verdrillung des sechsgliedrigen Ringes sorgt und die allenischen $\mathrm{H}$ somit einen Diederwinkel von $76.6^{\circ}$ aufweisen. Nichtsdestotrotz kann 27 eine wichtige Rolle spielen, wenn geeignete Substituenten, z.B. an Stelle des acetylenischen H, die biradikalische Struktur destabilisieren und 27 zur stabileren Konfiguration wird. Beim Schmittel-Produkt gibt es entsprechend den zwei Übergangszuständen $(\boldsymbol{E})$-132 und (Z)-132 auch zwei biradikalische Produkte $(\boldsymbol{E}) \mathbf{- 1 2 1}$ und $(\boldsymbol{Z}) \mathbf{- 1 2 1}$, bei denen die energetischen Verhältnisse uneinheitlich sind. Hierbei liegt (E)-121 mit 12.9 bzw. $8.8 \mathrm{kcal} \mathrm{mol}^{-1}$ für BLYP bzw. BD(T) etwas günstiger bzw. ungünstiger als (Z)-121, das $0.1 \mathrm{kcal} \mathrm{mol}^{-1}$ darüber bzw. $0.2 \mathrm{kcal} \mathrm{mol}^{-1}$ darunter liegt. Da der ÜZ zwischen den beiden Strukturen $(\boldsymbol{E})-\mathbf{1 2 1}$ und $(\boldsymbol{Z})$-121 kleiner als $5 \mathrm{kcal} \mathrm{mol}^{-1}$ sein sollte, können beide leicht ineinander übergehen. Die Struktur $(\boldsymbol{E})$-121 liegt auf beiden Niveaus $22.5 \mathrm{kcal} \mathrm{mol}^{-1}$ über dem Myers-Saito-Produkt 12. Auch beim Schmittel-Produkt gibt es ein geschlossenschaliges Produkt 135, das allerdings eine carbenoide Struktur aufweist und 4.9 bzw. $6.8 \mathrm{kcal} \mathrm{mol}^{-1}$ über $(\boldsymbol{E}) \mathbf{- 1 2 1}$ liegt. Auch hier ist $\mathbf{1 3 5}$ geometrisch unterscheidbar, da es von der planaren Geometrie abweicht und einen auf $112.5^{\circ}$ verkleinerten $\mathrm{H}^{\cdots \cdots} \mathrm{C} \cdots \mathrm{C}$ Winkel am carbenoiden Kohlenstoff gegenüber $134.9^{\circ}$ in $(\boldsymbol{E})-\mathbf{1 2 1}$ aufweist.

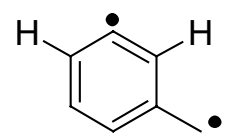

12<smiles>C=C1C=CC=CC1</smiles>

27

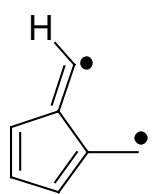

(E)-121

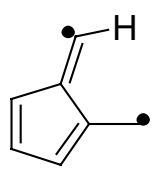

(Z)-121

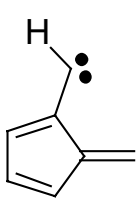

135

Abbildung 48. Offen- und geschlossenschalige Cyclisierungsprodukte des Eninallen 11 
Tabelle 10. Barrieren und Reaktionsenthalpien $\left(\Delta_{0} \mathrm{H}\right)$ der Cyclisierungspfade von Eninallen 11 (in kcal mol ${ }^{-1}$ )

\begin{tabular}{ccc}
\hline Struktur & BLYP/6-31G* & BD(T)/cc-pVDZ \\
\hline $\mathbf{1 1}$ & 0.0 & 0.0 \\
$\mathbf{3 3}$ & 18.8 & 20.7 \\
$\mathbf{1 2}$ & -9.6 & -13.6 \\
$\mathbf{2 7}$ & -4.4 & -10.3 \\
$(\boldsymbol{E}) \mathbf{- 1 3 2}$ & 30.2 & 33.3 \\
$(\mathbf{Z}) \mathbf{- 1 3 2}$ & 26.2 & 28.8 \\
$(\boldsymbol{E}) \mathbf{- 1 2 1}$ & 12.9 & 8.8 \\
$(\boldsymbol{Z}) \mathbf{- 1 2 1}$ & 13.0 & 8.6 \\
$\mathbf{1 3 5}$ & 17.8 & 15.6 \\
\hline
\end{tabular}

Die Standardbildungsenthalpie $\left(\Delta_{\mathrm{f}} \mathrm{H}^{\ominus}\right)$ von 12 wurde experimentell mit $103 \pm 3$ bestimmt, ${ }^{17}$ was durch die Theorie sehr gut reproduziert wird $\left(106.6 \pm 2 \mathrm{kcal} \mathrm{mol}^{-1}\right.$ für beide Methoden, Tab. 11). Zum Vergleich ist noch das Ergebnis der coupled cluster Methode CCSD(T) aufgenommen, an dem sich deutlich zeigt, daß auf HF basierende Ein-Referenz Methoden bei den vorliegenden Biradikalen an ihre Grenzen stoßen $\left(\sim 13 \mathrm{kcal} \mathrm{mol}^{-1}\right.$ Abweichung vom Experiment).

Tabelle 11. Vergleich von berechneter und experimentell ermittelter Standardbildungsenthalpie von 12 (in $\mathrm{kcal} \mathrm{mol}^{-1}$ ). Die experimentellen Standardbildungsenthalpien sind: 19.7 (Benzol), ${ }^{182} \quad 79.1 \pm 2 \quad$ (Phenylradikal) $^{183}$ und $48 \pm 2$ (Tolylrdikal) $^{184}$

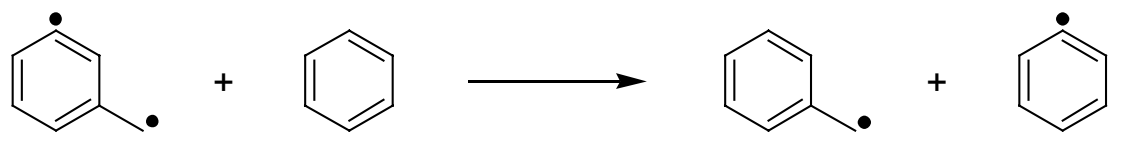

\begin{tabular}{ccc}
\hline Methode & $\Delta_{\mathrm{R}} \mathrm{H}_{0}$ & $\Delta_{\mathrm{f}} \mathrm{H}^{\ominus}$ \\
\hline BLYP/6-31G* & +0.8 & $106.6 \pm 2$ \\
CCSD(T)/cc-pVDZ & +17.3 & $90.1 \pm 2$ \\
BD(T)/cc-pVDZ & +0.8 & $106.6 \pm 2$ \\
Experiment & -4.4 & $103 \pm 3$ \\
\hline
\end{tabular}

${ }^{\mathrm{a}}$ Geometrien und ZPVE-Korrekturen aus BLYP/6-31G*

Die Standardbildungsenthalpie von $(\boldsymbol{E})$-121 konnte dann auf demselben Wege über eine isodesmische Gleichung erhalten werden, nachdem zuvor noch $\Delta_{\mathrm{f}} \mathrm{H}^{\ominus}$ von 2Methylfulven, die experimentell noch nicht bekannt ist, $\mathrm{zu} 45.4 \mathrm{kcal} \mathrm{mol}^{-1}$ bestimmt wurde 
(Tab. 12, 13). Die Standardbildungsenthalpie von (E)-121 wird auf der Basis des auf BD(T)Niveau erhaltenen Wertes mit $128.6 \pm 3 \mathrm{kcal} \mathrm{mol}^{-1}$ vorausgesagt. Auch hier zeigt sich wieder deutlich, daß CCSD(T) für die vorliegende Problemstellung nicht geeignet ist.

Tabelle 12. Bestimmung der Standardbildungsenthalpie von 2-Methylfulven (in $\mathrm{kcal} \mathrm{mol}^{-1}$ ). Die experimentellen Standardbildungsenthalpien sind: 53.6 (Fulven), ${ }^{182}$ -25.3 (Methylcyclopentan) ${ }^{182}$ und -18.4 (Cyclopentan) ${ }^{182}$

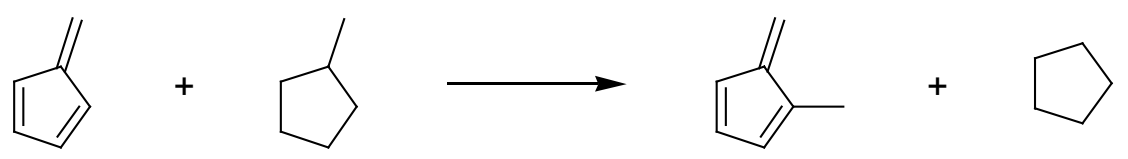

\begin{tabular}{ccc}
\hline Methode & $\Delta_{\mathrm{R}} \mathrm{H}_{0}$ & $\Delta_{\mathrm{f}} \mathrm{H}^{\ominus}$ \\
\hline BLYP/6-31G* & -2.7 & 44.0 \\
CCSD(T)/cc-pVDZ & -1.2 & 45.5 \\
BD(T)/cc-pVDZ & -1.3 & 45.4 \\
\hline
\end{tabular}

${ }^{\mathrm{a}}$ Geometrien und ZPVE-Korrekturen aus BLYP/6-31G*

Tabelle 13. Bestimmung der Standardbildungsenthalpie von (E)-132 (in kcal $\mathrm{mol}^{-1}$ ). Die experimentellen Standardbildungsenthalpien sind: $103 \pm 3(\mathbf{1 2}),{ }^{17} 12.0$ (Toluol) ${ }^{182}$ und 44.0 bzw. $45.5^{\text {b }}$ (2-Methylfulven)

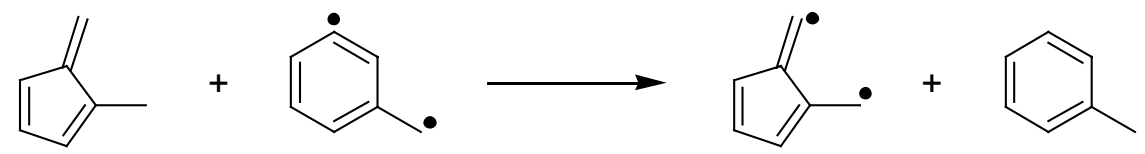

\begin{tabular}{ccc}
\hline Methode & $\Delta_{\mathrm{R}} \mathrm{H}_{0}$ & $\Delta_{\mathrm{f}} \mathrm{H}^{\ominus}$ \\
\hline BLYP/6-31G* & -9.6 & $125.4 \pm 3$ \\
$\mathrm{CCSD}(\mathrm{T}) / \mathrm{cc}^{*} \mathrm{pVDZ}{ }^{\mathrm{a}}$ & 11.9 & $148.4 \pm 3$ \\
$\mathrm{BD}(\mathrm{T}) / \mathrm{cc}-\mathrm{pVDZ}{ }^{\mathrm{a}}$ & -7.8 & $128.6 \pm 3$ \\
\hline
\end{tabular}

${ }^{\mathrm{a}}$ Geometrien und ZPVE-Korrekturen aus BLYP/6-31G*

${ }^{\mathrm{b}}$ Siehe Tabelle 12

Eine weitere wichtige Frage beschäftigt sich mit den Spindichten in den Produkten 12 und 121. Während 27 und 135 als geschlossenschalige Moleküle natürlich keine Einzelspindichten aufweisen, sind in $\mathbf{1 2}$ und $\mathbf{1 2 1}$ Delokalisationseffekte des $\boldsymbol{\pi}$-Radikals zu erwarten, und die Frage ist, welche Spinresonanzstruktur den größten Anteil hat (Abb. 49). Da die SingulettTriplett-Aufspaltungen sehr klein sind, ist zu erwarten, daß die Koeffizienten der Spindichten bei den offenschaligen Singuletts und den Tripletts praktisch gleich sind. 
<smiles></smiles>

$12_{\mathrm{SR} 1}$<smiles>C=C1C=CC(=O)C=C1</smiles>

$12_{\mathrm{SR} 2}$<smiles>C=C1C=CC(=O)C(C)C1</smiles>

$12_{\mathrm{SR} 3}$<smiles>C=C1C=CC(=O)C=C1C(=O)O</smiles>

$1_{\mathrm{SR} 4}$

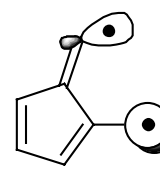

$121_{\text {SR1 }}$

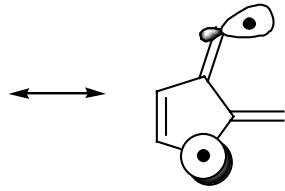

$121_{\text {SR2 }}$
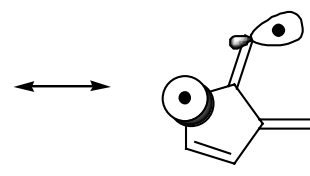

$121_{S R 3}$

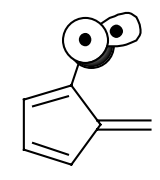

$121_{\text {SR4 }}$

Abbildung 49. Spinresonanzstrukturen für die offenschaligen 12 und 121

Während das $\sigma$-Radikal-Elektron auf sein $\mathrm{sp}^{2}$-Hybridorbital beschränkt ist und weder mit anderen Bindungen noch mit anderen Orbitalen, inklusive des $\pi$-Systems, wechselwirken kann, zeigt das $\pi$-Radikal-Elektron die Delokalisierungen, wie sie typisch für Benzyl- oder Allyl-Systeme sind. Die Konsequenzen für 12 sind deshalb nicht dramatisch, da das aromatische Elektronensextett nicht sehr effektiv mit dem Benzyl-Radikal wechselwirkt. Deshalb ist auch die Spinresonanz, wie sie in $\mathbf{1 2}_{\mathrm{SR} 1}$ dargestellt ist, die dominante Repräsentation der Spindichte für das Myers-Saito-Produkt, gefolgt von $\mathbf{1 2}_{\text {SR3 }}$ und danach $\mathbf{1 2}_{\mathrm{SR} 2}$ und $12_{\mathrm{SR} 4}$ zu gleichen Teilen (Abb. 50). Das Schmittel-Produkt zeigt jedoch ein ganz anderes Bild. Dort ist 121 $\mathbf{1}_{\text {SR1 }}$ die Repräsentation mit der geringsten Bedeutung und ist für die tatsächliche $\pi$-Radikaldichte unbedeutend, die am besten durch 121 $_{\text {SR3 }}$ und danach 121 $_{\text {SR2 }}$ beschrieben wird. Deshalb ist auch die Energie geringer als im Fulven-Biradikal 97 der $C^{1}$ $\mathrm{C}^{5}$-Endiincyclisierung, in der beide $\sigma$-Radikal-Elektronen in ihren Orbitalen lokalisiert sind, und folglich ist der Energieunterschied $30.8 \mathrm{kcal} \mathrm{mol}^{-1}$, der nicht durch unterschiedliche Stabilitäten der Edukte erklärt werden kann $\left(\Delta \mathrm{E}_{\text {Eninallen/Methylendiin }}=2.9 \mathrm{kcal} \mathrm{mol}^{-1}\right)$, sondern sich allein aus der $\pi$-Allylstabilisierung ergibt. 

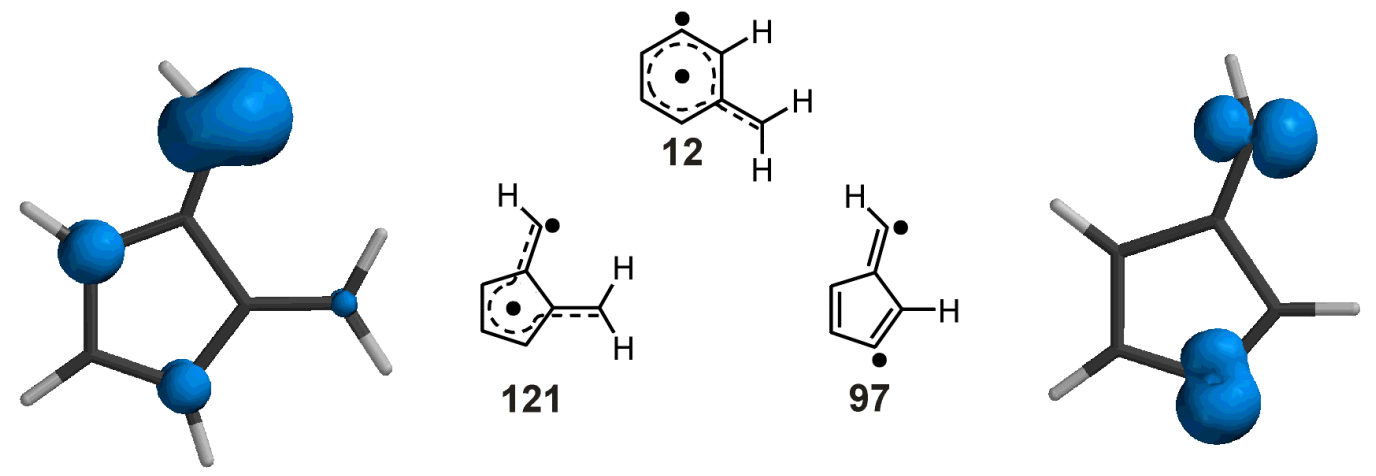

Abbildung 50. Triplett Spindichten im Myers-Saito- 12, Schmittel- 121 und Fulven-Biradikal 97

Andere Cyclisierungen des Eninallens 11 sind, wie schon erwähnt, nur zwischen den sp-hybridisierten Acetylen- und den $\mathrm{sp}^{2}$-hybridisierten Allenkohlenstoffatomen möglich und führen alle vier zu $\sigma, \sigma$-Biradikalen, die in der Energie entsprechend weit über den $\sigma, \pi$ Biradikalen stehen.

Die $C^{1}-C^{7}$-Cyclisierung zum siebengliedrigen Biradikal (Cycloheptatrien-Biradikal, 126) durch Bindungsbildung zwischen den endständigen Kohlenstoffatomen in $\mathbf{1 1}$ ist 32.9 kcal mol ${ }^{-1}$ endotherm und entsprechend ist $126 \sim 24 \mathrm{kcal} \mathrm{mol}^{-1}$ instabiler als das SchmittelProdukt 121 (Tab. 14). Da 121 bereits nicht als Produkt bei der Cyclisierung von 11 zu beobachten war, ist die Bildung von 126 ebenfalls unwahrscheinlich. Der Übergangszustand 130 (37.9 $\mathrm{kcal} \mathrm{mol}^{-1}$ ) liegt jedoch nur $\sim 9 \mathrm{kcal} \mathrm{mol}^{-1}$ über 132 für die Schmittel-Reaktion, und deshalb ist die Synthese substituierter Derivate von 126 zumindest nicht unmöglich, vorausgesetzt die richtigen Substituenten können gefunden werden. Der ÜZ 130 hat wie die der Schmittel- und Myers-Saito-Reaktion keinen Biradikalcharakter. Wie für das MyersSaito- und das Schmittel-Produkt gibt es auch für das Cycloheptatrien-Biradikal eine geschlossenschalige Lösung (136, Abb. 51). Da die Geometrien von 126 und 136 sich nicht wesentlich unterscheiden, ist 136 auch nur $\sim 2 \mathrm{kcal} \mathrm{mol}^{-1}$ höher in der Energie als sein offenschaliges Gegenstück (Tab. 14). 
<smiles></smiles>

27

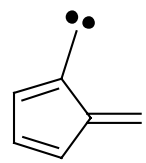

135<smiles>C1=CC=CCC=C1</smiles>

126

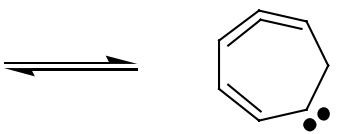

136

Abbildung 51. Geschlossenschalige Varianten aller Cyclisierungsmöglichkeiten von 11

Tabelle 14. Barrieren und Reaktionsenthalpien $\left(\Delta_{0} H\right)$ der Cyclisierungspfade von Eninallen 11 (in kcal mol ${ }^{-1}$ )

\begin{tabular}{ccc}
\hline Struktur & BLYP/6-31G* & BD(T)/cc-pVDZ \\
\hline $\mathbf{1 1}$ & 0.0 & 0.0 \\
$\mathbf{3 3}$ & 18.8 & 20.7 \\
$\mathbf{1 2}$ & -9.6 & -13.6 \\
$\mathbf{2 7}$ & -4.4 & -10.3 \\
$\mathbf{1 3 2}$ & 26.2 & 28.8 \\
$\mathbf{1 2 1}$ & 12.9 & 8.8 \\
$\mathbf{1 3 5}$ & 17.8 & 15.6 \\
$\mathbf{1 3 0}$ & 36.6 & 37.9 \\
$\mathbf{1 2 6}$ & 34.9 & 32.9 \\
$\mathbf{1 3 6}$ & 36.3 & 34.9 \\
\hline
\end{tabular}

${ }^{\mathrm{a}}$ Geometrien und ZPVE-Korrekturen aus BLYP/6-31G*

Andere Cyclisierungsarten für 11 führen nicht zu stabilen offenschaligen SingulettBiradikalen. Genaue Betrachtungen der $C^{3}-C^{7}$ und $C^{1}-C^{6}$ Cyclisierungs-Reaktionskoordinaten zeigen einen monotonen Energieanstieg für kleiner werdende C-C Abstände (Abb. 52); die $\mathrm{C}^{3}-\mathrm{C}^{6}$ Cyclisierung ist ebenfalls nicht zugänglich.

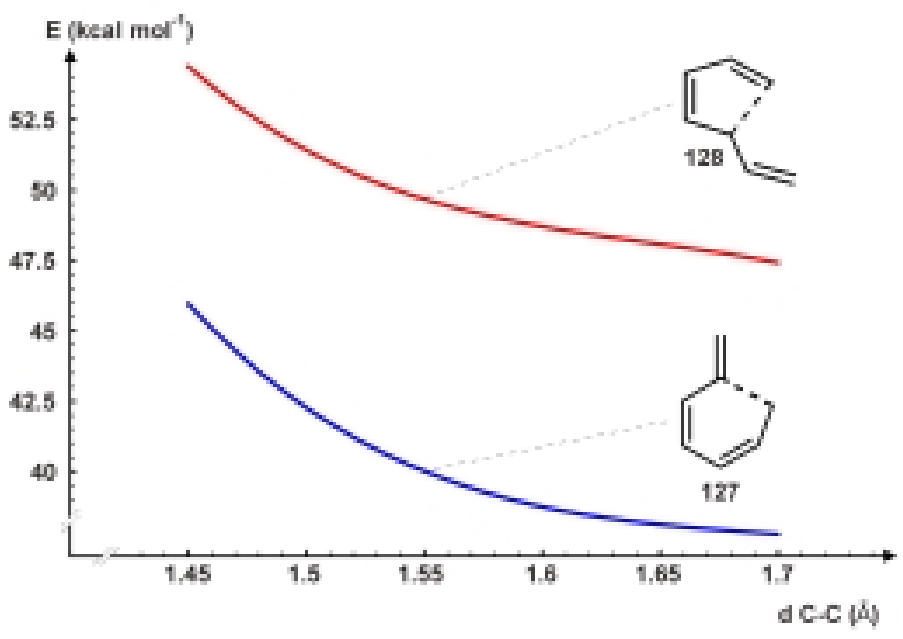

Abbildung 52. Energiehyperflächen der $C^{3}-C^{7}$ und $C^{1}-C^{6}$ Cyclisierungen auf BLYP/6-31G* 
Benzannelierung. Das benzannelierte Eninallen (1-Ethinyl-2-propa-1,2-dienylbenzol, 122) zeigt dieselben Cyclisierungsreaktionen wie das Stammsystem. Das benzannelierte Myers-Saito-Produkt 137 (-5.8 $\left.\mathrm{kcal} \mathrm{mol}^{-1}\right)$ liegt energetisch ungünstiger als das des Stammsystems, da die Aromatizität im Produkt durch den bereits vorhandenen Benzolring verringert ist (Abb. 53, 54; Tab. 15). Im geschlossenschaligen Produkt 138 ist die Aromatizität durch das ortho-chinoide System weitgehend aufgehoben, so daß die Energie 7.2 $\mathrm{kcal} \mathrm{mol}^{-1}$ höher liegt als in 137. Da das benzannelierte Schmittel-Produkt 139 nicht durch Cycloaromatisierung gebildet wird, ist die Energie mit $9.4 \mathrm{kcal} \mathrm{mol}^{-1}$ nahezu dieselbe wie im Stammsystem ( 0.6 kcal mol ${ }^{-1}$ stabiler, Abb. 54). Auch hier leidet das entsprechende geschlossenschalige Produkt 140 an der Aufhebung der Aromatizität und liegt $15.7 \mathrm{kcal} \mathrm{mol}^{-1}$ höher in der Energie. Barriere und Reaktionsenthalpie zum Benzocycloheptatrien-Biradikal 141 verändern sich wenig gegenüber der Stammreaktion. Im Gegensatz zur Stammreaktion hat der Übergangszustand jedoch Biradikalcharakter. Eine geschlossenschalige Variante existiert vom benzannelierten siebengliedrigen Ring nicht. Die fehlenden drei Reaktionspfade gibt es auch für 122 nicht.<smiles>Cc1ccc2ccccc2c1</smiles>

137<smiles></smiles>

138<smiles>C=C1C(C)=Cc2ccccc21</smiles>

139

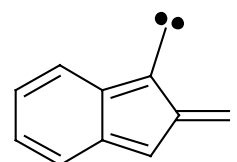

140<smiles>C1=Cc2ccccc2C=CC1</smiles>

141

Abbildung 53. Offen- und geschlossenschalige Cyclisierungsprodukte von 122

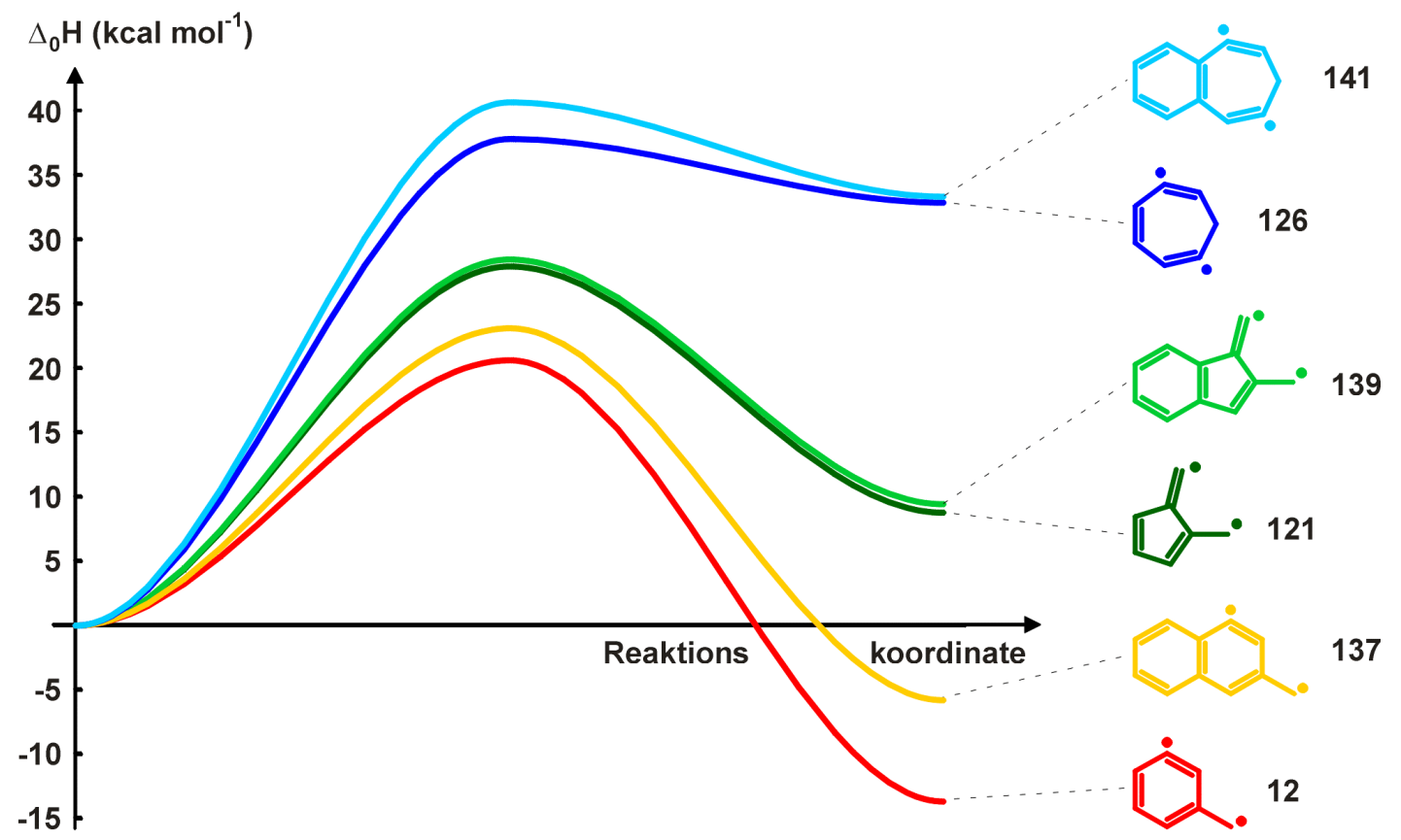

Abbildung 54. Vergleich der Potentialhyperflächen der Cyclisierungen von 11 und 122 
Tabelle 15. Barrieren und Reaktionsenthalpien $\left(\Delta_{0} \mathrm{H}\right)$ der Cyclisierungspfade des benzannelierten Eninallens 122 (in kcal $\mathrm{mol}^{-1}$ ).

\begin{tabular}{ccc}
\hline Struktur & BLYP/6-31G* & BD(T)/cc-pVDZ ${ }^{\mathrm{a}}$ \\
\hline $\mathbf{1 2 2}$ & 0.0 & 0.0 \\
$\mathbf{1 2 2} \rightarrow \mathbf{1 3 7}$ & 19.5 & 23.1 \\
$\mathbf{1 3 7}$ & -4.5 & -5.8 \\
$\mathbf{1 3 8}$ & 4.7 & 1.4 \\
$\mathbf{1 2 2} \rightarrow \mathbf{1 3 9}$ & 25.2 & 28.4 \\
$\mathbf{1 3 9}$ & 11.9 & 9.4 \\
$\mathbf{1 4 0}$ & 21.7 & 25.1 \\
$\mathbf{1 2 2} \rightarrow \mathbf{1 4 1}$ & 36.7 & 40.7 \\
$\mathbf{1 4 1}$ & 35.5 & 33.3 \\
${ }^{\mathrm{a}}$ Geometrien und ZPVE-Korrekturen aus BLYP/6-31G*.
\end{tabular}

Ringspannung. Wie bei den cyclischen Endiinen 99a-h sollte die Ringspannung in den cyclischen Eninallenen 123a-f eine Verringerung der Cyclisierungsbarriere und somit eine Reaktivitätserhöhung bewirken. Ein weiterer Aspekt ist die Möglichkeit, daß durch Ringspannungseffekte die Schmittel-Cyclisierung gegenüber der Myers-Saito-Cyclisierung favorisiert wird und statt der Benzocycloalken-Biradikalen 124a-f die 4H-Cyclopentacycloalken-Biradikale 125a-f gebildet werden (Abb. 54).

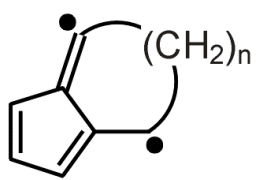

125a-f

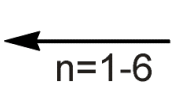

Abbildung 55. Ringschlußreaktionen der cyclischen Endiine 123a-f

Abbildung 55. Ringschlußreaktionen der cyclischen Endiine 123a-f

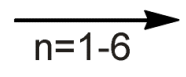

123a-f<smiles>CC12CCCCC1CCC21CCCCC1</smiles>

$124 a-f$

Der siebengliedrige Ring 123a, in dem der terminale acetylenische Kohlenstoff direkt mit dem allenischen verbunden ist, stellt einen Sonderfall dar, denn weder für das MyersSaito- (124a) noch für das Schmittel-Produkt 125a ergibt sich ein offenschaliges Biradikal. Beide Strukturen existieren nur als geschlossenschalige Moleküle. Mit einer Barriere von 9.1 $\mathrm{kcal} \mathrm{mol}^{-1} \mathrm{zu}$ 124a und einer Enthalpie von $9.0 \mathrm{kcal} \mathrm{mol}^{-1}$ ist die Rückreaktion sehr schnell und damit die Bildung des hochgespannten Benzocyclopropen-Biradikals 124a eher unwahrscheinlich (Tab. 16, Abb. 56). Das Schmittel-Produkt 125a scheint mit einer Barriere von $24.0 \mathrm{kcal} \mathrm{mol}^{-1}$ und einer Enthalpie von $14.0 \mathrm{kcal} \mathrm{mol}^{-1}$ experimentell erreichbar, ist jedoch ebenfalls sehr gespannt aufgrund des gebildeten viergliedrigen Ringes. 
Beim achtgliedrigen 123b sind beide Barrieren kleiner als die der Stammreaktionen. Die Barriere zu 125b ist dabei mit $15.2 \mathrm{kcal} \mathrm{mol}^{-1}$ um $2.6 \mathrm{kcal} \mathrm{mol}^{-1} \mathrm{kleiner}$ als die zu 124b. Mit nur $0.5 \mathrm{kcal} \mathrm{mol}^{-1}$ Reaktionsenthalpie für $\mathbf{1 2 5 b}$ sollte aus $\mathbf{1 2 3 b}$ ausschließlich das Schmittel-Produkt gebildet werden und nicht $\mathbf{1 2 4 b}$, das in seiner Enthalpie $3.3 \mathrm{kcal} \mathrm{mol}^{-1}$ über 125b liegt. Für beide Produkte 124b und 125b existiert jeweils auch ein geschlossenschaliges Pendant, die aber beide höher in der Energie liegen (5.7 bzw. $2.1 \mathrm{kcal} \mathrm{mol}^{-1}$ ).

Die Barrieren des neungliedrigen Rings 123c sind beide noch einmal niedriger als die

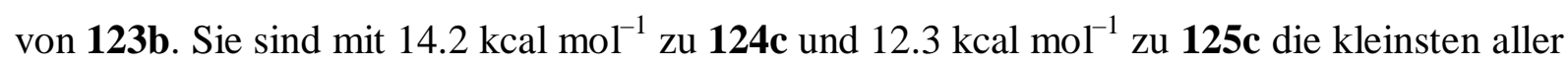
cyclischen Eninallene, da bei beiden Produkten die vorteilhafte Kombination von einem fünfund einem annelierten sechsgliedrigen Ring gebildet wird. Auch hier ist das SchmittelProdukt bevorzugt, es wird jedoch mit $4.2 \mathrm{kcal} \mathrm{mol}^{-1}$ endotherm gebildet, während $124 \mathrm{c}$ eine Exothermie von $-11.8 \mathrm{kcal} \mathrm{mol}^{-1}$ besitzt. Unter kinetischer Kontrolle sollte sich aber trotzdem bevorzugt 125c bilden. Die geschlossenschaligen Reaktionsprodukte haben etwa denselben Energieunterschied zu den offenschaligen wie bei $\mathbf{1 2 3 b}$.

Beim zehngliedrigen Ring 123d sind die Produktverhältnisse wie beim Stammsystem, obwohl beide Barrieren im Vergleich immer noch kleiner sind. Da jedoch die Barriere nach 124d $5.8 \mathrm{kcal} \mathrm{mol}^{-1}$ und die Reaktionsenthalpie $19.0 \mathrm{kcal} \mathrm{mol}^{-1}$ kleiner sind als die $\mathrm{zu}$ 125d, ist eine Bildung des Schmittel-Produktes ausgeschlossen. Zum elfgliedrigen 123e hin ist der Unterschied in der Barriere noch etwas größer geworden. Die Energien von 123e entsprechen im großen und ganzen denen des Stammsystems, während sie im zwölfgliedrigen 123f schon darüberliegen und hohe Temperaturen zum Cyclisieren benötigen. Nichtsdestotrotz wird auch dort das Myers-Saito-Produkt bevorzugt gebildet.

Bei den geschlossenschaligen Cyclisierungsprodukten von 123d-f ist anzumerken, daß der Energieunterschied bei den Myers-Saito-Produkten abnimmt (124c: $5.7 \mathrm{kcal} \mathrm{mol}^{-1}$; 124f: $2.5 \mathrm{kcal} \mathrm{mol}^{-1}$ ), während er bei den Schmittel-Produkten zunimmt (125d: $1.3 \mathrm{kcal} \mathrm{mol}^{-1}$; 125f: $6.1 \mathrm{kcal} \mathrm{mol}^{-1}$ ). Die biradikalischen Produkte sind aber in jedem Fall energetisch günstiger.

Die Ringe 123d-f können als Cyclisierungsprodukt auch Cycloheptatrien-Biradikale bilden, die jedoch mit Reaktionsenthalpien von 37.1, 42.0 und $41.3 \mathrm{kcal} \mathrm{mol}^{-1}$ weit oberhalb der anderen beiden Reaktionspfade liegen und experimentell wohl unzugänglich sein sollten. 
Tabelle 16. Barrieren und Reaktionsenthalpien $\left(\Delta \mathrm{G}_{298}\right)$ der Eninallene 123a-f, berechnet auf dem BLYP/6-31G* Nivau (in kcal mol ${ }^{-1}$ )

\begin{tabular}{lccccc}
\hline Ringgröße & & $\mathbf{1 2 3}$ & $\begin{array}{c}\mathbf{1 2 3} \rightarrow \mathbf{1 2 4} \\
\mathbf{1 2 3} \rightarrow \mathbf{1 2 5}\end{array}$ & $\begin{array}{c}\mathbf{1 2 4} \\
\mathbf{1 2 5}\end{array}$ & $\begin{array}{c}\text { Geschlossenschalige } \\
\text { Produkte }\end{array}$ \\
\hline 7-Ring & a & \multirow{2}{*}{0.0} & 9.1 & - & 9.0 \\
& & & 24.0 & - & 14.0 \\
8-Ring & b & \multirow{2}{*}{0.0} & 17.8 & 3.8 & 9.5 \\
& & & 15.2 & 0.5 & 2.6 \\
9-Ring & $\mathbf{c}$ & \multirow{2}{*}{0.0} & 14.2 & -11.8 & -6.1 \\
& & & 12.3 & 4.2 & 5.5 \\
10-Ring & $\mathbf{d}$ & \multirow{2}{*}{0.0} & 16.6 & -11.6 & -6.4 \\
& & & 22.4 & 7.4 & 12.9 \\
11-Ring & $\mathbf{e}$ & \multirow{2}{*}{0.0} & 18.3 & -3.7 & -2.0 \\
& & & 27.6 & 15.2 & 21.3 \\
12-Ring & $\mathbf{f}$ & \multirow{2}{*}{0.0} & 23.7 & -0.2 & 2.3 \\
& & & 28.4 & 26.0 & 30.0 \\
Stammsystem & $\mathbf{1 1}$ & \multirow{2}{*}{0.0} & 20.2 & -7.8 & -2.5 \\
& & & 27.7 & 14.2 & 19.4 \\
\hline
\end{tabular}

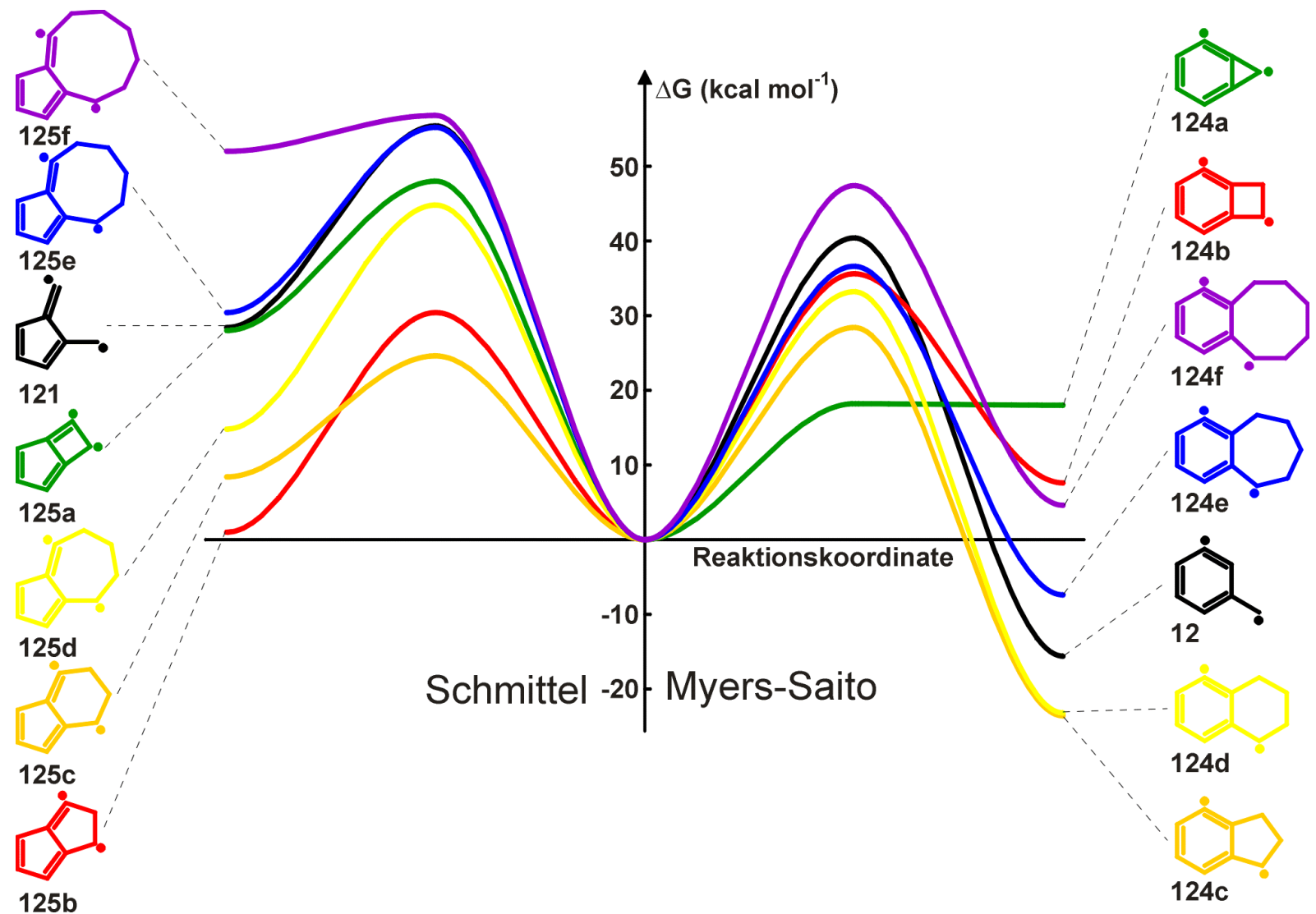

Abbildung 56. Vergleich der Barrieren und Enthalpien für die Cyclisierungen der Eninallene 123a-f im Vergleich mit dem Stammsystem 11 


\subsubsection{Die Cyclisierung von Hexa-1,3-dien-5-in ("Hopf-Cyclisierung")}

Die thermische Cyclisierung von Hexa-1,3-dien-5-in-Derivaten 142 ist von großer Wichtigkeit für die Synthese von schüsselförmigen Molekülen, wie z.B. Corannulen ${ }^{185-187} 143$ (Abb. 57) und Semibuckminsterfulleren. ${ }^{188}$ Die eingehende Untersuchung und das genaue Verständnis der Stammreaktion wird bei der Optimierung und Erweiterung dieser Synthesestrategie helfen.

Im Jahre 1969 konnten Hopf und Musso zeigen, daß Hexa-1,3-dien-5-in 89 thermisch zu Benzol 96 cycloisomerisiert. ${ }^{189}$ Die Reaktion beginnt ab einer Temperatur von $274^{\circ} \mathrm{C}$, bei der sich ein Isomerisierungsgleichgewicht zwischen $(\boldsymbol{E})-\mathbf{8 9}$ und $(\boldsymbol{Z})-\mathbf{8 9}$ einstellt. Aus rein geometrischer Sichtweise wurde dabei (Z)-89 als die Spezies benannt, die die Cyclisierung eingeht und nicht (E)-89 (Abb. 57).

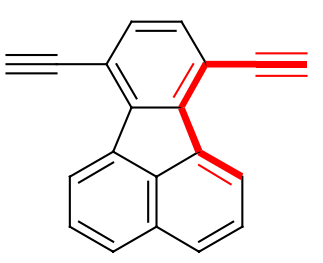

142

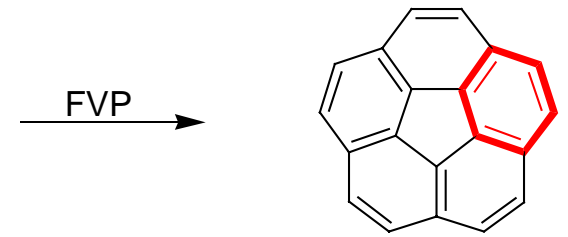

143

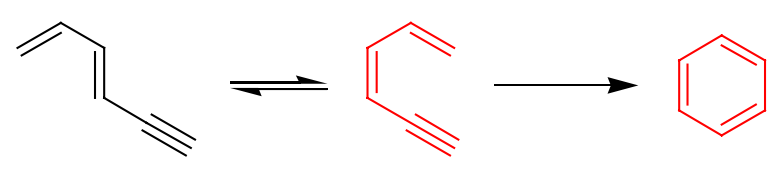

$\begin{array}{lll}(E)-89 & (Z)-89 & 96\end{array}$

Abbildung 57. Synthese des schüsselförmigen Corannulen 143 aus dem Hexa-1,3-dien-5-in-Derivat 142 und die thermische Cycloisomerisierung von $(Z)-89$ zu 96

Da 89 und 96 dieselbe Anzahl von Atomen besitzen, muß vor oder nach der Cyclisierung eine Wasserstoffverschiebung stattfinden. Abbildung 58 zeigt die wahrscheinlichsten Reaktionspfade, die die Strukturen 89 und 96 verbinden:

\section{[1,2]-H-Shift zum Vinyliden-Carben 144 und weiter zu 96}

- thermische Cyclisierung unter Umlagerung des $\pi$-Systems $\mathrm{zu}$ Isobenzol $(1,2,4-$ Cyclohexatrien) 90 und anschließendem H-Shift zu 96 thermische Cyclisierung zum Biradikal 91 und anschließendem H-Shift zu 96 Addition eines H-Radikals (145), Cyclisierung und anschließender Verlust von $\mathrm{H}^{\bullet} \mathrm{zu}^{96}$ 


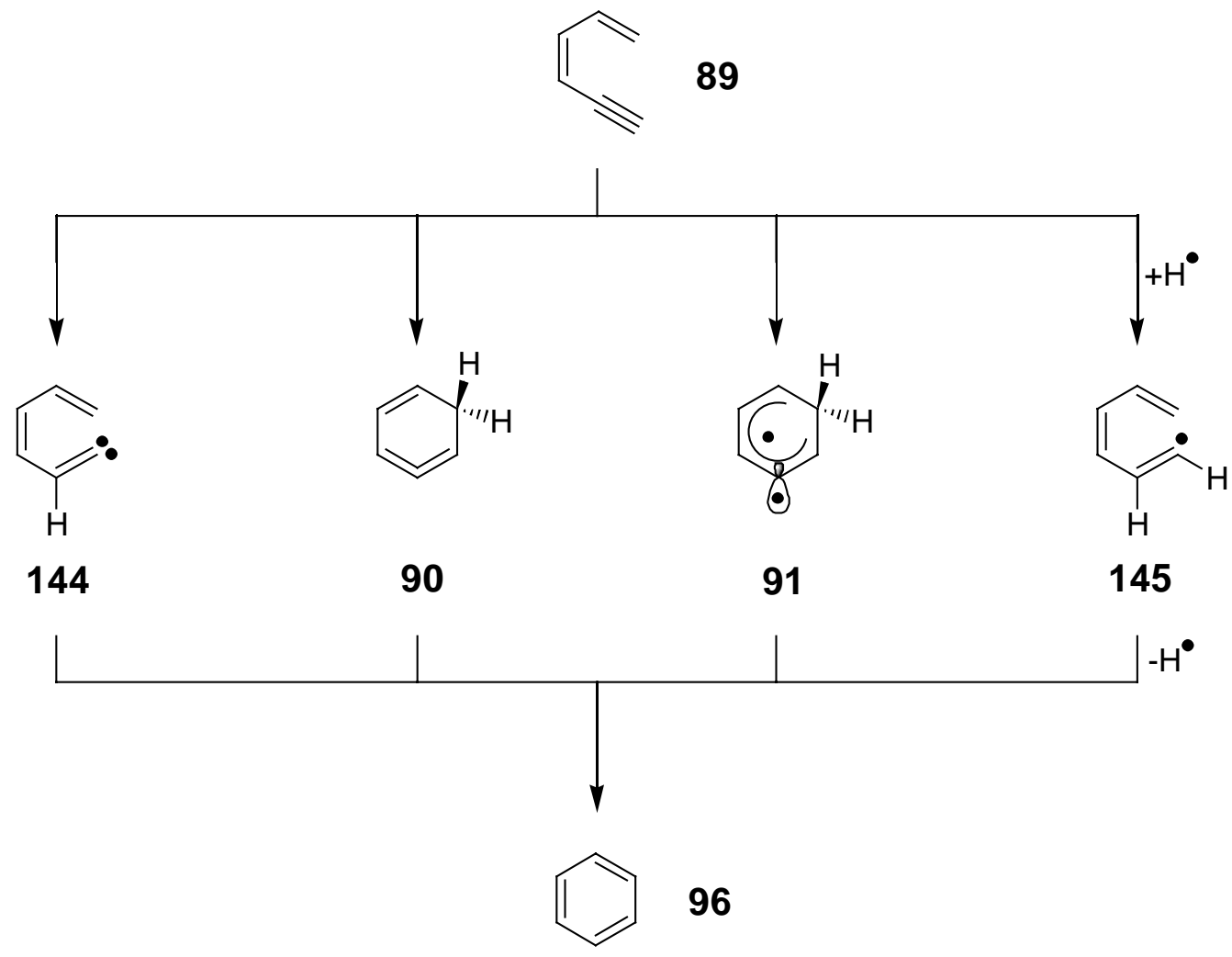

Abbildung 58. Reaktionspfade für die Cycloisomerisierung von 89 zu 96

Experimentelle und theoretische Studien berichten, daß bei niedrigeren Temperaturen (200-400 ${ }^{\circ} \mathrm{C}$ ) hauptsächlich die Intermediate 90 oder 91 gebildet werden. ${ }^{26,190,191}$ Die genaue Rolle von Allen 90 und Biradikal 91 ist allerdings noch unklar. ${ }^{192,193}$ Bei höheren Temperaturen sind Prozesse bevorzugt, die über Intermediate wie 144 und 145 gehen, was sich an der vermehrten Bildung von Pentafulven als Nebenprodukt bemerkbar macht. ${ }^{194-198}$

Im Hinblick auf die Bildung von Fulleren-ähnlichen Teilstrukturen oder "building blocks" ist der Effekt der Benzannelierung auf Barriere und Enthalpie ebenso entscheidend. Wie bereits beschrieben, erhöht die Benzannelierung die Barriere der Bergman-Reaktion des Endiins kaum, die Reaktionsenthalpie dagegen erhöht sie um fast $10 \mathrm{kcal} \mathrm{mol}^{-1} .{ }^{119,129,150,156,158}$ Da die Cycloisomerisierung von 89, im Gegensatz zur Bergman-Reaktion, offensichtlich kein einstufiger Prozeß ist, ist der Effekt der Benzannelierung nicht abzuschätzen.

Ein weiterer wichtiger Aspekt ist das Verhalten der Dienin-Einheit bei der Einbindung in ein carbocyclisches System. Die strukturell ähnlichen cyclischen Endiine 99a-h zeigen eine deutliche Abhängigkeit zwischen Ringspannung und Cyclisierungsbarriere. ${ }^{32,33}$ In Analogie zur Bergman-Reaktion von 99a-h sollten auch einige der kleineren Dienin-Ringe 146a-h eine niedrigere Barriere als das Stammsystem aufweisen (Abb. 59). 


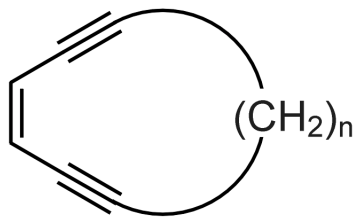

99a-f

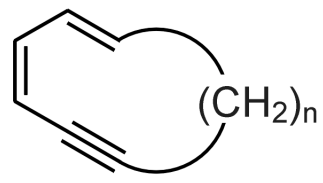

$146 a-h$

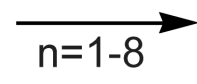<smiles>c1ccc2c(c1)CCCCC2</smiles>

112a-f

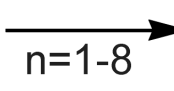<smiles>c1ccc2c(c1)CCCCC2</smiles>

147a-h

Abbildung 59. Schematische Darstellung der Endiin- (99a-h) bzw. Dienin-Ringverbindungen (146a-h) und deren Cyclisierungsprodukte (112a-h, 147a-h)

Die Synthese von Cycloocta-1,3-dien-5-in 146b ausgehend von Selendiazol 148 ist das erste Beispiel für ein cyclisches Dienin. ${ }^{199,200}$ Der hochgespannte Ring 146b konnte nach der Thermolyse von 148 bei $180{ }^{\circ} \mathrm{C}$ nur in Spuren detektiert werden, seine Bildung jedoch indirekt durch sein Cycloisomerisierungsprodukt Benzocyclobuten 147b bewiesen werden (Abb. ). Neben 146b ist das einzige andere in der Literatur erwähnte cyclische Dienin Cyclodeca-1,3-dien-5-in 146d, allerdings ist die NMR-spektroskopische Strukturzuweisung zweifelhaft. 201,202<smiles>C1=CCCc2nn[se]c2/C=C\CC1</smiles>

148

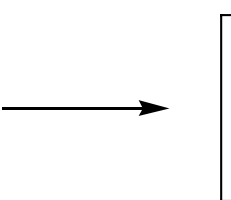

$146 b$<smiles>c1ccc2c(c1)CC2</smiles>

$147 b$

Abbildung 60. Synthese des nicht isolierbaren 146b ausgehend von Selendiazol 148 und dessen Cyclisierungsprodukt $147 \mathrm{~b}$

Im folgenden Kapitel wird die Cycloisomerisierung des Stammsystems (Z)-Hexa-1,3dien-5-in 89 zu Benzol 96 im Detail untersucht, einschließlich aller möglichen Zwischenstufen und Übergangszustände. Die berechneten Daten werden mit experimentellen verglichen und daraus sollen Rückschlüsse auf die Beteiligung von Isobenzol 90 oder dem Biradikal 91 gezogen werden. Weiterhin wird der Effekt der Benzannelierung auf die 
Cycloaromatisierung untersucht und Voraussagen für diese bisher nicht energetisch vermessene Reaktion gemacht.

Abschließend werden die Ringspannungseffekte auf die Cycloisomerisierung theoretisch auf der Basis der (E,Z)-Hexa-1,3-dien-5-in-Ringe 146a-h diskutiert und die Resultate dann den entsprechenden experimentellen Daten gegenübergestellt.

Stammsystem. Das Stammsystem (Z)-Hexa-1,3-dien-5-in 89 kann entlang verschiedener Pfade cycloisomerisieren, bei niedrigeren Temperaturen $\left(200-400^{\circ} \mathrm{C}\right)$ wird jedoch der elektrocyclische Ringschluß bevorzugt (via 90 oder 91, Abb. 61). Frühere Arbeiten zeigen uneinheitliche Ergebnisse, was das Intermediat auf diesem Reaktionspfad betrifft. Die ersten experimentellen Berichte schlagen Isobenzol 90 vor, ${ }^{203}$ eine erste semiempirische Studie gibt Hinweise darauf, daß das Biradikal 91 kein Grundzustand, sondern ein Übergangszustand zwischen den beiden enantiomeren Isobenzol-Strukturen $(\boldsymbol{S})$-90 und $(\boldsymbol{R})$ $90\left(C_{l}\right.$ Punktgruppe $)$ ist. ${ }^{204}$ Anhand der gemessenen Standardbildungsenthalpien wurde später das Biradikal 91 als Intermediat favorisiert. ${ }^{26}$

Eine sehr detaillierte B3LYP/6-31G* Studie hat sich vor kurzem dieser Fragestellung angenommen. ${ }^{205}$ Diese Berechnungen, die mit eigenen BLYP/6-31G* Resultaten sehr gut übereinstimmen, zeigen, daß das Biradikal 91 wirklich ein Übergangszustand für die Enantiomerisierung von 90 ist. Während sich die B3LYP Studie auf die Isomerisierung von 90 zu 96 konzentriert, wird hier eher auf die Reaktionen und Reaktivitäten von 89 eingegangen. Folglich cyclisiert Dienin 89 zu Isobenzol 90, das via 91 racemisiert (Abb. 61).

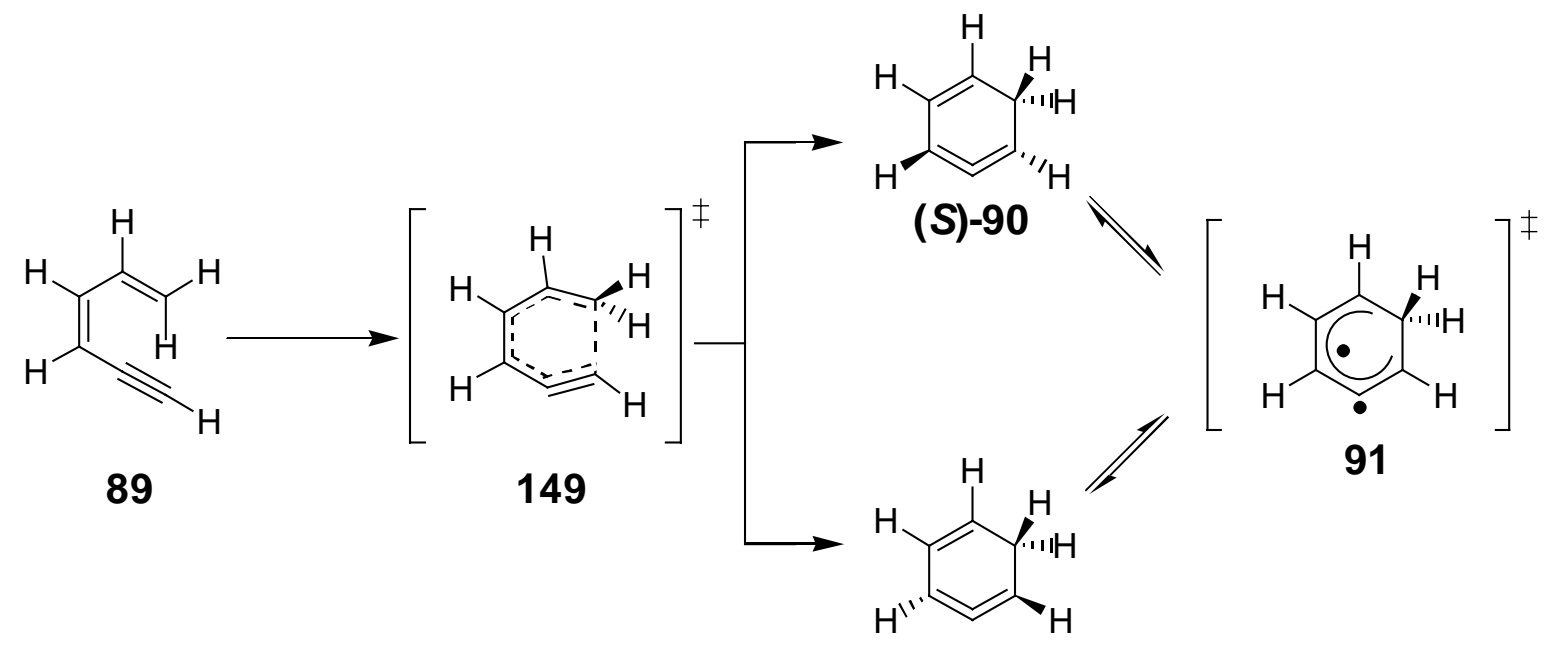

(R)-90

Abbildung 61. Cyclisierung von Dienin 89 zu den Isobenzolen $(\boldsymbol{S})-90$ und $(\boldsymbol{R})-90$, die durch den Biradikal-ÜZ 91 verbunden sind 
Die Barriere zwischen 89 und 90 ist $29.2 \mathrm{kcal} \mathrm{mol}^{-1}$ auf BLYP/6-31G* und $31.7 \mathrm{kcal}$ $\mathrm{mol}^{-1}$ auf BD(T)/cc-pVDZ Niveau, was mit dem experimentellen Wert von $30.3 \mathrm{kcal} \mathrm{mol}^{-1}$ sehr gut übereinstimmt (Tab. 17). Die Bildungsenthalpie des Zwischenproduktes wurde experimentell mit $20.4 \mathrm{kcal} \mathrm{mol}^{-1}$ bestimmt und korreliert damit am besten mit den Werten für das Biradikal 91 (BLYP: $18.5 \mathrm{kcal} \mathrm{mol}^{-1}$, BD(T): $17.1 \mathrm{kcal} \mathrm{mol}^{-1}$ ). Allerdings konnten erweiterte Berechnungen mit mehreren verschiedenen Methoden [BLYP, G96LYP, BPW91, HF, MP2, CASSCF $(8,8)$ mit einem 6-31G* Basissatz] zeigen, daß das planare 91 wirklich ein Übergangszustand ist (NImag $=1$ ), in dem der imaginäre Vektor und zusätzliche IRC (intrinsic reaction coordinates) Berechnungen darauf hindeuten, daß sich die allenischen Wasserstoffatome aus der Ebene heraus zu einem verdrehten Allen bewegen. Folgt man dem Pfad des Diederwinkels dieser Wasserstoffatome von $0^{\circ}(\mathbf{9 1})$ bis $98^{\circ}(\mathbf{9 0})$ indem man den Winkel konstant hält, den Rest des Moleküls mit BLYP/6-31G* optimiert und anschließend BD(T)/cc-pVDZ Einzelpunktenergien darauf berechnet, so zeigt sich die Natur des Übergangszustandes ohne jeden Zweifel (Abb. 62). Der Triplett-Zustand liegt $1.9 \mathrm{kcal} \mathrm{mol}^{-1}$ über dem offenschaligen Singulett und ist ein echtes Minimum. Zusätzlich zeigt eine IRC Berechnung von ÜZ 149, daß 89 und Allen 90 miteinander verbunden sind.

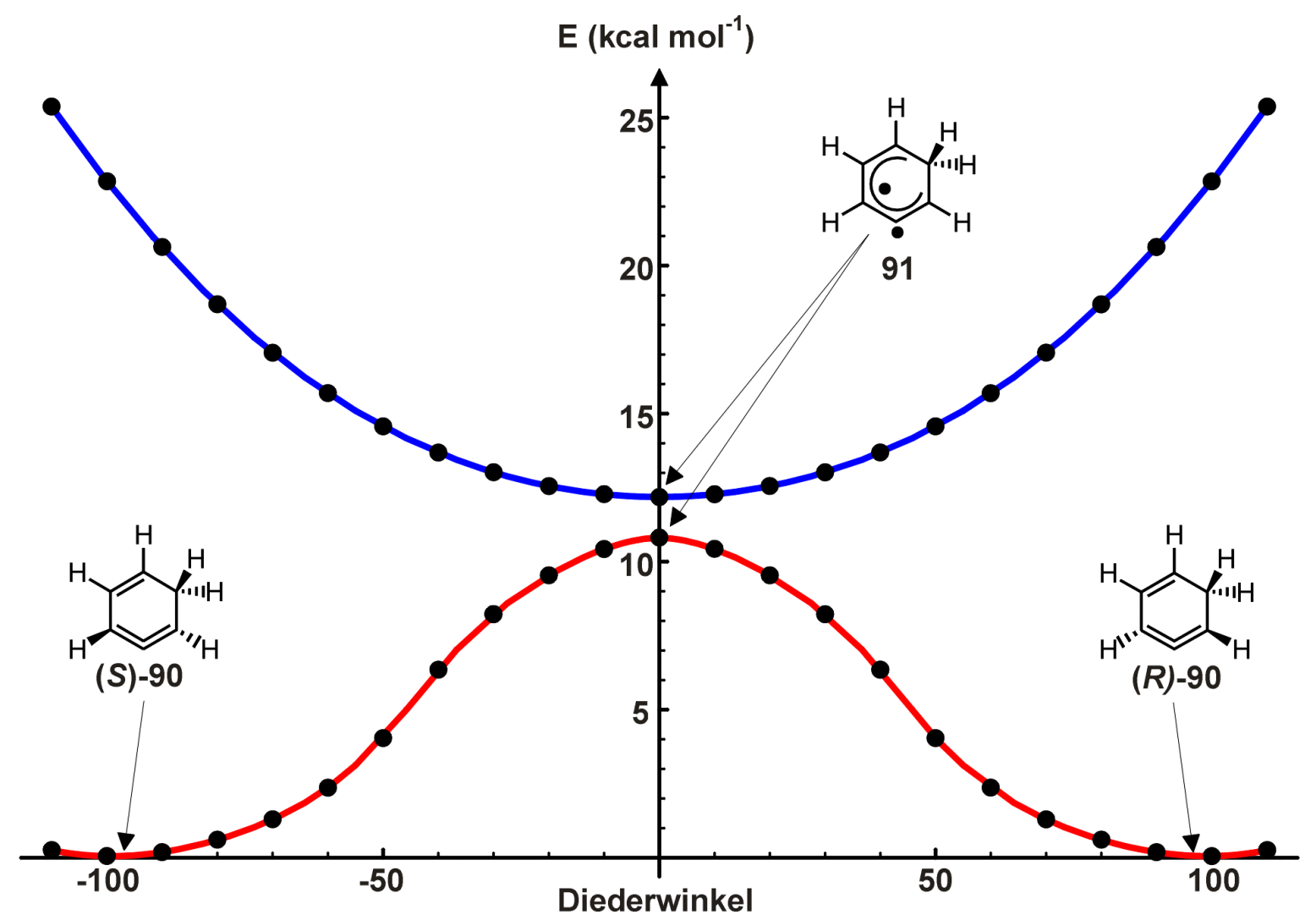

Abbildung 62. Singulett (rot) und Triplett (blau) Energiehyperflächen von 90 in Abhängigkeit vom Diederwinkel. Alle Punkte sind BD(T)/cc-pVDZ//BLYP/6-31G* Einzelpunktenergien 
Diese Situation erinnert an die Enantiomerisierung von 1,2,4,6-Cycloheptatetraen 150 via das Singulett Cycloheptatrienyliden 151 (Abb. 63). ${ }^{206-208}$ Das Carben 151 kann als Einzentren-Biradikal angesehen werden, das lange Zeit als Intermediat galt. Jetzt ist allerdings bekannt, daß nur der Triplett-Zustand ein Intermediat, alle Singuletts jedoch Übergangszustände sind.

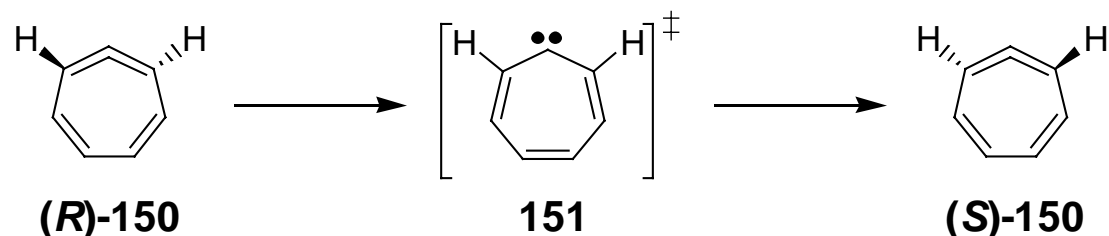

Abbildung 63. Racemisierung von 1,2,4,6-Cycloheptatetraen 150 über das planare Cycloheptatrienyliden 151

Im Gegensatz zu früheren Berechnungen liegt der Biradikal-ÜZ 91 höher in der Energie $\left(5.9 \mathrm{kcal} \mathrm{mol}^{-1}\right.$ auf BLYP und $7.9 \mathrm{kcal} \mathrm{mol}^{-1}$ auf BD(T) über 90 verglichen mit 2 kcal $\mathrm{mol}^{-1}$ ), die Racemisierung findet jedoch immer noch schnell statt. ${ }^{204,205}$ Die Differenz von ca. $10 \mathrm{kcal} \mathrm{mol}^{-1}$ zwischen Theorie und Experiment ist jedoch noch nicht geklärt, deshalb scheinen mehr Experimente zur Cyclisierung von 89 nötig. Für die Isomerisierung von 90 zu 96 sind mehrere Reaktionspfade denkbar (Abb. 64):

Direkte [1,5]-Wasserstoffverschiebung vom $\mathrm{sp}^{3}$-hybridisierten zum allenischen Kohlenstoff via Übergangszustand $154(\operatorname{Pfad} 1)$

[1,2]-Wasserstoffverschiebung vom $\mathrm{sp}^{3}$-hybridisierten Kohlenstoff via ÜZ 153 zu Biradikal-Intermediat 159 und anschließendem [1,4]-H Shift via 160 zu 96 (Pfad 2)

[1,2]-Wasserstoffverschiebung vom $\mathrm{sp}^{3}$-hybridisierten Kohlenstoff via ÜZ 152 zum allenischen Kohlenstoff unter Bildung des Intermediats 156/157 und anschließendem [1,2]-H Shift via 158 zu 96 (Pfad 3)

[1,2]-Wasserstoffverschiebung des allenischen Wasserstoffs via ÜZ 155 zum selben Intermediat 156/157 und anschließendem [1,2]-H Shift via 158 zu 96 (Pfad 4)

Wie bereits erwähnt, ist Pfad (3) der energetisch günstigste. Mit einer Barriere von $38.8 \mathrm{kcal} \mathrm{mol}^{-1}$ für BLYP und $38.4 \mathrm{kcal} \mathrm{mol}^{-1}$ für $\mathrm{BD}(\mathrm{T})$ ausgehend von $\mathbf{8 9}$ ergibt sich eine sehr gute Übereinstimmung mit dem experimentellen Wert von $40.5 \mathrm{kcal} \mathrm{mol}^{-1}$. Die Barriere des vielleicht offensichtlichsten Pfades (1) ist noch $\sim 16 \mathrm{kcal} \mathrm{mol}^{-1}$, die von Pfad (4) mehr als $34 \mathrm{kcal} \mathrm{mol}^{-1}$ höher als die Barriere für Pfad (3). Die erste Barriere in Pfad (2) ist nur $\sim 5 \mathrm{kcal}$ 
mol $^{-1}$ höher als in (3), eine zweite Barriere von jedoch mehr als $26 \mathrm{kcal} \mathrm{mol}^{-1}$ höher macht diese Reaktion ebenfalls energetisch ungünstig.

Die Struktur des Intermediats $\mathbf{1 5 6 / 1 5 7}$ ist schwer zu charakterisieren. Die BLYP Energien scheinen auf das Biradikal 156 hinzudeuten, während die BD(T)-Daten auf die carbenoide Struktur 157 als Grundzustand hinweisen. Weil sich die Energiedifferenzen innerhalb der Fehlergrenzen der Berechnungen befinden ist eine klare Aussage nicht möglich. Da die Singulett-Triplett-Lücke aber nur $1.9 \mathrm{kcal} \mathrm{mol}^{-1}$ groß ist, scheint eine biradikalische Beschreibung des Zwischenproduktes angezeigt. Die Barriere des zweiten [1,2]-H Shiftes ist sehr klein (BLYP: $4.4 \mathrm{kcal} \mathrm{mol}^{-1}$ über 156; $\mathrm{BD}(\mathrm{T})$ : $0.4 \mathrm{kcal} \mathrm{mol}^{-1}$ über 157) und war deshalb experimentell nicht zu bestimmen. Die Gesamt-Reaktionsenthalpie der Cycloisomerisierung von 89 zu 96 (Expt.: -64.9 kcal $\mathrm{mol}^{-1}$ ) stimmt wiederum gut mit den berechneten Werten überein (BLYP: $-65.1 \mathrm{kcal} \mathrm{mol}^{-1}$ und $\mathrm{BD}(\mathrm{T})$ : $-67.9 \mathrm{kcal} \mathrm{mol}^{-1}$ ).

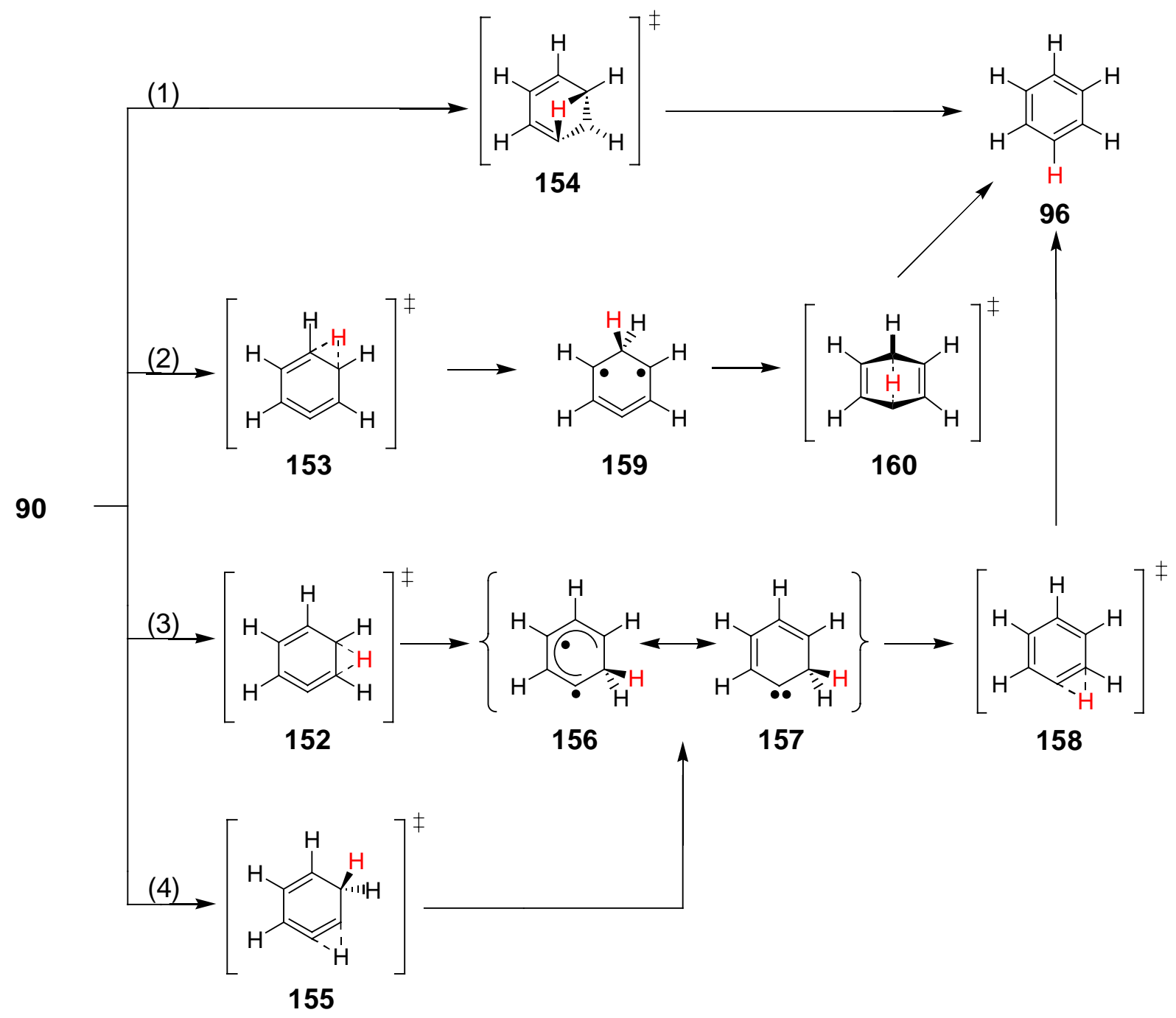

Abbildung 64. Isomerisierungs-Pfade von Isobenzol 90 zu Benzol 96 
Tabelle 17. Ergebnisse der berechneten Energien für die Cycloisomerisierung von 89 verglichen mit experimentellen Daten (alle Enthalpien in $\mathrm{kcal} \mathrm{mol}^{-1}$ ).

\begin{tabular}{cccc}
\hline Struktur & $\begin{array}{c}\Delta_{\mathrm{R}} \mathrm{H}_{298} / \Delta \mathrm{H}_{623}^{\ddagger} \\
\mathrm{BLYP} / 6-31 \mathrm{G}^{*}\end{array}$ & $\begin{array}{c}\Delta_{\mathrm{R}} \mathrm{H}_{298} / \Delta \mathrm{H}_{623}^{\ddagger} \\
\mathrm{BD}(\mathrm{T}) / \mathrm{cc}-\mathrm{pVDZ}\end{array}$ & Experiment ${ }^{26}$ \\
\hline $\mathbf{8 9}$ & 0.0 & 0.0 & 0.0 \\
$\mathbf{1 4 9}$ & 29.2 & 31.7 & 30.3 \\
$\mathbf{9 0}$ & 12.6 & 7.9 & 20.4 \\
$\mathbf{9 1}$ & 18.5 & 17.1 & \\
$\mathbf{1 5 2}$ & 38.8 & 38.4 & \\
$\mathbf{1 5 3}$ & 44.1 & 45.8 & 40.5 \\
$\mathbf{1 5 4}$ & 58.5 & 54.6 & \\
$\mathbf{1 5 5}$ & 74.4 & 75.0 & - \\
$\mathbf{1 5 6}$ & 19.8 & 22.0 & - \\
$\mathbf{1 5 7}$ & 20.6 & 21.3 & - \\
$\mathbf{1 5 8}$ & 24.2 & 21.7 & - \\
$\mathbf{1 5 9}$ & 21.5 & 22.6 & -64.9 \\
$\mathbf{1 6 0}$ & 65.3 & 58.8 & -67.9 \\
$\mathbf{9 6}$ & -65.1 & & \\
\hline
\end{tabular}

Benzannelierung. Für das benzannelierte System sind experimentelle Daten noch nicht verfügbar und deshalb sind genaue Voraussagen über die Cyclisierung von 161 äußerst nützlich. Die Benzannelierung beeinflußt die Cyclisierungsbarriere kaum (BLYP: $32.1 \mathrm{kcal}$ $\mathrm{mol}^{-1}, \mathrm{BD}(\mathrm{T}): 36.4 \mathrm{kcal} \mathrm{mol}^{-1}$ ) und liegt deshalb nur wenig höher als beim Stammsystem (Tab. 18). Der Effekt auf das Cyclisierungsprodukt 162 oder 163 ist hingegen gravierend. Das Biradikal 163 ist, im Gegensatz zum Stammsystem, kein ÜZ sondern ein stabiler Grundzustand und liegt in der Energie noch unterhalb der des Allens 162 (BLYP: 2.5 kcal $\mathrm{mol}^{-1}, \mathrm{BD}(\mathrm{T}): 2.1 \mathrm{kcal} \mathrm{mol}^{-1}$ ). Eine Erklärung dafür kann die Vergrößerung des System sein, über das sich das benzylische $\pi$-Elektron in 163 delokalisieren kann, während 162 seine Aromatizität beim Ausbilden eines ortho-chinoiden Systems einbüßt (Abb. 65). Verglichen mit dem Stammsystem haben beide Strukturen 162 und 163 eine höhere Energie. 
<smiles>C#Cc1ccccc1C=C</smiles>

161<smiles>C1=CCC=c2ccccc2=1</smiles>

162

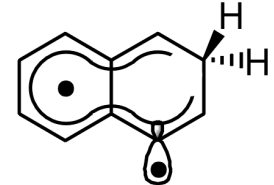

163

Abbildung 65. Das benzannelierte Dienin 161 und dessen Cyclisierungsprodukte 162 und 163

Die weitere Isomerisierung zu Naphthalin 164 findet ebenfalls über zwei aufeinanderfolgende [1,2]-Wasserstoffverschiebungen statt. Die Barrieren der anderen Reaktionspfade sind zu hoch, als daß sie eine Rolle im H-Transfer spielen könnten. Die höhere Barriere des zweistufigen Shifts (BLYP: $44.1 \mathrm{kcal} \mathrm{mol}^{-1}$, BD(T): $46.0 \mathrm{kcal} \mathrm{mol}^{-1}$ ) liegt $6 \mathrm{kcal} \mathrm{mol}^{-1}$ über der entsprechende des Stammsystems, was eine erhöhte Reaktionstemperatur bedeutet. Das Zwischenprodukt 165/166 stellt wiederum ein Problem dar (ein Biradikal 165 auf BLYP, auf BD(T) aber ein Carben 166). Die Barriere zwischen 165/166 und 164 ist sehr niedrig und kann experimentell nicht bestimmt werden. Die GesamtReaktionsenthalpie ist ein wenig kleiner als die der Stammreaktion $\left(\sim 60 \mathrm{kcal} \mathrm{mol}^{-1} v s . \sim 67\right.$ kcal $\mathrm{mol}^{-1}$ ), da im Edukt 161 schon ein Aromat vorhanden ist. Während 89 die volle aromatische Stabilisierungsenergie beim Ausbilden von 96 bekommt, ist bei 161 nur ein Teil der Energie zugänglich wenn aus dem schon aromatischen 161 Naphthalin 164 entsteht.

Tabelle 18. Ergebnisse der berechneten Energien für die Cycloisomerisierung von 161 (alle Enthalpien in $\mathrm{kcal} \mathrm{mol}^{-1}$ )

\begin{tabular}{ccc}
\hline \multirow{2}{*}{ Struktur } & $\begin{array}{c}\Delta_{\mathrm{R}} \mathrm{H}_{298} / \Delta \mathrm{H}_{298}^{\ddagger} \\
\text { BLYP/6-31G* }\end{array}$ & $\begin{array}{c}\Delta_{\mathrm{R}} \mathrm{H}_{298} / \Delta \mathrm{H}_{298}^{\ddagger} \\
\mathrm{BD}(\mathrm{T}) / \mathrm{cc}-\mathrm{pVDZ}\end{array}$ \\
\hline $\mathbf{1 6 1}$ & 0.0 & 0.0 \\
$\mathbf{1 6 1 \rightarrow \mathbf { 1 6 2 }}$ & 32.1 & 36.4 \\
$\mathbf{1 6 2}$ & 25.3 & 25.5 \\
$\mathbf{1 6 3}$ & 22.8 & 23.4 \\
$\mathbf{1 6 2} \rightarrow \mathbf{1 6 5}$ & 44.1 & 46.0 \\
$\mathbf{1 6 5}$ & 20.8 & 21.2 \\
$\mathbf{1 6 6}$ & 21.8 & 20.7 \\
$\mathbf{1 6 5} \rightarrow \mathbf{1 6 4}$ & 24.8 & 22.0 \\
$\mathbf{1 6 4}$ & -59.3 & -60.1 \\
\hline
\end{tabular}


Ringspannung. In Analogie zu den Endiinen 99a-h sollten die cyclischen Dienine 146a-h in ihrer Reaktivität stark von der Ringspannung beeinflußt werden. Wie experimentell bekannt, cyclisieren die neun- und zehngliedrigen Endiin-Ringe 99c und 99d bereits bei Raumtemperatur, während das Stammsystem hohe Temperaturen benötigt $\left(\mathrm{t}_{1 / 2} \approx 1 \mathrm{~h}\right.$ bei 155 ${ }^{\circ}$ C). ${ }^{32,33,119}$ Der Grund für die erhöhte Reaktivität ist der Abbau von Ringspannung in den Edukten hin $\mathrm{zu}$ den weniger gespannten Produkten. Entsprechend sollten zumindest die kleineren (und damit gespannteren) Dienine 146 bei niedrigeren Temperaturen cyclisieren als das Stammsystem 89.

Die folgende Untersuchung behandelt ausschließlich die (E,Z)-carbocyclischen Dienine 146a-h und deren Cyclisierungspfade, die zu den Benzocycloalkenen 147a-h führen, da diese Spezies in einer anderen Arbeitsgruppe synthetisiert und untersucht worden ist. $\mathrm{Zu}$ Vergleichszwecken wurden nur die Strukturen $(\boldsymbol{Z}, \boldsymbol{Z}) \mathbf{- 1 4 6 a - h}$ berechnet. Während 146a und 146b viel stabiler in der $(Z, Z)$ - als in der (E,Z)-Konformation sind (38.2 und $\left.16.1 \mathrm{kcal} \mathrm{mol}^{-1}\right)$, sind 146c und 146d nur noch wenig stabiler in der (Z,Z)-Form (7.7 und $\left.2.9 \mathrm{kcal} \mathrm{mol}^{-1}\right)$. Für 146e haben beide Isomere nahezu die gleiche Energie, während für die größeren Ringe 146f-h die $(E, Z)$-Konformation die stabilere ist.

Die (E,Z)-Isomere 146a-h können in der cis- und in der trans-Form auftreten (Abb. 66), wobei die kleinen Ringe erstere, die großen hingegen letztere Form bevorzugen. Die Cyclisierung zu 168a-h kann allerdings nur vom cis-Konformer aus stattfinden. Wie Rechnungen an dem 14-gliedrigen Ring 146h zeigen, ist die trans-Form nur $1.4 \mathrm{kcal} \mathrm{mol}^{-1}$ stabiler als die cis-Form, und die Barriere für die Umwandlung der Konformere ineinander beträgt nur $5.6 \mathrm{kcal} \mathrm{mol}^{-1}$. Da $\mathbf{1 4 6 h}$ als größter Ring natürlich die stabilste trans-Form besitzt, bevorzugen die kleineren Ringe die cis-Konformation oder haben eine niedrigere Barriere als 146h. Das bedeutet, daß die cis/trans-Isomerisierung vor der eigentlichen Cyclisierung kinetisch vernachlässigt werden kann.

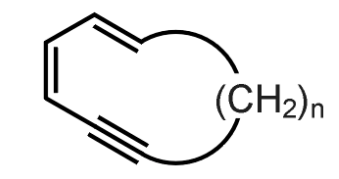

$\operatorname{cis}-(E, Z)-146 \mathrm{a}-\mathrm{h}$

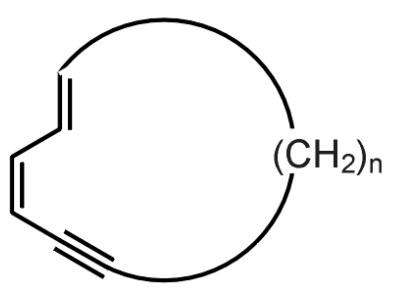

trans-(E,Z)-146a-h

Abbildung 66. Dienine 146a-h in cis- und trans-Konformation 
Generell ergeben die (E,Z)-Isomere 146a-h zunächst die trans-Allene trans-168a-h

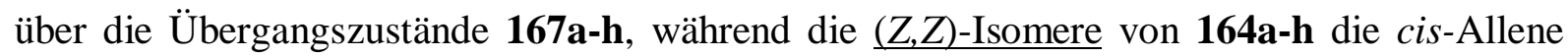
cis-168a-h ergeben. Die zwei diastereomeren Formen der Allene 168a-h sind über die Biradikal-ÜZ 169a-h miteinander verbunden (Abb. 67). Die trans-Allene 168a-h gehen dann durch [1,2]-H Shift in die Intermediate 171a-h über, die durch eine zweite [1,2]-H Verschiebung zu den entsprechenden Benzocycloalkenen 147a-h reagieren. Da die zweiten [1,2]-H Shifte eine wesentlich niedrigere Barriere haben als die ersten, sind sie kinetisch nicht relevant und werden deshalb nur in Tabelle 19 erwähnt.

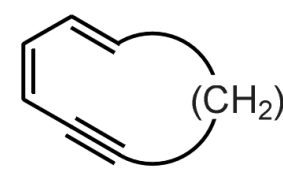

146a-h
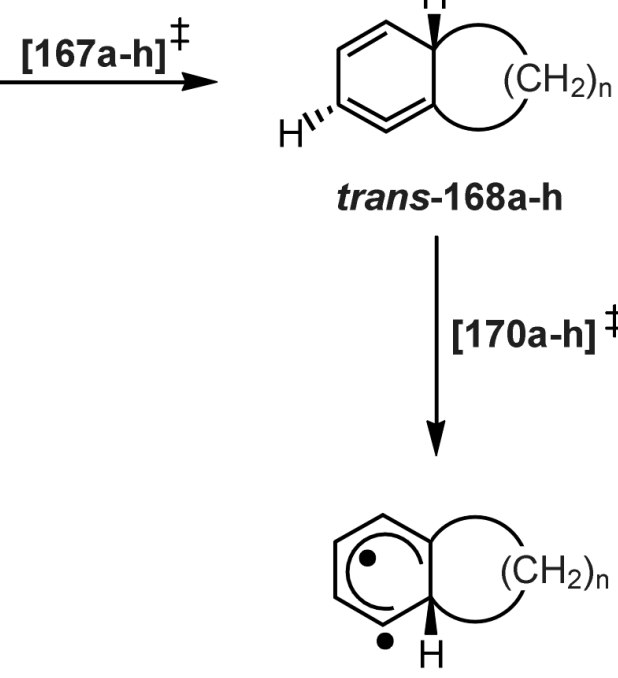

171a-h

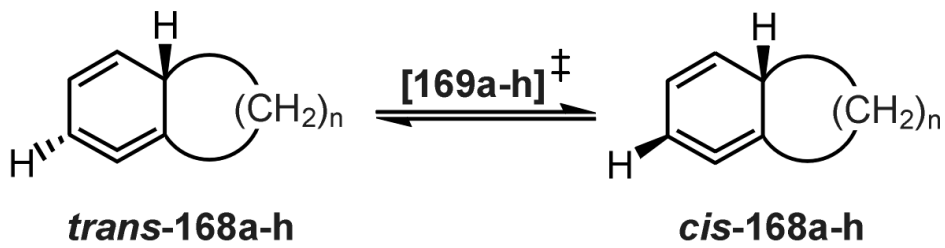

[170a-h] $\ddagger$

Abbildung 67. Die Cyclisierung der cyclischen Dienine 146a-h

Die Reaktionen des sieben- und achtgliedrigen Dienins 146a und 146b sind Spezialfälle und müssen separat von den anderen Ringen behandelt werden. Ein trans-Allen kann aus 146a wegen der großen Ringspannung eines hypothetischen trans-konfigurierten Cyclopropanringes nicht ausgebildet werden. Aus demselben Grund kann es auch den ÜZ 169a nicht geben. Da die Reaktion zu 171a nur ausgehend von trans-168a möglich ist, kann diese Reaktion nicht stattfinden und soll deshalb nicht weiter betrachtet werden. Das achtgliedrige 146b ist in der Lage das trans-Allen 168b auszubilden, ist jedoch ebenfalls hochgespannt, was die relativ hohe Barriere von $28.0 \mathrm{kcal} \mathrm{mol}^{-1}$ und eine Reaktionsenthalpie von $27.8 \mathrm{kcal} \mathrm{mol}^{-1}$ verständlich macht. Die Barriere des ersten [1,2]-H Shift ist mit $37.0 \mathrm{kcal}$ $\mathrm{mol}^{-1}$ etwas geringer als bei der Stammreaktion, was $\mathbf{1 4 6 \mathrm { b }}$ etwas reaktiver als 89 macht.

Der neungliedrige Ring 146c ist mit einer Cyclisierungsbarriere von $20.2 \mathrm{kcal} \mathrm{mol}^{-1}$ und der $\mathrm{H}$-Verschiebungsbarriere von $26.7 \mathrm{kcal} \mathrm{mol}^{-1}$ wesentlich reaktiver als 89. Nur die 
Energien des Allens trans-168c (14.4 $\left.\mathrm{kcal} \mathrm{mol}^{-1}\right)$ und 90 sind vergleichbar aufgrund der zusätzlichen Ringspannung des trans-konfigurierten fünfgliedrigen Cycloalkenringes in 168c (Abb. 68). Die Cyclisierungsbarriere des zehngliedrigen 146d (22.9 $\mathrm{kcal} \mathrm{mol}^{-1}$ ) ist wenig höher als die von 146c, während die H-Shift-Barriere mit $34.3 \mathrm{kcal} \mathrm{mol}^{-1}$ schon einen großen Unterschied zu der von 146c aufweist, jedoch immer noch weit unterhalb der von 89 liegt. Das Allen trans-168d hat kaum zusätzliche Ringspannung durch die trans-Konfiguration des Alkylrings und liegt deshalb unterhalb von trans-168c. Mit $12.1 \mathrm{kcal} \mathrm{mol}^{-1}$ ist trans-168d das energetisch günstigste aller Allene 168. Der elfgliedrige Ring 146e hat nahezu dieselben Energien wie das Stammsystem. Mit den Barrieren von $28.1 \mathrm{kcal} \mathrm{mol}^{-1}$ und $39.5 \mathrm{kcal} \mathrm{mol}^{-1}$ für Cyclisierung und $\mathrm{H}$-Verschiebung ist 146e nur wenig reaktiver als 89. Alle anderen cyclischen Dienine 146f-h haben höhere Barrieren als das Stammsystem und sind deshalb weniger reaktiv.

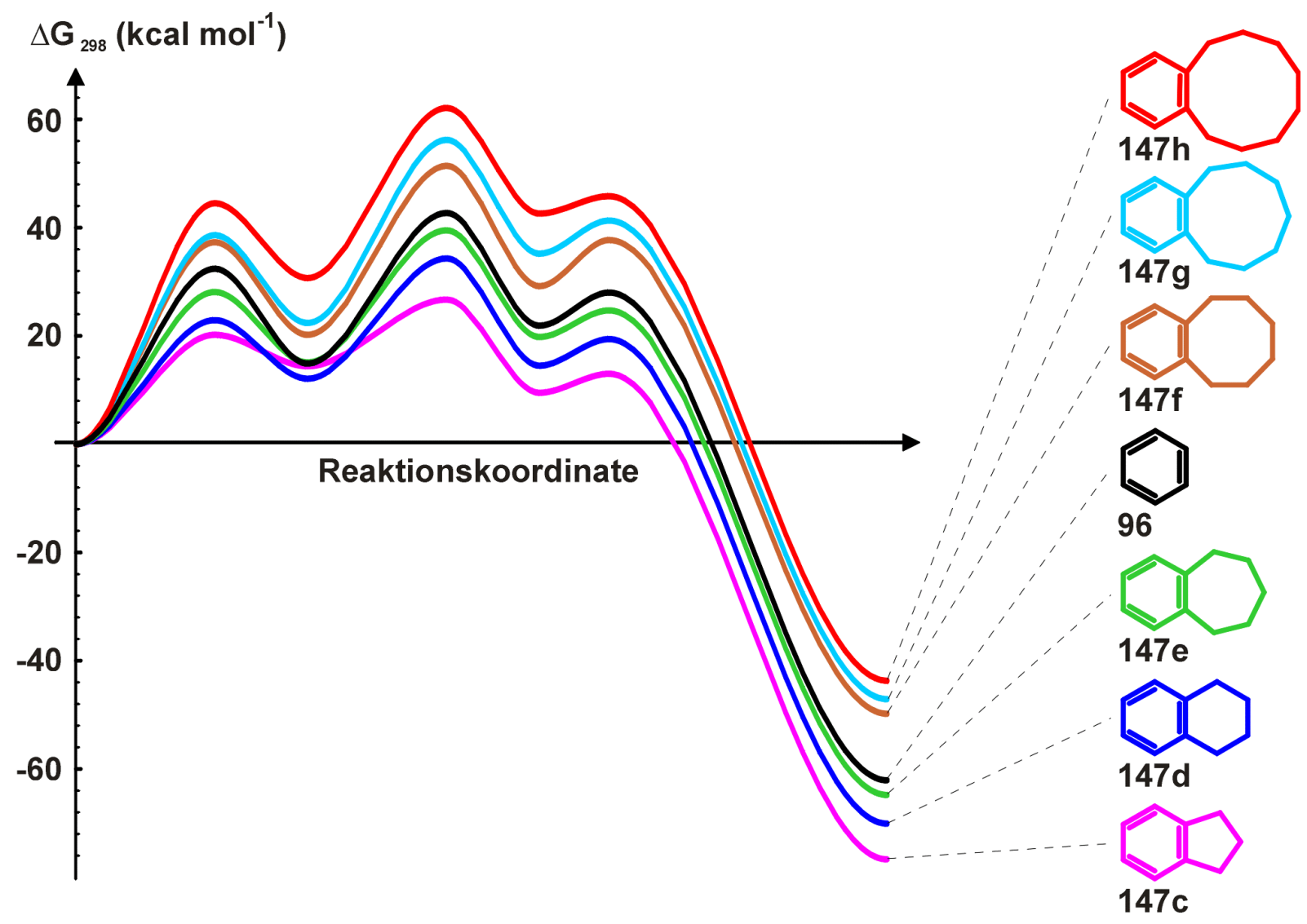

Abbildung 68. Energiehyperflächen der Cycloisomerisierungen von 146c-h to 147c-h 
Tabelle 19. Ergebnisse der berechneten Energien $\left(\Delta \mathrm{G}_{298}\right)$ für die Cycloisomerisierung von 146b-h (in kcal $\mathrm{mol}^{-1}$ )

\begin{tabular}{ccccccccc}
\hline Ringgröße & 8 & 9 & 10 & 11 & 12 & 13 & 14 & $\begin{array}{c}\text { Stamm- } \\
\text { system }\end{array}$ \\
\hline Struktur & $\mathbf{b}$ & $\mathbf{c}$ & $\mathbf{d}$ & $\mathbf{e}$ & $\mathbf{f}$ & $\mathbf{g}$ & $\mathbf{h}$ & 0.0 \\
\hline $\mathbf{1 4 6}$ & 0.0 & 0.0 & 0.0 & 0.0 & 0.0 & 0.0 & 0.0 & 0.0 \\
$\mathbf{1 6 7}$ & 28.0 & 20.2 & 22.9 & 28.1 & 37.3 & 38.6 & 44.5 & 32.4 \\
trans-168 & 27.8 & 14.4 & 12.1 & 15.1 & 20.2 & 22.4 & 30.7 & 14.9 \\
$\mathbf{1 6 9}$ & - & - & 13.8 & 24.3 & 33.5 & 37.5 & 42.4 & 21.1 \\
$\mathbf{1 7 0}$ & 37.0 & 26.7 & 34.3 & 39.5 & 51.4 & 56.2 & 62.1 & 42.7 \\
$\mathbf{1 7 1}$ & 15.9 & 9.5 & 14.5 & 21.4 & 29.2 & 34.8 & 42.6 & 21.9 \\
$\mathbf{1 7 2}$ & 23.1 & 13.0 & 19.4 & 24.7 & 37.7 & 41.3 & 45.8 & 28.0 \\
$\mathbf{1 4 7}$ & -65.8 & -76.7 & -70.1 & -64.8 & -49.8 & -47.1 & -43.7 & -62.1 \\
\hline
\end{tabular}

Experimentelle Ergebnisse. Die 1,3-Cyclodien-5-ine 146d-h konnten in Zusammenarbeit mit der Arbeitsgruppe von Herrn Prof. Hopf in einer fünfstufigen Synthese als farblose, flüchtige Flüssigkeiten in Gesamtausbeuten zwischen 2 und $22 \%$ erhalten werden. ${ }^{209}$

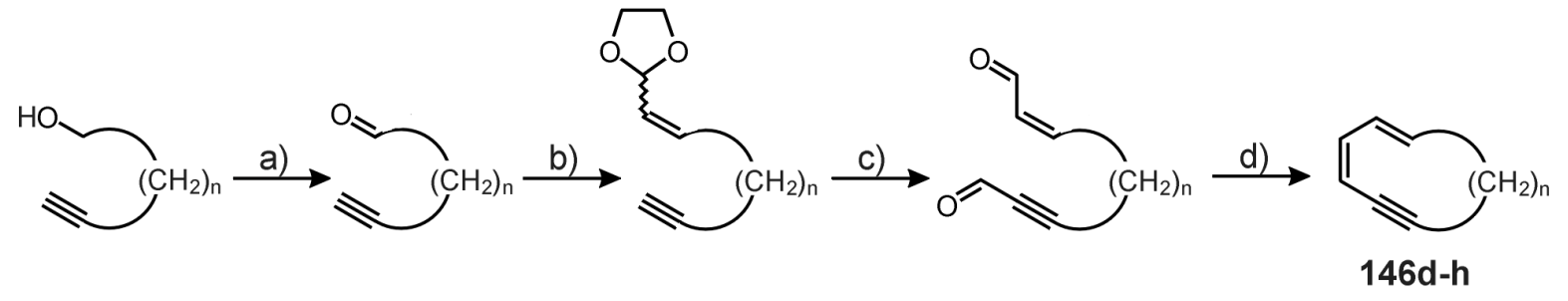

Abbildung 69. Synthese der 1,3-Cyclodien-5-ine 146d-h: a) $(\mathrm{COCl})_{2}, \mathrm{DMSO}, \mathrm{CH}_{2} \mathrm{Cl}_{2}, \mathrm{NEt}_{3},-60^{\circ} \mathrm{C}$; b) [1,3]Dioxolan-2-ylmethyl-triphenyl-phosphoniumbromid, KO- $t \mathrm{Bu}, \mathrm{THF}, \mathrm{RT}$; c) $n$-BuLi, Diethylether, $-50^{\circ} \mathrm{C}$, $\left.\mathrm{DMF},-70^{\circ} \mathrm{C}, \mathrm{d}\right) \mathrm{TiCl}_{3}(\mathrm{DME})_{1.5}, \mathrm{Zn} / \mathrm{Cu}$, DME, Rückfluß

Erste Experimente zur Thermocyclisierung von 146d-h wurden in $o-\mathrm{C}_{6} \mathrm{D}_{4} \mathrm{Cl}_{2}$-Lösung durchgeführt und NMR-spektroskopisch überwacht. Die Messungen zeigen deutlich eine Korrelation zwischen Ringgröße (und deshalb auch Ringspannung) und der Cyclisierungstemperatur. 1,3-Cyclodecadien-5-in 146d cyclisiert bereits bei Raumtemperatur, während die höheren Homologe wie erwartet höhere Temperaturen benötigen. Bei 1,3Cyclotetradecadien-5-in $\mathbf{1 4 6 h}$ konnte bis $210^{\circ} \mathrm{C}$ (Sdp. von o-Dichlorbenzol) keine Cyclisierung beobachtet werden (Tab. 20). 
Erste kinetische Messungen für 146d-f ergaben die Halbwertszeiten und damit die Reaktionsbarrieren für die Cyclisierung (Tab. 20). Wie erwartet handelt es sich dabei um Reaktionen erster Ordnung. Der Vergleich der experimentellen mit den berechneten Daten zeigt, daß die Cyclisierungs-Barrieren mit dem Experiment sehr gut übereinstimmen. Die anschließenden [1,2]-H Shifte, die alle höhere Barrieren haben als die Cyclisierung selbst, sind jedoch in der Berechnung viel zu hoch, um die Bildung von Benzocycloalkanen unterhalb von $200^{\circ} \mathrm{C}$ zuzulassen. Dieses Problem ist noch nicht vollständig gelöst. Eine Möglichkeit könnte sein, daß in den cyclischen Systemen 146a-h aufgrund der aliphatischen Ketten andere Wasserstoff-Verschiebungen auftreten können als im Stammsystem. Um diese Fragen beantworten zu können, sind weitere experimentelle und theoretische Arbeiten notwendig.

Tabelle 20. Cyclisierungstemperaturen und Resultate der kinetischen Experimente für 146d-h

\begin{tabular}{lcccc}
\hline Substanz & $\mathrm{T}_{\min }\left[{ }^{\circ} \mathrm{C}\right]$ & $\begin{array}{c}\text { Temperatur } \\
\mathrm{T}_{\text {therm. }}\left[{ }^{\circ} \mathrm{C}(\mathrm{K})\right]\end{array}$ & $10^{6} \mathrm{k}\left[\mathrm{s}^{-1}\right]$ & $\boldsymbol{\tau}_{1 / 2}[\mathrm{~h}]$ \\
\hline $\mathbf{1 4 6 d}\left(\mathrm{CDCl}_{3}\right)$ & r.t. & $40(313.15)$ & $7.1 \pm 0.3$ & $27.0 \pm 1.0$ \\
$\mathbf{1 4 6 e}\left(\mathrm{o}-\mathrm{C}_{6} \mathrm{D}_{4} \mathrm{Cl}_{2}\right)$ & 100 & $165(438.15)$ & $70 \pm 5$ & $2.8 \pm 0.2$ \\
$\mathbf{1 4 6 f}\left(\mathrm{o}-\mathrm{C}_{6} \mathrm{D}_{4} \mathrm{Cl}_{2}\right)$ & 150 & $165(438.15)$ & $3.5 \pm 0.1$ & $55.0 \pm 1.5$ \\
$\mathbf{1 4 6 g}$ & Spuren bei $210^{\circ} \mathrm{C}$ & - & - & - \\
$\mathbf{1 4 6 h}$ & keine Reaktion bis $210^{\circ} \mathrm{C}$ & - & - & - \\
\hline
\end{tabular}


2.4.4 Die Cyclisierung von Hexa-1,3,5-trien ("[ 6$]$-disrotatorische Cyclisierung")

Die Cyclisierung von Hexa-1,3,5-trien 7 wurde bei der Synthese von 7 aus $\mathbf{1 7 3}$ bei hohen Temperaturen entdeckt (Abb. 70). ${ }^{9,10}$ Als Nebenprodukt wurde dabei eine nicht unerhebliche Menge an $\mathbf{8}$ erhalten. Bei der thermischen Cyclisierung handelt es sich um einen disrotatorischen Vorgang, der für $(E, Z, E)$-Hexatriene 7 die entsprechenden cisCyclohexadiene 8 liefert.

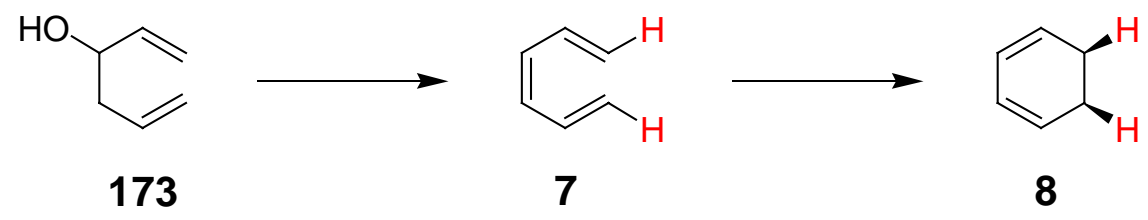

Abbildung 70. Darstellung und Cyclisierung von Hexa-1,3,5-trien 7

Stammsystem. Mit einer Barriere von $28.4 \mathrm{kcal} \mathrm{mol}^{-1}$ auf BLYP- und $31.4 \mathrm{kcal} \mathrm{mol}^{-1}$ auf BD(T)-Niveau wird das Experiment mit $29.0 \mathrm{kcal} \mathrm{mol}^{-1}$ gut reproduziert (Tab. 21).11 Damit benötigt 7 noch höhere Temperaturen zum Cyclisieren als die Bergman-Reaktion. Die Reaktionsenthalpie ist mit -8.3 bzw. $-15.5 \mathrm{kcal} \mathrm{mol}^{-1}$ (Experiment: $\left.-15.2 \mathrm{kcal} \mathrm{mol}^{-1}\right)^{11}$ jedoch günstiger als die der drei zuvor beschriebenen, da mit $\mathbf{8}$ weder ein offenschaliges, noch ein gespanntes cyclisches System entsteht.

Benzannelierung. Durch den Einfluß der Benzannelierung wird die Cyclisierungsbarriere von $174 \mathrm{um} \sim 8 \mathrm{kcal} \mathrm{mol}^{-1}$ angehoben und damit auch die Cyclisierungstemperatur im Vergleich zum Stammsystem gesteigert (Tab. 21). Der Effekt auf die Reaktionsenthalpie ist jedoch gravierender. Durch die Aufhebung der Aromatizität des Benzolringes durch das Ausbilden eines ortho-chinoiden Systems in 175 wird die Reaktion endotherm (BLYP: 3.8 kcal mol${ }^{-1}$; BD(T): $0.2 \mathrm{kcal} \mathrm{mol}^{-1}$ ) und zumindest nicht mehr vollständig ablaufen, da die Barrieren für hin und Rückreaktion praktisch gleich sind (Abb. 71).<smiles>C=Cc1ccccc1C=C</smiles>

174

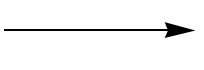<smiles>C1=c2ccccc2=CCC1</smiles>

175

Abbildung 71. Cyclisierung des benzannelierten 174 
Tabelle 21. Barrieren und Reaktionsenthalpien $\left(\Delta_{0} \mathrm{H}\right)$ der Cyclisierung der Triene $\mathbf{7}$ und $\mathbf{1 7 4}$ (in $\mathrm{kcal} \mathrm{mol}^{-1}$ )

\begin{tabular}{ccc}
\hline & BLYP/6-31G* & BD(T)/cc-pVDZ \\
\hline $\mathbf{7}$ & 0.0 & 0.0 \\
$\mathbf{7 \rightarrow \mathbf { 8 }}$ & 28.4 & 31.4 \\
$\mathbf{8}$ & -8.3 & -15.5 \\
\hline $\mathbf{1 7 4}$ & 0.0 & 0.0 \\
$\mathbf{1 7 4 \rightarrow \mathbf { 1 7 5 }}$ & 33.5 & 39.1 \\
$\mathbf{1 7 5}$ & 3.8 & 0.2 \\
\hline
\end{tabular}


2.4.5 Die Cyclisierung von Octa-1,2,4,6,7-pentaen

Dieses System ist bisher wenig untersucht und es existieren noch keine thermodynamischen Daten für die Cyclisierungen. Um so wichtiger ist es durch Berechnungen eine Vorhersage darüber zu machen, wie das Energieprofil für die Reaktion aussieht. Erste Hinweise auf den Cyclisierungsverlauf stammen von Bravermann, der die Cyclisierung des benzannelierten Endiallens 176 untersuchte und eine Cyclisierung mit anschließender H- bzw. D-Verschiebung unter Aromatisierung zu 177 beobachtete (Abb. 72). ${ }^{210}$ Neuere Erkenntnisse Stammen von Toda, der das Endiin 178 mit $\mathrm{SOCl}_{2}$ behandelte und in einem Schritt das Cyclisierungsprodukt 180 erhielt, das, wie er zeigen konnte, über die Zwischenstufe des Endiallens 179 reagierte (Abb. 72). ${ }^{211}$ Durch die fehlenden Wasserstoff-Atome in $\alpha$-Position zu den Allen-Bindungen schlägt die Reaktion hier einen anderen Aromatisierungspfad unter Ausbildung eines Cyclobutenrings ein. Auf diesem Wege konnte Toda eine der längsten $\mathrm{C}-\mathrm{C}-$ Einfachbindungen mit $1.72 \AA$ darstellen.<smiles></smiles>

176

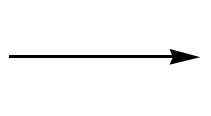

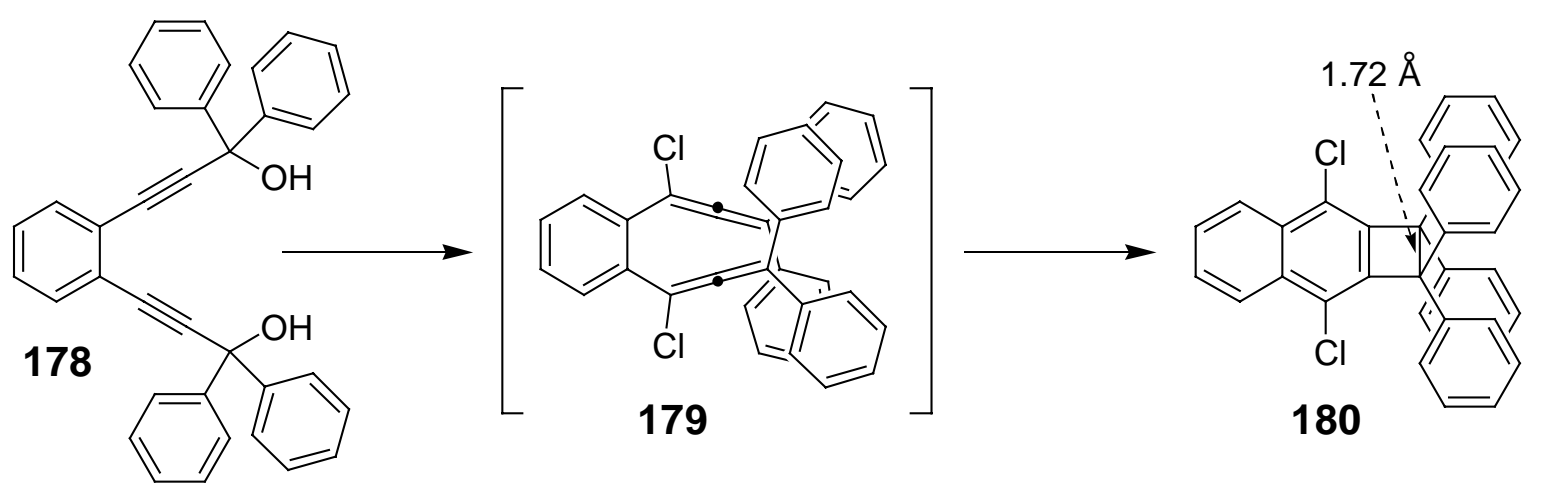

Abbildung 72. Cyclisierungsreaktionen benzannelierter Endiallen-Derivate

Stammsystem. Die Beispiele deuten an, daß beide Reaktionen zunächst zu einer ortho-chinoide Zwischenstufe 95 cyclisieren, von wo aus dann zwei unterschiedliche Aromatisierungsschritte folgen (Abb. 73). Im Stammsystem 94, das experimentell bisher nur als trans-Verbindung bekannt ist, kann nur die Stufe zum Benzocyclobuten 147b folgen. Obwohl der Cyclisierungsschritt von 94 das ortho-Chinon 95 ergibt wird im Gegensatz zu 
anderen ortho-chinoiden Stufen, die bisher besprochen wurden, keine Aromatizität zerstört oder herabgesetzt. Deshalb hat die Cyclisierung auch eine kleine Barriere $\left(17.3 \mathrm{kcal} \mathrm{mol}^{-1}\right.$ für beide Methoden) und eine hohe Exothermie (BLYP: $-39.7 \mathrm{kcal} \mathrm{mol}^{-1}$; BD(T): $-50.5 \mathrm{kcal}$ $\mathrm{mol}^{-1}$; Tab. 22). Der anschließende Aromatisierungsschritt unter Ausbildung des Cyclobutenringes 147b hat eine etwas höhere Barriere $\left(\sim 25 \mathrm{kcal} \mathrm{mol}^{-1}\right)$ ist aber durch die hohe Exothermie des ersten Schrittes leicht zu überwinden. Das aromatische Produkt 174b hat trotz der Ringspannung des viergliedrigen Ringes eine niedrigere Energie als das Zwischenprodukt 95 (BLYP: -50.0 kcal mol ${ }^{-1}$; BD(T): $-62.3 \mathrm{kcal} \mathrm{mol}^{-1}$ ).

Das tetramethylsubstituierte Cyclisieriungszwischenprodukt $\mathbf{1 8 1}$ aromatisiert jedoch wie im Experiment beobachtet ${ }^{210}$ zu 182 und nicht zum Benzocyclobuten 183, da die Barriere zu $1825.2 \mathrm{kcal} \mathrm{mol}^{-1}$ niedriger ist als zu 183 und $18211.0 \mathrm{kcal} \mathrm{mol}^{-1}$ exothermer gebildet wird.<smiles></smiles>

94<smiles>C=C(C)c1ccccc1C(C)C</smiles>
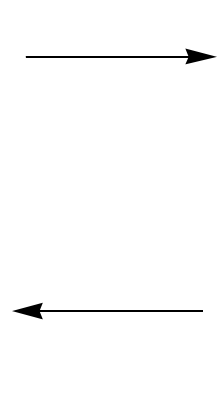<smiles>C=c1ccccc1=C</smiles>

95<smiles>CC(C)=c1ccccc1=C(C)C</smiles>

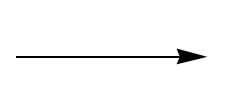<smiles>c1ccc2c(c1)CC2</smiles>

174b

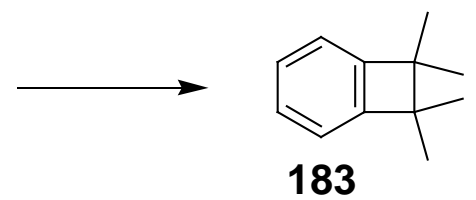

Abbildung 73. Cyclisierung des Endiallens 94 und Reaktionen des Tetramethyl-Zwischenproduktes 181

Benzannelierung. Auch das benzannelierte Endiallen 184 sollte mit einer Barriere von $18.7 \mathrm{kcal} \mathrm{mol}^{-1}$ bei wenig Wärmezufuhr cyclisieren (Tab. 22). Die Barriere ist nur geringfügig höher als im Stammsystem $\left(1.4 \mathrm{kcal} \mathrm{mol}^{-1}\right)$, die Reaktionsenthalpie jedoch mehr als $10 \mathrm{kcal} \mathrm{mol}^{-1}$, was auf die Aufhebung der Aromatizität in $\mathbf{1 8 5}$ zurückzuführen ist (Abb. 74). Die zweite Barriere ist mit $19.3 \mathrm{kcal} \mathrm{mol}^{-1}$ ebenfalls etwas erhöht, jedoch immer noch kleiner als die Reaktionsenthalpie zu 185. Die Gesamtreaktionsenthalpie zu 186 ist mit -47.6 kcal mol $^{-1}$ nur ein wenig höher als beim Stammsystem, was auf den bereits in $\mathbf{1 8 4}$ vorhandenen Aromaten zurückzuführen ist.

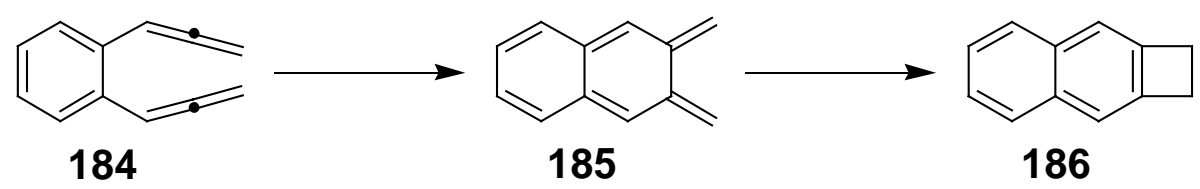


Tabelle 22. Barrieren und Reaktionsenthalpien $\left(\Delta_{0} \mathrm{H}\right)$ der Cyclisierung der Endiallene 94 und 184 (in kcal mol ${ }^{-1}$ )

\begin{tabular}{ccc}
\hline & BLYP/6-31G* & BD(T)/cc-pVDZ \\
\hline $\mathbf{9 4}$ & 0.0 & 0.0 \\
$\mathbf{9 4 \rightarrow \mathbf { 9 5 }}$ & 17.3 & 17.3 \\
$\mathbf{9 5}$ & -39.7 & -50.5 \\
$\mathbf{9 5} \rightarrow \mathbf{1 7 4 b}$ & -14.4 & -23.3 \\
$\mathbf{1 7 4 b}$ & -50.0 & -62.3 \\
\hline $\mathbf{1 8 4}$ & 0.0 & - \\
$\mathbf{1 8 4 \rightarrow \mathbf { 1 8 5 }}$ & 18.7 & - \\
$\mathbf{1 8 5}$ & -28.4 & - \\
$\mathbf{1 8 5} \rightarrow \mathbf{1 8 6}$ & -9.1 & - \\
$\mathbf{1 8 6}$ & -47.6 & - \\
\hline
\end{tabular}


2.4.6 Die Cyclisierung von Hepta-1,2,4,6-tetraen

Die Cyclisierung des Dienallen-Stammsystems ist noch kaum untersucht, da die Isolierung bisher nicht gelungen ist. Hinweise auf 92 kommen von IR-Messungen in der Argon-Matrix. ${ }^{212}$ Die Cyclisierung kann jedoch in situ ausgehend von 187 durchgeführt werden, bei der als Zwischenprodukt 92 entsteht. Als Produkte werden 5-Methylencyclohex1,3-dien 93 und in geringen Mengen Cycloheptatrien 188 erhalten (Abb. 75).212<smiles>C=C/C=C\C1CC1(Br)Br</smiles>

187

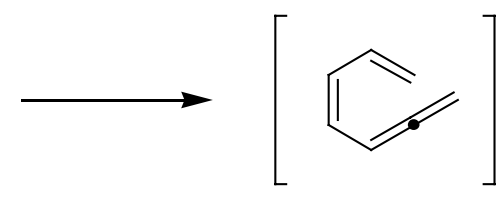

92

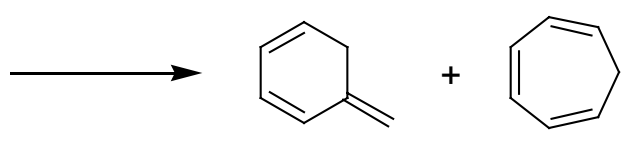

93
188

Abbildung 75. Cyclisierung des instabilen Dienallens 92 ausgehend vom Vorläufermolekül 187

Stammsystem. Das Stammsystem 92 hat mit 18.3 bzw. $19.4 \mathrm{kcal} \mathrm{mol}^{-1}$ eine kleine Barriere und ist deshalb bei Raumtemperatur unbeständig. Mit einer Reaktionsenthalpie von -23.0 bzw. $-31.5 \mathrm{kcal} \mathrm{mol}^{-1}$ wird 93 hoch exotherm gebildet (Abb. 76, Tab. 23). Eine Weiterreaktion und Aromatisierung zu Toluol 189 ist thermodynamisch möglich, da die Enthalpie noch einmal $30 \mathrm{kcal} \mathrm{mol}^{-1}$ abgesenkt würde. Tatsächlich wird bei der Reaktion in Spuren 189 gebildet. Die Barriere für die H-Übertragung ist jedoch fast $60 \mathrm{kcal} \mathrm{mol}^{-1}$ hoch und eine thermische Bildung von 189 daher auszuschließen und eher ein katalytischer $\mathrm{H}$ Transfer in Erwägung zu ziehen.<smiles>c1ccccc1</smiles>

92

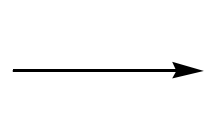<smiles>C=C1C=CC=CC1</smiles>

93

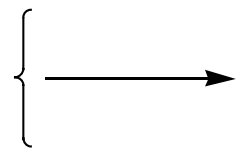<smiles>c1ccc(CCCCC2CC2)cc1</smiles>

189

Abbildung 76. Cyclisierung des Dienallen 92

Benzannelierung. Die Barriere des benzannelierten 190 ist mit $19.5 \mathrm{kcal} \mathrm{mol}^{-1}$ nur geringfügig höher als die des Stammsystems $\left(1.2 \mathrm{kcal} \mathrm{mol}^{-1}\right)$. Der experimentelle Wert von $19.6 \pm 0.56 \mathrm{kcal} \mathrm{mol}^{-1}$ wird jedoch sehr gut reproduziert. ${ }^{213}$ Das Produkt 191 bildet sich mit $11.2 \mathrm{kcal} \mathrm{mol}^{-1}$ immer noch exotherm, jedoch ist es $11.8 \mathrm{kcal} \mathrm{mol}^{-1}$ energiereicher als 93 (Abb. 77, Tab. 23). Die Weiterreaktion zu 2-Methylnaphthalin 192 wird auch hier in Spuren 
beobachtet, eine thermische Reaktion ist jedoch nicht möglich, obgleich die Barriere "nur" noch $47.5 \mathrm{kcal} \mathrm{mol}^{-1}$ beträgt.<smiles>C=C=Cc1ccccc1C=C</smiles>

190

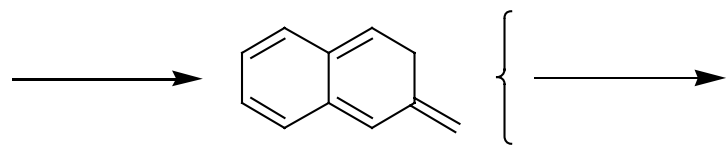

191<smiles>Cc1ccc2ccccc2c1</smiles>

192

Abbildung 77. Cyclisierung des benzannelierten Dienallen 190

Tabelle 23. Barrieren und Reaktionsenthalpien $\left(\Delta_{0} \mathrm{H}\right)$ der Cyclisierung der Dienallene 92 und 190 (in $\mathrm{kcal} \mathrm{mol}^{-1}$ )

\begin{tabular}{ccc}
\hline & BLYP/6-31G* & $\mathrm{BD}(\mathrm{T}) / \mathrm{cc}-\mathrm{pVDZ}$ \\
\hline $\mathbf{9 2}$ & 0.0 & 0.0 \\
$\mathbf{9 2} \rightarrow \mathbf{9 3}$ & 18.3 & 19.4 \\
$\mathbf{9 3}$ & -23.0 & -31.5 \\
$\mathbf{9 3} \rightarrow \mathbf{1 8 9}$ & 32.2 & 30.1 \\
$\mathbf{1 8 9}$ & -56.1 & -63.1 \\
$\mathbf{1 9 0}$ & 0.0 & - \\
$\mathbf{1 9 0} \rightarrow \mathbf{1 9 1}$ & 19.5 & - \\
$\mathbf{1 9 1}$ & -11.2 & - \\
$\mathbf{1 9 1} \rightarrow \mathbf{1 9 2}$ & 36.3 & - \\
$\mathbf{1 9 2}$ & -54.5 & - \\
\hline
\end{tabular}




\subsection{Schlußbetrachtung}

Im Rahmen des ersten, theoretischen Teils dieser Arbeit konnte gezeigt werden, daß die Reaktion, die als Cope-Umlagerung bekannt ist, nur ein Mitglied einer großen Familie von Umlagerungen ist. Alle Reaktionen dieser Cope-Familie haben einen mehr oder weniger aromatischen, sechsgliedrigen Übergangszustand bzw. ein Zwischenprodukt oder Endprodukt gemeinsam. Die Zwischen- und Endprodukte sind größtenteils offenschalige, biradikalische Systeme, die normalerweise schwer, durch die Kombination der gewählten, theoretischen Niveaus (BLYP/6-31G* und BD(T)/cc-pVDZ) aber sehr gut beschrieben werden können. Ein Vergleich mit bekannten experimentellen Daten konnte die hervorragende Qualität der theoretischen Berechnungen zeigen, auf deren Grundlage präzise Voraussagen über bisher nicht bekannte Reaktionen gemacht wurden.

Die Cope-Familie kann in zwei Familienzweige unterteilt werden, die sich nur durch die Verbindung der Einzelelemente unterscheiden (Abb. 78). In Familienzweig 1 werden die Einzelbausteine durch eine Einfachbindung verknüpft, was eine Vielfalt an verschiedenen Edukten und Produkten ergibt, die über sechsgliedrige Zustände ineinander übergehen können. Bei Familienzweig 2 wird die Verbindung der Bausteine über eine Doppelbindung hergestellt, was die Anzahl und die Auswahl der Bausteine deutlich einschränkt. Beide Familienzweige wurden im Rahmen dieser Arbeit im Detail untersucht.

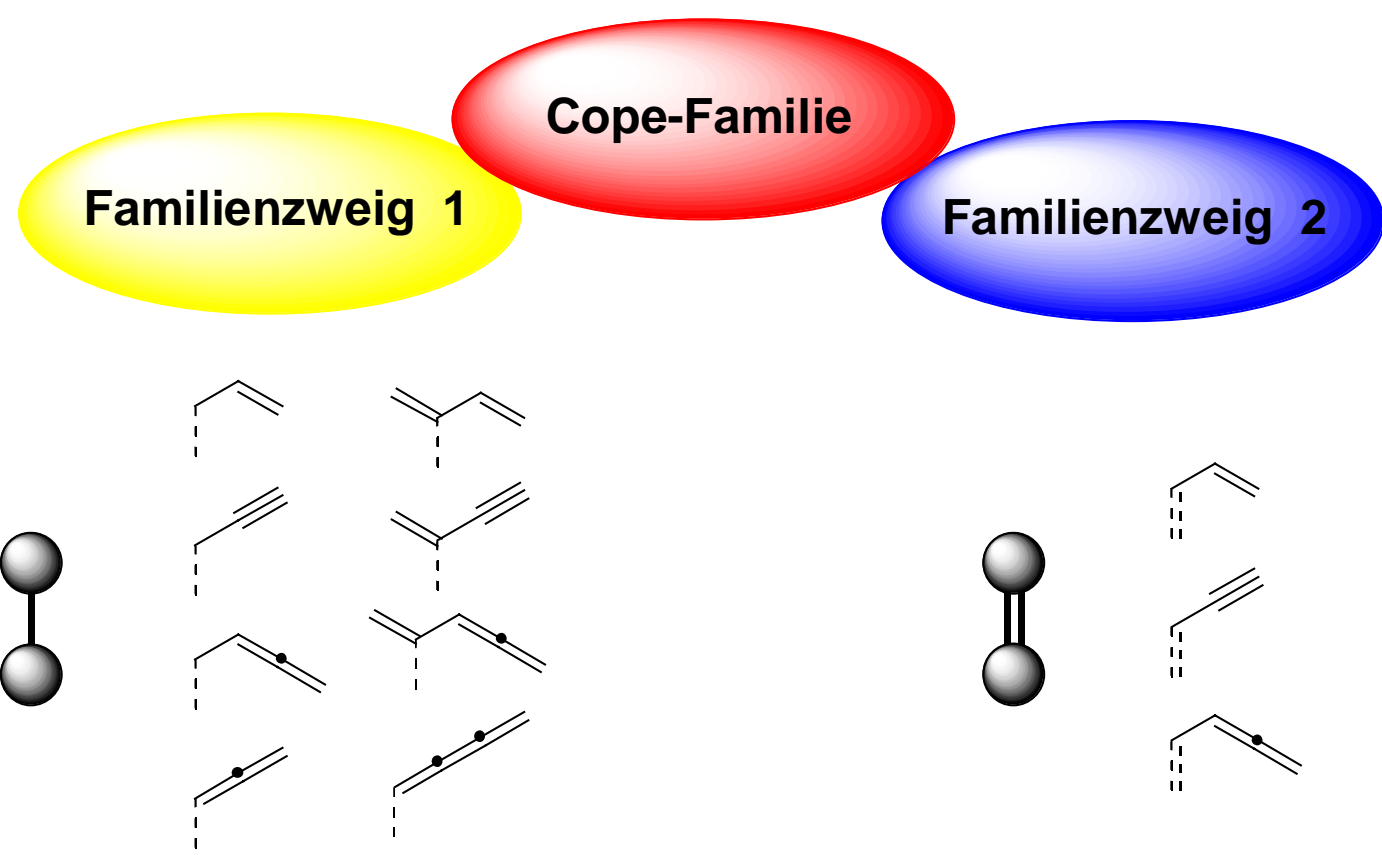

Abbildung 78. Schematische Darstellung der zwei Familienzweige der Cope-Familie 
Der Familienzweig 1 der Cope-Familie konnte in drei Teile gegliedert werden, wovon eine Untergruppe ausschließlich $\pi, \pi$-Biradikale bilden kann und deshalb auch keine Dreifachbindungen beinhaltet (Abb. 79). Die Reaktivität hängt dabei maßgeblich von der Stabilisierung des Zwischenproduktes ab, d.h. je besser das biradikalische Zwischenprodukt durch die exocyclischen Doppelbindungen stabilisiert wird, indem die $\pi$-Radikale delokalisiert werden, desto kleiner sind Barriere und Reaktionsenthalpie. Während 1 noch über einen konzertierten Prozeß bei hohen Temperaturen umlagert, findet die Cyclisierung von 54 zu 88 schon bei niedrigen Temperaturen und exotherm statt. Bei den Molekülen, die bei der Cyclisierung reine $\sigma, \sigma$-Biradikale bilden können, die also Dreifachbindungen und Allen- bzw. Kumuleneinheiten direkt an der zu brechenden bzw. zu knüpfenden Bindung haben, zeigt sich ein ähnliches Bild (Abb. 79). Während 45 und 59 konzertiert umlagern, bildet 69 eine echte biradikalische Zwischenstufe mit kleiner Barriere. Die Stabilisierung der Biradikale ist in diesem Fall nicht ganz so simpel wie bei den $\pi$, $\pi$-Biradikalen, kann aber über teilweise Aromatisierung durch die exocyclischen Doppelbindungen und durch die Bildung von Elektronomeren erklärt werden. Gemischte $\sigma, \pi$-Zustände haben in der Regel Energien zwischen oder nahe den reinen Zuständen. 


\section{Familienzweig 1}

$\pi, \pi$-Biradikale

$\sigma, \sigma$-Biradikale

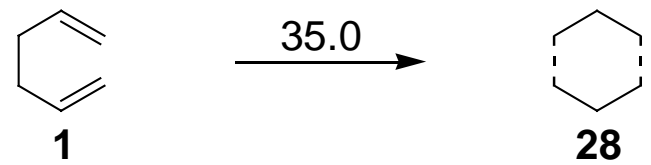

S2.5

42<smiles>C=CCC(=C)C=C</smiles>

43<smiles>C1=CCCC=C1</smiles>

34<smiles>CC1=CCCCC=C1</smiles>

52<smiles>C=C1C=CC(=C)CCCC1</smiles>

53<smiles>C=C=CC(=C)C(=C)C=C</smiles>

54

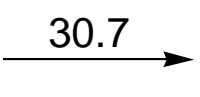

$\stackrel{28.6}{\longrightarrow}$

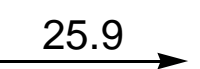

25.8

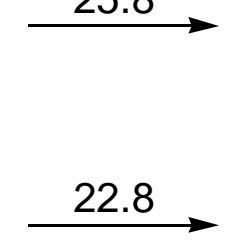

Abbildung 79. Umlagerungen des ersten Familienzweigs der Cope-Familie

In Familienzweig 2 gibt es insgesamt sechs Umlagerungsedukte (Abb. 80). Bei der Cyclisierung des Endiin 9 konnte neben der bekannten Bergman-Reaktion zu 1,4Didehydrobenzol 10 noch zwei weitere Cyclisierungen entdeckt werden. Die experimentellen Werte der Bergman-Cyclisierung konnten dabei sehr gut reproduziert werden, die Reaktionen zu Didehydrofulven 97 und Didehydrodimethylencyclobuten 103 sind jedoch aufgrund fehlender Stabilisierung hoch endotherm und deshalb experimentell noch nicht beobachtet worden. Bei der Cyclisierung des Eninallen 11 konnte, neben den bekannten Myers-Saito- 12 und Schmittel-Produkten 121, ein weiterer Ringschluß zum siebengliedrigen CycloheptatrienBiradikal 126 gefunden werden. Dieser ist, wie die Schmittel-Reaktion, im Stammsystem 
nicht zu beobachten, da der Myers-Saito-Pfad energetisch bevorzugt ist. Bei entsprechender Substitution kann es jedoch möglich sein, diesen Pfad, so wie bei der Schmittel-Cyclisierung, zur Hauptreaktion zu machen. Für die Hopf-Cyclisierung des Dienin 89 konnte der Cycloaromatisierungsmechanismus aufgeklärt werden, der mit den experimentell bestimmten Barrieren sehr gut übereinstimmt. Es wurden alle möglichen Reaktionspfade diskutiert und alle bis auf einen ausgeschlossen. Insbesondere konnte zweifelsfrei geklärt werden, daß das Intermediat in dieser Reaktion nicht das Biradikal 91, sondern das Isobenzol 90 ist. Die experimentelle Barriere und die Reaktionsenthalpie der Cyclisierung des Trien 7 konnten sehr genau berechnet werden, was wiederum für die Wahl der Methoden spricht. Die Cyclisierungen der, aufgrund der Instabilität der Edukte bisher nicht isolierten, Endiallen 94 und Dienallen 92 konnten beschrieben werden und thermische Folgereaktionen zu Benzocyclobuten 147b bzw. Toluol 189 daher bestätigt bzw. ausgeschlossen werden.

\section{Familienzweig 2}

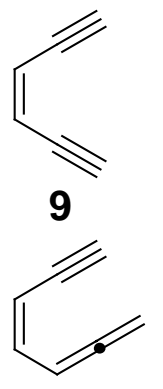

11<smiles>C#C/C=C\C</smiles>

89<smiles>c1ccccc1</smiles>

7<smiles>C1=CC=CC=CC=C1</smiles>

94<smiles>C=Cc1ccccc1</smiles>

92

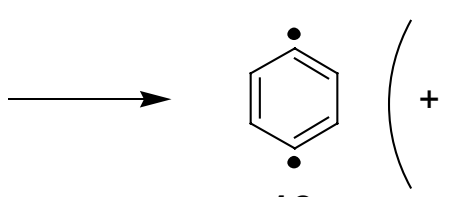

10

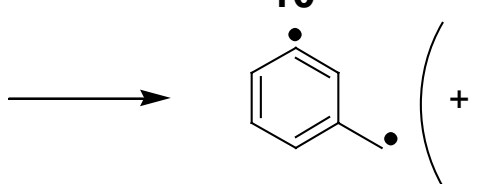

12

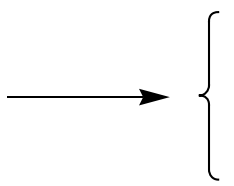<smiles>C1=CC=CCC=1</smiles>

90

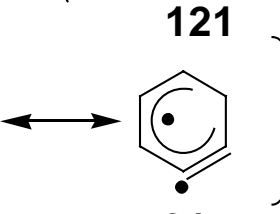

91

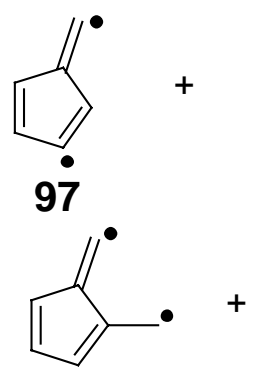

121

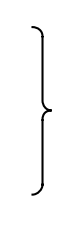

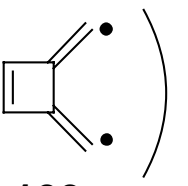

103<smiles>CC1=CCCC=CC=C1</smiles>

126<smiles>C1=CCCC=C1</smiles>

8<smiles>C=c1ccccc1=C</smiles>

95

147b<smiles>C=C1C=CC=CC1</smiles>

93

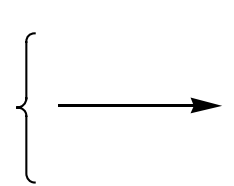

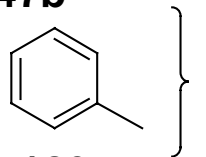

189

Abbildung 80. Cyclisierungen der Mitglieder des zweiten Cope-Familienzweigs 
Die benzannelierten Analoga zeigen alle dieselben Reaktionspfade wie die Stammsysteme, unterschiede bestehen nur in den Barrieren und den Reaktionsenthalpien. Während die Barrieren nur geringfügig erhöht sind, werden die Produkte erheblich weniger exotherm bzw. endothermer gebildet. Dafür gibt es zwei verschiedene Gründe: Bei den biradikalbildenden Cycloaromatisierungen des Endiin 98 und Eninallen 122 liegt es daran, daß im Edukt schon ein aromatischer Ring vorhanden ist und ein zweiter annelierter Ring weniger aromatische Stabilisierungsenergie liefert als ein einzelner, neu gebildeter Ring aus den Edukten (Abb. 81). Bei der Cycloaromatisierung von Dienin 161 spielt dieser Grund für die Gesamtreaktionsenthalpie zu 164 ebenfalls eine Rolle, für die reine Cyclisierung zu 162 jedoch, bei der kein neues aromatisches System ausgebildet wird, ist der Grund die Aufhebung der Aromatizität des im Edukt vorhandenen Benzolrings durch die Ausbildung eines ortho-chinoiden Systems. Dieser letzte Grund ist ebenfalls für die Energieerhöhung bei den Cyclisierungen von Trien 174, Endiallen 184 und Dienallen 190 verantwortlich.<smiles>C#Cc1ccccc1C#C</smiles>

98<smiles>C#Cc1ccccc1C=C=C</smiles>

122<smiles>C#Cc1ccccc1C=C</smiles>

161<smiles>C=Cc1ccccc1C=C</smiles>

174<smiles>C=C=Cc1ccccc1C=C=C</smiles>

184<smiles>C=C=Cc1ccccc1C=C</smiles>

190<smiles>Cc1cccc2ccccc12</smiles>

32<smiles>Cc1ccc2ccccc2c1</smiles>

137<smiles>C1=CCC=c2ccccc2=1</smiles>

162<smiles>c1ccc2ccccc2c1</smiles>

164

Abbildung 81. Cyclisierungen der benzannelierten Analoga des Familienzweigs 2 
Bei den cyclischen Varianten des Endiin 99, Eninallen 123 und Dienin 146 gilt es, drei verschiedene Fälle zu unterscheiden. Die sieben- und achtgliedrigen Ringe cyclisieren nicht, da hochgespannte Produkte zu erwarten sind. Die neun-, zehn- und teilweise elfgliedrigen Ringe erniedrigen die Barriere, verglichen mit dem Stammsystem, und erhöhen somit die Reaktivität. Alle größeren Ringe cyclisieren bei höheren Temperaturen als das Stammsystem, da sterisch und energetisch ungünstige, mittelgroße Cycloalkenringe gebildet werden müssen. Bei der Bergman-Reaktion konnte gezeigt werden, daß die Annelierung der Endiineinheit an Cycloalkenringe zu keinerlei Energieänderungen in der Cyclisierung führt, und daß bei fehlendem Radikalfänger die exocyclischen Endiine 113 über die Biradikale 112 in die endocyclischen Endiine 99 überführt werden können, jedoch nicht umgekehrt. Bei der Cyclisierung der Eninallene 123 ergab sich bei dem neun- und zehngliedrigen Ring eine mehr oder weniger ausgeprägte Bevorzugung des Schmittel-Produktes 125, während bei größeren Ringen, wie im Stammsystem, ausschließlich das Myers-Saito-Produkt 124 gebildet wird. Bei der Hopf-Cyclisierung der Dienin-Ringe 146 sind ebenfalls die Allene 147 das Zwischenprodukt und nicht die Biradikale. Experimentelle Cyclisierungsbarrieren zum Allen konnten bestätigt werden, die Gesamtbarriere war bei den Computerberechnungen jedoch viel höher als bei den Experimenten, was möglicherweise einen vom Stammsystem verschiedenen Umlagerungsmechanismus für die Carbocyclen bedeutet.

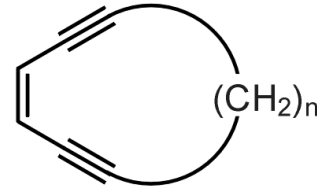

99a-h

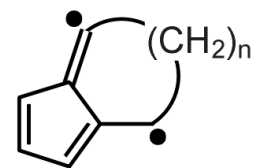

125a-f<smiles>C1#CCCCCCCC/C=C\C=C/1</smiles>

146a-h
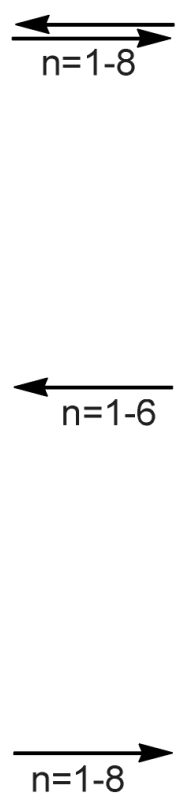

$n=1-8$

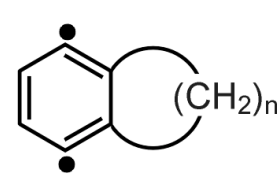

112a-h

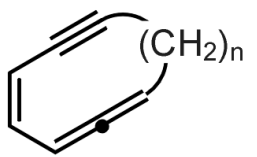

123a-f

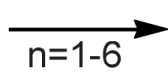

$\overrightarrow{n=1-8}$

147a-h
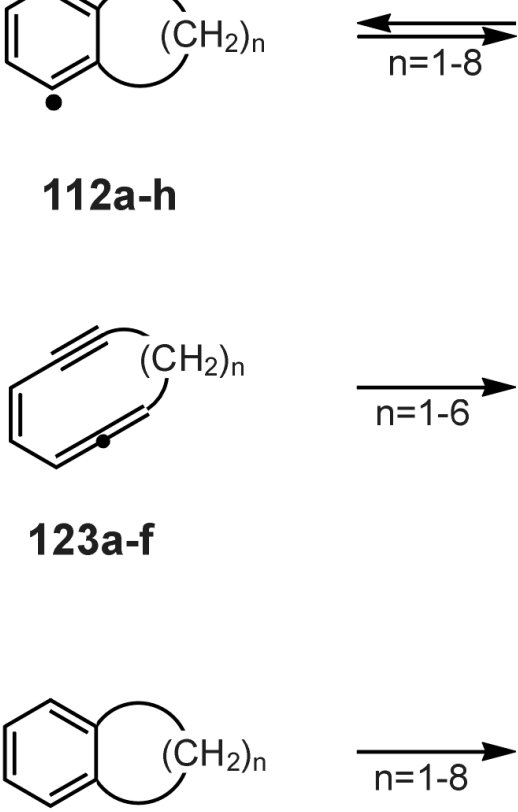<smiles>C#CC1=C(C#C)CCCC1</smiles>

113a-h<smiles>C[C@@H]1CCCc2ccccc21</smiles>

124a-f<smiles>C1=CC2=CCCCC2CC1</smiles>

147a-h

Abbildung 82. Cyclisierungen der cyclischen Analoga des Familienzweigs 2 
Bei der Bergman-Reaktion konnte eindringlich demonstriert werden, daß die Substitution der acetylenischen Wasserstoffatome durch funktionelle Gruppen einen entscheidenden Einfluß auf die Reaktivität hat (Abb. 83). Substituenten mit $\sigma$-akzeptierenden und $\pi$-donierenden Eigenschaften erniedrigen die Barriere und Enthalpie, während $\pi$ akzeptierende und $\sigma$-donierende Substituenten diese erhöhen. So ist das Difluor-Endiin 100rr bei Raumtemperatur instabil und cyclisiert exotherm zum Bergman-Produkt 117rr, während die Barriere des Diboranyl-Endiin 100aa um $10 \mathrm{kcal} \mathrm{mol}^{-1}$, die Reaktionsenthalpie sogar um $25 \mathrm{kcal} \mathrm{mol}^{-1}$ erhöht ist. Zusätzlich zu den elektronischen sind noch sterische und CoulombEffekte zu berücksichtigen. Das Diphenylendiin 100ee ist aufgrund seiner großen Substituenten im Bergman-Produkt 117ee sterisch gehindert und hat deshalb erhöhte Energiewerte. Im Diamino-Endiin 1171 ist der Substituent zwar recht klein und ein guter $\sigma$ Akzeptor, die beiden positiven Ladungen stoßen sich jedoch derart ab, daß Barriere und Enthalpie im Vergleich zum Stammsystem verdoppelt werden. Substitution eines vinylischen Wasserstoffatoms hat hingegen keine großen Auswirkungen auf die Energien.

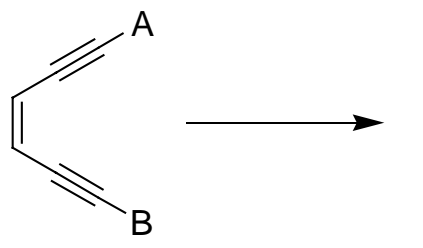

100a-t 100aa-tt

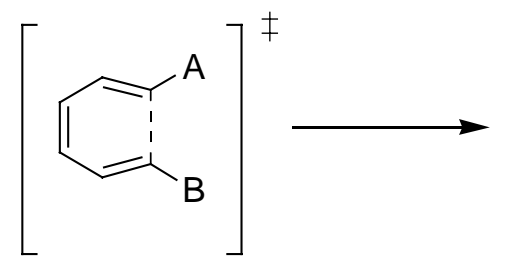

116a-t 116aa-tt

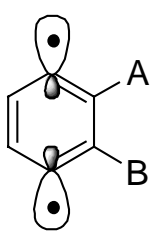

117a-t 117aa-tt

a-t: $\quad A=$ Substituent, $B=H$

aa-tt: $\quad \mathrm{A}=\mathrm{B}=$ Substituent

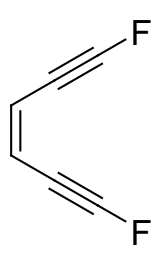

100rr

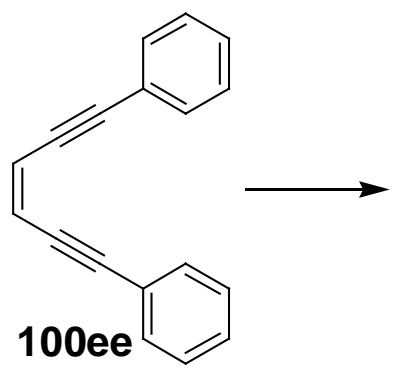<smiles>Fc1ccccc1F</smiles>

117rr

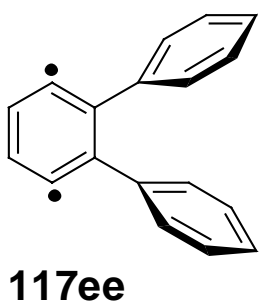<smiles>BC#C/C=C\C#CB</smiles>

100aa<smiles>Bc1ccccc1B</smiles>

$117 a a$

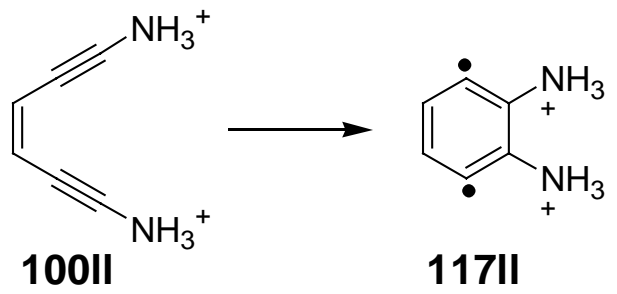

Abbildung 83. Substituierte Endiine und ihre Cyclisierung 
Im Rahmen des ersten, theoretischen Teils dieser Arbeit konnte ein umfassender Überblick über die Mitglieder und Reaktionen der Cope-Familie gegeben werden. Die Zusammenhänge zwischen allen Reaktion konnten deutlich aufgezeigt werden und präzise Voraussagen über noch nicht untersuchte Reaktionen wurden gemacht. Die Grundlagen für eine Systematisierung der Cope-Familie wurden geschaffen. Bei den Mitgliedern des zweiten Familienzweigs konnte gezeigt werden, daß die Benzannelierung grundsätzlich die Reaktivität herabsetzt, und daß sich sowohl durch Ringspannungseffekte als auch durch geeignete Substitution die Reaktivitäten der ungesättigten Verbindungen steuern lassen.

Neben der Bergman-Reaktion konnte darüber hinaus eine neue interessante Cyclisierung des Endiins zum Didehydrofulven gefunden werden. Diese ist jedoch hoch endotherm und konnte experimentell noch nicht beobachtet werden. Thermisch ist der einfache Zugang zu Fulvenderivaten 193 also nicht möglich, es wird das Bergman-Produkt 19 gebildet. Durch elektrophile Angriffe, z.B. durch Brom, ist das Verhältnis jedoch genau umgekehrt, es werden ausschließlich Fulven-Produkte 195 gebildet und keine sechsgliedrigen Aromaten 194 (Abb. 84). Diese ungewöhnlichen Reaktionen zu fulvenischen Produkten durch Addition von Brom, die, im Gegensatz zur Bergman-Reaktion, bei Raumtemperatur stattfinden, sollen im nächsten Teil dieser Arbeit eingehend untersucht werden.
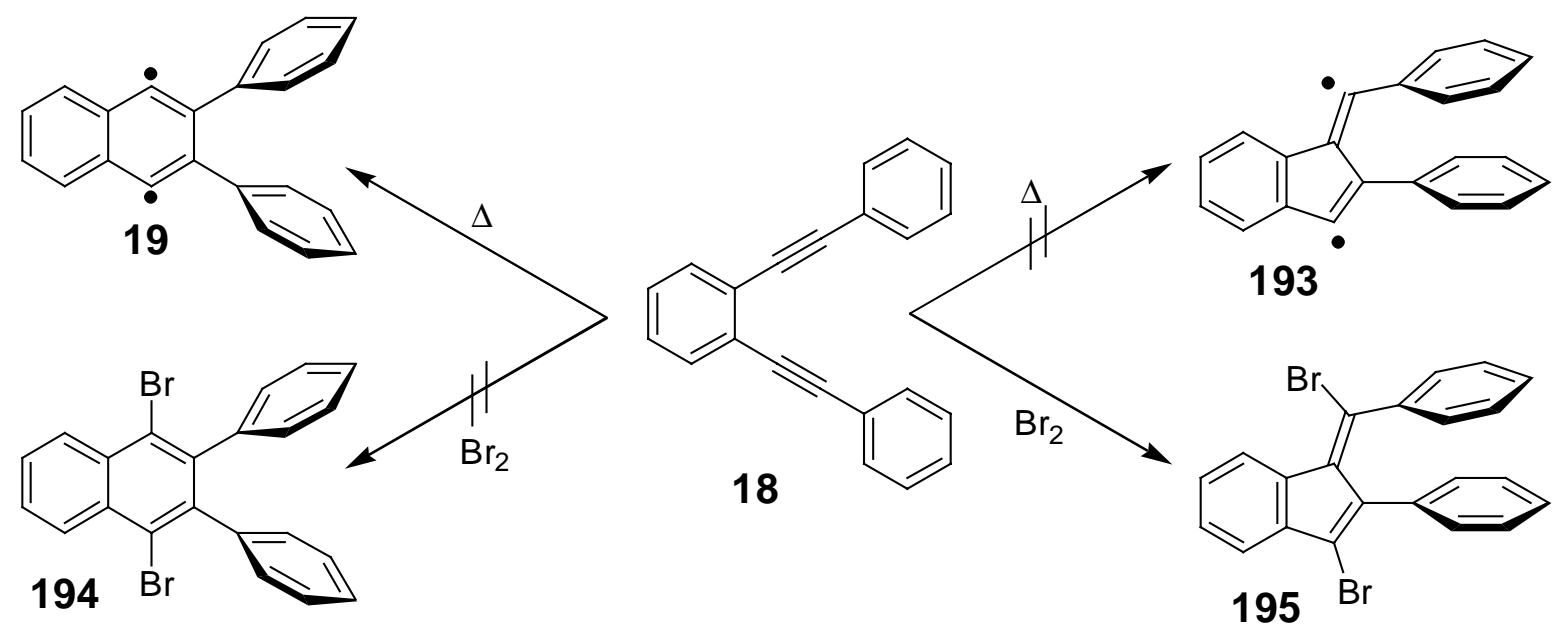

Abbildung 84. Gegenüberstellung von thermischer und elektrophil induzierter Cyclisierung des Endiin 18 


\section{Teil II: Experimente zur kationisch induzierten Cyclisierung}

\subsection{Grundlagen}

Der elektrophile Angriff von Brom an C-C-Mehrfachbindungen gehört $\mathrm{zu}$ den bekanntesten und am besten untersuchten Reaktionen in der organischen Chemie. Die wichtigsten Vertreter sind die elektrophile Addition an Doppel- und Dreifachbindungen sowie die elektrophile Substitution an Aromaten (Abb. 85). ${ }^{214}$ Allen Reaktionen gemeinsam ist die Ausbildung eines Bromoniumions in einem ersten Schritt, das dann entsprechend unter Addition bzw. Eliminierung eines Nucleophils weiterreagiert.<smiles>[R]C=C[2H]</smiles>
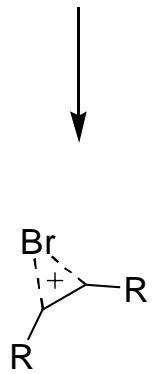
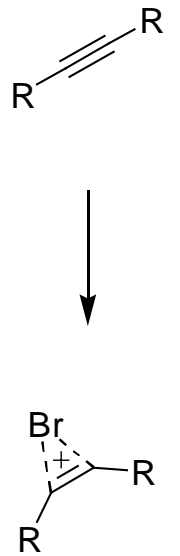<smiles>c1ccccc1</smiles>

$$
\downarrow
$$

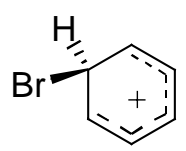

Abbildung 85. Bromoniumionen bei elektrophilen Angriffen

Die elektrophile Addition von Brom an eine Doppelbindung ist im Allgemeinen transselektiv, da der Angriff auf derselben Seite sterisch gehindert ist und die Addition daher von der anderen Seite aus erfolgt. ${ }^{214}$ Bei den Alkinen gilt dies entsprechend, so daß sich, je nach Substitution, vorwiegend trans-Dibromalkene bei der Addition von einem Äquivalent Brom bilden. Bei arylsubstituierten Alkinen ist die Situation allerdings etwas anders. Bei der Addition von " $\mathrm{Br}^{+"}$ an Diphenylacetylen 196 entsteht kein Bromoniumion 197, sondern das mesomeriestabilisierte Carbenium-Ion 198, das dann unter Addition von $\mathrm{Br}^{-}$ein Gemisch aus cis- 199 und trans-Dibromstilben 199 ergibt (Abb. 86). ${ }^{215}$ 


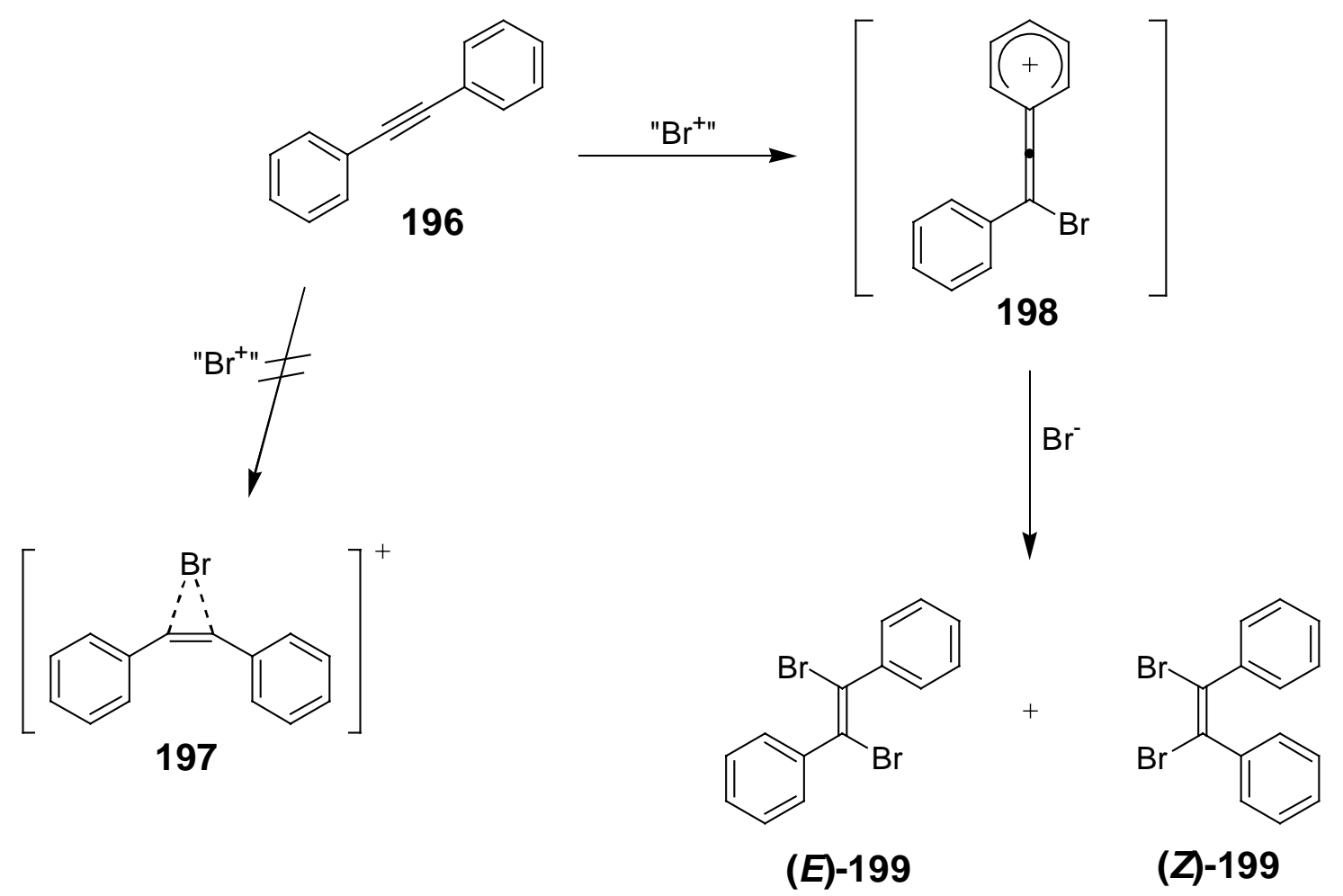

Abbildung 86. Mechanismus der elektrophilen Addition von Brom an Diphenylacetylen 196

Für die Addition von Brom an Moleküle mit benachbarten Dreifachbindungen, wie z.B. im 1,2-Bis(phenylethinyl)benzol 18, könnte man dem Additionsmechanismus nach Produkte des Typs $\mathbf{2 0 0}$ erwarten, die durch die Reaktion von Brom mit einer Dreifachbindung entstehen und die zweite acetylenische Bindung unberührt lassen (Abb. 87). Wie jedoch experimentell bekannt ist, interagieren die beiden Dreifachbindungen beim Erhitzten miteinander und bilden das Biradikal 19, das unter Abstraktion von zwei H-Atomen von einem geeigneten Donor 2,3-Diphenylnaphthalin ergibt (Bergman-Reaktion). ${ }^{12,14,48}$ Bei dem elektrophilen Angriff von Brom ist deshalb davon auszugehen, daß auch dort die benachbarten Dreifachbindungen miteinander reagieren und kein $\mathbf{2 0 0}$ erhalten wird. Theoretisch ist die Bildung von drei verschiedenen Ringsystemen durch den Angriff von "Br ${ }^{+"}$ auf eine der Dreifachbindungen und die anschließende Faltung und Interaktion mit der zweiten Dreifachbindung möglich (Abb. 87). Tatsächlich konnte Whitlock zeigen, daß weder das Bergman-ähnlich Produkt 194, noch das viergliedrige 201 gebildet wird, sondern ausschließlich das Diphenylbenzofulven-Produkt 195 als eine Mischung aus den $(E)$ - und $(Z)$ Isomeren. ${ }^{45}$ Ähnliche Produkte wurden auch durch elektrophile Addition von $\mathrm{HBr}$ und $\mathrm{I}_{2}$, sowie den nucleophilen Angriff von BuLi und radikalische Reaktionen mit Lithium und $\mathrm{PhSH}$ erhalten. ${ }^{45}$ Die Benzofulven-Cyclisierung scheint also eine prinzipielle Reaktion von $\mathbf{1 8} \mathrm{zu}$ sein, unabhängig von der angreifenden Spezies. 

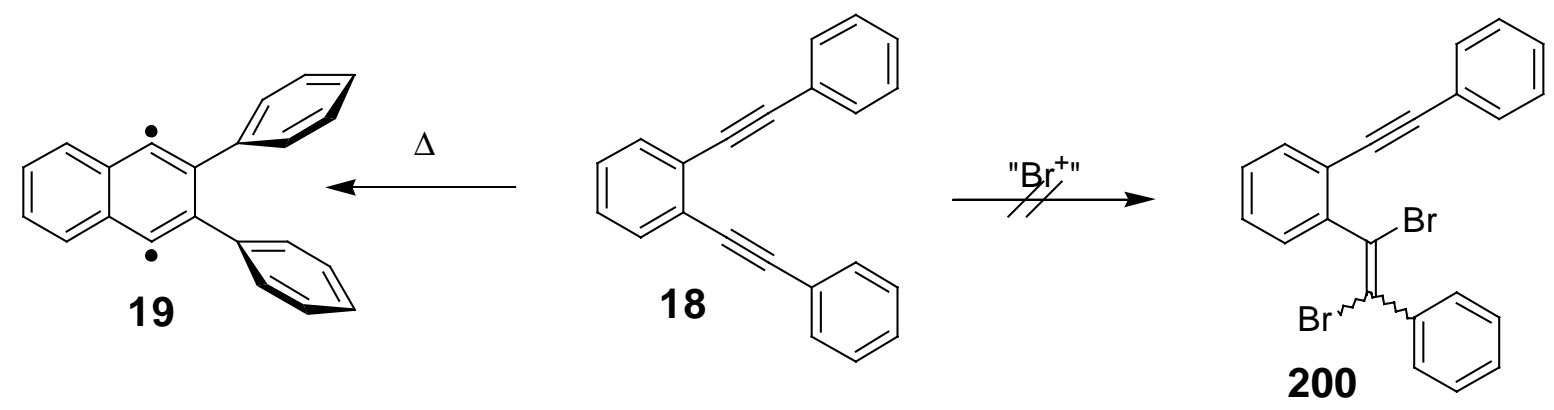

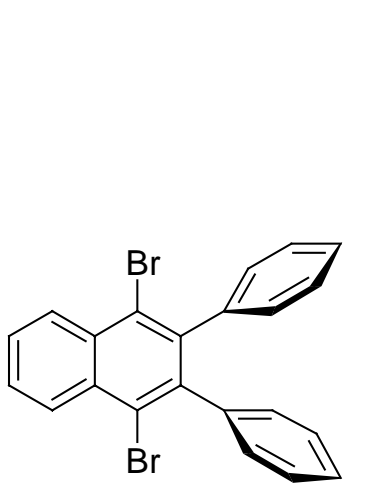

194
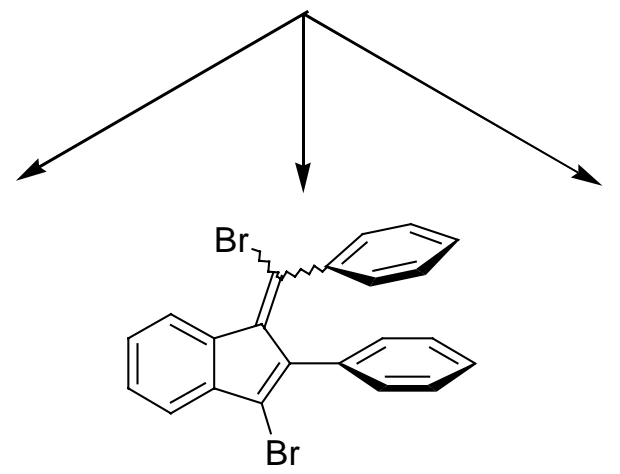

195

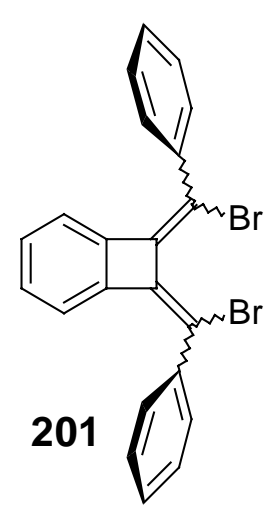

Abbildung 87. Mögliche Cyclisierungsarten von 1,2-Bis(phenylethinyl)-benzol 18

Durch zusätzliche Versuche mit $\mathrm{HBr}$ konnte gezeigt werden, daß der elektrophile Angriff auf die Dreifachbindung kernnah und nicht substituentennah erfolgt (Abb. 88). ${ }^{45}$ Daher ist auch die Bildung von 201 (ganz abgesehen von der Ringspannung) nicht möglich. Für den Angriff, die Faltung der Bindung und die Interaktion mit der zweiten Dreifachbindung wurde ein konzertierter Schritt vorgeschlagen, in dem sich die $\mathrm{Br}-\mathrm{C}$ - und die neue C-C-Bindung simultan bilden. Das gebildete resonanzstabilisierte Carbenium-Ion 202 kann dann von dem Nucleophil $\left(\mathrm{Br}^{-}\right)$von zwei Seiten angegriffen werden, was auch hier die Mischung aus cis- und trans-195 erklärt. Bromierungsversuche eines 1:1 Gemisches von 18 und Diphenylacetylen 196 mit einem Äquivalent Brom ergaben ausschließlich 195 und unreagiertes 196 als Produkte, jedoch kein Dibromstilben 199. ${ }^{45}$ Diese Beobachtung läßt die zwei Schlußfolgerungen zu, daß die Interaktion in $\mathbf{1 8}$ eindeutig die Reaktion gegenüber der normalen Addition beschleunigt, und daß der geschwindigkeitsbestimmende Schritt nicht die Trennung der Ladungen im $\mathrm{Br}_{2} \mathrm{zu} \mathrm{Br}^{+} "$ und $\mathrm{Br}^{-}$ist, sondern der elektrophile Angriff. 

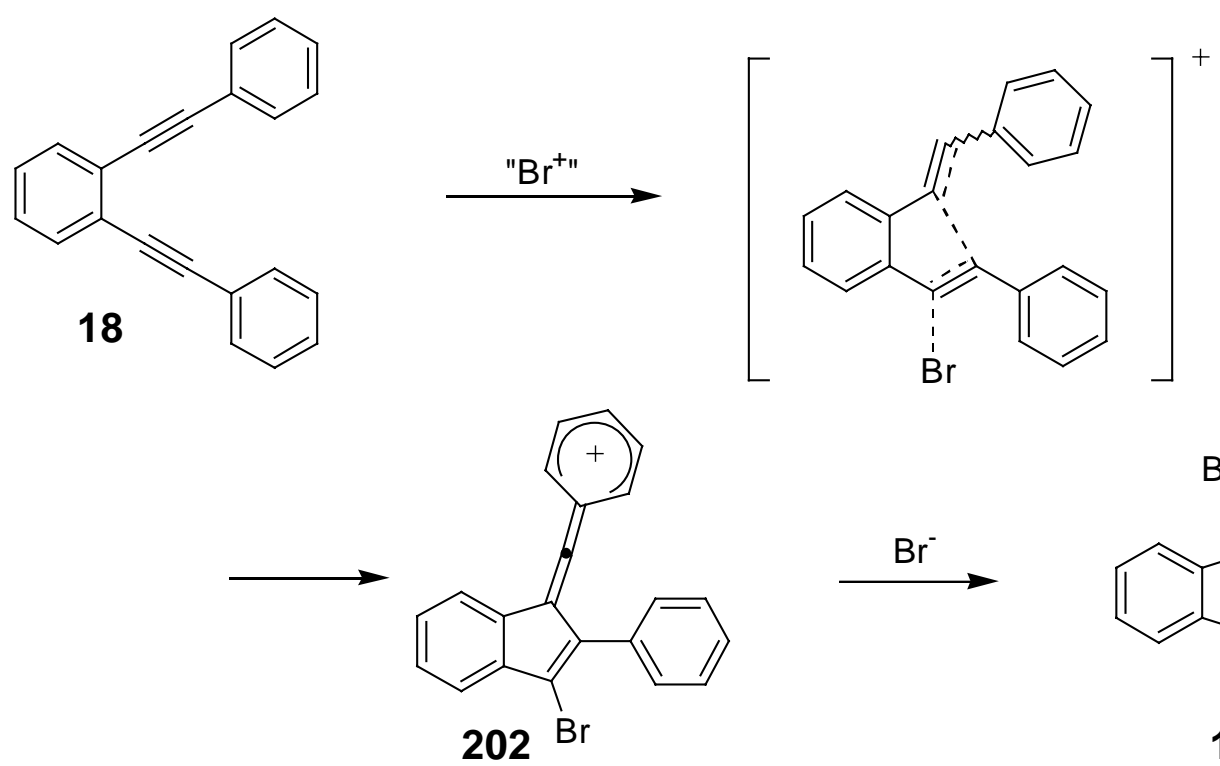

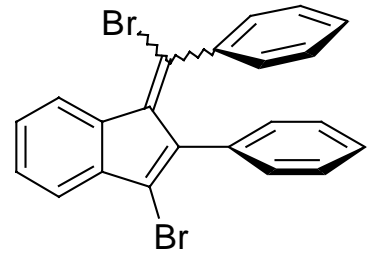

195

Abbildung 88. Cyclisierung von 1,2-Bis(phenylethinyl)-benzol 18

Eine ähnliche Cyclisierungsreaktion wurde für das 1,8-Bis(phenylethinyl)naphthalin 203 beobachtet. ${ }^{44}$ Obwohl die Acetylen-Gruppen hier ein C-Atom weiter voneinander entfernt sind und sich deshalb ein sechsgliedriger Ring 204 bilden könnte, wird wiederum nur die Cyclisierung zu einem fünfgliedrigen Ring beobachtet (Abb. 89). Für diesen Angriff wurde ein anderes Bromoniumion 205 als Zwischenprodukt vorgeschlagen, aus dem dann aus sterischen Gründen nur 206 entstehen sollte. ${ }^{44} \mathrm{Ob}$ es sich bei dem Produkt aber wirklich um 206 oder eins seiner Isomere handelt, konnte damals nicht zufriedenstellend geklärt werden. ${ }^{44}$

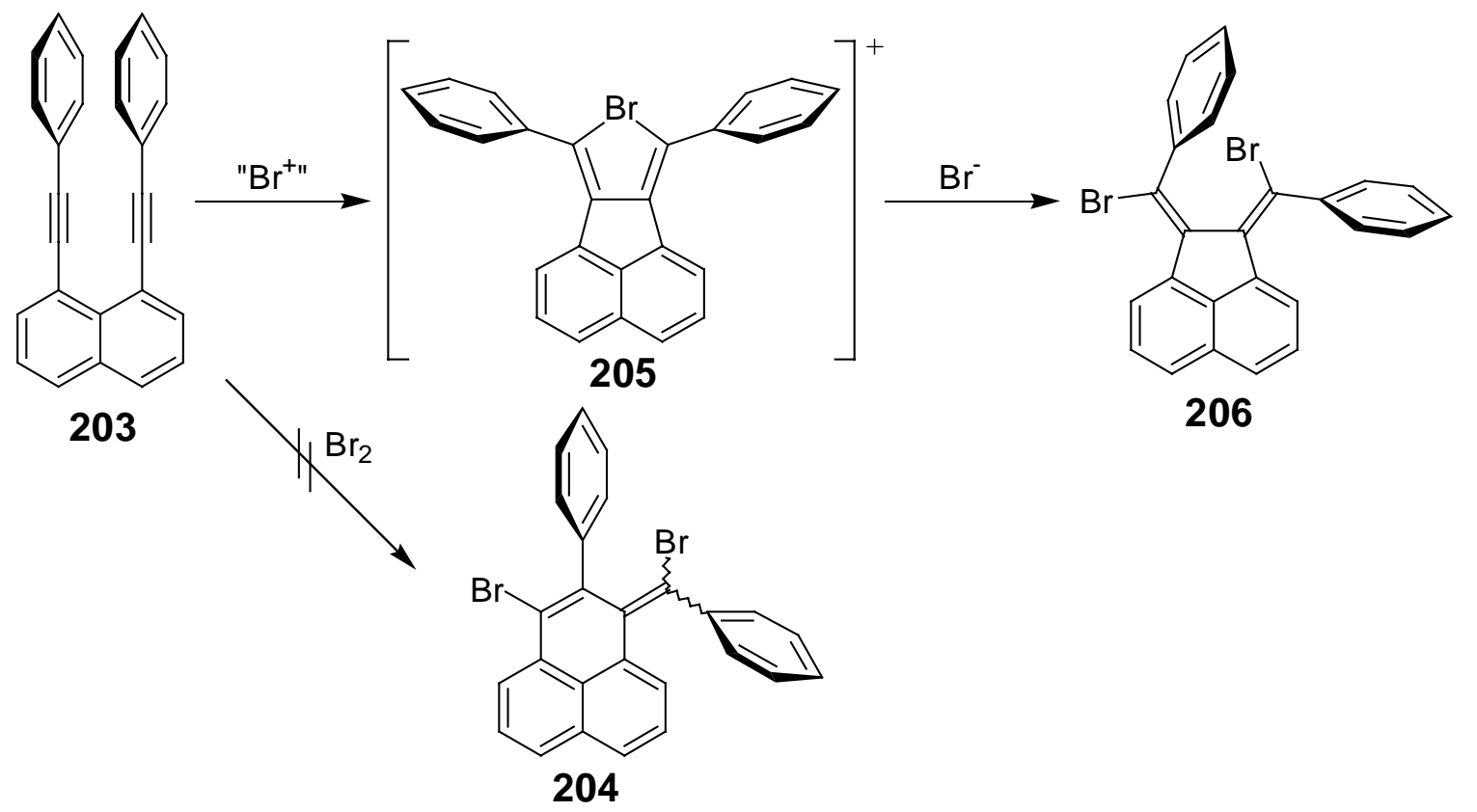

Abbildung 89. Cyclisierung von 1,8-Bis(phenylethinyl)naphthalin 203 
Die Reaktion von 1,8-Dipropinyl-naphthalin 207 mit Brom ergibt keine Cyclisierung, sondern ausschließlich das Tetrabromid 208, unabhängig von der Menge des zugegebenen Broms (Abb. 90). ${ }^{44}$ Zumindest im Naphthalin-Fall scheint die Anwesenheit der aromatischen Ringe an den Acetylen-Einheiten essenziell für eine Cyclisierung zu sein.<smiles>CC#Cc1cccc2cccc(C#CC)c12</smiles>

207

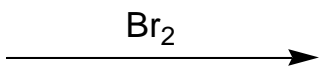

Abbildung 90. Die Reaktion von 1,8-Dipropinyl-naphthalin 207 mit Brom.<smiles>CC(Br)=C(Br)c1cccc2cccc(C(Br)=C(C)Br)c12</smiles>

208

Bei einer Erweiterung des Endiin-Gerüstes von 18 zu 2,2'-Bis(phenylethinyl)diphenylacetylen 209 sollte sich ein weiterer Cyclisierungsschritt im Anschluß an den von 18 ergeben und somit eine Kaskaden-"Zipper"-Reaktion ablaufen. ${ }^{46}$ Das Ergebnis bei der Addition eines Äquivalents Brom war jedoch nicht das gewünschte. Es wurde eine Mischung der beiden von der einfachen Cyclisierung abgeleiteten Benzofulvene 210 und 211 erhalten, zusammen mit dem Edukt und einem Tetrabrom-Produkt (Abb. 91). ${ }^{46}$ Mit einem hohen Überschuss an Brom konnte das Tetrabromid rein erhalten werden und ihm wurde die Struktur 212 zugeordnet. Die Zipper-Reaktion läuft offensichtlich nicht $a b$, da als Zwischenprodukt das energetisch ungünstige Kation 213 gebildet werden müßte, das nicht resonanzstabilsiert ist.

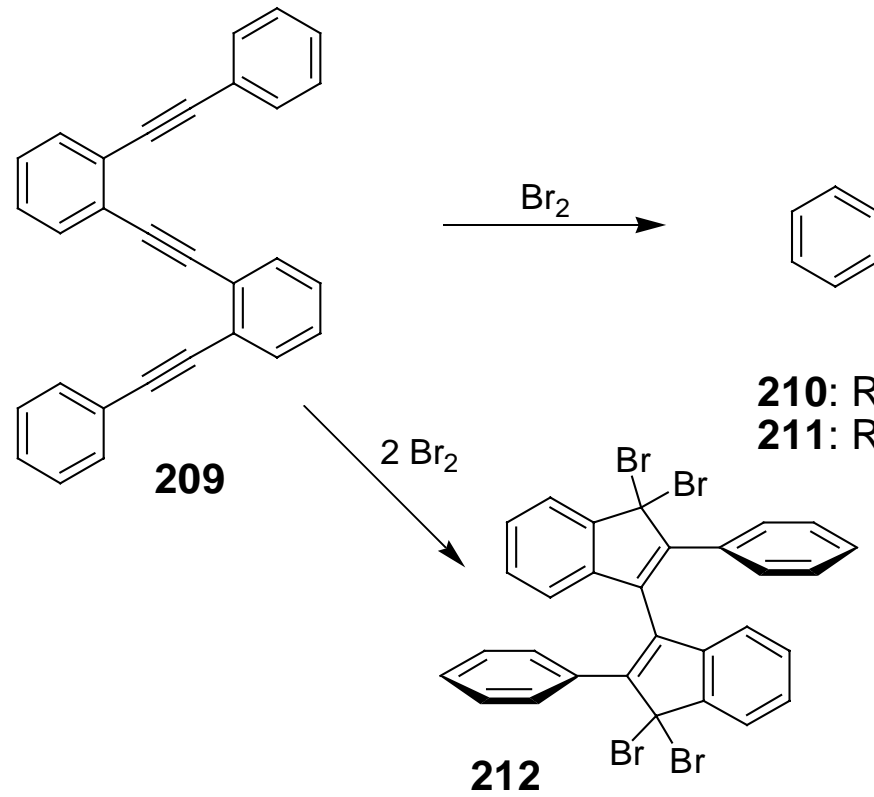

212

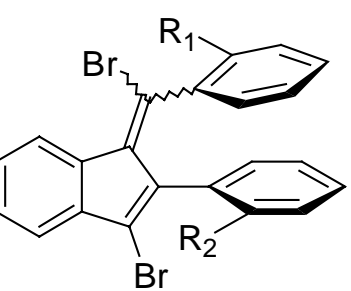

210: $\mathrm{R}_{1}=\mathrm{H} ; \mathrm{R}_{2}=\mathrm{C} \equiv \mathrm{C}-\mathrm{H}$

211: $R_{2}=H ; R_{1}=C \equiv C-H$<smiles>[Z13]c1ccccc1-c1c(-c2ccccc2)c(-c2ccccc2)cc2ccccc12</smiles> 
Ein ähnlicher Fall liegt bei der Cyclisierung von [4.4]Orthocyclopha-1,3,11,13-tetrain 214 vor (Abb. 92). ${ }^{47}$ In dieser cyclischen Variante des Endiins erfolgte eine doppelte IodAddition und eine zweifache Zipper-Reaktion zu dem Tetraiodid 215. Die Struktur konnte jedoch nicht vollständig charakterisiert werden, da die Löslichkeit von 215 für eine NMRspektroskopische Untersuchung zu gering war. Folgereaktionen von 215 zu 216, das eindeutig charakterisiert wurde, deuten jedoch stark auf die angegebene Struktur hin. ${ }^{47}$

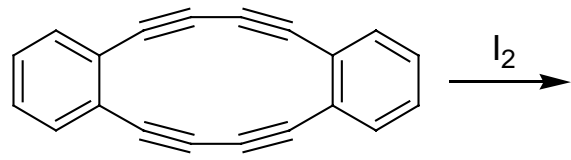

214

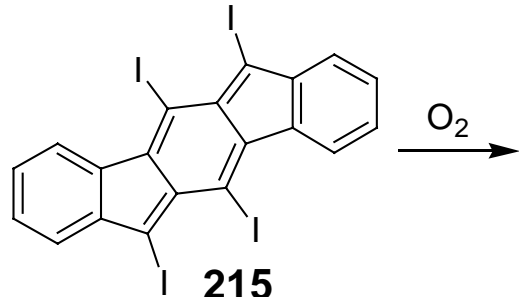

215

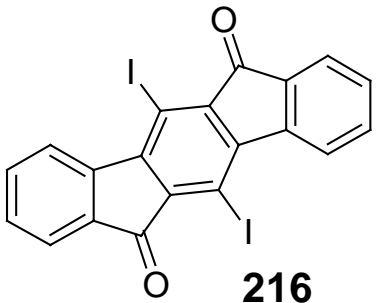

Abbildung 92. Cyclisierung von [4.4]Orthocyclopha-1,3,11,13-tetrain 214

Ein Beispiel für eine Methode zur Synthese von sechsgliedrigen Ringen durch elektrophil induzierte Cyclisierung ist die Reaktion des $\mathrm{I}(\mathrm{py})_{2} \mathrm{BF}_{4} / \mathrm{HBF}_{4}$ Systems mit 1,4Diphenylbut-1-in 217 bzw. 1,4-Diphenylbut-1-en 218. ${ }^{43}$ Hierbei wird zunächst "I ${ }^{+"}$ an die Doppel- bzw. Dreifachbindung addiert, und das so entstandene Kation greift dann den aromatischen Ring nach einem $\mathrm{S}_{\mathrm{E}} \mathrm{Ar}-\mathrm{Mechanismus}$ an, wobei die entsprechenden Benzocyclohexadien- 219 bzw. Benzocyclohexen-Derivate 220 entstehen (Abb. 93). ${ }^{43} \mathrm{Im}$ Falle von (Z)-218 entsteht sogar stereoselektiv nur cis-220. Obwohl bei der Cyclisierung von 218 theoretisch auch ein fünfgliedriger Ring entstehen könnte, konnte nur 220 als Produkt nachgewiesen werden. Auch im Falle einer unsubstituierten Doppelbindung wird nur der sechsgliedrige Ring erhalten. Für Cyclisierungen, die einen $\mathrm{S}_{\mathrm{E}} \mathrm{Ar}-\mathrm{Sch}$ ritt enthalten, ist die Reaktion zu Benzocyclohexen-Derivaten anscheinend ein allgemeines Prinzip.
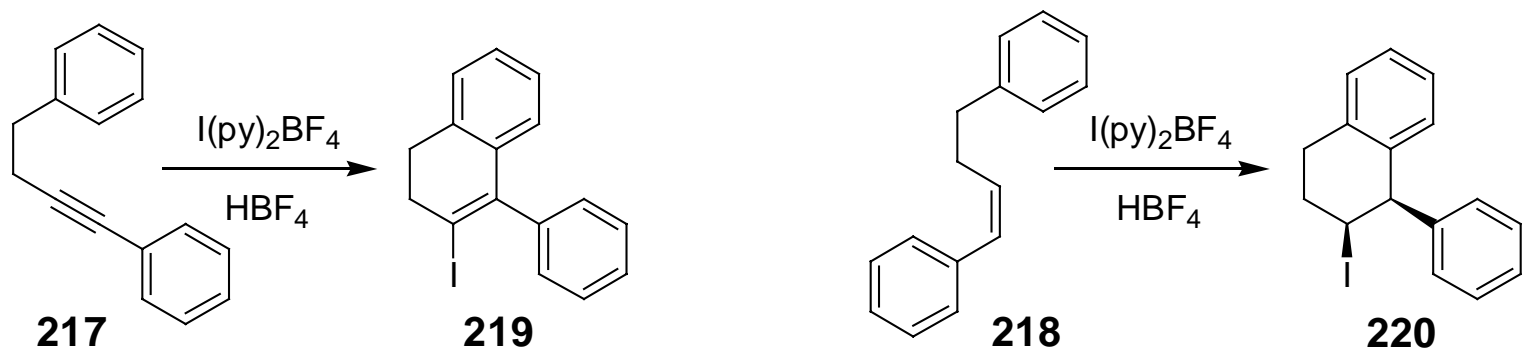

Abbildung 93. Cyclisierungen von 1,4-Diphenylbut-1-in 217 und 1,4-Diphenylbut-1-en 218 
Die gegebenen Beispiele zeigen, daß die Interaktion zwischen verschiedenen ungesättigten C-Atomen während eines elektrophilen Angriffes durchaus zu sehr unterschiedlichen Ergebnissen führen können. Um eine Systematik in die verschiedenen Reaktionen zu bringen und um eine Basis zu haben, auf der es möglich ist, Vorhersagen über die Reaktivität und Selektivität bei der Cyclisierung zu machen, wurden eine Reihe mehrfach ungesättigter Verbindungen dargestellt und der elektrophilen Reaktion mit Brom unterworfen. Ältere Ergebnisse wurden teilweise reproduziert, um deren NMR-spektroskopische Eigenschaften zu bestimmen und zum Vergleich bei höheren Homologen heranzuziehen. Im Folgenden werden zunächst die Synthesen der ungesättigten Edukte beschrieben, bevor im Weiteren auf deren Cyclisierungsverhalten gegenüber Brom eingegangen wird. 


\subsection{Synthese der Cyclisierungs-Ausgangsverbindungen}

Zur Einführung ungesättigter Gruppen gibt es einige wichtige organische StandardReaktionen, zu denen unter anderen die Eliminierung, die Wittig-, ${ }^{216}$ Heck-217-219 und Sonogashira-Reaktion ${ }^{220}$ gehören. Die in dieser Arbeit am häufigsten verwendete Reaktion ist die Sonogashira-Kupplung von terminalen Acetylenen mit Vinyl- oder Arylhalogeniden unter Kupfer(I)- und Pd-Katalyse. ${ }^{220}$ Dabei wird zunächst aus Bis(triphenylphosphin)palladium(II)-chlorid 221 durch reduktive Kupplung zweier Acetylene die reaktive Palladium-(0)Spezies 222 gebildet, die dann den Katalysecyclus in Gang setzt (Abb. 94). Durch oxidative Insertion in die Vinyl- oder Aryl-Halogen-Bindung ensteht die Palladium-(II)-Spezies 223, an der, durch Kupfer-(I) katalysiert, das Halogenatom durch den Acetylenrest ausgetauscht wird. Durch reduktive Eliminierung wird aus 224 das disubstituierte Acetylen frei und der Palladium-(0)-Katalysator 222 dabei zurückgebildet.

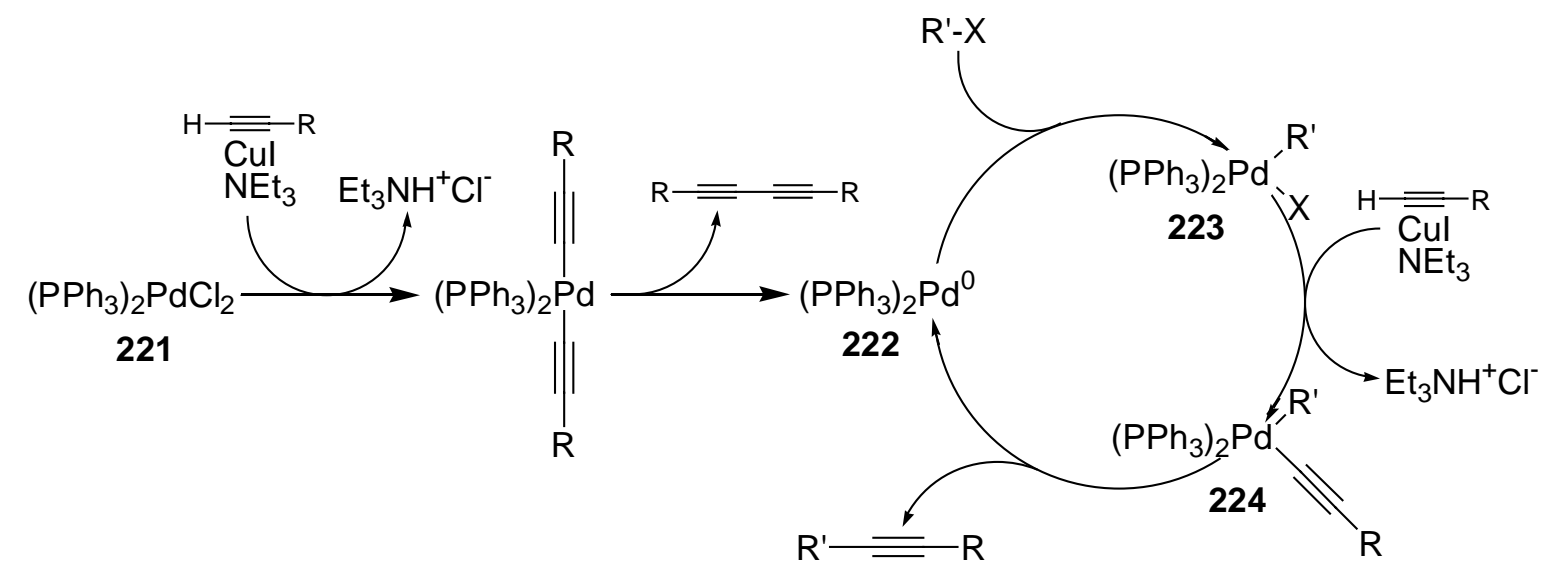

Abbildung 94. Der Katalysecyclus der Sonogashira-Reaktion

Für die Reihe der benzannelierten Enpolyine wurden zunächst die für die SonogashiraKupplung notwendigen Halogenbenzole nach literaturbekannten Verfahren dargestellt, sofern sie nicht erwerblich waren. Durch die Sandmeyer-Reaktion der entsprechenden bromierten Aniline 225, 226 und 227 und anschließendes Umkristallisieren aus Ethanol, konnten 1,2,3228 und 1,2,4-Tribrombenzol 229 sowie 1,2,3,5-Tetrabrombenzol 230 in guten bis mäßigen Ausbeuten erzielt werden (Abb. 95). ${ }^{221}$ Das 1,2,3,4-Tetrabrombenzol 231 konnte aus Hexabrombenzol 232 in 66\% Ausbeute durch Erhitzen mit einem großen Überschuß Hydrazin in Ethanol bis zum Verschwinden des schlecht löslichen 232 dargestellt werden. ${ }^{222}$ Während bei Hexachlorbenzol durch diese Reaktion zwei Chloratome in para-Stellung entfernt werden, sind es bei Hexabrombenzol aus sterischen Gründen zwei Bromatome in ortho-Stellung. ${ }^{222}$ 
Durch die Umgrignardisierung von Phenylmagnesiumbromid mit Hexabrombenzol 232 und anschließende saure Aufarbeitung wurde 1,2,3,4,5-Pentabrombenzol 233 in 18\% Ausbeute erhalten. ${ }^{223}$<smiles>[R]c1cc(Br)c(N)c(Br)c1</smiles>

225: $\mathrm{R}_{1}=\mathrm{Br} ; \mathrm{R}_{2}=\mathrm{H}$

226: $R_{1}=H ; R_{2}=B r$

227: $R_{1}=B r ; R_{2}=B r$<smiles>Brc1c(Br)c(Br)c(Br)c(Br)c1Br</smiles>

232<smiles>Brc1c(Br)c(Br)c(Br)c(Br)c1Br</smiles>

232

$$
\underset{\text { 1) } \mathrm{NaNO}}{\stackrel{\mathrm{CuBr} / \mathrm{HBr}}{\mathrm{HBr}}}
$$

228: $R_{1}=B r ; R_{2}=H ; 81 \%$

229: $\mathrm{R}_{1}=\mathrm{H} ; \mathrm{R}_{2}=\mathrm{Br} ; 70 \%$

230: $R_{1}=B r ; R_{2}=B r ; 23 \%$

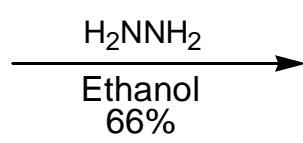

1) 1 Eq. $\mathrm{PhMgBr}$

2) $\mathrm{H}^{+} \quad 18 \%$<smiles>Brc1ccc(Br)c(Br)c1Br</smiles>

231<smiles>Brc1cc(Br)c(Br)c(Br)c1Br</smiles>

233

Abbildung 95. Darstellung der Brombenzole für die Reihe der benzannelierten Enpolyinen

Alle zwölf möglichen Bromide wurden dann nach der Sonogashira-Vorschrift mit Phenylacetylen in Triethylamin unter Rückfluß gekuppelt (Abb. 96, 97).220 Die Aufarbeitung erfolgte je nach Systemgröße durch einfaches Abfiltrieren für die Systeme mit eins bis drei Phenylethinyl-Gruppen, für die größeren Systeme durch Lösen in Chloroform und Ausschütteln mit verdünnter $\mathrm{HCl}$. Anschließend wurde das Lösungsmittel entfernt und aus Ethanol umkristallisiert. Die Ausbeuten waren gut bis sehr gut, mit Ausnahme des Hexakis(phenylethinyl)benzol 242, das aufgrund der geringen Löslichkeit von Hexabrombenzol nur eine Ausbeute von 18\% ergab. Die Verbindungen 22, 236, 238, 239 und 241 konnten dabei durch die Sonogashira-Kupplung erstmals dargestellt werden.
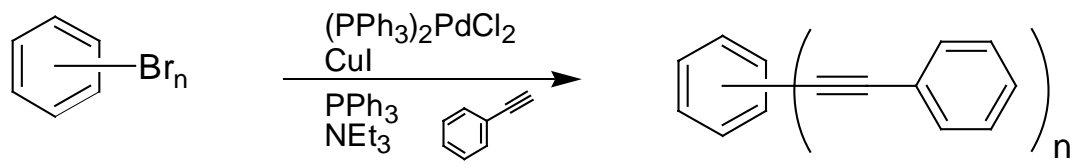

Abbildung 96. Sonogashira-Kupplung der Brombenzole 


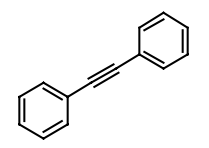

$19687 \%$

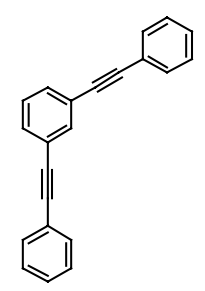

234 79\%

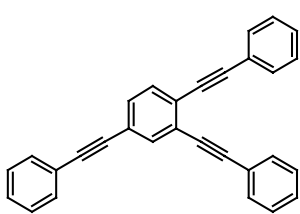

236 83\%

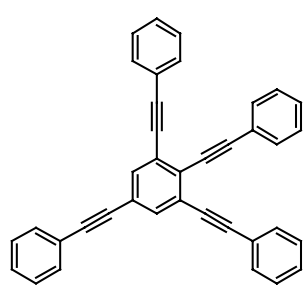

$23878 \%$

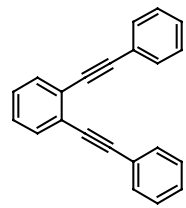

$1875 \%$

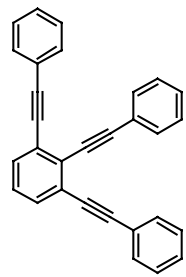

22 80\%

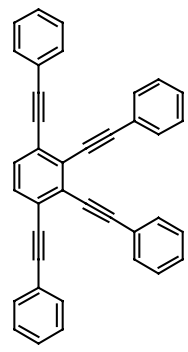

239 68\%<smiles>C(=C1C(=Cc2ccccc2)C(=Cc2ccccc2)C(c2ccccc2)C1=Cc1ccccc1)c1ccccc1</smiles>

$24174 \%$

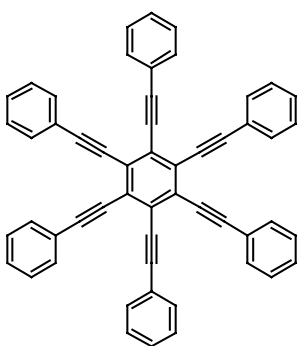

242 18\%

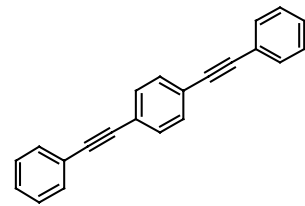

235 91\%

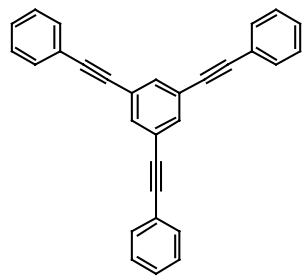

$23778 \%$

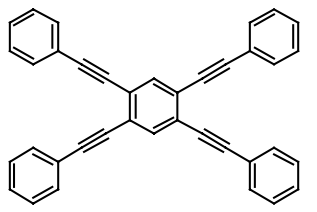

$24082 \%$ 
Durch Sonogashira-Kupplung aus den entsprechenden Bromiden und Phenylacetylen konnten auch die literaturbekannten Verbindungen 2-Vinyldiphenylacetylen 243 (88\%) und 2-(Phenylethinyl)-biphenyl 24 (79\%) dargestellt werden (Abb. 98). Das 2-Brombiphenyl wurde durch Sandmeyer-Reaktion aus 2-Aminobiphenyl erhalten. ${ }^{221}$<smiles>C=Cc1ccccc1C#Cc1ccccc1</smiles>

243<smiles>[Z6]#Cc1ccccc1-c1ccccc1</smiles>

24

Abbildung 98. Nach der Sonogashira-Vorschrift dargestellt: 2-Vinyldiphenylacetylen 243 und 2-(Phenylethinyl)-biphenyl 24

Aus 1-Bromnaphthalin 244 und Phenylacetylen wurde 1-(Phenylethinyl)naphthalin 245 in $88 \%$ Ausbeute erhalten (Abb. 99). Das 1,8-Bis(phenylethinyl)naphthalin 203 konnte ausgehend von 1,8-Diaminonaphthalin 246, wie in der Literatur beschrieben, dargestellt werden. ${ }^{44}$ Dafür wurde 246 zunächst durch Sandmeyer-Reaktion mit 37\% Ausbeute in das 1,8-Diiodnaphthalin 247 überführt ${ }^{224}$ und anschließend die Sonogashira-Kupplung mit Phenylacetylen durchgeführt, was 203 in einer Ausbeute von 22\% ergab. Die bekannte, jedoch nocheinmal selbst vermessene Kristallstruktur von 203 zeigt, daß die beiden Phenylringe um $60^{\circ}$ bzw. $63^{\circ}$ aus der Ebene des Naphthalin herausgedreht sind (Abb. 99).<smiles>Brc1cccc2ccccc12</smiles>

244<smiles>Nc1cccc2cccc(N)c12</smiles>

246

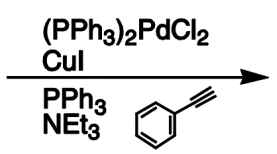

$\mathrm{NEt}_{3}$<smiles>C(#Cc1cccc2ccccc12)c1ccccc1</smiles>

245
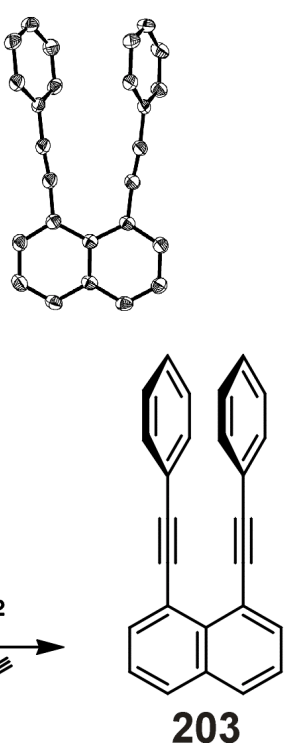

Abbildung 99. Darstellung von 1-(Phenylethinyl)naphthalin 245 und 1,8-Bis(phenylethinyl)naphthalin 203 und die Kristallstruktur von 203. 
Um das 1-(Phenylethinyl)-2-ethinylbenzol $248 \mathrm{zu}$ erhalten, wurde auf eine von der Literatur abweichende Strategie zurückgegriffen. Das trimethylsilylgeschützte Derivat von 248 wurde nicht aus schlecht zugänglichem 1-Iod-2-brombenzol oder aus 1,2-Dibrombenzol mit mäßigen Ausbeuten hergestellt und anschließend entschützt. Stattdessen diente 2Brombenzaldehyd 249 als Ausgangsmaterial, das standardmäßig mit Phenylacetylen mit einer Ausbeute von $80 \%$ zu 2-(Phenylethinyl)-benzaldehyd 250 gekuppelt wurde (Abb. 100). Dieses konnte dann mit einer abgewandelten Corey-Fuchs-Synthese in 248 überführt werden. ${ }^{225}$ Dazu wurde zunächst 251 durch die Reaktion von 250 mit $\mathrm{CBr}_{4} / \mathrm{PPh}_{3}$ in $88 \%$ Ausbeute erhalten, anschließend wurde mit LDA zu 248 umgesetzt (83\% Ausbeute).

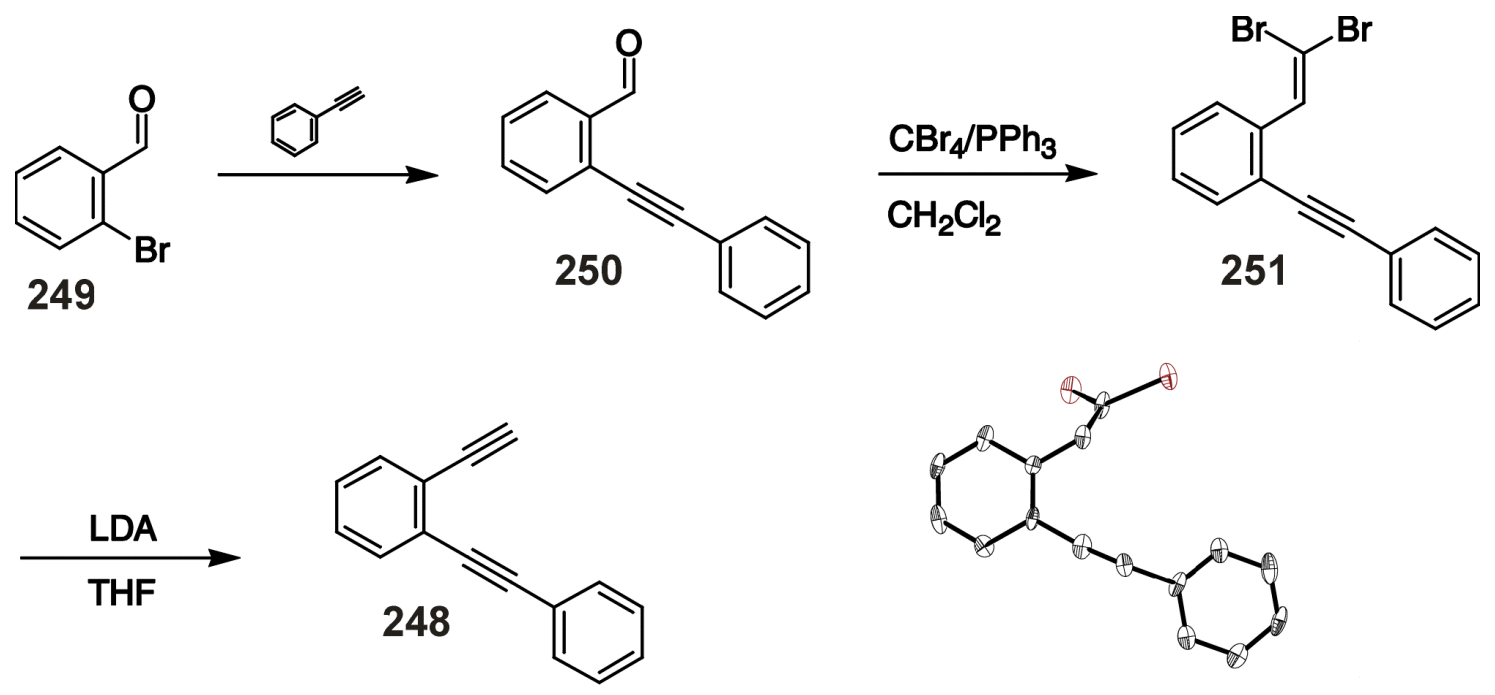

Abbildung 100. Darstellung von 1-(Phenylethinyl)-2-ethinylbenzol 249

Ausgehend von 248, konnten die drei neuen Moleküle 252, 253 und 23 synthetisiert werden (Abb. 101). Durch die Sonogashira-Kupplung mit 2-Brombiphenyl konnte 1-(2'Biphenylethinyl)-2-(phenylethinyl)benzol 252 mit einer Ausbeute von 27\% erhalten werden. Das 1-(4'-Phenylbuta-1',3'-diinyl)-2-(phenylethinyl)benzol 253 war durch eine CadiotChodkiewicz-Kupplung mit Bromphenylacetylen unter Kupfer-(I)-Katalyse mit 52\% Ausbeute zugänglich. 226 Das formale Dimere von 249, 2',2'-(Phenylethinyl)-1,4-diphenylbuta1,3-diin 23, wurde durch eine der Glaser-Reaktion ${ }^{227}$ verwandten Eglinton-Kupplung 228 unter Zugabe einer äquimolaren Menge Kupfer-(II)-acetat (Ausbeute 75\%) dargestellt. Die Kristallstruktur von 23 zeigt, daß alle Atome nahezu in einer Ebene liegen (Abb. 101). Während die Phenylringe der Butadiineinheit etwas gegeneinander versetzt sind, gleichermaßen eine Stufe mit zwei parallelen Phenylringen bilden, die einen Diederwinkel von 5. $7^{\circ}$ aufweisen, sind die Phenylringe der Acetyleneinheiten gleichsinnig um 5.6 gegen die Butadiin-Phenylringe verdreht. 


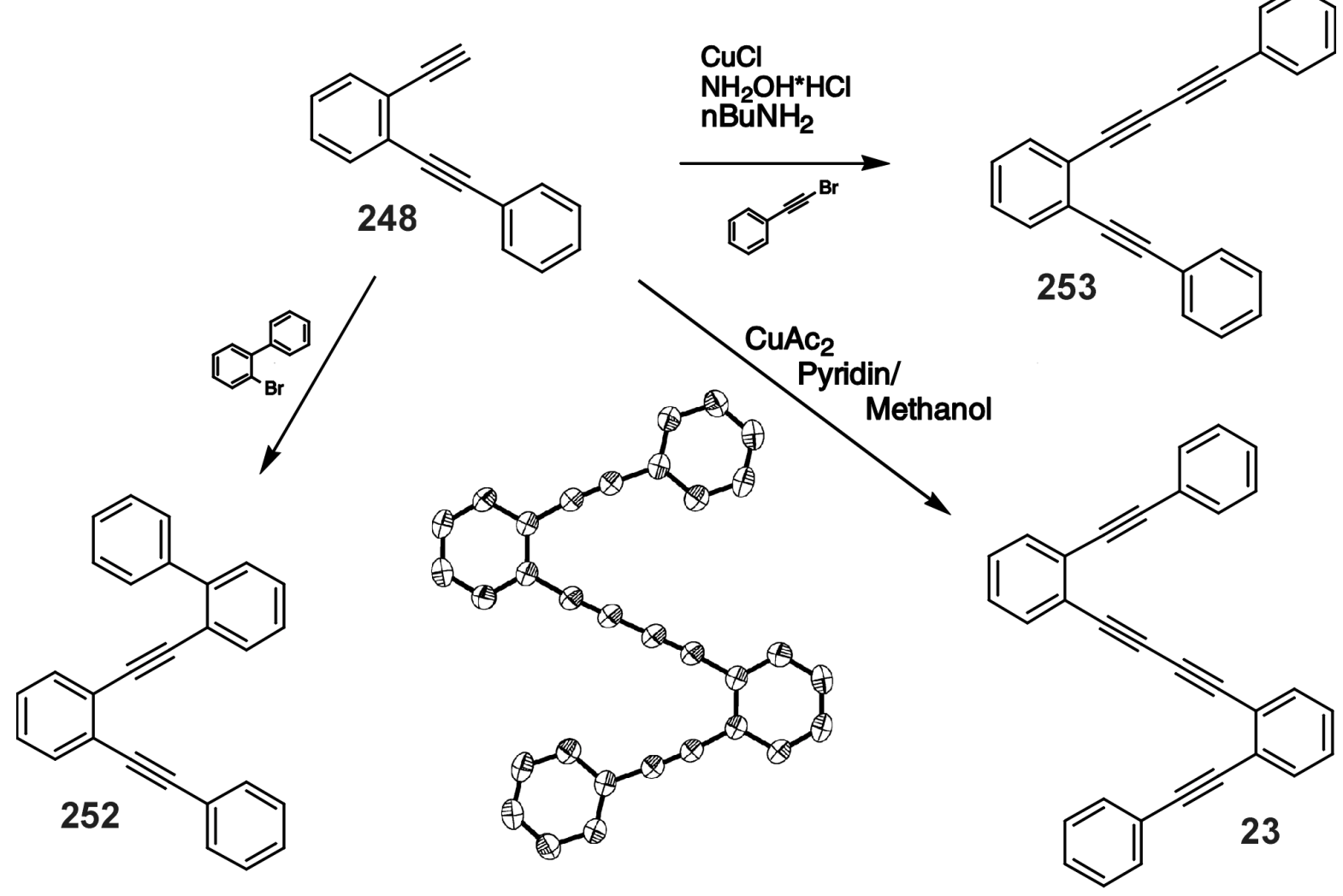

Abbildung 101. Synthese von 1-(2'-Biphenylethinyl)-2-(phenylethinyl)benzol 252, 1-(4'-Phenylbuta-1',3'diinyl)-2-(phenylethinyl)benzol 253 und 2',2'-(Phenylethinyl)-1,4-diphenylbuta-1,3-diin 23 und die Kristallstruktur von 23

Wie in der Literatur beschrieben, wurde durch Eglinton-Kupplung 1,4-Diphenylbuta1,3-diin 254 durch die Dimerisierung von Phenylacetylen in 92\% Ausbeute erhalten (Abb. 102). ${ }^{229}$

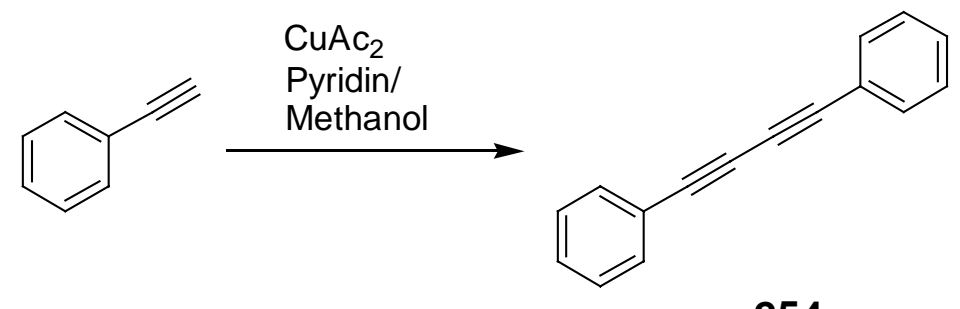

254

Abbildung 102. Darstellung von 1,4-Diphenylbuta-1,3-diin 254

Durch die Kupplung von 2-Methyl-but-3-in-2-ol mit 1,2-Dibrombenzol zu 255 und anschließender baseninduzierter Retro-Favorskii Reaktion, konnte 1,2-Diethinylbenzol 98 in $56 \%$ Gesamtausbeute nach einer Standardmethode hergestellt werden (Abb. 103). ${ }^{230}$ Vom Zwischenprodukt 255 konnte eine bis dato unbekannte Kristallstruktur erhalten werden, bei 
der man deutlich sehen kann, daß die Hydroxy-Gruppen nicht untereinander wechselwirken, sondern mit denen von Nachbarmolekülen.
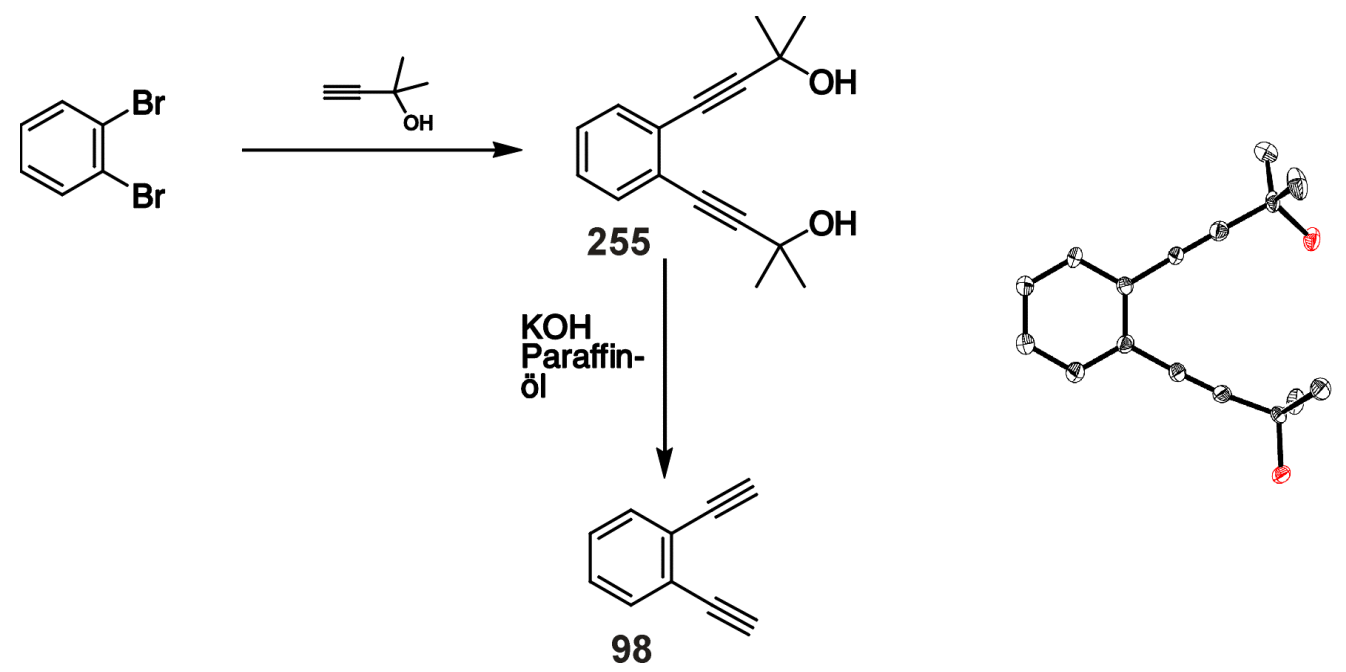

Abbildung 103. Darstellung von 1,2-Diethinylbenzol 98 und Kristallstruktur des Zwischenproduktes 255

Das bereits bekannte, aber nicht vollständig charakterisierte 1,2-Bis(4'-phenylbuta1',3'-diinyl)-benzol 256, konnte durch eine Cadiot-Chodkiewicz-Kupplung mit Bromphenylacetylen an 98 mit 74\% Ausbeute dargestellt werden (Abb. 104). ${ }^{231}$ Die Kristallstruktur zeigt, $\mathrm{da} ß$ beide Butadiin-Phenylringe konrotatorisch aus der Ebene gedreht sind, einer $73^{\circ}$, der andere $76^{\circ}$.<smiles>C#Cc1ccccc1C#C</smiles>

98
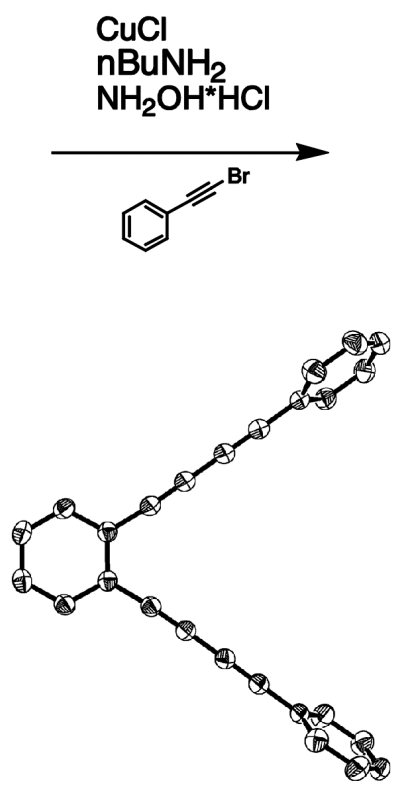

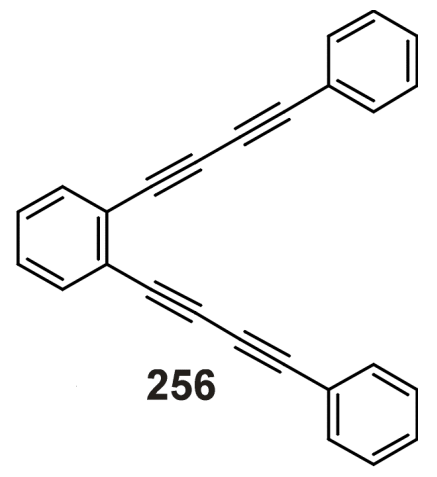

Abbildung 104. Darstellung und Kristallstruktur von 1,2-Bis(4'-phenylbuta-1',3'-diinyl)-benzol 256 
Um zu überprüfen, ob der elektrophile Angriff von Brom auf 1,4-Diphenylbut-1-in 217 ebenso wie das bereits beschriebene System $\mathrm{I}(\mathrm{py})_{2} \mathrm{BF}_{4} / \mathrm{HBF}_{4}$ eine Cyclisierung einleitet, ${ }^{43}$ wurde 217 aus 3-Phenylpropanal 257 über die Zwischenstufen 1,1-Dibrom-4-phenylbut-1-en 258 und 4-Phenylbut-1-in 259 durch eine Corey-Fuchs-Synthese und anschließende Sonogashira-Reaktion mit einer Gesamtausbeute von $41 \%$ über alle Schritte dargestellt (Abb. 105).<smiles>O=CCCc1ccccc1</smiles>

257

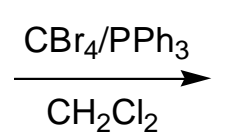

$\mathrm{CH}_{2} \mathrm{Cl}_{2}$<smiles>BrC(Br)=CCCc1ccccc1</smiles>

258
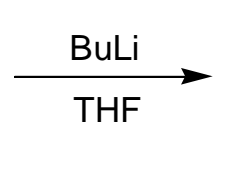<smiles>C#CCCc1ccccc1</smiles>

259

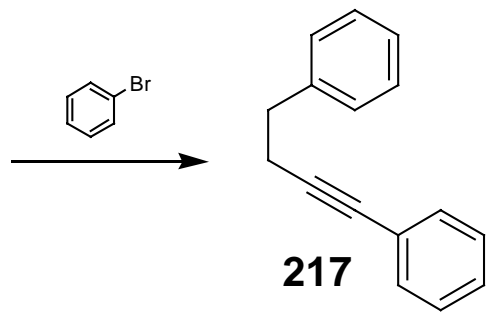

Abbildung 105. Darstellung von 1,4-Diphenylbut-1-in 217

Zur Einführung von Doppelbindungen wurde die Wittig-Reaktion mit THF als Lösungsmittel und Butyl-Lithium in Hexan als Base verwendet. Durch die Reaktion von 250 mit Benzyl-triphenylphosphoniumbromid konnte das bekannte aber nicht vollständig charakterisierte $(E)-2$-(Phenylethinyl)stilben 260 in einer Ausbeute von 63\% dargestellt werden (Abb. 106).232 Während ansonsten bei der Wittig-Reaktion cis-/trans-Gemische auftreten, wird von $\mathbf{2 6 0}$ ausschließlich das trans-Isomer gebildet, was vermutlich am Einfluß der Phenylethinylgruppe auf die Wittig-Zwischenprodukte liegt. An sterischen Einflüssen liegt es auch, daß bei der Reaktion von 250 mit Diphenylmethyltriphenylphosphoniumbromid die neue Verbindung 1-(Phenylethinyl)-2-(2',2'-diphenylvinyl)benzol 25 nur in 19\% Ausbeute gebildet wurde.<smiles>O=[N+]([O-])C#Cc1ccccc1/C=C/c1ccccc1</smiles>

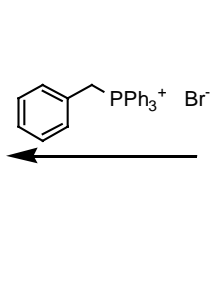

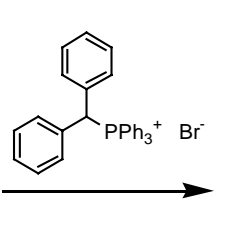<smiles>[C+]#Cc1ccccc1C=C(c1ccccc1)c1ccccc1</smiles>

Abbildung 106. Darstellung von (E)-2'-(Phenylethinyl)stilben 260 und 1-(Phenylethinyl)-2-(2',2'diphenylvinyl)benzol 25 
Ebenfalls durch die Wittig-Reaktion konnten die bekannten Verbindungen 2-Methyl5-phenylpent-2-en 261 und 1,1,4-Triphenylbut-1-en 262 aus (3-Phenylpropyl)-triphenylphosphoniumbromid und Aceton bzw. Benzophenon in $44 \%$ bzw. $72 \%$ Ausbeute erhalten werden (Abb. 107).<smiles>CC(C)=CCCc1ccccc1</smiles>

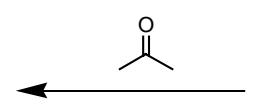

261<smiles>Br[PbH2]CCc1ccccc1</smiles><smiles>Cc1ccc(C(=O)c2ccccc2)cc1</smiles><smiles>C1CCCCC1</smiles><smiles>C(CCc1ccccc1)=C(c1ccccc1)c1ccccc1</smiles>

262

Abbildung 107. Darstellung von 2-Methyl-5-phenylpent-2-en 261 und 1,1,4-Triphenylbut-1-en 262 


\subsection{Kationisch induzierte Additions- und Cyclisierungsreaktionen}

Die kationisch induzierten Reaktionen wurden alle durch Zutropfen eines Äquivalents einer 0.4 molaren Bromlösung in Chloroform zu dem entsprechenden Eduktmolekül bei Raumtemperatur durchgeführt. Die Aufarbeitung erfolgte durch Ausschütteln mit Natriumsulfit-Lösung, Waschen der organischen Phase mit Wasser, Trocknen über Magnesiumsulfat und Entfernen des Lösungsmittels.

Die einfachste der durchgeführten Reaktionen, die Addition von Brom an Phenylacetylen 263, zeigt, daß die Reaktion bei einfacher Aromatensubstitution noch über ein Bromoniumion ablaufen muß, da die Produkte trans-selektiv gebildet werden $(E: Z=95: 5$, Abb. 108). Ein Kontrollexperiment mit 1-Phenyl-1-octin beweist, daß auch mit einem Aromaten- und einem Alkylsubstituenten die Dreifachbindung immer noch selektiv trans addiert $(E: Z=90: 10)$.

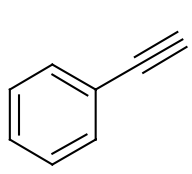

263

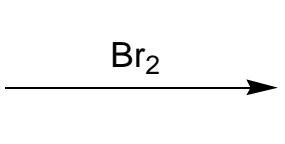

(E)-264 95\%<smiles>Br/C=C(\Br)c1ccccc1</smiles>

(Z)-264 5\%

Abbildung 108. Addition von Brom an Phenylacetylen 263

Bei doppelter Aromatensubstitution läuft die Addition nicht mehr über das Bromoniumion als Zwischenstufe ab, sondern es wird ein offenes, resonanzstabilisiertes Benzylkation gebildet, das von beiden Seiten aus durch $\mathrm{Br}^{-}$angegriffen werden kann. Die Addition von Brom an Diphenylacetylen ergibt demnach ein nahezu äquimolares Gemisch von cis- und trans-Dibromstilben $(E: Z=60: 40$, Abb. 109).

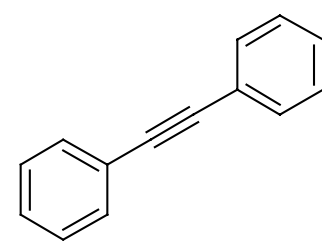

196
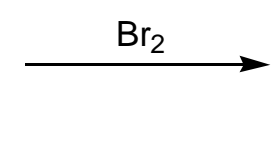

(E)-199 60\%<smiles>BrC(=C(Br)c1ccccc1)c1ccccc1</smiles><smiles>BrC(=C(Br)c1ccccc1)c1ccccc1</smiles>

(Z)-199 40\%

Abbildung 109. Addition von Brom an Diphenylacetylen 196 
In der Literatur existieren wenige spektroskopische Daten über die isomeren Dibromstilbene 199. In einer Quelle werden nur ${ }^{1} \mathrm{H}$-Verschiebungen angegeben, die mit den hier für (E)-199 gemessenen übereinstimmen, es gibt dort jedoch zusätzlich eine Kristallstruktur, die eindeutig (E)-199 zeigt. ${ }^{233}$ In eine zweiten Quelle werden nur ${ }^{13} \mathrm{C}$ Verschiebungen für $(\boldsymbol{E})$-199 angegeben, die aber mit den hier gemessenen von $(\boldsymbol{E})$-199 nicht übereinstimmen, sondern mit $(Z)-199$, obwohl $(\boldsymbol{E})-199$ aus Diphenylacetylen 196 und Tetra$n$-butylammoniumtribromid hergestellt wurde, das stereoselektiv das anti-Addukt liefern sollte. ${ }^{215}$ Es besteht deshalb grundlegendes Interesse, diese widersprüchlichen Ergebnisse in Einklang zu bringen. Die kristallographische Vermessung eines Einkristalls von (E)-199 war leider nicht erfolgreich, der direkte Beweis durch die Verknüpfung der Kristallstruktur mit akkuraten NMR-Daten konnte so nicht geführt werden. Allerdings konnte ein naher Verwandter von 196 auf dieselbe Art bromiert werden und ergab eine Mischung aus (E)-265 und (Z)-265 im Verhältnis 60:40 (Abb. 110). Aus dem Gemisch konnte (E)-265 durch fraktionierte Kristallisation abgetrennt und untersucht werden. Zusätzlich gelang es, einen Einkristall zu züchten und eine Röntgenstruktur zu erhalten. Diese zeigt klar, daß es sich um ein trans-Produkt handelt, bei dem die beiden Doppelbindungs-Kohlenstoffatome im ${ }^{13} \mathrm{C}$ NMR eine Verschiebung von 118.48 und 117.34 ppm zeigen. Dieser Wert stimmt mit der Verschiebung von 118.05 ppm des Dibromstilbens (E)-199 überein, die Zuordnung ist also in der zweiten Literaturquelle trotz stereoselektiver Reaktionsführung offensichtlich nicht richtig. Unterstützt wird diese Tatsache noch durch eigene ab initio Berechnungen der NMRVerschiebungen, die aufgrund der relativistischen Effekte des Broms zwar keine guten absoluten Werte liefern, für die Doppelbindungs-C aber brauchbare relative Werte produzieren. Auch hier ist (Z)-199 zu tieferem Feld verschoben als (E)-199. 

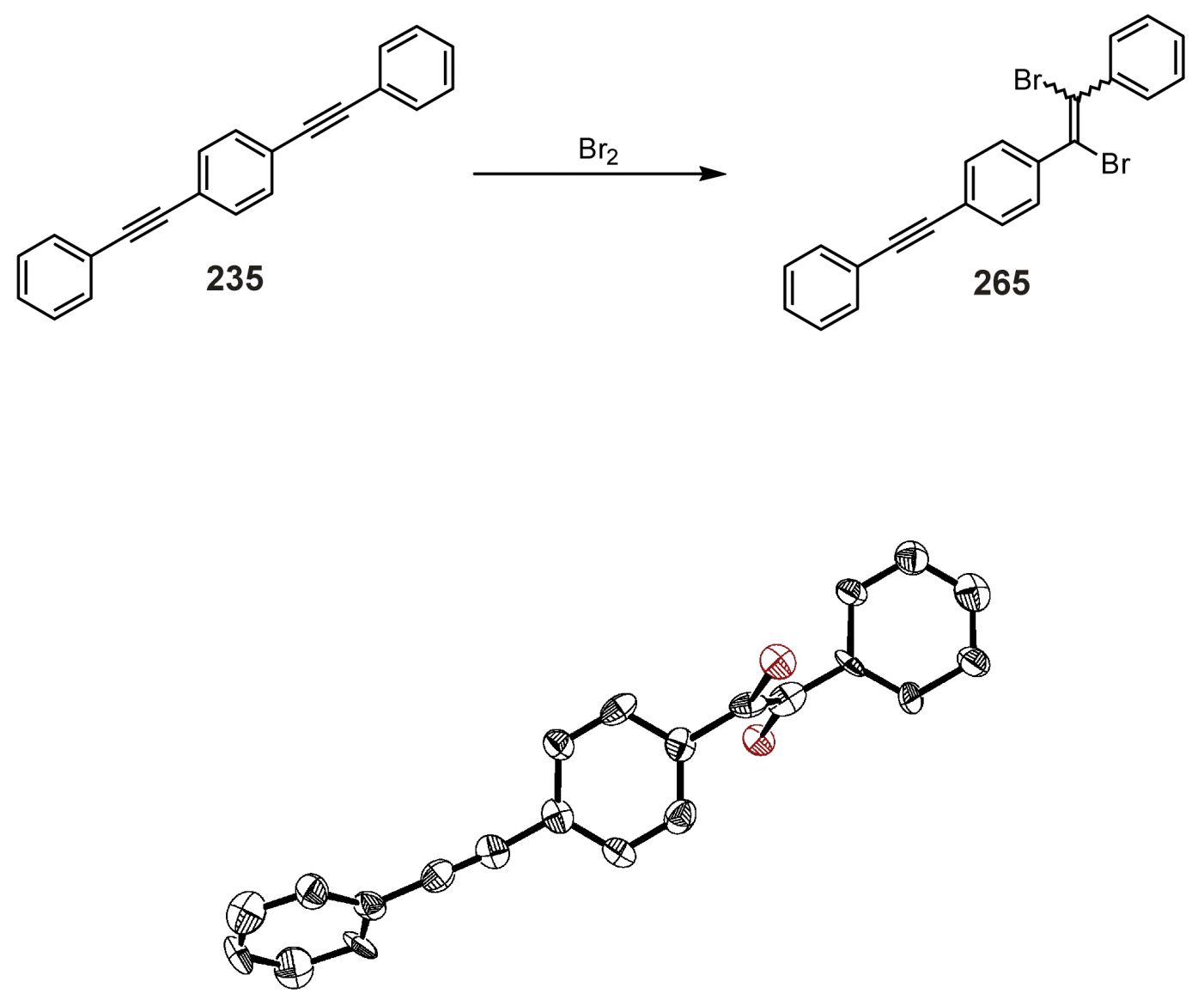

Abbildung 110. Addition von Brom an 1,3-Bis(phenylethinyl)benzol 235 und Kristallstruktur von (E)-265

Wie schon aus der Literatur bekannt, wird Brom nicht einfach nur an 1,2Bis(phenylethinyl)benzol 18 addiert, sondern die beiden Acetyleneinheiten interagieren miteinander, was zu der Cyclisierung zu den Benzofulvenen (E)-195 und (Z)-195 im Verhältnis 85:15 führt (Abb. 111). ${ }^{45}$ Die Isomere können auf einfachem Weg durch Umkristallisieren aus Ethanol bzw. Hexan rein erhalten werden. Durch Säure wird jedoch die Isomerisierung an der Doppelbindung katalysiert und es wird wieder das Gleichgewicht von (E)- und (Z)-195 hergestellt. Selbst in $\mathrm{CDCl}_{3}$, das Spuren von $\mathrm{HCl}$ enthält, findet die Umlagerung statt. Auf die spezielle ${ }^{1} \mathrm{H}-\mathrm{NMR}$-spektroskopische Eigenschaft des Wasserstoffatoms an C-7 (Abb. 111, rot markiert) wurde in der Literatur bereits hingewiesen, aufgrund der damals schlechten NMR-Auflösung konnten jedoch keine verläßlichen Daten erhalten werden. ${ }^{45}$ Es wurde spekuliert, daß die Nähe zu dem elektronenreichen Brom in (E)-195 eine Tieffeldverschiebung zu 8.80 ppm auslöst, während die Nähe zu dem Ringstrom des Phenylrings in (Z)-195 eine Verschiebung zu hohem Feld (6.15 ppm) ergibt. Diese Vermutungen konnten mittels eines hochaufgelösten ${ }^{1} \mathrm{H}-\mathrm{NMR}-$ Spektrums, sowie erstmals 
aufgenommenen ${ }^{13} \mathrm{C}$ - und 2D-NMR-Spektren bestätigt und durch die bis dato unbekannte Kristallstrukturanalyse eines gezüchteten Einkristalls von $(\boldsymbol{E})$-195 untermauert werden. Die Eigenschaften dieser intramolekularen Protonen-"Sonde" wird auch in weiteren Strukturanalysen herangezogen, um Aussagen über die Stellung der Substituenten an der exocyclischen Doppelbindung zu machen.
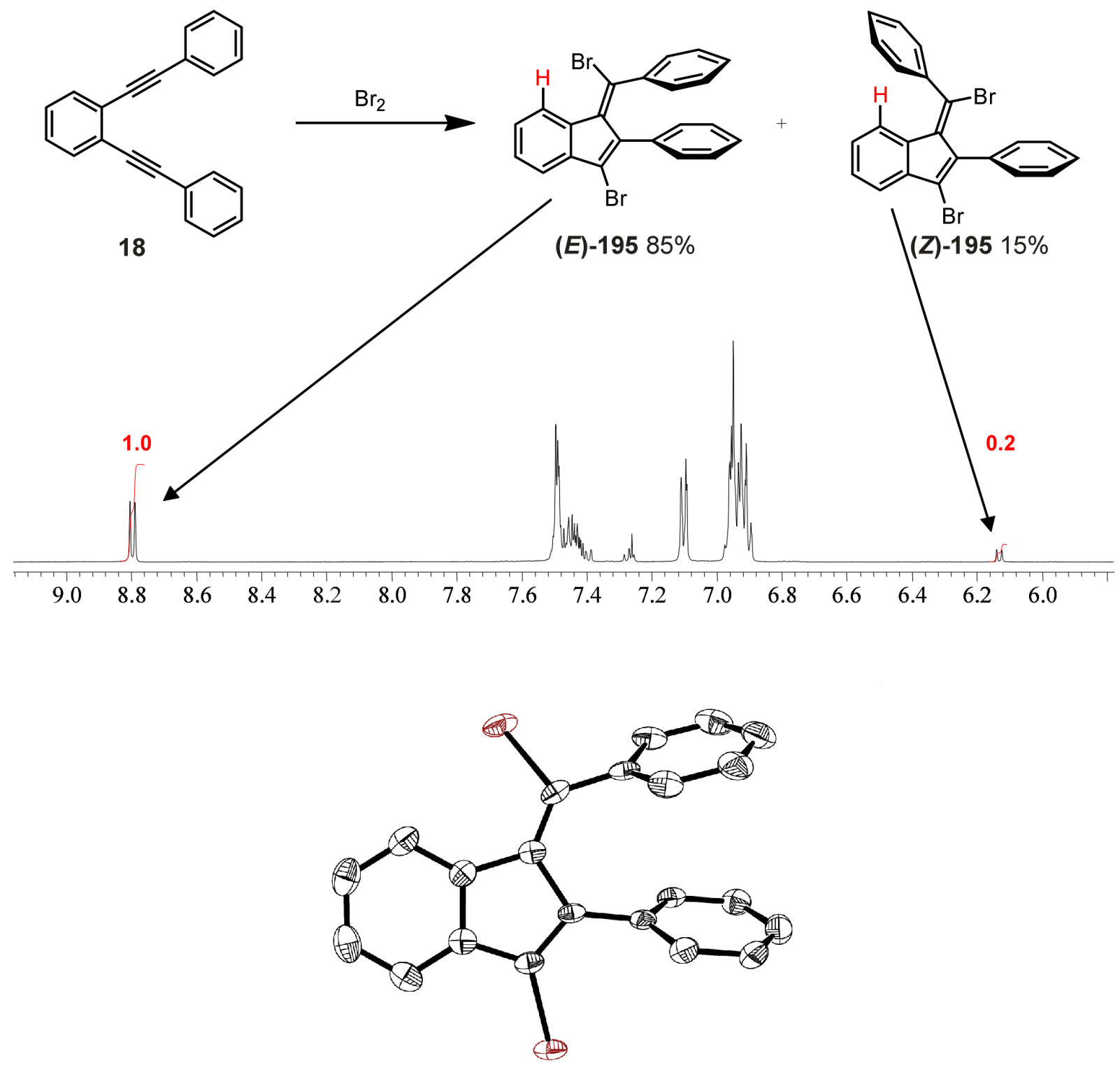

Abbildung 111. Cyclisierung von 1,2-Bis(phenylethinyl)benzol 18, ${ }^{1} \mathrm{H}$-NMR-Spektrum der Reaktionsmischung und Kristallstruktur von $(\boldsymbol{E})$-195

Bei der Addition von Brom an das nächsthöhere Endiin-Homolog 1,2,3Tris(phenylethinyl)benzol 22 ergaben sich große Probleme bei der Trennung der bromierten Produkte. Aus dem ${ }^{1}$ H-NMR konnte auf mindestens drei verschiedene Produkte geschlossen werden (Abb. 112, unteres Spektrum). Anhand der Verschiebungen des "Sonden"-Protons, 
wie sie auch bei den Cyclisierungsprodukten von $\mathbf{1 8}$ auftraten, wurden den Signalen bei 6.02 und 8.16 ppm die Strukturen (E)-266 und (Z)-266 zugeordnet. Durch fraktionierte Kristallisation konnte (Z)-266 abgetrennt werden, die beiden Signale bei 8.20 und 8.26 ppm wurden jedoch in unverändertem Verhältnis zueinander und zum Hauptprodukt wiedergefunden (1:1:4). Durch präparatives Säulen waren die Bromide nicht zu trennen, vielmehr bildete sich während der Trennung durch die sauren Eigenschaften des Kieselgels (Z)-266 aus (E)-266. Eine Trennung konnte schließlich durch präparative HPLC erreicht werden, wobei durch die schlechte Löslichkeit der Bromide in Acetonitril jeweils nur einige Milligramm auf die Säule appliziert werden konnten. Anhand der sauberen ${ }^{1} \mathrm{H}-,{ }^{13} \mathrm{C}$ - und 2D-NMR-Spektren konnte dem isolierten Produkt die Formel von (E)-266 zugeordnet werden (Abb. 112, oberes Spektrum). Die Probe wurde dabei in $\mathrm{CD}_{2} \mathrm{Cl}_{2}$ vermessen, um bei der lange Meßzeit, die durch die geringe Konzentration der Probe obligat war, die Isomerisierung zu (Z)-266 zu vermeiden. Die anderen Produkte konnten nicht rein erhalten werden. Aufgrund seiner offensichtlichen Ähnlichkeit $\mathrm{zu}$ dem isolierten Produkt wurde dem unbekannten Nebenprodukt vorläufig die Formel 267 zugeschrieben.
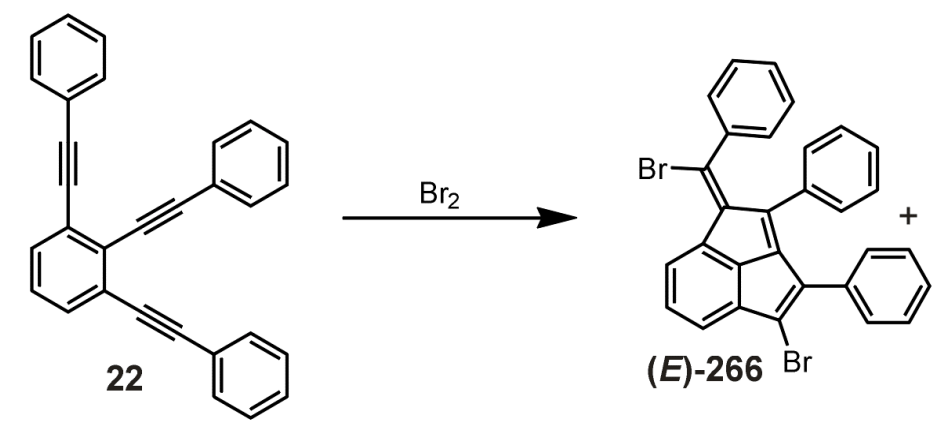

(Z)-266<smiles>Brc1c(-c2ccccc2)c(-c2ccccc2)c(-c2ccccc2)c2c(Br)cccc12</smiles>

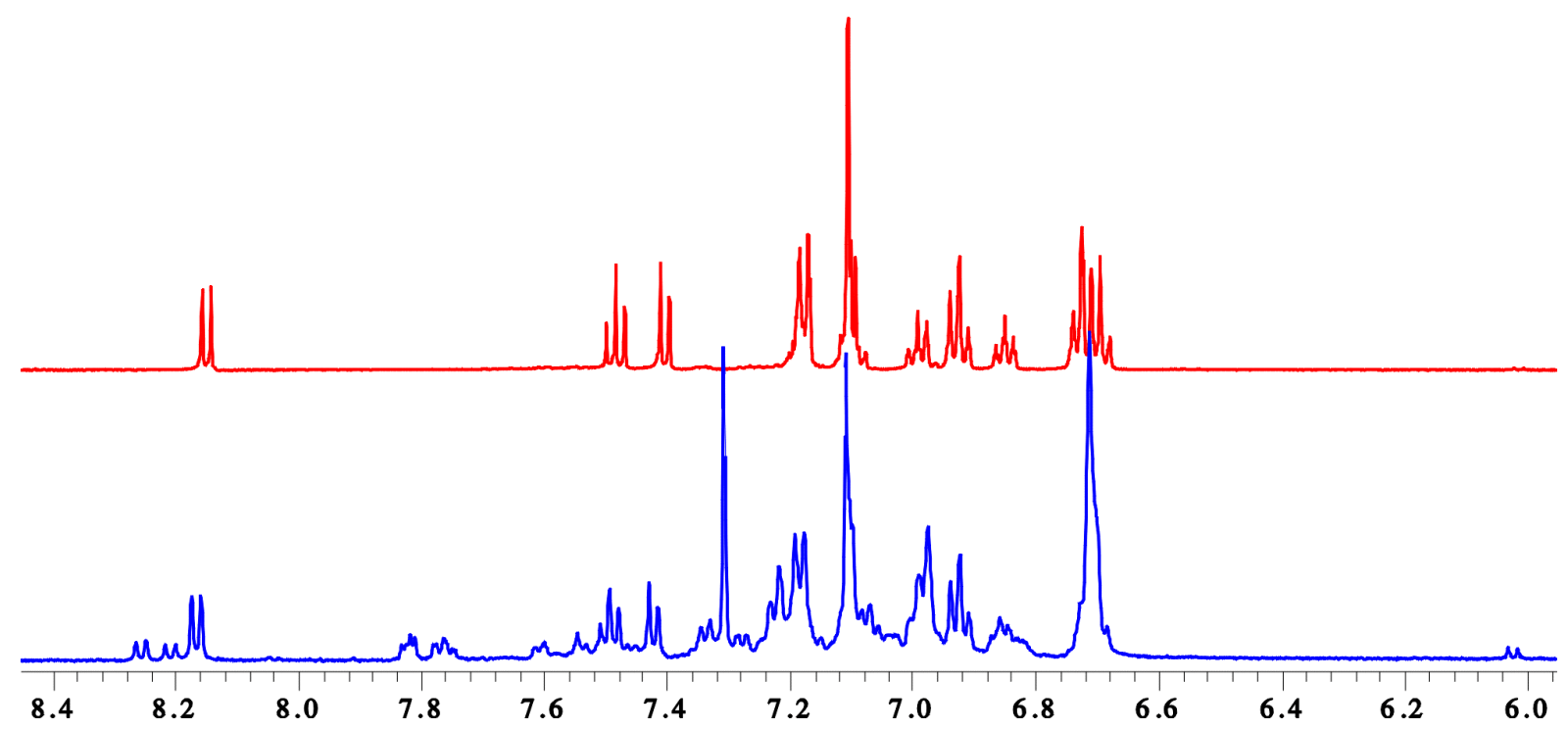

Abbildung 112. Cyclisierung von 1,2,3-Tris(phenylethinyl)benzol 18, das Rohproduktspektrum (unten) und das Spektrum des reinen $(\boldsymbol{E})-\mathbf{2 6 6}$ 
Das 1-Phenylethinylnaphthalin 245 wurde als Edukt für die Bromierung ausgewählt, um die Frage $\mathrm{zu}$ beantworten, ob sich auch fünfgliedrige Ringe bei einem elektrophilen Angriff, gefolgt von einer $\mathrm{S}_{\mathrm{E}} \mathrm{Ar}$ am Aromaten bilden können, oder ob sich wirklich nur sechsgliedrige Ringe ergeben, so wie in der Einleitung behauptet. Die Reaktion von $\mathbf{2 4 5}$ und Brom ergab tatsächlich nur $(\boldsymbol{E})$-267 und (Z)-267 als Additionsprodukt an die Dreifachbindung und keine Reaktionskaskade zu 268 (Abb. 113). Das Verhältnis von trans- zu cis-Isomer ist dabei 60 : 40, wie bei der Bromierung von 196.

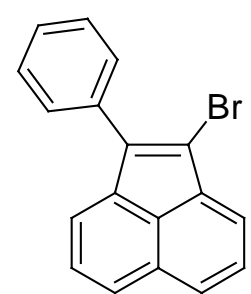

268

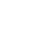

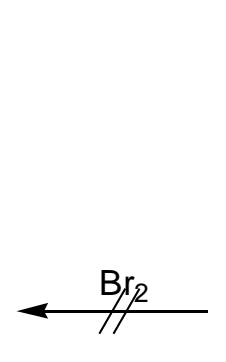

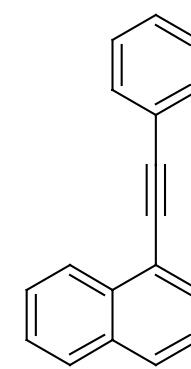

245

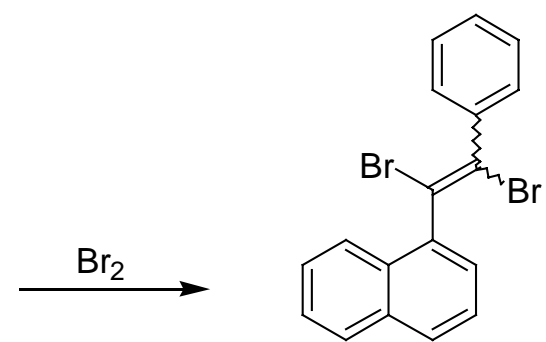

267

Abbildung 113. Addition von Brom an 1-Phenylethinylnaphthalin 245

Beim Versuch die spektroskopischen Daten des Reaktionsproduktes von 1,8Bis(phenylethinyl)naphthalin 203 zu vervollständigen, konnte als Hauptprodukt nicht eines der isomeren Acenapthenderivate 206, wie in der Literatur beschrieben, ${ }^{44}$ beobachtet werden. Vielmehr wurde das einfache Additionsprodukt 269 gefunden, das sich im ${ }^{13} \mathrm{C}-\mathrm{NMR}$ Spektrum durch seine, vom Edukt verschiedenen, Acetylenkohlenstoff-Signale verrät (Abb. 114). Das Produkt konnte nicht vollständig gereinigt werden, es ist jedoch klar, daß es sich dabei nicht um 206 handelt, zumal es sich um einen, wie auch in der Literatur beschrieben, ${ }^{44}$ farblosen Feststoff handelt, das entsprechende 206 ohne Bromatome jedoch bereits orange ist und deshalb die Verbindung 206 selbst ebenfalls farbig sein sollte. Die in der gleichen Quelle beschriebene Reaktion von $\mathbf{2 0 3}$ mit Bromwasserstoff ergibt nur das zweifache Additionsprodukt, obwohl auch hier ein elektrophiler Angriff vorliegt. Die experimentellen Daten wurden offensichtlich nicht richtig interpretiert, es sind allerdings zur Absicherung noch weitere Experimente nötig. 


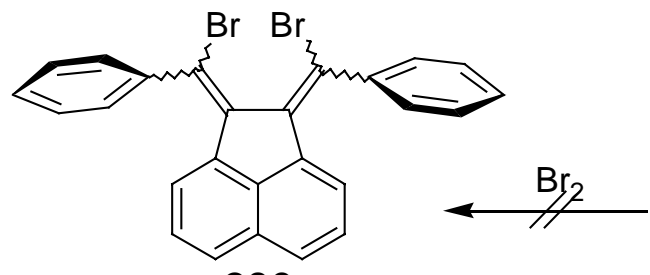

206

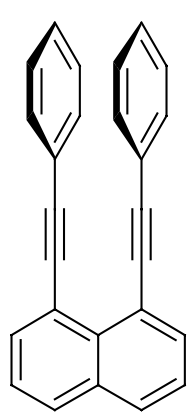

203

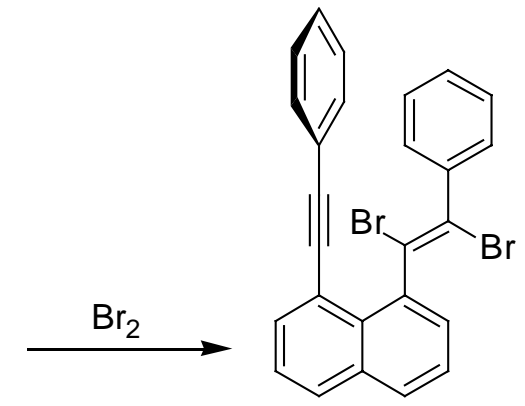

269

Abbildung 114. Reaktion von 1,8-Bis(phenylethinyl)naphthalin 203 mit Brom

Die bekannte Iodierung von 1,4-Diphenylbut-1-in 217 mit $\mathrm{I}(\mathrm{py})_{2} \mathrm{BF}_{4} / \mathrm{HBF}_{4}$ wurde bereits vorgestellt. ${ }^{43}$ Die einfache Zugabe von Brom statt des komplizierten Iodosylkomplexes führt jedoch zum gleichen Ergebnis, einer Cyclisierung zu 270 (Abb. 115). Der Angriff des "Br ${ }^{+"}$ erzeugt in diesem Fall ein stabilisiertes Benzylkation, das dann den anderen Aromaten nach einem elektrophilen, aromatischen Substitutionsmechanismus ( $\left.\mathrm{S}_{\mathrm{E}} \mathrm{Ar}\right)$ angreift. Im Gegensatz zu allen vorherigen Reaktionen enthält das Zielmolekül 270 nur ein Bromatom, es handelt sich also eher um eine Kaskaden-Substitution. Im Einklang mit den BaldwinRegeln ${ }^{234}$ wird in diesem Fall ein sechsgliedriger Ring mit endocyclischer Doppelbindung einem fünfgliedrigen mit exocyclischer Doppelbindung vorgezogen.
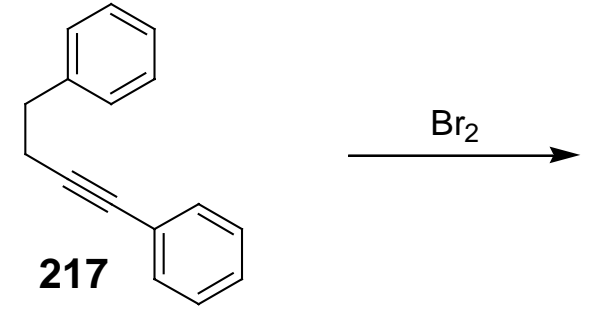

Abbildung 115. Cyclisierung von 1,4-Diphenylbut-1-in 217<smiles>BrC1=C(c2ccccc2)c2ccccc2CC1</smiles>

270

Die Cyclisierung von 2-Phenylethinyl-biphenyl 24 läuft analog der vorgenannten Cyclisierung von $217 \mathrm{ab}$, da man 24 als benzanneliertes Derivat von 217 ansehen kann. Die Reaktion mit Brom führt zu 9-Brom-10-phenylphenanthren 271, das schon auf anderem, komplizierterem Wege über die entsprechende Substitution am Phenanthrenkörper dargestellt werden konnte, dessen spektroskopische Eigenschaften jedoch noch nicht bekannt sind (Abb. 116). ${ }^{235}$ Die Kristallstrukturanalyse von 271 zeigt, daß die annelierten Ringe alle, wie zu erwarten, in einer Ebene liegen, und daß der Phenylsubstituent $74^{\circ}$ gegen die Ebene verdreht ist, um Platz für den Bromsubstituenten zu schaffen. 
<smiles>[Z10]c1c(-c2ccccc2)c2ccccc2c2ccccc12</smiles>

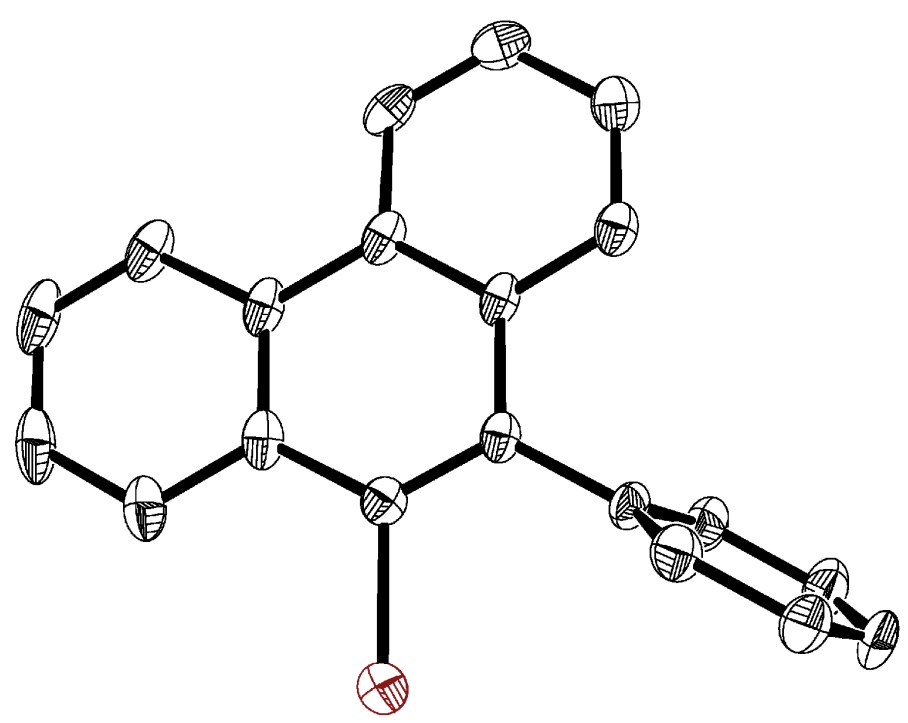

Abbildung 116. Cyclisierung von 2-Phenylethinyl-biphenyl 24 und Kristallstruktur von 271

Das Produkt 271 der Cyclisierung von 24 hat dasselbe Strukturelement wie das Edukt von 24, nämlich 2-Brombiphenyl. Dieser Vergleich legt es nahe, die Reaktionsfolge in einer Art "Selbstreplikation" fortzuführen. Deshalb wurde 271 wiederum nach der SonogashiraVorschrift mit Phenylacetylen gekuppelt, um 9-Phenyl-10-(phenylethinyl)-phenanthren 272 $\mathrm{zu}$ erhalten, das das gleiche Strukturelement wie das 2-Phenylethinyl-biphenyl $\mathbf{2 4}$ aufweist (Abb. 117). Die Kristallstrukturanalyse von 272 zeigt, daß alle Atome, bis auf die des Phenylsubstituenten, in einer Ebene liegen, die aber leicht verdreht ist $\left(<5^{\circ}\right)$. Der Phenylsubstituent selbst ist wie bei 271 um $76^{\circ}$ aus der Ebene gedreht.

Die Reaktion mit Brom ergibt 9-Brom-10-phenyl-benzo[g]chrysen 273, das wiederum die gleiche Struktur wie 2-Brombiphenyl und 271 aufweist. Leider konnte es weder durch präparatives Säulen noch durch mehrfaches Umkristallisieren von einem nicht zu bestimmenden Nebenprodukt getrennt werden. Eine weitere "selbstreplizierende" Stufe wurde nicht untersucht, es sollte ab dieser Stufe auch etwas schwieriger werden, denn das System müßte sich ab jetzt wie die Helicene aus der Ebene herausbewegen. 
<smiles>CC(C)C(C)Br</smiles>

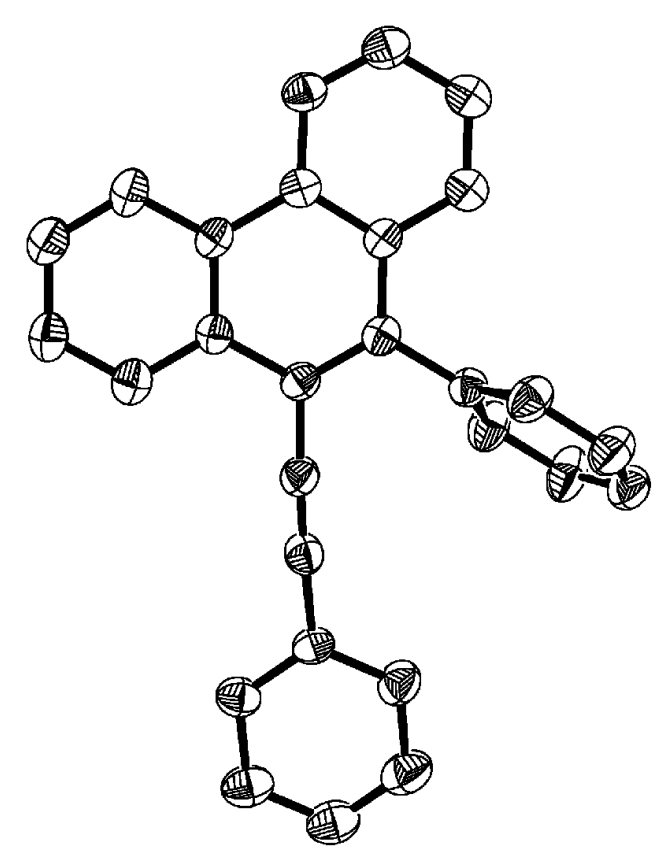

Abbildung 117. Cyclisierung von 9-Phenyl-10-(phenylethinyl)-phenanthren 272 und Kristallstruktur von 272

Bei der Reaktion von Brom mit 2-Vinyldiphenylacetylen 243, einem System ähnlich 24, bei dem ein Phenylring durch eine Vinylgruppe ersetzt ist, macht sich sowohl die Reaktivität der Doppelbindung als auch die fehlende Phenylsubstitution bemerkbar (Abb. 118). Das "Br" greift in diesem Fall die Doppelbindung zuerst an und zwar, nach der Regel von Markownikow, an der $=\mathrm{CH}_{2}$-Gruppe. Das gebildete stabilisierte Benzylkation könnte als Cyclisierung nur den viergliedrigen Ring 274, der wegen der hohen Ringspannung praktisch auszuschließen ist, oder den fünfgliedrigen Ring 275 bilden, der jedoch nicht durch Delokalisierung stabilisiert ist. Da beide Cyclisierungen unvorteilhaft sind, geschieht als Hauptreaktion die einfache Addition von Brom an die Doppelbindung zu 2-(1',2'Dibromethyl)diphenylacetylen 276 ohne Cyclisierung. 


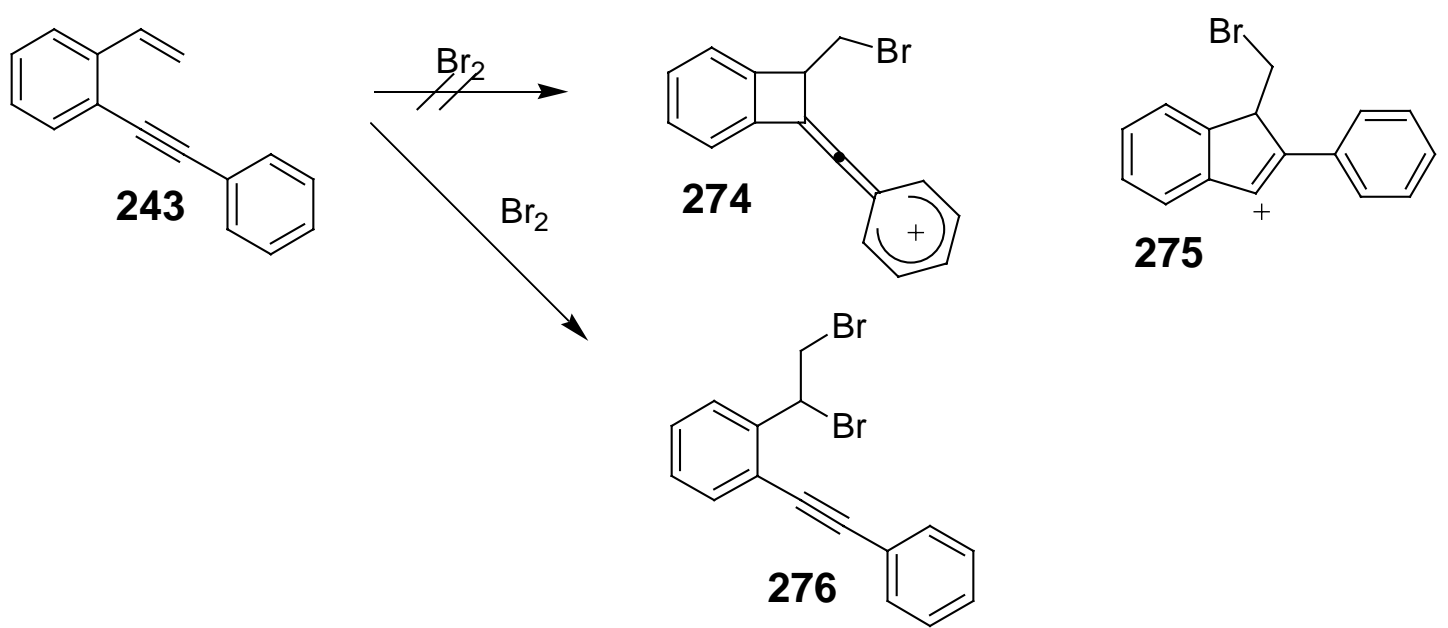

Abbildung 118. Reaktion von Brom mit 2-Vinyldiphenylacetylen 243

Die Reaktion von (E)-2'-(Phenylethinyl)stilben 260 liefert leider eine Anzahl untrennbarer Additions- und Cyclisierungsprodukte, da in 260 die Doppelbindung immer noch reaktiver als die Dreifachbindung ist. Ein Angriff des $" \mathrm{Br}^{+}$" an der Substituentenseite der Doppelbindung sollte wie zuvor bei $\mathbf{2 4 3}$ zu einem einfachen Additionsprodukt 277 führen (Abb. 119). Bei einem kernnahen Angriff sollte eine Cyclisierung stattfinden, wobei 278 als (E)- und (Z)-Isomer an der neuen Doppelbindung und zusätzlich als cis/trans-Isomer am neuen fünfgliedrigen Ring gebildet werden kann. Ein trotz der geringeren Reaktivität möglicher Angriff auf die Dreifachbindung sollte zu dem Cyclisierungsprodukt 279 führen. Die Ähnlichkeit aller dibromierten Produkte machte die Trennung jedoch unmöglich.

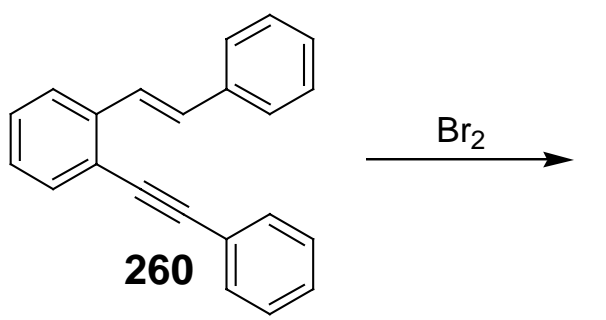<smiles>BrC(c1ccccc1)c1ccccc1C(Br)C(Br)c1ccccc1</smiles>

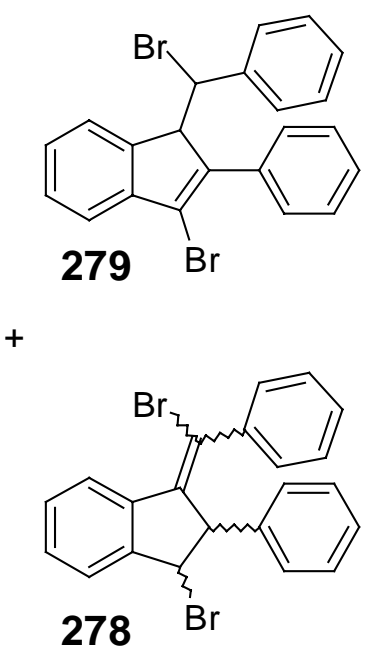

Abbildung 119. Reaktion von Brom mit (E)-2'-(Phenylethinyl)stilben 260 unter Bildung eines nicht trennbaren Gemisches der Dibromide 277, 278 und 279 
Bei der Reaktion von 2-(2',2'-diphenylvinyl)diphenylacetylen 25 mit Brom ergibt sich ein überraschendes Produkt (Abb. 120). Es findet weder die Addition an die Doppelbindung statt, noch reagiert das Brom überhaupt mit der Doppelbindung. In 25 ist die Dreifachbindung reaktiver als die Doppelbindung und wird elektrophil von $" \mathrm{Br}^{+} "$ angegriffen, um dann anschließend die Doppelbindung zu attackieren. Dieses cyclisierte Kation stabilisiert sich nicht durch die Addition von $\mathrm{Br}^{-}$, da die zwei Phenylgruppen des Vinylsubstituenten das Kohlenstoffatom an dem sie sitzen sterisch zu sehr abschirmen. Es findet statt dessen, wie bei den Aromaten, ein Additions-Eliminations-Mechanismus statt, bei dem das Kation ein Proton zur Stabilisierung abgibt, und es entsteht das triphenylsubstituierte Benzfulven 280. Die Kristallstrukturanalyse zeigt, daß die Benzfulven-Einheit planar ist, und daß der Phenylring in der 2-Position um $50^{\circ}$ aus der Ebene gedreht ist. Um der sterischen Spannung auszuweichen, ist die exocyclische Doppelbindung um $22^{\circ}$, die Phenylringe um $80^{\circ}$ bzw. $75^{\circ}$ gegen die Benzfulvenebene verdreht.
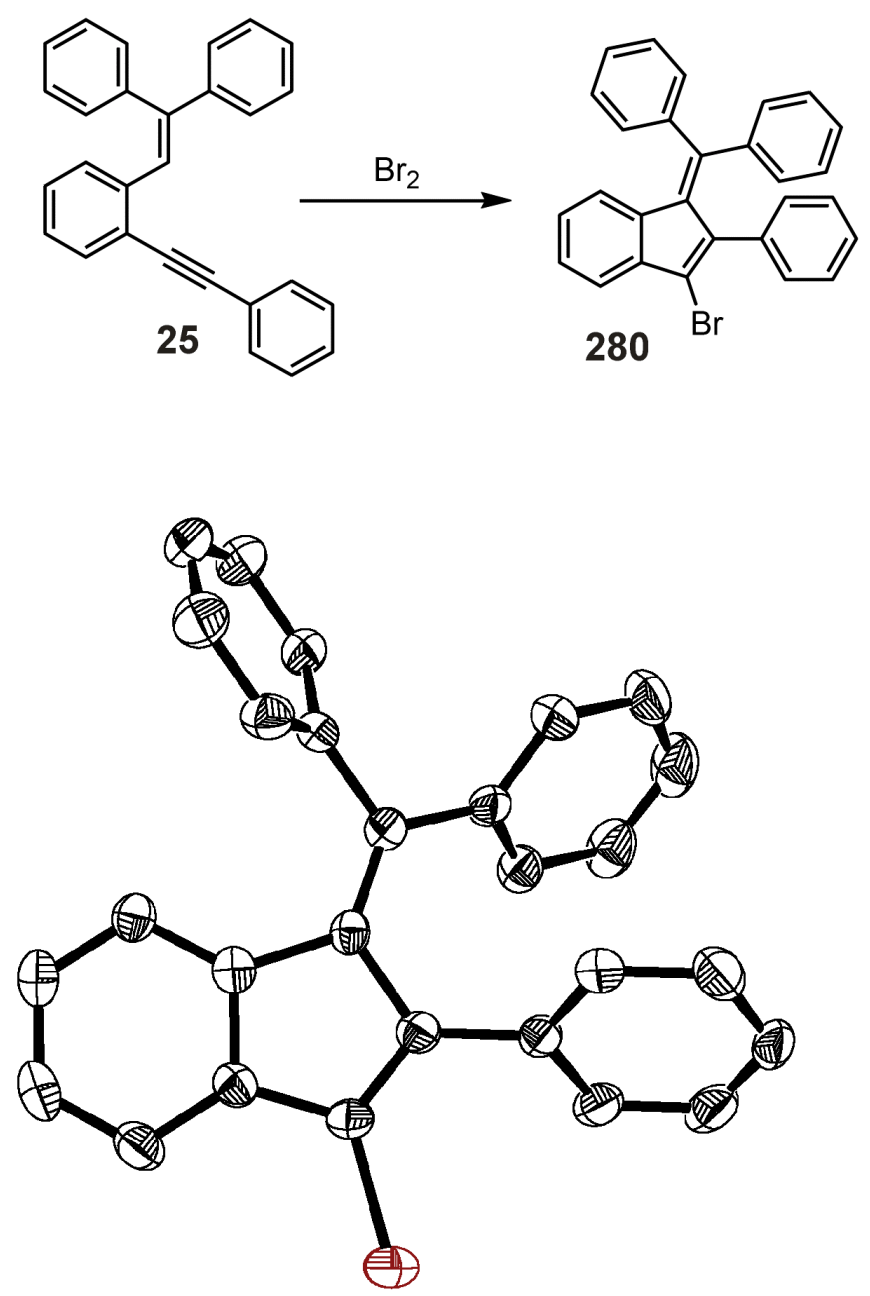

Abbildung 120. Cyclisierung von 2-(2',2'-diphenylvinyl)diphenylacetylen 25 und Kristallstruktur von 280 
Die Auswirkungen eines elektrophilen Angriffs auf Butadiin-Einheiten sind bisher wenig untersucht. Die Ergebnisse der Reaktion von Brom mit 1,4-Diphenylbuta-1,3-diin 254 stammen vom Anfang des letzten Jahrhunderts und charakterisieren die Produkte entsprechend schlecht. ${ }^{236}$ Immerhin wurde als Produkt bereits ein Tetrabromid beschrieben, das trotz äquimolarer Zugabe von Brom das Hauptprodukt war. Eine genauere Untersuchung mit modernen Mitteln zeigte, daß sich tatsächlich ein Gemisch aus dem Edukt, dem tetrabromierten Produkt 281 und wenig Verunreinigungen bildet (Abb. 121). Die Struktur konnte mit Hilfe der zuvor beschriebenen Zuordnung der ${ }^{13} \mathrm{C}-\mathrm{NMR}$-Signale zu $(E)$ - und $(Z)$ Dibromstilben 199 als (E,E)-1,2,3,4-Tetrabrom-1,4-diphenylbuta-1,3-dien 281 charakterisiert werden. Die Verunreinigungen könnten ein (E,Z)-Derivat von 281 sein, ein (Z,Z)-281 ist aus sterischen Gründen nicht möglich.

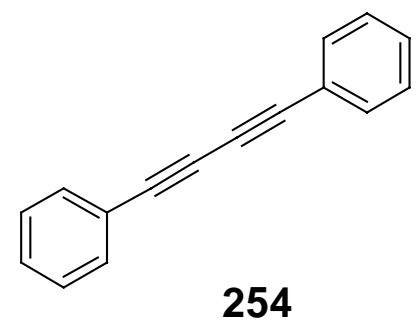

254
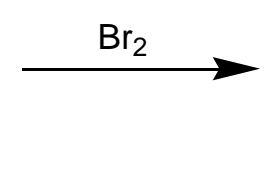<smiles>BrC(C(Br)=C(Br)c1ccccc1)=C(Br)c1ccccc1</smiles>

281

Abbildung 121. Addition von Brom an 1,4-Diphenylbuta-1,3-diin 254

Eine interessante Frage nach den Reaktivitätsunterschieden von Ethinyl- und Butadiinyl-Einheit stellt sich bei der Bromierung von 2',2'-Bis(phenylethinyl)-1,4diphenylbuta-1,3-diin 23. Aufgrund der vielfältigen Möglichkeiten zu Addieren und Cyclisieren ist eine große Zahl von di- und tetrabromierten Produkten $\mathrm{zu}$ erwarten. Tatsächlich wurde bei der Zugabe von einem Äquivalent Brom jedoch nur ein einziges, tetrabromiertes Produkt gebildet, im Gemisch mit nicht umgesetztem Edukt. Anhand des ${ }^{1} \mathrm{H}$ Signals bei 8.24 ppm und der Anzahl der ${ }^{13} \mathrm{C}$-Signale, die auf ein symmetrisches Produkt hindeuten, konnten alle Produkte, die an den exocyclischen Doppelbindungen (Z)Konfiguration haben, sowie alle unsymmetrischen Produkte ausgeschlossen werden. Übrig blieben die beiden Strukturen 282 und 283, die nicht aufgrund ihrer spektroskopischen Eigenschaften unterschieden werden konnten (Abb. 122). Die Röntgenstrukturanalyse eines Einkristalls der Probe zeigte eindeutig, daß es sich bei dem Produkt um 282 und nicht um 283 handelt. Somit ist auch klar, daß die Butadiinyl-Einheit eindeutig reaktiver ist als die EthinylEinheit. 

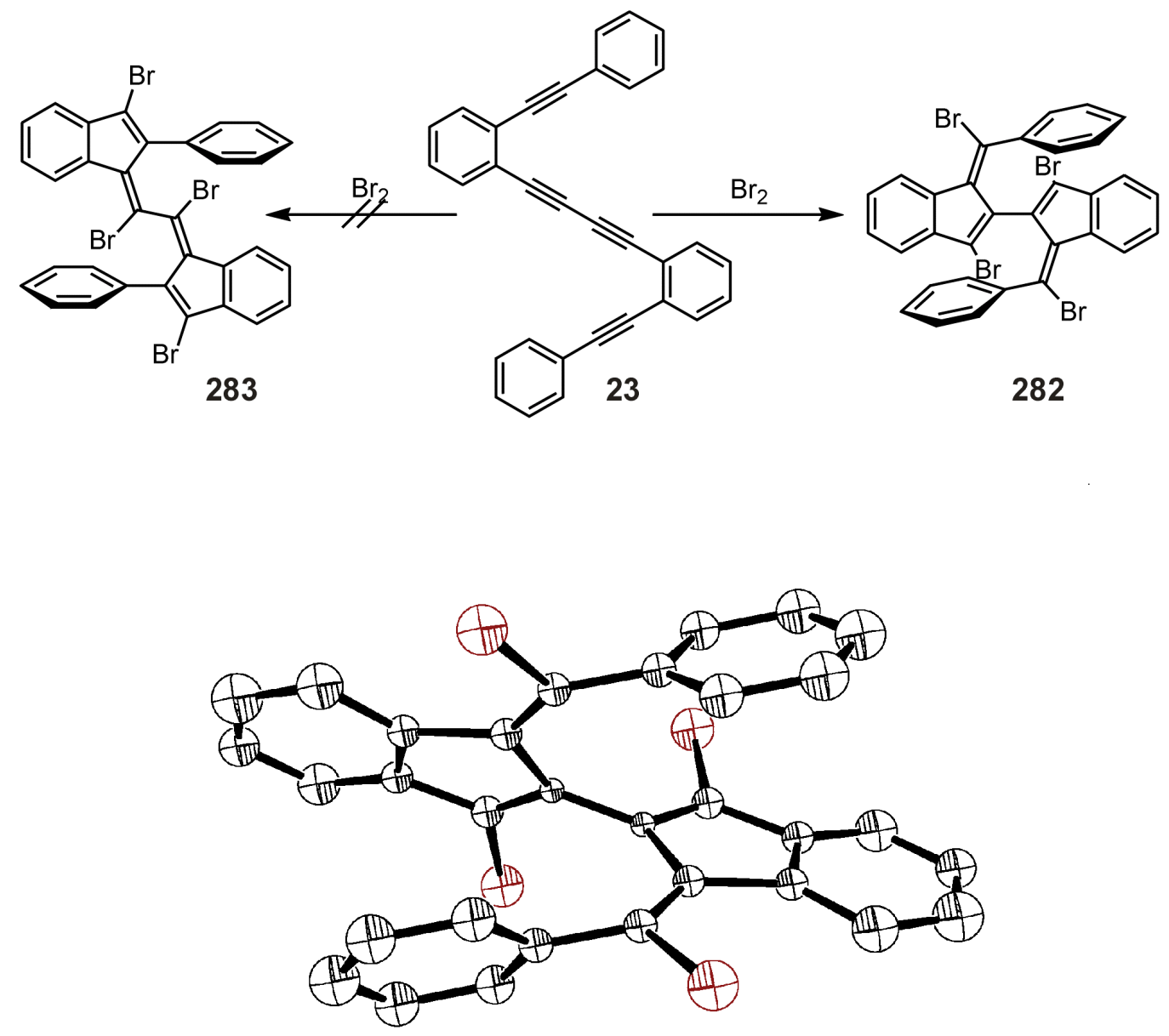

Abbildung 122. Cyclisierung von 2',2'-Bis(phenylethinyl)-1,4-diphenylbuta-1,3-diin 23 und Kristallstruktur von 282

Die elektrophil induzierte Kaskaden-Substitution am Aromaten wurde bereits in der Einleitung am Beispiel des 1,4-Diphenylbut-1-ens 218 vorgestellt, das mit $\mathrm{I}(\mathrm{py})_{2} \mathrm{BF}_{4} / \mathrm{HBF}_{4}$ 1-Phenyl-2-iodtetrahydronaphthalin 220 ergab. ${ }^{43}$ Auf demselben Weg konnte auch 4-Phenylbut-1-en in 2-Iodtetrahydronaphthalin überführt werden. ${ }^{43}$ Der Einfluß von Alkylsubstituenten an der Doppelbindung auf die Reaktion sollte am Beispiel des 2-Methyl-5phenylpent-2-en 261 untersucht werden. Bei der Zugabe von Brom ergab sich jedoch nicht das erwartete 1,1-Dimethyl-2-bromtetrahydronaphthalin $\mathbf{2 8 4}$, sondern nur das einfache Additionsprodukt 285 (Abb. 123). Offensichtlich ist $" \mathrm{Br}^{+} "$ nicht in der Lage, eine Cyclisierung einzuleiten, was einerseits an dessen elektrophilen Eigenschaften, andererseits an der fehlenden Stabilisierung des gebildeten Kations liegen kann. Auf jeden Fall ist die Addition eines $\mathrm{Br}^{-}$schneller als der Cyclisierungsschritt und es ergibt sich nur 285 als Reaktionsprodukt. 
<smiles>CC1(C)c2ccccc2CCC1Br</smiles>

284

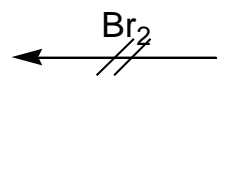<smiles>CC(C)=CCCc1ccccc1</smiles>

261

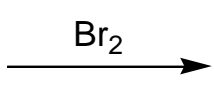<smiles>CC(C)(Br)C(Br)CCc1ccccc1</smiles>

285

Abbildung 123. Reaktion von 2-Methyl-5-phenylpent-2-en 261

Bei der Bromierung von 1,1,4-Triphenylbut-1-in 262 konnte ebenfalls kein Cyclisierungsprodukt 286 nachgewiesen werden, eine Addition fand hingegen auch nicht statt (Abb. 124). Durch die raumbeanspruchenden Phenylgruppen war eine Cyclisierung ohnehin eher unwahrscheinlich, wie von 2-(2',2'-diphenylvinyl)diphenylacetylen 25 jedoch bereits bekannt, ist die Diphenylethen-Gruppierung sehr wenig reaktiv (weniger als die Dreifachbindung im Fall von 25). Dementsprechend findet auch keine Addition statt, sondern ein, für die Diphenylethen-Gruppierung bekannter, Additions-Eliminierungs-Schritt, bei dem das olefinische Wasserstoffatom durch Brom ersetzt wird und 287 ergibt.<smiles>BrC1CCc2ccccc2C1(c1ccccc1)c1ccccc1</smiles>

286

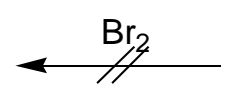<smiles>CCC=C(c1ccccc1)c1ccccc1</smiles>

262<smiles>BrC(CCc1ccccc1)=C(c1ccccc1)c1ccccc1</smiles>

287

Abbildung 124. Reaktion von 1,1,4-Triphenylbut-1-in 262

Die Reaktion von 1-(2'-Biphenylethinyl)-2-(phenylethinyl)benzol 252 mit Brom ergibt einen roten Feststoff, der nicht eindeutig charakterisiert werden konnte. Aufgrund der ${ }^{1} \mathrm{H}$ - und ${ }^{13} \mathrm{C}$-Verschiebungen wird dem Produkt vorerst die Formel 288 zugeordnet, die durch eine Aufeinanderfolge von kationisch induzierter Cyclisierung und Substitution entstanden sein kann (Abb. 125). Ein weiterer Hinweis kann die Farbe der Substanz sein, die ähnlich intensiv rot ist wie bei dem triphenylsubstituierte Benzfulven 280, das eindeutig charakterisiert werden konnte. 


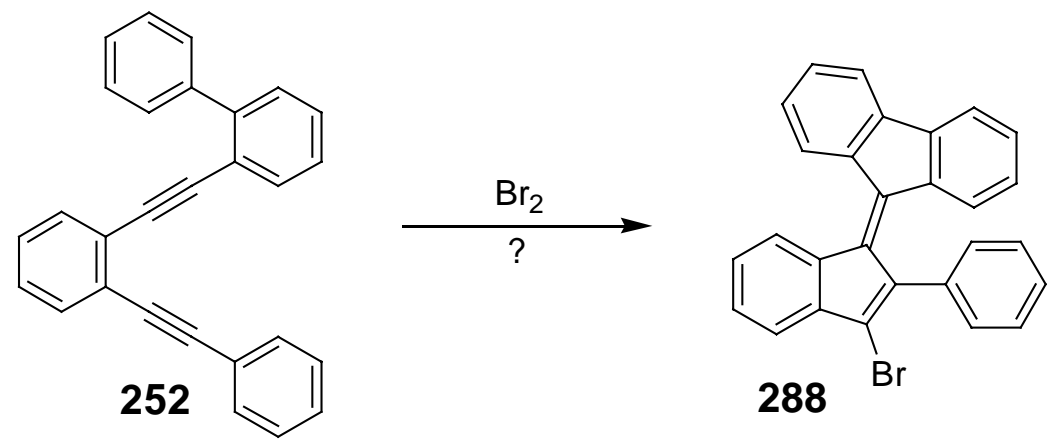

Abbildung 125. Mögliche Reaktion von 1-(2'-Biphenylethinyl)-2-(phenylethinyl)benzol 252 


\subsection{Schlußbetrachtung}

Im Rahmen des zweiten, praktischen Teils dieser Arbeit konnte gezeigt werden, daß die elektrophil induzierte, intramolekulare Interaktion zwischen ungesättigten Molekülbaugruppen eine interessante Vielfalt von cyclischen Produkten ergeben kann. Um eine Vorhersage über noch nicht durchgeführte Cyclisierungen machen zu können, müssen zuvor die Abfolge der Reaktivitäten der einzelnen ungesättigten Gruppen sowie der Einfluß der Substituenten und deren sterischer Effekte untersucht werden.

Zunächst konnte gezeigt werden, daß durch doppelte Aromatensubstitution die trans-Selektivität bei der Bromaddition an Dreifachbindungen durch die Bildung einer offenen Benzylkationzwischenstufe aufgehoben wird und sich eine nahezu äquimolare Mischung der cis- und trans-Isomere bildet. Die genaue Zuordnung der Isomere des Dibromstilbens 199 zu den NMR-Daten ist in der Literatur nicht eindeutig. Mit Hilfe der Kristallstruktur des verwandten 265 und dessen hochaufgelösten spektroskopischen Daten konnte die Zuordnung durch Transfer eindeutig vorgenommen werden und somit ohne jeden Zweifel gezeigt werden, daß die Daten in der Literatur falsch zitiert wurden.

Bei geeigneter ortho-ständiger Anordnung ungesättigter Gruppen kann eine Interaktion und damit eine Cyclisierung stattfinden. Beim 1,2-Bis(phenylethinyl)benzol 18 konnte in der Literatur gezeigt werden, daß die Dreifachbindungen durch den Angriff von Brom miteinander reagieren und sich eine Mischung der cis- und trans-Benzofulvene 195 bildet. Erstmals konnten diese komplett charakterisiert und deren Eindeutigkeit durch eine Kristallstruktur untermauert werden. Die Eigenschaften des „Sonden“-Protons, das zu tiefem Feld verschoben ist, wenn die Anordnung transoid ist und zu hohem Feld, wenn sie cisoid ist, konnten bestätigt werden. Auf diese Eigenschaft wurde für weitere Charakterisierungen fulvenischer Produkte zurückgegriffen.

Bei der Cyclisierung von 1,2,3-Tris(phenylethinyl)benzol 22 beispielsweise konnten zwei der drei Produkte sofort als die fulvenische Produkte 266 erkannt und nach der anspruchsvollen Trennung das (E)-266 durch 2D-Spektren identifiziert werden. Die Cyclisierungsprodukte höherer Homologe wie z.B. des 1,2,3,4-Tetrakis(phenylethinyl)benzols 239 konnte nicht getrennt und charakterisiert werden, die NMR-Spektren der Reaktionsmischungen deuten jedoch darauf hin, daß auch dort, neben diversen anderen Produkten, solche ,Zipper“-Fulvene entstanden sind. Während 266 jedoch noch planar ist müßte 289 schon aus der Ebene heraus und eine schüsselförmige, gewölbte Gestalt 
annehmen, was Energie kostet und deshalb die Reaktion entsprechend ungünstiger machen sollte (Abb. 126).

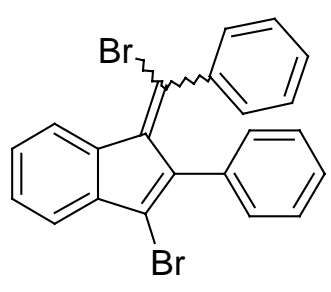

195

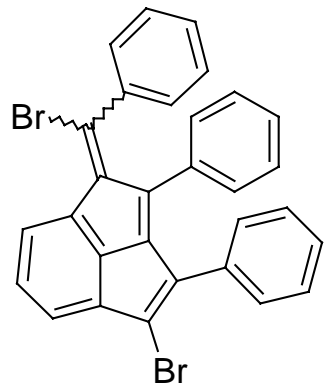

266

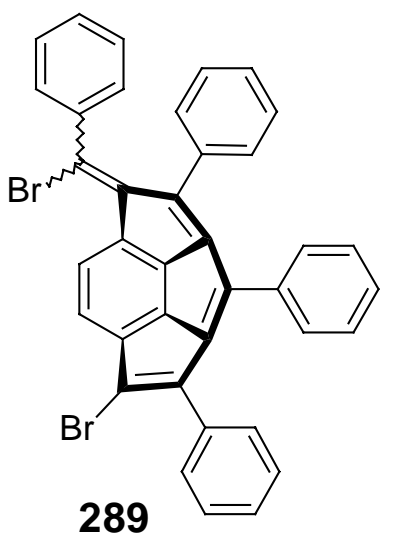

Abbildung 126. Die Reihe der "Zipper"-Cyclisierungsprodukte ortho-ständiger Enpolyine

Bei der Reaktion von 1-(Phenylethinyl)naphthalin 245 konnte kein Cyclisierungsprodukt festgestellt werden, es fand nur die normale Addition statt. Die in der Literatur beschriebene brominduzierte Cyclisierung von 1,8-Bis(phenylethinyl)benzol 203 konnte nicht nachvollzogen werden, das Hauptprodukt war ein Additionsprodukt an eine Dreifachbindung. Das Produkt wurde nicht rein erhalten werden, konnte aber anhand der Acetylensignale im ${ }^{13} \mathrm{C}$-NMR und durch Vergleich mit 267 identifiziert werden.

Sechsgliedrige Ringe werden gebildet, wenn ungesättigte Gruppen mit Phenylgruppen interagieren. Die Cyclisierung von 1,4-Diphenylbut-1-in 217 ergibt so das substituierte Dihydronaphthalin 270, das erstmals vollständig charakterisiert wurde, als einziges Produkt; cis/trans-Isomere gibt es hier natürlich nicht. Dieselbe Reaktion findet bei 2Phenylethinylbiphenyl 24 statt, das das Phenanthren 271 ergibt, welches in einer Art Selbstreplikation wieder mit Phenylacetylen umgesetzt und erneut cyclisiert werden konnte.

Substituenteneffekte konnten anhand der Reihe 2-Vinyldiphenylacetylen 243 - 2(Phenylvinyl)diphenylacetylen 260 - 2-(Diphenylvinyl)diphenylacetylen 25 studiert werden. Während 243 als Hauptprodukt nur das normale Additionsprodukt an die Doppelbindung ergibt, bildet 260 eine untrennbare Mischung von Additions- und Cyclisierungsprodukten. Verbindung 25 ergibt kein Additions-, sondern nur ein ungewöhnliches Cyclisierungsprodukt 280, in dem das vinylische $\mathrm{H}$ durch das entstandene Bromkation substituiert wird.

Weitere Substituenteneffekte sind bei der Reaktion von 261 und 262 zu beobachten. Während in 261 die Alkylgruppen zur Stabilisierung des Kations nicht ausreichen und sich deshalb nur das Additionsprodukt bildet, ist bei 262 wiederum keine Additions-, sondern nur 
eine Substitutionsreaktion zu 287 zu beobachten, während 218 mit einer Phenylgruppe als Substituent wie in der Literatur gezeigt, ein Cyclisierungsprodukt ergibt.

Als letztes wurden noch die Reaktivitätsunterschiede zwischen Ethinyl- und Butadiinyl-Gruppe verglichen. 1,4-Diphenylbutadiin 254 addiert selbst bei Zugabe einer äquimolaren Menge Brom zwei Äquivalente, so daß sich eine Mischung aus Tetrabromid 281 und Edukt ergibt. Die Sterochemie konnte durch die vorherige Lösung des $(E)$-(Z)-Rätsels bei Dibromstilben 199 und Transfer als $(\boldsymbol{E}, \boldsymbol{E})$-281 zugeordnet werden. Der direkte Vergleich der Ethinyl- und Butadiinyl-Gruppen in $\mathbf{2 3}$ zeigt eindeutig, daß die Butadiinyl-Grupppe reaktiver ist, da beide Angriffe dort erfolgen und nicht am Ethinyl-Teil. Es wird wiederum eine Mischung aus Edukt und Tetrabromid 282 erhalten, in dem die beiden Fulvenringe direkt miteinander verbunden sind.

Zusammenfassend kann man sagen, daß für die kationisch induzierten Cyclisierungen mehrfach ungesättigter Systeme folgende Regeln gelten:

- Aromatische Substitution an den ungesättigten Bausteinen ist essenziell, Alkylgruppen oder Wasserstoff als Substituenten ergeben hauptsächlich Additionsprodukte.

- Angriffe von bromierten Doppel- oder Dreifachbindungen an andere Doppel- oder Dreifachbindungen ergeben fünfgliedrige Ringe, Angriffe auf Phenylringe ergeben sechsgliedrige Ringe.

- Doppelte Aromatensubstitution an Doppelbindungen bewirkt durch sterische Behinderung, daß dort keine Addition stattfindet und ein an dieser Doppelbindung gebildetes Kation keine weitere Cyclisierung eingeht, sondern daß das vinylische $\mathrm{H}$ durch das entsprechende Kation (Alkyl oder Brom) substituiert wird.

- Sind Ethinyl- und Butadiinyl-Gruppen gleichzeitig in einem Molekül vorhanden wird stets die Butadiin-Einheit angegriffen.

Mit diesen Regeln sollte es möglich sein, das Ergebnis noch nicht durchgeführter Reaktionen vorherzusagen. Die Verbindung 253 sollte durch primären Angriff an die Butadiin-Einheit zuerst 290 ergeben und nicht das entsprechend andere Cyclisierungsprodukt, das durch Angriff an die Ethinyl-Einheit entsteht (Abb. 127). Interessant ist die mögliche Weiterreaktion zu dem Substituierten und benzannelierten Azulenderivat 291. Verbindung 292 hingegen sollte keine Cyclisierung eingehen, sondern lediglich ein oder zwei vinylische $\mathrm{H}$ durch Br substituieren. Die Verbindung 294 sollte, aufgrund der Alkylsubstituenten, nur Brom addieren und keine Cyclisierung eingehen. 
<smiles>O=S(=O)(O)C#Cc1ccccc1C#Cc1ccccc1C#CC1=C(Br)c2ccccc2C1=C(Br)c1ccccc1</smiles>

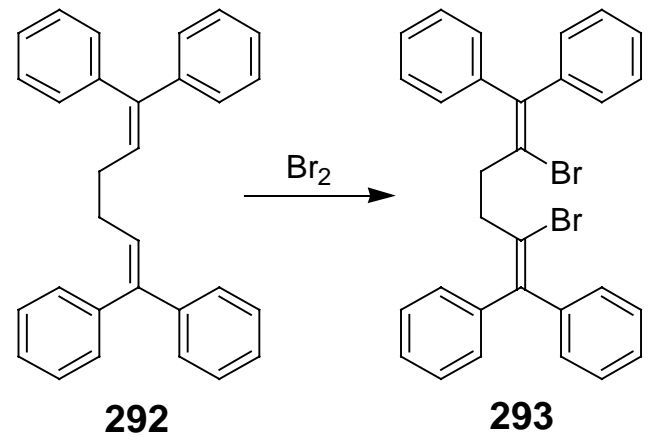

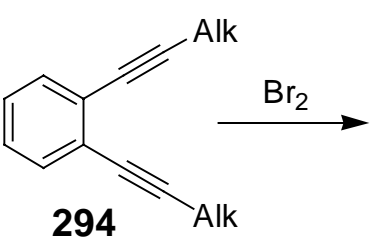<smiles>O=S(=O)(O)C#Cc1ccccc1C(Br)=C(Br)Br</smiles>

Abbildung 127. Verschieden Voraussagen für nicht durchgeführte Reaktionen

Interessant ist das Verhalten von Misch-Molekülen, die also einen cyclisierenden und einen nicht cyclisierenden Part beinhalten, wie z.B. 1-(Phenylethinyl)-2-octinylbenzol, oder cyclischer Varianten der Endiine, wie z.B. Tribenzo(12)annulen. Die Charakterisierung der Ergebnisse weiterer Cyclisierungen und die Verfeinerung der Cyclisierungsregeln sind Gegenstand weiterer Forschung. 


\section{Zusammenfassung}

Im Rahmen dieser Arbeit konnte ein wenig Ordnung in die unüberschaubare Vielfalt der "Cyclisierungsreaktionen mehrfach ungesättigter Systeme" gebracht werden. In einem ersten, theoretischen Teil konnte gezeigt werden, daß eine große Gruppe von thermischen Umlagerungen und Cyclisierungen über einen sechsgliedrigen Übergangszustand ablaufen oder zu cyclischen, sechsgliedrigen Produkten führen. Es konnte gezeigt werden, daß diese Reaktionen alle zu einer großen Familie gehören, deren bekanntestes Mitglied wohl die CopeUmlagerung von 1,5-Hexadien sein dürfte, weswegen diese Gruppe auch als Cope-Familie benannt wurde. Weiterhin konnte gezeigt werden, daß die Cope-Familie in zwei Untergruppen, Familienzweige genannt, unterteilt werden kann, und zwar in Zweig eins, in dem Moleküle zusammengefaßt sind, die teilweise über konzertierte Prozesse, teilweise über Zwischenprodukte ineinander umlagern und einen Zweig zwei, zu dem die Verbindungen gehören, die zu teils offenschaligen, teils geschlossenschaligen Produkten cyclisieren, ohne wieder zu öffnen. Für Zweig eins konnte dabei die Zuordnung der Umlagerungspartner vorgenommen werden, sowie deutlich Abhängigkeiten der Reaktivitäten, sowie des Auftretens eines biradikalischen Zwischenproduktes von der Radikalstabilisierung durch Delokalisierung und Aromatisierung beobachtet werden. Für Zweig zwei, der nur sechs Mitglieder hat, konnten die Stammreaktionen im Detail beschrieben und teilweise mit experimentellen Daten verglichen werden. Die gemessenen Werte wurden dabei durchweg gut bis sehr gut von den berechneten beschrieben. Auf dieser Basis aufbauend wurden teilweise neue Reaktionen der Stammsysteme vorhergesagt, teilweise die Reaktionsmechanismen geklärt. Der Effekt der Benzannelierung wurde im Detail betrachtet und eine Erklärung für die im Vergleich zum Stammsystem immer höheren Barrieren und Reaktionsenthalpien gegeben. Außerdem konnte gezeigt werden, daß die Inkorporation in ein carbocyclisches Ringsystem die Reaktivität durch Ringspannung erhöhen kann und daß dadurch und durch geeignete Substitution die Reaktion so gesteuert werden kann, daß sie kontrolliert bei physiologischer Temperatur ablaufen kann.

In einem zweiten Praktischen Teil konnte gezeigt werden, daß es neben den thermischen eine Vielfalt an kationisch induzierten Cyclisierungen an denselben Systemen geben kann, die zu ganz anderen Produkten führen. Es wurde eine sorgfältig ausgewählte Anzahl von mehrfach ungesättigten Systemen synthetisiert, dieser Cyclisierung unterworfen und anhand der teilweise überraschenden Ergebnisse eine Reaktivitätenreihenfolge 
aufgestellt, die es ermöglichen sollte, die Ergebnisse noch nicht durchgeführter Cyclisierungen vorherzusagen.

Auf beiden Gebieten, theoretisch sowie experimentell, wurde im Rahmen dieser Arbeit der Grundstein für eine systematische Erfassung und Beschreibung der Reaktionen mehrfach ungesättigter Systeme gelegt. Die Erweiterung und Verfeinerung der Systematik zum Zweck der akkuraten Vorhersage nicht bekannter Cyclisierungen ist Gegenstand weiterer Forschung. 


\section{Experimenteller Teil}

${ }^{1}$ H-NMR-Spektren wurden auf den Geräten AM 250 (250 MHz) der Firma Bruker und Unity INOVA 500 (500 MHz) der Firma Varian aufgenommen. Chemische Verschiebungen sind als $\delta$-Werte in ppm angegeben und beziehen sich auf $\delta=7.26$ für Chloroform. Charakterisierung der Signalaufspaltung: $\mathrm{s}=$ Singulett, $\mathrm{d}=$ Dublett, $\mathrm{t}=$ Triplett, $\mathrm{q}=$ Quartett, $\mathrm{m}=$ Multiplett .

${ }^{13}$ C-NMR-Spektren wurden auf den Geräten AM 250 (62.9 MHz) der Firma Bruker und Unity INOVA 500 (125.7 MHz) der Firma Varian aufgenommen. Chemische Verschiebungen sind als $\delta$-Werte in ppm angegeben und beziehen sich auf $\delta=77.0$ für Chloroform. Charakterisierung der Signale: $\mathrm{p}=$ primär, $\mathrm{s}=$ sekundär, $\mathrm{t}=$ tertiär, $\mathrm{q}=$ quartär.

Massenspektren wurden mit einem Spektrometer Modell MAT 311 der Firma Varian und einem Modell MAT 95 der Firma Finnigan aufgenommen.

Gaschromatographische Arbeiten und Analysen wurden an einem analytischen GC der Firma Hewlett Packert HP5890 II, ausgestattet mit Massendetektor HP5971A und der Säule HP Ultra 1 Säule (50 m x $0.2 \mathrm{~mm}$ x $0.33 \mathrm{~mm}$ Film) durchgeführt.

Säulenchromatographische Trennungen wurden an Merck Kieselgel 60 durchgeführt. Laufmittel wurden nur destilliert verwendet.

Dünschichtchromatographie (DC): Macherey Nagel Fertigfolien: Alugram ${ }^{\odot}$ SIL G/UV 254 ; Merck DC Fertigfolien: Kieselgel $60 \mathrm{~F}_{254}$ auf Aluminiumfolie. Detektion unter UV-Licht bei 254 nm oder durch Entwicklung mit Molybdatophosphorsäure Tauchreagenz (10 proz. Lsg. in Ethanol) und anschließendem Erwärmen.

Schmelzpunkte wurden mit einer Schmelzpunktapparatur nach Dr. Tottoli der Firma Büchi ermittelt; die gemessenen Schmelzpunkte sind unkorrigiert und wurden in verschlossenen Kapillaren bestimmt.

IR-Spektren wurden mit dem Spektrometer IFS 66 (FTIR) der Firma Perkin-Elmer 298 aufgenommen. 


\section{Synthesevorschriften}

\section{Synthese von Bromiden durch Sandmeyer-Reaktion: ${ }^{221}$}

Vorbereitung des Kupferkatalysators: In einem Rundkolben werden $15 \mathrm{mmol} \mathrm{CuSO}_{4}(2.4 \mathrm{~g})$ in $12 \mathrm{~mL}$ Wasser unter Erwärmen gelöst und mit $22.5 \mathrm{mmol} \mathrm{NaBr}(2.3 \mathrm{~g})$ versetzt. Unter Rühren werden $7.5 \mathrm{mmol} \mathrm{Na}_{2} \mathrm{SO}_{3}(0.95 \mathrm{~g})$ in $15 \mathrm{~mL}$ Wasser dazugetropft und die Reaktionsmischung abkühlen gelassen. Der entstandene Niederschlag wird mit Wasser gewaschen und anschließend in $6 \mathrm{~mL}$ konz. HBr gelöst.

In einer Mischung aus $3.5 \mathrm{~mL}$ konz. $\mathrm{HBr}$ und $3.5 \mathrm{~mL}$ Wasser werden $10 \mathrm{mmol}$ des entsprechenden Anilins suspendiert. Unter Kühlen werden $4 \mathrm{~mL}$ einer 2.5 molaren $\mathrm{NaNO}_{2}$ Lösung hinzugetropft. Die Lösung wird unverzüglich in die Kupfer-Katalysator-Lösung bei $0^{\circ} \mathrm{C}$ eingetragen und anschließend erwärmt, bis die Stickstoffentwicklung beendet ist. Die abgekühlte Reaktionsmischung wird dreimal mit $\mathrm{CH}_{2} \mathrm{Cl}_{2}$ extrahiert und die vereinigten organischen Phasen mit Wasser gewaschen. Abrotieren des Lösungsmittels und zweimaliges Umkristallisieren aus Ethanol ergibt das gewünschte Produkt.

\section{1,2,3-Tribrombenzol (230): 237,238}

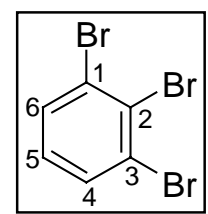

Ausgangsmaterial: 2,6-Dibromanilin 227 (2.5 g). Ausbeute: 2.5 g 227 (80.6\%) als farblose Kristalle. ${ }^{1}$ H-NMR: $\delta=7.57(\mathrm{~d}, 2 \mathrm{H}, 4-\mathrm{H}, 6-\mathrm{H}), 7.02(\mathrm{t}, 1 \mathrm{H}, 5-\mathrm{H})$. ${ }^{13}$ C-NMR: $\delta=132.47$ (t, 2C, C-4, C-6), 129.14 (t, 1C, C-5), 127.59 (q, 1C, C-2), 126.14 (q, 2C, C-1, C-3). MS (GC-MS) Massenpeak m/z = $311.8\left(\mathrm{M}^{+}\right) ; \mathrm{C}_{6} \mathrm{H}_{3} \mathrm{Br}_{3}$ (311.78).

\section{1,2,4-Tribrombenzol (229): 237}

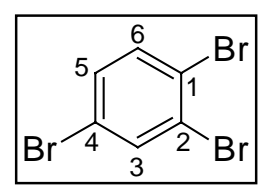

Ausgangsmaterial: 2,4-Dibromanilin 226 (2.5 g). Ausbeute: 2.2 g 229 (70\%) als farblose Kristalle. ${ }^{1}$ H-NMR: $\delta=7.76(\mathrm{~d}, 1 \mathrm{H}, 3-\mathrm{H}), 7.46(\mathrm{~d}, 1 \mathrm{H}, 6-\mathrm{H}), 7.28$ (dd, 1H, 5-H). ${ }^{13}$ C-NMR: $\delta=136.01$ (t, 1C, C-3), 134.59 (t, 1C, C-6), 131.66 (t, 1C, C-5), 125.77 (q, 1C, C-2), 123.71 (q, 1C, C-1), 121.35 (q, 1C, C-4). MS (GC-MS) Massenpeak m/z = $311.8\left(\mathrm{M}^{+}\right) ; \mathrm{C}_{6} \mathrm{H}_{3} \mathrm{Br}_{3}$ (311.78).

\section{1,2,3,5-Tetrabrombenzol (231): ${ }^{237}$}

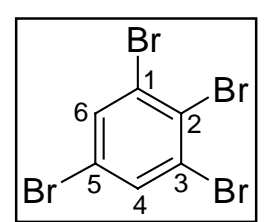

Ausgangsmaterial: 2,4,6-Tribromanilin 228 (3.3 g). Ausbeute: 0.9 g 231 (23\%) als farblose Nadeln. ${ }^{\mathbf{1}} \mathbf{H}-\mathbf{N M R}: \delta=7.71$ (s, 2H, 4-H, 6-H). ${ }^{\mathbf{1 3}} \mathbf{C}-\mathbf{N M R}$ : $\delta=134.82$ (t, 2C, C-4, C-6), 126.74 (q, 1C, C-2), 126.56 (q, 2C, C-1, C-3), 121.38 (q, 1C, C-5). MS (GC-MS) Massenpeak m/z = $389.8\left(\mathrm{M}^{+}\right) ; \mathrm{C}_{6} \mathrm{H}_{2} \mathrm{Br}_{4}$

(389.69). 


\section{2-Brombiphenyl: 239}

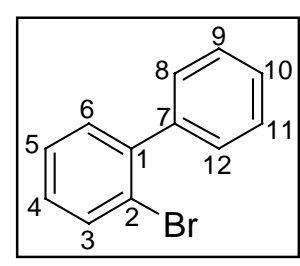

Ausgangsmaterial: 2-Aminobiphenyl (1.7 g). Ausbeute: $1.5 \mathrm{~g}(69.7 \%)$ als gelbliche Flüssigkeit. ${ }^{1} \mathbf{H}-\mathbf{N M R}: \delta=7.69(\mathrm{~m}, 1 \mathrm{H}, 6-\mathrm{H}), 7.50(\mathrm{~m}, 1 \mathrm{H}, 3-\mathrm{H})$, 7.44 (m, 5H, 8-H, 12-H, 9-H, 11-H, 10-H) 7.35 (m, 2H, 4-H, 5-H). ${ }^{13} \mathbf{C}-$ NMR: $\delta=142.53$ (q, 1C, C-1), 141.06 (q, 1C, C-7), 133.07 (t, 1C, C-3), 131.25 (t, 1C, C-5), 129.35 (t, 2C, C-8, C-12), 128.69 (t, 1C, C-10), 127.94 (t, 2C, C-9, C-11), 127.57 (t, 1C, C-4), 127.34 (t, 1C, C-5), 122.60 (q, 1C, C-2). MS (GC-MS) Massenpeak m/z $=232.0\left(\mathrm{M}^{+}\right) ; \mathrm{C}_{12} \mathrm{H}_{9} \mathrm{Br}(231.99)$.

\section{Synthese von 1,2,3,4-Tetrabrombenzol (232): 222}

Eine Suspension von 10 mmol Hexabrombenzol 233 (5.5 g) in einer Mischung aus $100 \mathrm{~mL}$ Ethanol und $100 \mathrm{~mL}$ Hydrazinhydrat wird unter Rückfluß erhitzt, bis der Niederschlag verschwunden ist. Die erkaltete Lösung wird in $500 \mathrm{~mL}$ Wasser eingetragen und der entstandene Niederschlag abgesaugt, mit Wasser gewaschen, getrocknet und aus Ethanol umkistallisieren.

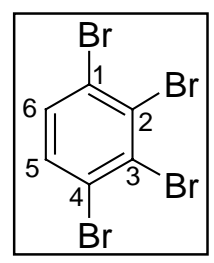

Ausbeute: 2.6 g 232 (66\%) als farblose Kristalle. ${ }^{1} \mathbf{H}-\mathbf{N M R}: \delta=7.45$ (s, 2H, 5-H, 6-H). ${ }^{13}$ C-NMR: $\delta=132.73$ (t, 2C, C-5, C-6), 129.07 (q, 2C, C-2, C-3), 124.75 (q, 2C, C-1, C-4). MS (GC-MS) Massenpeak m/z = $389.7\left(\mathrm{M}^{+}\right) ; \mathrm{C}_{6} \mathrm{H}_{2} \mathrm{Br}_{4}$ (389.69).

\section{Synthese von 1,2,3,4,5-Pentabrombenzol (234): 223}

Aus $20 \mathrm{mmol}$ Magnesium (0.5 g) und $20 \mathrm{mmol}$ Brombenzol $(3.2 \mathrm{~g} / 2.1 \mathrm{~mL})$ in $20 \mathrm{~mL}$ THF wird eine Grignard-Lösung hergestellt, die bei $0{ }^{\circ} \mathrm{C} \mathrm{zu}$ einer Suspension von $20 \mathrm{mmol}$ Hexabrombenzol 233 (11 g) in 20 mL THF getropft wird. Nach vierstündigem Rühren bei RT wird die Suspension hydrolysiert und der entstandene Feststoff abgesaugt. Durch Extraktion mit heißem Ethanol wird 234 von nicht reagiertem Hexabrombenzol abgetrennt. Der nach dem Erkalten ausgefallene Feststoff wird abgesaugt und nochmals aus Ethanol umkristallisiert.

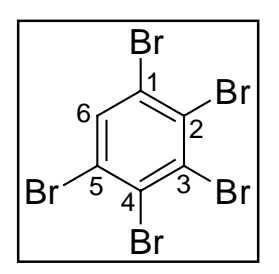

Ausbeute: 1.7 g 234 (18\%) als farblose Kristalle. ${ }^{1} \mathbf{H}-\mathbf{N M R}: \delta=7.91$ (s, 1H, 6-H). ${ }^{13}$ C-NMR: $\delta=135.55$ (t, 1C, C-6), 129.97 (q, 1C, C-3), 127.90 (q, 2C, C-2, C-4), 124.76 (q, 2C, C-1, C-5). MS (GC-MS) Massenpeak m/z = 467.7 $\left(\mathrm{M}^{+}\right) ; \mathrm{C}_{6} \mathrm{HBr}_{5}(467.60)$. 


\section{Synthese von 1,8-Diiodnapthalin (247):224}

$\mathrm{Zu}$ einer auf $-20{ }^{\circ} \mathrm{C}$ gekühlten Suspension von 177 mmol 1,8-Diaminonaphthalin 246 (28 g) in $325 \mathrm{~mL} 6.9$ molarer $\mathrm{H}_{2} \mathrm{SO}_{4}$ wird langsam eine Lösung von $521 \mathrm{mmol} \mathrm{NaNO}_{2}(36 \mathrm{~g})$ in 150 $\mathrm{mL}$ Wasser getropft, so daß die Temperatur $-15^{\circ} \mathrm{C}$ nicht übersteigt. Anschließend werden bei der selben Temperatur $1.08 \mathrm{~mol} \mathrm{KI}(180 \mathrm{~g})$ in $150 \mathrm{~mL}$ Wasser hinzugetropft, die Reaktionmischung wird langsam auf RT erwärmt und anschließend $1 \mathrm{~h}$ unter Rückfluß erhitzt. Nach dem Abkühlen wird bis zur neutralen Reaktion festes $\mathrm{NaOH}$ zugegeben und die Lösung filtriert. Die erhaltenen schwarzen Brocken werden mehrfach mit siedendem Ether extrahiert (mind. $1 \mathrm{~L}$ ) und die vereinigten organischen Phasen mit gesättigter $\mathrm{Na}_{2} \mathrm{SO}_{3}$-Lösung Iod-frei gewaschen. Die organische Phase wird getrocknet und das Lösungsmittel abgezogen. Der Rückstand wird aus Ethanol umkristallisiert.

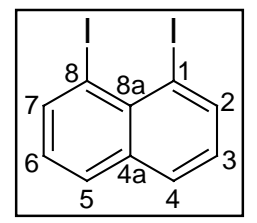

Ausbeute: $18.1 \mathrm{~g} 247$ (37\%) als brauner Feststoff. ${ }^{\mathbf{1}} \mathbf{H}-\mathbf{N M R}: \delta=8.41$ (m, 2H, 2-H, 7-H), 7.83 (m, 2H, 4-H, 5-H), 7.05 (m, 2H, 3-H, 6-H). ${ }^{13} \mathbf{C}-\mathbf{N M R}: \delta=$ 143.93 (t, 2C, C-2, C-7), 137.33 (q, 1C, C-8a), 135.65 (q, 1C, C-4a), 130.95 (t, 2C, C-4, C-5), 126.86 (t, 2C, C-3, C-6), 95.99 (q, 2C, C-1, C-8). MS (GC-MS) Massenpeak $\mathrm{m} / \mathrm{z}=379.9\left(\mathrm{M}^{+}\right) ; \mathrm{C}_{10} \mathrm{H}_{6} \mathrm{I}_{2}$ (379.86).

Bis(triphenylphosphin)palladium-(II)-chlorid (221): 240 Eine Lösung aus 5.6 mmol $\mathrm{PdCl}_{2}$ (1 g) und $12.4 \mathrm{mmol} \mathrm{PPh}_{3}(3.25 \mathrm{~g})$ in $30 \mathrm{~mL}$ Benzonitril wird schrittweise auf $180{ }^{\circ} \mathrm{C}$ erhitzt. Die klare tiefrote Lösung wird langsam auf RT abgekühlt, der ausgefallene gelbe Feststoff abfiltriert und mit Ether gewaschen. Ausbeute: 3.7 g 221 (93\%) als gelbe Kristalle.

\section{Synthese der Acetylene durch Palladium-katalysierte Sonogashira-Kupplung: ${ }^{220}$}

In einem Dreihalskolben mit Rückflußkühler/Blasenzähler und Gaseinlaß werden Halogenid, Acetylen und Triphenylphosphin in Triethylamin vorgelegt. Die Mischung wird dreimal evakuiert und dreimal mit Argon belüftet. Anschließend werden Bis(triphenylphosphin)palladium-(II)-chlorid und Kupfer-(I)-iodid hinzugegeben und die Reaktionsmischung über nacht zum Rückfluß erhitzt. Die Aufarbeitung erfolgt auf zwei verschiedene Arten: A) Nach dem Abkühlen wird die Reaktionsmischung filtriert und der Rückstand mit Ether gewaschen. B) Nach dem Abkühlen wird die Reaktionsmischung auf eine Mischung aus $1 \mathrm{~N} \mathrm{HCl}$ und Eis gegeben, mit $\mathrm{CHCl}_{3}$ extrahiert und die organische Phase getrocknet. Das Lösungsmittel wird in beiden Fällen am Rotationsverdampfer abgezogen. Feste Produkte werden aus Ethanol umkristallisiert, flüssige Produkte werden destilliert oder an Kieselgel gesäult. 


\section{Diphenylacetylen (196): 241}

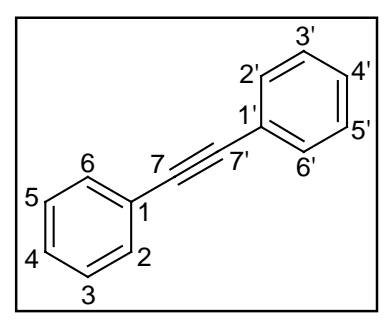

Ausgangsmaterialien: $50 \mathrm{mmol}$ Brombenzol (7.9 g/5.2 mL), $60 \mathrm{mmol}$ Phenylacetylen $(6.0 \mathrm{~g} / 6.6 \mathrm{~mL}), 150 \mathrm{mg} \mathrm{PPh}_{3}, 50 \mathrm{~mL} \mathrm{NEt} 3,50 \mathrm{mg}$ $\left(\mathrm{PPh}_{3}\right)_{2} \mathrm{PdCl}_{2}, 50 \mathrm{mg} \mathrm{CuI}$. Aufarbeitung nach Variante A. Ausbeute: 7.7 g 196 (87\%) als gelbliche Kristalle. ${ }^{1}$ H-NMR: $\delta=7.65$ (m, 4H, 2,2'-H, 6,6'-H), 7.42 (m, 6H, 3,3'-H, 5,5'-H, 4,4'-H). ${ }^{13} \mathbf{C}-\mathbf{N M R}: \delta=$ 131.53 (t, 4C, C-2,2', C-6,6'), 128.28 (t, 4C, C-3,3', C-5,5'), 128.20 (t, 2C, C-2,2'), 123.18 (q, 2C, C-1,1'), 89.37 (q, 2C, C-7,7'). MS (GC-MS) Massenpeak m/z = $178.2\left(\mathrm{M}^{+}\right) ; \mathrm{C}_{14} \mathrm{H}_{10}$ (178.08).

\section{1,2-Bis(phenylethinyl)benzol (18): ${ }^{242}$}

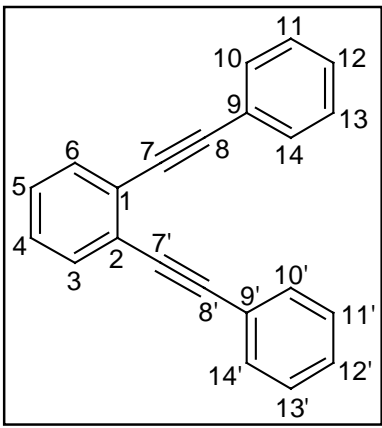

Ausgangsmaterialien: 50 mmol 1,2-Dibrombenzol (11.8 g/6 mL), 110 mmol Phenylacetylen (11.0 g/12.1 mL), $300 \mathrm{mg} \mathrm{PPh}$, $100 \mathrm{~mL} \mathrm{NEt}_{3}$, $100 \mathrm{mg}\left(\mathrm{PPh}_{3}\right)_{2} \mathrm{PdCl}_{2}, 100 \mathrm{mg}$ CuI. Aufarbeitung nach Variante A. Ausbeute: $10.4 \mathrm{~g} \mathbf{1 8}$ (75\%) als oranger Feststoff. ${ }^{\mathbf{1}} \mathbf{H}-\mathbf{N M R}: \delta=7.67$ $\left(\mathrm{m}, 4 \mathrm{H}, 10,10^{\prime}-\mathrm{H}, 14,14^{\prime}-\mathrm{H}\right), 7.64(\mathrm{~m}, 2 \mathrm{H}, 3-\mathrm{H}, 6-\mathrm{H}), 7.41$ (m, 6H, 11,11'-H, 13,13'-H, 12,12'-H), 7.36 (m, 2H, 4-H, 5-H). 13C-NMR: $\delta$ $=131.70$ (t, 2C, C-3, C-6), 131.57 (t, 4C, C-10,10', C-14,14'), 128.37 (t, 2C, C-12,12'), 128.30 (t, 4C, C-11,11', C-13,13'), 127.94 (t, 2C, C-4, C-5), 125.73 (q, 2C, C-1, C-2), 123.20 (q, 2C, C-9,9'), 93.56 (q, 2C, C-8,8'), 88.29 (q, 2C, C-7,7'). MS (GC-MS) Massenpeak m/z = 278.1 $\left(\mathrm{M}^{+}\right) ; \mathrm{C}_{22} \mathrm{H}_{14}(278.11)$.

\section{1,3-Bis(phenylethinyl)benzol (234): ${ }^{243}$}

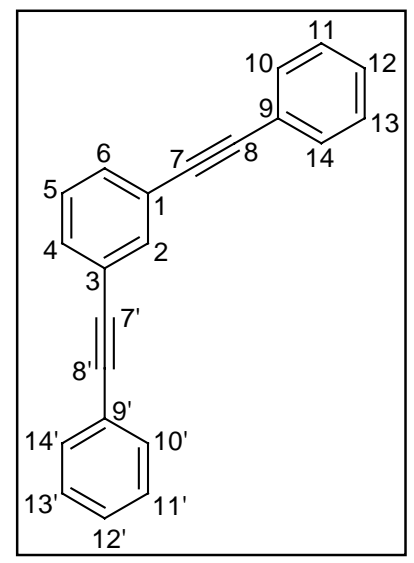

Ausgangsmaterialien: $25 \mathrm{mmol}$ 1,3-Dibrombenzol (5.9 g/3 mL), 60 mmol Phenylacetylen $(6.0 \mathrm{~g} / 6.6 \mathrm{~mL}), 300 \mathrm{mg} \mathrm{PPh}_{3}, 50 \mathrm{~mL} \mathrm{NEt}$, $100 \mathrm{mg}\left(\mathrm{PPh}_{3}\right)_{2} \mathrm{PdCl}_{2}, 100 \mathrm{mg}$ CuI. Aufarbeitung nach Variante A. Ausbeute: 5.5 g 234 (79\%) als farbloser Feststoff. ${ }^{1}$ H-NMR: $\delta=$ 7.76 (m, 1H, 2-H), 7.57 (m, 4H, 10,10'-H, 14,14'-H), 7.52 (m, 2H, 4H, 6-H), 7.38 (m, 7H, 11,11'-H, 13,13'-H, 12,12'-H). ${ }^{13} \mathbf{C}-\mathbf{N M R}: \delta=$ 134.56 (t, 1C, C-2), 131.62 (t, 4C, C-10,10', C-14,14'), 131.24 (t, 2C, C-4, C-6), 128.42 (t, 3C, C-12,12', C-5), 128.35 (t, 4C, C-11,11', C13,13'), 123.57 (q, 2C, C-1, C-3), 122.95 (q, 2C, C-9,9'), 89.95 (q, 2C, C-8, '8), 88.52 (q, 2C, C-7,7'). MS (70 eV) Massenpeak m/z = $278.1\left(\mathrm{M}^{+}\right) ; \mathrm{C}_{22} \mathrm{H}_{14}(278.11)$. 


\section{1,4-Bis(phenylethinyl)benzol (235): 244}

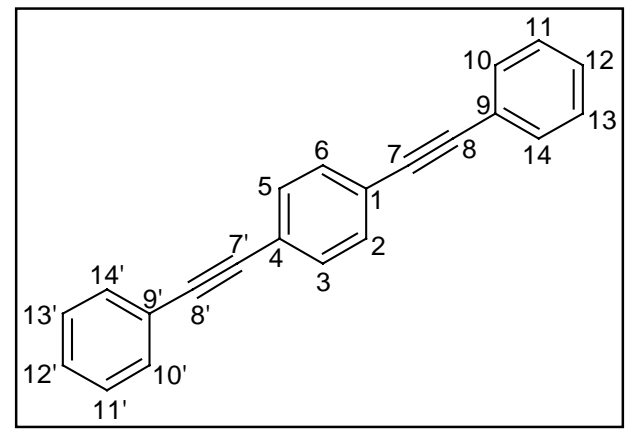

Ausgangsmaterialien: $30 \mathrm{mmol}$ 1,4-Dibrombenzol (7.1 g), $70 \mathrm{mmol}$ Phenylacetylen $(7.0 \mathrm{~g} / 7.7 \mathrm{~mL}), 150 \mathrm{mg}$ $\mathrm{PPh}_{3}, 50 \mathrm{~mL} \mathrm{NEt} 3,50 \mathrm{mg}\left(\mathrm{PPh}_{3}\right)_{2} \mathrm{PdCl}_{2}, 50 \mathrm{mg} \mathrm{CuI}$. Aufarbeitung nach Variante B. Ausbeute: 7.6 g 235 $(91 \%)$ als farbloser Feststoff. ${ }^{1}$ H-NMR: $\delta=7.54(\mathrm{~m}$, $\left.8 \mathrm{H}, 2-\mathrm{H}, 3-\mathrm{H}, 5-\mathrm{H}, 6 \mathrm{H}, 10,10^{\prime}-\mathrm{H}, 14,14 '-\mathrm{H}\right), 7.36$ (m, 6H, 11,11'-H, 13,13'-H, 12,12'-H). ${ }^{13}$ C-NMR: $\delta=131.61$ (t, 4C, C-2, C-3, C-5, C-6/C-10,10', C-14,14'), 131.52 (t, 4C, C-2, C-3, C-5, C-6/C-10,10', C-14,14'), 128.45 (t, 2C, C-12,12'), 128.38 (t, 4C, C-11,11', C-13,13'), 123.06 (q, 2C, C-1, C-4/C-9,9'), 122.99 (q, 2C, C-1, C-4/C9,9'), 91.20 (q, 2C, C-7,7'/C-8,8'), 89.08 (q, 2C, C-7,7'/C-8,8'). MS (70 eV) Massenpeak m/z = $278.1\left(\mathrm{M}^{+}\right) ; \mathrm{C}_{22} \mathrm{H}_{14}(278.11)$.

\section{1,2,3-Tris(phenylethinyl)benzol (22):}

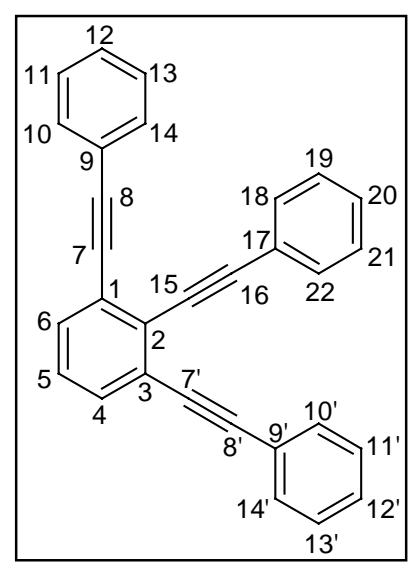

Ausgangsmaterialien: 5 mmol 1,2,3-Tribrombenzol 230 (1.6 g), 20 mmol Phenylacetylen $(2.0 \mathrm{~g} / 2.2 \mathrm{~mL}), 75 \mathrm{mg} \mathrm{PPh}, 25 \mathrm{~mL} \mathrm{NEt}_{3}, 25$ $\mathrm{mg}\left(\mathrm{PPh}_{3}\right)_{2} \mathrm{PdCl}_{2}, 25 \mathrm{mg} \mathrm{CuI}$. Aufarbeitung nach Variante A. Ausbeute: $1.5 \mathrm{~g} 22(80 \%)$ als farbloser Feststoff vom Schmelzpunkt $11{ }^{\circ} \mathrm{C} .{ }^{1} \mathbf{H}-\mathbf{N M R}: \delta=7.64$ (m, 6H, 10,10'-H, 14,14'-H, 18-H, 22-H), 7.55 (d, 2H, 4-H, 6-H), 7.39 (m, 9H, 11,11'-H, 13,13'-H, 12,12'-H, 19-H, 21-H, 20-H), $7.30(\mathrm{t}, 1 \mathrm{H}, 5-\mathrm{H}) .{ }^{13} \mathbf{C}-\mathbf{N M R}: \delta=131.68(\mathrm{t}, 6 \mathrm{C}$,

C-10,10', C-14,14', C-18, C-22), 131.32 (t, 2C, C-4, C-6), 128.55 (t, 1C, C-20), 128.54 (t, 2C, C-12,12'), 128.37 (t, 6C, C-11,11', C-13,13', C-19, C-21), 128.02 (q, 1C, C-2), 127.57 (t, 1C, C-5), 126.17 (q, 2C, C-1, C-3), 123.39 (q, 1C, C-17), 123.12 (q, 2C, C9,9'), 97.89 (q, 1C, C-16), 93.85 (q, 2C, C-8,8'), 88.02 (q, 2C, C-7,7'), 87.44 (q, 1C, C-15). IR (KBr, Preßling) v = 3059, 3033, 2206, 1596,1570, 1556, 1493, 1455, 1442, 1428, 1177 , 1158, 1073, 1026, 920, 813, 797, 764, 756, 739, $689 \mathrm{~cm}^{-1} . \mathbf{M S}(70 \mathrm{eV}) \mathrm{m} / \mathrm{z}(\%): 378(100)$ [M+', 351 (7), 300 (8), 262 (8), 189 (4). HRMS Gef.: 378.1409, Ber.: $\mathrm{C}_{30} \mathrm{H}_{18}$ 378.1409. 


\section{1,2,4-Tris(phenylethinyl)benzol (236):}

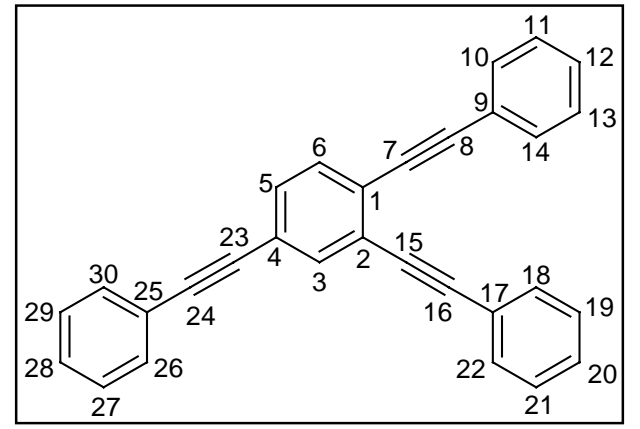

Ausgangsmaterialien: $11 \mathrm{mmol}$ 1,2,4-Tribrombenzol 229 (3.4 g), $50 \mathrm{mmol}$ Phenylacetylen $(5.0 \mathrm{~g} / 5.5 \mathrm{~mL})$, $150 \mathrm{mg} \mathrm{PPh}_{3}, 50 \mathrm{~mL} \mathrm{NEt}{ }_{3}, 50 \mathrm{mg}\left(\mathrm{PPh}_{3}\right)_{2} \mathrm{PdCl}_{2}, 50 \mathrm{mg}$ CuI. Aufarbeitung nach Variante A. Ausbeute: $3.5 \mathrm{~g}$ $236(83 \%)$ als gelblichen Feststoff vom Schmelzpunkt 108-110 ${ }^{\circ} \mathrm{C} .{ }^{1} \mathbf{H}$-NMR: $\delta=7.78(\mathrm{~d}, 1 \mathrm{H}, 3-\mathrm{H}), 7.58(\mathrm{~m}$, 6H, 10-H, 14-H, 18-H, 22-H, 26-H, 30-H), 7.55 (d, 1H, 6-H), 7.49 (dd, 1H, 5-H), 7.38 (m, 9H, 11-H, 13-H, 12-H, 19-H, 21-H, 20-H, 27-H, 29-H, 28-H). ${ }^{13}$ C-NMR: $\delta=134.68$ (t, 1C, C-3), 131.75 (t, 1C), 131.66 (t, 2C), 131.63 (t, 1C), 130.86 (t, 1C), 128.58 (t, 3C), 128.39 (t, 6C), 126.02 (q, 1C), 125.35 (q, 1C), 123.09 (q, 1C), 123.02 (q, 2C), 122.79 (q, 1C), 95.24 (q, 1C), 94.03 (q, 1C), 91.60 (q, 1C), 88.38 (q, 1C), 88.16 (q, 1C), 87.55 (q, 1C). IR (KBr, Preßling) $v=3050,3019,2212,1597,1572,1532,1509$, 1500, 1491, 1441, 1400, 1311, 1279, 1258, 1178, 1157, 1118, 1096, 1069, 1027, 915, 890, 835, 751, 750, $689 \mathrm{~cm}^{-1}$. MS (70 eV) m/z (\%): 378 (100) [M+], 306 (10), 277 (25), 262 (20), 229 (4), 201 (4), 189 (8), 183 (16), 152 (5), 108 (4), 77 (7). $\mathrm{C}_{30} \mathrm{H}_{18}$ (378.76). HRMS Gef.: 378.1410, Ber.: $\mathrm{C}_{30} \mathrm{H}_{18} 378.1409$.

\section{1,3,5-Tris(phenylethinyl)benzol (237): ${ }^{244}$}

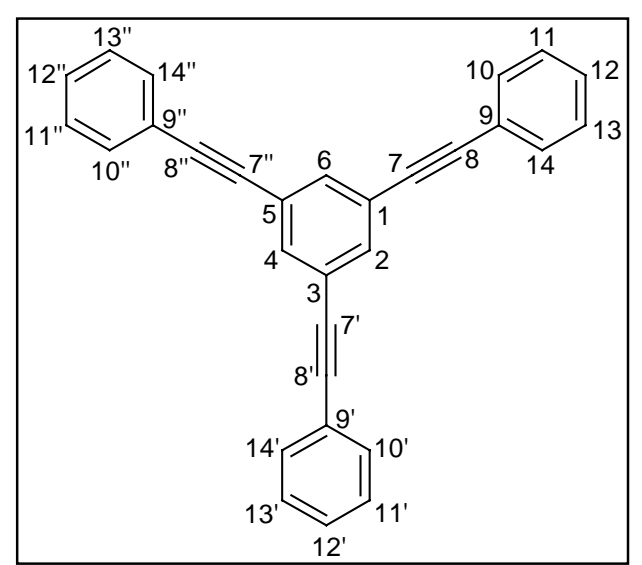

Ausgangsmaterialien: $11 \mathrm{mmol}$ 1,2,4-Tribrombenzol $(3.4 \mathrm{~g}), 50 \mathrm{mmol}$ Phenylacetylen $(5.0 \mathrm{~g} / 5.5 \mathrm{~mL}), 150$ $\mathrm{mg} \mathrm{PPh}, 50 \mathrm{~mL} \mathrm{NEt}_{3}, 50 \mathrm{mg}\left(\mathrm{PPh}_{3}\right)_{2} \mathrm{PdCl}_{2}, 50 \mathrm{mg} \mathrm{CuI}$. Aufarbeitung nach Variante A. Ausbeute: $3.5 \mathrm{~g} 237$ (83\%) als gelblichen Feststoff. ${ }^{1}$ H-NMR: $\delta=7.67(\mathrm{~s}$, 3H, 2-H, 4-H, 6-H), 7.55 (m, 6H, 10,10',10"-H, 14,14',14"-H), 7.38 (m, 9H, 11,11',11"-H, 13,13',13"--H, 12,12',12"-H). ${ }^{13}$ C-NMR: $\delta=134.01$ (t, 3C, C-2, C-4, C-6), 131.68 (t, 6C, C-10,10',10", C14,14',14"), 128.60 (t, 3C, C-12,12',12"), 128.40 (t, 6C, C-11,11',11", C-13,13',13"), 123.99 (q, 3C, C-1, C-3, C-5), 122.75 (q, 3C, C-9,9',9"), 90.48 (q, 3C, C-8,8',8"), 87.79 (q, 3C, C-7,7',7"). MS (70 eV) Massenpeak m/z = 378.2 ( $\left.\mathrm{M}^{+}\right) ; \mathrm{C}_{30} \mathrm{H}_{18}$ (378.14). 


\section{1,2,3,4-Tetrakis(phenylethinyl)benzol (239):}

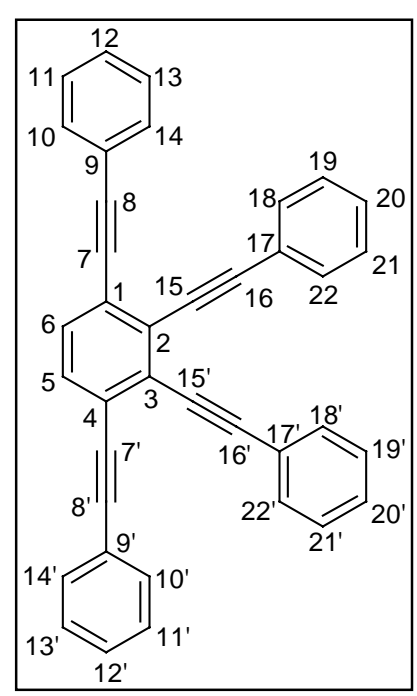

Ausgangsmaterialien: 2 mmol 1,2,3,4-Tetrabrombenzol 232 (0.8 g), 10 mmol Phenylacetylen $(1.0 \mathrm{~g} / 1.1 \mathrm{~mL}), 75 \mathrm{mg} \mathrm{PPh} 3,25 \mathrm{~mL} \mathrm{NEt}_{3}$, $25 \mathrm{mg}\left(\mathrm{PPh}_{3}\right)_{2} \mathrm{PdCl}_{2}, 25 \mathrm{mg} \mathrm{CuI}$. Aufarbeitung nach Variante B. Ausbeute: 0.7 g 239 (68\%) als farblosen Feststoff vom Schmelzpunkt $138^{\circ} \mathrm{C} .{ }^{1} \mathbf{H}-\mathbf{N M R}: \delta=7.62\left(\mathrm{~m}, 8 \mathrm{H}, 10,10^{\prime}-\mathrm{H}, 14,14 '-\mathrm{H}, 18,18^{\prime}-\right.$ H, 22,22'-H), 7.50 (s, 2H, 5-H, 6-H), 7.37 (m, 12H, 11,11'-H, 13,13'H, 19,19'-H, 21,21'-H, 12,12'-H, 20,20'-H). ${ }^{13} \mathbf{C}-\mathbf{N M R}: \delta=131.71(\mathrm{t}$, 4C, C-18,18', C-22,22'), 131.68 (t, 4C, C-10,10', C-14,14'), 130.77 (t, 2C, C-5, C-6), 128.65 (t, 4C, C-12,12', C-20,20'), 128.37 (t, 8C, C-11,11', C-13,13', C-19,19', C-21,21'), 128.26 (q, 2C, C-2, C-3), 125.54 (q, 2C, C-1, C-4), 123.22 (q, 2C, C-17,17'), 122.94 (q, 2C, C-9,9'), 98.10 (q, 2C, C16,16'), 95.36 (q, 2C, C-8,8'), 88.06 (q, 2C, C-7,7'), 87.25 (q, 2C, C-15,15'). IR (KBr, Preßling) $v=3047,3018,2962$, 2208, 1596, 1570, 1494, 1455, 1441, 1409, 1261, 1096, 1069, 1027, 910, 839, 802, 753, $685 \mathrm{~cm}^{-1}$. MS (70 eV) m/z (\%): 478 (100) [ $\left.\mathrm{M}^{+}\right], 400(8), 378(12)$, 237 (6), 200 (3). $\mathrm{C}_{38} \mathrm{H}_{22}$ (478.85). HRMS Gef.: 478.1722, Ber.: $\mathrm{C}_{30} \mathrm{H}_{18} 478.1721$.

\section{1,2,3,5-Tetrakis(phenylethinyl)benzol (238):}

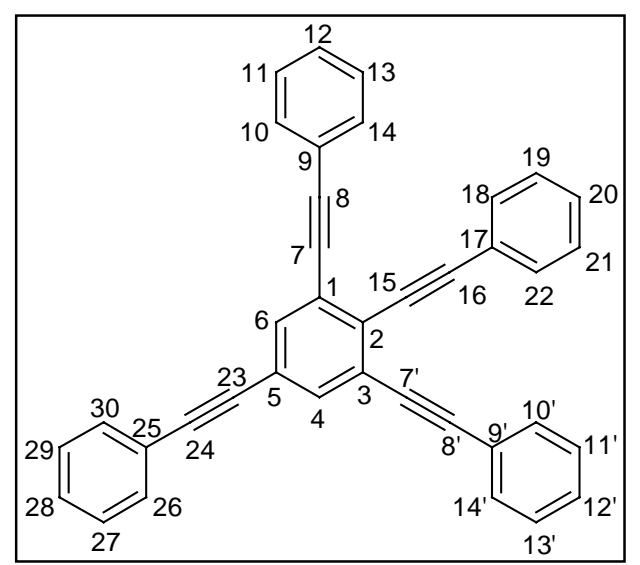

Ausgangsmaterialien: 4 mmol 1,2,3,5-Tetrabrombenzol 231 (1.6 g), 20 mmol Phenylacetylen ( $2.0 \mathrm{~g} / 2.2 \mathrm{~mL}), 75$ $\mathrm{mg} \mathrm{PPh}, 25 \mathrm{~mL} \mathrm{NEt}_{3}, 25 \mathrm{mg}\left(\mathrm{PPh}_{3}\right)_{2} \mathrm{PdCl}_{2}, 25 \mathrm{mg} \mathrm{CuI}$. Aufarbeitung nach Variante B. Ausbeute: $1.5 \mathrm{~g} 238$ (78\%) als farblosen Feststoff vom Schmelzpunkt 131 ${ }^{\circ} \mathrm{C} .{ }^{1}$ H-NMR: $\delta=7.70(\mathrm{~s}, 2 \mathrm{H}, 4-\mathrm{H}, 6-\mathrm{H}), 7.62(\mathrm{~m}, 6 \mathrm{H}$, $10,10 '-\mathrm{H}, 14,14 '-\mathrm{H}, 18-\mathrm{H}, 22-\mathrm{H}), 7.56$ (m, 2H, 26-H, 30-H), 7.36 (m, 12H, 11,11'-H, 13,13'-H, 19-H, 21-H, 27-H, 29-H, 12,12'-H, 20-H, 28-H). ${ }^{13}$ C-NMR: $\delta=133.98$ (t, 2C, C-4, C-6), 131.72 (t, 6C, C10,10', C-14,14', C-18, C-22), 131.67 (m, 2C, C-26, C-30), 128.70 (t, 4C, C-12,12', C-20, C28), 128.41 (t, 8C, C-11,11', C-13,13', C-19, C-21, C-27, C-29), 127.38 (q, 1C, C-2), 126.41 (q, 2C, C-1, C-3), 123.21 (q, 1C, C-5), 122.94 (q, 1C, C-17), 122.90 (q, 2C, C-9,9'), 122.63 (q, 1C, C-25), 99.34 (q, 1C, C-16), 94.23 (q, 2C, C-8,8'), 91.91 (q, 1C, C-24), 87.76 (q, 1C, C15), 87.38 (q, 1C, C-23), 87.35 (q, 2C, C-7,7'). IR (KBr, Preßling) v = 3079, 3051, 3018, $2210,1597,1581,1570,1534,1495,1489,1442,1401,1068,1027,906,882,753,745,683$ 


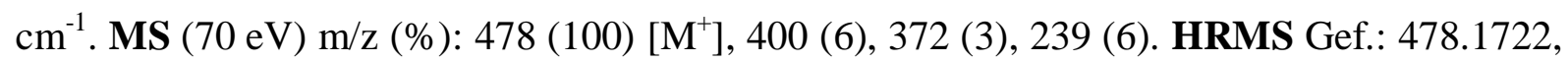
Ber.: $\mathrm{C}_{30} \mathrm{H}_{18} 478.1721$.

\section{1,2,4,5-Tetrakis(phenylethinyl)benzol (240): 245}

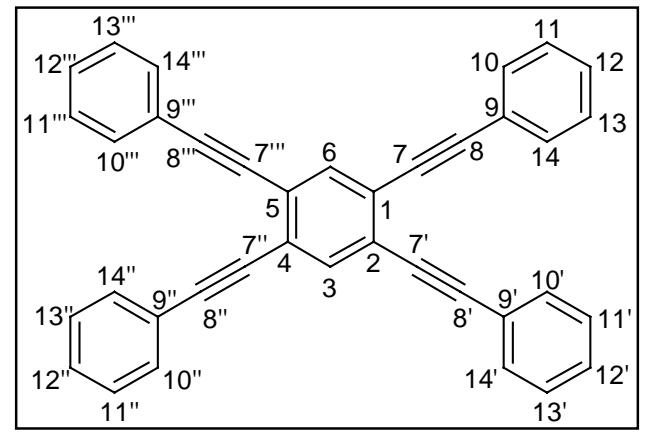

Ausgangsmaterialien: $10 \mathrm{mmol}$ 1,2,4,5-Tetrabrombenzol (3.9 g), $45 \mathrm{mmol}$ Phenylacetylen $(4.5 \mathrm{~g} / 5 \mathrm{~mL})$, $150 \mathrm{mg} \mathrm{PPh}, 50 \mathrm{~mL} \mathrm{NEt}_{3}, 50 \mathrm{mg}\left(\mathrm{PPh}_{3}\right)_{2} \mathrm{PdCl}_{2}, 50 \mathrm{mg}$ CuI. Aufarbeitung nach Variante B. Ausbeute: $3.9 \mathrm{~g}$ $240(82 \%)$ als farblosen Feststoff. ${ }^{1}$ H-NMR: $\delta=7.79$ (s, 2H, 3-H, 6-H), 7.60 (m, 8H, 10,10',10",10"'-H, 14,14',14",14'"-H), 7.38 (m, 12H, 11,11',11",11'"-H, 13,13',13",13"'-H, 12,12',12",12'"-H). ${ }^{13} \mathbf{C}-$ NMR: $\delta=134.85$ (t, 2C, C-3, C-6), 131.70 (t, 8C, C-10,10',10",10"', C-14,14',14",14"'), 128.71 (t, 4C, C-12,12',12",12"'), 128.41 (t, 8C, C-11,11',11",11"', C-13,13',13",13"'), 125.28 (q, 4C, C-1, C-2, C-4, C-5), 122.91 (q, 4C, C-9,9',9",9"'), 95.44 (q, 4C, C-8,8',8",8"'), 87.52 (q, 4C, C-7,7',7",7"'). MS (70 eV) Massenpeak m/z = 478.2 ( $\left.{ }^{+}\right) ; \mathrm{C}_{38} \mathrm{H}_{22}(478.17)$.

\section{1,2,3,4,5-Pentakis(phenylethinyl)benzol (241):}

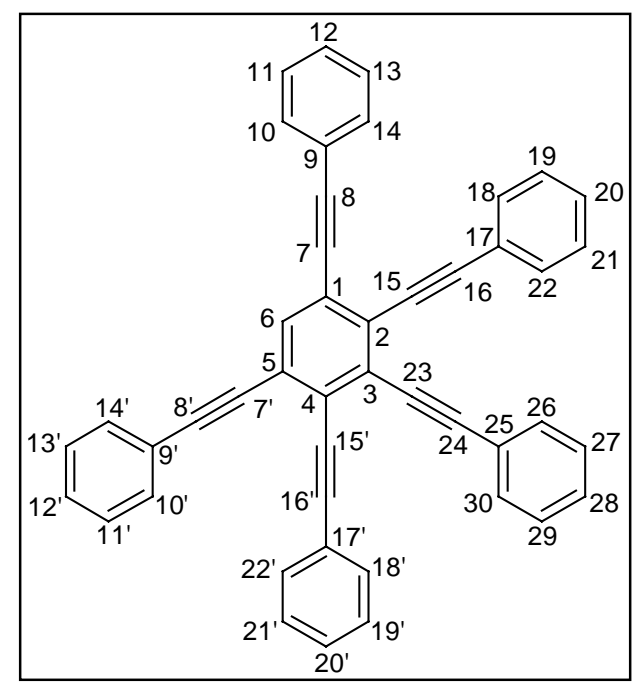

Ausgangsmaterialien: $2 \mathrm{mmol}$ 1,2,3,4,5-Pentabrombenzol 234 (0.95 g), $15 \mathrm{mmol}$ Phenylacetylen (1.5 g/1.7 $\mathrm{mL}), 75 \mathrm{mg} \mathrm{PPh}_{3}, 25 \mathrm{~mL} \mathrm{NEt}_{3}+5 \mathrm{~mL} \mathrm{DMF}, 25 \mathrm{mg}$ $\left(\mathrm{PPh}_{3}\right)_{2} \mathrm{PdCl}_{2}, 25 \mathrm{mg}$ CuI. Aufarbeitung nach Variante B. Ausbeute: 0.85 g 241 (74\%) als hellbraunen Feststoff vom Schmelzpunkt $162^{\circ} \mathrm{C} .{ }^{1} \mathbf{H}$-NMR: $\delta=7.72$ (s, $1 \mathrm{H}, 6-\mathrm{H}), 7.61\left(\mathrm{~m}, 10 \mathrm{H}, 10,10^{\prime}-\mathrm{H}, 18,18^{\prime}-\mathrm{H}, 26-\mathrm{H}\right.$, $\left.14,14^{\prime}-\mathrm{H}, 22,222^{\prime}-\mathrm{H}, 30-\mathrm{H}\right), 7.38$ (m, 15H, 11,11'-H, 19,19'-H, 27-H, 13,13'-H, 21,21'-H, 29'-H, 12,12'-H, 20,20'-H, 28-H). ${ }^{13} \mathbf{C}-\mathbf{N M R}: \delta=133.84(\mathrm{t}, 1 \mathrm{C}, \mathrm{C}-6)$, 131.77 (t, 10C, C-10,10', C-18,18', C-26, C-14,14', C-22,22', C-30), 128.78 (t, 5C, C-12,12', C-20,20', C-28), 128.59 (q, 1C, C-3), 128.43 (t, 10C, C-11,11', C-19,19', C-27, C-13,13', C21,21', C-29'), 127.50 (q, 2C, C-2, C-4), 125.31 (q, 2C, C-1, C-5), 123.18 (q, 3C, C-17, 17', C25), 122.8818 (q, 2C, C-9,9'), 99.36 (q, 2C, C-16,16'), 98.28 (q, 1C, C-24), 95.48 (q, 2C, C8,8'), 87.48 (q, 2C, C-7,7'/C-15,15'), 87.33 (q, 2C, C-7,7'/C-15,15'), 87.10 (q, 1C, C-23). IR (KBr, Preßling) $v=3052,3033,2205,1679,1596,1569,1514,1492,1441,1408,1383$, 1087, 1068, 1024, 912, 875, 751, $688 \mathrm{~cm}^{-1} . \mathbf{M S}(70 \mathrm{eV}) \mathrm{m} / \mathrm{z}(\%): 578$ (100) $\left[\mathrm{M}^{+}\right], 491(8)$, 
478 (50), 372 (8), 361 (8), 289 (4), 249 (4), 183 (25), 135 (6), 124 (10), 102 (15). HRMS Gef.: 578.2035, Ber.: $\mathrm{C}_{46} \mathrm{H}_{26}(578.2034)$.

\section{1,2,3,4,5,6-Hexakis(phenylethinyl)benzol (242): 245}

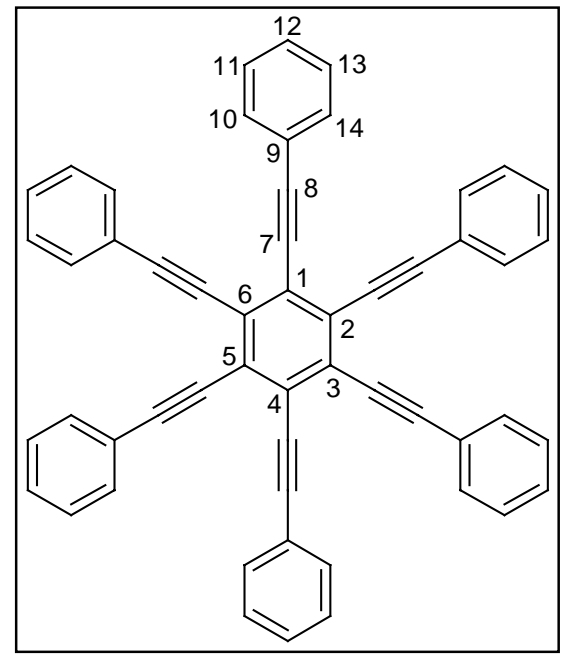

Ausgangsmaterialien: $10 \mathrm{mmol}$ 1,2,3,4,5,6-Hexabrombenzol 233 (5.5 g), $70 \mathrm{mmol}$ Phenylacetylen (7.0 g/7.7 $\mathrm{mL}$ ), $150 \mathrm{mg} \mathrm{PPh}_{3}, 50 \mathrm{~mL} \mathrm{NEt}{ }_{3}+5 \mathrm{~mL} \mathrm{DMF}, 50 \mathrm{mg}$ $\left(\mathrm{PPh}_{3}\right)_{2} \mathrm{PdCl}_{2}, 50 \mathrm{mg} \mathrm{CuI}$. Aufarbeitung nach Variante B. Ausbeute: $1.2 \mathrm{~g} 242$ (18\%) als gelben Feststoff. ${ }^{\mathbf{1}} \mathbf{H}$-NMR: $\delta=7.66$ (m, 12H, 10-10"'"-H, 14-14"'"-H), 7.37 (m, 18H, 11-11"'"-H, 13-13"'"-H, 12-12"'"-H). ${ }^{13} \mathbf{C}-\mathbf{N M R}: \delta=131.83$ (t, 12C, C-10-10'"'", C-14-14"'"), 128.92 (t, 6C, C-12-12"'"'), 128.48 (t, 12C, C-11-11"'", C-13-13"'"'), 127.49 (q, 6C, C-16), 123.13 (q, 6C, C-9-9"'"), 99.39 (q, 6C, C-8-8'"'), 87.26 (q, 6C, C-7-7"'"'). MS (70 eV) Massenpeak $\mathrm{m} / \mathrm{z}=678.0\left(\mathrm{M}^{+}\right) ; \mathrm{C}_{54} \mathrm{H}_{30}(678.23)$.

\section{1-(Phenylethinyl)naphthalin (245): 244}

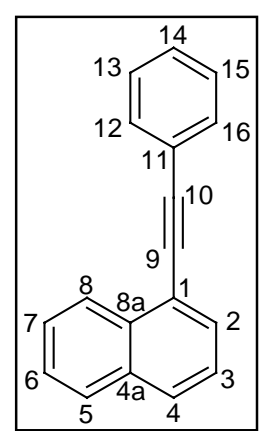

Ausgangsmaterialien: $20 \mathrm{mmol}$ 1-Bromnaphthalin $244(4.2 \mathrm{~g} / 2.5 \mathrm{~mL}), 25$ mmol Phenylacetylen $(2.5 \mathrm{~g} / 2.8 \mathrm{~mL}), 150 \mathrm{mg} \mathrm{PPh}_{3}, 50 \mathrm{~mL} \mathrm{NEt}_{3}, 50 \mathrm{mg}$ $\left(\mathrm{PPh}_{3}\right)_{2} \mathrm{PdCl}_{2}, 50 \mathrm{mg} \mathrm{CuI}$. Aufarbeitung nach Variante A. Die Reinigung erfolgte durch Säulen an Kieselgel mit Pentan $\left(\mathrm{R}_{\mathrm{f}}=0.19\right)$. Ausbeute: $4.0 \mathrm{~g}$ $245(87.7 \%)$ als farblose Flüssigkeit. ${ }^{1} \mathbf{H}-\mathbf{N M R}: \delta=8.57(\mathrm{~m}, 1 \mathrm{H}, 8-\mathrm{H}), 7.90$ (m, 3H, 2-H, 4-H, 5-H), 7.75 (m, 2H, 12-H, 16-H), 7.58 (m, 3H, 3-H, 6-H, 7H), 7.46 (m, 3H, 13-H, 15-H, 14-H). ${ }^{13}$ C-NMR: $\delta=133.36$ (q, 1C, C-8a), 133.30 (q, 1C, C4a), 131.76 (t, 2C, C-12, C-16), 130.46 (t, 1C, C-2), 128.86 (t, 1C, C-14), 128.52 (t, 2C, C13, C-15), 128.47 (t, 1C, C-4), 128.41 (t, 1C, C-5), 126.87 (t, 1C, C-7), 126.52 (t, 1C, C-8), 126.30 (t, 1C, C-6), 125.36 (t, 1C, C-3), 123.49 (q, 1C, C-1), 120.99 (q, 1C, C-11), 94.46 (q, 1C, C-10), 87.68 (q, 1C, C-9). MS (GC-MS) Massenpeak m/z = 228.2 (M+); $\mathrm{C}_{18} \mathrm{H}_{12}$ (228.09). 


\section{1,8-Bis(phenylethinyl)naphthalin (203): 44,246}

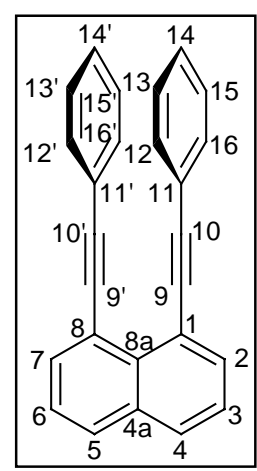

Ausgangsmaterialien: 5 mmol 1,8-Diiodnaphthalin 247 (1.9 g), $10 \mathrm{mmol}$ Phenylacetylen $(1.0 \mathrm{~g} / 1.1 \mathrm{~mL}), 75 \mathrm{mg} \mathrm{PPh}_{3}, 25 \mathrm{~mL} \mathrm{NEt}_{3}, 25 \mathrm{mg}$ $\left(\mathrm{PPh}_{3}\right)_{2} \mathrm{PdCl}_{2}, 25 \mathrm{mg} \mathrm{CuI}$. Aufarbeitung nach Variante A. Ausbeute: $0.4 \mathrm{~g}$ 203 (22\%) als hellbraunen Feststoff. ${ }^{1} \mathbf{H}-\mathbf{N M R}: \delta=7.87$ (m, 2H, 2-H, 7-H), $7.82(\mathrm{~m}, 2 \mathrm{H}, 4-\mathrm{H}, 5-\mathrm{H}), 7.45$ (m, 2H, 3-H, 6-H), 7.35 (m, 4H, 12,12'-H, 16,16'-H), 7.19 (m, 2H, 14,14'-H), 7.11 (m, 4H, 13,13'-H, 15,15'-H). ${ }^{13} \mathbf{C}-$ NMR: $\delta=134.88$ (t, 2C, C-2, C-7), 134.09 (q, 1C, C-8a), 131.55 (t, 4C, C12,12', C-16-16'), 131.39 (q, 1C, C-4a), 129.58 (t, 2C, C-4, C-5), 127.88 (t, 4C, C-13,13', C15,15'), 127.83 (t, 2C, C-14,14'), 125.55 (t, 2C, C-3, C-6), 123.71 (q, 2C, C-11,11'), 120.81 (q, 2C, C-1, C-8), 96.62 (q, 2C, C-10,10'), 89.69 (q, 2C, C-9,9'). MS (70 eV) Massenpeak m/z = $328.2\left(\mathrm{M}^{+}\right) ; \mathrm{C}_{26} \mathrm{H}_{16}(328.13)$.

\section{2-(Phenylethinyl)biphenyl (24): ${ }^{247}$}

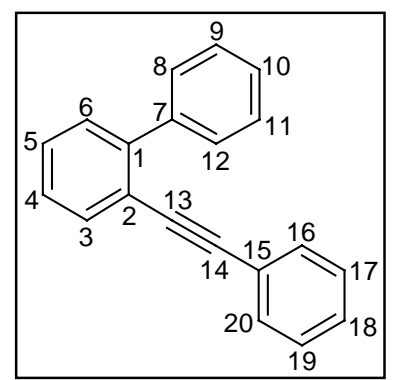

Ausgangsmaterialien: $10 \mathrm{mmol}$ 2-Brombiphenyl (2.3 g), $20 \mathrm{mmol}$ Phenylacetylen $(2.0 \mathrm{~g} / 2.2 \mathrm{~mL}), 75 \mathrm{mg} \mathrm{PPh}_{3}, 25 \mathrm{~mL} \mathrm{NEt}{ }_{3}, 25 \mathrm{mg}$ $\left(\mathrm{PPh}_{3}\right)_{2} \mathrm{PdCl}_{2}, 25 \mathrm{mg}$ CuI. Aufarbeitung nach Variante A, Säulen an Kieselgel mit Pentan:Ether = 99:1. Ausbeute: 2.0 g 24 (79\%) als oranges Öl. ${ }^{1} \mathbf{H}-\mathbf{N M R}: \delta=7.80(\mathrm{~m}, 3 \mathrm{H}, 6-\mathrm{H}, 8-\mathrm{H}, 12-\mathrm{H}), 7.61-7.36$ (m, 11H, 3-H, 4-H, 5-H, 9-H, 11-H, 10-H, 16-H, 20-H, 17-H, 19-H,

18-H). ${ }^{13}$ C-NMR: $\delta=143.81$ (q, 1C, C-1), 140.46 (q, 1C, C-7), 132.78 (t, 1C, C-3), 131.27 (t, 2C, C-16, C-20), 129.40 (t, 1C, C-6), 129.33 (t, 2C, C-8, C-12), 128.46 (t, 1C, C-18), 128.18 (t, 2C, C-17, C-19), 128.03 (t, 1C, C-10), 127.82 (t, 2C, C-9, C-11), 127.40 (t, 1C, C-5), 126.99 (t, 1C, C-4), 123.37 (q, 1C, C-2), 121.48 (q, 1C, C-15), 92.18 (q, 1C, C-14), 89.38 (q, 1C, C-13). MS (GC-MS) Massenpeak m/z = $254.2\left(\mathrm{M}^{+}\right) ; \mathrm{C}_{20} \mathrm{H}_{14}(254.11)$.

\section{2-(Phenylethinyl)benzaldehyd (250): 248}

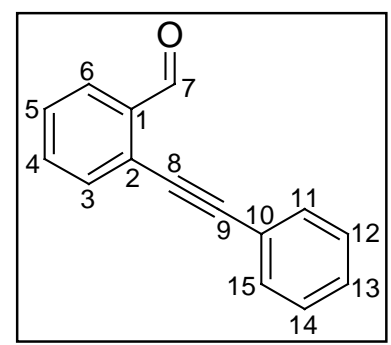

Ausgangsmaterialien: $50 \mathrm{mmol}$ 2-Brombenzaldehyd 249 (9.25 g/5.9 $\mathrm{mL}), 60 \mathrm{mmol}$ Phenylacetylen $(6.0 \mathrm{~g} / 6.6 \mathrm{~mL}), 150 \mathrm{mg} \mathrm{PPh}, 50 \mathrm{~mL}$ $\mathrm{NEt}_{3}, 50 \mathrm{mg}\left(\mathrm{PPh}_{3}\right)_{2} \mathrm{PdCl}_{2}, 50 \mathrm{mg} \mathrm{CuI}$. Aufarbeitung nach Variante A. Ausbeute: 8.2 g 250 (80\%) als gelbe Flüssigkeit. ${ }^{\mathbf{1}} \mathbf{H}-\mathbf{N M R}: \delta=10.66$ (s, 1H, 7-H), $7.95(\mathrm{~m}, 1 \mathrm{H}, 6-\mathrm{H}), 7.64(\mathrm{~m}, 1 \mathrm{H}, 4-\mathrm{H}), 7.57$ (m, 3H, 3-H, 11-H, 15-H), 7.45 (m, 1H, 5-H), 7.39 (m, 3H, 12-H, 14-H, 13-H). ${ }^{13}$ C-NMR: $\delta=191.67(\mathrm{t}$, 1C, C-7), 135.74 (q, 1C, C-1), 133.75 (t, 1C, C-4), 133.16 (t, 1C, C-3), 131.62 (t, 2C, C-11, 
C-15), 129.03 (t, 1C, C-6), 128.57 (t, 1C, C-13), 128.48 (t, 2C, C-12, C-14), 127.19 (t, 1C, C-5), 126.82 (q, 1C, C-2), 122.25 (q, 1C, C-10), 96.27 (q, 1C, C-9), 84.83 (q, 1C, C-8). MS (GC-MS) Massenpeak m/z = $206.1\left(\mathrm{M}^{+}\right) ; \mathrm{C}_{15} \mathrm{H}_{10} \mathrm{O}$ (206.07).

\section{2-(Phenylethinyl)styrol (243): ${ }^{249}$}

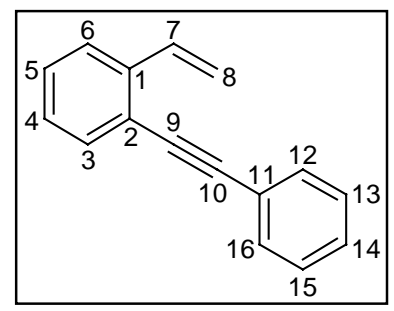

Ausgangsmaterialien: $2 \mathrm{mmol}$ 2-Bromstyrol (0.37 g/0.25 mL), 2.5 mmol Phenylacetylen $(2.5 \mathrm{~g} / 2.8 \mathrm{~mL}), 75 \mathrm{mg} \mathrm{PPh}_{3}, 25 \mathrm{~mL} \mathrm{NEt}_{3}, 25$ mg $\left(\mathrm{PPh}_{3}\right)_{2} \mathrm{PdCl}_{2}, 25 \mathrm{mg} \mathrm{CuI}$. Aufarbeitung nach Variante A. Säulen an Kieselgel mit Pentan $\left(\mathrm{R}_{\mathrm{f}}=0.21\right)$. Ausbeute: $0.36 \mathrm{~g} 243(88.2 \%)$ als gelbes Öl. ${ }^{1}$ H-NMR: $\delta=7.63(\mathrm{~m}, 4 \mathrm{H}, 3-\mathrm{H}, 6-\mathrm{H}, 12-\mathrm{H}, 16-\mathrm{H}), 7.40$ (m, 4H, 4-H, 5-H, 13-H, 15-H), 7.34 (m, 2H, 14-H, 7-H), $5.93\left(\mathrm{dd}, 1 \mathrm{H}\right.$, cis-8-H, ${ }^{2} \mathrm{~J}=0.8,{ }^{3} \mathrm{~J}=$ 17.6), 5.46 (dd, $1 \mathrm{H}$, trans-8-H, $\left.{ }^{2} \mathrm{~J}=0.8,{ }^{3} \mathrm{~J}=11.0\right) .{ }^{13} \mathrm{C}-\mathbf{N M R}: \delta=138.91$ (q, 1C, C-1), 134.87 (t, 1C, C-7), 132.45 (t, 1C, C-3), 131.48 (t, 2C, C-12, C-16), 128.40 (t, 1C, C-14), 128.31 (t, 2C, C-13, C-15), 128.28 (t, 1C, C-5), 127.45 (t, 1C, C-4), 124.61 (t, 1C, C-6), 123.25 (q, 1C, C-2), 121.87 (q, 1C, C-11), 115.56 (q, 1C, C-8), 93.96 (q, 1C, C-10), 87.72 (q, 1C, C-9). MS (GC-MS) Massenpeak m/z = 204.1 ( $\left.\mathrm{M}^{+}\right) ; \mathrm{C}_{16} \mathrm{H}_{12}$ (204.09).

\section{1-(2'-Biphenylethinyl)-2-(phenylethinyl)benzol (252):}

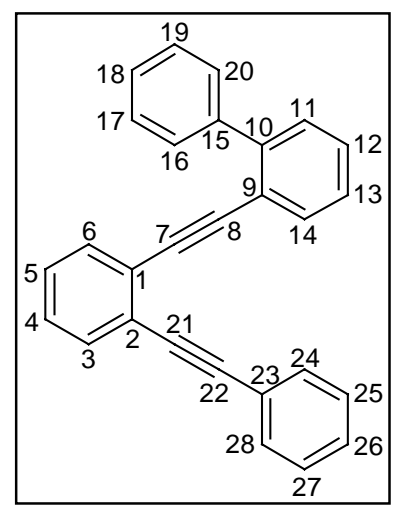

Ausgangsmaterialien: $12.5 \mathrm{mmol}$ 1-Phenylethinyl-2-ethinylbenzol 248 (2.5 g), $12.5 \mathrm{mmol}$ 2-Brombiphenyl (2.9 g), $150 \mathrm{mg} \mathrm{PPh}_{3}, 50 \mathrm{~mL}$ $\mathrm{NEt}_{3}, 50 \mathrm{mg}\left(\mathrm{PPh}_{3}\right)_{2} \mathrm{PdCl}_{2}, 50 \mathrm{mg} \mathrm{CuI}$. Aufarbeitung nach Variante A. Säulen an Kieselgel mit Pentan:Ether $=95: 5\left(R_{f}=0.3\right)$. Ausbeute: 1.2 g 252 (27\%) als gelbes Öl. ${ }^{1} \mathbf{H}$-NMR: $\delta=7.73$ (m, 3H, 14-H, 17H, 19-H), 7.55 (m, 3H, 3-H, 24-H, 28-H), 7.43 (m, 4H, 11-H, 16-H, 20-H, 18-H), 7.3 (m, 8H, 4-H, 5-H, 6-H, 12-H, 13-H, 26-H, 27-H, 26H). ${ }^{13}$ C-NMR: $\delta=143.65$ (q, 1C, C-15), 140.29 (q, 1C, C-10), 133.29 (t, 1C, C-14), 131.86 (t, 1C, C-3/C-6), 131.81 (t, 1C, C-3/C-6), 131.67 (t, 2C, C-24, C-28), 129.49 (t, 1C, C-11). 129.33 (t, 2C, C-17, C-19), 128.70 (t, 1C, C-4/C-5/C-12/C-13/C-18/C-26), 128.36 (t, 1C, C4/C-5/C-12/C-13/C-18/C-26), 128.31 (t, 2C, C-25, C-27), 127.88 (t, 2C, C-16, C-20), 127.86 (t, 1C, C-4/C-5/C-12/C-13/C-18/C-26), 27.82 (t, 1C, C-4/C-5/C-12/C-13/C-18/C-26), 127.45 (t, 1C, C-4/C-5/C-12/C-13/C-18/C-26), 126.99 (t, 1C, C-4/C-5/C-12/C-13/C-18/C-26), 125.88 (q, 1C, C-23), 125.37 (q, 1C, C-1/C-2), 123.24 (q, 1C, C-1/C-2), 121.50 (q, 1C, C-9), 93.32 (q, 1C, C-8/C-22), 93.29 (q, 1C, C-8/C-22), 91.03 (q, 1C, C-21), 88.59 (q, 1C, C-7). IR (KBr, Film) $v=3059$, 3030, 2214, 1953, 1896, 1819, 1712, 1598, 1493, 1482, 1442, 1312, 1161, 
1089, 1070, 1026, 1008, 950, 915, 870, 752, $699 \mathrm{~cm}^{-1}$. MS (70 eV) m/z (\%): $354(100)\left[\mathrm{M}^{+}\right]$, 352 (68), 339 (12), 326 (8), 277 (20), 252 (6), 201 (2), 136 (5). HRMS Gef.: 354.1409, Ber.: $\mathrm{C}_{28} \mathrm{H}_{18}$ (354.1409).

\section{1,4-Diphenyl-but-1-in (217): ${ }^{250}$}

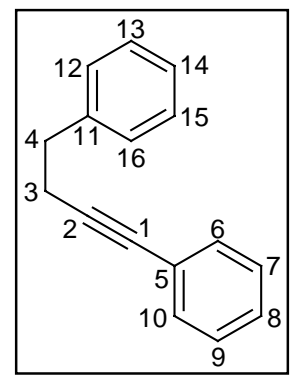

Ausgangsmaterialien: $20 \mathrm{mmol}$ 4-Phenyl-but-1-in 259 (2.6 g), $25 \mathrm{mmol}$ Brombenzol (3.9 g/2.6 mL), $150 \mathrm{mg} \mathrm{PPh}_{3}, 50 \mathrm{~mL} \mathrm{NEt} 3,50 \mathrm{mg}$ $\left(\mathrm{PPh}_{3}\right)_{2} \mathrm{PdCl}_{2}, 50 \mathrm{mg} \mathrm{CuI}$. Aufarbeitung nach Variante A. Säulen an Kieselgel mit Pentan $\left(\mathrm{R}_{\mathrm{f}}=0.36\right)$. Ausbeute: $3.4 \mathrm{~g} 217(82 \%)$ als farblose Flüssigkeit. ${ }^{1} \mathbf{H}-\mathbf{N M R}: \delta=7.42(\mathrm{~m}, 2 \mathrm{H}, 6-\mathrm{H}, 10-\mathrm{H}), 7.32(\mathrm{~m}, 8 \mathrm{H}, 7-\mathrm{H}, 9-\mathrm{H}$, 8-H, 12-H, 16-H, 13-H, 15-H, 14-H), 2.97 (t, 2H, 4-H, $\left.{ }^{3} J=7.3 \mathrm{~Hz}\right), 2.73$ (t, 2H, 3-H, $\left.{ }^{3} J=7.3 \mathrm{~Hz}\right) .{ }^{13} \mathrm{C}-\mathrm{NMR}: \delta=140.64$ (q, 1C, C-11), 131.47 (t, 2C, C-6, C-10), 128.49 (t, 2C, C-13, C-15), 128.33 (t, 2C, C-12, C-16), 128.15 (t, 2C, C-7, C-9), 127.58 (t, 1C, C-8), 126.26 (t, 1C, C-14), 123.77 (q, 1C, C-5), 89.45 (q, 1C, C-2), 81.26 (q, 1C, C-1), 35.13 (s, 1C, C-4), 21.65 (s, 1C, C-3). MS (GC-MS) Massenpeak m/z = 206.1 ( $\left.\mathrm{M}^{+}\right) ; \mathrm{C}_{16} \mathrm{H}_{14}(206.11)$.

\section{1,2-Bis(3-hydroxy-3-methylbutin-1-yl)benzol (255):230}

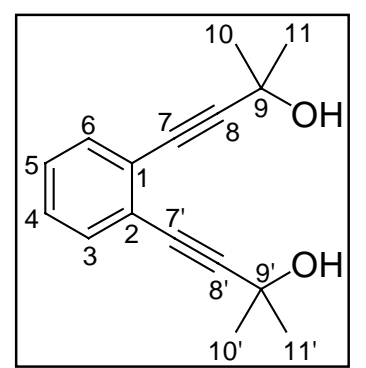

Ausgangsmaterialien: $100 \mathrm{mmol}$ 1,2-Dibrombenzol (23.6 g/11.9 mL), 250 mmol 2-Methyl-but-3-in-2-ol (21.0 g/24.4 mL), $750 \mathrm{mg} \mathrm{PPh}_{3}, 250$ $\mathrm{mL} \mathrm{NEt}{ }_{3}, 250 \mathrm{mg}\left(\mathrm{PPh}_{3}\right)_{2} \mathrm{PdCl}_{2}, 250 \mathrm{mg} \mathrm{CuI}$. Aufarbeitung nach Variante A. Säulen an Kieselgel mit Pentan:Ether $=60: 40\left(R_{\mathrm{f}}=0.22\right)$. Ausbeute: $23.0 \mathrm{~g} 255$ (95\%) als gelben Feststoff. ${ }^{1}$ H-NMR: $\delta=7.32(\mathrm{~m}$, 2H, 3-H, 6-H), 7.12 (m, 2H, 4-H, 5-H), 1.60 (s, 12H, 10,10'-C, 11,11'-C). ${ }^{13}$ C-NMR: $\delta=$ 131.08 (t, 2C, C-3, C-6), 127.49 (t, 2C, C-4, C-5), 125.25 (q, 2C, C-1, C-2), 98.01 (q, 2C, C8,8'), 80.45 (q, 2C, C-7,7'), 65.29 (q, 2C, C-9,9'), 31.22 (p, 6C, C-10,10', C-11,11'). MS (GCMS) Massenpeak m/z $=242.3\left(\mathrm{M}^{+}\right) ; \mathrm{C}_{16} \mathrm{H}_{18} \mathrm{O}_{2}(242.13)$. 


\section{0-(Phenylethinyl)-9-phenylphenathren (272):}

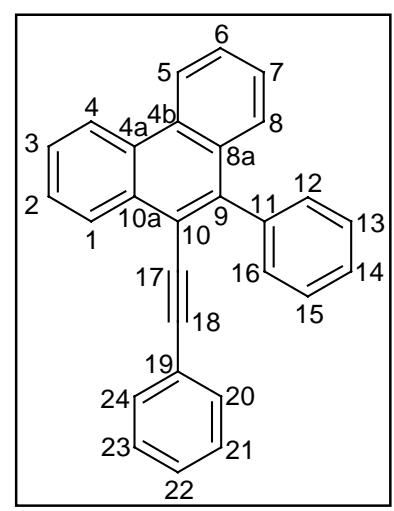

Ausgangsmaterialien: 3 mmol 9-Brom-10-phenylphenathren 271 (1.0 g), 10 mmol Phenylacetylen (1.0 g/1.1 mL), $75 \mathrm{mg} \mathrm{PPh}_{3}, 25 \mathrm{~mL}$ $\mathrm{NEt}_{3}, 25 \mathrm{mg}\left(\mathrm{PPh}_{3}\right)_{2} \mathrm{PdCl}_{2}, 25 \mathrm{mg} \mathrm{CuI}$. Aufarbeitung nach Variante B. Säulen an Kieselgel mit Pentan:Ether $=95: 5\left(R_{\mathrm{f}}=0.35\right)$. Ausbeute: $0.66 \mathrm{~g} 272(62 \%)$ als farblose Kristalle vom Schmelzpunkt $119^{\circ} \mathrm{C}$. ${ }^{1}$ H-NMR: $\delta=8.77(\mathrm{~m}, 2 \mathrm{H}, 4-\mathrm{H}, 5-\mathrm{H}), 8.70(\mathrm{~m}, 1 \mathrm{H}, 8-\mathrm{H}), 7.74(\mathrm{~m}$, 4G, Ar-H), 7.58 (m, 6-H, Ar-H), 7.30 (m, 5H, Ar-H). ${ }^{13}$ C-NMR: $\delta=$ 142.93 (q, 1C, C-9), 139.73 (q, 1C, C-11), 131.38 (t, 2C, C-20, C-24), 131.30 (q, 1C, C-10a), 130.65 (t+q, 3C, C-12, C-16, C-8a), 130.16 (q, 1C, C-4a/4b), 129.65 (q, 1C, C-4a/4b), 128.21 (t, 2C, C-Ar), 128.12 (t, 1C, C-Ar), 128.02 (t, 2C, C-Ar), 127.74 (t, 1C, C-Ar), 127.47 (t, 1C, C-Ar), 127.32 (t, 1C, C-Ar), 127.28 (t, 1C, C-Ar), 127.14 (t, 1C, CAr), 127.09 (t, 1C, C-Ar), 127.02 (t, 1C, C-Ar), 126.72 (t, 1C, C-Ar), 123.43 (q, 1C, C-10), 122.62 (t, 2C, C-4, C-5), 119.01 (q, 1C, C-18), 98.18 (q, 1C, C-18), 87.74 (q, 1C, C-17). IR (KBr, Preßling) $v=3079$, 3055, 3022, 2208, 1956, 1931, 1810, 1594, 1569, 1487, 1448, $1441,1418,1333,1235,1174,1155,1070,1038,1024,998,910,857,836,775,764,732$,

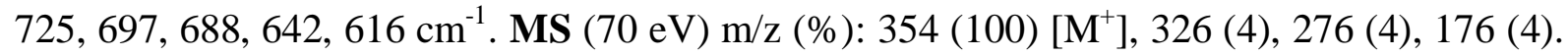

HRMS Gef.: 354.1409, Ber.: $\mathrm{C}_{28} \mathrm{H}_{18}$ (354.1409).

Synthese der Dibromolefine durch Corey-Fuchs Synthese: ${ }^{225} \mathrm{Zu}$ einer auf $0{ }^{\circ} \mathrm{C}$ gekühlten Lösung des entsprechenden Aldehyds und $\mathrm{CBr}_{4}$ in $\mathrm{CH}_{2} \mathrm{Cl}_{2}$ wird $\mathrm{PPh}_{3}$ in vier Portionen hinzugegeben und die Lösung zwei Stunden bei RT rühren gelassen. Anschließend wird das Lösungsmittel am Rotationsverdampfer entfernt und der Rückstand in Hexan aufgeschlämmt. Durch Vakuumfiltration wird der Feststoff abgetrennt und gut mit Hexan gewaschen, anschließend das Lösungsmittel abgezogen. Die Dibromolefine wurden ohne weitere Reinigung weiterverarbeitet.

\section{(2,2-Dibromvinyl)benzol: 251}

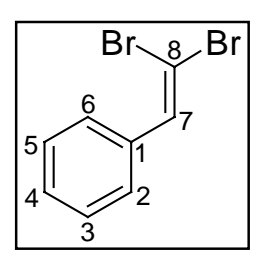

Ausgangsmaterialien: $50 \mathrm{mmol}$ Benzaldehyd $(5.3 \mathrm{~g} / 5.1 \mathrm{~mL}), 52 \mathrm{mmol} \mathrm{CBr}_{4}$ (17.2 g), $150 \mathrm{~mL} \mathrm{CH} \mathrm{Cl}_{2}, 104 \mathrm{mmol} \mathrm{PPh}_{3}(27.3 \mathrm{~g})$. Ausbeute: $10.6 \mathrm{~g}(80.7 \%)$ als farbloser Feststoff. ${ }^{1} \mathbf{H}-\mathbf{N M R}: \delta=7.53(\mathrm{~m}, 2 \mathrm{H}, 2-\mathrm{H}, 6-\mathrm{H}), 7.49$ (s, 1H, 7H), 7.37 (m, 3H, 3-H, 5-H, 4-H). ${ }^{13} \mathbf{C}-\mathbf{N M R}: \delta=136.83(\mathrm{t}, 1 \mathrm{C}, \mathrm{C}-7), 135.27$ (q, 1C, C-1), 128.52 (t, 1C, C-4), 128.38 (t, 2C, C-3, C-5), 128.35 (t, 2C, C-2, C-6), 89.58 (q, 1C, C-8). MS (GC-MS) Massenpeak m/z = $269.9\left(\mathrm{M}^{+}\right) ; \mathrm{C}_{8} \mathrm{H}_{6} \mathrm{Br}_{2}$ (259.88). 


\section{1,1-Dibrom-4-phenyl-but-1-en (258): 252}

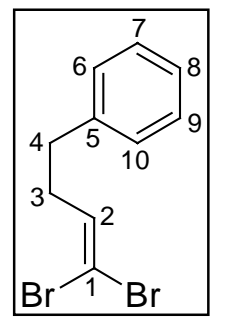

Ausgangsmaterialien: 50 mmol 3-Phenylpropionaldehyd 257 (6.7 g/6.7 mL), 52 mmol CBr 4 (17.2 g), $150 \mathrm{~mL} \mathrm{CH}_{2} \mathrm{Cl}_{2}, 104 \mathrm{mmol} \mathrm{PPh}_{3}(27.6 \mathrm{~g})$. Ausbeute: $11.7 \mathrm{~g}$ $258(81 \%)$ als gelbes Öl. ${ }^{1} \mathbf{H}-\mathbf{N M R}: \delta=7.33(\mathrm{~m}, 2 \mathrm{H}, 6-\mathrm{H}, 10-\mathrm{H}), 7.21(\mathrm{~m}, 3 \mathrm{H}$, 7-H, 9-H, 8-H), $6.43\left(\mathrm{t}, 1 \mathrm{H}, 2-\mathrm{H},{ }^{3} \mathrm{~J}=7.2 \mathrm{~Hz}\right), 2.76\left(\mathrm{t}, 2 \mathrm{H}, 4-\mathrm{H},{ }^{3} \mathrm{~J}=7.7 \mathrm{~Hz}\right)$, $2.45\left(\mathrm{dt}, 2 \mathrm{H}, 3-\mathrm{H},{ }^{3} \mathrm{~J}=7.2,7.7 \mathrm{~Hz}\right) .{ }^{13} \mathbf{C}-\mathbf{N M R}: \delta=140.46(\mathrm{q}, 1 \mathrm{C}, \mathrm{C}-5), 137.56$ (t, 1C, C-2), 128.46 (t, 2C, C-6, C-10), 128.32 (t, 2C, C-7, C-9), 126.21 (t, 1C, C-8), 89.43 (q, 1C, C-1), 34.61 (s, 1C, C-4), 33.79 (s, 1C, C-3). MS (GC-MS) Massenpeak m/z = $287.8\left(\mathrm{M}^{+}\right)$; $\mathrm{C}_{10} \mathrm{H}_{10} \mathrm{Br}_{2}$ (287.91).

\section{1-(2,2-Dibromvinyl)-2-(phenylethinyl)benzol (251):}

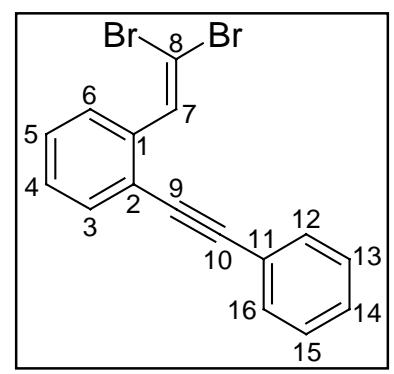

Ausgangsmaterialien: $36 \mathrm{mmol}$ 2-(Phenylethinyl)-benzaldehyd 250 (7.4 g), $38 \mathrm{mmol} \mathrm{CBr}_{4}$ (12.6 g), $100 \mathrm{~mL} \mathrm{CH}_{2} \mathrm{Cl}_{2}, 76 \mathrm{mmol} \mathrm{PPh}_{3}$ (19.9 g). Ausbeute: 11.4 g 251 (87.5\%) als gelbe Kristalle vom

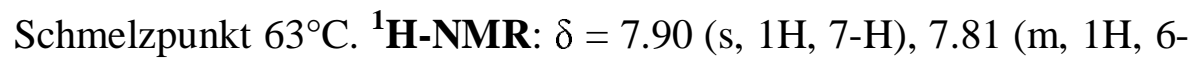
H), 7.59 (m, 3H, 3-H, 12-H, 16-H), 7.38 (m, 5H, 5-H, 4-H, 13-H, 15H, 14-H). ${ }^{13} \mathbf{C}-\mathbf{N M R}: \delta=137.17(\mathrm{q}, 1 \mathrm{C}, \mathrm{C}-1), 135.83$ (t, 1C, C-7), 132.01 (t, 1C, C-3), 131.54 (t, 2C, C-12, C-16), 128.59 (t, 1C, C-14), 128.40 (t, 2C, C-13, C15), 128.24 (t, 1C, C-6), 128.00 (t, 2C, C-4, C-5), 122.80 (q, 1C, C-2), 122.70 (q, 1C, C-11), 95.14 (q, 1C, C-10), 91.56 (q, 1C, C-8), 87.16 (q, 1C, C-9). IR (KBr, Preßling) v = 3055, 3013, 2213, 1598, 1490, 1441, 1260, 1153, 1120, 1089, 1069, 1025, 944, 914, 884, 862, 847, 832, 752, 726, $688 \mathrm{~cm}^{-1}$. MS (70 eV) m/z (\%): 364 (2), 362 (4), 360 (2) [M+ $], 283$ (25), 281 (25), 202 (100), 189 (5), 150 (4), 101 (15). HRMS Gef.: 359.9148, Ber.: $\mathrm{C}_{16} \mathrm{H}_{10} \mathrm{Br}_{2}$ (359.9149).

\section{1-Phenylethinyl-2-ethinylbenzol (248): ${ }^{242}$}

$\mathrm{Zu}$ einer auf $-78^{\circ} \mathrm{C}$ gekühlten Lösung von $30 \mathrm{mmol}$ Diisopropylamin $(3.0 \mathrm{~g} / 4.2 \mathrm{~mL})$ in 30 $\mathrm{mL}$ abs. THF werden $25 \mathrm{mmol} \mathrm{BuLi}$ (15.6 mL einer 1.6 molaren Lösung in Hexan) getropft. Die Lösung wird eine Stunde gerührt, anschließend werden 10 mmol 1-(2,2-Dibromvinyl)-2(phenylethinyl)-benzol (251, $3.6 \mathrm{~g})$ in $10 \mathrm{~mL}$ abs. THF hinzugetropft. Die Lösung wird eine Stunde bei $-78{ }^{\circ} \mathrm{C}$, eine weitere Stunde bei RT gerührt und anschließend mit Wasser gequencht. Die Mischung wird zweimal mit $\mathrm{CH}_{2} \mathrm{Cl}_{2}$ extrahiert, die vereinigten organischen Phasen getrocknet und das Lösungsmittel abgezogen. Die Reinigung erfolgte durch Säulen an Kieselgel mit Pentan:Ether = 90:10 $\left(\mathrm{R}_{\mathrm{f}}=0.42\right)$. 


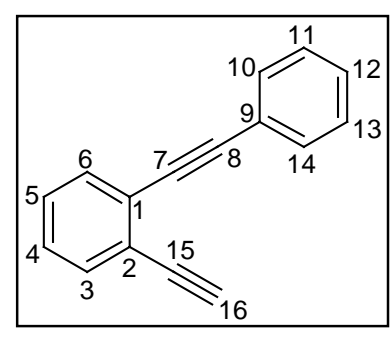

Ausbeute: $1.67 \mathrm{~g} 248$ ( 82.7\%) als gelbes Öl. ${ }^{1} \mathbf{H}-\mathbf{N M R}: \delta=7.58(\mathrm{~m}$, $4 \mathrm{H}, 6-\mathrm{H}, 10-\mathrm{H}, 14-\mathrm{H}), 7.33$ (m, 5H, 4-H, 5-H, 11- H, 13-H, 12-H), $3.40(\mathrm{~s}, 1 \mathrm{H}, 16-\mathrm{H}) .{ }^{13} \mathrm{C}-\mathbf{N M R}: \delta=132.50(\mathrm{t}, 1 \mathrm{C}, \mathrm{C}-6), 131.68(\mathrm{t}, 3 \mathrm{C}$, C-3, C-10, C-14), 128.48 (t, 1C, C-12), 128.44 (t, 1C, C-5), 128.27 (t, 2C, C-11, C-13), 127.86 (t, 1C, C-4), 126.19 (q, 1C, C-2), 124.52 (q, 1C, C-9), 123.03 (q, 1C, C-1), 93.47 (q, 1C, C-8), 87.79 (q, 1C, C-7), 82.11 (q, 1C, C-15), 81.13 (t, 1C, C-16). MS (GC-MS) Massenpeak m/z = $202.0\left(\mathrm{M}^{+}\right) ; \mathrm{C}_{16} \mathrm{H}_{10}$ (202.08).

\section{4-Phenyl-but-1-in (259): 253}

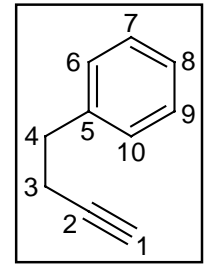

$\mathrm{Zu}$ einer auf $-78{ }^{\circ} \mathrm{C}$ gekühlten Lösung von 40 mmol 1,1-Dibrom-4-phenyl-but-1en 258 (11.6 g) in $100 \mathrm{~mL}$ abs. THF werden $90 \mathrm{mmol}$ BuLi-Lsg. 1.6 M in Hexan $(56.3 \mathrm{ml})$ getropft. Die Lösung wird eine Stunde bei $-78^{\circ} \mathrm{C}$, eine weitere Stunde bei RT gerührt und anschließend mit Wasser gequencht. Die Mischung wird zweimal mit Ether extrahiert, die vereinigten organischen Phasen getrocknet und das Lösungsmittel abgezogen. Die Reinigung erfolgte durch Destillation. Ausbeute: 3.3 g 259 $(62 \%)$ als farblose Flüssigkeit. ${ }^{1} \mathbf{H}-\mathbf{N M R}: \delta=7.31(\mathrm{~m}, 2 \mathrm{H}, 6-\mathrm{H}, 10-\mathrm{H}), 7.25$ (m, 3H, 7-H, 9$\mathrm{H}, 8-\mathrm{H}), 2.87\left(\mathrm{t}, 2 \mathrm{H}, 4-\mathrm{H},{ }^{3} \mathrm{~J}=7.5 \mathrm{~Hz}\right), 2.51\left(\mathrm{dt}, 2 \mathrm{H}, 3-\mathrm{H},{ }^{3} \mathrm{~J}=7.5,{ }^{4} \mathrm{~J}=2.7 \mathrm{~Hz}\right), 2.00(\mathrm{t}, 1 \mathrm{H}$, $\left.1-\mathrm{H},{ }^{4} J=2.7 \mathrm{~Hz}\right) .{ }^{13} \mathrm{C}-\mathrm{NMR}: \delta=140.37$ (q, 1C, C-5), 128.38 (t, 4C, C-6, C-10, C-7, C-9), 126.33 (t, 1C, C-8), 83.77 (q, 1C, C-2), 68.88 (t, 1C, C-1), 34.80 (s, 1C, C-4), 20.55 (s, 1C, C3). MS (GC-MS) Massenpeak m/z = $130.1\left(\mathrm{M}^{+}\right) ; \mathrm{C}_{10} \mathrm{H}_{10}$ (130.08).

Bromethinylbenzol: ${ }^{254} \mathrm{Zu}$ einer Mischung aus $50 \mathrm{mmol}$ (2,2-Dibromvinyl)benzol (13.5 g) und 5 mmol Benzyltrimethylammoniumchlorid $(0.9 \mathrm{~g})$ in $50 \mathrm{~mL} \mathrm{CH}_{2} \mathrm{Cl}_{2}$ werden unter Eiskühlung $50 \mathrm{~mL}$ einer 60\%igen wässrigen $\mathrm{KOH}$-Lösung zugegeben und $2 \mathrm{~h}$ bei RT rühren gelassen. Die organische Phase wird abgetrennt, zweimal mit Wasser gewaschen und über $\mathrm{Na}_{2} \mathrm{SO}_{4}$ getrocknet. Das Lösungsmittel wird abgezogen und das Produkt durch Destillation gereinigt.

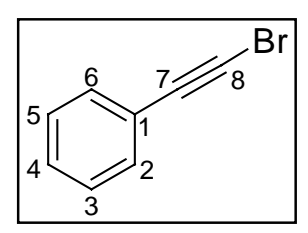

Ausbeute: $7.3 \mathrm{~g}(80.7 \%)$ als farblose Flüssigkeit. ${ }^{1} \mathbf{H}-\mathbf{N M R}: \delta=7.47(\mathrm{~m}$, 2H, 2-H, 6-H), 7.35 (m, 3H, 3-H, 5-H, 4-H). ${ }^{13} \mathbf{C}-\mathbf{N M R}: \delta=131.96$ (t, 2C, C-2, C-6), 128.65 (t, 1C, C-4), 128.30 (t, 2C, C-3, C-5), 122.63 (q, 1C, C1), 79.99 (q, 1C, C-7), 49.73 (q, 1C, C-8). MS (GC-MS) Massenpeak m/z = $180.1\left(\mathrm{M}^{+}\right) ; \mathrm{C}_{8} \mathrm{H}_{5} \mathrm{Br}(179.96)$. 
1,2-Diethinylbenzol (98): 230 Eine Suspension von $50 \mathrm{mmol}$ 1,2-Bis(3-hydroxy-3methylbutin-1-yl)benzol (255, $12.1 \mathrm{~g})$ und $2.5 \mathrm{~g}$ feingepulvertem $\mathrm{KOH}$ in $50 \mathrm{ml}$ Paraffinöl wird bei einem Druck von 0.1 Torr auf $180^{\circ} \mathrm{C}$ erhitzt und die leichtflüchtigen Produkte in einer Kühlfalle ausgefroren. Mitentstandenes Aceton wird abgezogen und das Produkt im Vakuum destilliert.

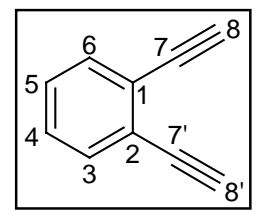

Ausbeute: 3.6 g 98 (58.7\%) als blaßgelbe Flüssigkeit. ${ }^{1}$ H-NMR: $\delta=7.52(\mathrm{~m}$, 2H, 3-H, 6-H), 7.31 (m, 2H, 4-H, 5-H), 3.35 (s, 2H, 8,8'-H). ${ }^{13} \mathbf{C}-\mathbf{N M R}: \delta=$ 132.58 (t, 2C, C-3, C-6), 128.49 (t, 2C, C-4, C-5), 124.96 (q, 2C, C-1, C-2), 81.77 (q, 2H, C-7,7'), 81.16 (t, 2H, C-8,8'). MS (GC-MS) Massenpeak m/z = $126.1\left(\mathrm{M}^{+}\right)$; $\mathrm{C}_{10} \mathrm{H}_{6}(126.05)$.

\section{Synthese der Butadiine durch Kupfer-katalysierte Cadiot-Chodkiewicz-Kupplung:231}

Eine farblose Lösung von Hydroxylaminhydrochlorid und $\mathrm{CuCl}$ in einer Mischung aus nButylamin und Ethanol (4:1) wird hergestellt und anschließend das Acetylen hinzugegeben, woraufhin die Lösung gelb und trüb wird. Eine Lösung von Bromphenylacetylen in nButylamin und Ethanol (4:1) wird unter Eiskühlung langsam zugetropft und $3 \mathrm{~h}$ bei RT gerührt. Kaliumcyanid wird hinzugegeben, das Lösungsmittel abgezogen und der Rückstand mit Ether extrahiert. Der Ether wird abgezogen und der Rückstand umkristallisiert.

\section{1-(Phenylethinyl)-2-(phenylbuta-1,3-diin-1-yl)benzol (253):}

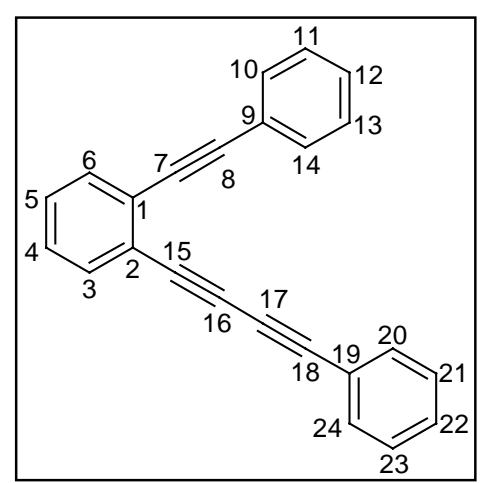

Ausgangsmaterialien: $7.5 \mathrm{mmol}$ Hydroxylaminhydrochlorid (0.52 g), $0.2 \mathrm{mmol} \mathrm{CuCl}(21 \mathrm{mg}), 20 \mathrm{ml} \mathrm{n}-\mathrm{C}_{4} \mathrm{H}_{9} \mathrm{NH}_{2}$-EtOH-Mix, 5 mmol 1-(Phenylethinyl)-2-ethinylbenzol 248 (1.0 g), $6 \mathrm{mmol}$ Bromphenylacetylen (1.1 g), $20 \mathrm{~mL} \mathrm{n}-\mathrm{C}_{4} \mathrm{H}_{9} \mathrm{NH}_{2}$-EtOH-Mix, 0.5 g KCN. Ausbeute: 0.8 g 253 (52\%) als gelber Feststoff vom Schmelzpunkt $51{ }^{\circ} \mathrm{C} .{ }^{1} \mathbf{H}-\mathbf{N M R}: \delta=7.68(\mathrm{~m}, 2 \mathrm{H}, 10-\mathrm{H}, 14-\mathrm{H})$, 7.59 (m, 4H, 3-H, 6-H, 20-H, 24-H), 7.36 (m, 8H, 4-H, 5-H, 11-

H, 13-H, 12-H, 21-H, 23-H, 22-H). ${ }^{13} \mathbf{C}-\mathbf{N M R}: \delta=132.59$ (t, 1C, C-3), 132.41 (t, 2C, C-20, C-24), 131.79 (t, 2C, C-10, C-14), 131.58 (t, 1C, C-6), 129.18 (t, 1C, C-22), 128.77 (t, 1C, C4), 128.49 (t, 1C, C-12), 128.36 (t, 2C, C-21, C-23), 128.26 (t, 2C, C-11, C-13), 127.86 (t, 1C, C-5), 126.96 (q, 1C, C-1), 124.36 (q, 1C, C-2), 22.93 (q, 1C, C-9), 121.66 (q, 1C, C-19), 94.23 (q, 1C, C-8), 87.75 (q, 1C, C-7), 82.62 (q, 1C, C-16), 80.21 (q, 1C, C-15), 77.78 (q, 1C, C-17), 74.01 (q, 1C, C-18). IR (KBr, Preßling) v = 3057, 3020, 2213, 1596, 1568, 1489, $1470,1440,1272,1174,1156,1096,1069,1024,949,919,848,755,688 \mathrm{~cm}^{-1}$. MS $(70 \mathrm{eV})$ 
m/z (\%): 302 (100) [M+], 274 (3), 202 (2), 151 (2). HRMS Gef.: 302.1096, Ber.: $\mathrm{C}_{28} \mathrm{H}_{18}$ (302.1096).

\section{1,2-Bis(4-phenylbuta-1,3-diin-1-yl)benzol (256):231}

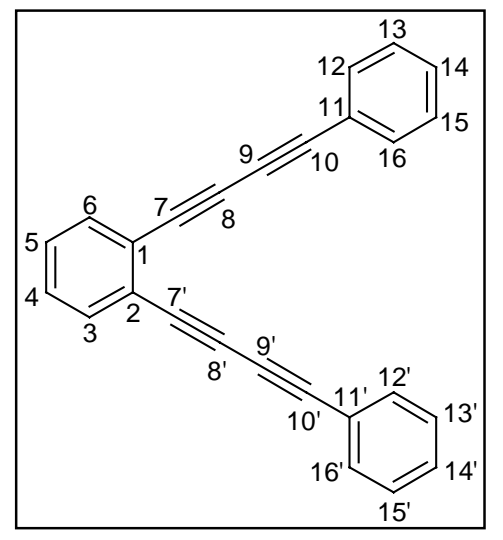

Ausgangsmaterialien: $15 \mathrm{mmol}$ Hydroxyl-aminhydrochlorid $(1.04 \mathrm{~g}), 0.2 \mathrm{mmol} \mathrm{CuCl}(21 \mathrm{mg}), 20 \mathrm{ml} \mathrm{n}-\mathrm{C}_{4} \mathrm{H}_{9} \mathrm{NH}_{2}-\mathrm{EtOH}-$ Mix, 5 mmol 1,2-Diethinylbenzol 98 (0.63 g), $13 \mathrm{mmol}$

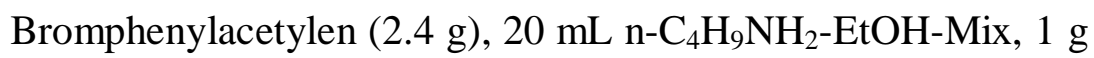
KCN. Aus Ethanol umkristallisiert. Ausbeute: 1.2 g 256 (74\%) als gelbe Kristalle. ${ }^{1} \mathbf{H}-\mathbf{N M R}: \delta=7.56$ (m, 6H, 3-H, 6-H, 12,12'H, 16,16'-H), 7.38 (m, 8H, 4-H, 5-H, 13,13'-H, 15,15'-H, 14,14'H). ${ }^{13}$ C-NMR: $\delta=133.18(\mathrm{t}, 2 \mathrm{C}, \mathrm{C}-3, \mathrm{C}-6), 132.53(\mathrm{t}, 4 \mathrm{C}, \mathrm{C}-$ 12,12', C-16,16'), 129.32 (t, 2C, C-4, C-5), 128.79 (t, 2C, C-14,14'), 128.41 (t, 4C, C-13,13', C-15,15'), 125.24 (q, 2C, C-1, C-2), 121.59 (q, 2C, C-11,11'), 83.09 (q, 2C, C-8,8'), 79.44 (q, 2C, C-7,7'), 78.05 (q, 2C, C-9,9'), 73.99 (q, 2C, C-10,10'). MS (70 eV) Massenpeak m/z = $326.1\left(\mathrm{M}^{+}\right) ; \mathrm{C}_{26} \mathrm{H}_{14}(326.11)$.

\section{Synthese der Butadiine durch Eglinton-Kupplung: ${ }^{255}$}

Eine Lösung aus $\mathrm{CuAc}_{2}$ in Pyridin:Methanol:Ether = 3:3:1 wird hergestellt und zum Rückfluß erhitzt. Das entsprechende Acetylen wird in Ether gelöst und hinzugetropft. Die Lösung wird 3 h unter Rückflüß gehalten, dann abgekühlt und das Lösungsmittel bis auf einen kleinen Rest abgezogen. Dieser wird mit 4N HCl angesäuert, die wässrige Phase mit Ether ausgeschüttelt und die organische Phase über $\mathrm{MgSO}_{4}$ getrocknet. Nach dem Abrotieren des Lösungsmittels wird einmal umkristallisiert.

\section{1,4-Diphenylbuta-1,3-diin (254): 244}

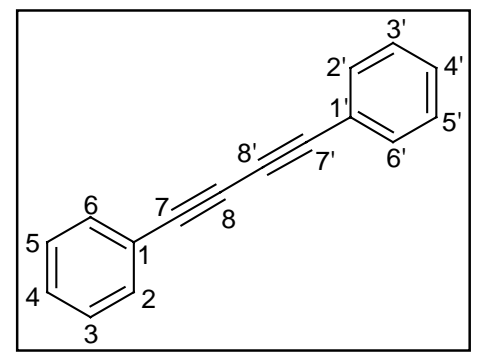

Ausgangsmaterialien: $80 \mathrm{mmol} \quad \mathrm{CuAc}_{2} \quad\left(\begin{array}{lll}16 & \mathrm{~g}\end{array}\right), 210 \mathrm{ml}$ Pyridin:Methanol:Ether-Mix, 40 mmol Phenylacetylen (4.0 g/4.4 $\mathrm{mL})$ in $50 \mathrm{ml}$ Ether. Aus Ethanol umkristallisiert. Ausbeute: 3.7 g $254(91.6 \%)$ als farblose Kristalle. ${ }^{1} \mathbf{H}-\mathbf{N M R}: \delta=7.57(\mathrm{~m}, 4 \mathrm{H}$, 2,2'-H, 6,6'-H), 7.39 (m, 4H, 3,3'-H, 5,5'-H), 7.37 (m, 2H, 4,4'H). ${ }^{13}$ C-NMR: $\delta=132.42$ (t, 4C, C-2,2', C-6,6'), 129.16 (t, 2C, C-4,4'), 128.38 (t, 4C, C-3,3', C-5,5'), 121.66 (q, 2C, C-1,1'), 81.51 (q, 2C, C-8,8'), 73.88 (q, 2C, C-7,7'). MS (GC-MS) Massenpeak m/z = 202.1 $\left(\mathrm{M}^{+}\right) ; \mathrm{C}_{16} \mathrm{H}_{10}(202.08)$. 


\section{2,2'-Bis(phenylethinyl)diphenylbutadiin (23):}

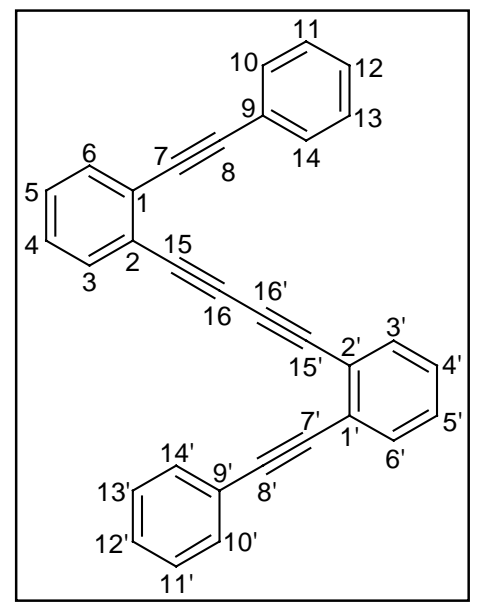

Ausgangsmaterialien: $\quad 20 \quad$ mmol $\quad \mathrm{CuAc}_{2} \quad\left(\begin{array}{lllll}4 & \mathrm{~g}\end{array}\right), \quad 70 \mathrm{ml}$ Pyridin:Methanol:Ether-Mix, $10 \mathrm{mmol}$ 1-(Phenylethinyl)-2ethinylbenzol $248(2.0 \mathrm{~g})$ in $10 \mathrm{ml}$ Ether. Aus Ethanol umkristallisiert. Ausbeute: 1.5 g 23 (75\%) als hellbraune Kristalle vom Schmelzpunkt $130^{\circ} \mathrm{C} .{ }^{1} \mathbf{H}-\mathbf{N M R}: \delta=7.58(\mathrm{~m}, 8 \mathrm{H}$, 3,3'-H, 6,6'-H, 10,10'-H, 14,14'-H), 7.32 (m, 10H, 4,4'-H, 5,5'-H, 11,11'-H, 13,13'-H, 12,12'-H). ${ }^{\mathbf{1 3}} \mathbf{C}-\mathbf{N M R}: \delta=132.79$ (t, 2C, C3,3'), 131.84 (t, 4C, C-10,10', C-14,14'), 131.69 (t, 2C, C-6,6'), 128.88 (t, 2C, C-4,4'), $128.46(t, 2 \mathrm{C}, \mathrm{C}-12,12$ '), 128.27 (t, 4C, C11,11', C-13,13'), 127.90 (t, 2C, C-5,5'), 127.07 (q, 2C, C-1,1'), 124.47 (q, 2C, C-9,9'), 122.87 (q, 2C, C-2,2'), 94.42 (q, 2C, C-8,8'), 87.71 (q, 2C, C-7,7'), 81.38 (q, 2C, C-16,16'), 77.91 (q, 2C, C-15,15'). IR (KBr, Preßling) v = 3074, 3057, 3022, 2216, 1943, 1910, 1878, 1799, 1684, 1653, 1597, 1567, 1492, 1466, 1441, 1195, 1181, 1155, 1089, 1068, 1024, 940, 911, 758, 747,

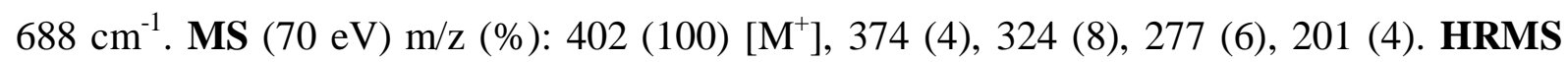
Gef.: 402.1408, Ber.: $\mathrm{C}_{28} \mathrm{H}_{18}$ (402.1409).

\section{Darstellung der Phosphoniumbromide: 221}

In einem Kolben mit Rückflußkühler werden Bromid und Triphenylphosphan in dem entsprechenden Lösungsmittel gelöst und 48h unter Rückfluß erhitzt. Mit Toluol als Lösungsmittel wird das ausgefallene Phosphonium-Salz abgesaugt, mit Benzol gewaschen und im Vakuum getrocknet. Mit DMF als Lösungsmittel wird das Lösungsmittel am Rotationsverdampfer unter Vakuum abgezogen, das Phosphonium-Salz aus Hexan/ $\mathrm{CH}_{2} \mathrm{Cl}_{2}$ ausgefällt, abgezogen, mit Benzol gewaschen und im Vakuum getrocknet.

\section{Benzyl-triphenylphosphoniumbromid:256}

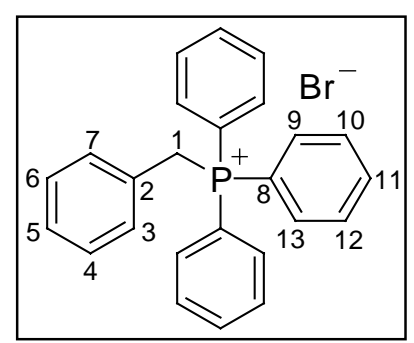

Ausgangsmaterialien: $50 \mathrm{mmol}$ Benzylbromid $(8.6 \mathrm{~g} / 5.9 \mathrm{~mL}), 50$ mmol $\mathrm{PPh}_{3}(13.1 \mathrm{~g}), 75 \mathrm{~mL}$ Toluol. Ausbeute: $20.4 \mathrm{~g}(94.2 \%)$ als farbloses Pulver. ${ }^{\mathbf{1}} \mathbf{H}-\mathbf{N M R}: \delta=7.63\left(\mathrm{~m}, 15 \mathrm{H}, 9,9^{\prime}, 9^{\prime \prime}-\mathrm{H}, 13,13\right.$ ',13"H, 10,10',10"-H, 12,12',12"-H, 11,11',11"-H), 7.14 (m, 1H, 5-H), $7.00(\mathrm{~m}, 4 \mathrm{H}, 3-\mathrm{H}, 7-\mathrm{H}, 4-\mathrm{H}, 6-\mathrm{H}), 5.19\left(\mathrm{~d}, 2 \mathrm{H}, 1-\mathrm{H},{ }^{2} \mathrm{~J}(\mathrm{H}, \mathrm{P})=14.35\right.$ Hz). ${ }^{13} \mathrm{C}-\mathrm{NMR}: \delta=134.87\left(\mathrm{t}, 3 \mathrm{C}, \mathrm{C}-11,11\right.$ ',11", $\left.{ }^{4} \mathrm{~J}(\mathrm{C}, \mathrm{P})=2.9 \mathrm{~Hz}\right), 134.06(\mathrm{t}, 6 \mathrm{C}, \mathrm{C}-$ 10,10',10", C-12,12',12", $\left.{ }^{3} \mathrm{~J}(\mathrm{C}, \mathrm{P})=9.8 \mathrm{~Hz}\right), 131.18$ (t, 2C, C-4, C-6, $\left.{ }^{4} \mathrm{~J}(\mathrm{C}, \mathrm{P})=5.5 \mathrm{~Hz}\right)$, 129.97 (t, 6C, C-9,9',9", C-13,13',13", $\left.{ }^{2} \mathrm{~J}(\mathrm{C}, \mathrm{P})=12.5 \mathrm{~Hz}\right), 128.61\left(\mathrm{t}, 2 \mathrm{C}, \mathrm{C}-3, \mathrm{C}-7,{ }^{3} \mathrm{~J}(\mathrm{C}, \mathrm{P})=\right.$ 
$3.4 \mathrm{~Hz}), 128.22\left(\mathrm{t}, 1 \mathrm{C}, \mathrm{C}-5,{ }^{5} \mathrm{~J}(\mathrm{C}, \mathrm{P})=3.9 \mathrm{~Hz}\right), 126.79\left(\mathrm{q}, 1 \mathrm{C}, \mathrm{C}-2,{ }^{2} \mathrm{~J}(\mathrm{C}, \mathrm{P})=8.6 \mathrm{~Hz}\right)$, $117.33\left(\mathrm{q}, 3 \mathrm{C}, \mathrm{C}-8,8^{\prime}, 8^{\prime \prime},{ }^{1} \mathrm{~J}(\mathrm{C}, \mathrm{P})=85.7 \mathrm{~Hz}\right), 30.57\left(\mathrm{~s}, 1 \mathrm{C}, \mathrm{C}-1,{ }^{1} \mathrm{~J}(\mathrm{C}, \mathrm{P})=46.8 \mathrm{~Hz}\right)$.

\section{(3-Phenylpropyl)-triphenylphosphoniumbromid:}

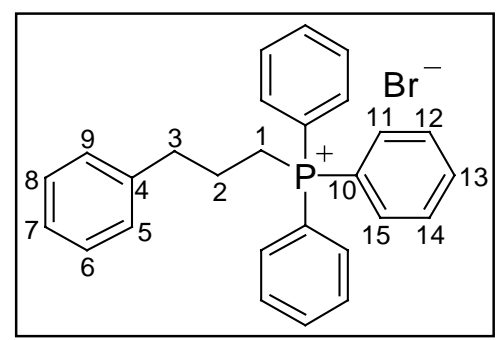

Ausgangsmaterialien: $100 \mathrm{mmol}$ (3-Brompropyl)benzol (19.9 $\mathrm{g} / 15.1 \mathrm{~mL}), 100 \mathrm{mmol} \mathrm{PPh}_{3}(26.2 \mathrm{~g}), 150 \mathrm{~mL}$ Toluol. Ausbeute: $40.6 \mathrm{~g}(88 \%)$ als farbloses Pulver. ${ }^{\mathbf{1}} \mathbf{H}-\mathbf{N M R}: \delta=$ 7.54 (m, 15H, 11,11',11"-H, 15,15',15"-H, 12,12',12"-H, 14,14',14"-H, 13,13',13"-H), 7.05 (m, 5H, 5-H, 9-H, 6-H, 8-H, 7-H), 3.58 (m, 2H, 1-H), 2.84 (m, 2H, 3-H), 1.81 (m, 2H, 2-H). ${ }^{13}$ C-NMR: $\delta=139.53$ (q, 1C, C-4), 134.59 (t, 3C, C-13,13',13", $\left.{ }^{4} \mathrm{~J}(\mathrm{C}, \mathrm{P})=2.9 \mathrm{~Hz}\right), 132.00$ (t, 6C, C-12,12',12", C$\left.14,14^{\prime}, 14^{\prime \prime},{ }^{3} \mathrm{~J}(\mathrm{C}, \mathrm{P})=10.0 \mathrm{~Hz}\right), 130.01$ (t, 6C, C-11,11',11", C-15,15',15", $\left.{ }^{2} \mathrm{~J}(\mathrm{C}, \mathrm{P})=12.5 \mathrm{~Hz}\right)$, 128.36 (t, 2C, C-6, C-8), 128.06 (t, 1C, C-7), 125.87 (t, 2C, C-5, C-9), 117.42 (q, 3C, C$\left.10,10^{\prime}, 10^{\prime \prime},{ }^{1} \mathrm{~J}(\mathrm{C}, \mathrm{P})=86.1 \mathrm{~Hz}\right), 35.05\left(\mathrm{~s}, 1 \mathrm{C}, \mathrm{C}-3,{ }^{3} \mathrm{~J}(\mathrm{C}, \mathrm{P})=16.6 \mathrm{~Hz}\right), 23.99\left(\mathrm{~s}, 1 \mathrm{C}, \mathrm{C}-2,{ }^{2} \mathrm{~J}\right.$ $(\mathrm{C}, \mathrm{P})=3.7 \mathrm{~Hz}), 20.99\left(\mathrm{~s}, \mathrm{C}-1,{ }^{1} \mathrm{~J}(\mathrm{C}, \mathrm{P})=51.1 \mathrm{~Hz}\right)$.

\section{Diphenylmethyl-triphenyl-phosphoniumbromid:}

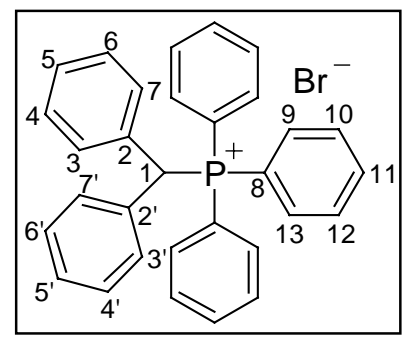

Ausgangsmaterialien: $50 \mathrm{mmol}$ Bromdiphenylmethan (13.1 g), 50 mmol $\mathrm{PPh}_{3}(13.1 \mathrm{~g}), 75 \mathrm{~mL}$ Toluol. Ausbeute: $20.9 \mathrm{~g}(82 \%)$ als farbloses Pulver. ${ }^{1} \mathbf{H}$-NMR: $\delta=7.86\left(\mathrm{~d}, 1 \mathrm{H}, 1-\mathrm{H},{ }^{2} \mathrm{~J}(\mathrm{H}, \mathrm{P})=17.7\right.$ Hz), 7.63 (m, 9H, 10,10',10"-H, 12,12',12"-H, 11,11',11"-H), 7.47 (m, $10 \mathrm{H}, 3,3^{\prime}-\mathrm{H}, 7,7^{\prime}-\mathrm{H}, 9,9^{\prime}, 9^{\prime \prime}-\mathrm{H}, 13,13^{\prime}, 13$ '-H), 7.10 (m, 6H, 4,4'-H, 6,6'-H, 5,5'-H). ${ }^{13} \mathrm{C}-\mathrm{NMR}: \delta=134.74\left(\mathrm{t}, 6 \mathrm{C}, \mathrm{C}-10,10^{\prime}, 10^{\prime \prime}, \mathrm{C}-12,12^{\prime}, 12^{\prime \prime},{ }^{3} \mathrm{~J}(\mathrm{C}, \mathrm{P})=9.2 \mathrm{~Hz}\right)$, 134.61 (t, 3C, C-11,11',11", $\left.{ }^{4} \mathrm{~J}(\mathrm{C}, \mathrm{P})=7.6 \mathrm{~Hz}\right), 132.89$ (q, 2C, C-2,2' , $\left.{ }^{2} \mathrm{~J}(\mathrm{C}, \mathrm{P})=2.7 \mathrm{~Hz}\right)$, $130.73\left(\mathrm{t}, 4 \mathrm{C}, \mathrm{C}-3,3^{\prime}, \mathrm{C}-7,7^{\prime},{ }^{3} \mathrm{~J}(\mathrm{C}, \mathrm{P})=6.8 \mathrm{~Hz}\right), 129.64$ (t, 6C, C-9,9',9", C-13,13',13", ${ }^{2} \mathrm{~J}$ $(\mathrm{C}, \mathrm{P})=12.3 \mathrm{~Hz}), 128.65$ (t, 4C, C-4,4', C-6,6'), 128.34 (t, 2C, C-5,5'), 118.05 (q, 3C, C$\left.8,8^{\prime}, 8^{\prime \prime},{ }^{1} \mathrm{~J}(\mathrm{C}, \mathrm{P})=82.2 \mathrm{~Hz}\right), 45.20\left(\mathrm{t}, 1 \mathrm{C}, \mathrm{C}-1,{ }^{1} \mathrm{~J}(\mathrm{C}, \mathrm{P})=42.3 \mathrm{~Hz}\right)$.

\section{Olefinsynthese nach Wittig: ${ }^{257}$}

$\mathrm{Zu}$ einer Suspension des Phosphoniumsalzes in absolutem THF wird bei RT 1.6 M BuLiLösung zugetropft und 30 Min. gerührt. Anschließend wird die entsprechende Carbonylverbindung in absolutem THF hinzugetropft und noch einmal $2 \mathrm{~h}$ gerührt. Wasser wird hinzugegeben und die Mischung mit Ether extrahiert. Die organische Phase wird über $\mathrm{MgSO}_{4}$ getrocknet und der Ether abgezogen. Der Rückstand wird in Pentan suspendiert, der 
Feststoff abfiltriert und das Filtrat eingeengt. Der Rückstad wird über eine Kieselgelsäule gereinigt.

\section{2-Methyl-5-phenyl-pent-2-en (261): 258}

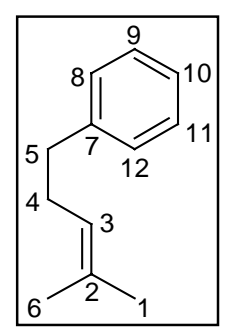

Ausgangsmaterialien: $30 \mathrm{mmol}$ (3-Phenylpropyl)-triphenylphosphoniumbromid $(13.8 \mathrm{~g})$ in $100 \mathrm{~mL}$ THF, $30 \mathrm{mmol}$ BuLi-Lsg. $1.6 \mathrm{M}$ in Hexan (18.75 ml), 40 mmol Aceton $(2.3 \mathrm{~g} / 3 \mathrm{~mL})$ in $20 \mathrm{~mL}$ THF. Ausbeute: $2.1 \mathrm{~g} 261(44 \%)$ als farblose Flüssigkeit. ${ }^{1} \mathbf{H}-\mathbf{N M R}: \delta=7.28(\mathrm{~m}, 2 \mathrm{H}, 9-\mathrm{H}, 11-\mathrm{H}), 7.21(\mathrm{~m}, 3 \mathrm{H}, 8-\mathrm{H}$, $12-\mathrm{H}, 10-\mathrm{H}), 5.21\left(\mathrm{tt}, 1 \mathrm{H}, 3-\mathrm{H},{ }^{3} \mathrm{~J}=7.1 ;{ }^{4} \mathrm{~J}=1.4 \mathrm{~Hz}\right), 2.65\left(\mathrm{dt}, 2 \mathrm{H}, 5-\mathrm{H},{ }^{3} \mathrm{~J}=7.4\right.$; $\left.{ }^{4} \mathrm{~J}=1.4 \mathrm{~Hz}\right), 2.32\left(\mathrm{dt}, 2 \mathrm{H}, 4-\mathrm{H},{ }^{3} \mathrm{~J}=7.1 ; 7.4 \mathrm{~Hz}\right), 1.71(\mathrm{~s}, 3 \mathrm{H}, 1-\mathrm{H} / 6-\mathrm{H}), 1.59$ (s, 3H, 1-H/6H). ${ }^{13}$ C-NMR: $\delta=142.35$ (q, 1C, C-7), 132.07 (q, 1C, C-2), 128.38 (t, 2C, C-9, C-11), 128.16 (t, 2C, C-8, C-12), 125.60 (t, 1C, C-10), 123.67 (t, 1C, C-3), 36.10 (s, 1C, C-5), 30.03 (s, 1C, C-4), 25.64 (p, 1C, C-1/C-6), 17.59 (p, 1C, C-1/C-6). MS (GC-MS) Massenpeak m/z = $160.1\left(\mathrm{M}^{+}\right) ; \mathrm{C}_{12} \mathrm{H}_{16}(160.13)$.

\section{1,1,4-Triphenyl-but-1-en (262): 253}

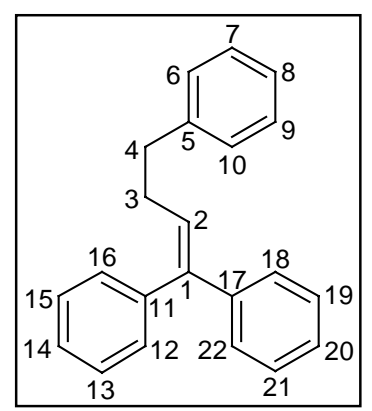

Ausgangsmaterialien: $20 \mathrm{mmol}$ (3-Phenylpropyl)-triphenylphosphoniumbromid (9.2 g) in $80 \mathrm{~mL}$ THF, $25 \mathrm{mmol}$ BuLi-Lsg. 1.6 $\mathrm{M}$ in Hexan (15.6 ml), $25 \mathrm{mmol}$ Benzophenon (4.6 g) in $40 \mathrm{~mL}$ THF. Ausbeute: $4.1 \mathrm{~g} 262$ (72\%) als farblose Flüssigkeit. ${ }^{1} \mathbf{H}$-NMR: $\delta=7.27$ $\left(\mathrm{m}, 15 \mathrm{H}\right.$, alle Ar-H), $6.17\left(\mathrm{t}, 1 \mathrm{H}, 2-\mathrm{H},{ }^{3} \mathrm{~J}=7.4 \mathrm{~Hz}\right), 2.80\left(\mathrm{t}, 2 \mathrm{H}, 4-\mathrm{H},{ }^{3} \mathrm{~J}\right.$ $=7.6 \mathrm{~Hz}), 2.49\left(\mathrm{dt}, 2 \mathrm{H}, 3-\mathrm{H},{ }^{3} \mathrm{~J}=7.4,7.6 \mathrm{~Hz}\right) .{ }^{13} \mathbf{C}-\mathbf{N M R}: \delta=142.57$ (q, 1C, C-17), 142.14 (q, 1C, C-11), 141.59 (q, 1C, C-5), 139.97 (q, 1C, C-1), 129.75 (t, 2C, C-12, C-16), 128.74 (t, 1C, C-2), 128.46 (t, 2C, C-7, C-9), 128.23 (t, 2C, C-6, C-10), 128.07 (t, 2C, C-19, C-21), 128.01 (t, 2C, C-13, C-15), 127.15 (t, 2C, C-18, C-22), 126.84 (t, 1C, C14/C-20), 126.81 (t, 1C, C-14/C-20), 125.78 (t, 1C, C-8), 36.11 (s, 1C, C-4), 31.60 (s, 1C, C3). MS (70 eV) Massenpeak m/z = $284.3\left(\mathrm{M}^{+}\right) ; \mathrm{C}_{22} \mathrm{H}_{20}(284.16)$. 


\section{(E)-1-(Phenylethinyl)-2-(phenylvinyl)benzol (260):232}

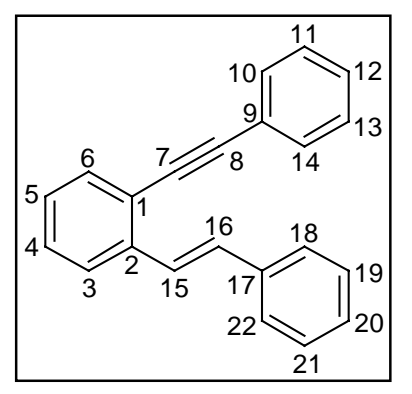

Ausgangsmaterialien: $10 \mathrm{mmol}$ Benzyl-triphenylphosphoniumbromid (4.3 g) in $40 \mathrm{~mL}$ THF, $10 \mathrm{mmol}$ BuLi-Lsg. 1.6 M in Hexan (6.3 mL), 10 mmol 2-(Phenylethinyl)benzaldehyd 250 (2.1 g) in $20 \mathrm{~mL}$ THF. Ausbeute: $1.8 \mathrm{~g} \mathbf{2 6 0}(63 \%)$ als farbloser Feststoff. ${ }^{1} \mathbf{H}$-NMR: $\delta=7.75$ $(\mathrm{m}, 2 \mathrm{H}, 6-\mathrm{H}, 15-\mathrm{H}), 7.61(\mathrm{~m}, 5 \mathrm{H}, 3-\mathrm{H}, 10-\mathrm{H}, 14-\mathrm{H}, 18-\mathrm{H}, 22-\mathrm{H})$, 7.46-7.21 (m, 11H, 4-H, 5-H, 11-H, 13-H, 12-H, 19-H, 21-H, 20-H).

${ }^{13}$ C-NMR: $\delta=138.67$ (q, 1C, C-2), 137.35 (q, 1C, C-17), 132.68 (t, 1C, C-6), 131.49 (t, 2C, C-10, C-14), 130.29 (t, 1C, C-16), 128.73 (t, 2C, C-19, C-21), 128.52 (t, 1C, C-4/C-5/C-15), 128.42 (t, 2C, C-11, C-13), 128.35 (t, 1C, C-12), 127.84 (t, 1C, C-20), 127.22 (t, 1C, C-4/C5/C-15), 126.69 (t, 3C, C-18, C-22, C-3), 124.73 (t, 1C, C-4/C-5/C-15), 123.31 (q, 1C, C-1), 122.14 (q, 1C, C-9), 94.39 (q, 1C, C-8), 87.92 (q, 1C, C-7). MS (70 eV) Massenpeak m/z = $280.1\left(\mathrm{M}^{+}\right) ; \mathrm{C}_{22} \mathrm{H}_{16}(280.13)$.

\section{1-(Phenylethinyl)-2-(2',2'-diphenylvinyl)benzol (25):}

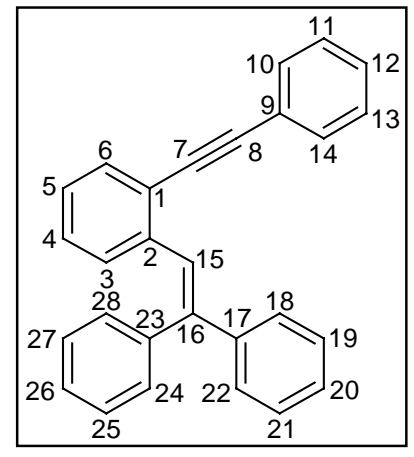

Ausgangsmaterialien: $10 \mathrm{mmol}$ Diphenylmethyl-triphenylphosphoniumbromid $(5.1 \mathrm{~g})$ in $40 \mathrm{~mL}$ THF, $10 \mathrm{mmol}$ BuLi-Lsg. 1.6 $\mathrm{M}$ in Hexan (6.3 ml), 10 mmol 2-(Phenylethinyl)benzaldehyd 250 (2.1 g) in $20 \mathrm{~mL}$ THF. Ausbeute: $0.6 \mathrm{~g} 25$ (17\%) als farbloser Feststoff vom Schmelzpunkt $86^{\circ} \mathrm{C} .{ }^{1} \mathbf{H}-\mathbf{N M R}: \delta=7.52(\mathrm{~m}, 4 \mathrm{H}, 6-\mathrm{H}, 10-\mathrm{H}, 14-\mathrm{H}$, 15-H), 7.43 (m, 2H, 18-H, 22-H), 7.34 (m, 8H, 11-H, 13-H, 12-H, 19-H, 21-H, 25-H, 27-H, 26-H), 7.23 (m, 3H, 20-H, 24-H, 28-H), $7.12(\mathrm{~m}, 1-\mathrm{H}, 5-\mathrm{H}), 6.79(\mathrm{~m}, 1-\mathrm{H}, 4-\mathrm{H}), 6.86(\mathrm{~m}, 1-\mathrm{H}, 3-\mathrm{H}) .{ }^{13} \mathbf{C}-\mathbf{N M R}: \delta=143.82$ (q, 1C, C17/C-23), 143.24 (q, 1C, C-17/C-23), 140.19 (q, 1C, C-16), 139.44 (q, 1C, C-2), 132.18 (t, 1C, C-6), 131.53 (t, 2C, C-10, C-14), 130.58 (t, 2C, C-24, C-28), 129.16 (t, 1C, C-3), 128.43 (t, 2C, C-11, C-13), 128.29 (q, 2C, C-19, C-21), 128.21 (t, 1C, C-12), 128.18 (t, 2C, C-25, C27), 127.86 (t, 2C, C-18, C-22), 127.61 (t, 1C, C-20/C-26), 127.49 (t, 1C, C-4), 127.41 (t, 1C, C-20/C-26), 126, 49 (t, 1C, C-5), 126.42 (t, 1C, C-15), 123.67 (q, 1C, C-1), 123.33 (q, 1C, C9), 94.39 (q, 1C, C-8), 88.43 (q, 1C, C-7). IR (KBr, Preßling) v = 3076, 3046, 1958, 1884, 1596, 1570, 1490, 1467, 1441, 1354, 1275, 1177, 1162, 1087, 1074, 1028, 998, 954, 922, 915, 863, 850, 776, 758, $702 \mathrm{~cm}^{-1}$. MS (70 eV) m/z (\%): 356 (16) $\left[\mathrm{M}^{+}\right], 280$ (24), 202 (100), 178 (5), 168 (24). HRMS Gef.: 356.1565, Ber.: $\mathrm{C}_{28} \mathrm{H}_{20}$ (356.1565). 
Bromierungen: $\mathrm{Zu}$ einer Lösung des entsprechenden Acetylens in $25 \mathrm{ml} \mathrm{CHCl}_{3}$ werden pro eingesetztem mmol $2.5 \mathrm{~mL}$ einer 0.4 molaren Stammlösung von Brom in $\mathrm{CHCl}_{3}$ hinzugetropft. Die Reaktion wird 2 h bei RT unter Lichtausschluß gerührt, anschließend wird die Reaktionsmischung gegen ges. $\mathrm{Na}_{2} \mathrm{SO}_{3}$-Lösung und zweimal gegen Wasser ausgeschüttelt, über $\mathrm{Na}_{2} \mathrm{SO}_{4}$ getrocknet und das Lösungsmittel abgezogen. Aufarbeitung entweder durch Umkristallisieren (A), durch Säulenchromatographie (B) oder durch präparative HPLC (C).

\section{Bromierung von Phenylacetylen (263): 215}

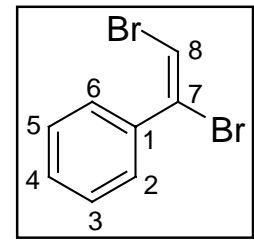

Ausgangsmaterialien: 263 (5 mmol, 500 mg). Produkte: 95\% (E)-(1,2Dibromvinyl)benzol (E)-264, 5\% (Z)-(1,2-Dibromvinyl)benzol (Z)-264. (E)264: gelbe Kristalle. ${ }^{1}$ H-NMR: $\delta=7.52$ (m, 2H, 3-H, 5-H), 7.38 (m, 3H, 2-H, 6-H, 4-H), $6.82(\mathrm{~s}, 1 \mathrm{H}, 8-\mathrm{H}) .{ }^{13} \mathbf{C}-\mathbf{N M R}: \delta=136.98(\mathrm{q}, 1 \mathrm{C}, \mathrm{C}-1), 129.38(\mathrm{t}$, 1C, C-4), 129.12 (t, 2C, C-3, C-5), 128.24 (t, 2C, C-2, C-6), 121.29 (q, 1C, C-7), 103.01 (t, $1 \mathrm{C}, \mathrm{C}-8)$. MS (GC-MS) Massenpeak $\mathrm{m} / \mathrm{z}=260\left(\mathrm{C}_{8} \mathrm{H}_{6} \mathrm{Br}_{2} 259.88\right)$.

\section{Bromierung von Diphenylacetylen (196): 215,233}

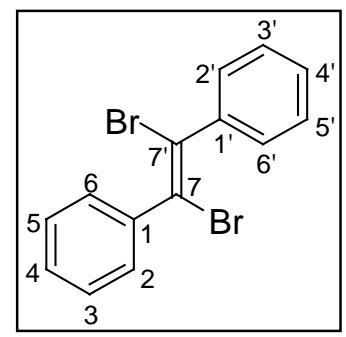

Ausgangsmaterialien: 196 (5 mmol, $900 \mathrm{mg}$ ). Produkte: 60\% $\alpha, \alpha^{\prime}-$ Dibrom-cis-stilben (Z)-199, $40 \% \alpha, \alpha^{\prime}$-Dibrom-trans-stilben $(\boldsymbol{E})$-199. (E)199: farblose Kristalle. ${ }^{1} \mathbf{H}-\mathbf{N M R}: \delta=7.56$ (m, 4H, 3,3'-H, 5,5'-H), 7.43 (m, 6H, 2,2'-H, 6,6'-H, 4,4'-H). ${ }^{13}$ C-NMR: $\delta=140.73$ (q, 2C, C-1,1'), 129.05 (t, 4C, C-3,3', C-5,5'), 128.90 (t, 2C, C-4,4'), 128.36 (t, 4C, C-

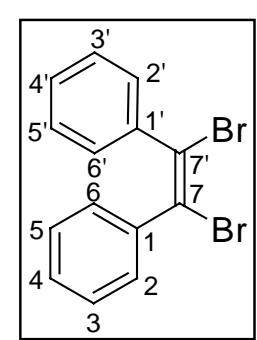

2,2', C-6,6'), 118.04 (q, 2C, C-7,7'). MS (GC-MS) Massenpeak m/z = 336.1 $\left(\mathrm{C}_{14} \mathrm{H}_{10} \mathrm{Br}_{2}\right.$ 335.95). (Z)-199: farblose Kristalle. ${ }^{1} \mathbf{H}-\mathbf{N M R}: \delta=7.20-7.12(\mathrm{~m}$, 10H, alle Ar-H). ${ }^{13}$ C-NMR: $\delta=139.37$ (q, 2C, C-1,1'), 129.74 (t, 4C, C-2,2', C-6,6'), 128.31 (t, 2C, C-4,4'), 128.00 (t, 4C, C-3,3', C-5,5'), 125.66 (q, 2C, C-7,7'). MS (GC-MS) Massenpeak m/z = $336.1\left(\mathrm{C}_{14} \mathrm{H}_{10} \mathrm{Br}_{2} 335.95\right)$. 


\section{Bromierung von 1,2-Bis(phenylethinyl)benzol (18): 45}

Ausgangsmaterialien: 18 (3 mmol, 830 mg). Produkte: 85\% (E)-3-Brom-1-( $\alpha$-brombenzyliden)-2-phenyl-inden (E)-195, 15\% (Z)-3-Brom-1-( $\alpha$-brom-benzyliden)-2-phenyl-inden

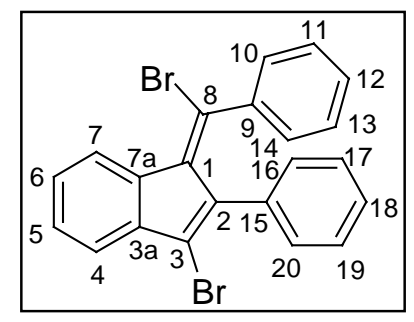
(Z)-195. (E)-195: gelbe Kristalle. ${ }^{1} \mathbf{H}-\mathbf{N M R}: \delta=8.84(\mathrm{~d}, 1 \mathrm{H}, 7-\mathrm{H})$, 7.52 (m, 3H, 4-H, 5-H, 6-H), 7.14 (m, 2H, Ar-H), 6.99 (m, 8H, ArH). ${ }^{13}$ C-NMR: $\delta=141.10$ (q, 1C, C-3a), 140.65 (q, 1C, C-9/C-15), 139.71 (q, 1C, C-9/C-15), 137.95 (q, 1C, C-1), 135.52 (q, 1C, C-7a), 134.82 (q, 1C, C-2), 130.74 (q, 1C, C-8), 130.35 (t, 2C, C-10/C-16, C-14/C-20), 129.51 (t, 2C, C-10/C-16, C-14/C-20), 128.81 (t, 1C, C-5), 128.61 (t, 2C, C11/C-17, C-13/C-19), 127.08 (t, 2C, C-11/C-17, C-13/C-19), 127.06 (t, 1C, C-12/C-18), 126.95 (t, 1C, C-6), 126.42 (t, 1C, C-12/C-18), 126.25 (q, 1C, C-3), 124.46 (t, 1C, C-7),

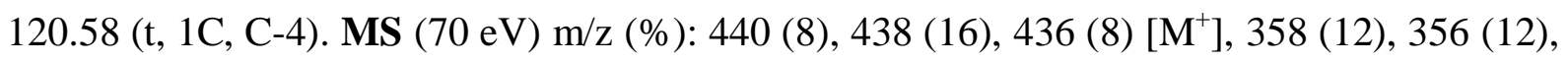
278 (100), 138 (12). HRMS Gef.: 435.9462, Ber.: $\mathrm{C}_{22} \mathrm{H}_{14} \mathrm{Br}_{2}$ 435.9462. (Z)-195: gelber

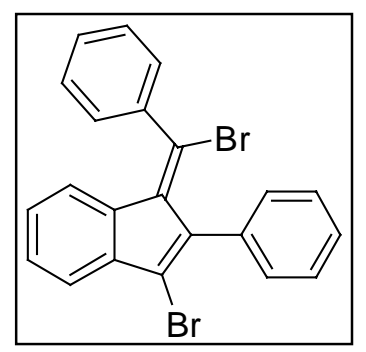

Feststoff. ${ }^{1} \mathbf{H}-\mathbf{N M R}: \delta=7.46(\mathrm{~m}, 10 \mathrm{H}, 10-\mathrm{H}, 14-\mathrm{H}, 16-\mathrm{H}, 20-\mathrm{H}, 11-\mathrm{H}$, 13-H, 17-H, 19-H, 12-H, 18-H), 7.38 (d, 1H, 4-H), 7.25 (dd, 1H, 5-H), 6.89 (dd, 1H, 6-H), $6.12(\mathrm{~d}, 1 \mathrm{H}, 7-\mathrm{H}) .{ }^{13} \mathrm{C}-\mathbf{N M R}: \delta=142.29(\mathrm{q}, 1 \mathrm{C})$, 140.68 (q, 1C), 139.95 (q, 1C), 137.93 (q, 1C), 136.11 (q, 1C), 134.79 (q, 1C), $130.30(\mathrm{t}, 2 \mathrm{C}), 129.61(\mathrm{t}, 1 \mathrm{C}), 129.04(\mathrm{t}, 2 \mathrm{C}), 128.63(\mathrm{q}, 1 \mathrm{C})$,

128.57 (t, 2C), 128.46 (q, 1C), 128.19 (t, 2C), 127.88 (t, 1C), 127.79 (t, 1C), 126.66 (t, 1C), 122.99 (t, 1C), $120.16(t, 1 C)$. MS (70 eV) Massenpeak m/z = $436\left(\mathrm{M}^{+}\right) ;\left(\mathrm{C}_{22} \mathrm{H}_{14} \mathrm{Br}_{2} 435.99\right)$.

\section{Bromierung von 1,4-Bis(phenylethinyl)benzol (235):}

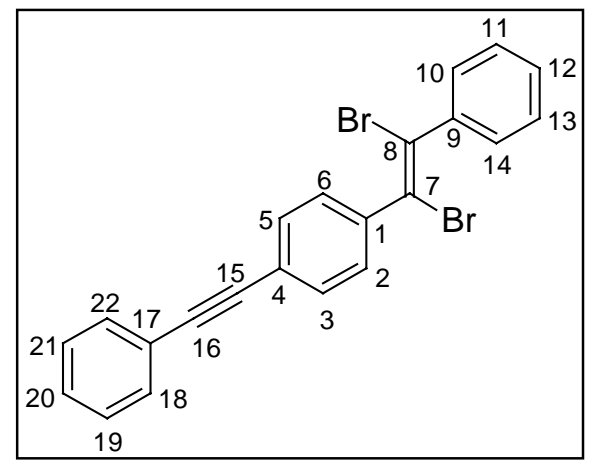

Ausgangsmaterialien: 235 ( $3 \mathrm{mmol}, 830 \mathrm{mg}$ ). Produkte: 60\% (E)-1-(1,2-Dibrom-2-phenylvinyl)-4-phenylethinylbenzol (E)-265, 40\% (Z)-1-(1,2-Dibrom-2-phenyl-vinyl)4-phenylethinyl-benzol $\quad(\boldsymbol{E})-265 . \quad(\boldsymbol{E})-265: \quad$ farblose Kristalle vom Schmelzpunkt $177^{\circ} \mathrm{C} .{ }^{1}$ H-NMR: $\delta=7.55$ (m, 2H, 3-H, 5-H), 7.51 (m, 6H, 11-H, 13-H, 12-H, 18-H, 22-H, 20-H), 7.41 (m, 2H, 2-H, 6-H), 7.34 (m, 4H, 10-H,

14-H, 19-H, 21-H). ${ }^{13}$ C-NMR: $\delta=140.62$ (q, 1C, C-9), 140.30 (q, 1C, C-1), 131.67 (t, 2C, C3/C-18, C-5/C-22), 131.5267 (t, 2C, C-3/C-18, C-5/C-22), 129.25 (t, 2C, C-11/C-19, C13/21), 129.02 (t, 2C, C-11/C-19, C-13/21), 129.00 (t, 1C, C-12/C-20), 128.49 (t, 1C, C-12/C20), 128.40 (t, 2C, C-6/C-10, C-2/14), 128.39 (t, 2C, C-6/C-10, C-2/14), 123.93 (q, 1C, C-17), 122.98 (q, 1C, C-4), 118.48 (q, 1C, C-8), 117.34 (q, 1C, C-7), 90.66 (q, 1C, C-16), 88.86 (q, 
1C, C-15). IR (KBr, Preßling) v = 3078, 3055, 1954, 1925, 1601, 1572, 1502, 1485, 1441, 1403, 1276, 1244, 1104, 1069, 1019, 914, 856, 835, 826, 758, 751, 732, 694, 671, $613 \mathrm{~cm}^{-1}$.

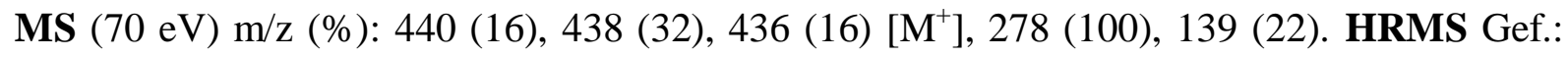
435.9462, Ber.: $\mathrm{C}_{22} \mathrm{H}_{14} \mathrm{Br}_{2}$ (435.9462).

\section{Bromierung von 1,2,3-Tris(phenylethinyl)benzol (22):}

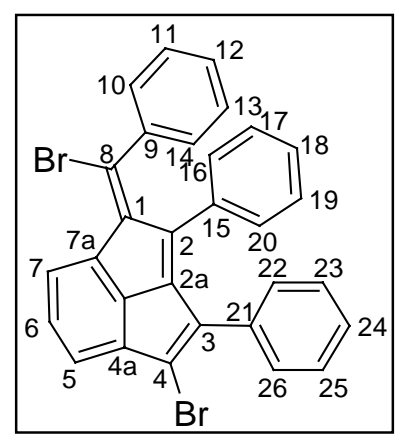

Ausgangsmaterialien: 22 (2 mmol, $750 \mathrm{mg})$. Produkt: 266 orangeroter Feststoff vom Schmelzpunkt $201^{\circ} \mathrm{C} .{ }^{1} \mathbf{H}-\mathbf{N M R}: \delta=8.10(\mathrm{~d}, 1 \mathrm{H}, 7-\mathrm{H})$, $7.43(\mathrm{t}, 1 \mathrm{H}, 6-\mathrm{H}), 7.35(\mathrm{~d}, 1 \mathrm{H}, 5-\mathrm{H}), 7.13(\mathrm{~m}, 3 \mathrm{H}-\mathrm{Ar}-\mathrm{H}), 7.05$ (m, 4H, Ar-H), 6.94 (m, 1H, Ar-H), 6.88 (m, 2H, Ar-H), 6.80 (m, 1H, Ar$\mathrm{H}), 6.68$ (m, 4H, Ar-H). ${ }^{13} \mathrm{C}-\mathbf{N M R}: \delta=148.37$ (q, 1C, C-7b), 145.70 (q, 1C, C-2a), 142.13 (q, 1C, C-9/C-15/C21), 140.51 (q, 1C, C-9/C15/C21), 139.85 (q, 1C, C-9/C-15/C21), 136.17 (q, 1C), 135.50 (q, 1C), 134.57 (q, 1C), 133.39 (q, 1C), 131.35 (q, 1C, C-7a), 130.64 (t, 2C), 129.95 (t, 2C), 129.86 (t, 2C), 129.77 (q, 1C, C-4a), 129.35 (t, 1C), 127.77 (t, 2C), 127.72 (t, 1C), 127.62 (t, 1C), 127.58 (t, 2C), 127.28 (q, 1C, C-4), 127.22 (t, 2C), 127.07 (t, 1C, C-6), 123.74 (t, 1C, C7), 119.58 (t, 1C, C-5). IR (KBr, Preßling) $v=3056,3015,2962,2924,1740,1653,1599$, 1582, 1559, 1484, 1457, 1440, 1350, 1261, 1198, 1088, 1044, 1019, 866, 802, 790, 755, 696,

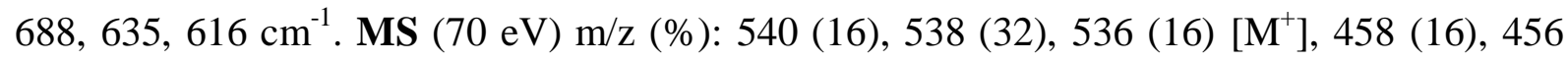
(16), 378 (100), 350 (4), 300 (6), 237 (8), 189 (16), 121 (28). HRMS Gef.: 535.9775, Ber.: $\mathrm{C}_{30} \mathrm{H}_{18} \mathrm{Br}_{2}$ (535.9775).

\section{Bromierung von 1-Phenylethinylnapthalin (245):}

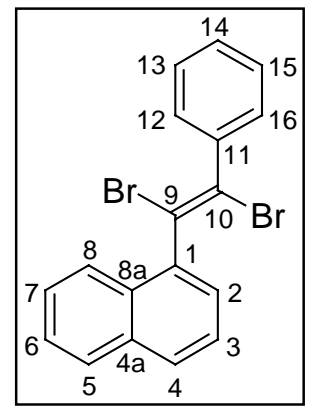

Ausgangsmaterialien: 245 (5 mmol, 1.1 g). Produkte: 60\% (E)-267, 40\% (Z)-267. (E)-267: farblose Kristalle vom Schmelzpunkt $148-152^{\circ} \mathrm{C} .{ }^{\mathbf{1}} \mathbf{H}-$ NMR: $\delta=8.10(\mathrm{~m}, 1 \mathrm{H}, 8-\mathrm{H}), 7.94(\mathrm{~m}, 2 \mathrm{H}, 4-\mathrm{H}, 5-\mathrm{H}), 7.7-7.4(\mathrm{~m}, 9 \mathrm{H}, \mathrm{Ar}-$ H). ${ }^{13}$ C-NMR: $\delta=140.11$ (q, 1C, C-11), 138.22 (q, 1C, C-1), 133.76 (q, 1C, C-4a), 129.43 (t, 1C, C-Ar), 129.36 (q, 1C, C-8a), 129.22 (t, 2C, C-13, C-15), 129.11 (t, 1C, C-Ar), 128.62 (t, 1C, C-Ar), 128.43 (t, 2C, C-12, C16), 126.89 (t, 1C, C-Ar), 126.70 (t, 1C, C-Ar), 126.41 (t, 1C, C-Ar), 125.53 (t, 1C, C-Ar), 124.70 (t, 1C, C-Ar), 121.05 (q, 1C, C-9), 116.14 (q, 1C, C-10). IR (KBr, Preßling) v = 3073, 3052, 3027, 1589, 1577, 1508, 1488, 1443, 1389, 1337, 1238, 1213, 1070, 1017, 868, 799, 777, 764, 739, 693, 681, 652, $625 \mathrm{~cm}^{-1}$. MS (70 eV) m/z (\%): 390 (8), 388 (16), $386(8)\left[\mathrm{M}^{+}\right]$, 308 (4), 228 (100), 202 (6), 114 (12). HRMS Gef.: 356.1565, Ber.: $\mathrm{C}_{18} \mathrm{H}_{12} \mathrm{Br}_{2}$ (356.1565). 


\section{Bromierung von 1,4-Diphenylbut-1-in (217): 259,260}

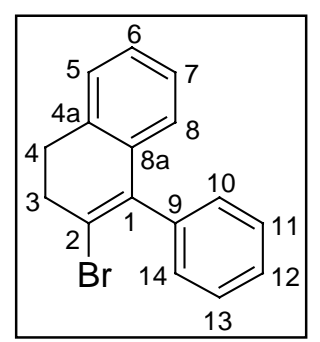

Ausgangsmaterialien: 217 (5 mmol, 1.0 g). Produkt: 270, blaßgelber Feststoff vom Schmelzpunkt 52-54 ${ }^{\circ} \mathrm{C} .{ }^{1} \mathbf{H}-\mathbf{N M R}: \delta=7.46(\mathrm{~m}, 3 \mathrm{H}, 10-\mathrm{H}$, 14-H, 12-H), 7.29 (m, 2H, 11-H, 13-H), 7.20 (m, 2H, 6-H, 7-H), 7.09 (m, 1H, 5-H), $6.69(\mathrm{~d}, 1 \mathrm{H}, 8-\mathrm{H}), 3.06(\mathrm{~m}, 4 \mathrm{H}, 3-\mathrm{H}, 4-\mathrm{H}) .{ }^{13} \mathbf{C}-\mathbf{N M R}: \delta=$ 139.58 (q, 1C, C-9), 138.31 (q, 1C, C-1), 135.70 (q, 1C, C-4a), 134.05 (q, 1C, C-8a), 129.66 (t, 2C, C-11, C-13), 128.32 (t, 2C, C-10, C-14), 127.43 (t, 1C, C-6), 127.27 (t, 1C, C-12), 127.13 (t, 1C, C-5), 126.49 (t, 1C, C-8), 125.98 (t, 1C, C-7), 123.44 (q, 1C, C2), 35.10 (s, 1C, C-4), 29.49 (s, 1C, C-3). IR (KBr, Preßling) v = 3054, 3019, 3008, 2948, 2938, 2890, 2825, 1617, 1597, 1489, 1479, 1442, 1419, 1109, 1072, 1059, 960, 857, 799, 765,

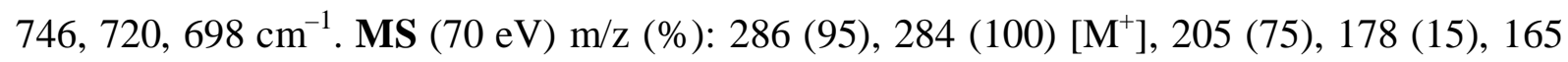
(8), 127 (12), 101 (20), 91 (12). HRMS Gef.: 284.0201, Ber.: $\mathrm{C}_{28} \mathrm{H}_{20}$ (284.0201).

\section{Bromierung von 2-(Phenylethinyl)biphenyl (24):235}

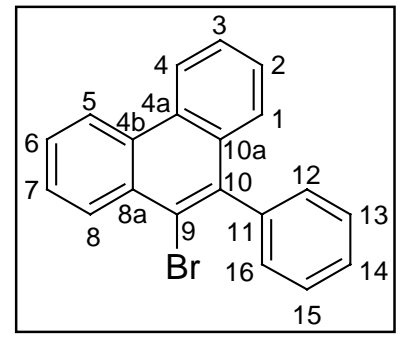

Ausgangsmaterialien: 24 (3 mmol, $760 \mathrm{mg}$ ). Produkt: 271, farblose Kristalle vom Schmelzpunkt $111^{\circ} \mathrm{C} .{ }^{1} \mathbf{H}-\mathbf{N M R}: \delta=8.75(\mathrm{~m}, 2 \mathrm{H}, 4-\mathrm{H}$, 5-H), 8.57 (m, 1H, 8-H), 7.75 (m, 2H, 6-H, 7-H), 7.68 (m, 1H, 1-H), $7.58(\mathrm{~m}, 3 \mathrm{H}, 13-\mathrm{H}, 15-\mathrm{H}, 14-\mathrm{H}), 7.47$ (m, 2H, 2-H, 3-H), $7.38(\mathrm{~m}$, 2H, 12-H, 16-H). ${ }^{13} \mathbf{C}-\mathbf{N M R}: \delta=140.77$ (q, 1C, C-10), 139.37 (q, 1C, C-11), 132.28 (q, 1C, C-8a), 130.65 (q, 1C, C-10a), 130.15 (q, 1C, C-4a), 129.77 (t, 2C, C-13, C-15), 129.38 (q, 1C, C-4b), 128.68 (t, 1C, C-8), 128.23 (t, 2C, C-12, C-16), 127.66 (t, 1C, C3), 127.53 (t, 1C, C-7), 127.49 (t, 1C, C-14), 127.21 (t, 1C, C-6), 126.82 (t, 1C, C-2), 126.64 (t, 1C, C-1), 123.39 (q, 1C, C-9), 122.43 (t, 1C, C-4/C-5), 122.40 (t, 1C, C-4/C-5). IR (KBr, Preßling) $v=3076,3048,3022,1600,1583,1564,1493,1482,1446,1439$, 1416, 1273, 1174 , 1156, 1102, 1070, 1046, 1029, 958, 875, 767, 753, 733, 722, 696, $619 \mathrm{~cm}^{-1} . \mathbf{M S}(70 \mathrm{eV}) \mathrm{m} / \mathrm{z}$ (\%): 334 (70), 332 (70) [M+], 252 (100), 224 (8), 126 (28) 113 (10). HRMS Gef.: 332.0201, Ber.: $\mathrm{C}_{20} \mathrm{H}_{13} \mathrm{Br}(332.0201)$. 


\section{Bromierung von 1-Phenylethinyl-2-vinylbenzol (243):}

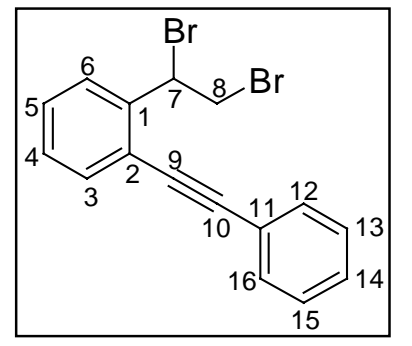

Ausgangsmaterialien: 243 ( $1 \mathrm{mmol}, 200 \mathrm{mg}$ ). Produkt: 276, gelbes Ö1 ${ }^{1}$ H-NMR: $\delta=7.60$ (m, 4H, 3-H, 6-H, 12-H, 16-H), 7.41 (m, 5H, 4-H, $5-\mathrm{H}, 13-\mathrm{H}, 15-\mathrm{H}, 14-\mathrm{H}), 5.87\left(\mathrm{dd}, 1 \mathrm{H}, 7-\mathrm{H},{ }^{3} \mathrm{~J}=10.3,5.8 \mathrm{~Hz}\right), 4.18$ $(\mathrm{m}, 2 \mathrm{H}, 8-\mathrm{H}) .{ }^{13} \mathrm{C}-\mathbf{N M R}: \delta=139.54$ (q, 1C, C-1), 132.56 (t, 1C, C-3), 131.55 (t, 2C, C-12, C-16), 128.95 (t, 1C, C-4/C-5/C-14), 128.76 (t, 1C, C-4/C-5/C-14), 128.71 (t, 1C, C-4/C-5/C-14), 128.42 (t, 2C, C-13, C-15), 126.92 (t, 1C, C-6), 122.95 (q, 1C, C-2), 122.69 (q, 1C, C-11), 95.74 (q, 1C, C-10), 86.16 (q, 1C, C-9), 48.16 (t, 1C, C-7), 34.16 (s, 1C, C-8). IR (KBr, Film) v = 3061, 3030, 2978, 2215, 1953, 1808, 1692, 1600, 1572, 1493, 1442, 1280, 1231, 1195, 1128, 1070, 1026, 911, 753, $690 \mathrm{~cm}^{-}$

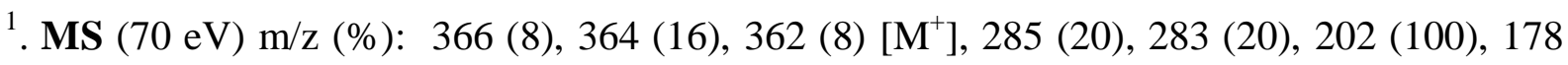
(16), 122 (12), 105 (20). HRMS Gef.: 361.9306, Ber.: $\mathrm{C}_{16} \mathrm{H}_{12} \mathrm{Br}_{2}$ (361.9306).

\section{Bromierung von (E)-1-(Phenylethinyl)-2-(2',2'-diphenylvinyl)benzol (25):}

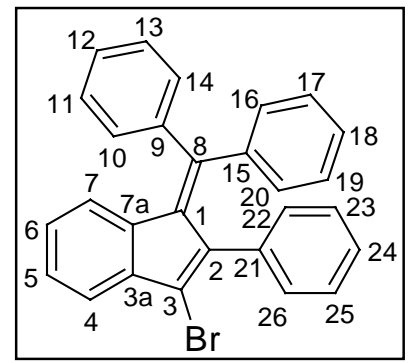

Ausgangsmaterialien: 25 (1 mmol, $360 \mathrm{mg}$ ). Produkt: 280, rote Kristalle vom Schmelzpunkt $195^{\circ} \mathrm{C} .{ }^{1} \mathbf{H}-\mathbf{N M R}: \delta=7.50(\mathrm{~m}, 4 \mathrm{H}, 4-$ $\mathrm{H}, \mathrm{Ar}-\mathrm{H}), 7.42$ (m, 2H, Ar-H), 7.30 (m, 1H, 5-H), 7.13 (m, 2H, Ar$\mathrm{H}), 6.96(\mathrm{~m}, 9 \mathrm{H}, 6-\mathrm{H}, \mathrm{Ar}-\mathrm{H}), 6.39(\mathrm{~m}, 1 \mathrm{H}, 7-\mathrm{H}) .{ }^{13} \mathbf{C}-\mathbf{N M R}: \delta=$ 149.50 (q, 1C, C-8), 142.85 (q, 1C, C-9), 140.86 (q, 1C, C-15/C-21), 140.68 (q, 1C, C-3a), 140.14 (q, 1C, C-15/C-21), 136.57 (q, 1C, C-7a), 136.40 (q, 1C, C-1/C2), 135.76 (q, 1C, C-1/C-2), 132.06 (t, 2C, C-Ar), 130.80 (t, 2C, C-Ar), 130.36 (t, 2C, C-Ar), 128.98 (t, 1C, C-12/C-18/C-24), 128.63 (t, 2C, C-Ar), 127.99 (t, 1C, C-6), 127.29 (t, 1C, C5), 127.07 (t, 2C, C-Ar), 126.98 (t, 2C, C-Ar), 126.18 (t, 1C, C-12/C-18/C-24), 126.10 (t, 1C, C-12/C-18/C-24), 125.78 (q, 1C, C-3), 123.12 (t, 1C, C-7), 120.04 (t, 1C, C-4). IR (KBr, Preßling) $v=3075,3055,3026,1952,1603,1575,1551,1541,1488,1481,1455,1438,1345$, 1334, 1286, 1269, 1235, 1179, 1154, 1076, 12027, 952, 933, 846, 782, 774, 757, 723, 702, 624, $615 \mathrm{~cm}^{-1}$. MS (70 eV) m/z (\%): 436 (100), 434 (95) [ $\left.\mathrm{M}^{+}\right], 355$ (40), 278 (24), 178 (16). HRMS Gef.: 434.0670, Ber.: $\mathrm{C}_{28} \mathrm{H}_{19} \mathrm{Br}$ (434.0670). 


\section{Bromierung von 1,4-Diphenylbuta-1,3-diin (254): 236}

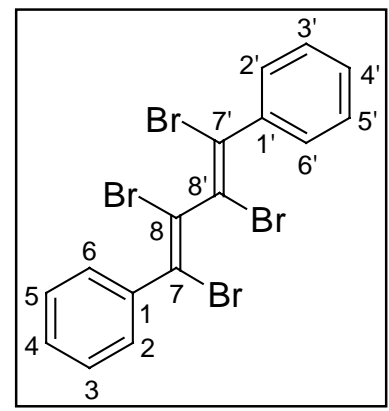

Ausgangsmaterialien: 254 (5 mmol, 1.0 g). Produkt: 281, gelbe Kristalle vom Schmelzpunkt $165^{\circ} \mathrm{C} .{ }^{1} \mathbf{H}-\mathbf{N M R}: \delta=7.57$ (m, 4H, 3,3'H, 5,5'-H), 7.43 (m, 6H, 2,2'-H, 6,6'-H, 4,4'-H). ${ }^{13}$ C-NMR: $\delta=$ 138.51 (q, 2C, C-1,1'), 139.54 (t, 2C, C-4,4'), 128.93 (t, 4C, C-3,3', C-5,5') 128.29 (t, 4C, C-2,2', C-6,6'), 122.68 (q, 2C, C-7,7'), 116.22 (q, 2C, C-8,8'). IR (KBr, Preßling) v = 3075, 3055, 3014, 1954, 1486, 1442, 1276, 1210, 1161, 1099, 1069, 1024, 998, 925, 886, 883, 764, 723, 695, $677 \mathrm{~cm}^{-1}$. MS

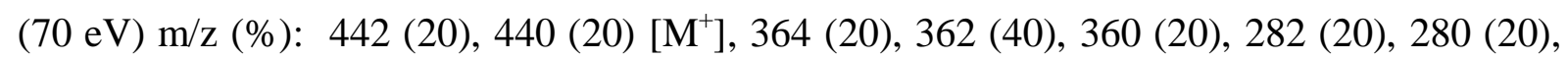
202 (100), 101 (24). HRMS Gef.: 517.7516, Ber.: $\mathrm{C}_{16} \mathrm{H}_{10} \mathrm{Br}_{4}$ (517.7516).

\section{Bromierung von 2',2'-Bis(phenylethinyl)-1,4-diphenylbuta-1,3-diin (23):}

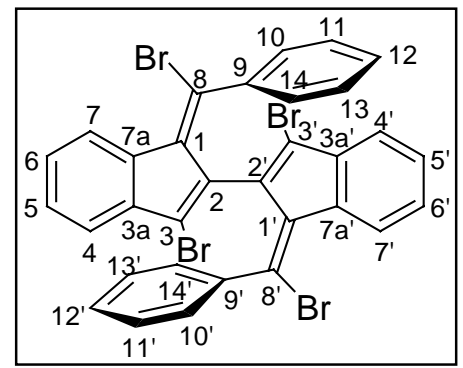

Ausgangsmaterialien: $\mathbf{2 3}$ ( $2 \mathrm{mmol}, 800 \mathrm{mg})$. Produkt: 282, orange Kristalle vom Schmelzpunkt $222^{\circ} \mathrm{C} .{ }^{1} \mathbf{H}-\mathbf{N M R}: \delta=8.24(\mathrm{~m}, 2 \mathrm{H}$, 7,7'-H), 7.57 (m, 2H, 4,4'-H), 7.30 (m, 2H, 5,5'-H), 7.22 (m, 6H, 11,11'-H, 13,13'-H, 12,12'-H), 7.01 (m, 2H, 6,6'-H), 6.65 (m, 4H, 10,10'-H, 14,14'-H). ${ }^{13} \mathbf{C}-\mathbf{N M R}: \delta=140.80$ (q, 2C, C-3a,3a'), 140.42 (q, 2C, C-9,9'), 138.45 (q, 2C, C-1,1'), 135.34 (q, 2C, C7a,7a'), 133.31 (q, 2C, C-2,2'), 130.28 (q, 2C, C-8,8'), 129.96 (t, 2C, C-Ar), 129.61 (q, 2C, C3,3'), 128.91 (t, 2C, C-5,5'), 128.37 (t, 2C, C-Ar), 128.14 (t, 2C, C-Ar), 127.09 (t, 2C, C-6,6'), 126.78 (t, 2C, C-Ar), 125.69 (t, 2C, C-Ar), 123.69 (t, 2C, C-7,7'), 120.13 (t, 2C, C-4,4'). IR $(\mathrm{KBr}$, Preßling) $v=3050,1947,1082,1596,1577,1486,1447,1440,1347,1270,1260$, 1230, 1165, 1098, 1076, 1026, 966, 942, 926, 894, 859, 762, 752, 738, 705, 694, 659, 645, $628 \mathrm{~cm}^{-1}$. MS (70 eV) m/z (\%): 726 (10), 724 (50), 722 (72), 720 (50), 718 (10) [M+], 355 (40), 278 (24), 178 (16). HRMS Gef.: 717. 8142, Ber.: $\mathrm{C}_{32} \mathrm{H}_{18} \mathrm{Br}_{4}$ (717.8142).

\section{Bromierung von 2-Methyl-5-phenylpent-2-en (261):}

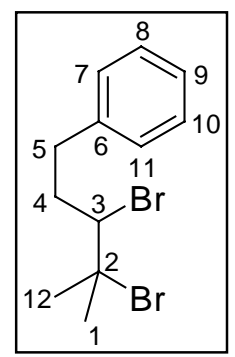

Ausgangsmaterialien: 261 (5 mmol, $800 \mathrm{mg}$ ). Produkt: 285, farblose Flüssigkeit. ${ }^{1} \mathbf{H}-\mathbf{N M R}: \delta=7.27$ (m, 5H, 7-H, 11-H, 8-H, 10-H, 9-H), 4.17 (m, 1H, 3-H), $3.28(\mathrm{~m}, 1 \mathrm{H}, 4-\mathrm{H}), 3.08(\mathrm{~m}, 2-\mathrm{H}, 5-\mathrm{H}), 2.17(\mathrm{~m}, 1 \mathrm{H}, 4-\mathrm{H}), 1.98(\mathrm{~s}$, $3 \mathrm{H}, 1-\mathrm{H} / 12 \mathrm{H}), 1.83$ (s, 3H, 1-H/12H). ${ }^{13} \mathrm{C}-\mathbf{N M R}: \delta=140.26$ (q, 1C, C-6), 128.49 (t, 2C, C-8, C-10), 128.38 (t, 2C, C-7, C-11), 126.11 (t, 1C, C-9), 68.25 (q, 1C, C-2), 65.68 (t, 1C, C-3), 37.16 (s, 1C, C-5), 35.10 (p, 1C, C-1/C-12), 34.09 (s, 1C, C4), 28.21 (p, 1C, C-1/C-12). MS (70 eV) m/z (\%): 322 (2), 320 (4), 318 (2) [M+1, 240 (4), 238 
(4), 159 (100), 143 (20), 128 (8), 117 (18), 91 (70), 69 (20). HRMS Gef.: 317.9619, Ber.: $\mathrm{C}_{12} \mathrm{H}_{16} \mathrm{Br}_{2}$ (317.9619).

\section{Bromierung von 1,1,4-Triphenylbut-1-en (262):}

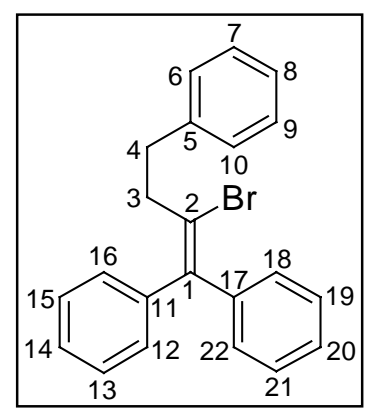

Ausgangsmaterialien: 262 (5 mmol, 1.4 g). Produkt: 287, gelber Feststoff vom Schmelzpunkt $44^{\circ} \mathrm{C} .{ }^{1} \mathbf{H}-\mathbf{N M R}: \delta=7.25(\mathrm{~m}, 11 \mathrm{H}, \mathrm{Ar}-$ $\mathrm{H}), 7.14(\mathrm{~m}, 2 \mathrm{H}, \mathrm{Ar}-\mathrm{H}), 6.89(\mathrm{~m}, 2 \mathrm{H}, \mathrm{Ar}-\mathrm{H}), 3.01\left(\mathrm{t}, 2 \mathrm{H}, 4-\mathrm{H},{ }^{3} \mathrm{~J}=7.4\right.$ $\mathrm{Hz}$ ), 2.85 (t, 2H, 3-H, $\left.{ }^{3} \mathrm{~J}=7.4 \mathrm{~Hz}\right) .{ }^{13} \mathbf{C}-\mathbf{N M R}: \delta=143.09(\mathrm{q}, 1 \mathrm{C}, \mathrm{C}-$ 1/C-5), 142.88 (q, 1C, C-1/C-5), 140.65 (q, 1C, C-11/C-17), 140.43 (q, 1C, C-11/C-17), 128.80 (t, 4C, C-Ar), 128.49 (t, 2C, C-Ar), 128.27 (t, 2C, C-Ar), 128.18 (t, 2C, C-Ar), 127.99 (t, 2C, C-Ar), 127.10 (t, 2C, C-14, C-20), 126.59 (q, 1C, C-2), 126.12 (t, 1C, C-8), 40.38 (s, 1C, C-4), 34.79 (S, 1C, C-3). IR (KBr, Preßling) v = 3050, 3024, 2946, 2924, 2859, 1941, 1803, 1632, 1595, 1491, 1453, 1443, 1423, 1167, 1153, 1074, 1052, 1029, 1008, 866, 763, 748, 704, $695 \mathrm{~cm}^{-1} . \mathbf{M S}(70 \mathrm{eV}) \mathrm{m} / \mathrm{z}(\%): 364$ (30), 362 (30) $\left[\mathrm{M}^{+}\right], 271$ (30), 269 (30), 192 (100), 165 (15), 91 (20). HRMS Gef.: 362.0670, Ber.: $\mathrm{C}_{22} \mathrm{H}_{19} \mathrm{Br}(362.0670)$. 


\section{Literaturverzeichnis}

1) Cope, A. C.; Hardy, E. M. J. Am. Chem. Soc. 1940, 62, 441.

2) Doering, W. v. E.; Toscano, V. G.; Beasley, G. H. Tetrahedron 1971, 27, 5299-3506.

3) Claisen, L. Ber. 1912, 45, 3157.

4) Claisen, L. Ber. 1925, 58, 275.

5) Claisen, L.; Tietze, E. Ber. 1926, 59, 2344.

6) Diels, O.; Alder, K. Ann. 1928, 460, 98.

7) Diels, O.; Alder, K.; Lubbert, W.; Naujokas, E.; Querberitz, F.; Rohl, K.; Segeberg, H. Ann. 1929, 470, 62.

8) Diels, O.; Alder, K. Ber. 1929, 62, 2081.

9) Butz, L. W.; Butz, E. W. J.; Gaddis, A. M. J. Org. Chem. 1940, 5, 171, 180.

10) Butz, L. W. J. Am. Chem. Soc. 1942, 64, 1978.

11) Doering, W. v. E.; Roth, W. R.; Bauer, F.; Boenke, M.; Breuckmann, R. Chem. Ber. 1991, 124, 1461-1470.

12) Bergman, R. G. Acc. Chem. Res. 1973, 6, 25-31.

13) Darby, N.; Kim, C. U.; Salaün, J. A.; Shelton, K. W.; Takada, S.; Masamune, S. Chem. Comm. 1971, 1516-1517.

14) Jones, R. R.; Bergman, R. G. J. Am. Chem. Soc. 1972, 94, 660-661.

15) Wong, H. N. C.; Sondheimer, F. Tetrahedron Lett. 1980, 21, 217-220.

16) Myers, A. G.; Kuo, E. Y.; Finney, N. S. J. Am. Chem. Soc. 1989, 111, 8057-8059.

17) Myers, A. G.; Dragovich, P. S.; Kuo, E. Y. J. Am. Chem. Soc. 1992, 114, 9369-9386.

18) Saito, I.; Nagata, R.; Yamanaka, H.; Okazaki, E. Tetrahedron Lett. 1989, 30(37), 4995-4998.

19) Saito, I.; Nagata, R.; Yamanaka, H.; Murahashi, E. Tetrahedron Lett. 1990, 31(20), 2907-2910.

20) Maier, M. E. Synlett 1995, 13-26.

21) Nicolaou, K. C.; Dai, W.-M. Angew. Chem. 1991, 103, 1453-1481.

22) Nicolaou, K. C.; Smith, A. L.; Wendeborn, S. V.; Hwang, C.-K. J. Am. Chem. Soc. 1991, 113, 3106-3114.

23) Nicolaou, K. Chem. Brit. 1994, 33-36.

24) Smith, A. L.; Nicolaou, K. C. J. Med. Chem. 1996, 39, 2103-2117.

25) Grissom, J. W.; Gunawardena, G. U.; Klingberg, D.; Huang, D. Tetrahedron 1996, 52(19), 6453-6518. 
26) Roth, W. R.; Hopf, H.; Horn, C. Chem. Ber. 1994, 127, 1765-1779.

27) Casazza, A. M.; Kelly, S. L. Enediyne Antibiotics as Antitumor Agents; Marcel Dekker: New York, 1995; Vol. 14.

28) Nicolaou, K. C.; Dai, W.-M.; Hog, Y. P.; Tsay, S.-C.; Baldridge, K. K.; Siegel, J. S. J. Am. Chem. Soc. 1993, 115, 7944-7953.

29) Brückner, R.; Suffert, J.; Abraham, E.; Raeppel, S. Liebigs Ann. 1996, 447-456.

30) Tykwinski, R. R. Chem. Commun. 1999, 905-906.

31) Snyder, J. P. J. Am. Chem. Soc. 1990, 112, 5367-5369.

32) Schreiner, P. R. Chem. Commun. 1998, 4, 483-484.

33) Schreiner, P. R. J. Am. Chem. Soc. 1998, 120, 4184-4190.

34) Semmelhack, M. F.; Neu, T.; Foubelo, F. J. Org. Chem. 1994, 59, 5038-5047.

35) Mita, T.; Kawata, S.; Hirama, M. Chem. Lett. 1998, 959-960.

36) Nicolaou, K. C.; Zuccarello, G.; Ogawa, Y.; Schweiger, E. J.; Kumazawa, T. J. Am. Chem. Soc. 1988, 110, 4866-4868.

37) Basak, A.; Shain, J. C. Tetrahedron Lett. 1998, 39, 3029-3030.

38) Basak, A.; Shain, J. C.; Khamrai, U. K.; Rudra, K. R.; Basak, A. J. Chem. Soc., Perkin Trans. 1 2000, 1955-1964.

39) König, B. Eur. J. Org. Chem. 2000, 381-385.

40) König, B.; Pitsch, W. J. Org. Chem. 1996, 61, 4258-4261.

41) Warner, B. P.; Millar, S. P.; Broene, R. D.; Buchwald, S. L. Science 1995, 269, 814816.

42) Prall, M.; Wittkopp, A.; Fokin, A. A.; Schreiner, P. R. J. Comput. Chem. 2001, 22, 1605-1614.

43) Barluenga, J.; Gonzàlez, P. J. C.; Asensio, G. Angew. Chem. 1988, 100, 1604-1605.

44) Bossenbroeck, B.; Sanders, D. C.; Curry, H. M.; Shechter, H. J. Am. Chem. Soc. 1969, 91, 371-379.

45) Whitlock, H. W. J.; Sandvick, P. E.; Overman, L. E.; Reichardt, P. B. J. Org. Chem. 1969, 34, 879-886.

46) Whitlock, B. J.; Whitlock, H. W. J. Org. Chem. 1972, 37, 3559-3561.

47) Zhou, Q.; Caroll, P. J.; Swager, T. M. J. Org. Chem. 1994, 59, 1294-1301.

48) Schmittel, M.; Kiau, S. Chem. Lett. 1995, 953-954.

49) Hopf, H. Classics in Hydrocarbon Chemistry; Wiley-VCH: Weinheim, 2000.

50) Houk, K. N.; Li, Y.; Evanseck, J. D. Angew. Chem. Int. Ed. Engl. 1992, 31, 682-708.

51) Kraka, E.; Cremer, D. Chem. Phys. Lett. 1993, 216, 333-340. 
52) Kraka, E.; Cremer, D. J. Am. Chem. Soc. 1994, 116, 4929-4936.

53) Kraka, E.; Cremer, D. J. Am. Chem. Soc. 2000, 122, 8245-8264.

54) Sander, W. Acc. Chem. Res. 1999, 32, 669-676.

55) Cramer, C. J. J. Am. Chem. Soc. 1998, 120, 6261-6269.

56) Cramer, C. J.; Nash, J. J.; Squires, R. R. Chem. Phys. Lett. 1997, 277, 311-320.

57) Gräfenstein, J.; Hjerpe, A. M.; Kraka, E.; Cremer, D. J. Phys. Chem. A 2000, 104, 1748-1761.

58) Pople, J. A.; Nesbet, R. K. J. Chem. Phys. 1954, 23, 571-574.

59) Roothan, C. C. J. Rev. Mod. Phys. 1951, 23, 69.

60) Parr, R. G.; Yang, W. Density Functional Theory of Atoms and Molecules; Oxford University Press: New York, 1989.

61) Hohenberg, P.; Kohn, W. Phys. Rev. B 1964, 136, 864-871.

62) Kohn, W.; Sham, L. J. Phys. Rev. A 1965, 140, 1133-1138.

63) Becke, A. D. Phys. Rev. A 1988, 38, 3098-3100.

64) Slater, J. C. The Self-Consistent Field for Molecular and Solids; McGraw-Hill: New York, 1974; Vol. 4.

65) Gill, P. M. W. Mol. Phys. 1996, 89, 433.

66) Adamo, C.; Barone, V. J. Comp. Chem. 1998, 19, 418-429.

67) Becke, A. D. J. Chem. Phys. 1993, 98, 5648-5652.

68) Miehlich, B.; Savin, A.; Stoll, H.; Preuss, H. Chem. Phys. Lett. 1989, 157, 200-206.

69) Lee, C.; Yang, W.; Parr, R. G. Phys. Rev. B 1988, 37, 785-789.

70) Perdew, J. P.; Wang, Y. Phys. Rev. B 1992, 23, 12947-12954.

71) Vosko, S. H.; Wilk, L.; Nusair, M. Canadian J. Phys. 1980, 58, 1200-1211.

72) Møller, C.; Plesset, M. S. Phys. Rev. 1934, 98, 5648.

73) Hegarty, D.; Robb, M. A. Mol. Phys. 1979, 38, 1795-1812.

74) Koga, N.; Morokuma, K. J. Am. Chem. Soc. 1991, 113, 1907-1911.

75) Eade, R. H. E.; Robb, M. A. Chem. Phys. Lett. 1981, 83, 362-368.

76) Habas, M. P.; Dargelos, A. Chem. Phys. 1995, 199, 177-182.

77) Turker, L. Theochem-J. Mol. Struct. 1999, 491, 275-280.

78) Purvis, G. D.; Bartlett, R. J. J. Chem. Phys. 1982, 76, 1910-1918.

79) Raghavachari, K.; Trucks, G. W.; Pople, J. A.; Head-Gordon, M. Chem. Phys. Lett. 1989, 157, 479-483.

80) Handy, N. C.; Pople, J. A.; Head-Gordon, M.; Raghavachari, K.; Trucks, G. W. Chem. Phys. Lett. 1989, 164, 185-192. 
81) Dykstra, C. E. Chem. Phys. Lett. 1977, 45, 466-469.

82) Brueckner, K. A. Phys. Rev. 1954, 96, 508.

83) Crawford, T. D.; Lee, T. J.; Handy, N. C.; Schaefer III, H. F. J. Chem. Phys. 1997, 107, 9980-9984.

84) Jensen, F. Introduction to Computational Chemistry; John Wiley \& Sons: Chichester, 1999.

85) Dunning, T. H. J. Chem. Phys. 1989, 90, 1007-1023.

86) Frisch, M. J.; Trucks, G. W.; Schlegel, H. B.; Gill, P. M. W.; B. G. Johnson; Robb, M. A.; Cheeseman, J. R.; Keith, T.; Petersson, G. A.; Montgomery, J. A.; Raghavachari, K.; Al-Laham, M. A.; Zakrzewski, V. G.; Ortiz, J. V.; Foresman, J. B.; Cioslowski, J.; Stefanov, B. B.; Nanayakkara, A.; Challacombe, M.; Peng, C. Y.; Ayala, P. Y.; Chen, W.; Wong, M. W.; Andres, J. L.; Replogle, E. S.; Gomperts, R.; Martin, R. L.; Fox, D. J.; Binkley, J. S.; Defrees, D. J.; Baker, J.; Stewart, J. P.; Head-Gordon, M.; Gonzalez, C.; Pople, J. A. Gaussian 94, Revision D.3; Gaussian, Inc.: Pittsburgh PA, 1995.

87) Frisch, M. J.; Trucks, G. W.; Schlegel, H. B.; G. E. Scuseria; Robb, M. A.; Cheeseman, J. R.; Zakrzewski, V. G.; J. A. Montgomery, J.; Stratmann, R. E.; Burant, J. C.; Dapprich, S.; J. M. Millam; Daniels, A. D.; Kudin, K. N.; Strain, M. C.; Farkas, O.; Tomasi, J.; Barone, V.; Cossi, M.; Cammi, R.; Mennucci, B.; Pomelli, C.; Adamo, C.; Clifford, S.; Ochterski, J.; Petersson, G. A.; Ayala, P. Y.; Cui, Q.; Morokuma, K.; Malick, D. K.; Rabuck, A. D.; Raghavachari, K.; Foresman, J. B.; Cioslowski, J.; Ortiz, J. V.; Stefanov, B. B.; Liu, G.; Liashenko, A.; Piskorz, P.; Komaromi, I.; Gomperts, R.; Martin, R. L.; Fox, D. J.; Keith, T.; Al-Laham, M. A.; Peng, C. Y.; Nanayakkara, A.; Gonzalez, C.; Challacombe, M.; Gill, P. M. W.; Johnson, B.; Chen, W.; Wong, M. W.; Andres, J. L.; Gonzalez, C.; Head-Gordon, M.; Replogle, E. S.; Pople, J. A. Gaussian 98, Revision A.9; Gaussian, Inc.: Pittsburgh PA, 1999.

88) Allen, W. D.; Horner, D. A.; DeKock, R. L.; Remmington, R. B.; Schaefer III, H. F. Chem. Phys. 1989, 133, 11-45.

89) Burton, N. A.; Yamaguchi, Y.; Alberts, I. L.; Schaefer III, H. F. J. Chem. Phys. 1991, 95,7466 .

90) Barnes, L. A.; Lindh, R. Chem. Phys. Lett. 1994, 223, 207-214.

91) Crawford, T. D.; Stanton, J. F.; Allen, W. D.; H. F. Schaefer, I. J. Chem. Phys. 1997, 107, 10626-10632.

92) Dehareng, D.; Dive, G. J. Comp. Chem. 2000, 21, 483-504. 
93) Sherrill, C. D.; Krylov, A. I.; Byrd, E. F. C.; Head-Gordon, M. J. Chem. Phys. 1998, 109, 4171-4181.

94) Sherrill, C. D.; Lee, M. S.; Head-Gordon, M. Chem. Phys. Lett. 1999, 301, 425-430.

95) Wenthold, P. G.; Squires, R. R.; Lineberger, W. C. J. Am. Chem. Soc. 1998, 120, 5279-5290.

96) Wenthold, P. G.; Wierschke, S. G.; Nash, J. J.; Squires, R. R. J. Am. Chem. Soc. 1994, 116, 7378-7392.

97) McWeeny, R.; Dierksen, G. J. Chem. Phys. 1968, 49, 4852-4856.

98) Krishnan, R.; Pople, J. A. Int. J. Quant. Chem. 1978, 14, 91-100.

99) Malmqvist, P.-A.; Roos, B. O. Chem. Phys. Lett. 1989, 155, 189.

100) Squires, R. R.; Cramer, C., J. J. Phys. Chem. A 1998, 102, 9072-9081.

101) McLean, A. D.; Lengsfield, B. H.; Pacansky, J.; Ellinger, Y. J. Chem. Phys. 1985, 83, 3567-3576.

102) Lindh, R.; Barnes, L. A. J. Chem. Phys. 1994, 100, 224-237.

103) Engelbrecht, L.; Liu, B. J. Chem. Phys. 1983, 78, 3097-3106.

104) Xie, Y.; Schaefer III, H. F.; Fu, X.-Y.; Liu, R.-Z. J. Chem. Phys. 1999, 111, 25322541.

105) Gauss, J. Coupled-Cluster Theory; Schleyer, P. v. R., Allinger, N. L., Clark, T., Gasteiger, J., Kollman, P. A., Schaefer III, H. F. and Schreiner, P. R., Ed.; John Wiley \& Sons: Chichester, 1998; Vol. 1, pp 615-636.

106) Bartlett, R. J.; Watts, J. D.; Kucharski, S. A.; Noga, J. J. Chem. Phys. Lett. 1989, 165, 513-522.

107) Gill, P. M. W. Density Functional Theory (DFT), Hartree-Fock (HF), and the SelfConsistent Field; Schleyer, P. v. R., Allinger, N. L., Clark, T., Gasteiger, J., Kollman, P. A., Schaefer III, H. F. and Schreiner, P. R., Ed.; John Wiley \& Sons: Chichester, 1998; Vol. 1, pp 678-689.

108) Perdew, J. P.; Savin, A.; Burke, K. Phys. Rev. A 1995, 51, 4531-4540.

109) Kozlowski, P. M.; Rauhut, G.; Pulay, P. J. Chem. Phys. 1995, 103, 5650-5661.

110) Ayala, P. Y.; Schlegel, H. B. J. Chem. Phys. 1998, 108, 7560-7567.

111) Archibong, E. F.; St-Amant, A. Chem. Phys. Lett. 1998, 284, 331-338.

112) Duarte, H. A.; Proynov, E.; Salahub, D. R. J. Chem. Phys. 1998, 109, 26-35.

113) Wang, S. G.; Schwarz, W. H. E. J. Chem. Phys. 1996, 105, 4641.

114) Beno, B. R.; Fennen, J.; Houk, K. N.; Lindner, H. J.; Hafner, K. J. Am. Chem. Soc 1998, $120,10490$. 
115) Haller, J.; Beno, B. R.; Houk, K. N. J. Am. Chem. Soc 1998, 120, 6468.

116) Goldstein, E.; Beno, B. R.; Houk, K. N. J. Am. Chem. Soc. 1996, 118, 6036-6043.

117) Goddard, J. D.; Chen, X.; Orlova, G. J. Phys. Chem. A 1999, 103, 4078-4084.

118) Garavelli, M.; Bernardi, F.; Olivucci, M.; Robb, M. J. Am. Chem. Soc. 1998, 120, 10210.

119) Chen, W.-H.; Chang, N.-Y.; Yu, C.-H. J. Phys. Chem. 1998, 102, 2584-2593.

120) Gräfenstein, J.; Kraka, E.; Cremer, D. Chem. Phys. Lett. 1998, 288, 593-602.

121) Bettinger, H. F.; Schleyer, P. v. R.; Schreiner, P. R.; Schaefer III, H. F. Computational Analyses of Prototype Carbene Structures and Reactions; Davidson, E. L., Ed.; World Scientific Press: Singapore, 1997, pp 89-171.

122) Goddard, J. D.; Orlova, G. J. Chem. Phys. 1999, 17, 7705-7712.

123) Cremer, D.; Kraka, E.; Szalay, P. G. Chem. Phys. Lett. 1998, 292, 97-109.

124) Schreiner, P. R.; Prall, M. J. Am. Chem. Soc. 1999, 121, 8615-8627.

125) Adamo, C.; Barone, V. Chem. Phys. Lett. 1997, 274, 242-250.

126) Perdew, J. P. Phys. Rev. B 1986, 33, 8822-8824.

127) Perdew, J. P.; Zunger, A. Phys. Rev. B 1981, 23, 5048-5079.

128) Hariharan, P. C.; Pople, J. A. Theoret. Chimica Acta 1973, 28, 213.

129) Roth, W. R.; Hopf, H.; Wasser, T.; Zimmermann, H.; Werner, C. Liebigs Ann. 1996, 1691-1695.

130) Skattebøl, L.; Solomon, S. J. Am. Chem. Soc. 1965, 87, 4506-4513.

131) Roth, W. R.; Wollweber, D.; Offerhaus, R.; Rekowski, V.; Lennartz, H.-W. Chem. Ber. 1993, 126, 2701-2716.

132) Roth, W. R.; Scholz, B. P.; Breuckmann, R.; Jelich, K.; Lennartz, H.-W. Chem. Ber. 1982, 115, 1934-1946.

133) Huntsman, W. D. J. Am. Chem. Soc. 1966, 88, 5846-5850.

134) Roth, W. R.; Bauer, F.; Braun, K.; Offerhaus, R. Angew. Chem. 1989, 101, 1092-1094.

135) Zimmermann, G.; Nuechter, M.; Remmler, M.; Findeisen, M.; Hopf, H. Chem. Ber. 1994, 127, 1747-1754.

136) Roth, W. R.; Bartmann, M.; Maier, G.; Reisenauer, H. P.; Sustmann, R. Angew. Chem. 1987, 99, 271-272.

137) Doering, W. V.; Wang, Y. H. J. Am. Chem. Soc. 1999, 121, 10112-10118.

138) Hrovat, D. A.; Duncan, J. A.; Borden, W. T. J. Am. Chem. Soc. 1999, 121, 169-175.

139) Hrovat, D. A.; Beno, B. R.; Lange, H.; Yoo, H.-Y.; Houk, K. N.; Borden, W. T. J. Am. Chem. Soc. 1999, 122, 10529-10537. 
140) Jiao, H.; Nagelkerke, R.; Kurtz, H. A.; Williams, R. V.; Borden, W. T.; Schleyer, P. v. R. J. Am. Chem. Soc. 1997, 119, 5921-5929.

141) Roth, W. R.; Schaffers, T.; Heiber, M. Chem. Ber. Recl. 1992, 125, 739-749.

142) Sakai, S. Int. J. Quant. Chem. 2000, 80.

143) Staroverov, V. N.; Davidson, E. R. J. Am. Chem. Soc. 2000, 122, 186-187.

144) Staroverov, V. N.; Davidson, E. R. J. Am. Chem. Soc. 2000, 122, 7377-7385.

145) Wiest, O.; Black, K. A.; Houk, K. N. J. Am. Chem. Soc. 1994, 116, 10336-10337.

146) Wenthold, P. G. J. Chem. Soc., Perkin T. 2 1999, 11, 2357-2363.

147) Galbraith, J. M.; Schreiner, P. R.; Harris, N.; Wei, W.; Wittkopp, A.; Shaik, S. Chem. Eur. J. 2000, 1615-1628.

148) Marquardt, R.; Balster, A.; Sander, W.; Kraka, E.; Cremer, D.; Radziszewski, J. G. Angew. Chem. 1998, 110, 1001-1005.

149) König, B.; Pitsch, W.; Klein, M.; Kröner, J.; Prall, M.; Schreiner, P. R. J. Org. Chem. 2001, 66, 1742-1746.

150) Kaneko, T.; Takahashi, M.; Hirama, M. Tetrahedron Lett. 1999, 40, 2015-2018.

151) Lindh, R.; Persson, B. J. J. Am. Chem. Soc. 1994, 116, 4963-4969.

152) Lindh, R.; Lee, T. J.; Bernhardsson, A.; Persson, B. J.; Karlström, G. J. Am. Chem. Soc. 1995, 117, 7186-7194.

153) Lindh, R.; Schütz, M. Chem. Phys. Lett. 1996, 258, 409-415.

154) Lindh, R.; Ryde, U.; Schütz, M. Theor. Chem. Acc. 1997, 97, 203-210.

155) Chakraborty, M.; Tessier, C. A.; Youngs, W. J. J. Org. Chem. 1999, 64, 2947-2949.

156) Koseki, S.; Fujimura, Y.; Hirama, M. J. Phys. Chem. A 1999, 103, 7672-7675.

157) Jones, G. B.; Warner, P. M. J. Am. Chem. Soc. 2001, 123, 2134-2145.

158) Prall, M.; Wittkopp, A.; Schreiner, P. R. J. Phys. Chem. A 2001, 105, 9265-9274.

159) Holweger, W.; Hanack, M. Chem. Ber. 1984, 117, 3004-3020.

160) Bleckmann, W.; Hanack, M. Chem. Ber. 1984, 117, 3021-3033.

161) John, J. A.; Tour, J. M. Tetrahedron 1997, 53, 15515-15534.

162) Vollhardt, K. P. C.; Winn, L. S. Tetrahedron Lett. 1985, 26, 709-712.

163) McQuilkin, R. M.; Garratt, P. J.; Sondheimer, F. J. Am. Chem. Soc. 1970, 92, 66826683.

164) Walker, J. A.; Bitler, S. P.; Wudl, F. J.Org.Chem. 1984, 49, 4733-4734.

165) Märk1, G.; Hennig, R.; Nöth, H. Liebigs Ann./Recueil 1997, 121-125.

166) Bowles, D. M.; Anthony, J. E. Org. Lett. 2000, 2, 85-87.

167) Ishida, N.; Miyazaki, K.; Kumagai, K. M.; Rikimaru, M. J. Antibiot. 1965, 18, 68-76. 
168) Goldberg, I. H. Free Radicals Biol. Med. 1987, 3, 41.

169) Povirk, L. F.; Dattagupta, N.; Warf, B. C.; Goldberg, I. H. Biochemistry 1981, 20, 4007.

170) Roth, W. R.; Ruf, G.; Ford, P. W. Chem. Ber. 1974, 107, 48-52.

171) Schleyer, P. v. R.; Jiao, H. Pure Appl. Chem. 1996, 68, 209-218.

172) Schmittel, M.; Strittmatter, M.; Kiau, S. Tetrahedron Lett. 1995, 36(28), 4975-4978.

173) Schmittel, M.; Kiau, S.; Siebert, T.; Strittmatter, M. Tetrahedron Lett. 1996, 37(43), 7691-7694.

174) Schmittel, M.; Strittmatter, M.; Kiau, S. Angew. Chem. 1996, 108(16), 1952-1954.

175) Schmittel, M.; Keller, M.; Kiau, S.; Strittmatter, M. Chem. Eur. J. 1997, 3(5), 807816.

176) Schmittel, M.; Maywald, M.; Strittmatter, M. Synlett 1997, 165-166.

177) Schmittel, M.; Steffen, J.-P.; Auer, D.; Maywald, M. Tetrahedron Lett. 1997, 38(35), 6177-6180.

178) Engels, B.; Hanrath, M. J. Am. Chem. Soc. 1998, 120, 6356-6361.

179) Engels, B.; Lennartz, C.; Hanrath, M.; Schmittel, M.; Marc, S. Angew. Chem. 1998, 110, 2067-2070.

180) Gillmann, T.; Hülsen, T.; Massa, W.; Wocadlo, S. Synlett 1995, 1257-1259.

181) Rodriguez, A.; Garcia, J. G.; Ramos, B.; Pratt, L. M. Tetrahedron Lett. 1995, 36(41), 7391-7394.

182) Lias, S. G.; Bartmess, J. E.; Liebman, J. F.; Holmes, J. L.; Levin, R. D.; Mallam, W. G. J. Phys. Chem. Ref. Data 1988, 17 (S1), 1-872.

183) Wenthold, P. G.; Wierschke, S. G.; Nash, J. J.; Squires, R. R. J. Am. Chem. Soc. 1993, $115,12611-12612$.

184) McMillen, D. F.; Golden, D. M. Annu. Rev. Phys. Chem. 1982, 33, 493-523.

185) Scott, L. T.; Chemg, P.-C.; Hashemi, M. M.; Bratcher, M. S.; Meyer, D. T.; Warren, H. B. J. Am. Chem. Soc. 1997, 119, 10963-10968.

186) Scott, L. T.; Hashemi, M. M.; Meyer, D. T.; Warren, H. B. J. Am. Chem. Soc. 1991, 113, 7083-7083.

187) Zimmermann, G.; Nuechter, U.; Hagen, S.; Nuechter, M. Tetrahedron Lett. 1994, 35, 4747-4750.

188) Rabideau, P. W.; Abdourazak, A. H.; Folsom, H. E.; Marcinow, Z.; Sygula, A.; Sygula, R. J. Am. Chem. Soc. 1994, 116, 7891-7892.

189) Hopf, H.; Musso, H. Angew. Chem. 1969, 81, 704-704. 
190) Roth, W. R.; Hopf, H.; Horn, C. Chem. Ber. 1994, 127, 1781-1795.

191) Hopf, H.; Berger, H.; Zimmermann, G.; Nüchter, U.; Jones, P. G.; Dix, I. Angew. Chem. 1997, 109, 1236-1238.

192) Christl, M.; Groetsch, S. Eur. J. Org. Chem. 2000, 1871-1874.

193) Christl, M.; Drinkuth, S. Eur. J. Org. Chem. 1998, 237-241.

194) Zimmermann, G.; Nüchter, M.; Remmler, M.; Findeisen, M.; Hopf, H.; Ernst, L.; Mlynek, C. Chem. Ber. 1994, 127, 1747-1753.

195) Nüchter, U.; Zimmermann, G.; Francke, V.; Hopf, H. Liebigs Ann./Recueil 1997, 1505-1515.

196) Schulz, K.; Hofmann, J.; Zimmermann, G. Liebigs Ann./Recueil 1997, 2535-2539.

197) Hofmann, J.; Schulz, K.; Altmann, A.; Findeisen, M.; Zimmermann, G. Liebigs Ann./Recueil 1997, 2541-2548.

198) Zimmermann, G. Eur. J. Org. Chem. 2001, 457-471.

199) Meier, H.; Hanold, N.; Kolshorn, H. Angew. Chem. 1982, 94, 67-68.

200) Hanold, N.; Meier, H. Chem. Ber. 1985, 118, 198-209.

201) Hanack, M.; Rieth, R. J. Chem. Soc., Chem. Comm. 1985, 21, 1487-1489.

202) Hanack, M.; Rieth, R. Chem. Ber. 1987, 120, 1659-1666.

203) Christl, M.; Braun, M.; Müller, G. Angew. Chem. 1992, 104, 471-473.

204) Janoschek, R. Angew. Chem. 1992, 104, 473-475.

205) Navarro-Vázquez, A.; Rodríguez, D.; Castedo, L.; Domínguez, D.; Saá, C. persönliche Mitteilung 2001.

206) Schreiner, P. R.; Karney, W. L.; Schleyer, P. v. R.; Borden, W. T.; Hamilton, T. P.; Schaefer III, H. F. J. Org. Chem. 1996, 61, 7030-7039.

207) Wong, M. W.; Wentrup, C. J. Org. Chem. 1996, 61, 7022-7029.

208) Matzinger, S.; Bally, T.; Patterson, E. V.; McMahon, R. J. J. Am. Chem. Soc. 1996, $118,1535-1542$.

209) Hopf, H.; Krüger, A. Chem. Eur. J. 2001, 7, 4378-4385.

210) Braverman, S.; Duar, Y. Tetrahedron Lett. 1978, 1493-1496.

211) Toda, F.; Tanaka, K.; Sano, I.; Isozaki, T. Angew. Chem. 1994, 106, 1856-1858.

212) Fleischhauer, I.; Brinker, U. H. Tetrahedron Lett. 1983, 24, 3205-3208.

213) Brinker, U. H.; Wilk, G.; Gomann, K. Angew. Chem. 1983, 95, 892-893.

214) Christen, H. R.; Vögtle, F. Organische Chemie; Salle: Frankfurt a.M., 1992.

215) Bianchini, R.; Chiappe, C.; Lo Moro, G.; Lenoir, D.; Lemmen, P.; Goldberg, N. Chem. Eur. J. 1999, 5, 1570-1580. 
216) Wittig, G.; Geissler, G. Justus Liebigs Ann. Chem. 1953, 580, 44-57.

217) Heck, R. F.; Dieck, H. A. J. Am. Chem. Soc. 1974, 96, 1133-1136.

218) Heck, R. F. Org. React. 1982, 27, 345-390.

219) Heck, R. F. Palladium Reagents in Organic Syntheses; Academic Press: New York, 1985.

220) Sonogashira, K.; Tohda, Y.; Hagihara, N. Tetrahedron Lett. 1975, 50, 4467-4470.

221) Becker, H. G. O.; Gluch, R.; Berger, W.; Mayer, R.; Domschke, G.; Müller, K.; Fanghänel, G.; Pavel, D.; Faust, J.; Schmidt, H.; Fischer, M.; Schollberg, K.; Gentz, F.; Schwetlick, K.; Gewald, K.; Seiler, E.; Zeppenfeld, G. Organikum: organischchemisches Grundpraktikum; Barth, Dt. Verl. der Wiss.: Leipzig; Berlin; Heidelberg, 1993.

222) Collins, I.; Suschitzky, H. J. Chem. Soc. (C) 1969, 2337-2341.

223) Smith, C. F.; Moore, G. J.; Tamborski, C. J. Organomet. Chem. 1971, 33, C21-C24.

224) House, H. O.; Campbell, W. J.; Koepsell, D. G. J. Org. Chem. 1972, 37, 1003-1008.

225) Corey, E. J.; Fuchs, P. L. Tetrahedron Lett. 1972, 36, 3769-3772.

226) Chodkiewicz, W. Ann. Chim. (Paris) 1957, 13, 819-869.

227) Glaser, C. Ber. Dtsch. Chem. Ges. 1869, 2, 422-424.

228) Eglinton, G.; Galbraith, A. R. Chem. Ind. (London) 1956, 737-738.

229) Campbell, I. D.; Eglinton, G. Org. Synth. Coll. Vol. V 1973, 517.

230) König, B.; Pitsch, W.; Klein, M.; Vasold, R.; Prall, M.; Schreiner, P. R. J. Org. Chem. 2001, 66, 1742-1746.

231) Behr, O. M.; Eglinton, G.; Lardy, I. A.; Raphael, R. A. J. Chem. Soc. 1964, 11471154.

232) Muenzenmaier, W.; Straub, H. Synthesis 1976, 49-51.

233) Espenson, J. H.; Zhu, Z.; Zauche, T. H. J. Org. Chem. 1999, 64, 1191-1196.

234) Baldwin, J. E. J. Chem. Soc., Chem. Commun. 1976, 734-736.

235) Koelsch J. Am. Chem. Soc. 1934, 56, 480-482.

236) Straus Justus Liebigs Ann. Chem. 1905, 342, 257.

237) Takeuchi, Y.; Furuyama, H.; Fukushi, S.; Fujiwara, S. J. Chem. Soc., Perkin Trans. 2 1985, 175-178.

238) Rajca, A.; Safronov, A.; Rajca, S.; Ross, C. R.; Stezowski, J. J. J. Am. Chem. Soc. 1996, 118, 7272-7279.

239) Darses, S.; Jeffery, T.; Brayer, J.-L.; Demoute, J.-P.; Genet, J.-P. Bull. Soc. Chim. Fr. 1996, 133, 1095-1102. 
240) Miyaura, N.; Suzuki, A. Org. Synth. 1990, 68, 130-137.

241) Katritzky, A. R.; Wang, J.; Karodia, N.; Li, J. J. Org. Chem. 1997, 62, 4142-4147.

242) Grubbs, R. H.; Kratz, D. Chem. Ber. 1993, 126, 149-158.

243) Kawase, T.; Ueda, N.; Darabi, H. R.; Oda, M. Angew. Chem. 1996, 108, 1658-1660.

244) Chow, H.-F.; Wan, C.-W.; Low, K.-H.; Yeung, Y.-Y. J. Org. Chem. 2001, 66, 19101913.

245) Tao, W.; Nesbitt, S.; Heck, R. F. J. Org. Chem. 1990, 55, 63-69.

246) Komatsu, K.; Arai, M.; Hattori, Y.; Fukuyama, K.; Katsube, Y.; Okamoto, K. J. Org. Chem. 1987, 52, 2183-2192.

247) Watanabe, S.-I.; Yamamoto, K.; Itagaki, Y.; Iwamura, T.; Iwama, T.; Kataoka, T. Tetrahedron 2000, 56, 855-864.

248) Padwa, A.; Weingarten, M. D. J. Org. Chem. 2000, 65, 3722-3732.

249) Brouw, P. M. o. d.; Laarhoven, W. H. J. Chem. Soc., Perkin Trans. 2 1982, 795-800.

250) Ma, S.; Wang, L. J. Org. Chem. 1998, 63, 3497-3498.

251) Tanaka, R.; Zheng, S.-Q.; Kawaguchi, K.; Tanaka, T. J. Chem. Soc., Perkin Trans. 2 1980, 1714-1720.

252) Renaud, J.-L.; Aubert, C.; Malacria, M. Tetrahedron 1999, 55, 5113-5128.

253) Uno, H.; Sakamoto, K.; Semba, F.; Suzuki, H. Bull. Chem. Soc. Jpn. 1992, 65, 210217.

254) Lin, S.-T.; Lee, C.-C.; Liang, D. W. Tetrahedron 2000, 56, 9619-9623.

255) Behr, O. M.; Eglinton, G.; Galbraith, A. R.; Raphael, R. A. J. Chem. Soc. 1960, 36143625 .

256) Kiddle, J. J. Tetrahedron Lett. 2000, 41, 1339-1342.

257) Snider, B. B.; Jackson, A. C. J. Org. Chem. 1983, 48, 1471-1774.

258) Kropp, P. J.; Breton, G. W.; Craig, S. L.; Crawford, S. D.; Durland, W. F. J. Org. Chem. 1995, 60, 4146-4152.

259) Wittig, G.; Hellwinkel, D. Chem. Ber. 1964, 769-788.

260) Stille, J. K.; Newsom, R. A. J. Org. Chem. 1961, 26, 1375-1379. 


\section{Anhang}

\subsection{Abbildungen der ${ }^{1}$ H-NMR Spektren}

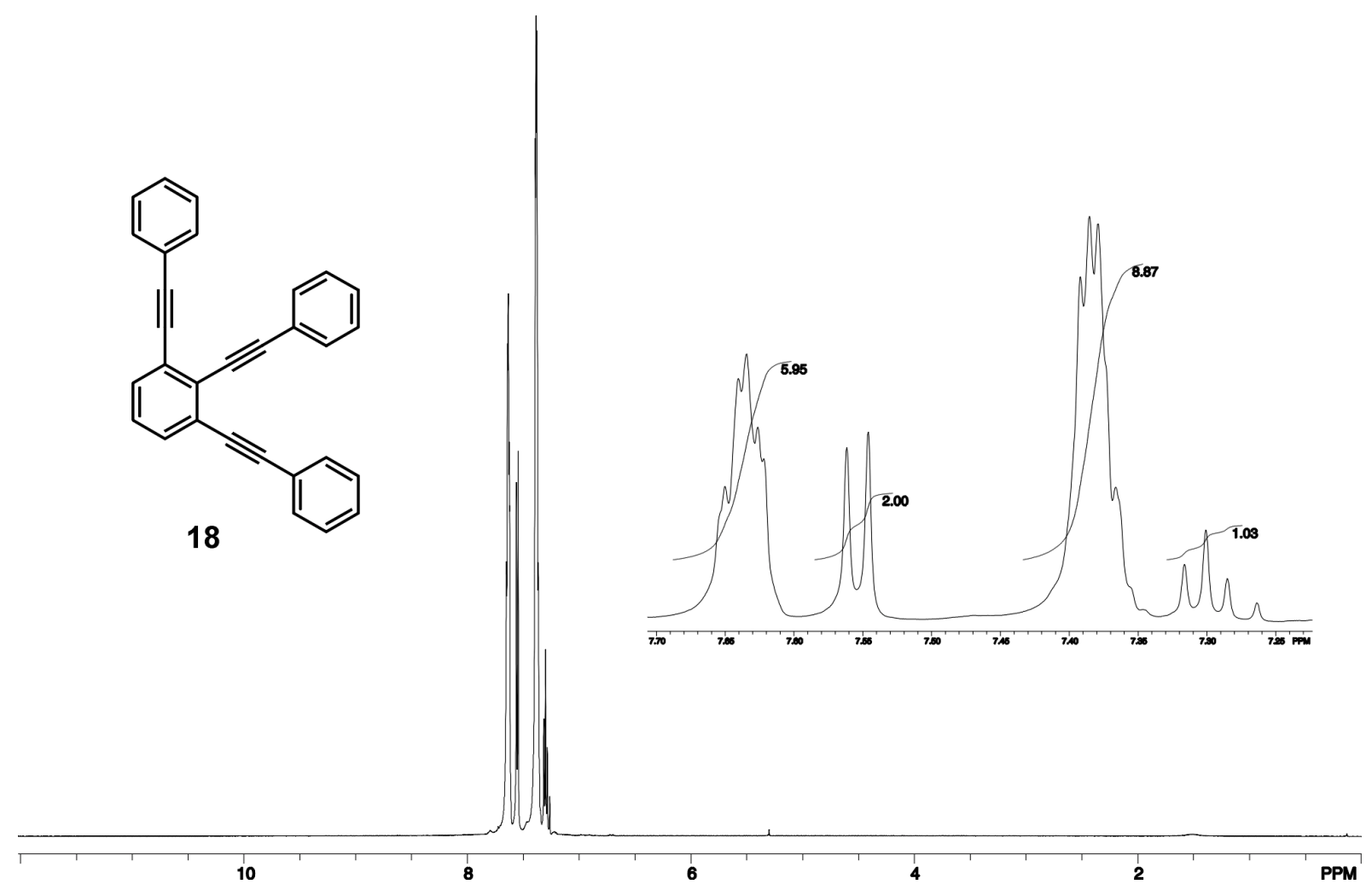

1,2,3-Tris(phenylethinyl)benzol 18

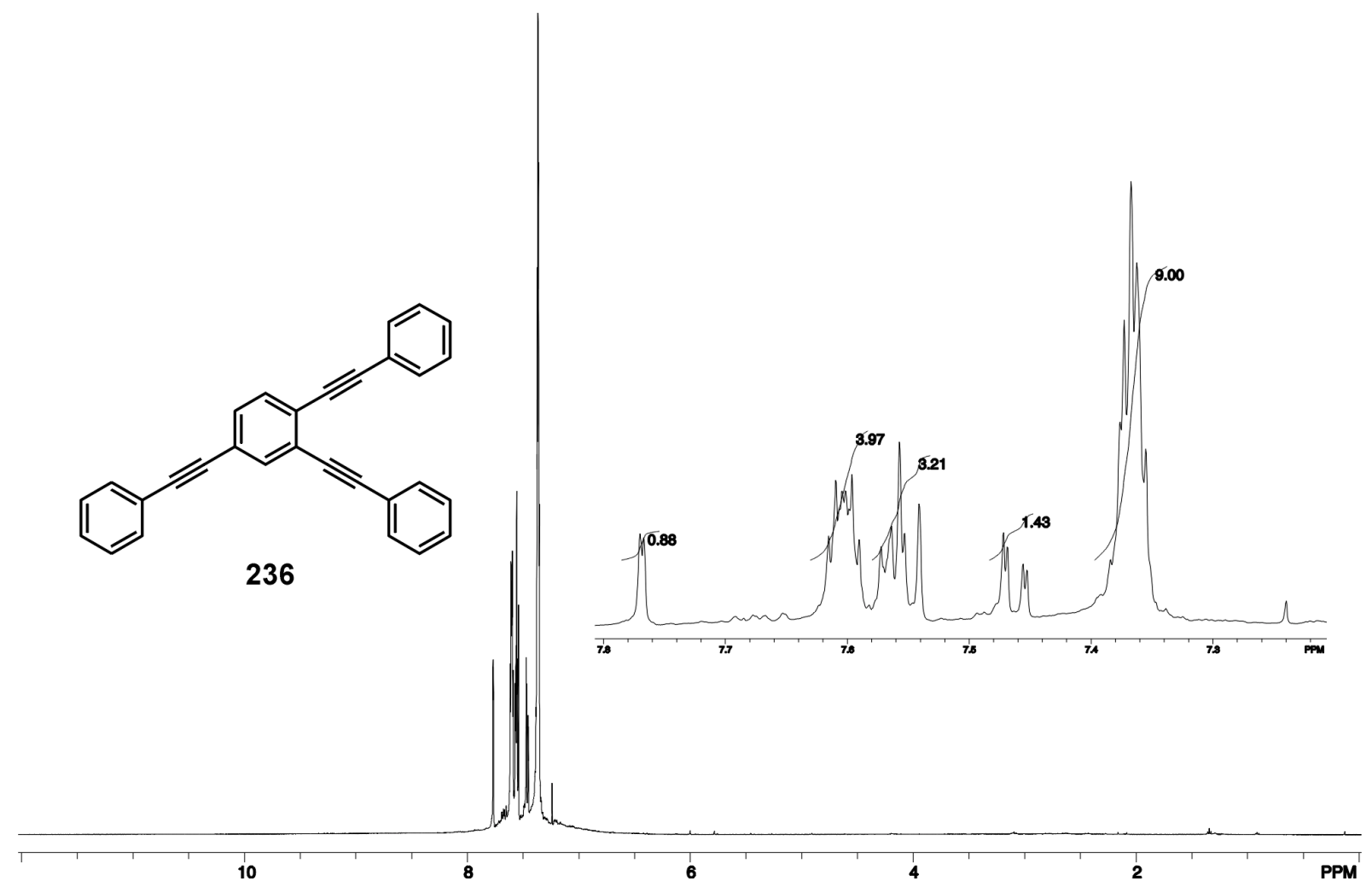

1,2,4-Tris(phenylethinyl)benzol 236 


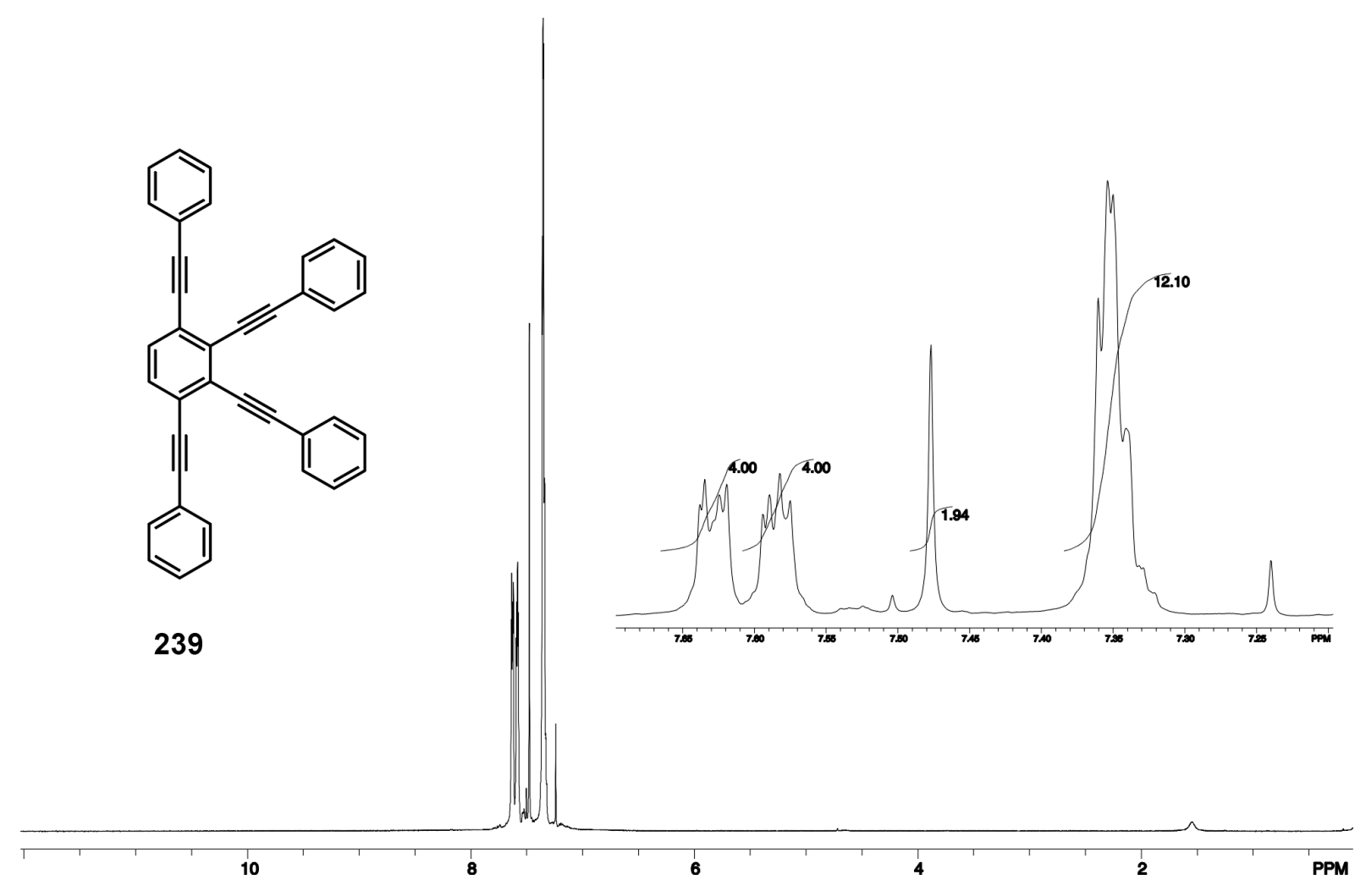

1,2,3,4-Tetrakis(phenylethinyl)benzol 239

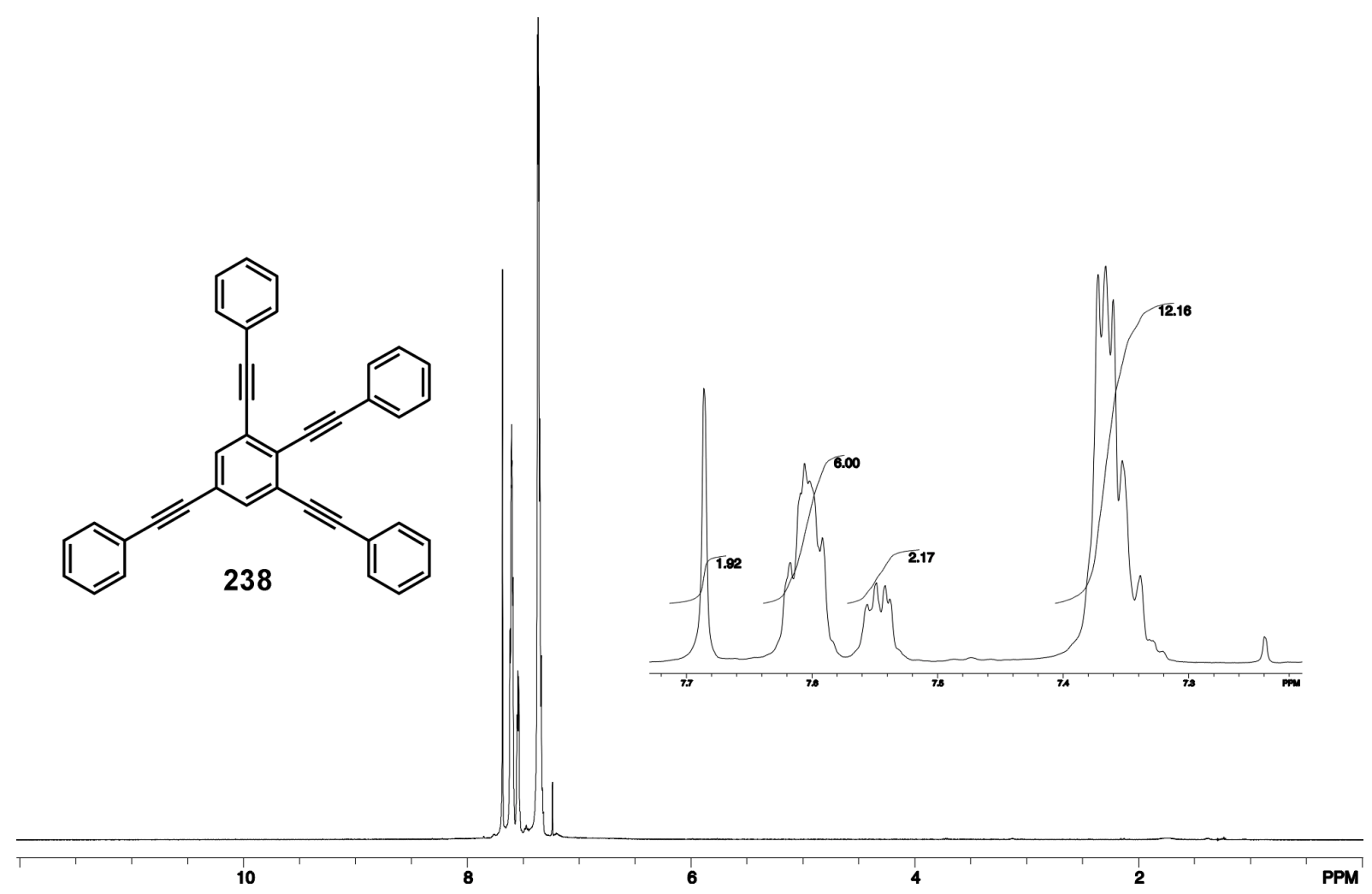

1,2,3,5-Tetrakis(phenylethinyl)benzol 238 


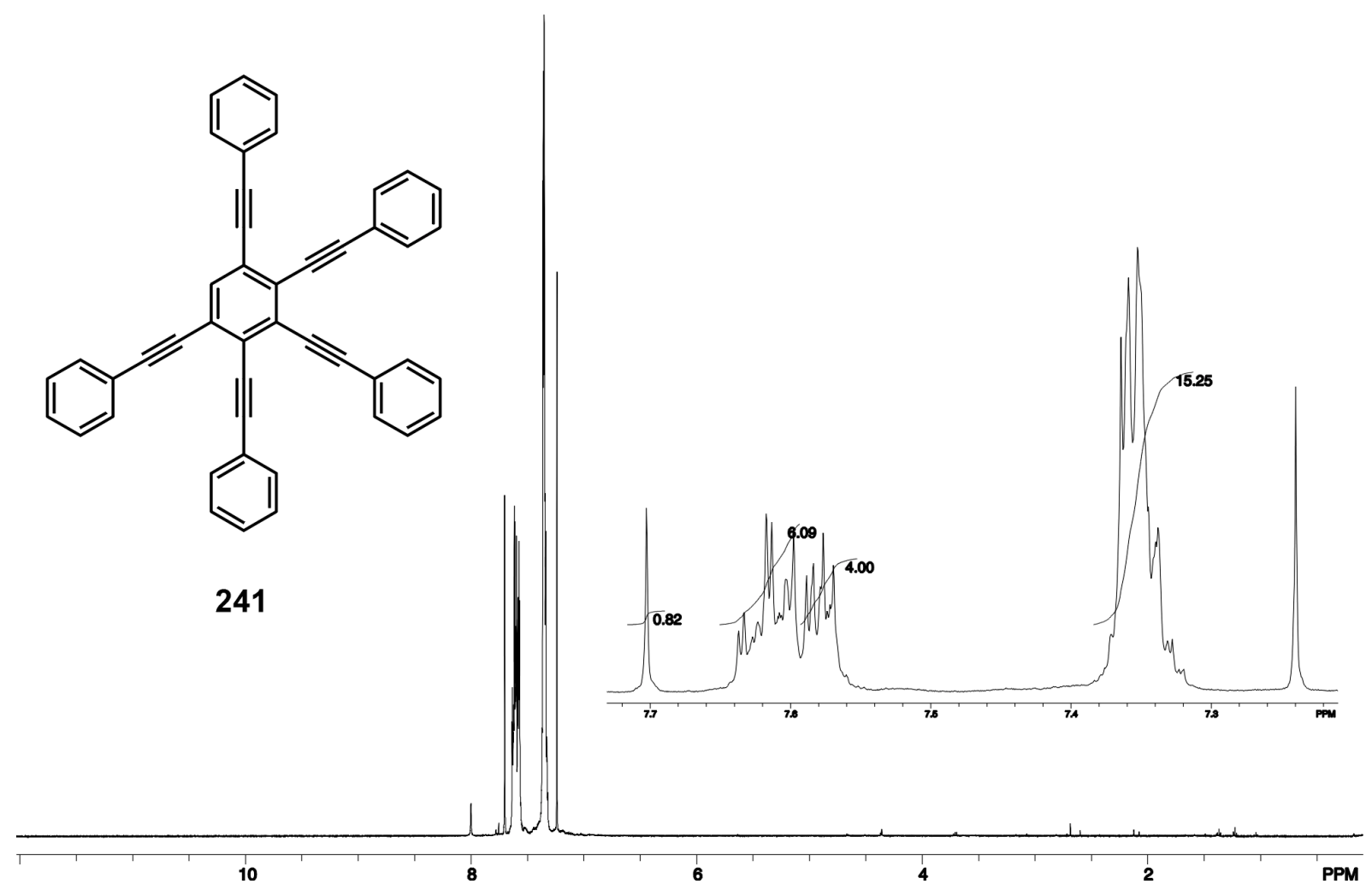

1,2,3,4,5-Pentakis(phenylethinyl)benzol 241

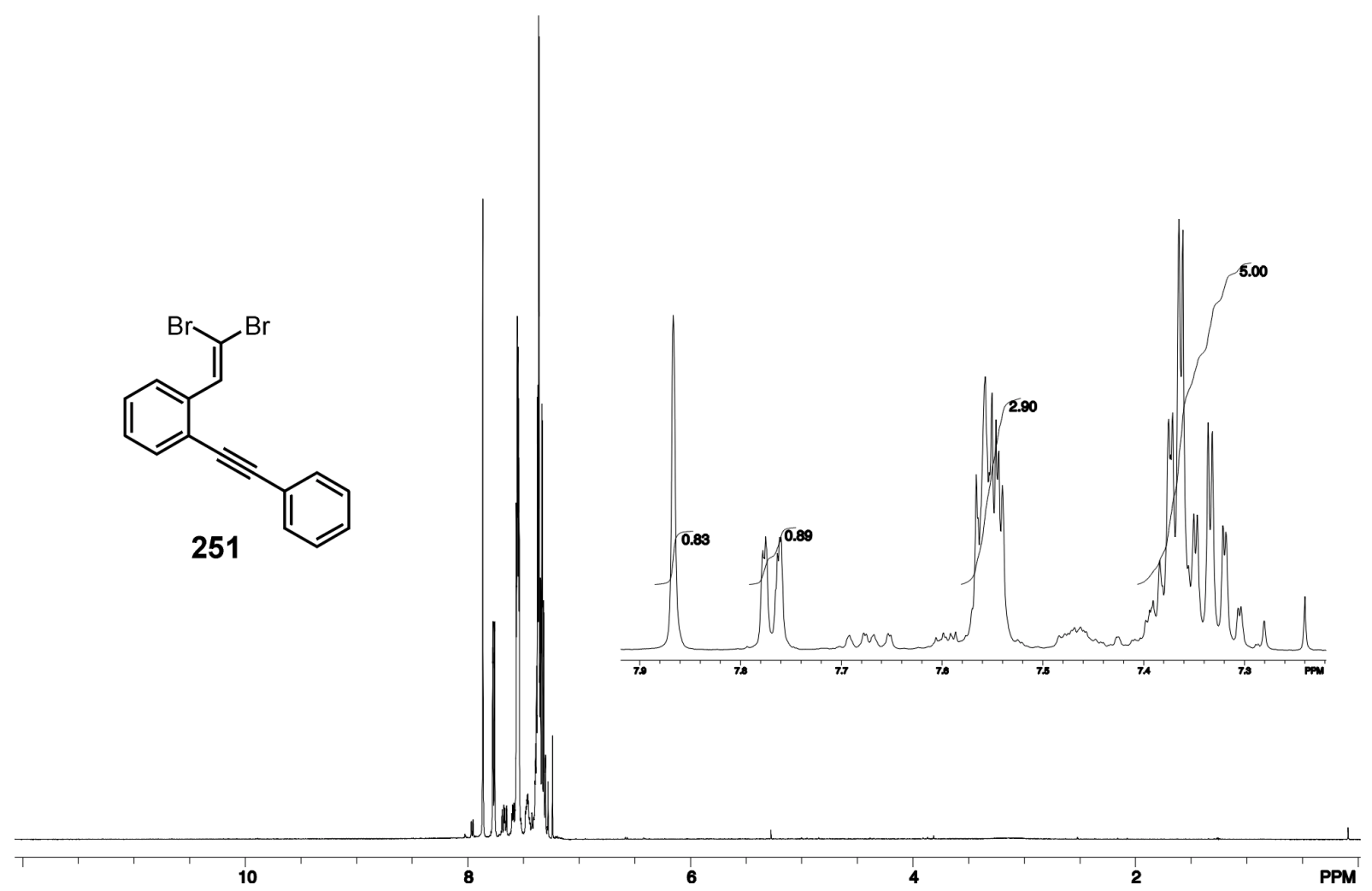

2-(2',2'-Dibromvinyl)diphenylacetylen 251 


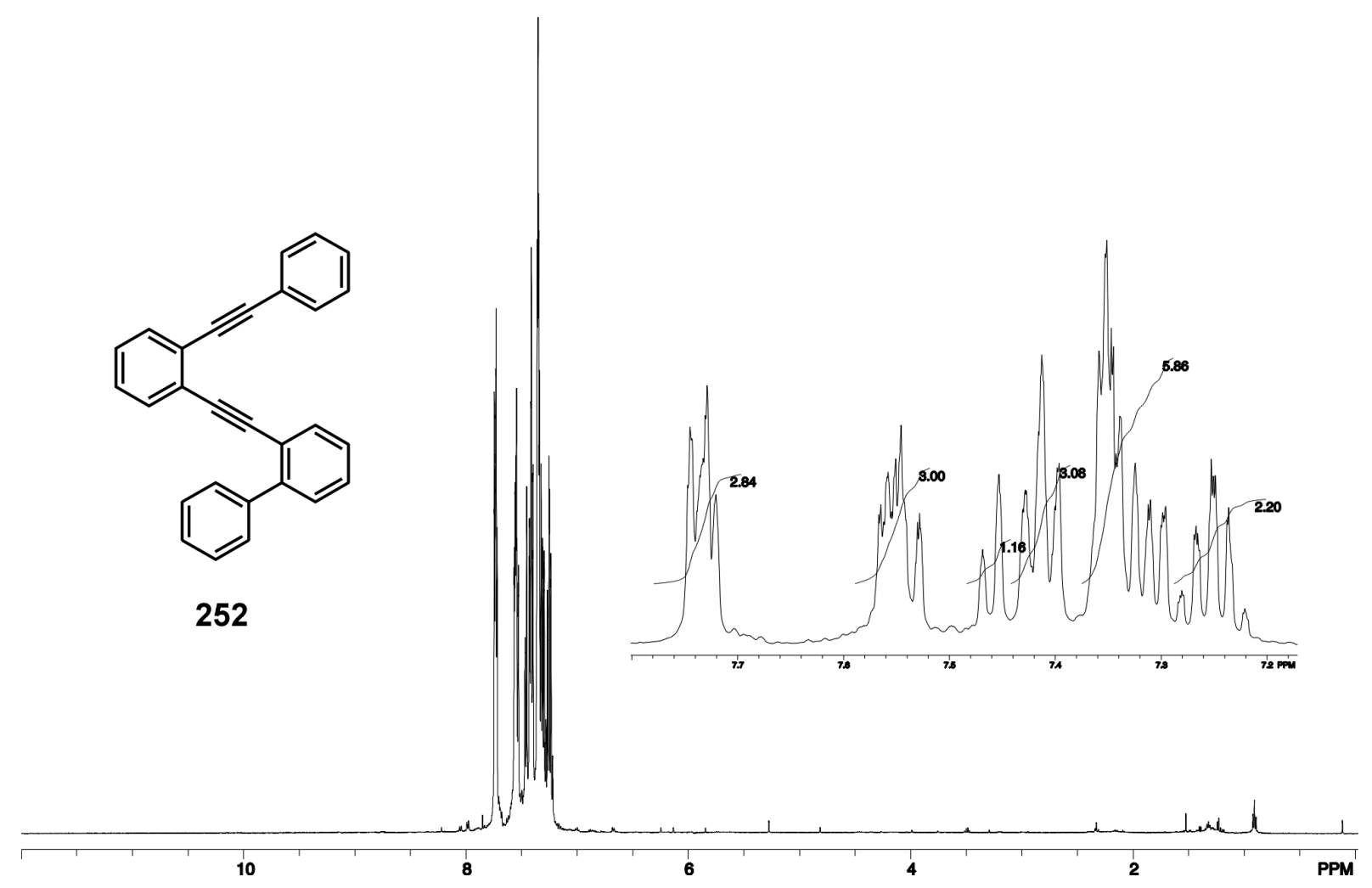

1-(2'-Biphenylethinyl)-2-(phenylethinyl)benzol 252

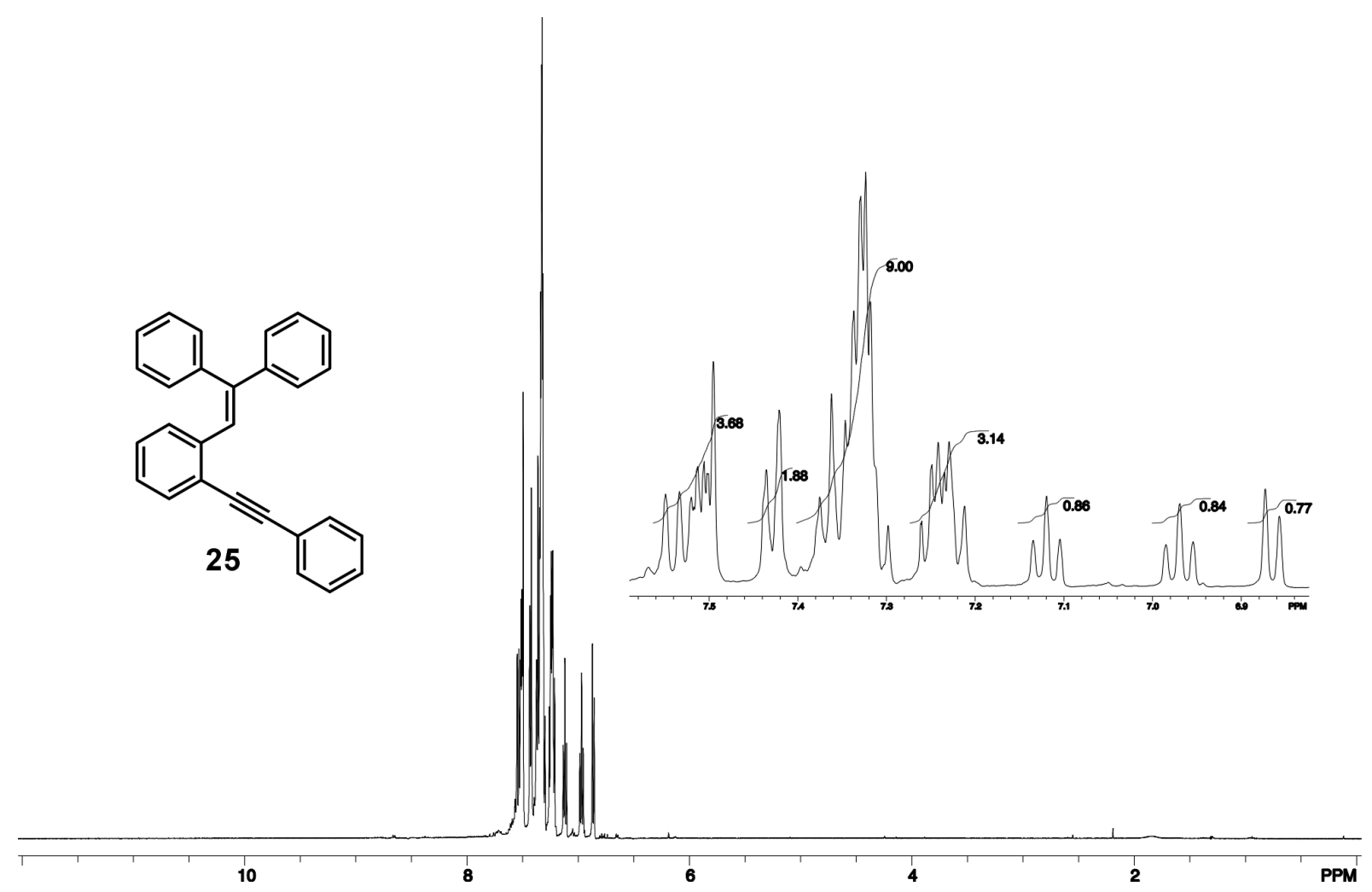

2-(2',2'-Diphenylvinyl)diphenylacetylen $\mathbf{2 5}$ 


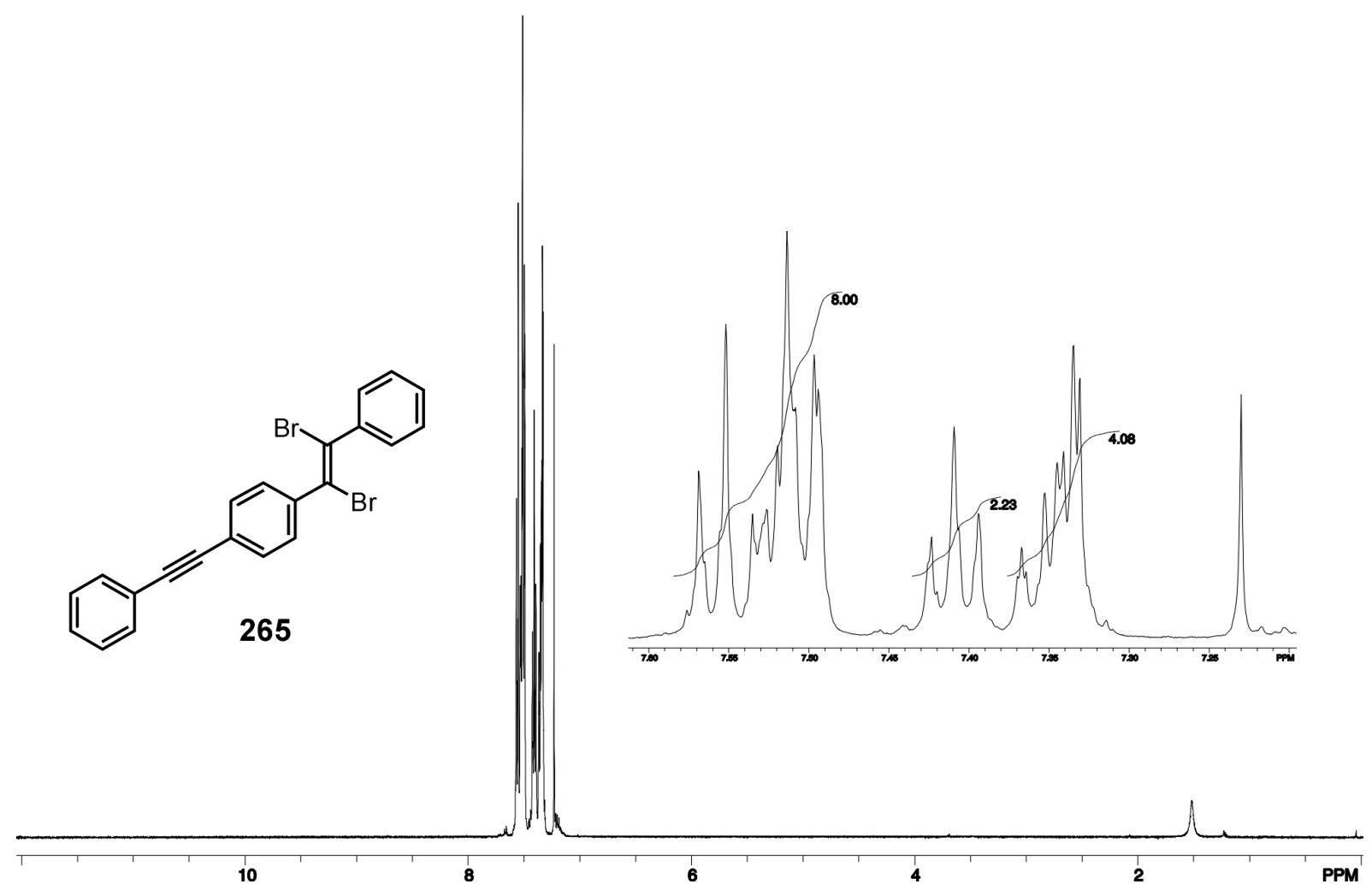

4-(Phenylethinyl)dibromstilben 265<smiles>C[R6](C)(=O)C1=CC=Cc2c3c(-c4ccccc4)c(c1c2-c1ccccc1)C(c1ccccc1)=C3Br</smiles>
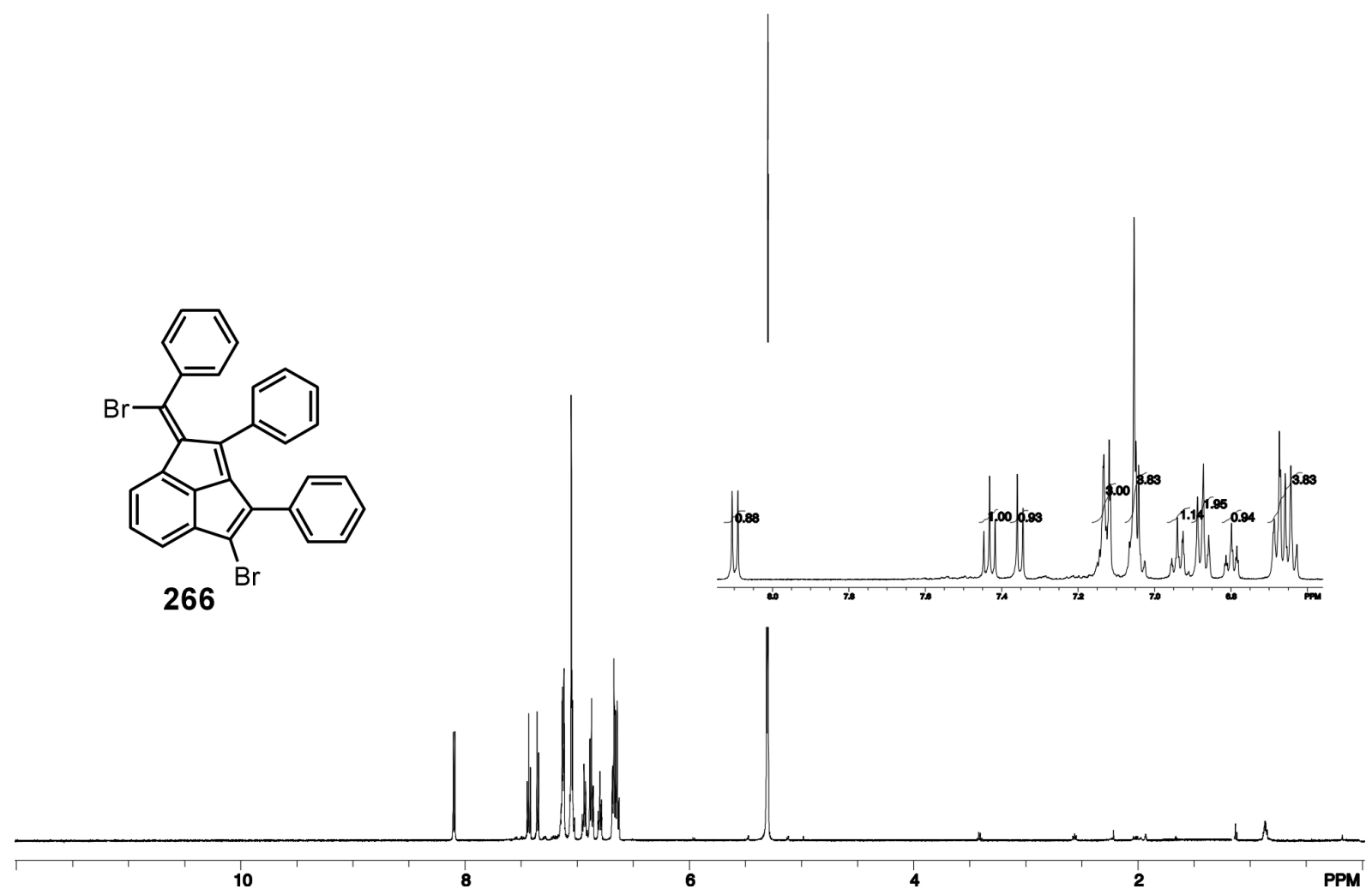

1-(Bromphenylmethylen)-2,3-diphenyl-4-brom-1H-cyclopenta $[c d]$ indene 266 


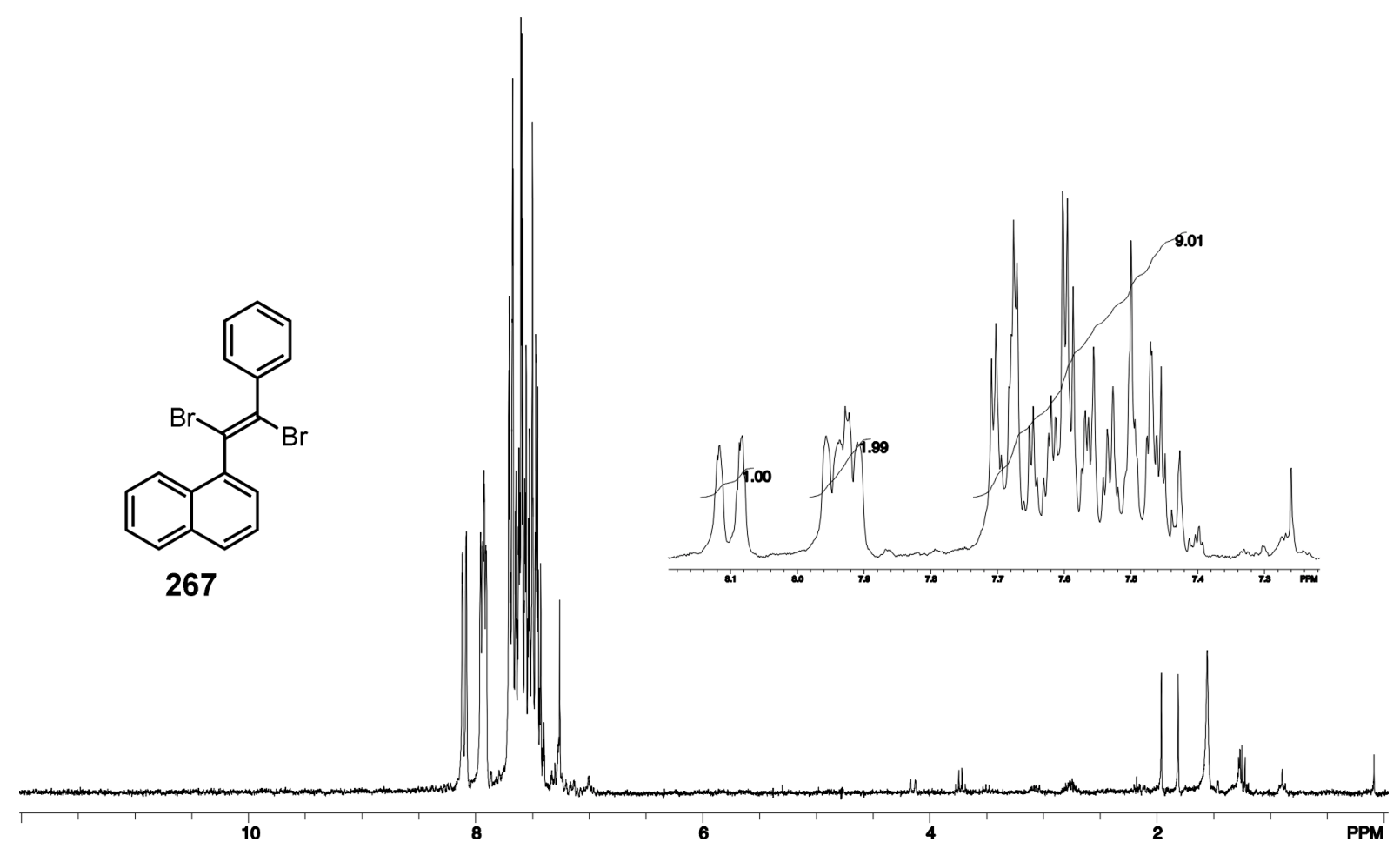

1-(1,2-Dibrom-2-phenylvinyl)-naphthalin 267

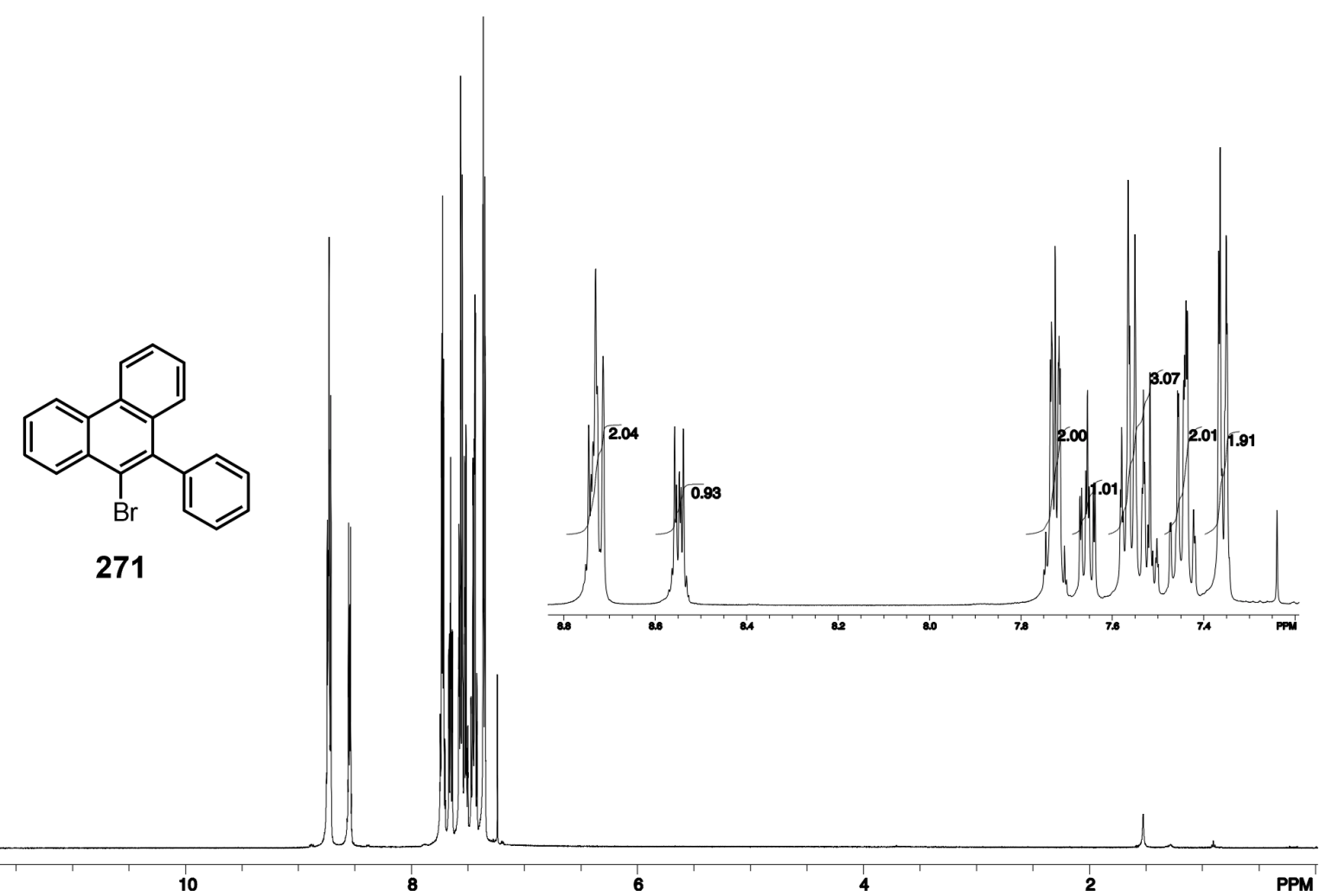

9-Brom-10-phenylphenanthren 271 


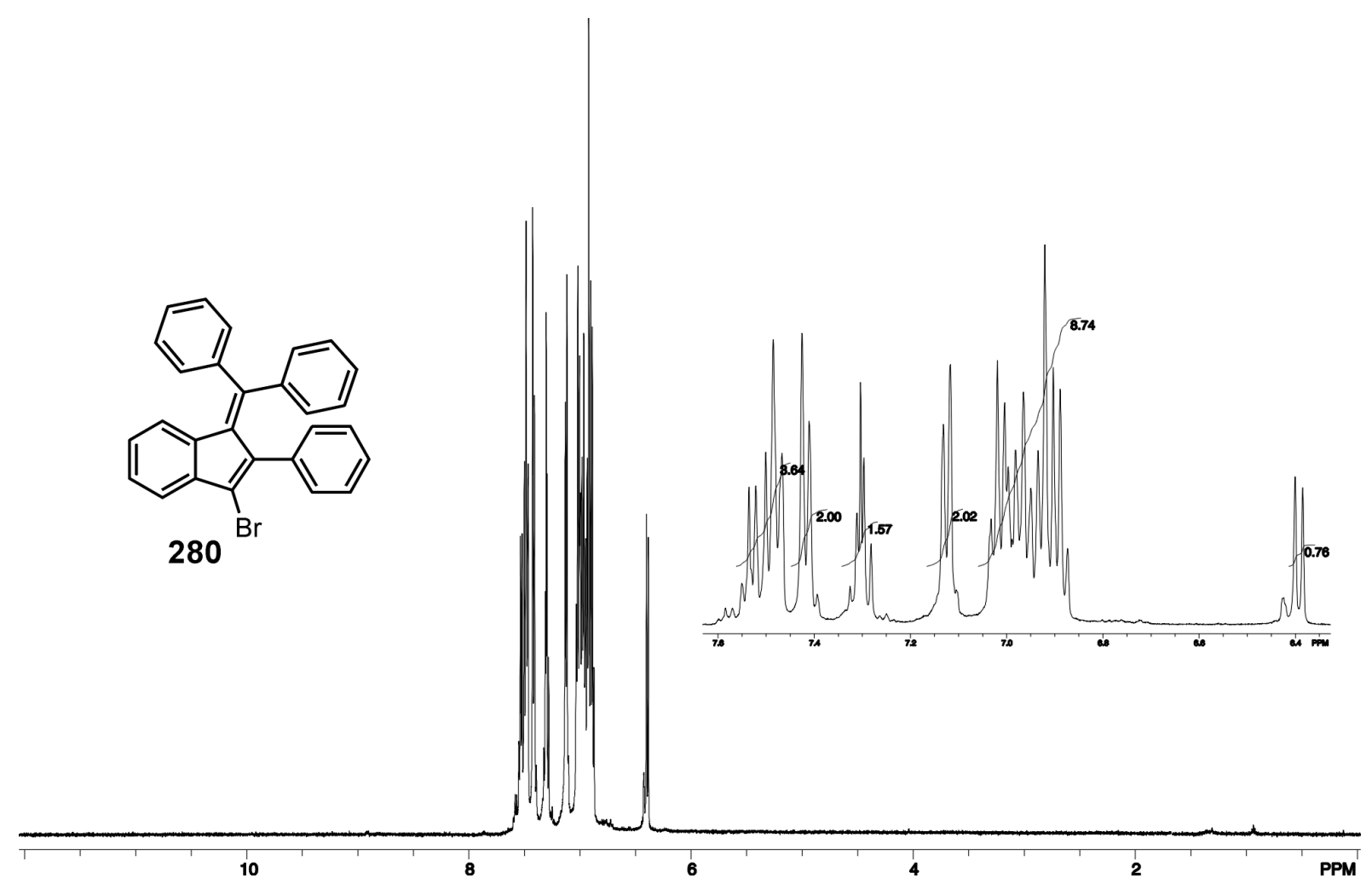

1-(Diphenylmethylen)-2phenyl-3-brom-1H-inden 280

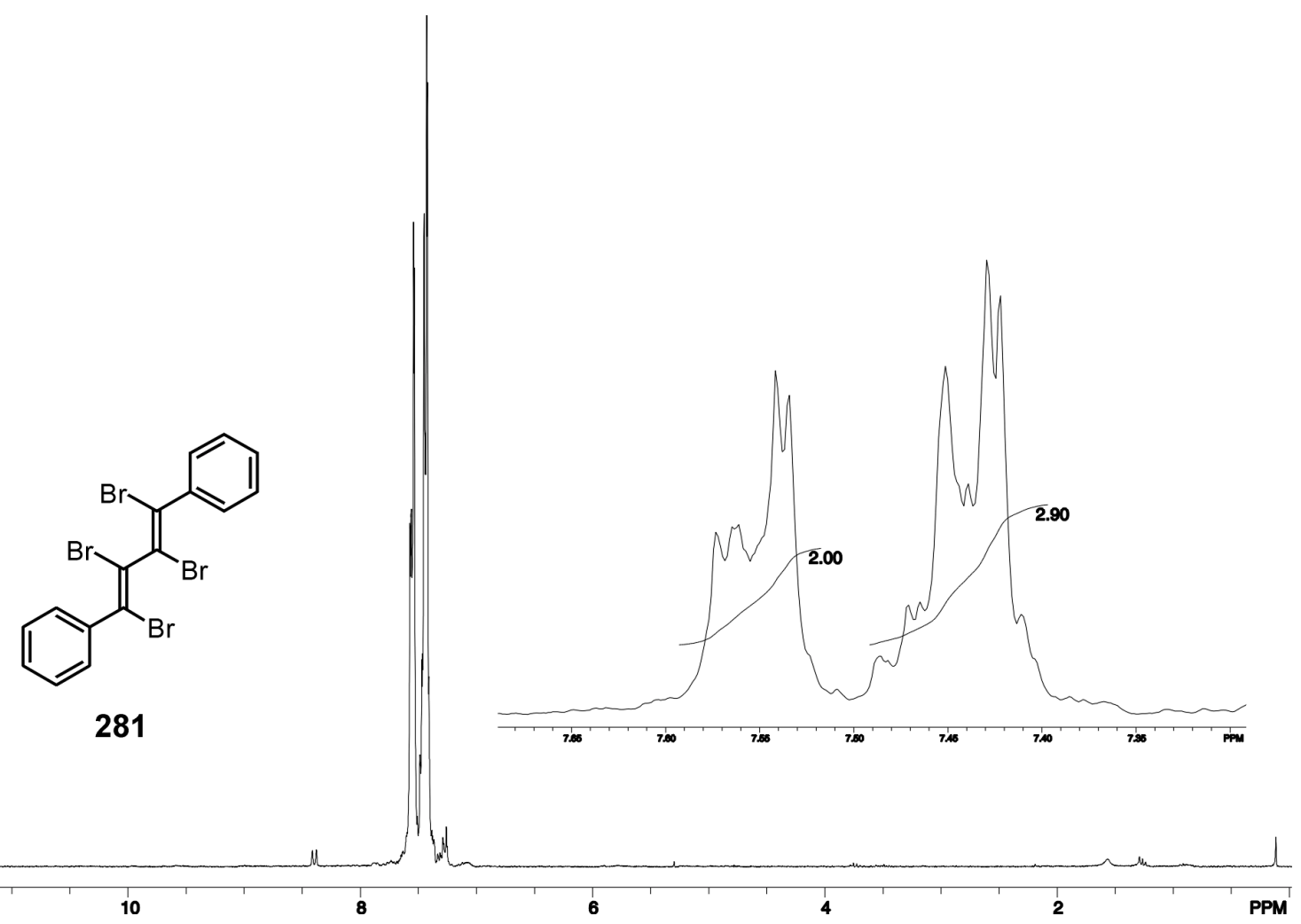

1,2,3,4-Tetrabrom-1,4-diphenylbuta-1,3-dien 281 


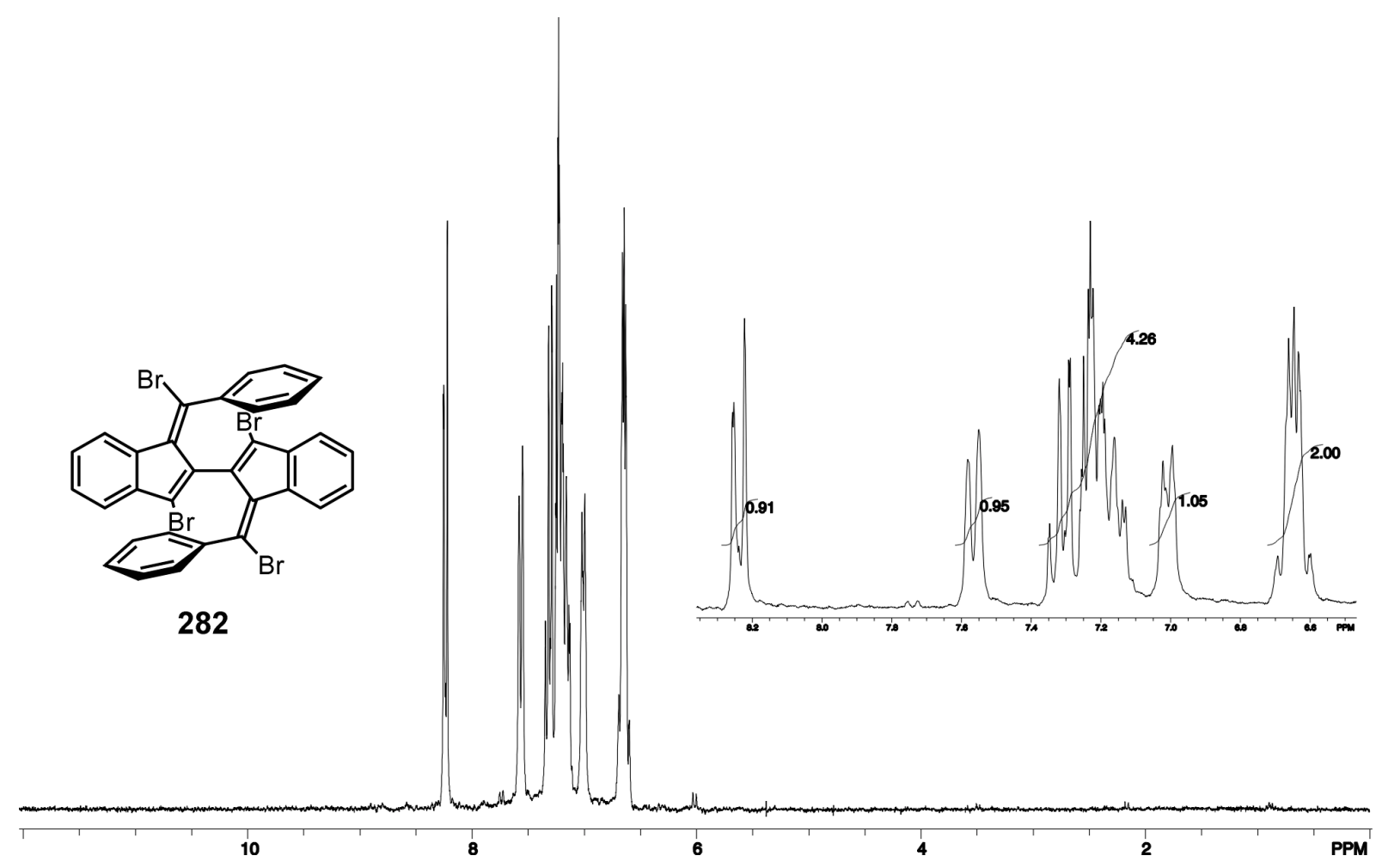

2,2'-Bis[(1-bromphenylmethylen)-3-brom- $1 H$-inden] 282

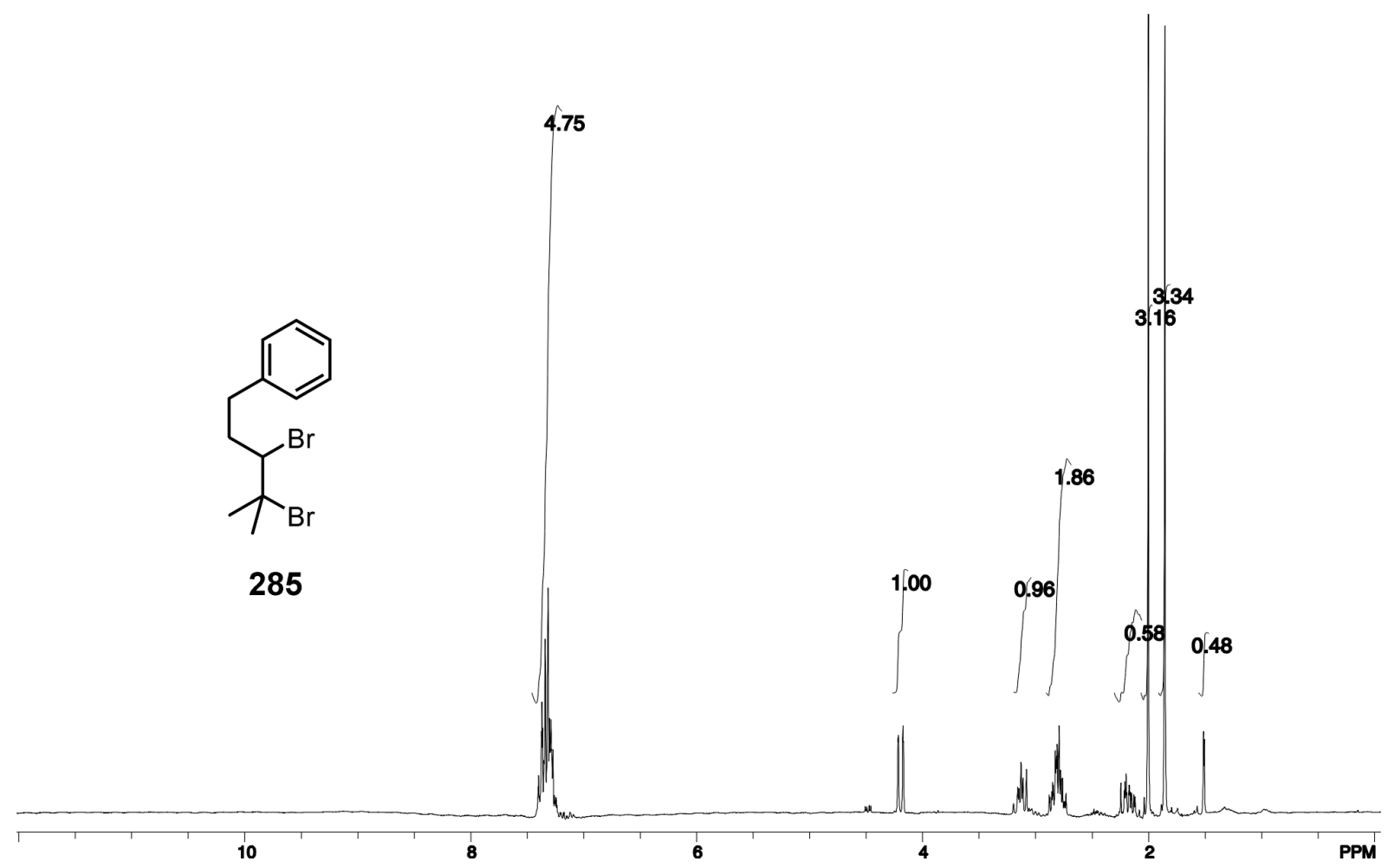

2,3-Dibrom-2-methyl-5-phenylpentan 285 


\subsection{Abbildung der ${ }^{13} \mathrm{C}$-NMR Spektren}

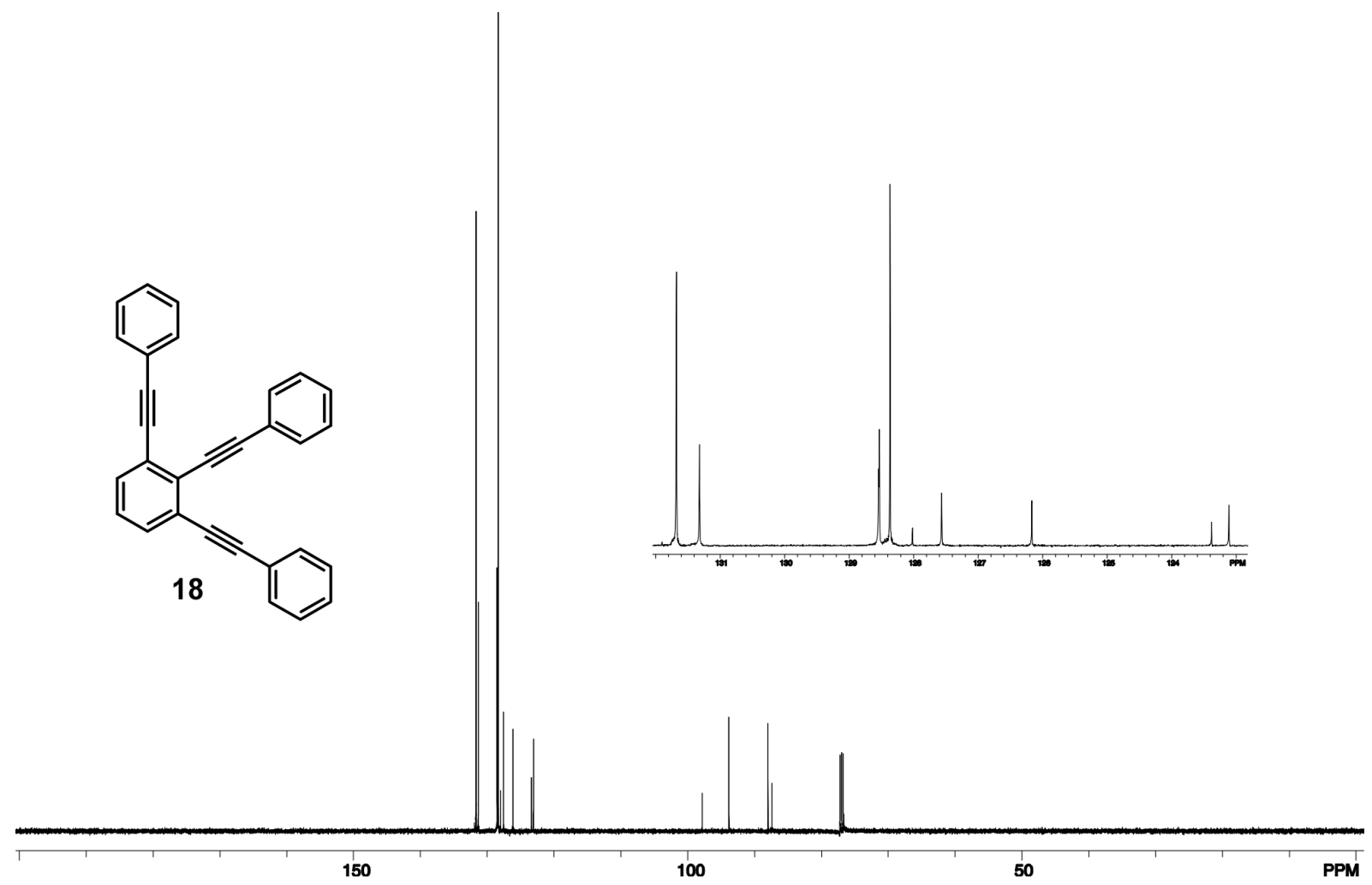

1,2,3-Tris(phenylethinyl)benzol 18

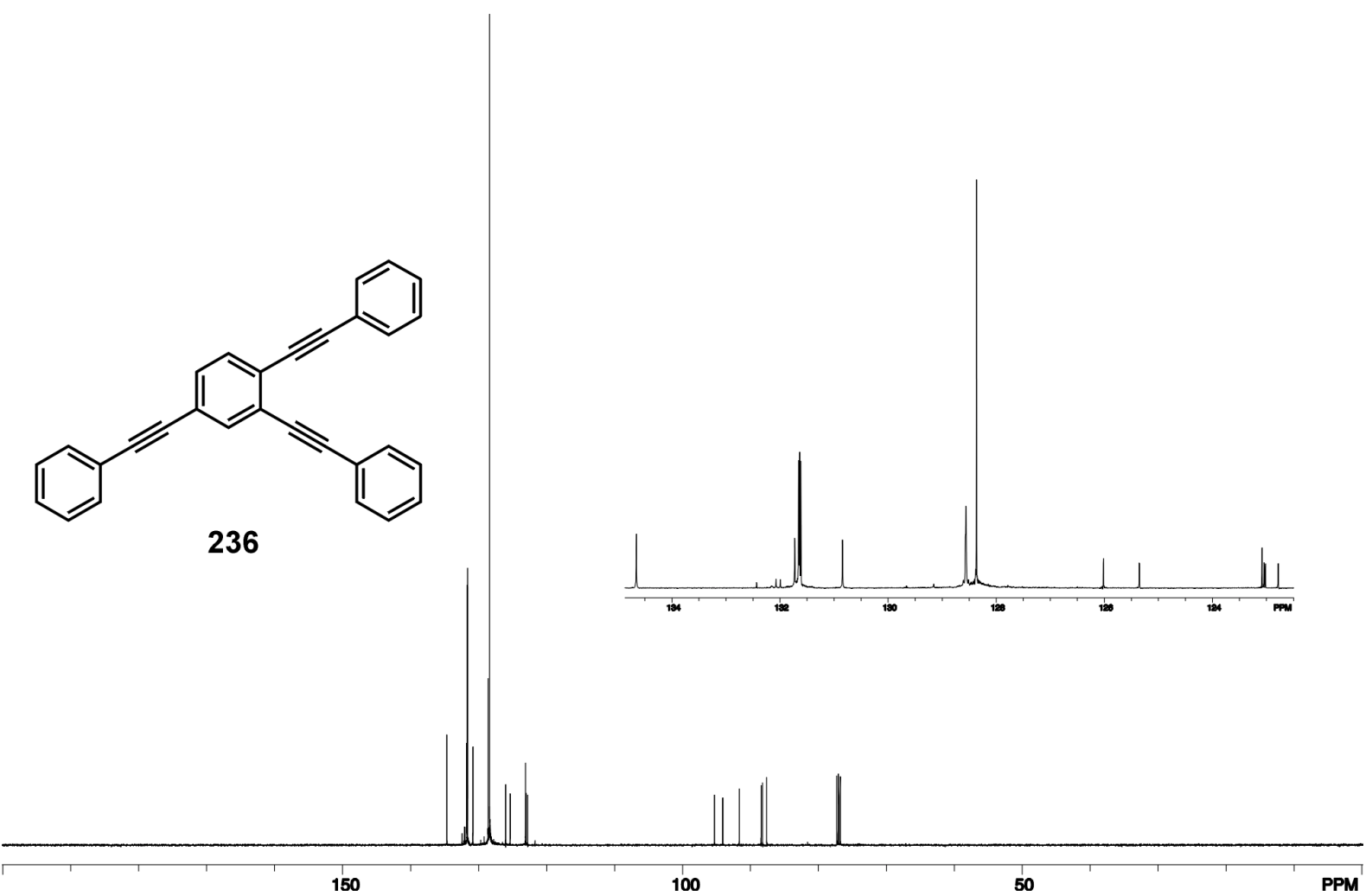

1,2,4-Tris(phenylethinyl)benzol 236 


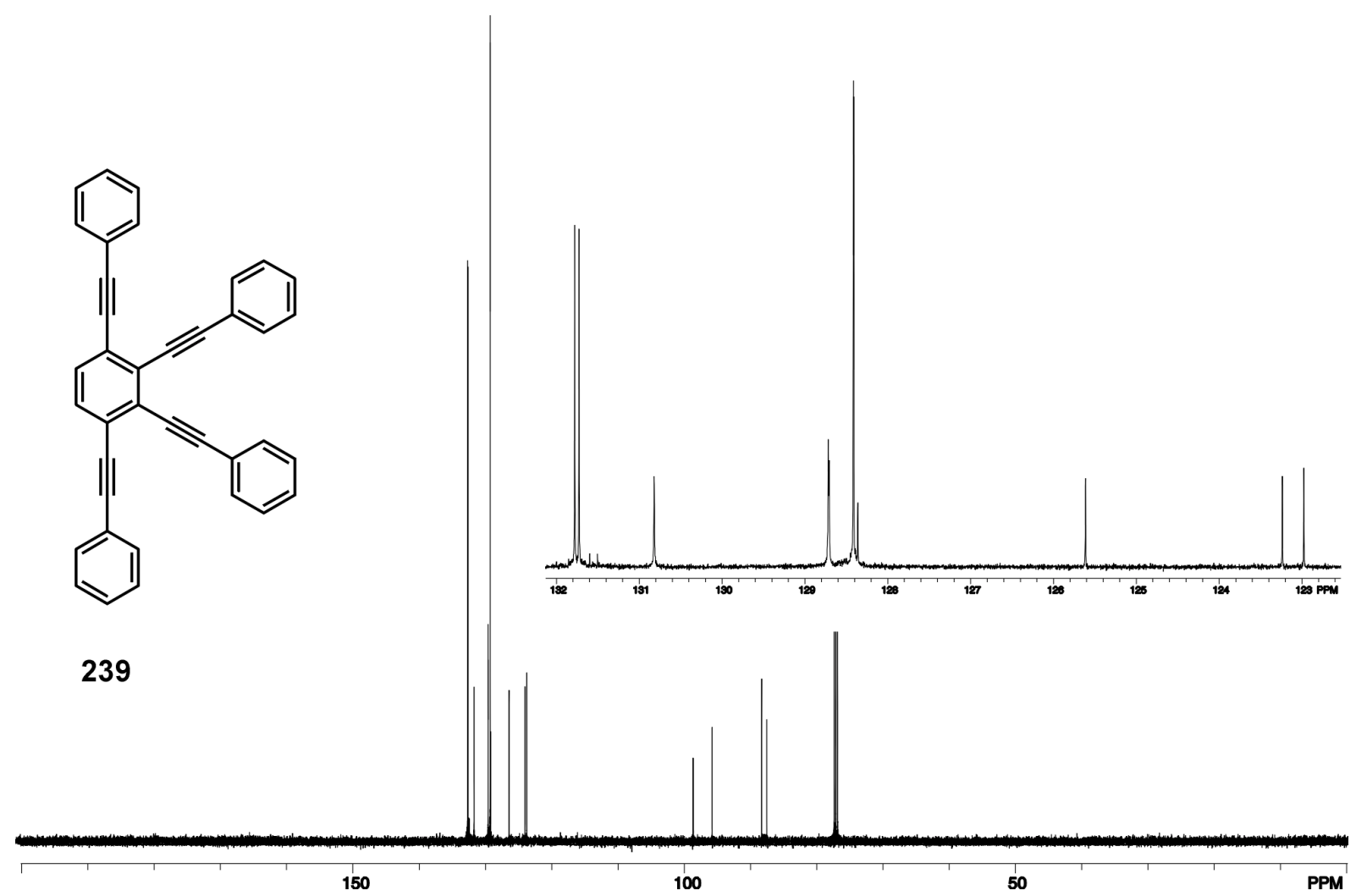

1,2,3,4-Tetrakis(phenylethinyl)benzol 239

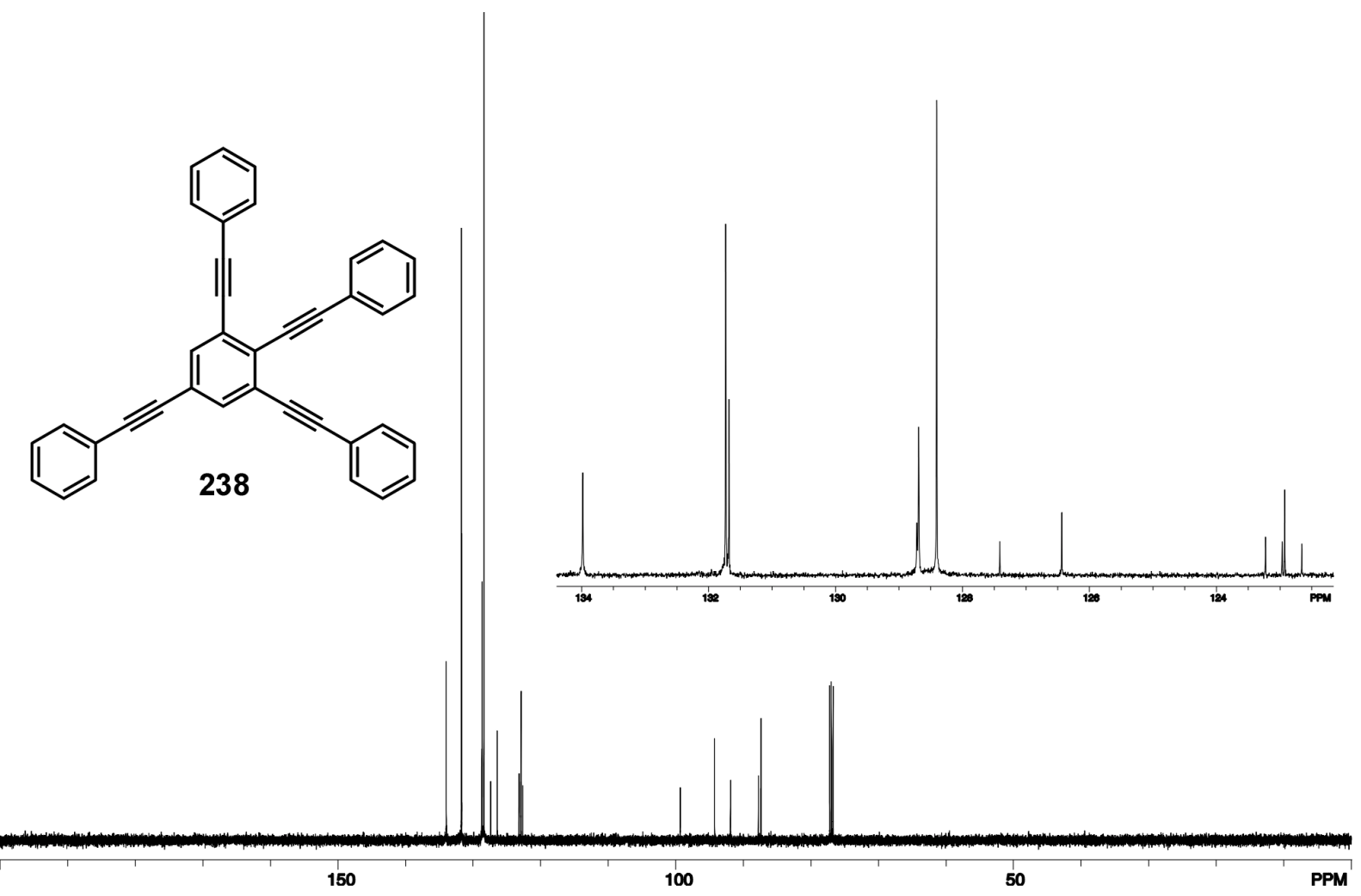

1,2,3,5-Tetrakis(phenylethinyl)benzol 238 


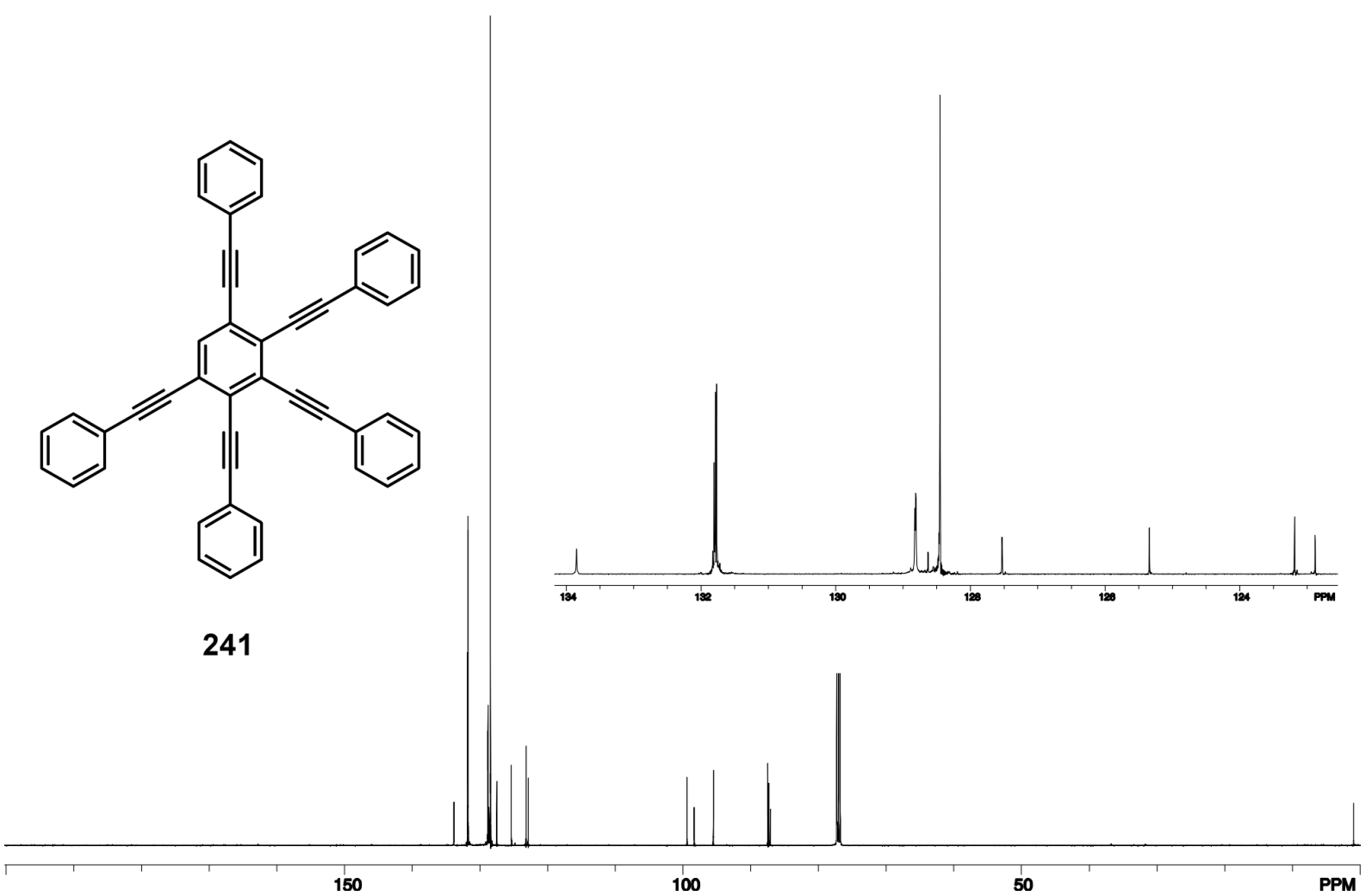

1,2,3,4,5-Pentakis(phenylethinyl)benzol 241

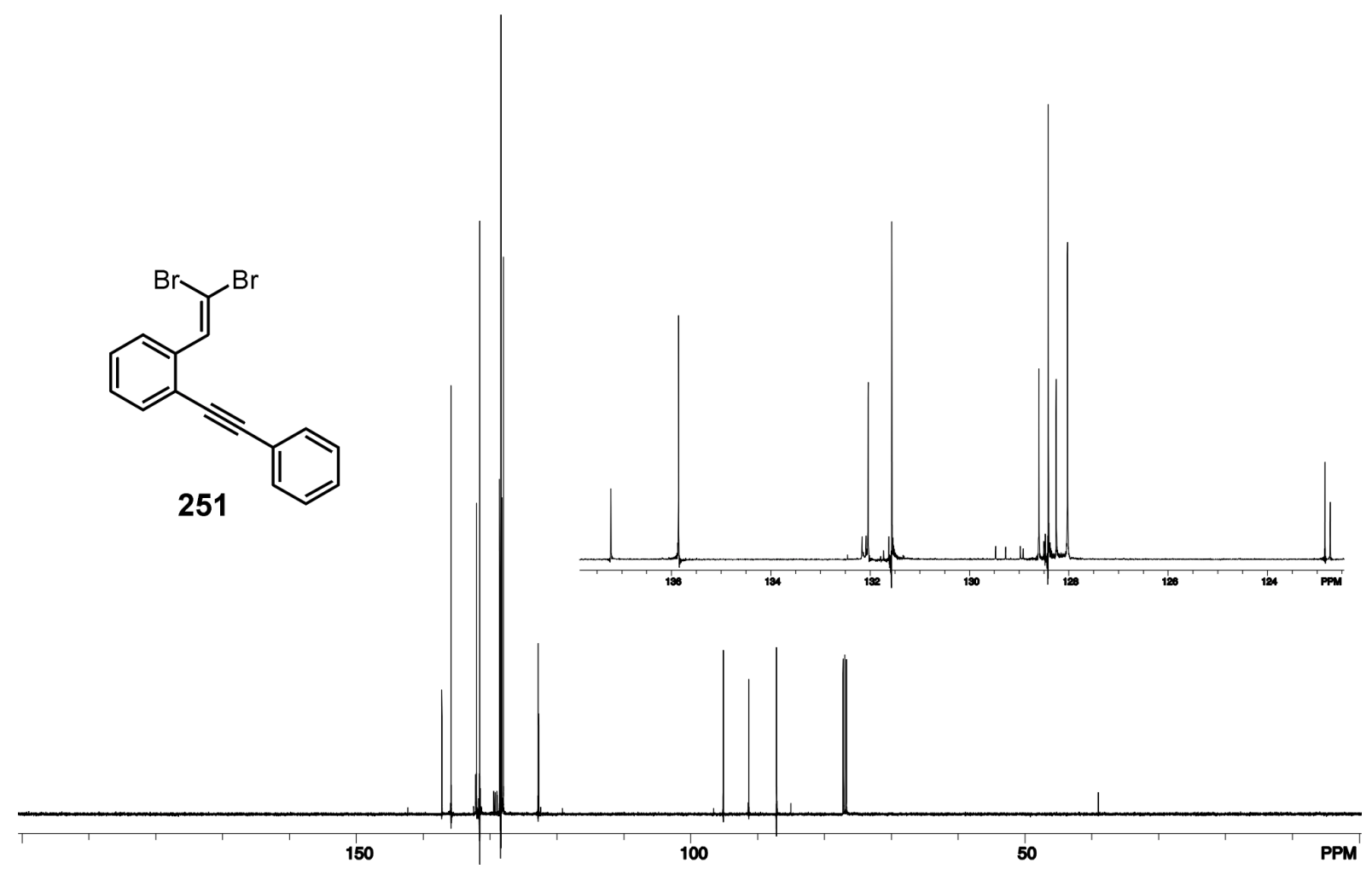

2-(2',2'-Dibromvinyl)diphenylacetylen 251 


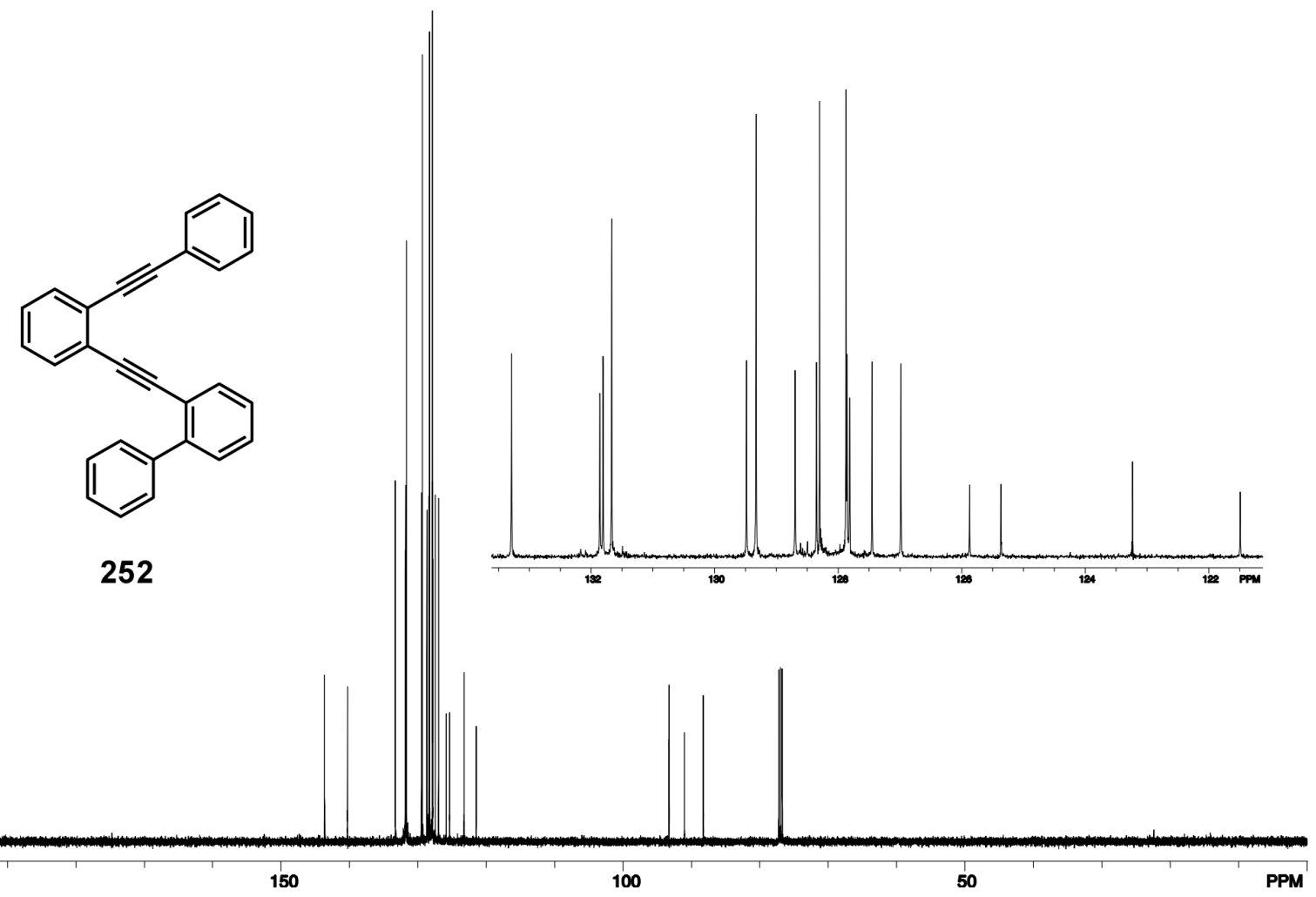

1-(2'-Biphenylethinyl)-2-(phenylethinyl)benzol 252

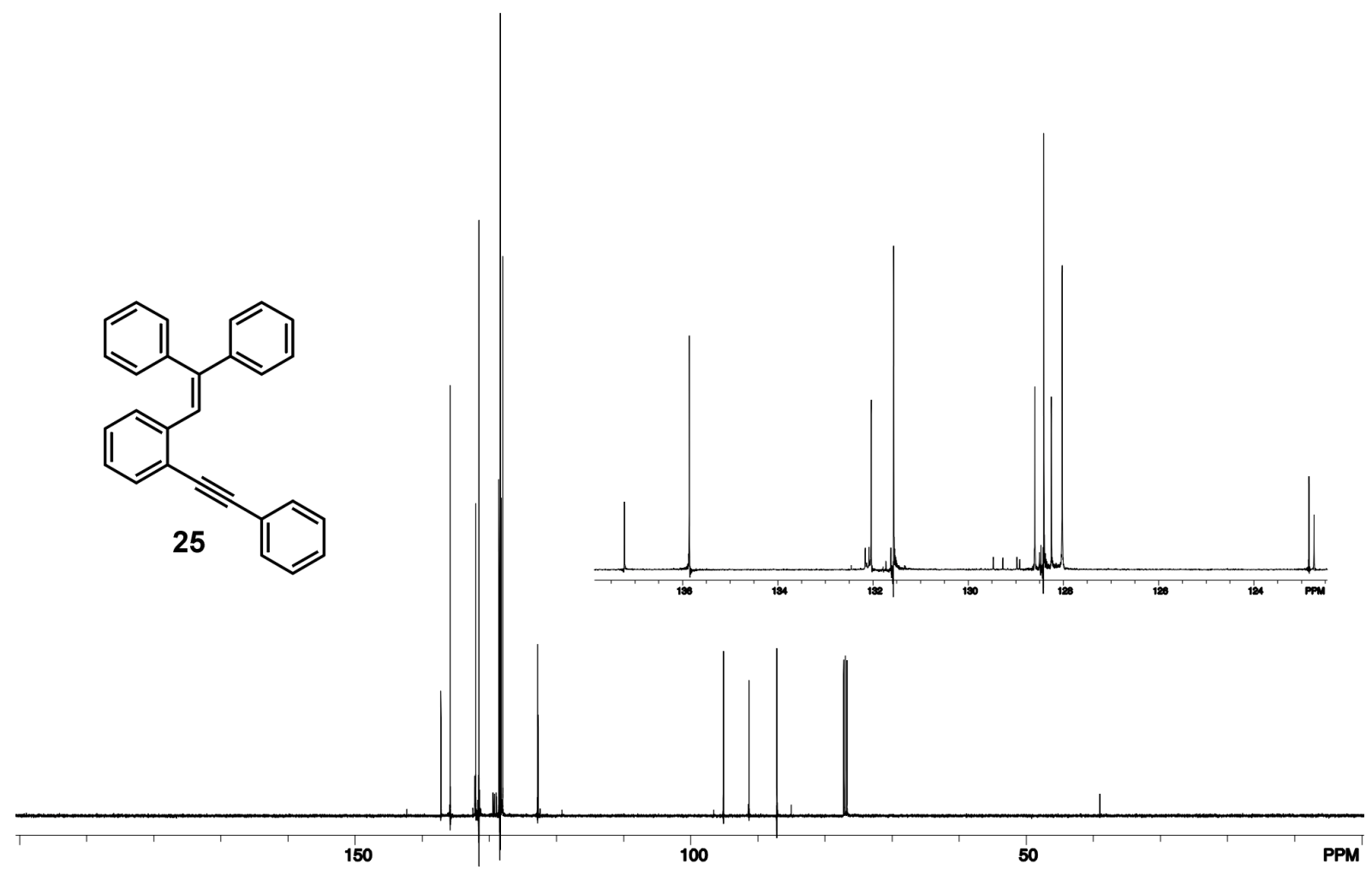

2-(2',2'-Diphenylvinyl)diphenylacetylen $\mathbf{2 5}$ 


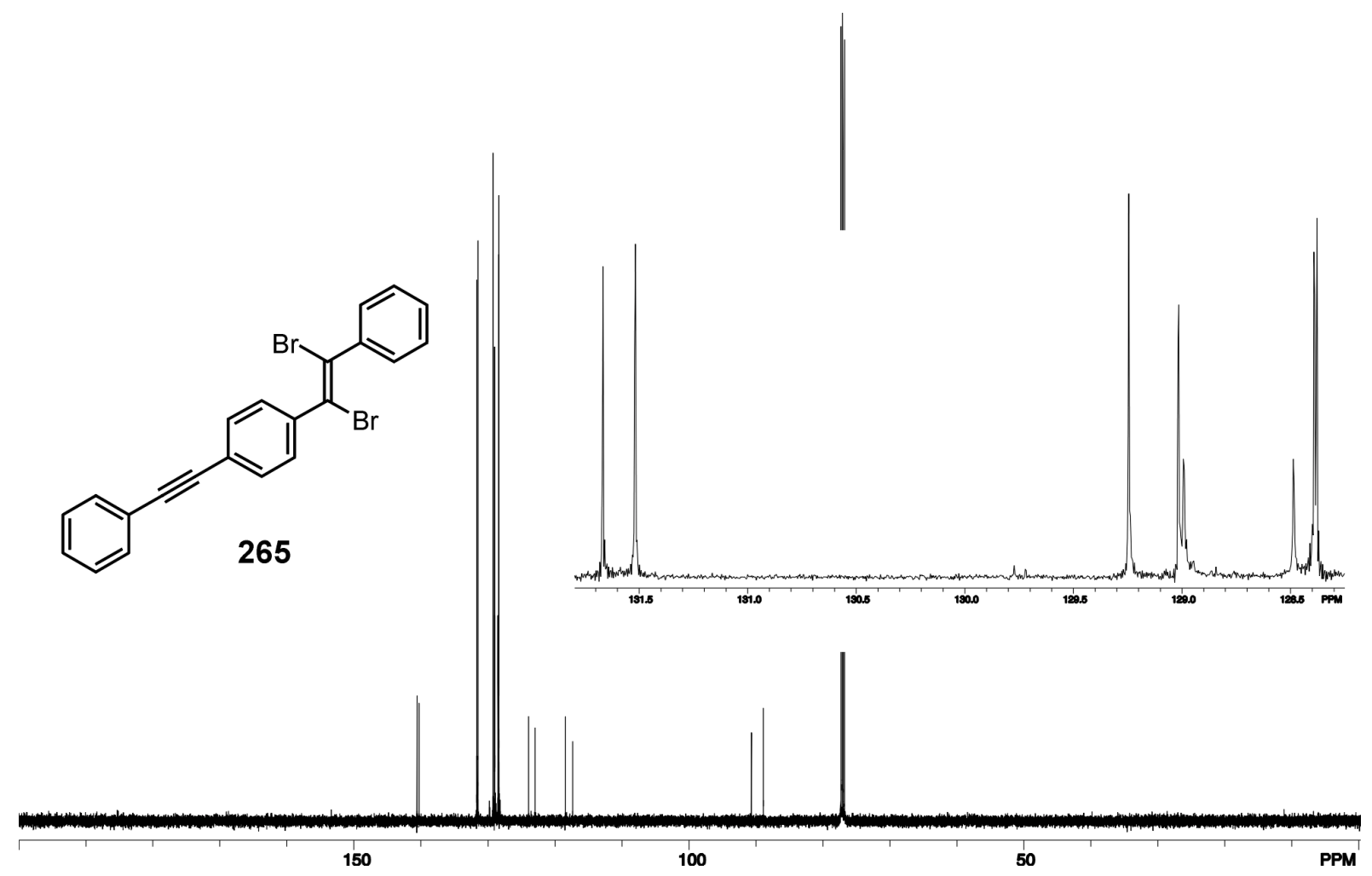

4-(Phenylethinyl)dibromstilben 265
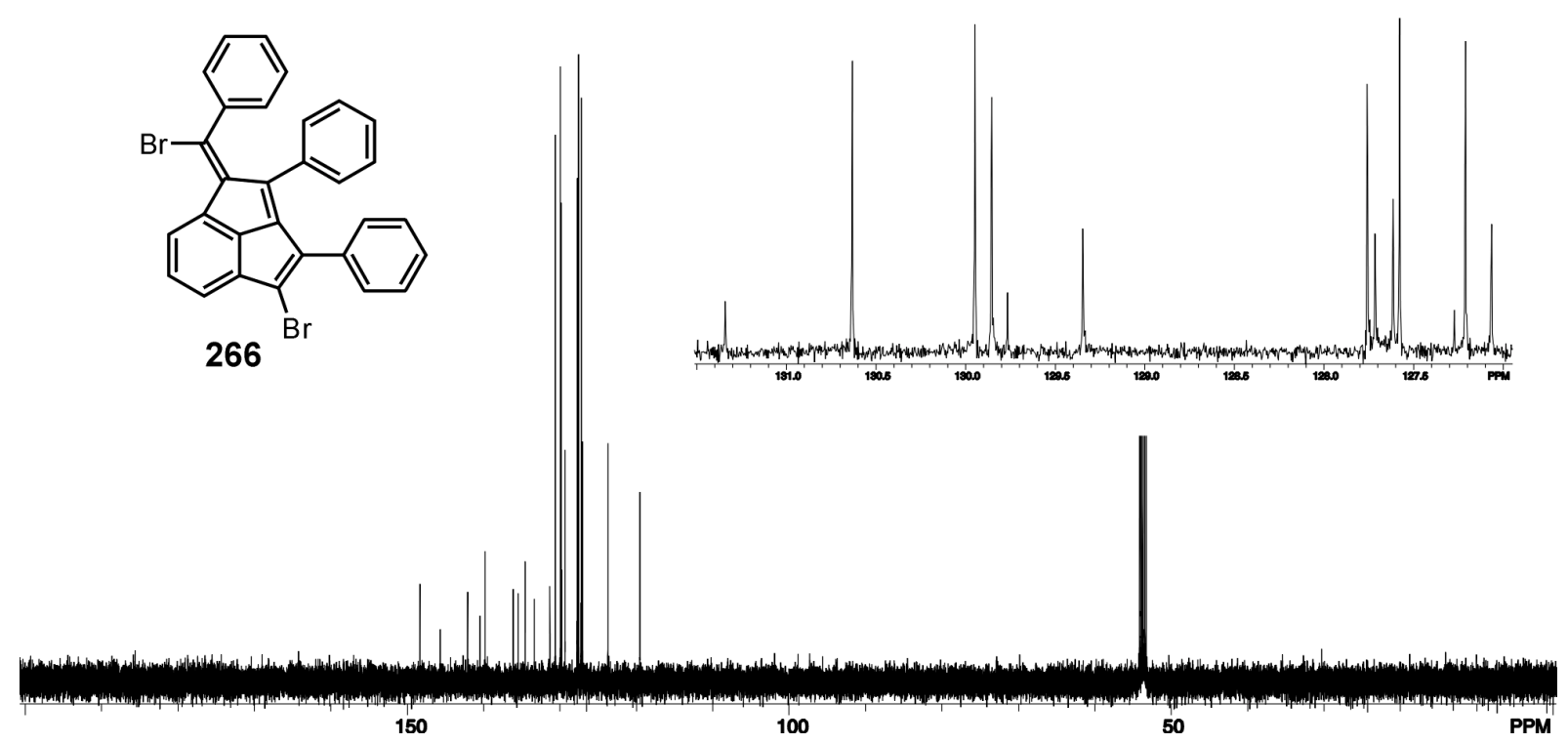

1-(Bromphenylmethylen)-2,3-diphenyl-4-brom-1H-cyclopenta[ $c d]$ indene 266 


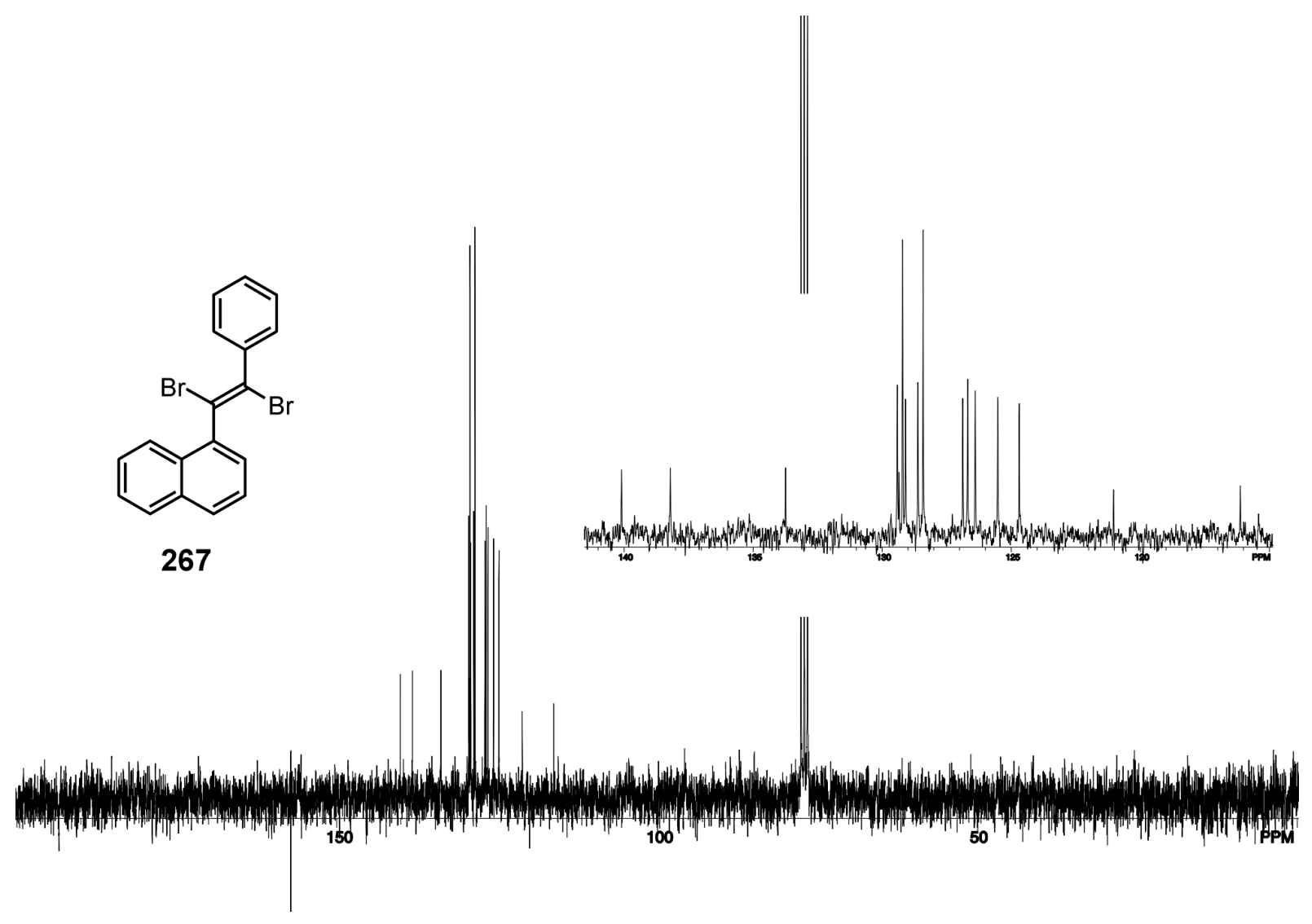

1-(1,2-Dibrom-2-phenylvinyl)-naphthalin 267

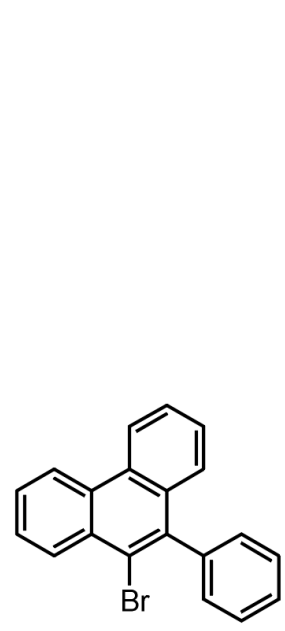

271
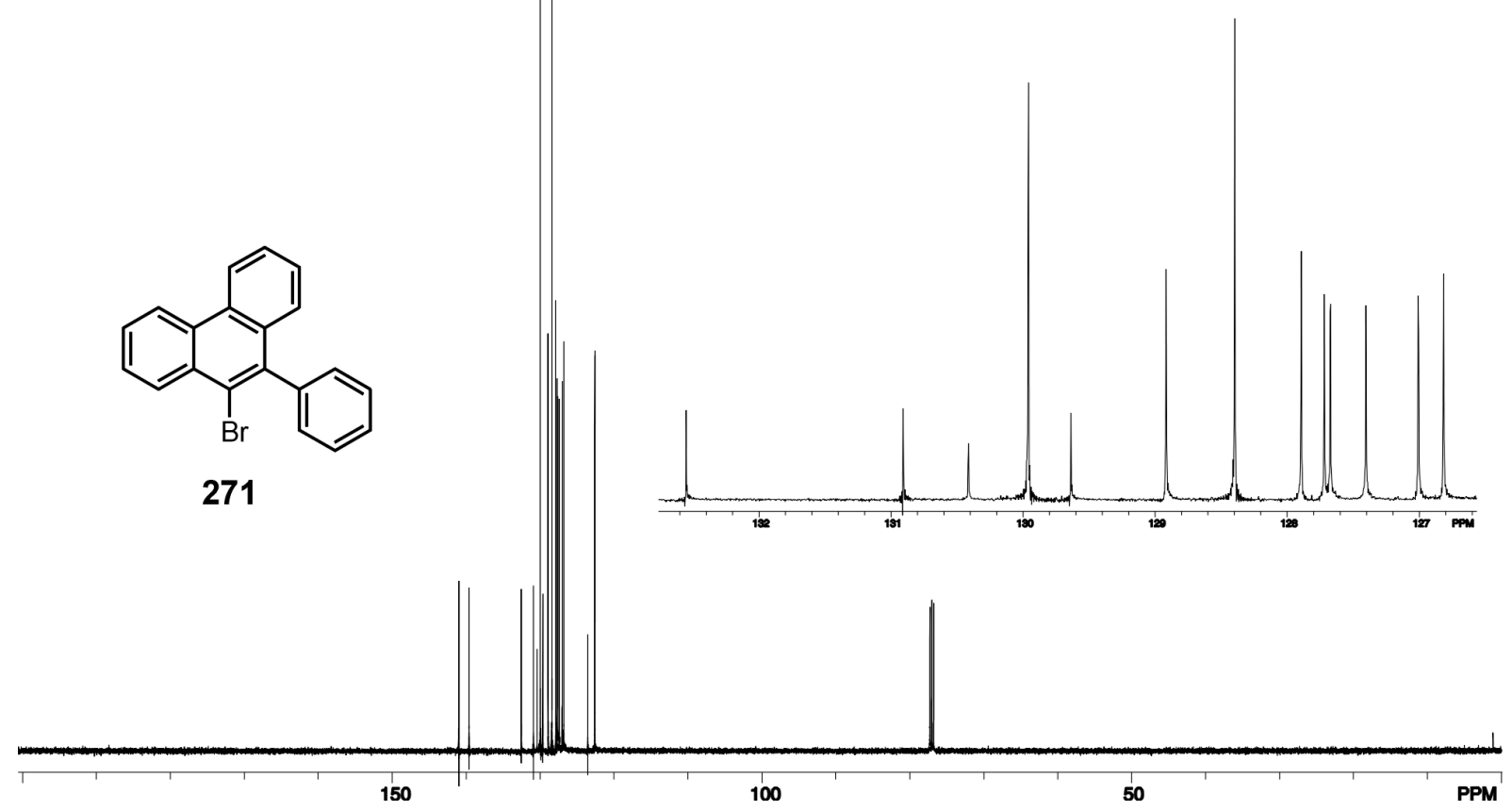

9-Brom-10-phenylphenanthren 271 


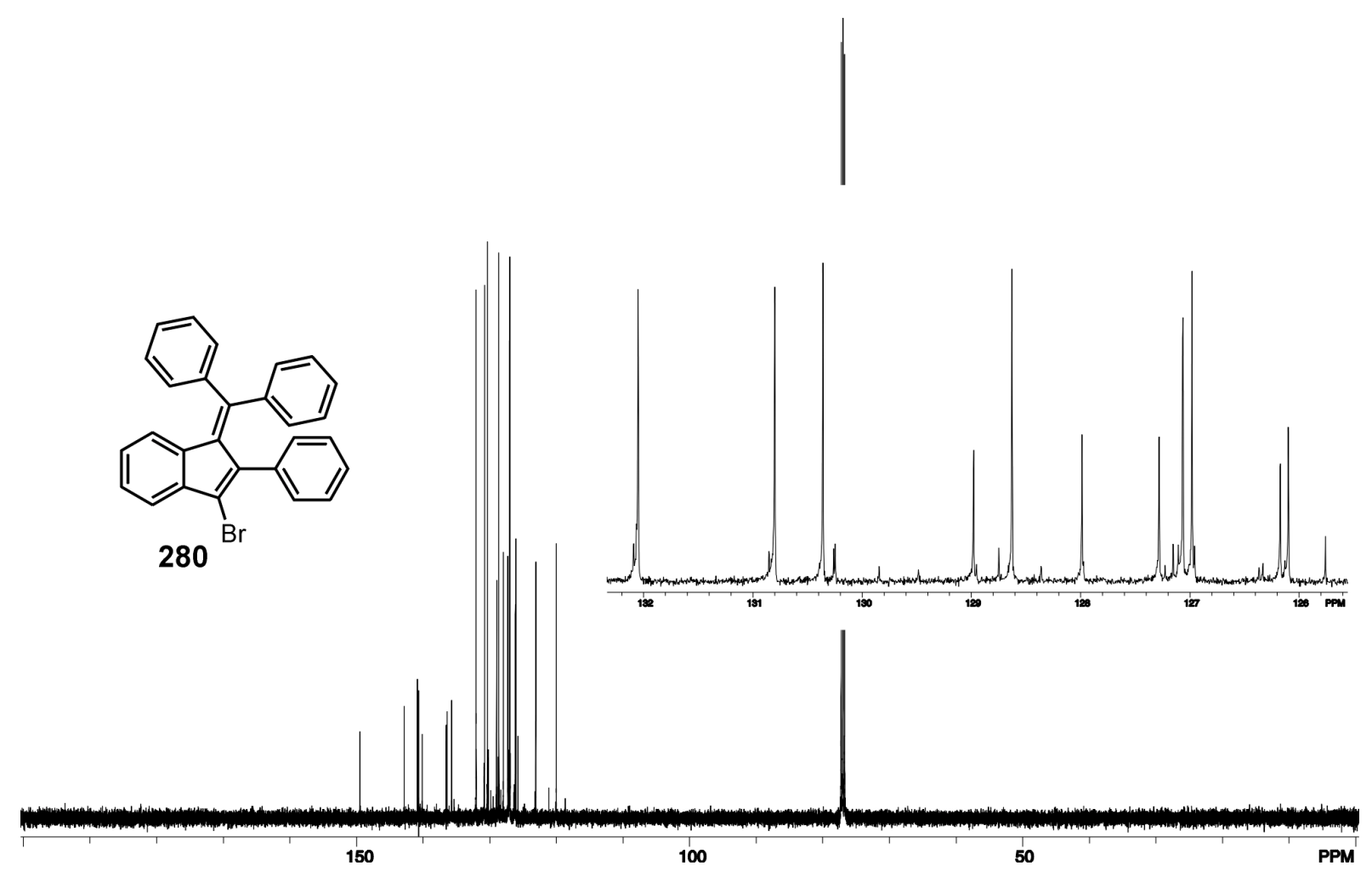

1-(Diphenylmethylen)-2phenyl-3-brom-1 $H$-inden 280

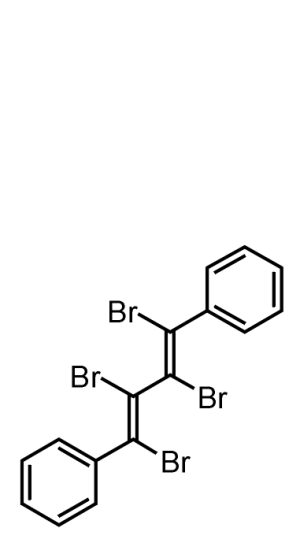

281

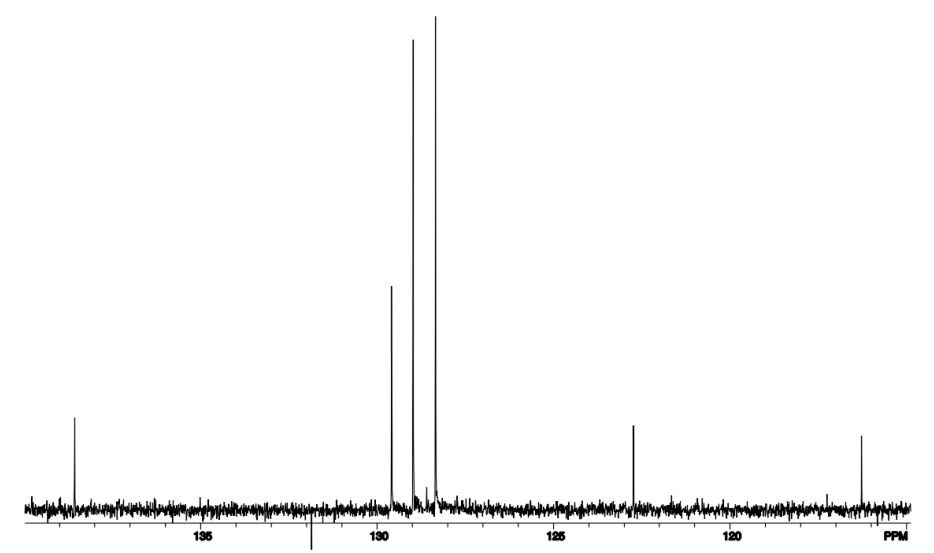

1,2,3,4-Tetrabrom-1,4-diphenylbuta-1,3-dien 281 


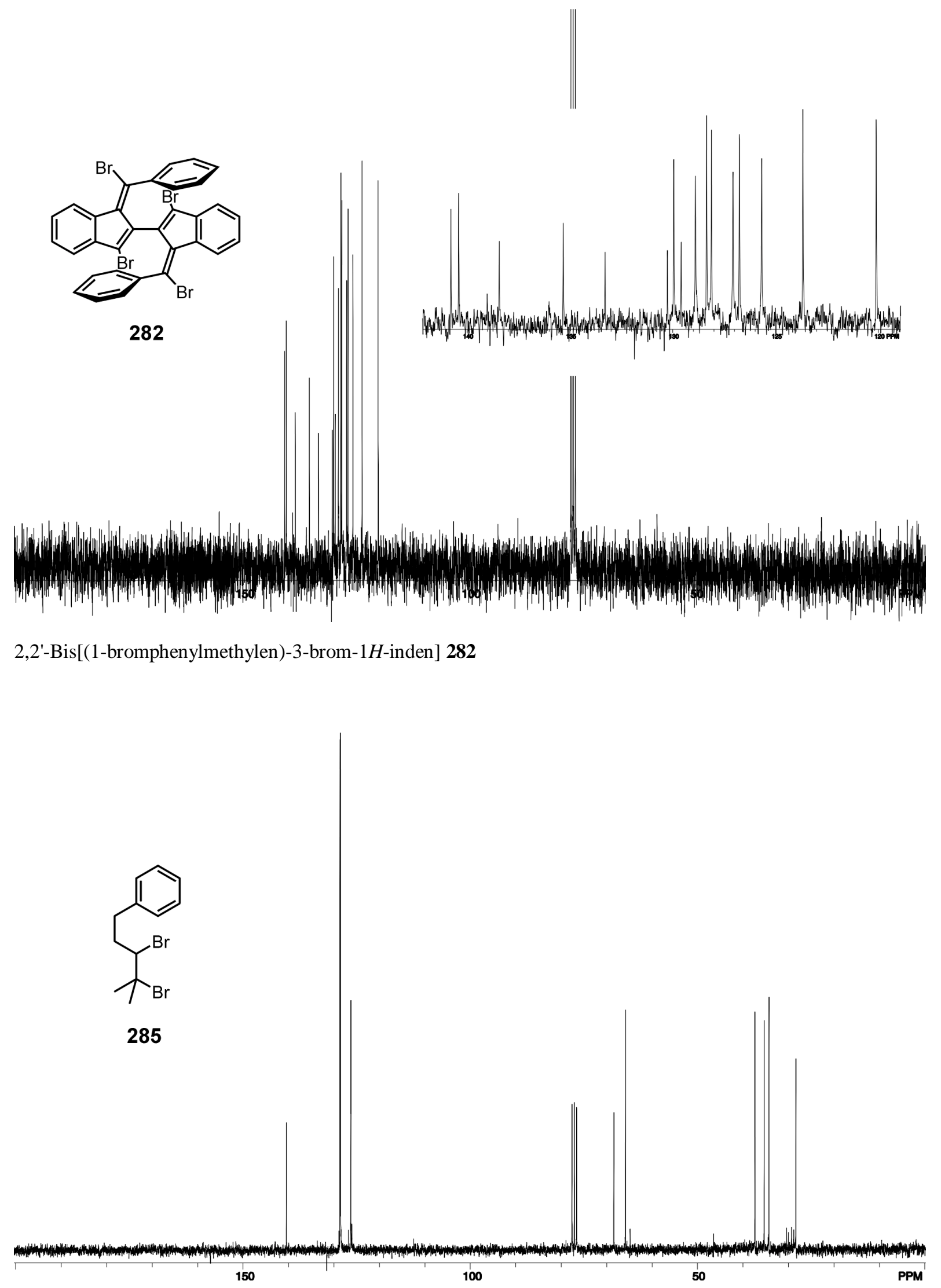

2,3-Dibrom-2-methyl-5-phenylpentan 285 


\subsection{Molekülverzeichnis}

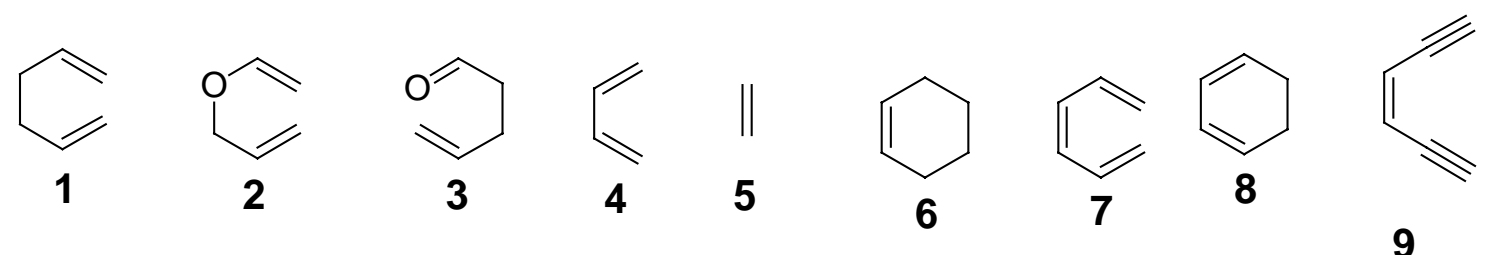<smiles>[C+]1=CC=CC=C1</smiles><smiles>C#C/C=C\C(=C)/C=C\C=C1C=C[C](C)C=C1</smiles>

10 11

12

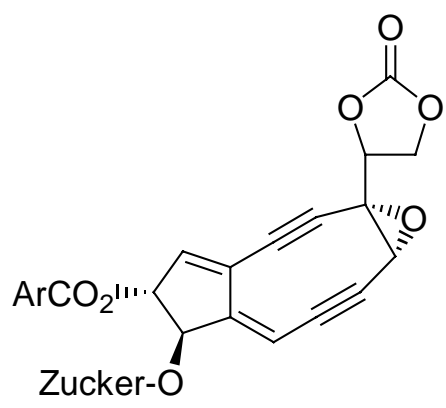

15
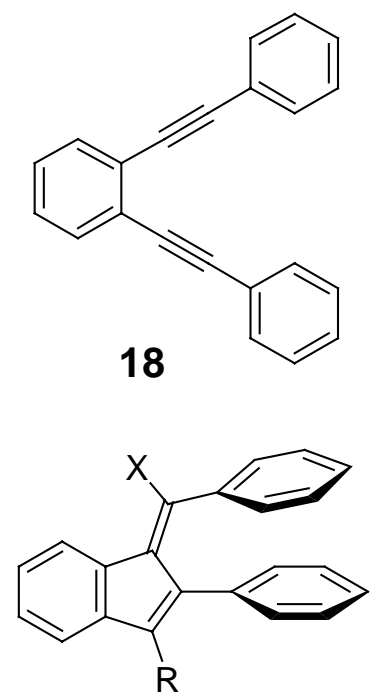

(Z)-21

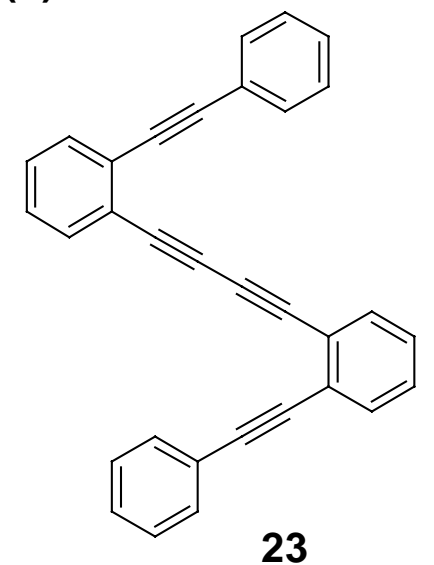

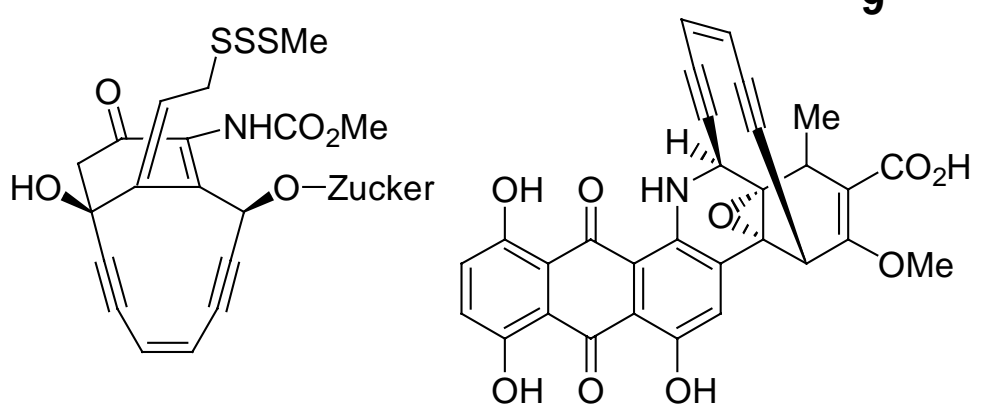

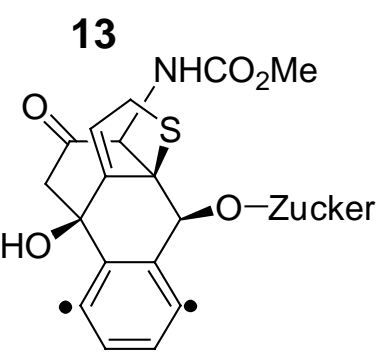

16
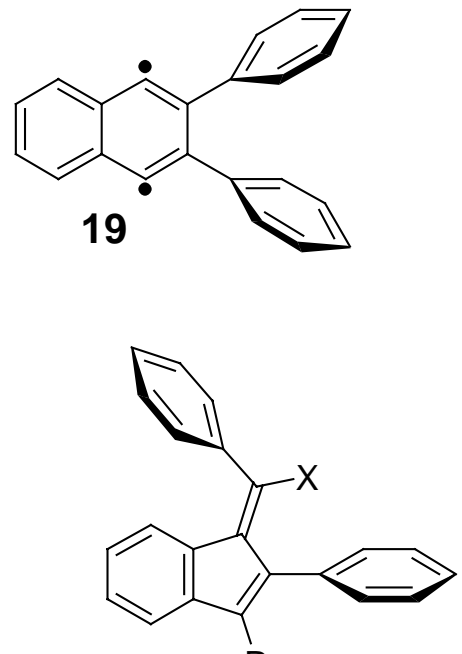

(E)-21

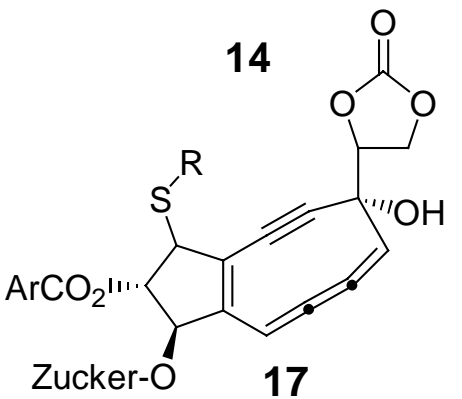

Zucker-O 17
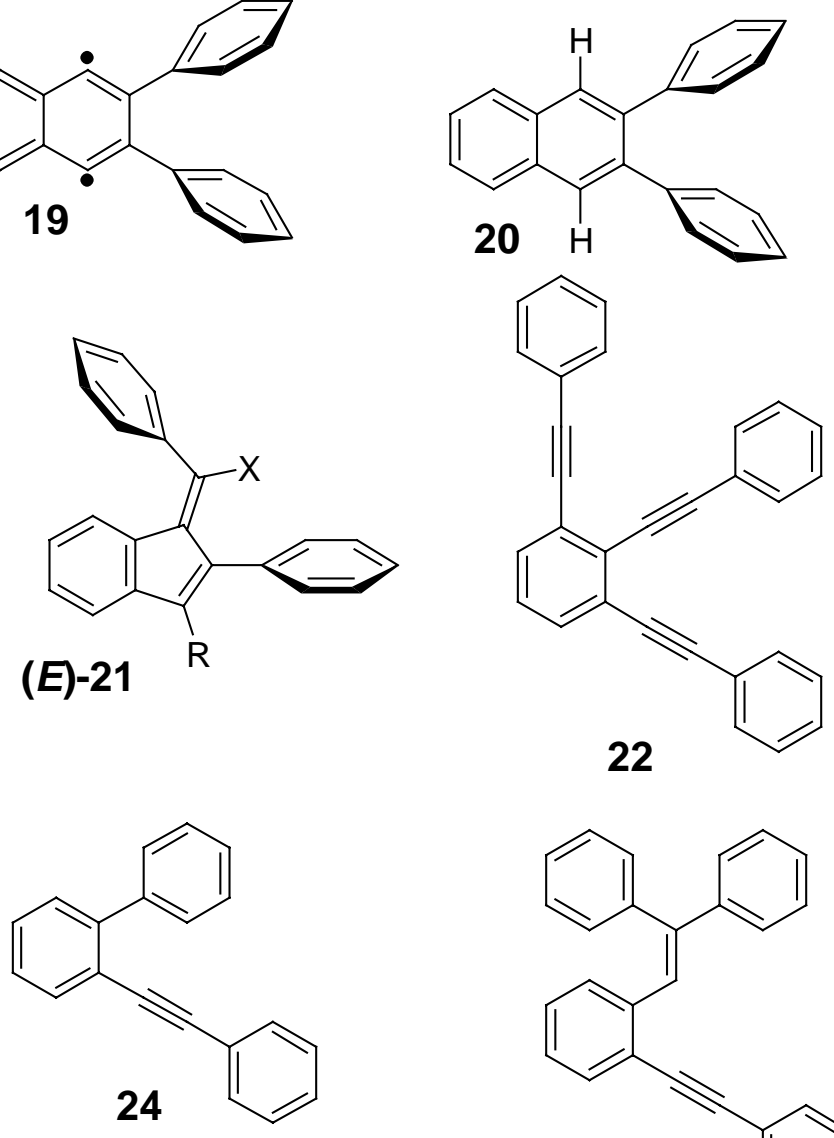

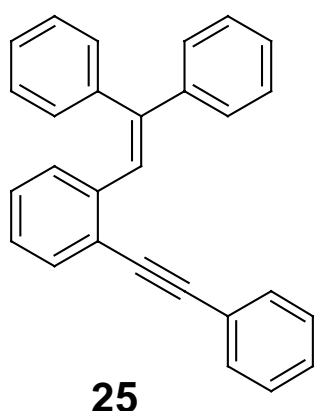




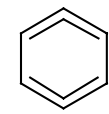

26

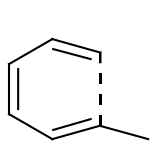

33<smiles>C#CC(=C)C(=C)C=C</smiles>

41<smiles>C=CC(=C)CC=C1CCCC1</smiles><smiles>C#CC(=C)CC=C</smiles><smiles>C#CCCC=C</smiles>

48
34

42

49

27<smiles>C1=CCCC=C1</smiles>

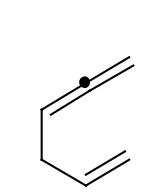

55<smiles>C1CCCCC1</smiles>

28

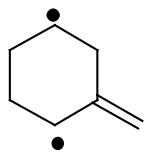

35<smiles>C1=CCCCC1</smiles>

29<smiles>c1ccccc1</smiles>

30<smiles>c1ccc2ccccc2c1</smiles>

31<smiles>Cc1cccc2ccccc12</smiles>

32<smiles>C=CCC(=C)C=C</smiles>

36<smiles>C=CC(=C)C(=C)C=C</smiles>

37<smiles>C#CCCC=C</smiles><smiles>C#CCC(=C)C=C</smiles>

38

39<smiles>C=CCC(=C)C=C</smiles>

43<smiles>C=CC(=C)C(=C)C=C</smiles>

44<smiles>C#CCCC#C</smiles>

$45 \quad 46$

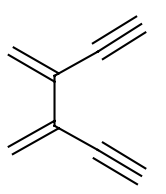

47<smiles>C#CCC(=C)C=C=C</smiles>

50<smiles>C#CC(=C)C(=C)C=C</smiles>

51<smiles>C=C=CC=CCCC(=C)C=CC=C</smiles>

52

53<smiles>C=C=CC(=C)C(=C)C=C</smiles>

54<smiles>C=CC(=C)C=C</smiles>

56<smiles>C=CCC=CC</smiles>

57

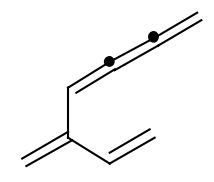

58<smiles>C#CCC=C</smiles>

59

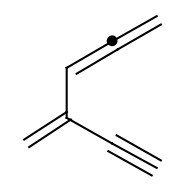

60<smiles>C#CCC=C=C</smiles>

61

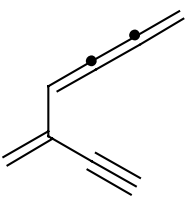

62

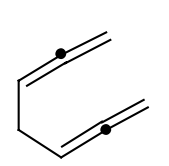

63

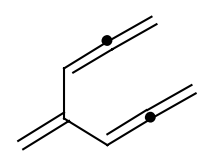

64

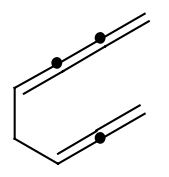

65

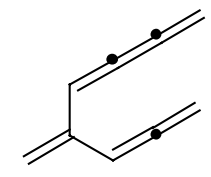

66

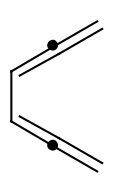

67

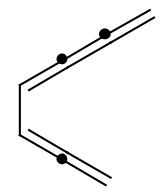

68
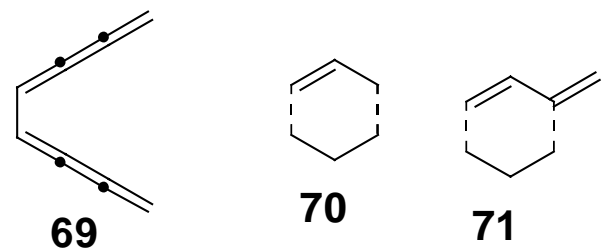<smiles>C=C1C[C](C)CC(=C)C1</smiles>

72<smiles>C1=CCCC=C1</smiles>

73<smiles>C=C1[C]CCC=C1</smiles>

74<smiles>C=C1C=CC=CC1</smiles>

75<smiles>C=C1[CH]CC(=C)C=C1</smiles>

82

76<smiles>C=C1C[CH]C(=C)C(=C)C1</smiles>

83<smiles>C=C1C=CCC(=C)C1C</smiles>

77<smiles>C=C1[CH]CC=CC1=C</smiles>

84<smiles>C1=CCC=CC1</smiles>

78<smiles>C=C1C=CCC=C1</smiles>

79<smiles>C=C1CCCCC1=C</smiles>

80<smiles>C=c1ccccc1=C</smiles>

85

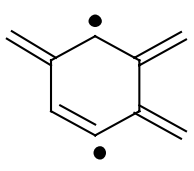

86

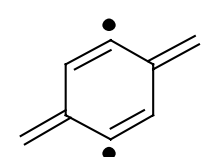

87<smiles>C=C1[CH]CC(=C)CC1</smiles>

81

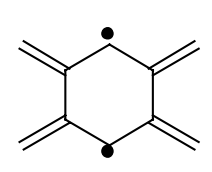

88 
<smiles>C#C/C=C\C=C</smiles>

89<smiles>C1=CC=CCC=1</smiles>

90<smiles>CC1C=CCCC1</smiles>

91<smiles>c1ccccc1</smiles>

92<smiles>C=C1C=CC=CC1</smiles>

93<smiles>C1=CC=CC=CC=C1</smiles>

94<smiles>C=c1ccccc1=C</smiles>

95<smiles>c1ccccc1</smiles>

96<smiles>C=C1C=CC=C1</smiles>

97<smiles>C#Cc1ccccc1C#C</smiles>

98<smiles>C1#CCCC#CC1</smiles>

99a-h $\mathrm{n}=1-8$

100a-t: $\mathrm{R}_{1}=\mathrm{BH}_{2}, \mathrm{BF}_{2}, \mathrm{AlH}_{2}, \mathrm{CH}_{3}, \mathrm{Ph}, \mathrm{CN}, \mathrm{Ac}, \mathrm{CF}_{3}$,

$\mathrm{SiH}_{3}, \mathrm{NH}_{2}, \mathrm{NH}_{3}+, \mathrm{NO}_{2}, \mathrm{PH}_{2}, \mathrm{OH}, \mathrm{OH}_{2}+$,

$\mathrm{SH}, \mathrm{F}, \mathrm{Cl}, \mathrm{Br}$

$\mathrm{R}_{2}=\mathrm{H}$

100aa-tt: $R_{1}=R_{2}=$ Subst

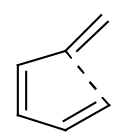

101

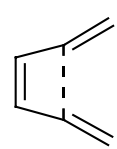

102

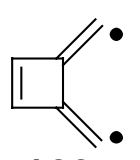

103<smiles>[2H]c1c(C)c(C)c(O)c2c1C#Cc1c(O)c(C)c(C)c([2H])c1C#C2</smiles>

104

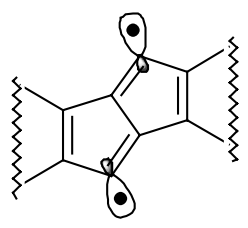

105<smiles>[R]c1c(C)c(C)c(O)c2c1-c1cc3c(C)c(C)c([R9])c(O)c3cc1-2</smiles>

106<smiles>C=C1C(=C)c2ccccc21</smiles>

107<smiles>C=C1C(=C)c2ccccc21</smiles>

108<smiles>C=C1C=Cc2ccccc21</smiles>

109<smiles></smiles>

110<smiles>[2H]C#C/C=C\C#C[2H]</smiles><smiles>C#CC1=C(C#C)C1CCCCCCc1ccccc1</smiles>

112a-h $n=1-8$

113a-h $n=1-8$<smiles>c1ccc2c(c1)CCCCCCCCCCC2</smiles>

114a-h $n=1-8$<smiles></smiles>

115a-h $n=1-8$

116a-t: $\mathrm{R}_{1}=\mathrm{BH}_{2}, \mathrm{BF}_{2}, \mathrm{AlH} \mathrm{H}_{2}, \mathrm{CH}_{3}, \mathrm{Ph}, \mathrm{CN}, \mathrm{Ac}, \mathrm{CF}_{3}$,

$\mathrm{SiH}_{3}, \mathrm{NH}_{2}, \mathrm{NH}_{3}+, \mathrm{NO}_{2}, \mathrm{PH}_{2}, \mathrm{OH}, \mathrm{OH}_{2}+$ $\mathrm{SH}, \mathrm{F}, \mathrm{Cl}, \mathrm{Br}$

$$
\mathrm{R}_{2}=\mathrm{H}
$$

116aa-tt: $R_{1}=R_{2}=$ Subst

117a-t: $\mathrm{R}_{1}=\mathrm{BH}_{2}, \mathrm{BF}_{2}, \mathrm{AlH}_{2}, \mathrm{CH}_{3}, \mathrm{Ph}, \mathrm{CN}, \mathrm{Ac}, \mathrm{CF}_{3}$,

$\mathrm{SiH}_{3}, \mathrm{NH}_{2}, \mathrm{NH}_{3}, \mathrm{NO}_{2}, \mathrm{PH}_{2}, \mathrm{OH}, \mathrm{OH}_{2}+$ $\mathrm{SH}, \mathrm{F}, \mathrm{Cl}, \mathrm{Br}$

$$
\mathrm{R}_{2}=\mathrm{H}
$$

117aa-tt: $R_{1}=R_{2}=$ Subst 
R

118aa-tt

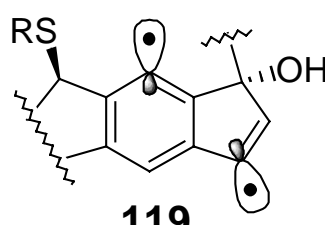

119

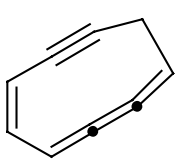

120<smiles>[R]C(C)=C1C=CC=C1C=O</smiles>

121

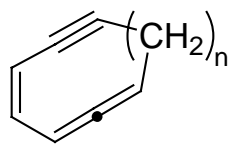<smiles>c1ccc2c(c1)CCC2</smiles>
124a-f $n=1-6 \quad$ 125a-f $n=1-6$<smiles>C1=CC2=CC3CCC2C3=C1</smiles>
123a-f $n=1-6$<smiles>C1=CC=CCC=C1</smiles>

126<smiles>C#Cc1ccccc1C=C</smiles>

122<smiles>C=C([18OH])C1C=C[C+]C=C1</smiles>

128<smiles>C=CC1C=CC1=C</smiles>

129<smiles>c1ccccc1</smiles>
130<smiles>C=C1C=CC=CC1</smiles>

131<smiles>C=C1C=CC=C1C</smiles>

139<smiles>C=C1C=CC=CC1</smiles>

127<smiles>Cc1ccc2ccccc2c1</smiles>

137<smiles></smiles>

138

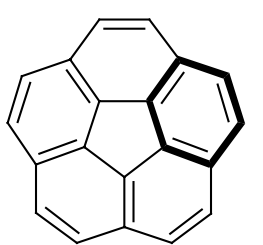

143

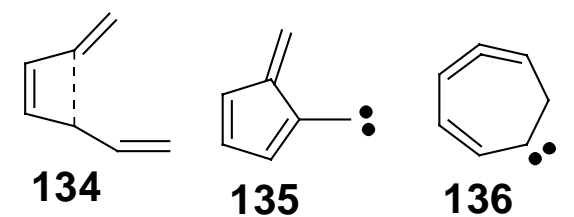<smiles>c1ccccc1</smiles>

144

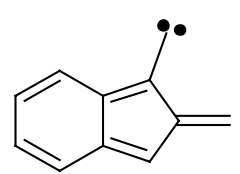

140<smiles>CC1=Cc2ccccc2C=CC1</smiles>

141
142

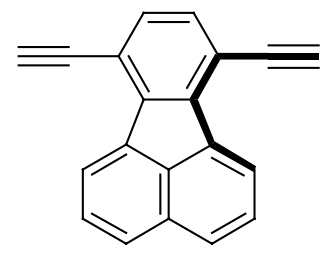

149

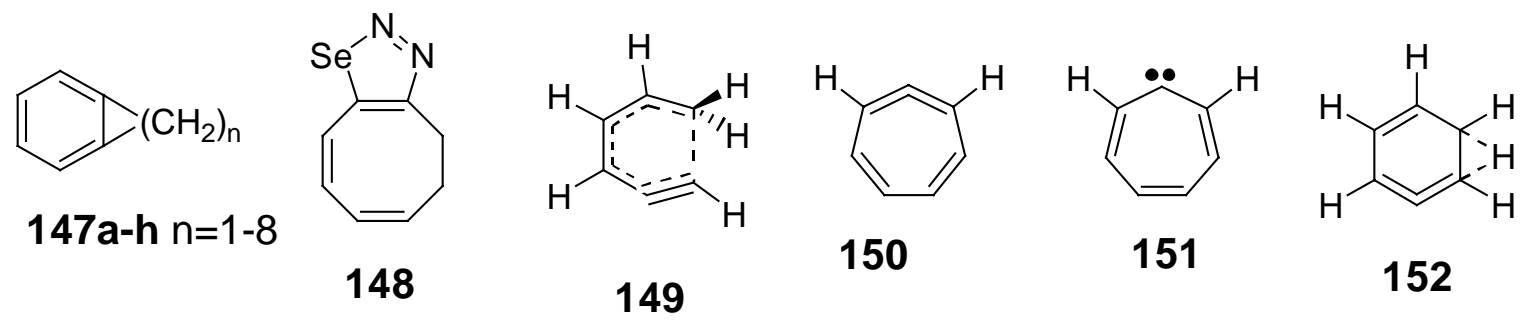

150

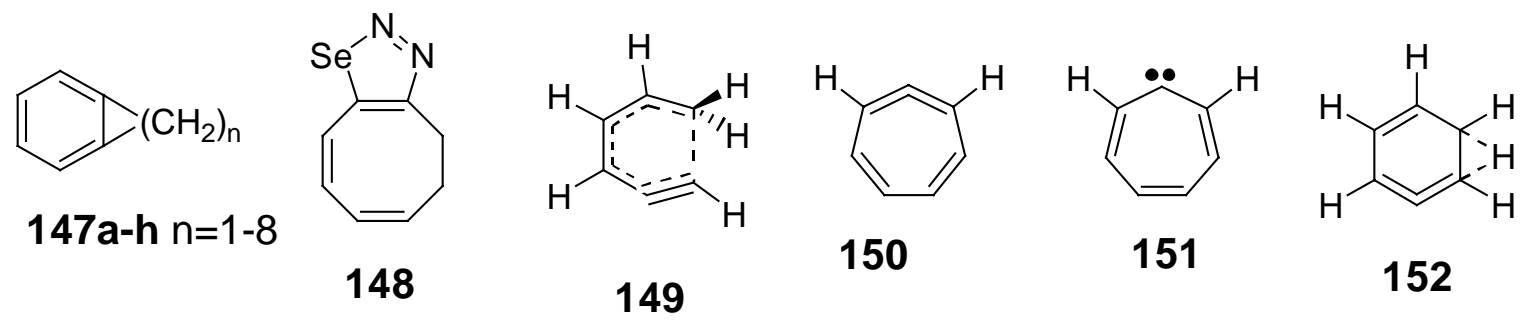<smiles>c1ccccc1</smiles>

145<smiles>c1ccccc1</smiles>

146a-h $n=1-8$<smiles>c1ccccc1</smiles>

153<smiles>c1ccc[cH+]cc1</smiles>

154<smiles>c1ccccc1</smiles>

155<smiles>C1=CC=CC=CC=C1</smiles>

156<smiles>C1=CC=CCC=C1</smiles>

157<smiles>C1=CC=CCC=C1</smiles>

159<smiles>C1=CCC=CC1</smiles>

160<smiles>C#Cc1ccccc1C=C</smiles>

161<smiles>C1=CCC=c2ccccc2=C1</smiles>

162<smiles>CC1=CC[C]c2ccccc21</smiles>

163<smiles>c1ccc2ccccc2c1</smiles>

164 


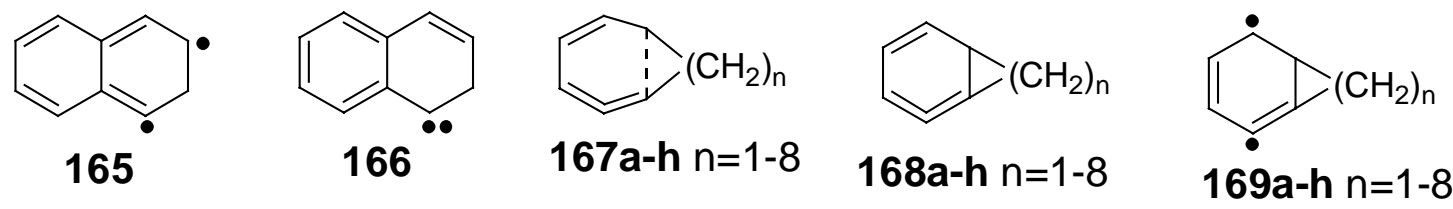

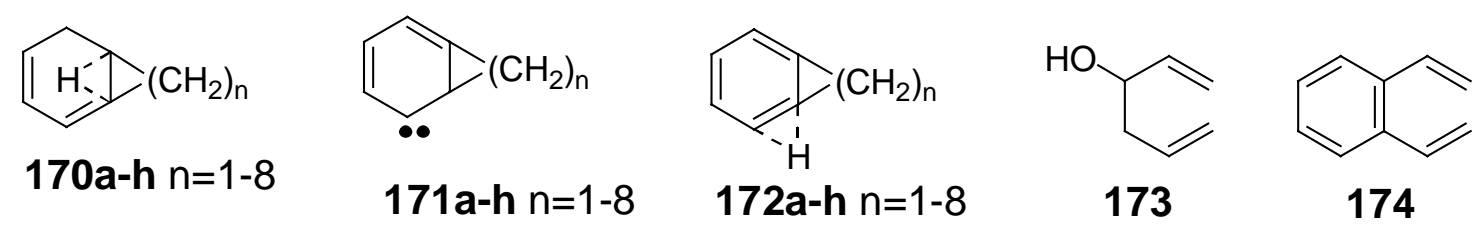<smiles>C1=c2ccccc2=CCC1</smiles>

175

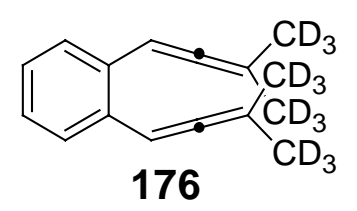

176

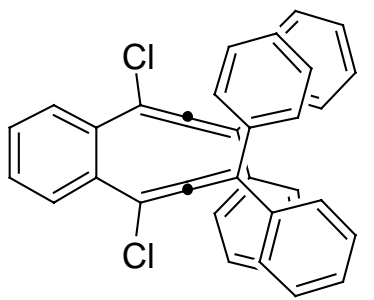

179<smiles>CC(C)=c1ccccc1=C(C)C</smiles>

181<smiles>C=C(C)c1ccccc1C(C)C</smiles><smiles>CC1(C)c2ccccc2C1(C)C</smiles>

183

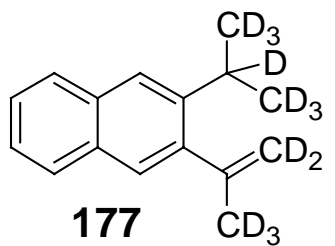<smiles>CC=CC#CC(O)(c1ccccc1)c1ccccc1</smiles><smiles>c1ccc2cc3c(cc2c1)CC3</smiles>

186<smiles>C=C/C=C\C1CC1(Br)Br</smiles>

187<smiles>C1=CC=CCC=C1</smiles>

188<smiles>C=C=Cc1ccccc1C=C</smiles>

184<smiles>C=c1cc2ccccc2cc1=C</smiles>

185<smiles></smiles>

193<smiles></smiles>
$194 \mathrm{Br}$<smiles>O=C(O)c1ccccc1</smiles><smiles>C=Cc1ccccc1C=CC=Cc1ccccc1[Hg]</smiles>

190
191<smiles>C(#Cc1ccccc1)c1ccccc1</smiles>

196<smiles>BrC1=C(c2ccccc2)C1c1ccccc1</smiles>

197<smiles>O=S(=O)([Sn])c1ccccc1-c1c(Br)c(-c2ccccc2)c(Br)c2ccccc12</smiles><smiles>BrC(=C(Br)c1ccccc1)c1ccccc1</smiles>

(E)-199 
<smiles>BrC(Br)=C(Br)c1ccccc1</smiles>

(Z)-199

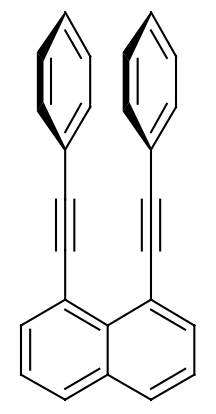

203<smiles>O=C(O)c1ccccc1C(Br)=C(Br)c1ccccc1C#Cc1ccccc1</smiles><smiles></smiles><smiles>O=[R20]C1=C(Br)C(=C=Cc2ccccc2)c2ccccc21</smiles><smiles>CC#Cc1cccc2cccc(C#CC)c12</smiles>

207<smiles></smiles>

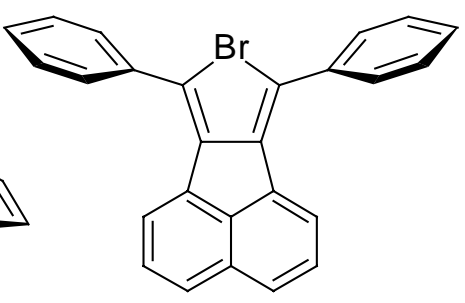

205

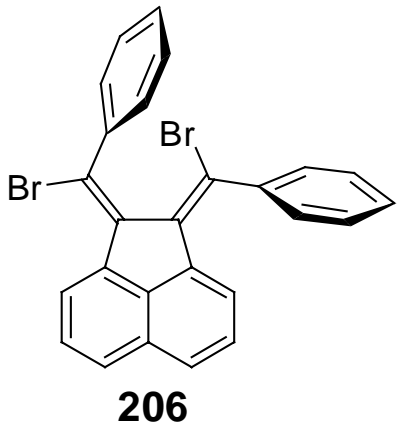<smiles>[R12]Cc1ccccc1C1=C(C2=C(c3ccccc3)C(Br)(Br)c3ccccc32)c2ccccc2C1(Br)Br</smiles><smiles>[Z15]CI1C2=C(I)c3ccccc3C2=c2c(I)c3c(c(I)c21)=C(I)c1ccccc1-3</smiles><smiles>IC1=C(c2ccccc2)c2ccccc2CC1</smiles>

219<smiles>CC(Br)=C(Br)c1cccc2cccc(C(Br)=C(C)Br)c12</smiles>

208
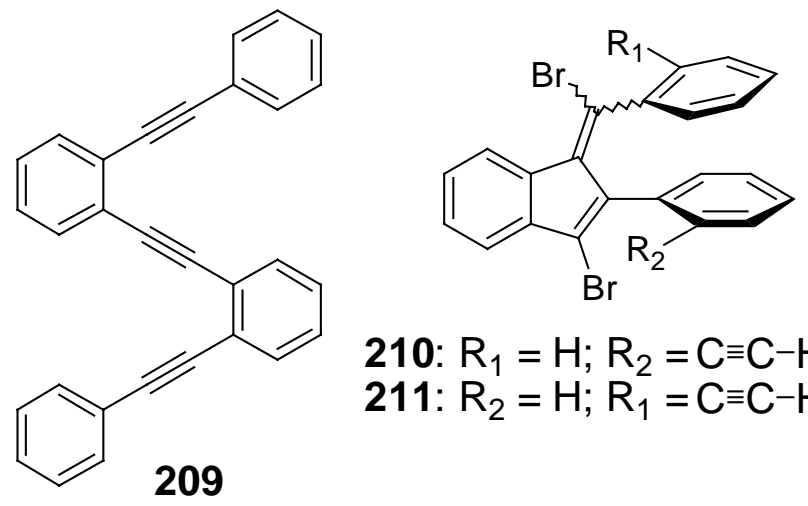

210: $R_{1}=H ; R_{2}=C \equiv C-H$

211: $R_{2}=H ; R_{1}=C \equiv C-H$

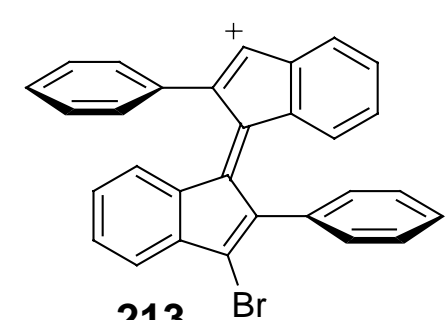

213<smiles>C#Cc1ccccc1C#CC#Cc1ccccc1C#CC#Cc1ccccc1</smiles>

214<smiles>[Z60]Cc1c2c(=O)c3ccccc3c2c(I)c2c(=O)c3ccccc3c12</smiles><smiles>C(#Cc1ccccc1)CCc1ccccc1</smiles><smiles>[123I]</smiles><smiles>IC1CCc2ccccc2[C@H]1c1ccccc1</smiles>

220
$\left(\mathrm{PPh}_{3}\right)_{2} \mathrm{PdCl}_{2}$
$\mathbf{2 2 1}$
$\left(\mathrm{PPh}_{3}\right)_{2} \mathrm{Pd}^{0}$

222 
<smiles>[R]c1cc([R2])c(N)c(Br)c1</smiles>

226: $R_{1}=B r ; R_{2}=H \quad$ 229: $R_{1}=B r ; R_{2}=H$ 227: $R_{1}=H ; R_{2}=B r \quad$ 230: $R_{1}=H ; R_{2}=B r$ 228: $R_{1}=B r ; R_{2}=B r \quad$ 231: $R_{1}=B r ; R_{2}=B r$<smiles>Brc1cc(Br)c(Br)c(Br)c1Br</smiles>

234<smiles>C(=C/c1cccc(Cc2ccccc2)c1)\c1ccccc1</smiles>

234

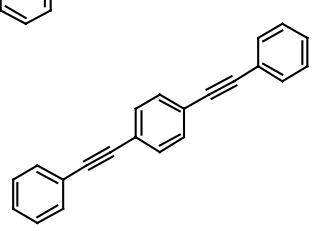

235<smiles>Brc1ccc(Br)c(Br)c1Br</smiles>

232<smiles>[R19]Br</smiles>

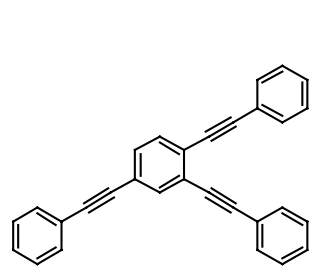

236<smiles>C(=Cc1ccccc1)C1=CC(=Cc2ccccc2)CC1=Cc1ccccc1</smiles>

237

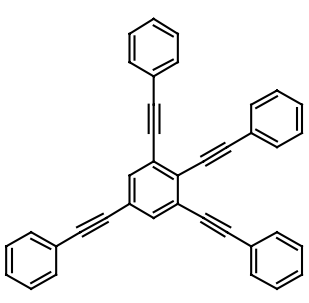

238<smiles>C(c1ccccc1)=c1c(=Cc2ccccc2)c(=Cc2ccccc2)c(=Cc2ccccc2)c(=Cc2ccccc2)c1=Cc1ccccc1</smiles>

242<smiles></smiles>

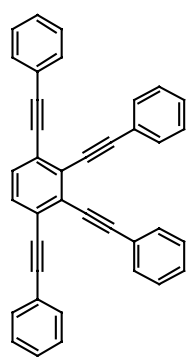

239<smiles>C=Cc1ccccc1C#Cc1ccccc1</smiles>

243

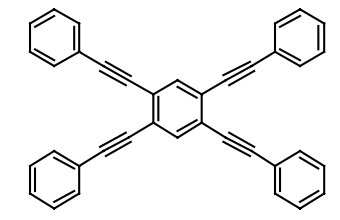

240<smiles>Brc1cccc2ccccc12</smiles>

244<smiles>C(=C1C(=Cc2ccccc2)C(=Cc2ccccc2)C(=Cc2ccccc2)C1=Cc1ccccc1)c1ccccc1</smiles>

$\mathrm{NH}_{2} \quad \mathrm{NH}_{2}$

241<smiles>Cc1cccc2cccc(C)c12</smiles>

246<smiles>Ic1cccc2cccc(I)c12</smiles>

247

245<smiles>BrC(Br)=Cc1ccccc1C#Cc1ccccc1</smiles><smiles>O=Cc1ccccc1Br</smiles><smiles>O=Cc1ccccc1C#Cc1ccccc1</smiles><smiles>C=CC(=C)C#CC#Cc1ccccc1C#Cc1ccccc1</smiles><smiles>CC(C)(C)OC(C)(C)O</smiles>

254

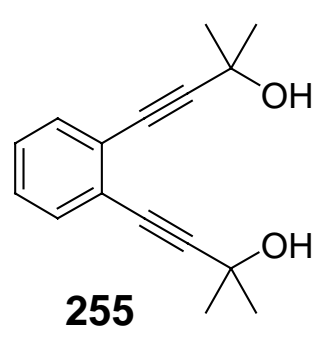



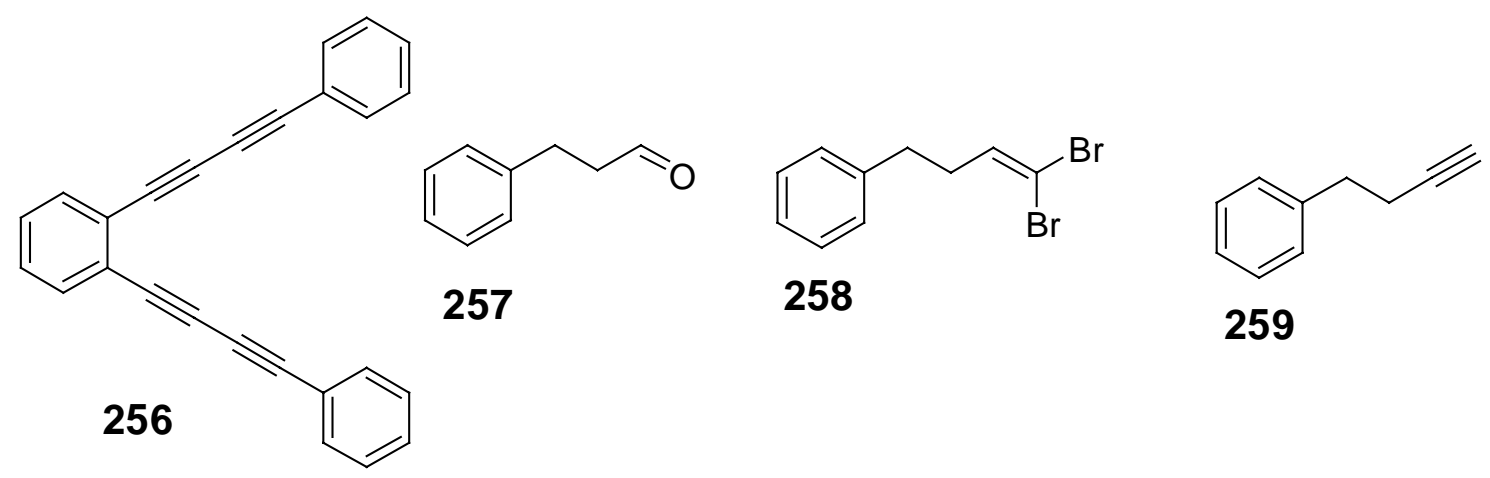<smiles>O=[N+]([O-])C#Cc1ccccc1/C=C/c1ccccc1</smiles><smiles>CC(C)=CCCc1ccccc1</smiles><smiles>C(Cc1ccccc1)=C(c1ccccc1)c1ccccc1</smiles><smiles>C#Cc1ccccc1</smiles>
263<smiles>O=C(O)c1ccccc1</smiles><smiles>O=S(=O)(O)c1ccc(C(Br)=C(Br)c2ccccc2)cc1</smiles>

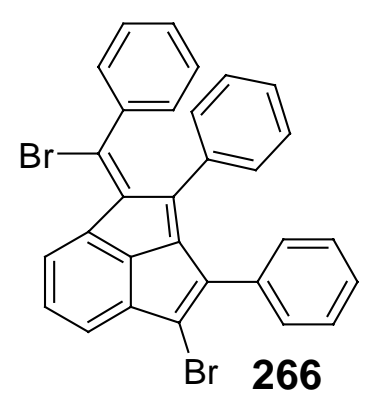<smiles></smiles><smiles>BrC(=C(Br)c1cccc2ccccc12)c1ccccc1</smiles>

267<smiles>Brc1c(-c2ccccc2)c2ccccc2c2ccccc12</smiles>

271<smiles>BrC1=C(c2ccccc2)c2cccc3cccc1c23</smiles>

268<smiles>BrC(=C(Br)c1cccc2cccc(C#Cc3ccccc3)c12)c1ccccc1</smiles>

269<smiles>BrC1=C(c2ccccc2)c2ccccc2CC1</smiles>

270<smiles>[R17]c1ccccc1-c1c(C#Cc2ccccc2)c2ccccc2c2ccccc12</smiles><smiles></smiles><smiles>[Z20]#CC1=C(CBr)C1=Cc1ccccc1</smiles> 
<smiles>O=[SH](=[W])c1ccccc1C#Cc1ccccc1C(Br)CBr</smiles><smiles>BrC1=C(c2ccccc2)C(C(Br)c2ccccc2)c2ccccc21</smiles><smiles></smiles><smiles>O=[R6]c1ccccc1/C(Br)=C(\Br)C(Br)=C1C(Br)=C(Br)c2ccccc21</smiles><smiles>CC1(C)c2ccccc2CCC1Br</smiles>
284<smiles>BrC(Br)=C(Br)C(Br)=C(Br)c1ccccc1</smiles>

281<smiles>BrC(=C1C(C2=C(Br)c3ccccc3C2=C(Br)c2ccccc2)=C(Br)c2ccccc21)c1ccccc1</smiles>

282<smiles>O=S(=O)(O)C1=C(c2ccccc2)C(=C2c3ccccc3-c3ccccc32)c2ccccc21</smiles><smiles>Brc1c(Br)c2c(Br)c3c(c(Br)c2c2ccccc12)-c1ccccc1-3</smiles><smiles>CC(Br)(Br)C(Br)CCc1ccccc1</smiles><smiles>BrC1CCc2ccccc2C1(c1ccccc1)c1ccccc1</smiles><smiles>BrC(CCc1ccccc1)=C(c1ccccc1)c1ccccc1</smiles>
286 287<smiles>C=CC(=C)c1c(Br)c2ccc3c(=C(Br)c4ccccc4)c(-c4ccccc4)c4c(-c5ccccc5)c1c2c43</smiles><smiles>BrC1=C(C#Cc2ccccc2)/C(=C(/Br)c2ccccc2)c2ccccc21</smiles>

290<smiles>C(CCC=C(c1ccccc1)c1ccccc1)=C(c1ccccc1)c1ccccc1</smiles>

292<smiles>BrC(CCC(Br)=C(c1ccccc1)c1ccccc1)=C(Br)c1ccccc1</smiles>

293<smiles>C#Cc1ccccc1C#C[AlH2]</smiles><smiles>O=S(=O)(Cl)C#Cc1ccccc1C(Br)=C([AlH2])Br</smiles> 


\subsection{Abkürzungen und Akronyme}

$\delta$

$\mu$

v

$\AA$

Abb.

abs.

äq

$\mathrm{au}$

ber.

BLYP

br. $\mathrm{s}$

bzw.

$\mathrm{C}$

CASSCF complete active space self consistent field; selbstkonsistentes Feld im vollständig aktiven Raum

$\mathrm{CC} \quad$ coupled cluster

$\mathrm{cc}$

$\mathrm{cm}^{-1}$

d bzw. $d$

DFT

$\mathrm{E}$

$\mathrm{e}$

FTIR

G

gef.

Gl.

$\mathrm{H}$

$\mathrm{h}$

$\mathrm{HF}$

HK

HOMO

$\mathrm{Hz}$

IR

IRC

$\mathrm{J}$

$\mathrm{k}$

K

Kat.

NMR-Skala der chemischen Verschiebung

Mikro

IR-Verschiebung in Wellenzahlen

Ångstrom

Abbildung

absolut

äquivalent

atomic unit; atomare (Massen)-Einheit

berechnet

Becke-Lee-Yang-Parr

breites Singulett

beziehungsweise

Celsius, Symmetriegruppe

correlation consistent; korrelationskonsistent

Wellenzahlen

Dublett, double; doppelt, deuterium, d-Orbitalfunktionen, days; Tage

density functional theory; Dichtefunktionaltheorie

Energie

Elektron, Elektronenladung, Eulersche-Konstante

Fourier transform infra red, Fourier-transformierte infra-rot

freie Gibbssche Enthalpie

Gefunden

Gleichung

freie Enthalpie

Stunde, Plancksche Konstante

Hartree-Fock

Hohenberg-Kohn

highest occupied molecular orbital; (energetisch) höchstes besetztes

Molekülorbital

Herz

Infrarot

intrinsic reaction coordinate; intrinsische Reaktionskoordinate

Joule

Kilo

Kelvin

Katalysator 
Konz. $\quad$ Konzentration

L Liter

Lit. $\quad$ Literatur

LUMO lowest unoccupied molecular orbital; (energetisch) niedrigstes unbesetztes Molekülorbital

m Multiplett

m Meter, meta, mili

$\min \quad$ Minute

MO molecular orbital; Molekülorbital

mol Einheit der Stoffmenge

MP2 second order Møller-Plesset perturbation theory; störungstheoretischer Ansatz zweiter Ordnung nach Møller-Plesset

n Normal

$\mathrm{p} \quad$ Polarisationsfunktionen, $\mathrm{p}$-Orbitalfunktionen

ppm parts per million

q Quartett

rev. Revision

$\mathrm{R}_{\mathrm{f}} \quad$ Retardierungsfaktor

RHF restricted Hartree-Fock; beschränkte Hartree-Fock

RT Raumtemperatur

s Singulett, Sekunde

SCF self consistent field; selbstkonsistentes Feld

Smp. Schmelzpunkt

sog. Sogenannt

t Triplett, triple; dreifach, Zeit

$\mathrm{T} \quad$ Temperatur

ÜZ Übergangszustand

UHF unrestricted Hartree-Fock; unbeschränkte Hartree-Fock

vs. versus; im Vergleich zu

VWN Vosko-Wilk-Nussair

Z Zeta $(\zeta)$

z. B. zum Beispiel

ZPVE zero point vibrational energy; Nullpunktsenergie 


\subsection{Publikationsliste}

(1) Stahl, F.; Moran, D.; Schleyer, P. v. R.; Prall, M.; Schreiner, P. R.; "Aromaticity of the Bergman, Myers-Saito, Schmittel, and directly related cyclizations of enediynes"; $J$. Org. Chem. 2002, 67 (5), 1453-1461.

(2) Prall, M.; Krüger, A.; Schreiner, P. R.; Hopf, H.; "The cyclization of parent and cyclic hexa-1,3-dien-5-ynes - A combined theoretical and experimental study"; Chem. Eur. J 2001, 7 (20), 4386-4394.

(3) Prall, M.; Wittkopp, A.; Schreiner, P. R.; "Can fulvenes form from enediynes? A systematic high-level computational study on parent and benzannelated enediyne and enyne-allene cyclizations"; J. Phys. Chem. A 2001, 105 (40), 9265-9274.

(4) Prall, M.; Wittkopp, A.; Fokin, A. A.; Schreiner, P. R.; "Substituent effects on the Bergman cyclization of (Z)-1,5-hexadiyne-3-enes: A systematic computational study"; J. Comput. Chem. 2001, 22 (13), 1605-1614.

(5) König, B.; Pitsch, W.; Klein, M.; Prall, M.; Schreiner, P. R.; "Carbonyl- and carboxylsubstituted enediynes: Synthesis, computations, and thermal reactivity"; J. Org. Chem. 2001, $66(5), 1742-1746$.

(6) Prall, M.; "Software news and comments - VMD: A graphical tool for the modern chemists"; J. Comput. Chem. 2001, 22 (1), 132-134.

(7) Wittkopp, A.; Prall, M.; Schreiner, P. R.; Schäfer III, H. F.; "Is $\mathrm{SH}_{4}$, the simplest 10-S4 sulfurane, observable?"; Phys. Chem. Chem. Phys. 2000, 2 (10), 2239-2244.

(8) Schreiner, P. R.; Prall, M.; "Myers-Saito versus $C^{2}-C^{6}$ ("Schmittel") cyclizations of parent and monocyclic enyne-allenes: Challenges to chemistry and computation"; $J$. Am. Chem. Soc. 1999, 121 (37), 8615-8627. 


\section{Danksagung}

Ich danke Herrn Prof. Dr. P. R. Schreiner für die Überlassung des Themas und seine fortwährende Unterstützung bei der Anfertigung dieser Arbeit. Weiterhin möchte ich Herrn Prof. Dr. A. Fokin für die Zusammenarbeit und die Einführung in die ukrainische Improvisationskunst danken. Mein Dank gilt weiterhin Herrn M. Noltemeyer für die Messung der Kristallstrukturen und Herrn O. Senge für seine Hilfe bei diversen Trennproblemen. Ganz besonderes bedanken möchte ich mich bei Dr. M. Maurer, der alle hochaufgelösten NMRSpektren angefertigt hat und mir auch sonst immer hilfreich zur Seite gestanden hat, und bei Dr. F. M. Gottschalk, der mich immer tatkräftig unterstützt hat. Den Doktoren Oliver Lauenstein und Alexander Wittkopp danke ich für die gute Zusammenarbeit, und daß sie mich auch nach Beendigung ihrer Promotionen nicht vergessen haben. Besonders Alexander danke ich für seine Freundschaft und die schöne, gemeinsame Zeit. Weiterhin möchte ich Prof. Dr. A. de Meijere für seine Unterstützung danken.

Als letztes möchte ich noch meiner Familie danken, die mir das Studium erst ermöglicht hat und ohne die ich nicht soweit gekommen wäre, und vor allem meiner Frau Antje, die mich mit Liebe und Geduld auf dem Weg durch das Studium begleitet hat. 


\section{Lebenslauf}

1971 Geboren am 07.09.1971 in Kassel

1978-1982 Besuch der Grundschule Brückenhof-Nordshausen in Kassel

1982-1988 Besuch der Mittelstufe an der Heinrich-Schütz-Schule in Kassel

1988-1991 Besuch der gymnasialen Oberstufenschule Jacob-Grimm-Schule in Kassel

1991 Erreichen der allgemeinen Hochschulreife (Note: 1.6)

1991-1992 Ableistung des Grundwehrdienstes

1992 Beginn des Studiums der Chemie an der Universität Gesamthochschule Kassel

1994 Diplomvorprüfung in Chemie mit sehr gut bestanden

1994-1997 Nebentätigkeit als Croupier für Black Jack und Poker in der Spielbank KasselWilhelmshöhe

1995 Dreimonatige berufspraktische Studien im chemischen Labor der Qualitätssicherung der AEG Hausgeräte AG in Kassel

1997 Im April Hochzeit mit der Zeitsoldatin Antje Prall, geb. Altenhofen

1997-1998 Diplomarbeit im AK von Prof. Dr. H. Frauenrath an der Universität Gesamthochschule Kassel zum Thema: Synthese und Epoxidierung von 4H-1,3Dioxinen: Vergleich mit der Reaktion von 5-Methyl-4H-1,3-Dioxinen. Diplomprüfung in Chemie mit sehr gut bestanden

1998 Beginn der Dissertation im AK von Prof. Dr. Peter R. Schreiner am Institut für organische Chemie der Universität Göttingen zum Thema: Cyclisierungsreaktionen mehrfach ungesättigter Systeme - Theorie und Experiment.

1999 Teilnahme an der Winter School on Organic Reactivity (WISOR VIII) in Brixen/Italien

2000-2002 Insgesamt vier Monate Forschungsaufenthalt an der University of Georgia in Athens/USA

2002 Promotionsprüfung in Chemie 